\title{
3D-Structure Clarifying Total Synthesis of the (Polyenoyl)tetramic Acid Militarinone B. A Highly Acid-Labile $N$-Protecting Group for Amides
}

\section{Christian Drescher ${ }^{\dagger}$, Reinhard Brückner* ${ }^{*}$,}

${ }^{\dagger}$ Institut für Organische Chemie

Albert-Ludwigs-Universität Freiburg

Albertstraße 21

79104 Freiburg im Breisgau

Germany

*reinhard.brueckner@organik.chemie.uni-freiburg.de 
Christian Drescher and Reinhard Brückner; 3D-Structure Clarifying Total Synthesis of the (Polyenoyl)tetramic Acid Militarinon B. A Highly Acid-Labile N-Protecting Group for Amides

\section{Table of Contents}

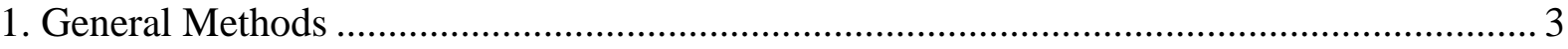

2. Graphical Index for the Experiments and Characterization Data...................................... 5

2.1 Synthesis of the Tetramic Acid (S)-17 Using die DMB-, $p$ NB-, DMNB- and TMB-

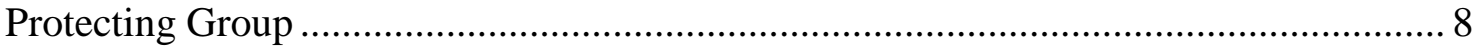

2.2 Synthesis of the two Diastereomers of the Western Block 10e ..................................53

2.3 Synthesis of the Tetramic Acid Militarinone B $[(S, S, R, R)-3]$ and its Epimer $(R, S, R, R)-3$

2.4 Chiral GLC Studies of the Enantiomeric Alcohol 8 and HPLC-Reports of the Tetramic Acid Militarinone B $[(S, S, R, R)-3]$ and its Epimer $(R, S, R, R)-3$

3. ${ }^{1} \mathrm{H}$ and ${ }^{13} \mathrm{C}$ NMR Spectra in Numerical Order

3.1 ${ }^{1} \mathrm{H}$ and ${ }^{13} \mathrm{C}$ NMR to the Synthesis of the Tetramic Acid $(S)$-17 Using die DMB-, $p$ NB-, DMNB- and TMB-Protecting Group.

$3.2{ }^{1} \mathrm{H}$ and ${ }^{13} \mathrm{C}$ NMR Spectra to the Synthesis of the two Western Blocks $(S, S)-10 \mathrm{e}$ and $(R, S)-10 \mathrm{e}$

$3.3{ }^{1} \mathrm{H}$ and ${ }^{13} \mathrm{C}$ NMR Spectra to the Synthesis of the Tetramic Acid Militarinone B $[(S, S, R, R)-\mathbf{3}]$ and its Epimer $(R, S, R, R)-\mathbf{3}$ 


\section{General Methods}

Working technique: All reactions not containing water were carried out under an $\mathrm{N}_{2}$-atmosphere. Reaction flasks were dried in an oven $\left(65^{\circ} \mathrm{C}\right)$ and in vacuo with a heat gun prior to use. Liquids were added with syringe and cannula through a rubber septum. Solids were added in a $\mathrm{N}_{2}$-counterflow. Reactions containing water were carried out without inert gas atmosphere. For reactions that require heating, it was used an oil bath.

Solvents: Tetrahydrofuran (THF) for reactions was distilled over potassium, diethyl ether $\left(\mathrm{Et}_{2} \mathrm{O}\right)$ over sodium-potassium alloy, and dichloromethane $\left(\mathrm{CH}_{2} \mathrm{Cl}_{2}\right)$ and triethyl amine $\left(\mathrm{NEt}_{3}\right)$ over $\mathrm{CaH}_{2}$ under an $\mathrm{N}_{2}$-atmosphere prior to use. Other solvents for that purpose were obtained commercially as "dry" or "extra dry" solvents and used without further purification. Cyclohexane $\left(c \mathrm{C}_{6} \mathrm{H}_{12}\right)$, ethyl acetate $(\mathrm{AcOEt})$, methanol $(\mathrm{MeOH})$, ethanol $(\mathrm{EtOH})$, dichloromethane $\left(\mathrm{CH}_{2} \mathrm{Cl}_{2}\right)$, and tert-butyl methyl ether $(t \mathrm{BuOMe})$ for workup and column chromatography were distilled using a rotary evaporator prior to use to remove high boiling fractions. Diethyl ether $\left(\mathrm{Et}_{2} \mathrm{O}\right)$, pentane, and chloroform $\left(\mathrm{CHCl}_{3}\right)$ for that purpose were obtained as p. a. grade solvents and used without further purification.

Organo-lithium reagents were stored in a refrigerator in Schlenk flasks with PTFE screw caps and PTFE valves and were titrated using $N$-pivaloyl-o-toluidine ${ }^{[1]}$ prior to use.

Chromatography: Thin layer chromatography (TLC) was used to monitor reactions and purification procedures. Merck silica plates with glass as supporting material (TLC Silicagel 60 $\mathrm{F}_{254}$ and TLC Silica gel 60 RP-18 F 254 ) were used. Chromatograms were, if applicable, marked in UV light at $254 \mathrm{~nm}$ and subsequently stained using one of the following stains: permanganate stain (0.65 g KMnO $\left.4,3.15 \mathrm{~g} \mathrm{~K}_{2} \mathrm{CO}_{3}, 125 \mathrm{~mL} \mathrm{H}_{2} \mathrm{O}\right)$ or cerium sulfate / molybdophosphoric acid (10 g Ce( $\left.\mathrm{SO}_{4}\right)_{2}, 25 \mathrm{~g}$ molybdophosphoric acid, $1 \mathrm{~L} \mathrm{H}_{2} \mathrm{O} ; 80 \mathrm{~mL}$ conc. $\mathrm{H}_{2} \mathrm{SO}_{4}$ ). Flash column chromatography ${ }^{[2]}$ was conducted on Macherey-Nagel \& Co silica gel $60^{\circledR}$ (230-400 mesh) and Sigma-Aldrich $\mathrm{C}_{18}$-Reversed phase silica gel. Chromatography conditions are documented at the respective experiment in the following manner: (solv1:solv2 $=\mathrm{a}: \mathrm{b} \stackrel{\mathrm{x}}{\longrightarrow} \mathrm{c}: \mathrm{d}, \mathrm{d} \times \mathrm{h} \mathrm{cm}$, $\mathrm{V} \mathrm{mL}$ ), which means: a column with the outer diameter $\mathrm{d} \mathrm{cm}$ is packed with $\mathrm{h} \mathrm{cm}$ silica gel. Fractions of the size $\mathrm{V} \mathrm{mL}$ are collected. The product is eluted with the solvents solv1 and solv2 in the ratio a:b. The ratio was changed at fraction $\mathrm{x}$ to $\mathrm{c}: \mathrm{d}$. 
Christian Drescher and Reinhard Brückner; 3D-Structure Clarifying Total Synthesis of the (Polyenoyl)tetramic Acid Militarinon B. A Highly Acid-Labile N-Protecting Group for Amides

Nuclear magnetic resonance spectroscopy: NMR spectra were recorded by Dr. M. Keller, F.

Reinbold, and M. Schonhard on a Bruker Avance 400 spectrometer $\left[{ }^{1} \mathrm{H}(400 \mathrm{MHz}),{ }^{13} \mathrm{C}\right.$ (100 MHz), DQF-COSY, edHSQC, and HMBC experiments] and a Bruker DRX 500 spectrometer $\left[{ }^{1} \mathrm{H}(500 \mathrm{MHz}),{ }^{13} \mathrm{C}(126 \mathrm{MHz}), \mathrm{DQF}-\mathrm{COSY}\right.$, edHSQC, and HMBC experiments] or by myself on a Varian Mercury VX 300 spectrometer $\left[{ }^{1} \mathrm{H}(300 \mathrm{MHz})\right]$ or on a Bruker Avance 300 spectrometer $\left[{ }^{1} \mathrm{H}(300 \mathrm{MHz})\right] .{ }^{1} \mathrm{H}$ NMR spectra were referenced internally to TMS or the solvent signal respectively $\left(\mathrm{CDCl}_{3}: 7.26 \mathrm{ppm}, \mathrm{C}_{6} \mathrm{D}_{6}: 7.16 \mathrm{ppm}, \mathrm{MeOD}: 3.30 \mathrm{ppm}, \mathrm{D}_{2} \mathrm{O}: 4.79\right.$ $\mathrm{ppm}) .{ }^{13} \mathrm{C}$ NMR spectra were referenced internally to the solvent signal $\left(\mathrm{CDCl}_{3}: 77.10 \mathrm{ppm}\right.$, $\left.\mathrm{C}_{6} \mathrm{D}_{6}: 128.00 \mathrm{ppm}, \mathrm{MeOD}: 49.00 \mathrm{ppm}\right) .{ }^{1} \mathrm{H}$ NMR data are reported as follows: chemical shift $\left(\delta\right.$ in $\mathrm{ppm}$ ), multiplicity ( $\mathrm{s}$ for singlet; $\mathrm{d}$ for doublet; $\mathrm{t}$ for triplet; $\mathrm{m}$ for multiplet; $\mathrm{m}_{\mathrm{c}}$ for symmetrical multiplet; br for broad signal), coupling constant(s) (Hz; ${ }^{3} J$ couplings unless otherwise noted), integral, and specific assignment. ${ }^{13} \mathrm{C}$ NMR data are reported in terms of chemical shift and assignment. For AB signals the high-field part was named A and the lowfield part B.

High resolution mass spectrometry: High resolution mass spectra were recorded by Dr. J. Wörth and C. Warth on a Thermo Scientific Exactive mass spectrometer equipped with an orbitrap (ion trap) analyzer. Ionization method: Electrospray ionization (ESI; spray voltage: 2.5-4.0 kV) or atmospheric pressure chemical ionization (APCI; spray current: $5 \mu \mathrm{A}$ ).

Melting points: Melting points were determined in a Büchi melting point apparatus using open glass capillaries.

IR spectroscopy: IR spectra were recorded on a Perkin Elmer Paragon 1000 FT-IR spectrometer for a film of the substance on a $\mathrm{NaCl}$ plate unless otherwise stated.

Specific rotations: Optical rotations were measured using a $341 \mathrm{MC}$ Perkin-Elmer polarimeter. The specific rotations $[\alpha]_{\lambda}^{\mathrm{T}}$ were calculated by the formula:

$$
[\alpha]_{\lambda}^{\mathrm{T}}=\frac{\alpha_{\text {observed }} \times 100}{c \times d}
$$

with $\mathrm{T}=$ temperature in ${ }^{\circ} \mathrm{C}, \alpha_{\text {observed }}=$ the experimentally observed optical rotation, $c=$ concentration in $\mathrm{g} / 100 \mathrm{~mL}, d=$ length of the cuvette in $\mathrm{dm}$. 
Christian Drescher and Reinhard Brückner; 3D-Structure Clarifying Total Synthesis of the (Polyenoyl)tetramic Acid Militarinon B. A Highly Acid-Labile N-Protecting Group for Amides

\section{Graphical Index for the Experiments and Characterization Data}

Content of Section 2.1 (pp. 8): Synthesis of the tetramic acid (S)-17 using die DMB-, $p$ NB-, DMNB- and TMB-protecting group:
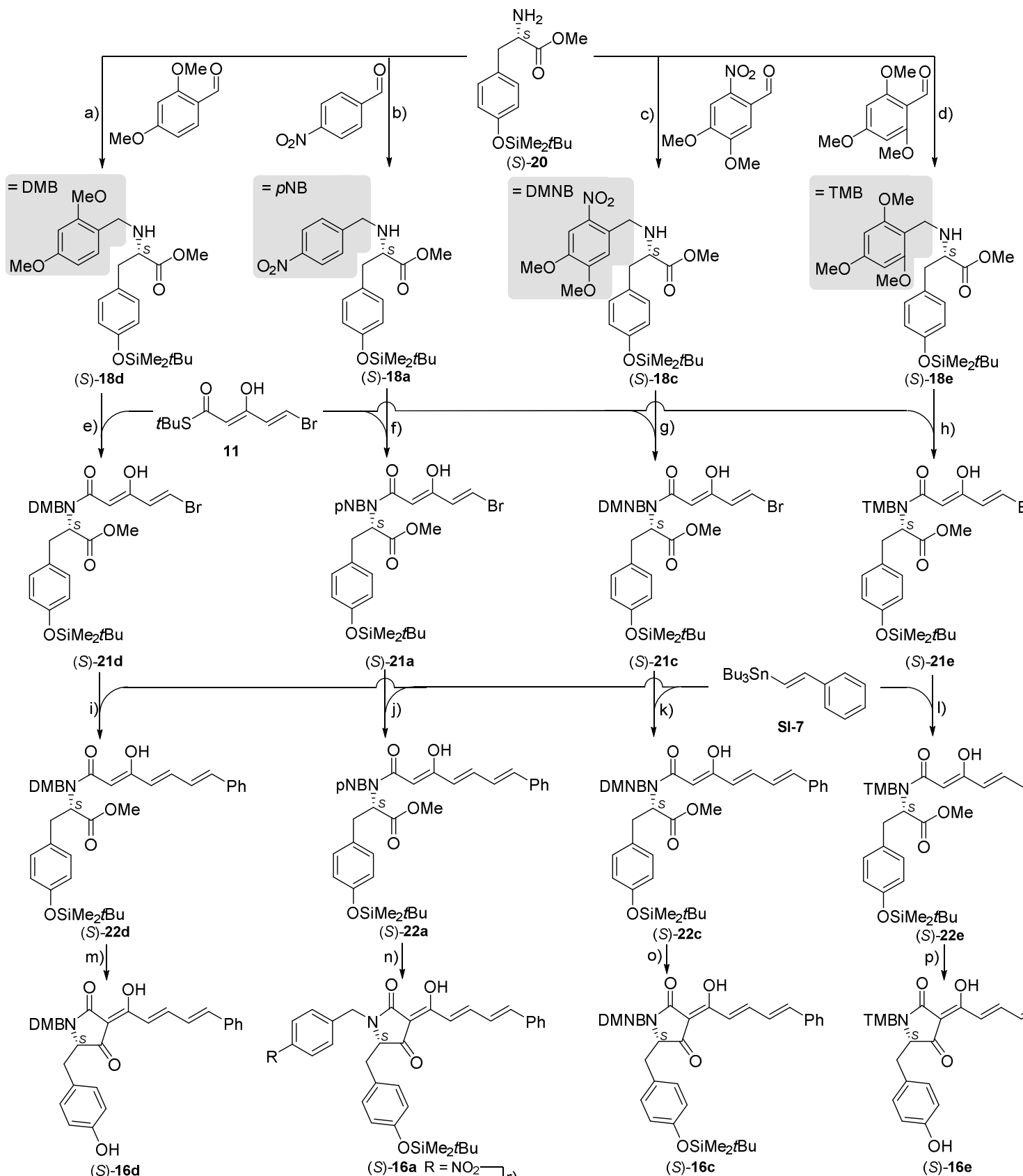
Christian Drescher and Reinhard Brückner; 3D-Structure Clarifying Total Synthesis of the (Polyenoyl)tetramic Acid Militarinon B. A Highly Acid-Labile N-Protecting Group for Amides

Content of Section 2.2 (pp. 53): Synthesis of the two diastereomers of the western block 10e:

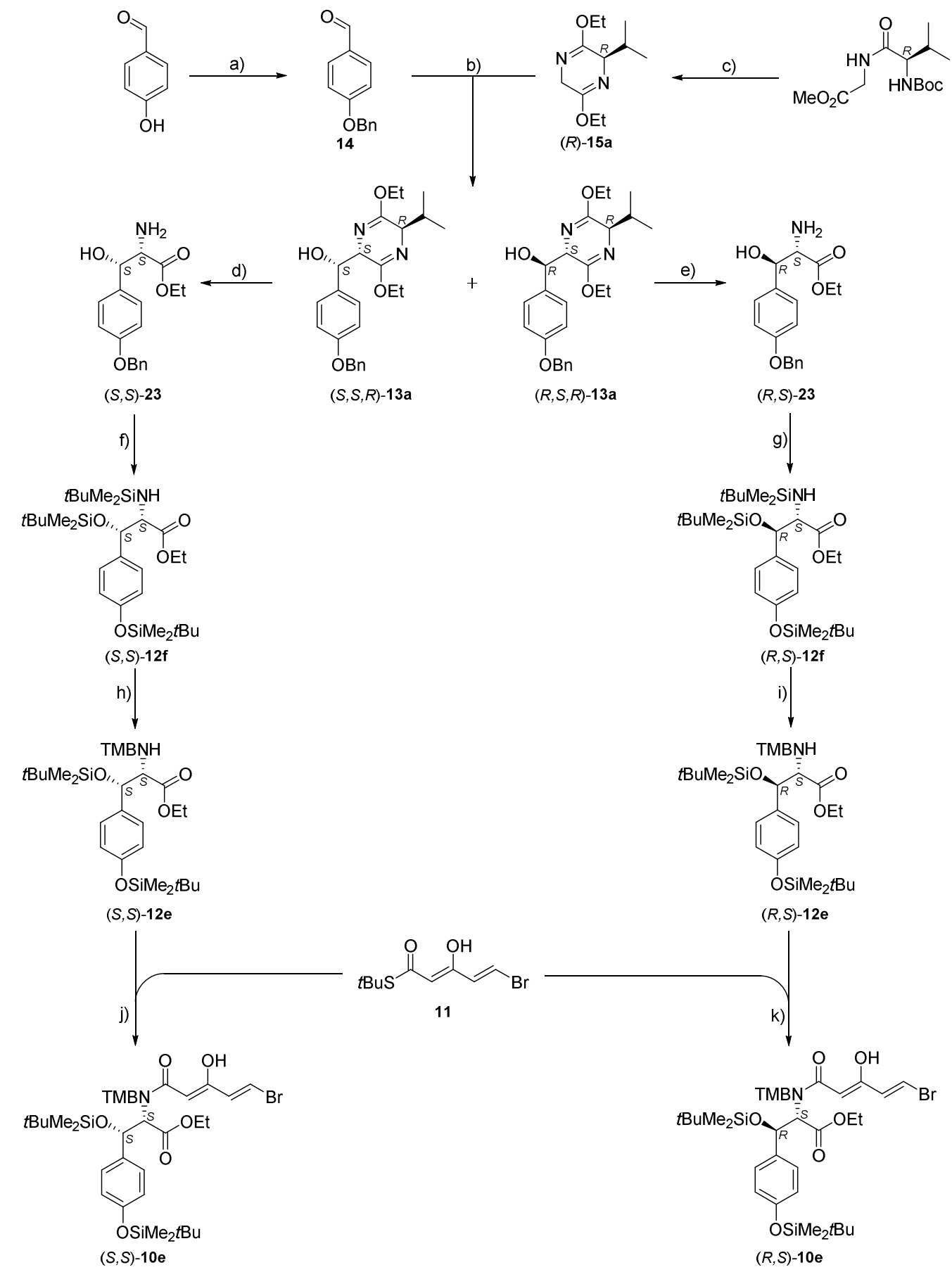


Christian Drescher and Reinhard Brückner; 3D-Structure Clarifying Total Synthesis of the (Polyenoyl)tetramic Acid Militarinon B. A Highly Acid-Labile N-Protecting Group for Amides

Content of Section 2.3 (pp. 73Fehler! Textmarke nicht definiert.): Synthesis of the Tetramic Acid Militarinone B [(S,S,R,R)-3] and its Epimer $(R, S, R, R)-3$ :
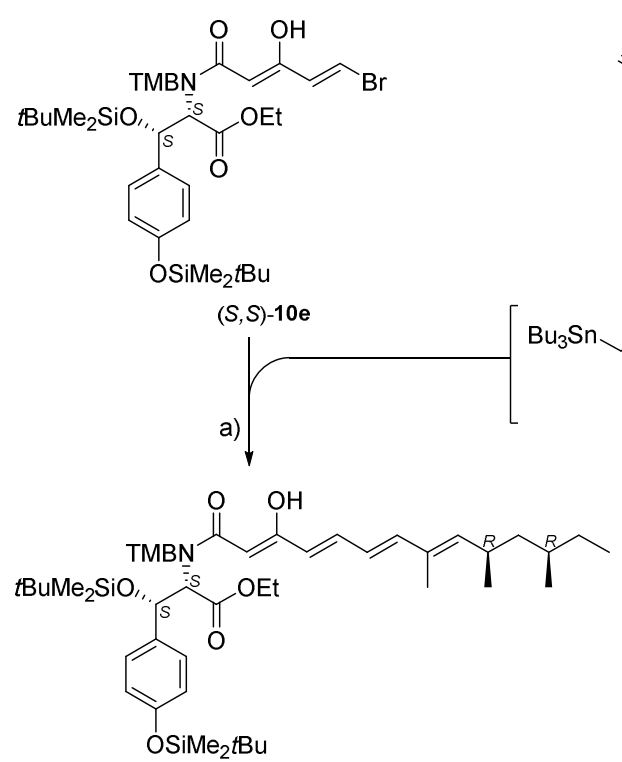

$(S, S, R, R)-\mathbf{2 6}$

C)

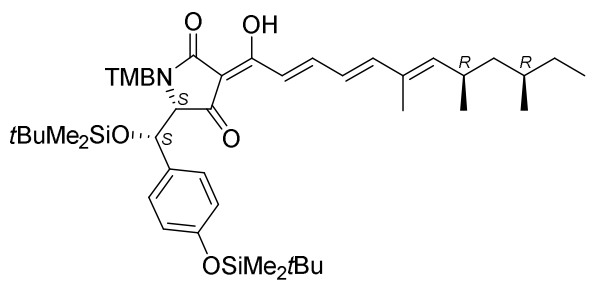

$(S, S, R, R)-27$

e)

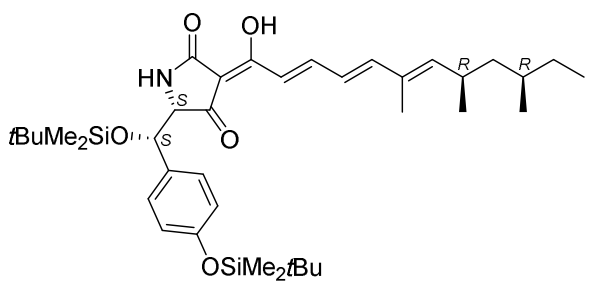

$(S, S, R, R)-\mathbf{2 8}$

g)

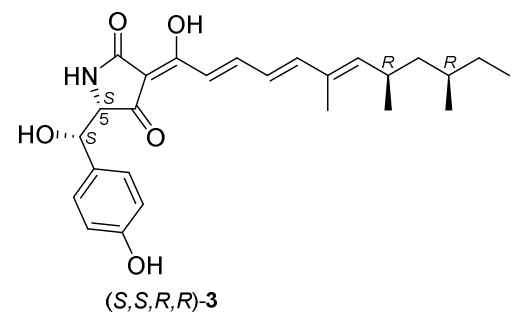

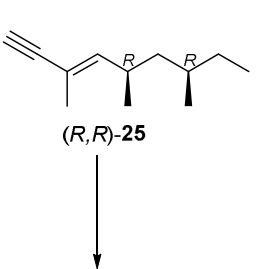

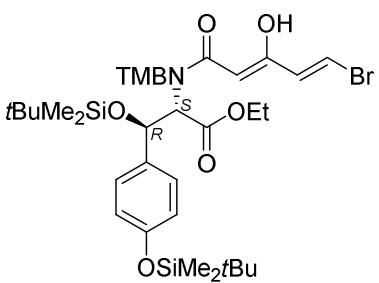

$(R, S)-10 \mathrm{e}$

$(R, R)-9$
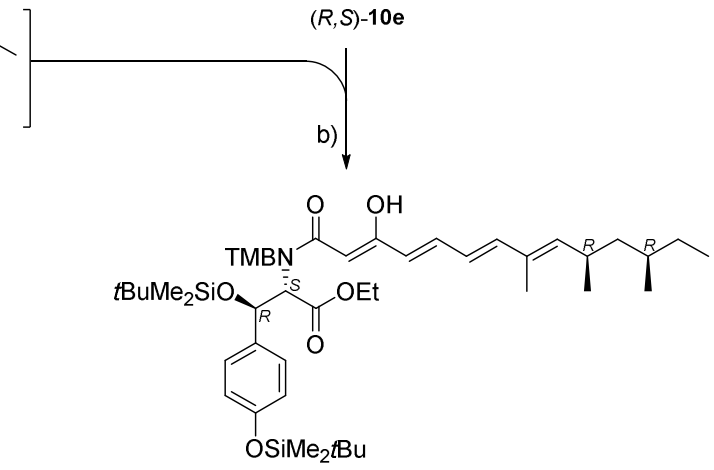

$(R, S, R, R)-26$

d)

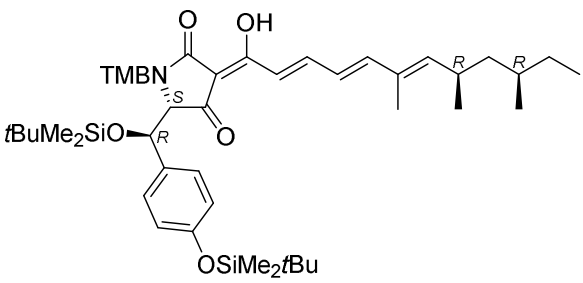

$(R, S, R, R)-27$

f)

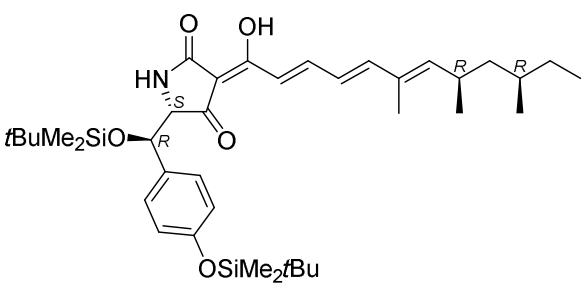

(R,S,R,R)-28

h)

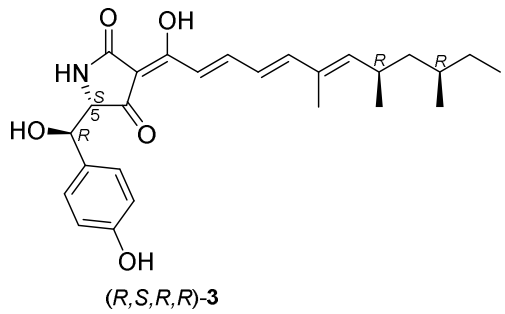




\title{
2.1 Synthesis of the Tetramic Acid (S)-17 Using die DMB-, $p$ NB-, DMNB- and TMB-Protecting Group
}

\author{
trans-3-Bromacrylic Acid (SI-1)
}<smiles>O=C(O)C=CBr</smiles>

\section{SI-1}

A mixture of propiolic acid $(6.02 \mathrm{~g}, 86.0 \mathrm{mmol})$ and aq. $\mathrm{HBr}$ solution $(8.9 \mathrm{M}, 35 \mathrm{~mL})$ was heated to reflux for $1.5 \mathrm{~h}$ and cooled to room temperature. Further cooling of the mixture to $0^{\circ} \mathrm{C}$ resulted in the formation of crystals, which were collected by filtration, washed with $\mathrm{H}_{2} \mathrm{O}(50$ $\mathrm{mL}$ ), and dried to leave the product SI-1 [9.67 g, 75\%, $64.5 \mathrm{mmol}$ (Lit. ${ }^{3}: 85 \%$ )] as brown needles.

$\mathbf{R}_{\mathbf{f}}\left(c \mathrm{C}_{6} \mathrm{H}_{12}: \mathrm{AcOEt}=4: 1\right)=0.35$

${ }^{1} \mathbf{H}$ NMR (300.13 MHz, $\left.\mathrm{CDCl}_{3}, \mathrm{DsBrAu} 18-30540,18.8 .2016\right): \delta=6.54\left(\mathrm{~d}, J_{2,3}=14.0 \mathrm{~Hz}, 1 \mathrm{H}\right.$, 2-H), $7.76\left(\mathrm{~d}, J_{3,2}=14.0 \mathrm{~Hz}, 1 \mathrm{H}, 3-\mathrm{H}\right), 8.56$ (br. s, 1H, 1-OH).

\section{$N$-Methylmethoxyamine (SI-2)}<smiles>CNOC</smiles>

\section{SI-2}

A solution of $N$-methylmethoxyamine hydrochloride $(6.05 \mathrm{~g}, 60.0 \mathrm{mmol})$ in 2-propanole: $\mathrm{H}_{2} \mathrm{O}$ $=(1: 1,20 \mathrm{~mL})$ was treated with $\mathrm{KOH}(85 \mathrm{wt} .-\%, 3.60 \mathrm{~g}, 54.5 \mathrm{mmol}, 0.9$ eq. $)$ at $-10^{\circ} \mathrm{C}$. The mixture has been distillated (bp. $1013 \mathrm{mbar}: 45-50^{\circ} \mathrm{C}$ ). The product $\left[1.52 \mathrm{~g}, 40 \%, 24.0\right.$ mmol (Lit. ${ }^{4}$ : $60 \%)$ ] was obtained as a colorless liquid.

\footnotetext{
${ }^{3}$ Y. Luo, I. D. Roy, A.G. E. Madec, H. W. Lam, Angew. Chem. Int. Ed. 2014, 53, 4186-4190.

${ }^{4}$ P. Beak, A. Basha, B. Kokko, D. Loo, J., Am. Chem. Soc. 1986, 108, 6016-6023.
} 
Christian Drescher and Reinhard Brückner; 3D-Structure Clarifying Total Synthesis of the (Polyenoyl)tetramic Acid Militarinon B. A Highly Acid-Labile N-Protecting Group for Amides

${ }^{1} \mathbf{H}$ NMR $\left(300.13 \mathrm{MHz}, \mathrm{CDCl}_{3} / \mathrm{CHCl}_{3}\right.$, sample contained 7 weight-\% of isopropanol with $\mathrm{d}$ at 1.20 and sep at 4.02, DsBrAp24-30930, 24.4.2018): $\delta=2.72$ (s, 3H, $\left.N-\mathrm{CH}_{3}\right), 3.53(\mathrm{~s}, 3 \mathrm{H}, O-$ $\mathrm{CH}_{3}$ ), 5.52 (br. s, $1 \mathrm{H}, N \mathrm{H}$ ).

\section{trans-3-Bromo- $N$-methoxy- $N$-methylacrylamide (SI-3)}

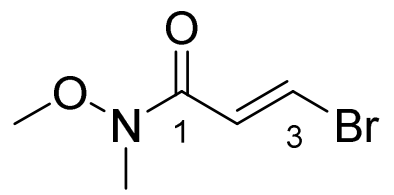

SI-3

At $0^{\circ} \mathrm{C}$ a solution of propylphosphoric acid anhydride in $\mathrm{CH}_{2} \mathrm{Cl}_{2}(54 \mathrm{wt} .-\%, 0.42 \mathrm{~mL}, 0.56 \mathrm{~g}$, $0.88 \mathrm{mmol}, 1.2$ eq.) was added dropwise to a solution of $N, O$-dimethylhydroxylamine SI-2 (60 $\mathrm{mg}, 0.88 \mathrm{mmol}, 1.2$ eq.), trans-3-bromoacrylic acid (SI-1) $(120 \mathrm{mg}, 0.80 \mathrm{mmol})$, and $\mathrm{N}$ methylmorpholine (180 $\mu \mathrm{L}, 160 \mathrm{mg} 1.60 \mathrm{mmol}, 2.0$ eq.) in $\mathrm{CH}_{2} \mathrm{Cl}_{2}(9 \mathrm{~mL})$. After $30 \mathrm{~min}$ the solution was diluted with $\mathrm{H}_{2} \mathrm{O}(3 \mathrm{~mL})$ und $\mathrm{HOAc}(\mathrm{pH}=3-4)$. The layers were separated, and the aq. layer was extracted with $\mathrm{CH}_{2} \mathrm{Cl}_{2}(3 \times 5 \mathrm{~mL})$. The combined organic layers were dried over $\mathrm{Na}_{2} \mathrm{SO}_{4}$ and concentrated under reduced pressure. Purification by flash chromatography (PE 30/50: $\mathrm{Et}_{2} \mathrm{O}=3: 1,1.5 \mathrm{~cm}, 10 \mathrm{~cm}, 8 \mathrm{~mL}$ ) afforded SI-3 [134 mg, 85\%, 0.68 mmol (Lit. ${ }^{5}$ : $86 \%)]$ as a pale brown oil.

$\mathbf{R}_{\mathbf{f}}\left(\mathrm{PE} 30 / 50: \mathrm{Et}_{2} \mathrm{O}=3: 1\right)=0.22$

${ }^{1} \mathbf{H}$ NMR (300.13 MHz, $\mathrm{CDCl}_{3} / \mathrm{CHCl}_{3}$, DsBrNo21-30560, 21.11.2019): $\delta=3.24$ (s, 3H, $N$ $\left.\mathrm{CH}_{3}\right), 3.72\left(\mathrm{~s}, 3 \mathrm{H}, O-\mathrm{CH}_{3}\right), 7.09\left(\mathrm{~d}, J_{2,3}=13.7 \mathrm{~Hz}, 1 \mathrm{H}, 2-\mathrm{H}\right), 7.58\left(\mathrm{~d}, J_{3,2}=13.7 \mathrm{~Hz}, 1 \mathrm{H}, 3-\mathrm{H}\right)$.

HRMS (ESI) m/z: [M+H] ${ }^{+}$Calcd for $\mathrm{C}_{5} \mathrm{H}_{8} \mathrm{BrNO}_{2}$ 193.9811; Found 193.9814

IR $(\mathrm{KBr}): \tilde{v}=3000,2950,2840,1735,1615,1590,1510,1465,1440,1420,1365,1290,1265$, $1210,1175,1160,1130,1080,1035,935,920,835,790,765,750,710,640 \mathrm{~cm}^{-1}$.

\footnotetext{
${ }^{5}$ M. L. Hofferberth, R. Brückner, Angew. Chem. 2014, 126, 7456-7462; Angew. Chem. Int. Ed. 2014, 53, 73287334.
} 
Christian Drescher and Reinhard Brückner; 3D-Structure Clarifying Total Synthesis of the (Polyenoyl)tetramic Acid Militarinon B. A Highly Acid-Labile N-Protecting Group for Amides

\section{$t$-Butylthioacetate (SI-4)}

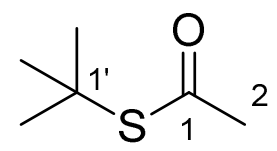

SI-4

Pyridine (2.89 g, $36.6 \mathrm{mmol}, 1.1$ eq.) was dissolved in chloroform $(13.3 \mathrm{~mL})$ at $0^{\circ} \mathrm{C}$. It was added $\mathrm{AcCl}\left(2.80 \mathrm{~g}, 36.6 \mathrm{mmol}, 1.1 \mathrm{eq}\right.$.). The mixture stirred for $30 \mathrm{~min}$ at $0^{\circ} \mathrm{C}$. $t \mathrm{BuSH}(3.00 \mathrm{~g}$, $33.3 \mathrm{mmol}$ ) was added over a period of $3 \mathrm{~h}$. The mixture was warmed to $25^{\circ} \mathrm{C}$ and stirred at this temperature for $12 \mathrm{~h}$. The mixture was diluted with $\mathrm{H}_{2} \mathrm{O}(15 \mathrm{~mL})$. The organic layer was separated and washed with $\mathrm{H}_{2} \mathrm{O}(10 \mathrm{~mL})$, aq. $\mathrm{H}_{2} \mathrm{SO}_{4}(10$ wt.- $\%, 10 \mathrm{~mL})$, sat. aq. $\mathrm{NaHCO}_{3}$ solution $(10 \mathrm{~mL})$ and again with $\mathrm{H}_{2} \mathrm{O}(10 \mathrm{~mL})$. The organic layer was tried over $\mathrm{K}_{2} \mathrm{CO}_{3}$ und the solvent was removed under reduced pressure. The residue (bp. $1013 \mathrm{mbar}: 130^{\circ} \mathrm{C}$ ) was distillated to get the product SI-4 [3.17 g, 72\%, $24.0 \mathrm{mmol}$ (Lit. ${ }^{6}: 81 \%$ )] as a pale yellow liquid.

${ }^{1} \mathbf{H}$ NMR (300.13 MHz, $\left.\mathrm{CDCl}_{3} / \mathrm{CHCl}_{3}, \mathrm{DsBrMz} 23-30370,23.3 .2015\right): \delta=1.46$ [s, 9H, 1'$\left.\left(\mathrm{CH}_{3}\right)_{3}\right], 2.23\left(\mathrm{~s}, 3 \mathrm{H}, 2-\mathrm{H}_{3}\right)$.

\section{S-tert-Butyl (E,E)-5-Bromo-3-hydroxypenta-2,4-dienethioate [enol-(11)]}

[pure (when crystallized)

or in an 85:15 mixture with the tautomeric]

S-tert-Butyl (E)-5-Bromo-3-oxopent-4-enethioate [keto-(11)]

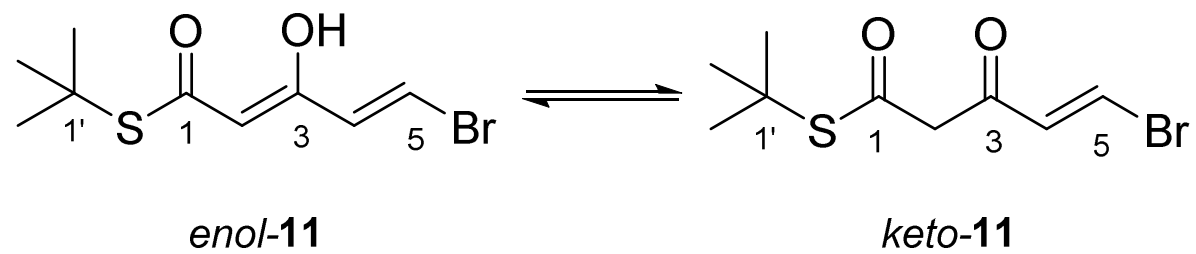

A solution of LiHMDS (1 M in hexane, $0.77 \mathrm{~mL}, 0.77 \mathrm{mmol}, 1.5$ eq.) in THF (1.2 mL) at $78^{\circ} \mathrm{C}$ was diluted with $t$ BuSAc (SI-4) $(99 \mathrm{mg}, 0.78 \mathrm{mmol}, 1.6$ eq.) in THF (0.9 mL). After $1 \mathrm{~h}$ $\mathrm{MgBr}_{2} \cdot \mathrm{OEt}_{2}$ (290 mg, $1.12 \mathrm{mmol}, 2.2$ eq.) was added in one portion. After $1 \mathrm{~h}$ a solution of Weinreb amide SI-3 (98 mg, $0.51 \mathrm{mmol})$ in THF $(0.9 \mathrm{~mL})$ was added tropwise. After $2 \mathrm{~h}$ the

${ }^{6}$ P. N. Rylander, D. S. Tarbe, J. Am. Chem. Soc., 1950, 72, 3021-3025. 
Christian Drescher and Reinhard Brückner; 3D-Structure Clarifying Total Synthesis of the (Polyenoyl)tetramic Acid Militarinon B. A Highly Acid-Labile N-Protecting Group for Amides

solution was diluted with $\mathrm{HOAc}(0.5 \mathrm{~mL})$ and sat. aq. $\mathrm{NH}_{4} \mathrm{Cl}$ solution $(3 \mathrm{~mL})$. The mixture was warmed to room temperature and extracted with $\mathrm{CH}_{2} \mathrm{Cl}_{2}(3 \times 10 \mathrm{~mL})$. The combined organic layers were dried over $\mathrm{Na}_{2} \mathrm{SO}_{4}$ and concentrated under reduced pressure. Purification by flash chromatography $\left(c \mathrm{C}_{6} \mathrm{H}_{12}: \mathrm{AcOEt}=75: 1,1.5 \mathrm{~cm}, 15 \mathrm{~cm}, 8 \mathrm{~mL}\right)$ afforded $11[101 \mathrm{mg}, 75 \%, 0.38$ mmol (Lit. $\left.{ }^{5}: 83 \%\right)$ ] as a red oil, which became solid (mp.: $\left.51^{\circ} \mathrm{C}\right)$ after $12 \mathrm{~h}$ at $-20^{\circ} \mathrm{C}$.

$\mathbf{R}_{\mathbf{f}}\left(c \mathrm{C}_{6} \mathrm{H}_{12}: \mathrm{AcOEt}=4: 1\right)=0.90$

${ }^{1} \mathbf{H}$ NMR $\left(300.13 \mathrm{MHz}, \mathrm{CDCl}_{3} / \mathrm{CHCl}_{3}\right.$, sample contained unknown impurity with a s at 1.46, DsBrNo26-30370, 26.11.2019): $\delta=1.48$ [s, 9H, 1'-( $\left.\mathrm{CH}_{3}\right)_{3}$ (keto-11)], 1.52 [s, 9H, 1'-( $\left(\mathrm{CH}_{3}\right)_{3}$ (enol-11)], 3.68 [s, 2H, 2- $\mathrm{H}_{2}$ (keto-11)], 5.32 [s, 1H, 2-H (enol-11)], 6.36 [dd, $J_{4,5}=13.6 \mathrm{~Hz}$, ${ }^{4} J_{4,3-\mathrm{OH}}=1.6 \mathrm{~Hz}, 1 \mathrm{H}, 4-\mathrm{H}($ enol-11) $], 6.90\left[\mathrm{~d}, J_{4,5}=14.0 \mathrm{~Hz}, 1 \mathrm{H}, 4-\mathrm{H}(\right.$ keto-11)], 7.27 [d, $J_{5,4}=13.6 \mathrm{~Hz}, 1 \mathrm{H}, 5-\mathrm{H}\left(\right.$ enol-11), $7.66\left[\mathrm{~d}, J_{5,4}=14.0 \mathrm{~Hz}, 1 \mathrm{H}, 5-\mathrm{H},(\right.$ keto-11) $], 12.44\left[\mathrm{~d},{ }^{4} J_{3-}\right.$ $\mathrm{OH}, 4=1.7 \mathrm{~Hz}, 1 \mathrm{H}, 3-\mathrm{OH}($ enol-11) $]$.

HRMS (ESI) m/z: [M-H] ${ }^{+}$Calcd for $\mathrm{C}_{9} \mathrm{H}_{12} \mathrm{O}_{2} \mathrm{BrS} 262.9747$; Found 262.9746

IR (KBr): $\tilde{v}=3085,2965,2925,2865,1625,1575,1495,1475,1455,1400,1365,1325,1285$, $1205,1170,1075,1025,985,930,860,820,760,700,685,610,530,510 \mathrm{~cm}^{-1}$.

\section{Methyl (2S)-Amino-3-(4'-hydroxyphenyl)propanoate [(S)-SI-5]}<smiles>COC(=O)[C@H](N)Cc1ccc(O)cc1</smiles>

(S)-SI-5

Thionyl chloride (4.00 mL, $6.55 \mathrm{~g}, 55.0 \mathrm{mmol}, 1.8 \mathrm{eq}$.) was added dropwise to a suspension of $L$-tyrosine $(5.45 \mathrm{~g}, 30.1 \mathrm{mmol})$ in $\mathrm{MeOH}(100 \mathrm{~mL})$ at $0^{\circ} \mathrm{C}$. The mixture was allowed to warm to $25^{\circ} \mathrm{C}$ and stirred overnight. The solvent was removed under reduced pressure and the residue 
Christian Drescher and Reinhard Brückner; 3D-Structure Clarifying Total Synthesis of the (Polyenoyl)tetramic Acid Militarinon B. A Highly Acid-Labile N-Protecting Group for Amides

was washed with $\mathrm{Et}_{2} \mathrm{O}(50 \mathrm{~mL})$. The product $(S)-\mathbf{S I - 5}$ [5.58 g, 95\%, 28.6 mmol (Lit. $\left.{ }^{7}: 99 \%\right)$ ] was obtained as a white solid.

${ }^{1} \mathbf{H}-\mathbf{N M R}\left(300.13 \mathrm{MHz}, \mathrm{D}_{2} \mathrm{O} / \mathrm{H}_{2} \mathrm{O}\right.$, DsBrAp15-30500, 15.4.2015): $\delta=\mathrm{AB}$ signal $\left(\delta_{\mathrm{A}}=3.21\right.$, $\delta_{\mathrm{B}}=3.30, J_{\mathrm{AB}}=14.6 \mathrm{~Hz}$, in addition split by $\left.J_{3-\mathrm{H}^{\mathrm{A}}, 2}=5.8 \mathrm{~Hz}, J_{3-\mathrm{H}^{\mathrm{B}}, 2}=7.5 \mathrm{~Hz}, 2 \mathrm{H}, 3-\mathrm{H}_{2}\right), 3.87$ $\left(\mathrm{s}, 3 \mathrm{H}, 1-\mathrm{OCH}_{3}\right), 4.41\left(\mathrm{dd}, J_{2,3-\mathrm{H}^{\mathrm{A}}}=6.0 \mathrm{~Hz}, J_{2,3-\mathrm{H}^{\mathrm{B}}}=7.4 \mathrm{~Hz}, 1 \mathrm{H}, 2-\mathrm{H}\right), 6.93\left(\mathrm{~m}, J_{3^{\prime}, 5^{\prime}, 2^{\prime}, 6^{\prime}}=8.7 \mathrm{~Hz}\right.$,

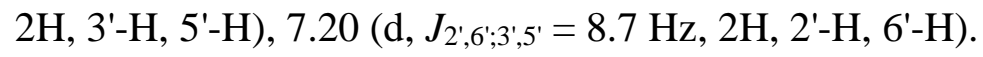

Specific rotation $(S)-273[a]_{D}^{20}=+13.1^{\circ}\left(\mathrm{c}=1.0\right.$ in $\left.\mathrm{H}_{2} \mathrm{O}\right)$.

\section{Methyl (2S)-2-Amino-3-\{4'-[(tert-butyldimethylsilyl)oxy]phenyl $\}$ propanoate $[(S)-20]$}<smiles>COC(=O)[C@H](N)Cc1ccc(O[Si](C)(C)C(C)(C)C)cc1</smiles>

(S)-20

A suspension of $L$-tyrosine methyl ester $(S)$-SI-5 (500 mg, $2.55 \mathrm{mmol})$ in $\mathrm{CH}_{2} \mathrm{Cl}_{2}(25 \mathrm{~mL})$ was treated with imidazole (438 mg, $6.34 \mathrm{mmol}, 2.5$ eq.) and $t \mathrm{BuMe}_{2} \mathrm{SiCl}$ (461 mg, $3.06 \mathrm{mmol}, 1.2$ eq.). The mixture stirred at $25^{\circ} \mathrm{C}$ for $14 \mathrm{~h}$ before being quenched with sat. aq. $\mathrm{NH}_{4} \mathrm{Cl}$ solution (25 mL). The layers were separated, and aq. layer was extracted with $\mathrm{CH}_{2} \mathrm{Cl}_{2}(3 \times 15 \mathrm{~mL})$. The combined organic layers were dried over $\mathrm{MgSO}_{4}$ and concentrated under reduced pressure. Purification by flash chromatography (AcOEt, $2.5 \mathrm{~cm}, 15 \mathrm{~cm}, 20 \mathrm{~mL}$ ) afforded $(S)-\mathbf{2 0}[667 \mathrm{mg}$, $84 \%, 2.14$ mmol (Lit. $\left.{ }^{8}: 87 \%\right)$ ] as a colorless oil.

$\mathbf{R}_{\mathbf{f}}(\mathrm{AcOEt})=0.45$

${ }^{1} \mathbf{H}-\mathbf{N M R}\left(300.13 \mathrm{MHz}, \mathrm{CDCl}_{3} / \mathrm{CHCl}_{3}\right.$, DsBrNo21-30550, 21.11.2019): $\delta=0.18$ [s, 6H, Si$\left(\mathrm{CH}_{3}\right)_{2}$ ], 0.97 [s, 9H, Si-C(CH$)_{3}$ ], 1.60 (br. s, $\left.2 \mathrm{H}, 2-\mathrm{NH}_{2}\right), \mathrm{AB}$ signal $\left(\delta_{\mathrm{A}}=2.81, \delta_{\mathrm{B}}=3.00\right.$,

\footnotetext{
${ }^{7}$ R. Liu, H. Sogawa, M. Shiotsuki, T. Masuda, F. Sanda, Polymer 2010, 51, 2255-2263.

${ }^{8}$ L. Raffier, O. Piva, Beilstein J. Org. Chem. 2011, 7, 151-155.
} 
Christian Drescher and Reinhard Brückner; 3D-Structure Clarifying Total Synthesis of the (Polyenoyl)tetramic Acid Militarinon B. A Highly Acid-Labile N-Protecting Group for Amides

$J_{\mathrm{AB}}=13.6 \mathrm{~Hz}$, in addition split by $\left.J_{3-\mathrm{H}^{\mathrm{A}}, 2}=5.4 \mathrm{~Hz}, J_{3-\mathrm{H}^{\mathrm{B}}, 2}=7.8 \mathrm{~Hz}, 2 \mathrm{H}, 3-\mathrm{H}_{2}\right), 3.69\left(\mathrm{dd}, J_{2,3-\mathrm{H}^{\mathrm{B}}}\right.$ $\left.=5.3 \mathrm{~Hz}, J_{2,3-\mathrm{H}^{\mathrm{A}}}=7.8 \mathrm{~Hz}, 1 \mathrm{H}, 2-\mathrm{H}\right), 3.70\left(\mathrm{~s}, 3 \mathrm{H}, 1-\mathrm{OCH}_{3}\right), 6.77\left(\mathrm{~d}, J_{3^{\prime}, 5^{\prime} ; 2^{\prime}, 6^{\prime}}=8.7 \mathrm{~Hz}, 2 \mathrm{H}, 3^{\prime}-\mathrm{H}\right.$, 5'-H), $7.03\left(\mathrm{~d}, J_{2^{\prime}, 6^{\prime} ; 3^{\prime}, 5^{\prime}}=8.6 \mathrm{~Hz}, 2 \mathrm{H}, 2^{\prime}-\mathrm{H}, 6^{\prime}-\mathrm{H}\right)$.

Specific rotation $(S)-20[a]_{D}^{20}=+4.9^{\circ}\left(\mathrm{c}=1.0\right.$ in $\left.\mathrm{CHCl}_{3}\right)$.

\section{Methyl (2S)-3-\{4'-[(tert-Butyldimethylsilyl)oxy]phenyl $\}-2-[(1 ", 5 "-$ dimethoxybenzyl)amino]propanoate $[(S)-18 d]$}<smiles>COC(=O)[C@H](Cc1ccc(O[Si](C)(C)C(C)(C)C)cc1)NCc1ccc(OC)cc1OC</smiles>

(S)-18d

The amine $(S)$-20 (250 mg, $0.80 \mathrm{mmol})$ was dissolved in $\mathrm{MeOH}(7.5 \mathrm{~mL})$. It was added HOAc (5 drops), 2,4-dimethoxybenzaldehyde (135 mg, $0.88 \mathrm{mmol}, 1.1$ eq.) and immediately (not more than $10 \mathrm{sec}) \mathrm{NaBH}_{3} \mathrm{CN}$ (79 mg, $1.20 \mathrm{mmol}, 1.5 \mathrm{eq}$.). The mixture was stirred at $25^{\circ} \mathrm{C}$ for $2.25 \mathrm{~h}$. The solvent was removed under reduced pressure. It was added $\mathrm{H}_{2} \mathrm{O}(5 \mathrm{~mL})$ and $\mathrm{Et}_{2} \mathrm{O}$ $(10 \mathrm{~mL})$. The layers were separated, and aq. layer was extracted with $\mathrm{Et}_{2} \mathrm{O}(3 \times 15 \mathrm{~mL})$. The combined organic layers were dried over $\mathrm{MgSO}_{4}$ and concentrated under reduced pressure. Purification by flash chromatography $\left(c \mathrm{C}_{6} \mathrm{H}_{12}: \mathrm{AcOEt}=2: 1,3.0 \mathrm{~cm}, 20 \mathrm{~cm}, 20 \mathrm{~mL}\right)$ afforded (S)-18d (260 mg, 70\%, $0.56 \mathrm{mmol}, 99.5 \%$ ee ) as a colorless oil.

$\mathbf{R}_{\mathbf{f}}\left(c \mathrm{C}_{6} \mathrm{H}_{12}: \mathrm{AcOEt}=2: 1\right)=0.35$

${ }^{1} \mathbf{H}-\mathbf{N M R}\left(500.10 \mathrm{MHz}, \mathrm{CDCl}_{3} / \mathrm{CHCl}_{3}\right.$, DsBrNo07-31160, 28.11.2019): $\delta=0.18$ [s, 6H, Si$\left.\left(\mathrm{CH}_{3}\right)_{2}\right], 0.97$ [s, 9H, Si-C $\left.\left(\mathrm{CH}_{3}\right)_{3}\right], 1.97$ (br. s, $\left.1 \mathrm{H}, N \mathrm{H}\right), \mathrm{AB}$ signal $\left(\delta_{\mathrm{A}}=2.85, \delta_{\mathrm{B}}=2.89\right.$, $J_{\mathrm{AB}}=13.4 \mathrm{~Hz}$, in addition split by $\left.J_{3-\mathrm{H}^{\mathrm{A}}, 2}=6.6 \mathrm{~Hz}, J_{3-\mathrm{H}^{\mathrm{B}}, 2}=7.6 \mathrm{~Hz}, 2 \mathrm{H}, 3-\mathrm{H}_{2}\right), 3.45(\mathrm{t}$, $\left.J_{2,3}=6.7 \mathrm{~Hz}, 1 \mathrm{H}, 2-\mathrm{H}\right), 3.59\left(\mathrm{~s}, 3 \mathrm{H}, 1-\mathrm{OCH}_{3}\right), \mathrm{AB}$ signal $\left(\delta_{\mathrm{A}}=3.61, \delta_{\mathrm{B}}=3.72, J_{\mathrm{AB}}=13.4 \mathrm{~Hz}\right.$, $\left.2 \mathrm{H}, \mathrm{N}-\mathrm{CH}_{2}\right), 3.68$ (s, 3H, 1"-- $\left.\mathrm{OCH}_{3}\right), 3.78$ (s, 3H, 5"--OCH $), 6.35-6.41$ (m, 2H, 4"-H, 6"-H), 6.67-6.76 (m, 2H, 3'-H, 5'-H), 6.94-7.05 (m, 1H, 3'-H), 6.94-7.05 (m, 2H, 2'-H, 6'-H). 
Christian Drescher and Reinhard Brückner; 3D-Structure Clarifying Total Synthesis of the (Polyenoyl)tetramic Acid Militarinon B. A Highly Acid-Labile N-Protecting Group for Amides

${ }^{13} \mathrm{C}-\mathrm{NMR}\left(125.75 \mathrm{MHz}, \mathrm{CDCl}_{3} / \mathrm{CHCl}_{3}\right.$, DsBrMi21-5076, 28.11.2019): $\delta=-4.4$ [Si-( $\left.\left(\mathrm{CH}_{3}\right)_{2}\right]$, $18.3\left[\mathrm{Si}-\mathrm{C}\left(\mathrm{CH}_{3}\right)_{3}\right], 25.8\left[\mathrm{Si}-\mathrm{C}\left(\mathrm{CH}_{3}\right)_{3}\right], 39.0(\mathrm{C}-3), 47.3\left(\mathrm{~N}-\mathrm{CH}_{2}\right), 51.6\left(1-\mathrm{OCH}_{3}\right), 55.2(1 "-$ $\mathrm{OCH}_{3}$ ), 55.4 (5"-OCH 3 ), 62.3 (C-2), 98.3 (C-4"), 103.9 (C-6"), 120.0 (C-3', C-5'), 120.2 (C-2"), 130.2 (C-1', C-2', C-6'), 130.4 (C-3"), 154.4 (C-4'), 158.7 (C-1"), 160.2 (C-5"), 175.1 (C-1).

edHSQC [,,short-range H,C-COSY“, 500.32 MHz/125.80 MHz, $\mathrm{CDCl}_{3} ; \delta\left({ }^{1} \mathrm{H}\right) \leftrightarrow \delta\left({ }^{13} \mathrm{C}\right): 0.18$ $\left[\mathrm{s}, 6 \mathrm{H}, \mathrm{Si}-\left(\mathrm{CH}_{3}\right)_{2}\right] \leftrightarrow-4.4\left[\mathrm{Si}-\left(\mathrm{CH}_{3}\right)_{2}\right], 0.98\left[\mathrm{~s}, \mathrm{Si}-\mathrm{C}\left(\mathrm{CH}_{3}\right)_{3}\right] \leftrightarrow 25.8\left[\mathrm{Si}-\mathrm{C}_{(}\left(\mathrm{CH}_{3}\right)_{3}\right], \mathrm{AB}$ signal $\left(\delta_{\mathrm{A}}=2.85, \delta_{\mathrm{B}}=2.89,3-\mathrm{H}_{2}\right) \leftrightarrow 39.0(\mathrm{C}-3), 3.45(\mathrm{t}, 2-\mathrm{H}) \leftrightarrow 62.3(\mathrm{C}-2), 3.59\left(\mathrm{~s}, 3 \mathrm{H}, 1-\mathrm{OCH}_{3}\right) \leftrightarrow$ $51.6\left(1-\mathrm{OCH}_{3}\right), \mathrm{AB}$ signal $\left(\delta_{\mathrm{A}}=3.61, \delta_{\mathrm{B}}=3.72, N-\mathrm{CH}_{2}\right) \leftrightarrow 47.3\left(N-\mathrm{CH}_{2}\right), 3.68\left(\mathrm{~s}, 1 "-\mathrm{OCH}_{3}\right)$ $\leftrightarrow 55.20\left(1 "-\mathrm{OCH}_{3}\right), 3.78\left(\mathrm{~s}, 5 "-\mathrm{OCH}_{3}\right) \leftrightarrow 55.40\left(5 "-\mathrm{OCH}_{3}\right), 6.35-6.41(\mathrm{~m}, 4$ "-H) ↔ $98.3(\mathrm{C}-$ 4"), 6.35-6.41 (m, 6"-H) 103.9 (C-6"), 6.67-6.76 (m, 3'-H, 5'-H) 120.0 (C-3', C-5'), 6.94$7.05\left(\mathrm{~m}, 3^{\prime \prime}-\mathrm{H}\right) \leftrightarrow 130.4\left(\mathrm{C}-3^{\prime \prime}\right), 6.94-7.05\left(\mathrm{~m}, 2^{\prime}-\mathrm{H}, 6^{\prime}-\mathrm{H}\right) \leftrightarrow 130.2$ (C-2', C-6').

Specific rotation $(S)-\mathbf{1 8 d}[a]_{D}^{20}=+14.9^{\circ}\left(\mathrm{c}=1.0\right.$ in $\left.\mathrm{CHCl}_{3}\right)$.

HRMS: (EI, $70 \mathrm{eV}): \mathrm{m} / \mathrm{z}=460.2517[\mathrm{M}+\mathrm{H}]^{+}$, corresponds to the molecular formula $\mathrm{C}_{25} \mathrm{H}_{38} \mathrm{O}_{5} \mathrm{NSi}(460.2514)\left\{[\mathrm{M}+\mathrm{H}]^{+}\right\}$with a deviation of $0.8 \mathrm{ppm}$.

IR (KBr): $\tilde{v}=3000,2955,2930,2860,1730,1610,1590,1510,1465,1440,1290,1260,1210$, $1155,1130,1040,915,840,780,690 \mathrm{~cm}^{-1}$.

Methyl (2'S)-2'-\{N-[(1Z,2Z,4E)-5-Bromo-3-hydroxy-1-oxoypenta-2,4-dienyl]- $N$-(1"',5"'dimethoxybenzyl) $\}-3$ '- $\left\{1^{\prime \prime}-[(\right.$ tert-butyldimethylsilyl)oxy $]$ phenyl $\}$ propanoate $[Z-e n o l-(S)$ 21d]

in equilibration with

Methyl (2'S)-2'-\{N-[(1E,2Z,4E)-5-Bromo-3-hydroxy-1-oxoypenta-2,4-dienyl]- $N$-(1"',5'"dimethoxybenzyl $)\}-3^{\prime}-\left\{1^{\prime \prime}-[(\right.$ tert-butyldimethylsilyl $)$ oxy $]$ phenyl $\}$ propanoate $[$ E-enol-(S)21d]

in equilibration with

Methyl (2'S)-2'-\{N-[(1Z,4E)-5-Bromo-1,3-dioxopent-4-enyl]- $N-\left(1{ }^{\prime \prime \prime}, 5\right.$ '"dimethoxybenzyl) $\}-3$ '- $\left\{\right.$ 1" $^{\prime \prime}-[($ tert-butyldimethylsilyl)oxy $]$ phenyl $\}$ propanoate $[$ Z-keto-(S)21d]

in equilibration with 
<smiles></smiles>

Z-enol-(S)-21d<smiles>C=CC=C</smiles><smiles>COC(=O)[C@H](Cc1ccc(O[Si](C)(C)C(C)(C)C)cc1)N(Cc1ccc(OC)cc1[O-])C(=O)CC(=O)/C=C/Br</smiles>

Z-keto-(S)-21d<smiles>COC(=O)[C@H](Cc1ccc(O[Si](C)(C)C(C)(C)C)cc1)N(Cc1ccc(OC)cc1OC)C(=O)/C=C(O)/C=C/Br</smiles>

E-enol-(S)-21d<smiles>[CH]=[IH]</smiles><smiles></smiles>

E-keto-(S)-21d

A solution of $N$-DMB-tyrosine methyl ester $(S)$-18d $(73.0 \mathrm{mg}, 220 \mu \mathrm{mol})$ and the $\beta$ ketothioester 11 (66.0 mg, $330 \mu \mathrm{mol}, 1.5$ eq.) in THF $(1.9 \mathrm{~mL})$ was treated with powdered molecular sieves $4 \AA(0.4 \mathrm{~g})$. The suspension stirred at $25^{\circ} \mathrm{C}$ for $30 \mathrm{~min}$. Then $\mathrm{AgO}_{2} \mathrm{CCF}_{3}(65.1$ $\mathrm{mg}, 330 \mu \mathrm{mol}, 1.5$ eq.) was added and the mixture was stirred for $20 \mathrm{~h}$ at $25^{\circ} \mathrm{C}$. The solvent was removed under reduced pressure and $\mathrm{Et}_{2} \mathrm{O}(10 \mathrm{~mL})$ was added to the resulting suspension. The mixture was filtered, and the filtrate was concentrated under reduced pressure. Purification by flash chromatography $\left(c \mathrm{C}_{6} \mathrm{H}_{12}\right.$ :AcOEt $\left.=5: 1,2.0 \mathrm{~cm}, 20 \mathrm{~cm}, 15 \mathrm{~mL}\right)$ afforded $(S)-\mathbf{2 1 d}$ $(65.2 \mathrm{mg}, 65 \%, 141 \mu \mathrm{mol}, 99 \% e e)$ as a red oil. It represented a mixture of two rotamers in a keto and an enol form each [Z-enol-(S)-21d:E-enol-(S)-21d:Z-keto-(S)-21d:E-keto-(S)21d $=84: 12: 2: 1 .^{9}{ }^{9}$ This ratio was determined by integrating the 5 -H signals [at $\delta=7.18(Z$ -

\footnotetext{
${ }^{9}$ That the main compound is Z-configured was determined by means of a NOE experiment.
} 
Christian Drescher and Reinhard Brückner; 3D-Structure Clarifying Total Synthesis of the (Polyenoyl)tetramic Acid Militarinon B. A Highly Acid-Labile N-Protecting Group for Amides

enol), 7.53 (E-keto), $7.60($ Z-keto $)$ and $7.68($ E-enol $) \mathrm{ppm}$ ] and compared with the 3-OH signals [14.00 (Z-enol) and $14.08($ E-enol)].

$\mathbf{R}_{\mathbf{f}}\left(c \mathrm{C}_{6} \mathrm{H}_{12}: \mathrm{AcOEt}=5: 1\right)=0.38$

Z-enol-(S)-21d:

${ }^{1} \mathbf{H}$-NMR $\left(500.10 \mathrm{MHz}, \mathrm{CDCl}_{3} / \mathrm{CHCl}_{3}\right.$, sample contained grease, $\mathrm{H}_{2} \mathrm{O}$, trace amounts of AcOEt and unknown impurity $\mathrm{m}$ at 5.05 and dd at 6.08, DsBrMi21-5070, 26.8.2015): $\delta=0.18[\mathrm{~s}, 6 \mathrm{H}$, $\left.\mathrm{Si}-\left(\mathrm{CH}_{3}\right)_{2}\right], 0.98$ [s, 9H, Si-C $\left.\left(\mathrm{CH}_{3}\right)_{3}\right]$, $\mathrm{AB}$ signal $\left(\delta_{\mathrm{A}}=3.12, \delta_{\mathrm{B}}=3.29, J_{\mathrm{AB}}=14.1 \mathrm{~Hz}\right.$, in addition split by $\left.J_{3^{\prime}-\mathrm{H}^{\mathrm{A}}, 2^{\prime}}=6.0 \mathrm{~Hz}, J_{3^{\prime}-\mathrm{H}^{\mathrm{B}}, 2^{\prime}}=9.0 \mathrm{~Hz}, 2 \mathrm{H}, 3^{\prime}-\mathrm{H}_{2}\right), 3.61$ (s, 3H, $\left.1^{\prime}-\mathrm{OCH}_{3}\right), 3.75$ (s, 3H, 1'"$\left.\mathrm{OCH}_{3}\right), 3.79(\mathrm{~s}, 3 \mathrm{H}, 5$ "'-OCH $), 3.90\left(\mathrm{~d},{ }^{2} J=17.2 \mathrm{~Hz}, 1 \mathrm{H}, 2^{\prime \prime}-\mathrm{C}-\mathrm{H}^{\mathrm{A}}\right), 4.32\left(\mathrm{~d},{ }^{2} J=17.2 \mathrm{~Hz}, 1 \mathrm{H}\right.$,

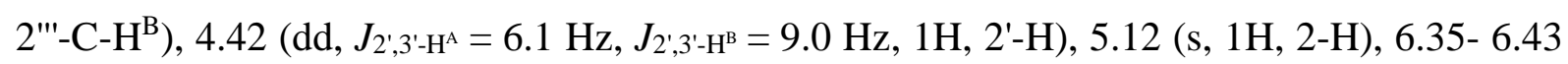
(m, 3H, 4'"-H, 6"'-H, 4-H), 6.70-6.74 (m, 2H, 2"-H, 6"-H), 6.93-6.98 (m, 2H, 3"-H, 5"-H), 6.93$6.98\left(\mathrm{~m}, 1 \mathrm{H}, 3^{\prime \prime}-\mathrm{H}\right), 7.18\left(\mathrm{~d}, J_{5,4}=13.6 \mathrm{~Hz}, 1 \mathrm{H}, 5-\mathrm{H}\right), 14.00\left(\mathrm{~d},{ }^{4} J_{3-\mathrm{OH}, 2}=1.5 \mathrm{~Hz}, 1 \mathrm{H}, 3-\mathrm{OH}\right)$.

${ }^{13} \mathrm{C}-\mathrm{NMR}\left(125.75 \mathrm{MHz}, \mathrm{CDCl}_{3} / \mathrm{CHCl}_{3}, \mathrm{DsBrMi} 21-5076\right): \delta=-4.4$ [Si- $\left.\left(\mathrm{CH}_{3}\right)_{2}\right], 18.3$ [Si$\left.C\left(\mathrm{CH}_{3}\right)_{3}\right], 25.8$ [Si-C $\left.\left(\mathrm{CH}_{3}\right)_{3}\right], 34.7\left(\mathrm{C}-3\right.$ '), $47.1\left(2^{\prime \prime \prime}-\mathrm{CH}_{2}\right), 52.3\left(1^{\prime}-\mathrm{OCH}_{3}\right), 55.2\left(1{ }^{\prime \prime}-\mathrm{OCH}_{3}\right), 55.5$ (5"'-OCH 3 ), 60.9 (C-2'), 90.7 (C-2), 98.3 (C-4"'), 103.9 (C-6"'), 116.3 (C-5), 116.6 (C-2'"), 120.2 (C-2", C-6"), 129.3 (C-3"'), 130.3 (C-3", C-5"), 130.5 (C-4"), 132.8 (C-4), 154.4 (C-1"), 157.9 (C-1"'), 160.7 (C-5"'), 166.9 (C-3), 171.1 (C-1'), 172.6 (C-1).

edHSQC [,,short-range H,C-COSY“, $500.32 \mathrm{MHz} / 125.80 \mathrm{MHz}, \mathrm{CDCl}_{3} ; \delta\left({ }^{1} \mathrm{H}\right) \leftrightarrow \delta\left({ }^{13} \mathrm{C}\right): 0.18$ $\left[\mathrm{s}, \mathrm{Si}-\left(\mathrm{CH}_{3}\right)_{2}\right] \leftrightarrow-4.4\left[\mathrm{Si}-\left(\mathrm{CH}_{3}\right)_{2}\right], 0.98\left[\mathrm{~s}, \mathrm{Si}-\mathrm{C}\left(\mathrm{CH}_{3}\right)_{3}\right] \leftrightarrow 25.8\left[\mathrm{Si}-\mathrm{C}\left(\mathrm{CH}_{3}\right)_{3}\right], \mathrm{AB}$ signal $\left(\delta_{\mathrm{A}}\right.$ $\left.=3.12, \delta_{\mathrm{B}}=3.29,3^{\prime}-\mathrm{H}_{2}\right) \leftrightarrow 34.7\left(\mathrm{C}-3{ }^{\prime}\right), 3.61\left(\mathrm{~s}, 1^{\prime}-\mathrm{OCH}_{3}\right) \leftrightarrow 52.3\left(1^{\prime}-\mathrm{OCH}_{3}\right), 3.75\left(\mathrm{~s}, 1^{\prime \prime}-\mathrm{OCH}_{3}\right)$

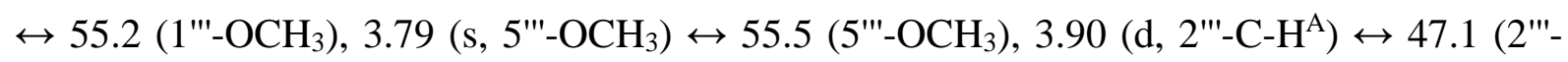
$\left.\mathrm{CH}_{2}\right), 4.32\left(\mathrm{~d}, 2^{2 '-}-\mathrm{C}-\mathrm{H}^{\mathrm{B}}\right) \leftrightarrow 47.1\left(2^{\prime \prime \prime}-\mathrm{CH}_{2}\right), 4.42\left(\mathrm{dd}, 2^{\prime}-\mathrm{H}\right) \leftrightarrow 60.9\left(\mathrm{C}-2^{\prime}\right), 5.12(\mathrm{~s}, 2-\mathrm{H}) \leftrightarrow 90.7$ (C-2), 6.35- 6.43 (m, 4'"-H) 98.3 (C-4"'), 6.43 (m, 6"'-H) 103.9 (C-6"'), 6.41 (m, 4-H) 132.8 (C-4), 6.70-6.74 (m, 2"-H, 6"-H) 120.2 (C-2", C-6"), 6.93-6.98 (m, 3"-H, 5"-H) 130.3 (C-3", C-5"), 6.93-6.98 (m, 3"'-H) 129.3 (C-3'"), $7.18(\mathrm{~d}, 5-\mathrm{H}) \leftrightarrow 116.3$ (C-5).

Specific rotation $(S)-21 d[a]_{D}^{20}=-189.3^{\circ}\left(\mathrm{c}=1.0\right.$ in $\left.\mathrm{CHCl}_{3}\right)$.

HRMS: (EI, $70 \mathrm{eV}): \mathrm{m} / \mathrm{z}=634.18298[\mathrm{M}+\mathrm{H}]^{+}$, corresponds to the molecular formula $\mathrm{C}_{30} \mathrm{H}_{41} \mathrm{O}_{7} \mathrm{NBrSi}(634.18302)\left\{[\mathrm{M}+\mathrm{H}]^{+}\right\}$with a deviation of $-0.1 \mathrm{ppm}$.

IR (KBr): $\tilde{v}=2955,2930,2855,1745,1635,1585,1470,1390,1290,1260,1210,1170,1160$, $1120,1040,915,840,780,695 \mathrm{~cm}^{-1}$. 
Christian Drescher and Reinhard Brückner; 3D-Structure Clarifying Total Synthesis of the (Polyenoyl)tetramic Acid Militarinon B. A Highly Acid-Labile N-Protecting Group for Amides

(E)-(2-Iodovinyl)benzene (SI-6)<smiles>IC=Cc1ccccc1</smiles>

SI-6

At $0^{\circ} \mathrm{C}$ a solution of DIBAL-H $(0.93 \mathrm{M}$ in hexane, $23.8 \mathrm{~mL}, 22.1 \mathrm{mmol}, 1.5$ eq. $)$ was added dropwise to a solution of $\mathrm{Cp}_{2} \mathrm{ZrCl}_{2}(6.50 \mathrm{~g}, 22.1 \mathrm{mmol}, 1.5 \mathrm{eq}$.) in THF (50 mL). The mixture was stirred for $30 \mathrm{~min}$ at this temperature. A solution of phenylacetylene $(1.65 \mathrm{~mL}, 1.50 \mathrm{~g}, 14.7$ mmol) in THF (19 mL) was added slowly, the mixture was warmed to $25^{\circ} \mathrm{C}$ and held this temperature for $1 \mathrm{~h}$. The mixture was cooled to $0^{\circ} \mathrm{C}$ and a solution of iodine $(7.41 \mathrm{~g}, 29.4 \mathrm{mmol}$, 2.0 eq. $)$ in THF $(30 \mathrm{~mL})$ was added. After $5 \mathrm{~min}$ at this temperature it was added aq. $\mathrm{HCl}(1 \mathrm{M}$, $40 \mathrm{~mL})$. The organic layer was separated, and the aq. layer was extracted with $\mathrm{Et}_{2} \mathrm{O}(3 \times 50 \mathrm{~mL})$. The combined organic layers were washed with aq. sat. $\mathrm{Na}_{2} \mathrm{~S}_{2} \mathrm{O}_{4}(50 \mathrm{~mL})$, aq. sat. $\mathrm{NaHCO}_{3}$ $(50 \mathrm{~mL})$ and brine $(50 \mathrm{~mL})$ and dried over $\mathrm{Na}_{2} \mathrm{SO}_{4}$. The solvent was removed under reduced pressure and the residue was purified by flash chromatography $\left(6.0 \mathrm{~cm}, 10 \mathrm{~cm}, c \mathrm{C}_{6} \mathrm{H}_{12}, 50 \mathrm{~mL}\right)$ to give the desired product SI-6 $(2.71 \mathrm{~g}, 80 \%, 11.8 \mathrm{mmol})$ as a yellow liquid.

$\mathbf{R}_{\mathbf{f}}\left(c \mathrm{C}_{6} \mathrm{H}_{12}\right): 0.50$

${ }^{1} \mathbf{H}$-NMR $\left(300.06 \mathrm{MHz}, \mathrm{CDCl}_{3} / \mathrm{CHCl}_{3}\right.$, sample contained 1 weight-\% $c \mathrm{C}_{6} \mathrm{H}_{12}$, DsBrJ12830960, 28.7.2015): $\delta=6.82\left(\mathrm{~d}, J_{1,2}=14.9 \mathrm{~Hz}, 1 \mathrm{H}, 1-\mathrm{H}\right), 7.27-7.35\left(\mathrm{~m}, 5 \mathrm{H}, \mathrm{Ar}-\mathrm{H}_{5}\right), 7.43\left(\mathrm{~d}, J_{2,1}\right.$ $=14.9 \mathrm{~Hz}, 1 \mathrm{H}, 2-\mathrm{H}) \mathrm{ppm}$.

\section{(E)-Tributyl(styryl)stannane (SI-7)}

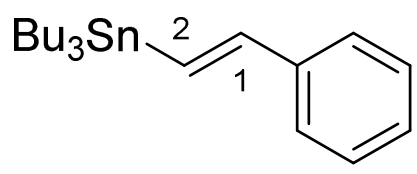

\section{SI-7}

At $-78^{\circ} \mathrm{C}$ a solution of SI-6 $(917 \mathrm{mg}, 3.99 \mathrm{mmol})$ in $\mathrm{Et}_{2} \mathrm{O}(19 \mathrm{~mL})$ was treated with $n \mathrm{BuLi}$ $\left(1.48 \mathrm{M}\right.$ in hexane, $4.05 \mathrm{~mL}, 5.99 \mathrm{mmol}, 1.5$ eq.) and stirred for $20 \mathrm{~min}$. Then $\mathrm{Bu}_{3} \mathrm{SnCl}$ (1.63 mL, $1.95 \mathrm{~g}, 6.00 \mathrm{mmol}, 1.5$ eq.) was added dropwise to the mixture. After stirring for 
Christian Drescher and Reinhard Brückner; 3D-Structure Clarifying Total Synthesis of the (Polyenoyl)tetramic Acid Militarinon B. A Highly Acid-Labile N-Protecting Group for Amides

$20 \mathrm{~min}$ at $-78^{\circ} \mathrm{C}$ the mixture was warmed to $25^{\circ} \mathrm{C}$ and held this temperature for $1 \mathrm{~h}$. It was added aq. sat $\mathrm{NaHCO}_{3}$-solution $(10 \mathrm{~mL})$. The organic layer was separated und the aq. layer was extracted with $\mathrm{Et}_{2} \mathrm{O}(3 \times 30 \mathrm{~mL})$. The combined organic layers were dried over $\mathrm{Na}_{2} \mathrm{SO}_{4}$ and the solvent was removed under reduced pressure. The residue was purified by flash chromatography $\left(4.0 \mathrm{~cm}, 20 \mathrm{~cm}, c \mathrm{C}_{6} \mathrm{H}_{12}: \mathrm{NEt}_{3}=100: 3,10 \mathrm{~mL}\right)$ to give the stannane SI-7 $(1.04 \mathrm{~g}, 2.63 \mathrm{mmol}, 66 \%)$ as a clear liquid.

$\mathbf{R}_{\mathbf{f}}\left(c \mathrm{C}_{6} \mathrm{H}_{12}\right): 0.88$

${ }^{\mathbf{1}} \mathbf{H}$-NMR $\left(300.06 \mathrm{MHz}, \mathrm{CDCl}_{3} / \mathrm{CHCl}_{3}\right.$, sample contained $\mathrm{SnBu}_{4}: \mathrm{m}$ at 0.77-0.99 and $\mathrm{m}$ at 1.241.60 superimposed by product signals, DsBrJ103-30660, 3.7.2017): $\delta=\left(\mathrm{t}, J_{\mathrm{CH}_{3}, \mathrm{CH}_{2}}=7.3 \mathrm{~Hz}\right.$, $9 \mathrm{H}, 3 \times \mathrm{SnCH}_{2} \mathrm{CH}_{2} \mathrm{CH}_{2} \mathrm{CH}_{3}$ ), 0.91-0.96 (m, 6H, $\left.3 \times \mathrm{SnCH}_{2} \mathrm{CH}_{2} \mathrm{CH}_{2} \mathrm{CH}_{3}\right), 1.24-1.40$ (m, 6H, $\left.3 \times \mathrm{SnCH}_{2} \mathrm{CH}_{2} \mathrm{CH}_{2} \mathrm{CH}_{3}\right), 1.45-1.60\left(\mathrm{~m}, 6 \mathrm{H}, 3 \times \mathrm{SnCH}_{2} \mathrm{CH}_{2} \mathrm{CH}_{2} \mathrm{CH}_{3}\right), 6.86\left(\mathrm{~m}_{\mathrm{c}}, 2 \mathrm{H}, 1-\mathrm{H}, 2-\mathrm{H}\right)$, 7.19-7.43 (m, 5H, Ar-H5) ppm.

Methyl (2'S)-2'-\{N-[(1Z,2Z,4E,6E)-3-Hydroxy-7-phenylhepta-2,4,6-trienamido]- $N$ $(1 " ', 5$ "'-dimethoxybenzyl $)\}-3$ '-\{1"-[(tert-butyldimethylsilyl)oxy]phenyl $\}$ propanoate $[Z$ enol-(S)-22d]

in equilibration with

Methyl (2'S)-2'-\{N-[(1E,2Z,4E,6E)-3-Hydroxy-7-phenylhepta-2,4,6-trienamido]- $N$ $(1 " ', 5$ "'-dimethoxybenzyl) $\}-3$ '-\{1"'-[(tert-butyldimethylsilyl)oxy]phenyl\}propanoate $[E-$ enol-(S)-22d]

in equilibration with

Methyl (2'S)-2'-\{N-[(1Z,4E,6E)-3-Oxo-7-phenylhepta-4,6-dienamido]- $N$-(1'"',5'"'dimethoxybenzyl)\}-3'-\{1"'-[(tert-butyldimethylsilyl)oxy]phenyl $\}$ propanoate $[$ Z-keto- $(S)$ 22d]

in equilibration with

Methyl (2'S)-2'-\{N-[(1E,4E,6E)-3-Oxo-7-phenylhepta-4,6-dienamido]- $N-\left(1{ }^{\prime \prime \prime}, 5\right.$ '"'dimethoxybenzyl) $\}-3$ '-\{1''-[(tert-butyldimethylsilyl)oxy $]$ phenyl $\}$ propanoate $[$ E-keto- $(S)$ 22d] 

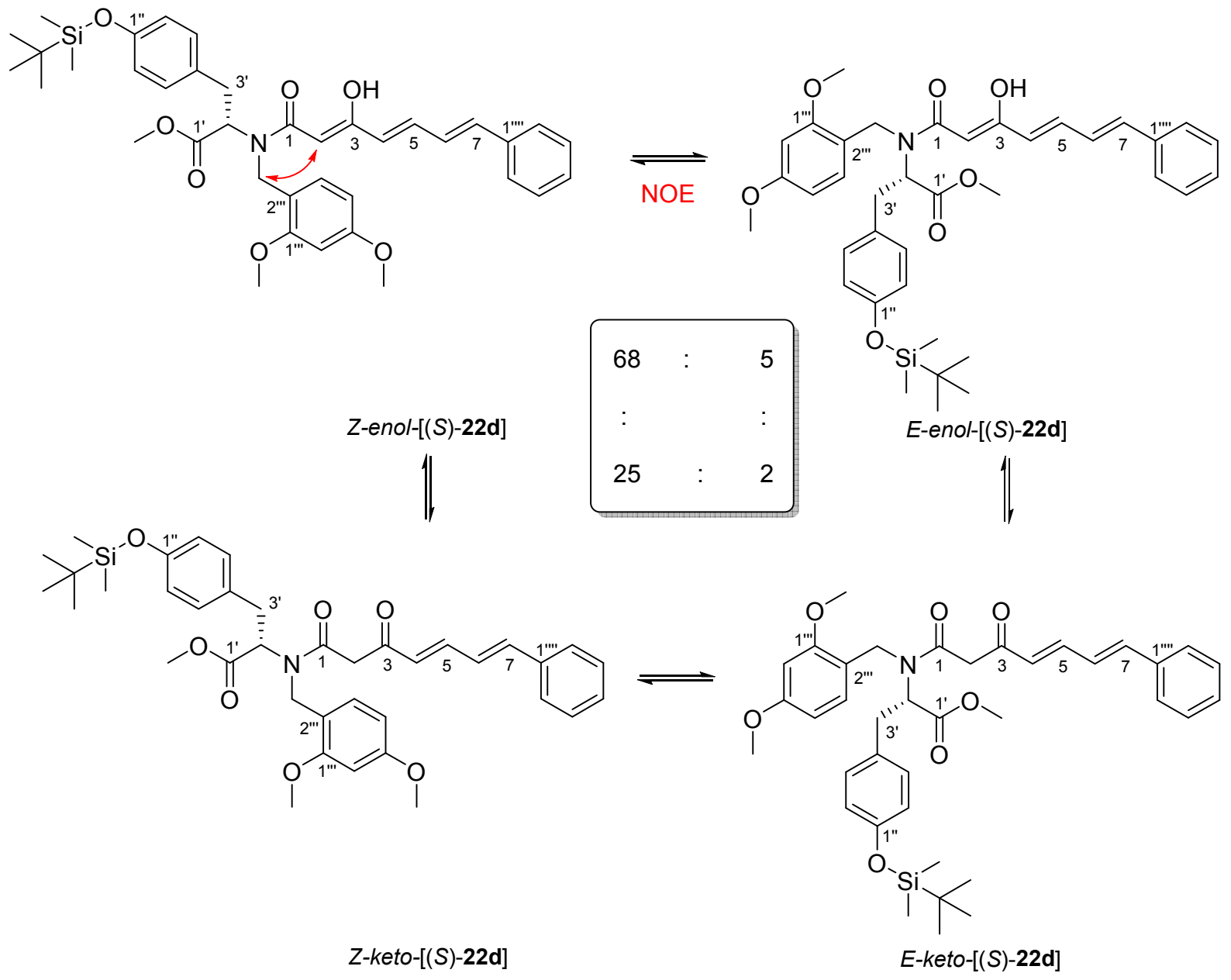

A solution of the western building block $(S)$-21d $(108 \mathrm{mg}, 172 \mu \mathrm{mol})$ and the stannane SI-7 (135 mg, $344 \mu \mathrm{mol}, 2.0$ eq.) in THF (4.0 mL) was deluded with $\operatorname{Pd}_{2}(\mathrm{dba})_{3}(8.0 \mathrm{mg}, 8.9 \mu \mathrm{mol}$, $5 \mathrm{~mol}-\%)$ and $\mathrm{AsPh}_{3}(8.1 \mathrm{mg}, 26.6 \mu \mathrm{mol}, 15 \mathrm{~mol}-\%)$. After $12 \mathrm{~h}$ at $25^{\circ} \mathrm{C}$ aq. KF solution $(8 \mathrm{M}$, $4.5 \mathrm{~mL}$ ) was added and it was sonicated in the ultrasonic bath for $1 \mathrm{~min}$. The layers were separated, and the organic layer was extracted with $\mathrm{Et}_{2} \mathrm{O}(3 \times 15 \mathrm{~mL})$. The combined organic layers were washed with brine $(10 \mathrm{~mL})$, tried over $\mathrm{Na}_{2} \mathrm{SO}_{4}$ and concentrated under reduced pressure. The residue was purified by flash chromatography $\left(c \mathrm{C}_{6} \mathrm{H}_{12}\right.$ :AcOEt $=5: 1,2.0 \mathrm{~cm}$, $20 \mathrm{~cm}, 10 \mathrm{~mL})$. The polyen $(S)-\mathbf{2 6 9}(83.0 \mathrm{mg}, 74 \%, 127.3 \mu \mathrm{mol})$ was obtained as a pale brown oil. It represented a mixture of two rotamers in a keto and an enol form each [Z-enol-(S)-22d:Eenol-(S)-22d:Z-keto-(S)-22d:E-keto- $(S)-22 d=68: 5: 25: 2] .{ }^{9}$ This ratio was determined by integrating the 4-H signals [at $\delta=5.88$ (E-keto), 5.96 (Z-enol), 6.02 (E-enol) and 6.44 (Z-keto) ppm] and compared with die 3-OH signals [14.08 (Z-enol) and 14.20 (E-enol) ppm].

$\mathbf{R}_{\mathbf{f}}\left(c \mathrm{C}_{6} \mathrm{H}_{12}: \mathrm{EtOAc}=4: 1\right): 0.32$

${ }^{1} \mathbf{H}-\mathrm{NMR}\left(500.10 \mathrm{MHz}, \mathrm{CDCl}_{3} / \mathrm{CHCl}_{3}\right.$, sample contained 3 weight-\% of $\mathrm{O}\left(\mathrm{SnBu}_{3}\right)_{2}$ with $\mathrm{t}$ at $0.92, \mathrm{~m}_{\mathrm{c}}$ at $1.30, \mathrm{~m}_{\mathrm{c}}$ at 1.35 and $\mathrm{m}_{\mathrm{c}}$ at $\left.1.63, \mathrm{DsBrFe} 04-500200,4.2 .2020\right): \delta=0.18[\mathrm{~s}, 6 \mathrm{H}, \mathrm{Si}-$ 
Christian Drescher and Reinhard Brückner; 3D-Structure Clarifying Total Synthesis of the (Polyenoyl)tetramic Acid Militarinon B. A Highly Acid-Labile N-Protecting Group for Amides

$\left.\left(\mathrm{CH}_{3}\right)_{2}\right], 0.98\left[\mathrm{~s}, 9 \mathrm{H}, \mathrm{Si}-\mathrm{C}\left(\mathrm{CH}_{3}\right)_{3}\right], \mathrm{AB}$ signal $\left(\delta_{\mathrm{A}}=3.14, \delta_{\mathrm{B}}=3.31, J_{\mathrm{AB}}=14.1 \mathrm{~Hz}\right.$ in addition split by $\left.J_{3^{\prime}-\mathrm{H}^{\mathrm{A}}, 2^{\prime}}=8.9 \mathrm{~Hz}, J_{3^{\prime}-\mathrm{H}^{\mathrm{B}}, 2^{\prime}}=6.0 \mathrm{~Hz}, 2 \mathrm{H}, 3^{\prime}-\mathrm{H}_{2}\right), 3.63\left(\mathrm{~s}, 3 \mathrm{H}, 1^{\prime}-\mathrm{OCH}_{3}\right), 3.76\left(\mathrm{~s}, 3 \mathrm{H}, 1^{\prime \prime}-\right.$ $\left.\mathrm{OCH}_{3}\right), 3.79$ (s, 3H, 5"'-OCH $), 3.95\left(\mathrm{~d},{ }^{2} J=17.4 \mathrm{~Hz}, 1 \mathrm{H}, 2\right.$ ''-C-H $\left.{ }^{\mathrm{A}}\right), 4.36\left(\mathrm{~d},{ }^{2} J=17.5 \mathrm{~Hz}, 1 \mathrm{H}\right.$,

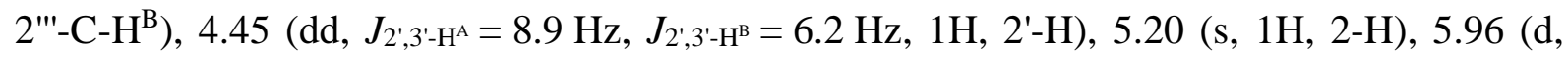
$\left.J_{4,5}=14.9 \mathrm{~Hz}, 1 \mathrm{H}, 4-\mathrm{H}\right), 6.36-6.42$ (m, 2H, 4"'-H, 6"'-H), 6.70-6.75 (m, 2H, 2"-H, 6"-H), 6.786.83 (m, 2H, 6-H, 7-H), 6.94-7.05 (m, 3H, 3"-H, 5"-H, 3"'-H), 7.21 (dd, J5,6 = 9.7 Hz, $\left.J_{5,4}=14.9 \mathrm{~Hz}, 1 \mathrm{H}, 5-\mathrm{H}\right), 7.25\left(\mathrm{dd}, J_{4^{\prime \prime \prime}, 3^{\prime \prime \prime}}=J_{4^{\prime \prime \prime}, 5^{\prime \prime \prime}}=7.4 \mathrm{~Hz}, 1 \mathrm{H}, 4{ }^{\prime \prime \prime '-H}\right), 7.32\left(\mathrm{dd}, J_{3^{\prime \prime \prime}, 4^{\prime \prime \prime \prime}}=J_{3^{\prime \prime \prime}, 2^{\prime \prime \prime}}\right.$ $\left.=7.5 \mathrm{~Hz}, 1 \mathrm{H}, 3^{\prime \prime \prime '-H}\right), 7.32$ (dd, $\left.J_{5^{\prime \prime \prime}, 4^{\prime \prime \prime}}=J_{5}{ }^{\prime \prime \prime}, 6^{\prime \prime \prime}=7.5 \mathrm{~Hz}, 1 \mathrm{H}, 5^{\prime \prime \prime '-H}\right), 7.41\left(\mathrm{~d}, J_{2^{\prime \prime \prime}, 3^{\prime \prime \prime}}=7.5 \mathrm{~Hz}, 1 \mathrm{H}\right.$, 2'"'-H), $7.41\left(\mathrm{~d}, J_{6 " ' ", 5 " '}=7.5 \mathrm{~Hz}, 1 \mathrm{H}, 66^{\prime \prime \prime}-\mathrm{H}\right), 14.08\left(\mathrm{~d}, J_{3-\mathrm{OH}, 2}=1.3 \mathrm{~Hz}, 1 \mathrm{H}, 3-\mathrm{OH}\right) \mathrm{ppm}$.

${ }^{13} \mathbf{C}$-NMR $\left(125.75 \mathrm{MHz}, \mathrm{CDCl}_{3} / \mathrm{CHCl}_{3}\right.$, sample contained $\mathrm{O}\left(\mathrm{SnBu}_{3}\right)_{2}$ at 13.6, 17.6, 26.9 and 27.9, DsBrMz03-5071, 7.3.2017): $\delta=-4.4$ [Si- $\left.\left(\mathrm{CH}_{3}\right)_{2}\right], 18.3\left[\mathrm{Si}-\mathrm{C}\left(\mathrm{CH}_{3}\right)_{3}\right], 25.8\left[\mathrm{Si}-\mathrm{C}\left(\mathrm{CH}_{3}\right)_{3}\right]$, 34.8 (C-3'), 46.9 (2'"-C), $52.2\left(1\right.$ '- $\left.\mathrm{OCH}_{3}\right), 55.2\left(1 " '-\mathrm{OCH}_{3}\right), 55.4\left(5^{\prime \prime}-\mathrm{OCH}_{3}\right), 60.8(\mathrm{C}-2$ '), 90.7 (C-2), 98.3 (C-4"'), 103.8 (C-6"'), 116.7 (C-2"'), 120.2 (C-2", C-6"), 126.9 (C-2"'", C-6"'"), 127.0 (C-4), 127.4 (C-1'"'), 127.6 (C-6), 128.4 (C-4'"'), 128.8 (C-3'"', C-5'"'), 129.3 (C-3'"'), 130.3 (C3", C-5"), 130.4 (C-4"), 136.1 (C-5), 137.3 (C-7), 154.4 (C-1"), 157.9 (C-1"'), 160.5 (C-5"'), 169.2 (C-3), $171.4\left(\mathrm{C}-1^{\prime}\right), 172.8$ (C-1) ppm.

edHSQC [,,short-range H,C-COSY“, 500.10 MHz/125.75 MHz, $\mathrm{CDCl}_{3} ; \delta\left({ }^{1} \mathrm{H}\right) \leftrightarrow \delta\left({ }^{13} \mathrm{C}\right): 0.18$ $\left[\mathrm{s}, \mathrm{Si}-\left(\mathrm{CH}_{3}\right)_{2}\right] \leftrightarrow-4.4\left[\mathrm{Si}-\left(\mathrm{CH}_{3}\right)_{2}\right], 0.98\left[\mathrm{~s}, \mathrm{Si}-\mathrm{C}\left(\mathrm{CH}_{3}\right)_{3}\right] \leftrightarrow 25.8\left[\mathrm{Si}-\mathrm{C}\left(\mathrm{CH}_{3}\right)_{3}\right], \mathrm{AB}$ signal $\left(\delta_{\mathrm{A}}=3.14, \delta_{\mathrm{B}}=3.31,3^{\prime}-\mathrm{H}_{2}\right) \leftrightarrow 34.8\left(\mathrm{C}-3\right.$ '), $3.63\left(\mathrm{~s}, 1^{\prime}-\mathrm{OCH}_{3}\right) \leftrightarrow 52.2\left(1^{\prime}-\mathrm{OCH}_{3}\right), 3.76\left(\mathrm{~s}, 1{ }^{\prime \prime}-\right.$

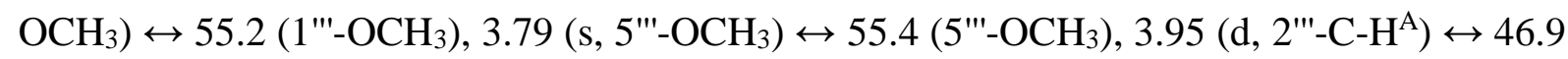
(2"'-C), $4.36\left(\mathrm{~d}, 2^{\prime \prime}-\mathrm{C}-\mathrm{H}^{\mathrm{B}}\right) \leftrightarrow 46.9$ (2'"-C), 4.45 (dd, 2'-H) $60.8(\mathrm{C}-2$ '), 5.20 (s, 2-H) 90.7 (C-2), $5.96(\mathrm{~d}, 4-\mathrm{H}) \leftrightarrow 127.0(\mathrm{C}-4), 6.36-6.42\left(\mathrm{~m}, 4\right.$ 4"'-H) $^{\circ} 98.3$ (C-4'"), 6.36-6.41 (m, 6"'-H) $\leftrightarrow 103.8$ (C-6"'), 6.70-6.75 (m, 2"-H, 6"-H) ↔ 120.2 (C-2", C-6"), 6.78-6.83 (m, 6-H) 127.6 (C-6), 6.78-6.83 (m, 7-H) ↔ 137.3 (C-7), 6.94-7.05 (m, 3"-H, 5"-H) ↔ 130.3 (C-3", C-5"), 6.94-7.05 (m, 3"'-H) 129.3 (C-3"'), 7.21 (dd, 5-H) 136.1 (C-5), 7.25 (dd, 4"'"-H) 128.4 (C-4"'"), 7.32 (dd, 3'"'-H, 5"'-H) 128.8 (C-3"'", C-5"'"), 7.41 (d, 2"'-H, 6'"'-H) 126.9 (C-2'"', C-6"').

HRMS: (EI, $70 \mathrm{eV}): \mathrm{m} / \mathrm{z}=658.3187[\mathrm{M}+\mathrm{H}]^{+}$, corresponds to the molecular formula $\mathrm{C}_{38} \mathrm{H}_{48} \mathrm{O}_{7} \mathrm{NSi}(658.3195)\left\{[\mathrm{M}+\mathrm{H}]^{+}\right\}$with a deviation of $-1.1 \mathrm{ppm}$.

Specific rotation $(S)-22 d[\alpha]_{D}^{20}=-264.9^{\circ}\left(\mathrm{c}=0.51\right.$ in $\left.\mathrm{CHCl}_{3}\right)$

IR (KBr): $\tilde{v}=3060,3025,3000,2955,2930,2860,1740,1630,1580,1510,1470,1390,1330$, $1340,1290,1260,1210,1160,1040,995,915,840 \mathrm{~cm}^{-1}$. 
Christian Drescher and Reinhard Brückner; 3D-Structure Clarifying Total Synthesis of the (Polyenoyl)tetramic Acid Militarinon B. A Highly Acid-Labile N-Protecting Group for Amides

(5S)-5-(1"'-Hydroxybenzyl)-1-(1'",5'"'-dimethoxybenzyl)-3-(1'Z,2' $E, 4$ ' $E)-1$ '-hydroxy-5phenylpenta-2',4"'-dien-1'-ylidene)pyrrolidine-2,4-dion [Z-enol-(S)-16d]

in equilibration with

(5S)-5-(1"'-Hydroxybenzyl)-1-(1'"',5'"'-dimethoxybenzyl)-3-(1'E,2'E,4'E)-1'-hydroxy-5phenylpenta-2',4"'-dien-1'-ylidene)pyrrolidine-2,4-dion [E-enol-(S)-16d]

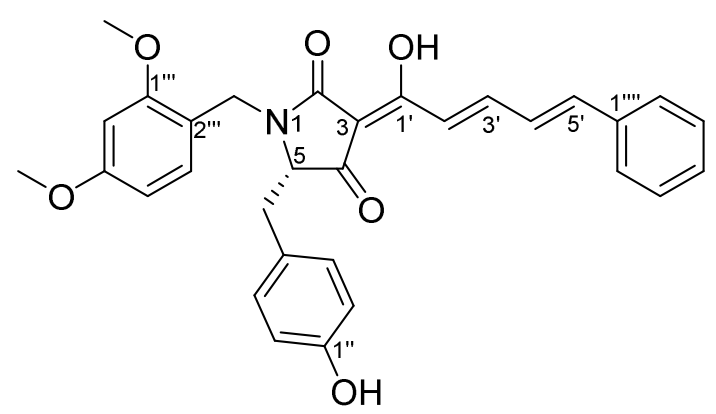

Z-enol-(S)-16d

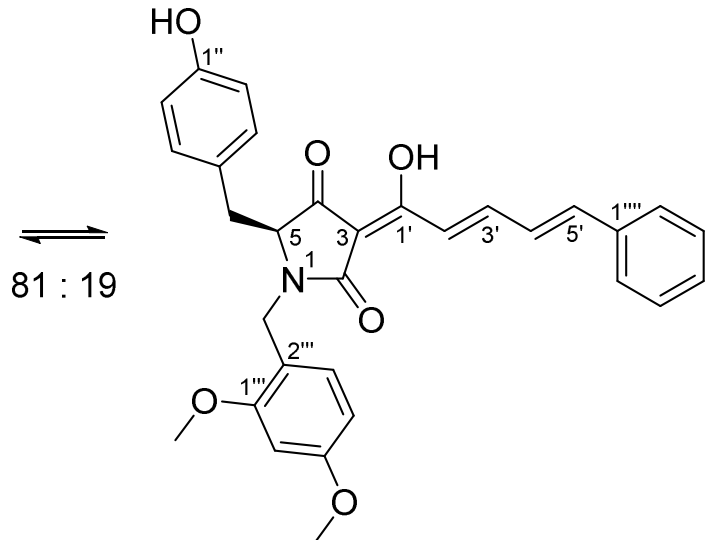

E-enol-(S)-16d

A solution of the polyen $(S)-\mathbf{2 2 d}(50 \mathrm{mg}, 76 \mu \mathrm{mol})$ in THF $(2.0 \mathrm{~mL})$ was deluded with TBAF ( $1 \mathrm{M}$ in THF, $380 \mu \mathrm{L}, 380 \mu \mathrm{mol}, 3.5$ eq.) at $25^{\circ} \mathrm{C}$. At this temperature the mixture was stirred for $1 \mathrm{~h}$. The solvent was removed under reduced pressure and the residue was obtained with $\mathrm{H}_{2} \mathrm{O}(1.0 \mathrm{~mL})$, aq. $\mathrm{H}_{2} \mathrm{SO}_{4}(4 \mathrm{M}, 1.0 \mathrm{~mL})$ and $\mathrm{Et}_{2} \mathrm{O}(5 \mathrm{~mL})$. The layers were separated, and the organic layer was extracted with $\mathrm{Et}_{2} \mathrm{O}(3 \times 10 \mathrm{~mL})$. The combined organic layers were tried over $\mathrm{Na}_{2} \mathrm{SO}_{4}$ and concentrated under reduced pressure. The residue was purified by reverse phase flash chromatography $\left(\mathrm{MeCN}: \mathrm{H}_{2} \mathrm{O}: \mathrm{TFA}=85: 15: 0.1,1.5 \mathrm{~cm}, 10 \mathrm{~cm}, 2 \mathrm{~mL}\right)$. The polyenoyltetramic acid $(S)$-16d $(27.6 \mathrm{mg}, 71 \%, 54.0 \mu \mathrm{mol})$ was obtained as a yellow powder (mp.: $85-87^{\circ} \mathrm{C}$ ). It was assigned as an $81: 19$ mixture of the $Z$ - and the $E$-enol of the title compounds $(S)$-16d. This ratio was determined by integrating the triplets of the respective 5-H [3.87 (Z-enol) and $4.01($ E-enol)].

$\mathbf{R}_{\mathbf{f}}\left(\mathrm{MeCN}: \mathrm{H}_{2} \mathrm{O}: \mathrm{TFA}=85: 15: 0.1\right): 0.27$

${ }^{1} \mathbf{H}-\mathbf{N M R}\left(500.10 \mathrm{MHz}, \mathrm{CDCl}_{3} / \mathrm{CHCl}_{3}\right.$, sample contained trace amounts of MeCN, DsBrFe04500300, 4.2.2020): $\delta=3.11\left(\mathrm{~m}_{\mathrm{c}}, 2 \mathrm{H}, 5-\mathrm{CH}_{2}\right), 3.79$ (s, 3H, 1'"--OCH $), 3.80$ (s, 3H, 5'"--OCH 3 ), $3.87\left(\mathrm{t}, J_{5,5-\mathrm{CH}_{2}}=4.5 \mathrm{~Hz}, 1 \mathrm{H}, 5-\mathrm{H}\right), 4.24\left(\mathrm{~d},{ }^{2} J=17.7 \mathrm{~Hz}, 1 \mathrm{H}, 2^{2}{ }^{\prime \prime}-\mathrm{C}-\mathrm{H}^{\mathrm{A}}\right), 5.03\left(\mathrm{~d},{ }^{2} J=17.7 \mathrm{~Hz}\right.$, 1H, 2'"-C-H ${ }^{\mathrm{B}}$ ), 6.42-6.46 (m, 2H, 4"'-H, 6"'-H), 6.65-6.68 (m, 2H, 2"-H, 6"-H), 6.92-7.02 (m,

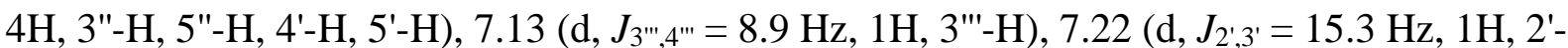


Christian Drescher and Reinhard Brückner; 3D-Structure Clarifying Total Synthesis of the (Polyenoyl)tetramic Acid Militarinon B. A Highly Acid-Labile N-Protecting Group for Amides

H), 7.30-7.38 (m, 3H, 3"'"-H, 4'"'-H, 5'"'-H), 7.43-7.51 (m, 2H, 2"'"-H, 6'"'-H), 7.54 (dd, $\left.J_{3^{\prime}, 4^{\prime}}=9.2 \mathrm{~Hz}, J_{3^{\prime}, 2^{\prime}}=15.0 \mathrm{~Hz}, 1 \mathrm{H}, 3^{\prime}-\mathrm{H}\right) \mathrm{ppm}$.

${ }^{13}$ C-NMR (125.75 MHz, $\left.\mathrm{CDCl}_{3} / \mathrm{CHCl}_{3}, \mathrm{DsBrFe} 04-500304,4.2 .2020\right): \delta=33.9$ (C-3'), 38.5 (2"'-C), 54.5 (1"'--OCH3), 54.5 (5'"--OCH3), 65.2 (C-5), 98.6 (C-4"'), 101.1 (C-3), 104.5 (C-6"'), 115.4 (C-2", C-6"), 116.2 (C-2'"), 121.8 (C-2'), 127.4 (C-4'), 127.6 (C-2'"', C-6'"'), 129.0 (C-3'"', C-5"'"), 129.6 (C-4'"'), 130.9 (C-3", C-5"), 131.6 (C-3"'), 136.1 (C-1"'"), 142.5 (C-5'), 144.4 (C3'), 154.9 (C-1"), 158.7 (C-1'"), 161.0 (C-5"'), 172.8 (C-1'), 173.7 (C-2), 194.9 (C-4) ppm.

edHSQC [,,short-range H,C-COSY“, $500.10 \mathrm{MHz} / 125.75 \mathrm{MHz}, \mathrm{CDCl}_{3} ; \delta\left({ }^{1} \mathrm{H}\right) \leftrightarrow \delta\left({ }^{13} \mathrm{C}\right): 3.11$ $\left(\mathrm{m}_{\mathrm{c}}, 5-\mathrm{CH}_{2}\right) \leftrightarrow 33.9\left(5-\mathrm{CH}_{2}\right), 3.79\left(\mathrm{~s}, 1^{\prime \prime \prime}-\mathrm{OCH}_{3}\right) \leftrightarrow 55.5\left(1^{\prime \prime \prime}-\mathrm{OCH}_{3}\right), 3.80\left(\mathrm{~s}, 5^{\prime \prime}-\mathrm{OCH}_{3}\right) \leftrightarrow 55.5$

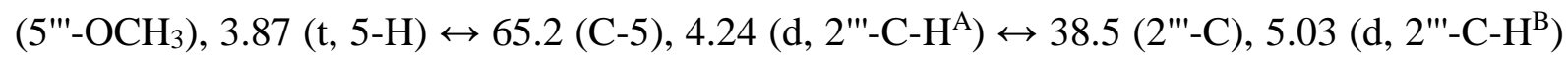
$\leftrightarrow 38.5$ (2'"-C), 6.42-6.46 (m, 4'"-H) ↔ 98.6 (C-4'"), 6.42-6.46 (m, 6"'-H) 104.5 (C-6"'), 6.65-6.73 (m, 2"-H, 6"-H) 115.4 (C-2", C-6"), 6.94-6.99 (m, 3"-H, 5"-H) 130.7 (C-2", C6"), 6.92-7.02 (m, 3"-H, 5"-H) 130.9 (C-3", C-5"), 6.92-7.02 (m, 4'-H) 127.4 (C-4'), 6.92$7.02\left(\mathrm{~m}, 5^{\prime}-\mathrm{H}\right) \leftrightarrow 142.5\left(\mathrm{C}-5^{\prime}\right), 7.13\left(\mathrm{~d}, 3^{\prime \prime \prime}-\mathrm{H}\right) \leftrightarrow 131.6\left(\mathrm{C}-3^{\prime \prime \prime}\right), 7.22\left(\mathrm{~d}, 2^{\prime}-\mathrm{H}\right) \leftrightarrow 121.8\left(\mathrm{C}-2^{\prime}\right)$, 127.0 (C-4"), 7.30-7.38 (m, 3'"'-H, 5"'"-H) ↔ 129.0 (C-3'"', C-5"'"), 7.30-7.38 (m, 4"'--H) ↔ 129.6 (C-4'"'), 7.43-7.51 (m, 2"'-H, 6'"'-H) ↔ 127.6 (C-2'"', C-6'"'), 7.54 (dd, 3'-H) 144.4 (C3').

HRMS: $(\mathrm{EI}, 70 \mathrm{eV}): \mathrm{m} / \mathrm{z}=510.1920[\mathrm{M}-\mathrm{H}]^{+}$, corresponds to the molecular formula $\mathrm{C}_{31} \mathrm{H}_{28} \mathrm{O}_{6} \mathrm{~N}$ $(510.1922)\left\{[\mathrm{M}-\mathrm{H}]^{+}\right\}$with a deviation of $-0.4 \mathrm{ppm}$.

Specific rotation $(S)-\mathbf{1 6 d}[\alpha]_{D}^{20}=-1243.7^{\circ}\left(\mathrm{c}=0.5\right.$ in $\left.\mathrm{CHCl}_{3}\right)$

IR (KBr): $\tilde{v}=2980,2935,2865,2805,1965,1695,1610,1555,1510,1450,1385,1245,1210$, $1225,1155,1125,1075,1035,1000,935,840 \mathrm{~cm}^{-1}$. 
(5S)-5-(1'-Hydroxybenzyl)-3-(1'Z,2' E, 4' $E)-1^{\prime}$-hydroxy-5-phenylpenta-2',4'-dien-1'ylidene)pyrrolidine-2,4-dion [Z-enol-(S)-17]

in equilibration with

(5S)-5-(1'-Hydroxybenzyl)-3-(1'E,2' $\left.E, 4^{\prime} E\right)-1$ '-hydroxy-5-phenylpenta-2',4'-dien-1'ylidene)pyrrolidine-2,4-dion [E-enol-(S)-17]
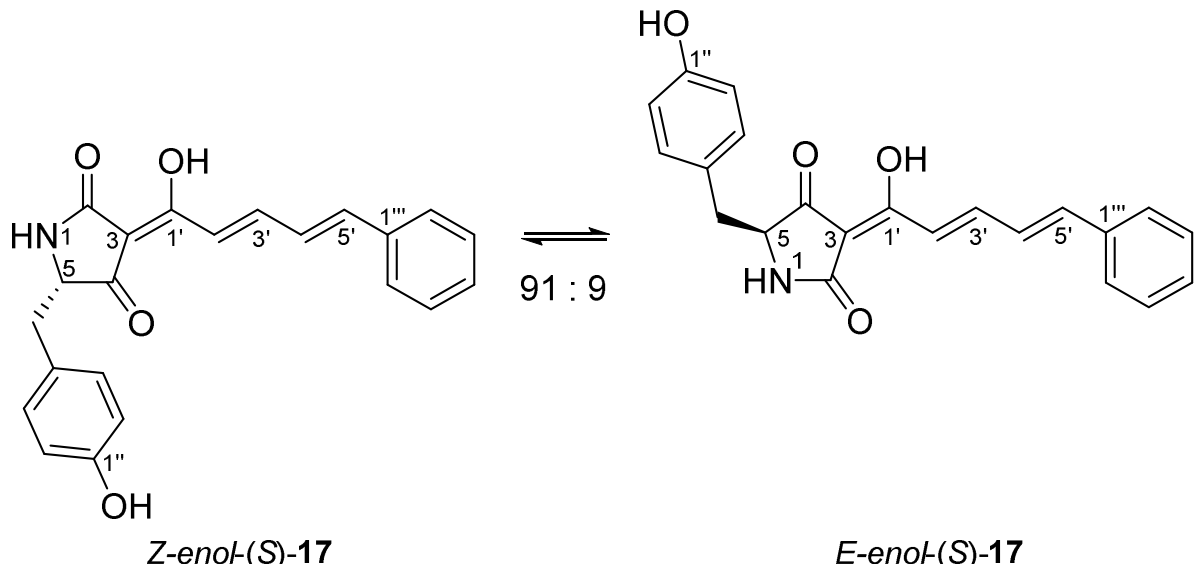

Method A: The tetramic acid $(S)-\mathbf{1 6 d}(27.6 \mathrm{mg}, 54.0 \mu \mathrm{mol})$ was dissolved in $\mathrm{CH}_{2} \mathrm{Cl}_{2}(2.0 \mathrm{~mL})$. First Thioanisole $(310 \mu \mathrm{L}, 318 \mathrm{mg}, 2.70 \mathrm{mmol}, 50$ eq. $)$ and then $\mathrm{F}_{3} \mathrm{CCO}_{2} \mathrm{H}(0.5 \mathrm{~mL})$ was added at $0^{\circ} \mathrm{C}$. Die temperature was warmed up to $25^{\circ} \mathrm{C}$ and held this temperature for $1 \mathrm{~h}$. The mixture was diluted with $\mathrm{H}_{2} \mathrm{O}(2 \mathrm{~mL})$. The layers were separated, and the aq. layer was extracted with $\mathrm{CH}_{2} \mathrm{Cl}_{2}(3 \times 10 \mathrm{~mL})$. The combined organic layers were washed with brine $(5 \mathrm{~mL})$ and tried over $\mathrm{Na}_{2} \mathrm{SO}_{4}$. The solvent was removed under reduced pressure and to the residue was added $\mathrm{MeOH}(3 \mathrm{~mL})$. The resulting suspension was filtered off and the filtrate was concentrated under reduced pressure. The residue was purified by reverse phase flash chromatography $\left(\mathrm{MeCN}: \mathrm{H}_{2} \mathrm{O}: \mathrm{TFA}=75: 25: 0.1,1.5 \mathrm{~cm}, 10 \mathrm{~cm}, 2 \mathrm{~mL}\right)$. The polyenoyltetramic acid $(S)-\mathbf{1 7}$ (15.6 mg, 80\%, $43.2 \mu \mathrm{mol})$ was obtained as a yellow powder. It was assigned as a 91:9 mixture of the $Z$ - and the $E$-enol of the title compounds $(S)$-17. This ratio was determined by integrating dd's of the respective 3'-H [7.62 (Z-enol) and 8.04 (E-enol)].

Method B: The tetramic acid $(S)$-16e $(40.0 \mathrm{mg}, 73.9 \mu \mathrm{mol})$ was dissolved in $\mathrm{CH}_{2} \mathrm{Cl}_{2}(5.0 \mathrm{~mL})$. $\mathrm{F}_{3} \mathrm{CCO}_{2} \mathrm{H}(50 \mu \mathrm{L})$ was added at $25^{\circ} \mathrm{C}$. Die temperature was held this temperature for $5 \mathrm{~h}$. The mixture was diluted with $\mathrm{H}_{2} \mathrm{O}(2 \mathrm{~mL})$. The layers were separated, and the aq. layer was extracted with $\mathrm{CH}_{2} \mathrm{Cl}_{2}(3 \times 10 \mathrm{~mL})$. The combined organic layers were washed with brine $(5 \mathrm{~mL})$ and tried over $\mathrm{Na}_{2} \mathrm{SO}_{4}$. The solvent was removed under reduced pressure and to the residue was added $\mathrm{MeOH}(3 \mathrm{~mL})$. The resulting suspension was filtered off and the filtrate was 
Christian Drescher and Reinhard Brückner; 3D-Structure Clarifying Total Synthesis of the (Polyenoyl)tetramic Acid Militarinon B. A Highly Acid-Labile N-Protecting Group for Amides

concentrated under reduced pressure. The residue was purified by reverse phase flash chromatography $\left(\mathrm{MeCN}: \mathrm{H}_{2} \mathrm{O}: \mathrm{TFA}=75: 25: 0.1,1.5 \mathrm{~cm}, 10 \mathrm{~cm}, 2 \mathrm{~mL}\right)$. The polyenoyltetramic acid $(S)-17(24.8 \mathrm{mg}, 93 \%, 68.7 \mu \mathrm{mol})$ was obtained as a yellow powder. It was assigned as a 91:9 mixture of the $Z$ - and the $E$-enol of the title compounds $(S)-\mathbf{1 7}$. This ratio was determined by integrating dd's of the respective 3'-H [7.62 (Z-enol) and 8.04 (E-enol)].

$\mathbf{R}_{\mathbf{F}}\left(\mathrm{MeCN}: \mathrm{H}_{2} \mathrm{O}: \mathrm{TFA}=75: 25: 0.1\right): 0.24$

${ }^{1} \mathbf{H}-\mathrm{NMR}$ (500.10 MHz, MeOD/MeOH, sample contained trace amounts of MeCN, DsBrFe04500400, 4.2.2020): $\delta=\mathrm{AB}$ signal $\left(\delta_{\mathrm{A}}=2.87, \delta_{\mathrm{B}}=2.99, J_{\mathrm{AB}}=14.2 \mathrm{~Hz}\right.$ in addition split by $J_{5-}$ $\left.\mathrm{CH}^{\mathrm{A}, 2^{\prime}}=4.4 \mathrm{~Hz}, J_{5-\mathrm{CH}^{\mathrm{B}}, 2^{\prime}}=6.1 \mathrm{~Hz}, 2 \mathrm{H}, 5-\mathrm{CH}_{2}\right), 4.05$ (br. s, 1H, 5-H), 6.64-6.68 (m, 2H, 2"-H, 6"H), 6.96-7.00 (m, 3H, 3"-H, 5"-H), 7.05-7.15 (m, 2H, 4'-H, 5'-H), 7.23 (d, J $2^{\prime}, 3^{\prime}=15.3 \mathrm{~Hz}, 1 \mathrm{H}$, 2'-H), 7.29-7.39 (m, 3H, 3"'-H, 4'"-H, 5"'-H), 7.51-7.57 (m, 2H, 2"'-H, 6"'-H), 7.62 (dd, $\left.J_{3^{\prime}, 4^{\prime}}=9.9 \mathrm{~Hz}, J_{3^{\prime}, 2^{\prime}}=15.0 \mathrm{~Hz}, 1 \mathrm{H}, 3^{\prime}-\mathrm{H}\right) \mathrm{ppm}$.

${ }^{13}$ C-NMR (125.75 MHz, $\left.\mathrm{CDCl}_{3} / \mathrm{CHCl}_{3}, \mathrm{DsBrFe} 04-500404,4.2 .2020\right): \delta=37.6$ (5- $\left.\delta \mathrm{CH}_{2}\right), 64.4$ (C-5), 101.8 (C-3), 116.1 (C-2", C-6"), 122.2 (C-2'), 127.2 (C-4"), 128.3 (C-4'), 128.8 (C-2'", C-6"'), 130.0 (C-3"', C-5"'), 130.6 (C-4"'), 131.9 (C-3", C-5"), 137.5 (C-1"'), 144.4 (C-5'), 146.2 (C-3'), 157.3 (C-1"), 174.9 (C-1'), 197.5 (C-4) ppm.

edHSQC [,,short-range H,C-COSY“, 500.10 MHz/125.75 MHz, $\mathrm{CDCl}_{3} ; \delta\left({ }^{1} \mathrm{H}\right) \leftrightarrow \delta\left({ }^{13} \mathrm{C}\right)$ : AB signal $\left(\delta_{\mathrm{A}}=2.87, \delta_{\mathrm{B}}=2.99,5-\mathrm{CH}_{2}\right) \leftrightarrow 37.6\left(5-\mathrm{CH}_{2}\right), 4.05$ (br. s, 5-H) $64.4(\mathrm{C}-5), 6.64-6.68$ (m, 2"-H, 6"-H) ↔ 116.1 (C-2", C-6"), 6.96-7.00 (m, 3"-H, 5"-H) ↔ 131.9 (C-3", C-5"), 7.05$7.15\left(\mathrm{~m}, 4^{\prime}-\mathrm{H}\right) \leftrightarrow 128.3\left(\mathrm{C}-4^{\prime}\right), 7.05-7.15\left(\mathrm{~m}, 5^{\prime}-\mathrm{H}\right) \leftrightarrow 144.4\left(\mathrm{C}-5^{\prime}\right), 7.23\left(\mathrm{~d}, 2^{\prime}-\mathrm{H}\right) \leftrightarrow 122.2(\mathrm{C}-$ 2'), 7.29-7.39 (m, 3'"-H, 5"'-H) ↔ 130.0 (C-3"', C-5"'), 7.29-7.39 (m, 4"'-H) ↔ 130.6 (C-4'"), 7.51-7.57 (m, 2'"-H, 6"'-H) 128.8 (C-2"', C-6"'), 7.62 (dd, 3'-H) 146.2 (C-3').

HRMS: (EI, $70 \mathrm{eV}): \mathrm{m} / \mathrm{z}=360.1242[\mathrm{M}-\mathrm{H}]^{+}$, corresponds to the molecular formula $\mathrm{C}_{22} \mathrm{H}_{18} \mathrm{O}_{4} \mathrm{~N}$ $(360.1241)\left\{[\mathrm{M}-\mathrm{H}]^{+}\right\}$with a deviation of $0.3 \mathrm{ppm}$.

Specific rotation $(S)-17[\alpha]_{D}^{20}=-745.9^{\circ}(\mathrm{c}=0.3$ in $\mathrm{MeOH})$

IR (KBr): $\tilde{v}=2955,2925,2870,2855,2065,1645,1615,1435,1365,1290,1260,1210,1155$, $1110,1070,1050,1005,950,895 \mathrm{~cm}^{-1}$. 


\section{Methyl (2S)-3-\{1'-[(tert-Butyldimethylsilyl)oxy]phenyl\}-2-[(1'- nitrobenzyl)amino]propanoate $[(S)-18 a]$}

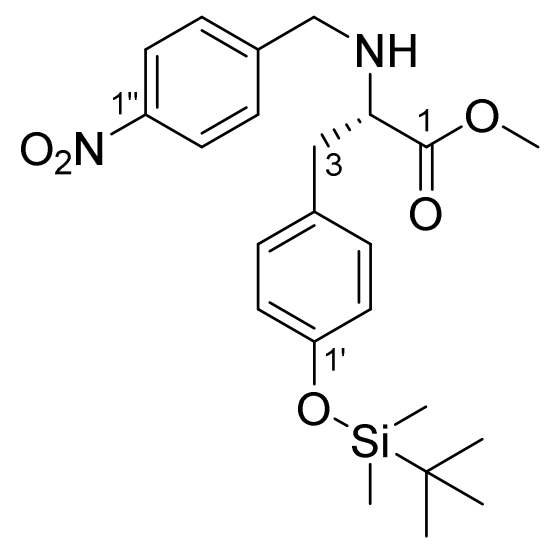

(S)-18a

The amine $(S)$-20 (500 mg, $1.60 \mathrm{mmol})$ was dissolved in $\mathrm{MeOH}(15 \mathrm{~mL})$. It was added HOAc (12 drops) and 4-nitrobenzaldehyd (246 mg, $1.76 \mathrm{mmol}, 1.1 \mathrm{eq}$.). After $10 \mathrm{~min}$ at $25^{\circ} \mathrm{C}$ it was added $\mathrm{NaBH}_{3} \mathrm{CN}$ (178 mg, $2.40 \mathrm{mmol}, 1.5$ eq.). The mixture was stirred at $25^{\circ} \mathrm{C}$ for $6 \mathrm{~h}$. The solvent was removed under reduced pressure. It was added $\mathrm{H}_{2} \mathrm{O}(10 \mathrm{~mL})$ and $\mathrm{Et}_{2} \mathrm{O}(20 \mathrm{~mL})$. The layers were separated, and aq. layer was extracted with $\mathrm{Et}_{2} \mathrm{O}(3 \times 25 \mathrm{~mL})$. The combined organic layers were dried over $\mathrm{MgSO}_{4}$ and concentrated under reduced pressure. Purification by flash chromatography $\left(c \mathrm{C}_{6} \mathrm{H}_{12}\right.$ :AcOEt $\left.=5: 1,3.5 \mathrm{~cm}, 20 \mathrm{~cm}, 30 \mathrm{~mL}\right)$ afforded $(S)-\mathbf{2 8 2}$ (445 mg, 62\%, $0.92 \mathrm{mmol}$ ) as a colorless oil.

$\mathbf{R}_{\mathbf{f}}\left(c \mathrm{C}_{6} \mathrm{H}_{12}: \mathrm{EtOAc}=5: 1\right): 0.28$

${ }^{1} \mathbf{H}-\mathbf{N M R}\left(500.42 \mathrm{MHz}, \mathrm{CDCl}_{3} / \mathrm{CHCl}_{3}\right.$, DsBrOk30-500500, 2.11.2020): $\delta=0.19$ [s, 6H, 1'-O$\left.\mathrm{Si}-\left(\mathrm{CH}_{3}\right)_{2}\right], 0.98$ [s, 9H, 1'-O-Si-C $\left.\left(\mathrm{CH}_{3}\right)_{3}\right], 1.84$ (br. s, $\left.1 \mathrm{H}, N \mathrm{H}\right), \mathrm{AB}$ signal $\left(\delta_{\mathrm{A}}=2.85, \delta_{\mathrm{B}}=2.93\right.$, $J_{\mathrm{AB}}=13.5 \mathrm{~Hz}$, in addition split by $\left.J_{3-\mathrm{H}^{\mathrm{A}}, 2}=6.0 \mathrm{~Hz}, J_{3-\mathrm{H}^{\mathrm{B}}, 2}=7.7 \mathrm{~Hz}, 2 \mathrm{H}, 3-\mathrm{H}_{2}\right), 3.42$ (dd, $\left.J_{2,3-\mathrm{H}^{\mathrm{A}}}=6.0 \mathrm{~Hz}, J_{2,3-\mathrm{H}^{\mathrm{B}}}=7.7 \mathrm{~Hz}, 1 \mathrm{H}, 2-\mathrm{H}\right), 3.67\left(\mathrm{~s}, 3 \mathrm{H}, 1-\mathrm{OCH}_{3}\right), 3.69$ (d, ${ }^{2} J=14.6 \mathrm{~Hz}, 1 \mathrm{H}, 4$ "$\left.\mathrm{CH}^{\mathrm{A}}\right), 3.93\left(\mathrm{~d},{ }^{2} J=14.6 \mathrm{~Hz}, 1 \mathrm{H}, 4^{\prime \prime}-\mathrm{CH}^{\mathrm{B}}\right), 6.75-6.78$ (m, 2H, 2'-H, 6'-H), 7.00-7.03 (m, 2H, 3'H, 5'-H), 7.34-7.38 (m, 2H, 3"-H, 5"-H), 8.10-8.13 (m, 2H, 2"-H, 6"-H) ppm.

${ }^{13}$ C-NMR (125.75 MHz, $\left.\mathrm{CDCl}_{3} / \mathrm{CHCl}_{3}, \mathrm{DsBrOk} 30-500504,2.11 .2020\right): \delta=-4.3$ [1'-O-Si$\left(\mathrm{CH}_{3}\right)_{2}$ ], 18.3 [1'-O-Si- $C\left(\mathrm{CH}_{3}\right)_{3}$ ], 25.8 [1'-O-Si-C $\left(\mathrm{CH}_{3}\right)_{3}$ ], 39.1 (C-3), 51.3 (4"-- $\left.\mathrm{CH}_{2}\right), 51.9$ (1$\mathrm{OCH}_{3}$ ), 62.3 (C-2), 120.1 (C-2', C-6'), 123.6 (C-2", C-6"), 128.6 (C-3", C-5"), 129.8 (C-4'), 130.3 (C-3', C-5'), 147.2 (C-1"), 147.6 (C-4"), 154.7 (C-1'), 175.0 (C-1) ppm. 
Christian Drescher and Reinhard Brückner; 3D-Structure Clarifying Total Synthesis of the (Polyenoyl)tetramic Acid Militarinon B. A Highly Acid-Labile N-Protecting Group for Amides

edHSQC [,short-range H,C-COSY“, $\left.500.42 \mathrm{MHz} / 125.75 \mathrm{MHz}, \mathrm{CDCl}_{3} ; \delta\left({ }^{1} \mathrm{H}\right) \leftrightarrow \delta\left({ }^{13} \mathrm{C}\right)\right]$ : 0.19 [s, 1'-O-Si- $\left.\left(\mathrm{CH}_{3}\right)_{2}\right] \leftrightarrow-4.3$ [1'-O-Si- $\left.\left(\mathrm{CH}_{3}\right)_{2}\right], 0.98$ [s, 1'-O-Si-C $\left.\left(\mathrm{CH}_{3}\right)_{3}\right] \leftrightarrow 25.8$ [1'-O-Si$\left.\mathrm{C}\left(\mathrm{CH}_{3}\right)_{3}\right]$, AB signal $\left(\delta_{\mathrm{A}}=2.85, \delta_{\mathrm{B}}=2.93,3-\mathrm{H}_{2}\right) \leftrightarrow 39.1(\mathrm{C}-3), 3.42(\mathrm{dd}, 2-\mathrm{H}) \leftrightarrow 62.3(\mathrm{C}-2)$, $3.67\left(\mathrm{~s}, 1-\mathrm{OCH}_{3}\right) \leftrightarrow 51.9\left(1-\mathrm{OCH}_{3}\right), 3.69\left(\mathrm{~d}, 4 "-\mathrm{CH}^{\mathrm{A}}\right) \leftrightarrow 51.3\left(4 "-\mathrm{CH}_{2}\right), 3.93\left(\mathrm{~d}, 4\right.$ "- $\left.\mathrm{CH}^{\mathrm{B}}\right) \leftrightarrow$ $51.3\left(4^{\prime \prime}-\mathrm{CH}_{2}\right), 6.75-6.78\left(\mathrm{~m}, 2^{\prime}-\mathrm{H}, 66^{\prime}-\mathrm{H}\right) \leftrightarrow 120.1$ (C-2', C-6'), 7.00-7.03 (m, 3'-H, 5'-H) ↔ 130.3 (C-3', C-5'), 7.34-7.38 (m, 3"-H, 5"-H) ↔ 128.6 (C-3", C-5"), 8.10-8.13 (m, 2"-H, 6"-H) $\leftrightarrow 123.6$ (C-2", C-6").

Specific rotation $(S)-\mathbf{1 8 a}[\alpha]_{D}^{20}=-0.3^{\circ}\left(\mathrm{c}=0.78\right.$ in $\left.\mathrm{CHCl}_{3}\right)$

HRMS: (EI, $70 \mathrm{eV}): \mathrm{m} / \mathrm{z}=445.2157[\mathrm{M}+\mathrm{H}]^{+}$, corresponds to the molecular formula $\mathrm{C}_{23} \mathrm{H}_{33} \mathrm{O}_{5} \mathrm{~N}_{2} \mathrm{Si}(445.2153)\left\{[\mathrm{M}+\mathrm{H}]^{+}\right\}$with a deviation of $0.9 \mathrm{ppm}$.

IR (KBr): $\tilde{v}=3435,2955,2930,2860,1735,1645,1510,1345,1255,1200,1170,1105,910$, $840,780,735 \mathrm{~cm}^{-1}$.

Methyl (2'S)-2' - $\{N$-[(1Z,2Z,4E)-5-Bromo-3-hydroxy-1-oxoypenta-2,4-dienyl]- $N$-(1'"'nitrobenzyl) $\}-3$ '- $\left\{1^{\prime \prime}-[(\right.$ tert-butyldimethylsilyl)oxy]phenyl $\}$ propanoate [Z-enol-(S)-21a] in equilibration with

Methyl (2'S)-2'- $\left\{N-[(1 E, 2 Z, 4 E)-5-B r o m o-3-h y d r o x y-1-o x o y p e n t a-2,4-d i e n y l]-N-\left(1{ }^{\prime \prime \prime}-\right.\right.$ nitrobenzyl) $\}-3 '-\left\{1^{\prime \prime}-[(\right.$ tert-butyldimethylsilyl)oxy]phenyl $\}$ propanoate $[$ E-enol-(S)-21a] in equilibration with

Methyl (2'S)-2' -\{N-[(1Z,4E)-5-Bromo-1,3-dioxopent-4-enyl]-N-(1'"'-nitrobenzyl) $\}-3$ '- $\{1$ '"[(tert-butyldimethylsilyl)oxy]phenyl $\}$ propanoate $[$ Z-keto-(S)-21a]

in equilibration with

Methyl (2'S)-2'-\{N-[(1E,4E)-5-Bromo-1,3-dioxopent-4-enyl]-N-(1"'-nitrobenzyl) $\}-3$ '- $\left\{1^{\prime \prime}\right.$ [(tert-butyldimethylsilyl)oxy]phenyl $\}$ propanoate $[$ E-keto-(S)-21a] 


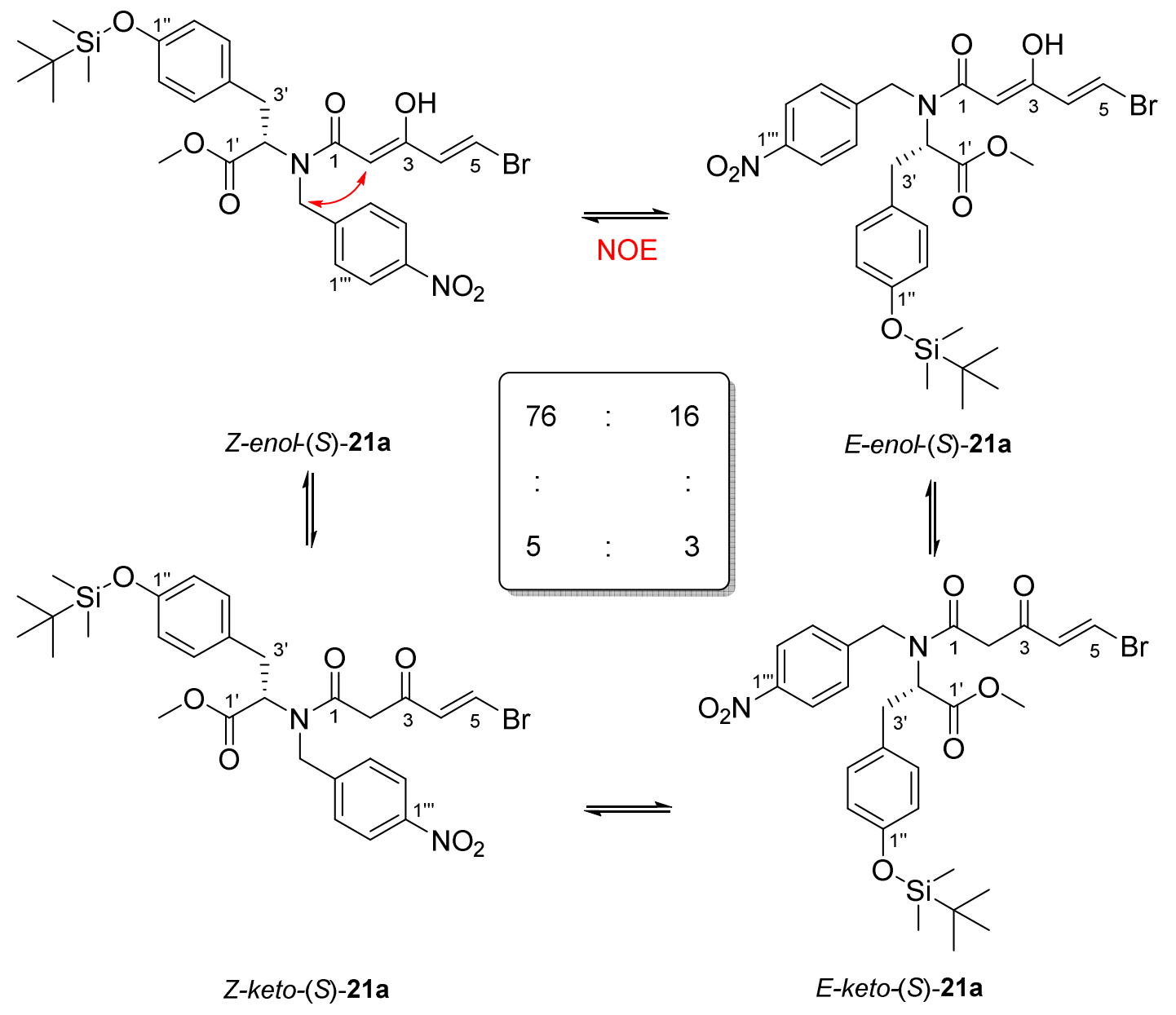

A solution of $N$ - $p$ NB-tyrosine methyl ester $(S)$-18a $(150 \mathrm{mg}, 465 \mu \mathrm{mol})$ and the $\beta$-ketothioester 11 (139 mg, $696 \mu \mathrm{mol}, 1.5$ eq.) in THF (4.5 mL) was treated with powdered molecular sieves $4 \AA(0.8 \mathrm{~g})$. The suspension stirred at $25^{\circ} \mathrm{C}$ for $30 \mathrm{~min}$. Then $\mathrm{AgO}_{2} \mathrm{CCF}_{3}(137 \mathrm{mg}, 696 \mu \mathrm{mol}$, 1.5 eq.) was added and the mixture was stirred for $22 \mathrm{~h}$ at $25^{\circ} \mathrm{C}$. The solvent was removed under reduced pressure and $\mathrm{Et}_{2} \mathrm{O}(10 \mathrm{~mL})$ was added to the resulting suspension. The mixture was filtered, and the filtrate was concentrated under reduced pressure. Purification by flash chromatography $\left(c \mathrm{C}_{6} \mathrm{H}_{12}: \mathrm{AcOEt}=8: 1,2.5 \mathrm{~cm}, 20 \mathrm{~cm}, 20 \mathrm{~mL}\right)$ afforded $(S)-21 \mathbf{a}(121 \mathrm{mg}, 58 \%$, $264 \mu \mathrm{mol})$ as a red oil. It represented a mixture of two rotamers in a keto and an enol form each [Z-enol-(S)-21a:E-enol-(S)-21a:Z-keto- $(S)-21 \mathbf{a}:$ E-keto- $(S)-21 \mathbf{a}=76: 16: 5: 3] .{ }^{9}$ This ratio was determined by integrating the 5-H signals [at $\delta=7.75$ (Z-keto) and 7.63 (E-keto)] ppm], by integrating the 4-H signals [at $\delta=6.33$ (Z-enol) and 6.46 (E-enol) $\mathrm{ppm}$ ] and by integrating the 3-OH signals [14.00 (Z-enol) and 14.08 (E-enol)].

$\mathbf{R}_{\mathbf{f}}\left(c \mathrm{C}_{6} \mathrm{H}_{12}: \mathrm{EtOAc}=8: 1\right): 0.35$

${ }^{1} \mathbf{H}$-NMR $\left(500.42 \mathrm{MHz}, \mathrm{CDCl}_{3} / \mathrm{CHCl}_{3}\right.$, sample contained 1 weight- $\%$ of $c \mathrm{C}_{6} \mathrm{H}_{12}$ and sample contained grease with $\mathrm{s}$ at 1.26, DsBrOk30-500600, 2.11.2020): $\delta=0.19$ [s, 6H, 1"-O-Si- 
Christian Drescher and Reinhard Brückner; 3D-Structure Clarifying Total Synthesis of the (Polyenoyl)tetramic Acid Militarinon B. A Highly Acid-Labile N-Protecting Group for Amides

$\left.\left(\mathrm{CH}_{3}\right)_{2}\right], 0.99$ [s, 9H, 1"-O-Si-C $\left.\left(\mathrm{CH}_{3}\right)_{3}\right], \mathrm{AB}$ signal $\left(\delta_{\mathrm{A}}=3.21, \delta_{\mathrm{B}}=3.30, J_{\mathrm{AB}}=14.3 \mathrm{~Hz}\right.$, in addition split by $\left.J_{3^{\prime}-\mathrm{H}^{\mathrm{A}}, 2^{\prime}}=5.5 \mathrm{~Hz}, J_{3^{\prime}-\mathrm{H}^{\mathrm{B}}, 2^{\prime}}=10.2 \mathrm{~Hz}, 2 \mathrm{H}, 3^{\prime}-\mathrm{H}_{2}\right), 3.70$ (s, 3H, 1'-OCH $), 3.99$ (d,

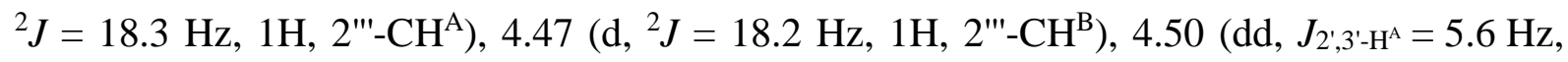
$\left.J_{2^{\prime}, 3^{\prime}-\mathrm{H}^{\mathrm{B}}}=10.1 \mathrm{~Hz}, 1 \mathrm{H}, 2^{\prime}-\mathrm{H}\right), 4.84(\mathrm{~s}, 1 \mathrm{H}, 2-\mathrm{H}), 6.33\left(\mathrm{~d}, J_{4,5}=13.5 \mathrm{~Hz}, 1 \mathrm{H}, 4-\mathrm{H}\right), 6.72-6.78$ (m, 2H, 2"-H, 6"-H), 6.95-7.00 (m, 2H, 3"-H, 5"-H), 7.23 (d, J5,4 = 13.5 Hz, 1H, 5-H), 7.28-7.38 (m, 2H, 3-H"', 5-H"'), 8.10-8.14 (m, 2H, 2-H'", 6-H"'), 13.90 (s, 1H, 3-OH) ppm.

${ }^{13}$ C-NMR (125.75 MHz, $\mathrm{CDCl}_{3} / \mathrm{CHCl}_{3}$, DsBrOk30-500604, 2.11.2020): $\delta=-4.3$ [1"-O-Si$\left(\mathrm{CH}_{3}\right)_{2}$ ], 18.3 [1"-O-Si- $C\left(\mathrm{CH}_{3}\right)_{3}$ ], 25.8 [1"-O-Si-C $\left(\mathrm{CH}_{3}\right)_{3}$ ], $34.1\left(\mathrm{C}-3\right.$ '), $51.6\left(4^{\prime \prime \prime-}-\mathrm{CH}_{2}\right), 52.5$ (1'$\left.\mathrm{OCH}_{3}\right), 61.7$ (C-2'), 89.6 (C-2), 118.0 (C-5), 120.5 (C-2", C-6"), 123.9 (C-2'", C-6"'), 127.5 (C3"', C-5"'), 130.1 (C-3", C-5"), 130.3 (C-4"), 132.2 (C-4), 143.7 (C-4"'), 147.5 (C-1"'), 154.9 (C1"), 168.1 (C-3), 170.6 (C-1'), 172.8 (C-1) ppm.

edHSQC [,,short-range H,C-COSY“, $\left.500.42 \mathrm{MHz} / 125.75 \mathrm{MHz}, \mathrm{CDCl}_{3} ; \delta\left({ }^{1} \mathrm{H}\right) \leftrightarrow \delta\left({ }^{13} \mathrm{C}\right)\right]$ : 0.19 [s, 1"-O-Si- $\left.\left(\mathrm{CH}_{3}\right)_{2}\right] \leftrightarrow-4.3$ [1"-O-Si- $\left.\left(\mathrm{CH}_{3}\right)_{2}\right], 0.99$ [s, 1"-O-Si-C $\left.\left(\mathrm{CH}_{3}\right)_{3}\right] \leftrightarrow 25.8$ [1"-O-Si$\left.\mathrm{C}\left(\mathrm{CH}_{3}\right)_{3}\right]$, AB signal $\left(\delta_{\mathrm{A}}=3.21, \delta_{\mathrm{B}}=3.30,3^{\prime}-\mathrm{H}_{2}\right) \leftrightarrow 34.6\left(\mathrm{C}-33^{\prime}\right), 3.70\left(\mathrm{~s}, 1^{\prime}-\mathrm{OCH}_{3}\right) \leftrightarrow 52.5\left(1^{\prime}-\right.$ $\left.\mathrm{OCH}_{3}\right), 3.99\left(\mathrm{~d}, 2^{\prime \prime \prime}-\mathrm{CH}^{\mathrm{A}}\right) \leftrightarrow 51.6\left(4^{\prime \prime \prime}-\mathrm{CH}_{2}\right), 4.50\left(\mathrm{dd}, 2^{\prime}-\mathrm{H}\right) \leftrightarrow 61.7\left(\mathrm{C}-2^{\prime}\right), 4.84(\mathrm{~s}, 2-\mathrm{H}) \leftrightarrow$ 89.6 (C-2), 6.33 (d, 4-H) 132.2 (C-4), 6.72-6.78 (m, 2"-H, 6"-H) 120.5 (C-2", C-6"), 6.957.00 (m, 3"-H, 5"-H) 130.2 (C-3", C-5"), 7.23 (d, 5-H) ↔ 118.0 (C-5), 7.28-7.38 (m, 3-H'", $\left.5-\mathrm{H}^{\prime \prime \prime}\right) \leftrightarrow 127.5$ (C-3"', C-5"'), 8.10-8.14 (m, 2-H"', 6-H'") 123.9 (C-2"', C-6"').

Specific rotation $(S)-21 \mathrm{a}[\alpha]_{D}^{20}=-170.2^{\circ}\left(\mathrm{c}=1.15\right.$ in $\left.\mathrm{CHCl}_{3}\right)$

HRMS: (EI, $70 \mathrm{eV}): \mathrm{m} / \mathrm{z}=617.1330[\mathrm{M}-\mathrm{H}]^{-}$, corresponds to the molecular formula $\mathrm{C}_{28} \mathrm{H}_{34} \mathrm{O}_{7} \mathrm{~N}_{2} \mathrm{SiBr}(617.1324)\left\{[\mathrm{M}-\mathrm{H}]^{-}\right\}$with a deviation of $0.9 \mathrm{ppm}$.

IR (KBr): $\tilde{v}=3435,2955,2085,1740,1635,1510,1470,1345,1255,1170,910 \mathrm{~cm}^{-1}$.

Methyl (2'S)-2'-\{N-[(1Z,2Z,4E,6E)-3-Hydroxy-7-phenylhepta-2,4,6-trienamido]- $N$-(1'"'nitrobenzyl)\}-3'-\{1'-[(tert-butyldimethylsilyl)oxy]phenyl $\}$ propanoate [Z-enol-(S)-279]

in equilibration with

Methyl (2'S)-2'-\{N-[(1E,2Z,4E,6E)-3-Hydroxy-7-phenylhepta-2,4,6-trienamido]- $N$-(1"'nitrobenzyl)\}-3'-\{1"'-[(tert-butyldimethylsilyl)oxy]phenyl $\}$ propanoate [E-enol-(S)-279]

in equilibration with 
Christian Drescher and Reinhard Brückner; 3D-Structure Clarifying Total Synthesis of the (Polyenoyl)tetramic Acid Militarinon B. A Highly Acid-Labile N-Protecting Group for Amides

Methyl (2'S)-2'-\{N-[(1Z,4E,6E)-3-Oxo-7-phenylhepta-4,6-dienamido]- $N$-(1'"'nitrobenzyl)\}-3'-\{1"'-[(tert-butyldimethylsilyl)oxy]phenyl\}propanoate [Z-keto-(S)-279]

in equilibration with

Methyl (2'S)-2'-\{N-[(1E,4E,6E)-3-Oxo-7-phenylhepta-4,6-dienamido]- $N$-(1'"'nitrobenzyl)\}-3'-\{1'"-[(tert-butyldimethylsilyl)oxy]phenyl $\}$ propanoate [E-keto-(S)-279]

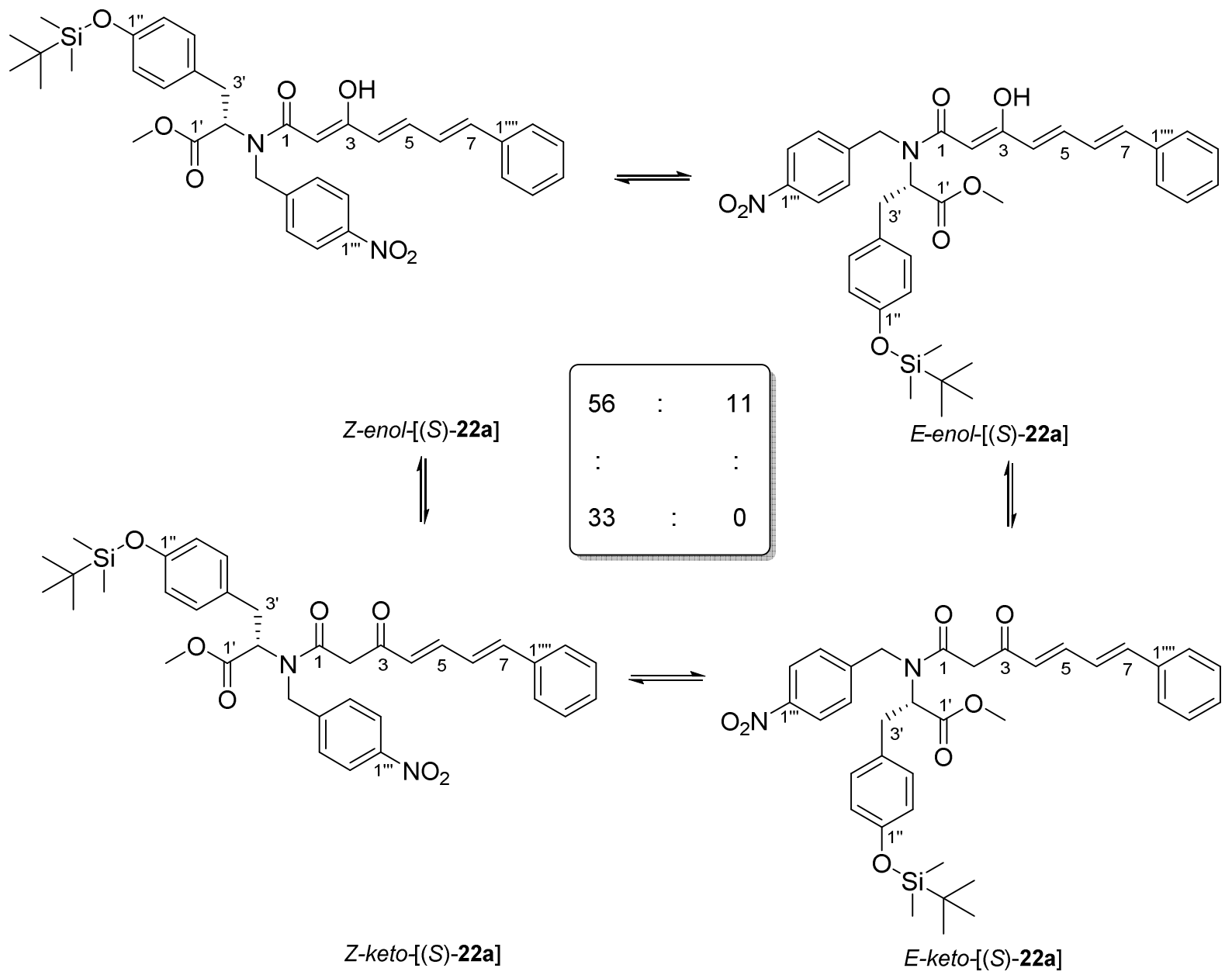

A solution of the western building block $(S)$-21a $(105 \mathrm{mg}, 172 \mu \mathrm{mol})$ and the stannane SI-7 (135 mg, $344 \mu \mathrm{mol}, 2.0$ eq.) in THF $(5.0 \mathrm{~mL})$ was deluded with $\operatorname{Pd}_{2}(\mathrm{dba})_{3}(16.0 \mathrm{mg}, 17.5 \mu \mathrm{mol}$, $10 \mathrm{~mol}-\%)$ and $\mathrm{AsPh}_{3}(13.5 \mathrm{mg}, 44.3 \mu \mathrm{mol}, 25 \mathrm{~mol}-\%)$. After $20 \mathrm{~h}$ at $25^{\circ} \mathrm{C}$ aq. KF solution $(8 \mathrm{M}, 4.5 \mathrm{~mL})$ was added and it was sonicated in the ultrasonic bath for $1 \mathrm{~min}$. The layers were separated, and the organic layer was extracted with $\mathrm{Et}_{2} \mathrm{O}(3 \times 20 \mathrm{~mL})$. The combined organic layers were washed with brine $(10 \mathrm{~mL})$, tried over $\mathrm{Na}_{2} \mathrm{SO}_{4}$ and concentrated under reduced pressure. The residue was purified by flash chromatography $\left(c \mathrm{C}_{6} \mathrm{H}_{12}\right.$ :AcOEt $=7: 1,2.0 \mathrm{~cm}$, $20 \mathrm{~cm}, 10 \mathrm{~mL})$. The polyen $(S)-22 \mathrm{a}(74 \mathrm{mg}, 68 \%, 117 \mu \mathrm{mol})$ was obtained as a pale brown oil. It represented a mixture of two rotamers in a keto and an enol form each [Z-enol-(S)-22a:E- 
Christian Drescher and Reinhard Brückner; 3D-Structure Clarifying Total Synthesis of the (Polyenoyl)tetramic Acid Militarinon B. A Highly Acid-Labile N-Protecting Group for Amides

enol-(S)-22a:Z-keto- $(S)-22 a: E-k e t o-(S)-22 a=56: 11: 33:] .{ }^{10}$ This ratio was determined by integrating the 4-H signals [at $\delta=5.86(Z$-enol), 6.02 (E-enol) ppm] and the 2'-H [at $\delta=3.42$ (Z-keto) ppm].

$\mathbf{R}_{\mathbf{f}}\left(c \mathrm{C}_{6} \mathrm{H}_{12}: \mathrm{EtOAc}=7: 1\right): 0.33$

${ }^{1} \mathrm{H}-\mathrm{NMR}\left(500.42 \mathrm{MHz}, \mathrm{CDCl}_{3} / \mathrm{CHCl}_{3}\right.$, sample contained 1 weight-\% of $\left(\mathrm{Bu}_{3} \mathrm{Sn}\right)_{2} \mathrm{O}$, DsBrOk30-500700, 2.11.2020): $\delta=0.20$ [s, 6H, 1"-O-Si- $\left.\left(\mathrm{CH}_{3}\right)_{2}\right], 0.99$ [s, 9H, 1"-O-Si$\left.\mathrm{C}\left(\mathrm{CH}_{3}\right)_{3}\right], \mathrm{AB}$ signal $\left(\delta_{\mathrm{A}}=3.22, \delta_{\mathrm{B}}=3.31, J_{\mathrm{AB}}=14.3 \mathrm{~Hz}\right.$, in addition split by $J_{3^{\prime}-\mathrm{H}^{\mathrm{A}}, 2^{\prime}}=5.7 \mathrm{~Hz}$, $\left.J_{3^{\prime}-\mathrm{H}^{\mathrm{B}}, 2^{\prime}}=10.2 \mathrm{~Hz}, 2 \mathrm{H}, 3^{\prime}-\mathrm{H}_{2}\right), 3.74\left(\mathrm{~s}, 3 \mathrm{H}, 1^{\prime}-\mathrm{OCH}_{3}\right), 4.01\left(\mathrm{~d},{ }^{2} J=18.3 \mathrm{~Hz}, 1 \mathrm{H}, 4^{\prime \prime}-\mathrm{CH}^{\mathrm{A}}\right), 4.47-$ $4.54\left(\mathrm{~m}, 2 \mathrm{H}, 2^{\prime}-\mathrm{H}, 4^{\prime \prime}-\mathrm{CH}^{\mathrm{B}}\right), 4.90(\mathrm{~s}, 1 \mathrm{H}, 2-\mathrm{H}), 5.86$ (d, J4,5 = $\left.15.1 \mathrm{~Hz}, 1 \mathrm{H}, 4-\mathrm{H}\right), 6.73-6.81$ (m, 4H, 6-H, 7-H, 2"-H, 6"-H), 6.97-7.04 (m, 2H, 3"-H, 5"-H), 7.20-7.25 (m, 1H, 5-H), 7.24-7.51 (m, 7H, 3"'-H, 5'"-H, 2"'--H, 3"'"-H, 4'"'-H, 5"'--H, 6"'--H), 8.10-8.16 (m, 2H, 2"'-H, 6"'-H), 13.96 $\left(\mathrm{d}, J_{3-\mathrm{OH}, 4}=1.4 \mathrm{~Hz}, 1-\mathrm{H}, 3-\mathrm{OH}\right) \mathrm{ppm}$.

${ }^{13} \mathrm{C}$-NMR $\left(125.75 \mathrm{MHz}, \mathrm{CDCl}_{3} / \mathrm{CHCl}_{3}\right.$, sample contained $\left(\mathrm{Bu}_{3} \mathrm{Sn}\right)_{2} \mathrm{O}, \mathrm{DsBrOk} 30-500704$, 2.11.2020): $\delta=-4.3$ [1"-O-Si- $\left(\mathrm{CH}_{3}\right)_{2}$ ], 18.3 [1"-O-Si- $C\left(\mathrm{CH}_{3}\right)_{3}$ ], 25.8 [1"-O-Si-C $\left(\mathrm{CH}_{3}\right)_{3}$ ], 34.8 (C-3'), 51.6 (4'"--CH2), 52.5 (1'-OCH 3 ), 61.6 (C-2'), 89.5 (C-2), 120.5 (C-2", C-6"), 123.6 (C2"', C-6"'), 126.2 (C-4), 127.0 (C-2'"', C-6'"'), 127.2 (C-6), 127.6 (C-1"'), 127.6 (C-3"', C-5"'), 128.6 (C-4'"'), 128.8 (C-3'"', C-5'"'), 130.2 (C-3", C-5"), 130.3 (C-4"), 137.3 (C-5), 138.2 (C-7), 144.2 (C-4"'), 147.6 (C-1"'), 154.8 (C-1"), 170.5 (C-3), 173.0 (C-1), 175.0 (C-1') ppm.

edHSQC [,,short-range H,C-COSY“, $\left.500.42 \mathrm{MHz} / 125.75 \mathrm{MHz}, \mathrm{CDCl}_{3} ; \delta\left({ }^{1} \mathrm{H}\right) \leftrightarrow \delta\left({ }^{13} \mathrm{C}\right)\right]$ : 0.20 [s, 1"-O-Si- $\left.\left(\mathrm{CH}_{3}\right)_{2}\right] \leftrightarrow-4.3$ [1"-O-Si- $\left.\left(\mathrm{CH}_{3}\right)_{2}\right], 0.99$ [s, 1"-O-Si-C $\left.\left(\mathrm{CH}_{3}\right)_{3}\right] \leftrightarrow 25.8$ [1"-O-Si$\left.\mathrm{C}\left(\mathrm{CH}_{3}\right)_{3}\right]$, AB signal $\left(\delta_{\mathrm{A}}=3.22, \delta_{\mathrm{B}}=3.31,3^{\prime}-\mathrm{H}_{2}\right) \leftrightarrow 34.8\left(\mathrm{C}-33^{\prime}\right), 3.74\left(\mathrm{~s}, 1^{\prime}-\mathrm{OCH}_{3}\right) \leftrightarrow 52.5\left(1^{\prime}-\right.$

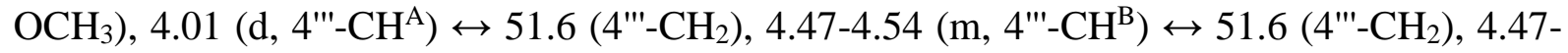
$4.54\left(\mathrm{~m}, 2^{\prime}-\mathrm{H}\right) \leftrightarrow 61.6\left(\mathrm{C}-2^{\prime}\right), 4.90(\mathrm{~s}, 2-\mathrm{H}) \leftrightarrow 89.5(\mathrm{C}-2), 5.86(\mathrm{~d}, 4-\mathrm{H}) \leftrightarrow 126.2(\mathrm{C}-4), 6.73-$ $6.81\left(\mathrm{~m}, 2^{\prime \prime}-\mathrm{H}, 6 "-\mathrm{H}\right) \leftrightarrow 120.5$ (C-2", C-6"), 6.73-6.81 (m, 6-H) ↔ 127.2 (C-6), 6.73-6.81 (m, 7-H) 138.2 (C-7), 6.97-7.04 (m, 3"-H, 5"-H) ↔ 130.2 (C-3", C-5"), 7.20-7.25 (m, 5-H) 137.3 (C-5), 7.24-7.51 (m, 3"'-H, 5'"-H) 127.6 (C-3"', C-5"'), 7.24-7.51 (m, 2'"'-H, 6"''-H) 127.0 (C-2"'", C-6'"'), 7.24-7.51 (m, 3'"'-H, 5"'-H) 128.8 (C-3"'", C-5"'"), 7.24-7.51 (m, 4"'-H) $\leftrightarrow 128.6$ (C-4"'"), 8.10-8.16 (m, 2"'-H, 6"'-H) 123.6 (C-2'", C-6"').

Specific rotation $(S)-279[\alpha]_{D}^{20}=-232.4^{\circ}\left(\mathrm{c}=0.59\right.$ in $\left.\mathrm{CHCl}_{3}\right)$

\footnotetext{
${ }^{10}$ An NOE experiment was not performed, but it can be assumed that the main compound is also Z-configured.
} 
Christian Drescher and Reinhard Brückner; 3D-Structure Clarifying Total Synthesis of the (Polyenoyl)tetramic Acid Militarinon B. A Highly Acid-Labile N-Protecting Group for Amides

HRMS: (EI, $70 \mathrm{eV}): \mathrm{m} / \mathrm{z}=677.2464[\mathrm{M}+\mathrm{Cl}]^{-}$, corresponds to the molecular formula $\mathrm{C}_{36} \mathrm{H}_{42} \mathrm{O}_{7} \mathrm{~N}_{2} \mathrm{SiCl}(677.2455)\left\{[\mathrm{M}+\mathrm{Cl}]^{-}\right\}$with a deviation of $1.3 \mathrm{ppm}$.

IR (KBr): $\tilde{v}=3030,2955,2930,2860,1740,1630,1610,1580,1570,1510,1470,1390,1345$, $1255,1170,1105,995,950,915,840,780,750,690 \mathrm{~cm}^{-1}$.

(5S)-5-\{1'-[(tert-Butyldimethylsilyl)oxy]benzyl\}-1-(1'"'-nitrobenzyl)-3-(1'Z,2'E,4'E)-1'hydroxy-5-phenylpenta-2',4''-dien-1'-ylidene)pyrrolidine-2,4-dion [Z-enol-(S)-16a] in equilibration with

(5S)-5-\{1"'-[(tert-Butyldimethylsilyl)oxy]benzyl\}-1-(1'"'nitrobenzyl)-3-(1'E,2' E,4'E)-1'hydroxy-5-phenylpenta-2',4' '-dien-1'-ylidene)pyrrolidine-2,4-dion [E-enol-(S)-16a]

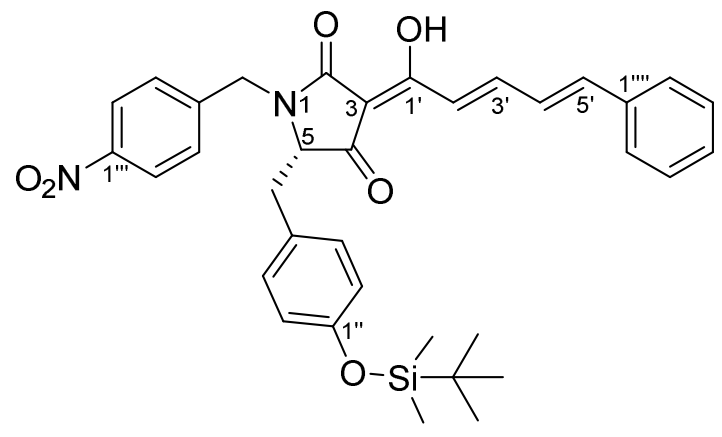

Z-enol-(S)-16a

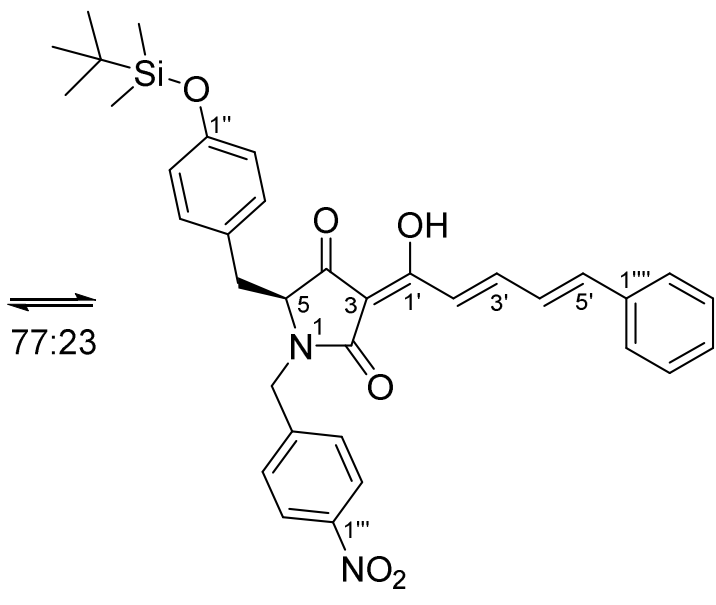

E-enol-(S)-16a

A solution of the polyen $(S)$-22a $(43 \mathrm{mg}, 69 \mu \mathrm{mol})$ in $\mathrm{MeOH}(8.0 \mathrm{~mL})$ was deluded with $\mathrm{NaOMe}\left(18.8 \mathrm{mg}, 345 \mu \mathrm{mol}, 5.0\right.$ eq.) at $25^{\circ} \mathrm{C}$. The mixture stirred at this temperature for $15 \mathrm{~min}$. It was added aq. citric acid (5 weight-\%, $5 \mathrm{~mL})$ and $\mathrm{Et}_{2} \mathrm{O}(15 \mathrm{~mL})$. The organic layer was separated, and the aq. layer was extracted with $\mathrm{Et}_{2} \mathrm{O}(3 \times 15 \mathrm{~mL})$. The organic layers were tried over $\mathrm{Na}_{2} \mathrm{SO}_{4}$ and the solvent was removed under reduced pressure. The residue was purified reverse phase flash chromatography $\left(\mathrm{MeCN}: \mathrm{H}_{2} \mathrm{O}: \mathrm{TFA}=\right.$ 95:5:0.1, $1.5 \mathrm{~cm}, 10 \mathrm{~cm}$, $2 \mathrm{~mL})$. The tetramic acid $(S)-\mathbf{1 6 a}(34.9 \mathrm{mg}, 85 \%, 59 \mu \mathrm{mol})$ was obtained as a yellow powder. It was assigned as a 77:23 mixture of the $Z$ - and the $E$-enol of the title compounds $(S)$-16a. This ratio was determined by integrating the triplets of the respective 5- $\mathrm{H}[\delta=3.80-3.85$ (Z-enol) and 3.94-3.97 (E-enol)]. 
Christian Drescher and Reinhard Brückner; 3D-Structure Clarifying Total Synthesis of the (Polyenoyl)tetramic Acid Militarinon B. A Highly Acid-Labile N-Protecting Group for Amides

$\mathbf{R}_{\mathbf{f}}\left(\mathrm{MeCN}: \mathrm{H}_{2} \mathrm{O}: \mathrm{TFA}=95: 5: 0.1\right): 0.39$

${ }^{1} \mathbf{H}-N M R$ (500.42 MHz, $\mathrm{CDCl}_{3} / \mathrm{CHCl}_{3}$, DsBrOk30-500800, 2.11.2020): $\delta=0.18$ [s, 6H, 1"-O$\mathrm{Si}-\left(\mathrm{CH}_{3}\right)_{2}$ ], 0.97 [s, 9H, 1"-O-Si-C $\left.\left(\mathrm{CH}_{3}\right)_{3}\right], \mathrm{AB}$ signal $\left(\delta_{\mathrm{A}}=2.93, \delta_{\mathrm{B}}=3.21, J_{\mathrm{AB}}=14.8 \mathrm{~Hz}\right.$, in addition split by $\left.J_{5-\mathrm{CH}^{\mathrm{A}}, 5}=3.7 \mathrm{~Hz}, J_{5-\mathrm{CH}^{\mathrm{B}}, 5}=7.0 \mathrm{~Hz}, 2 \mathrm{H}, 5-\mathrm{CH}_{2}\right), 3.80-3.85(\mathrm{~m}, 1 \mathrm{H}, 5-\mathrm{H}), 4.01$ $\left(\mathrm{d},{ }^{2} J=15.6 \mathrm{~Hz}, 1 \mathrm{H}, 4^{\prime \prime}-\mathrm{CH}^{\mathrm{A}}\right), 5.19\left(\mathrm{~d},{ }^{2} J=15.2 \mathrm{~Hz}, 1 \mathrm{H}, 2^{\prime \prime}-\mathrm{CH}^{\mathrm{B}}\right), 6.72-6.77$ (m, 2H, 2"-H, 6"H), 6.93-6.98 (m, 2H, 3"-H, 5"-H), 6.99-7.09 (m, 2H, 4'-H, 5'-H), 7.13-7.17 (m, 2H, 3"'-H, 5'"H), 7.30 (d, $\left.J_{2^{\prime}, 3^{\prime}}=15.2 \mathrm{~Hz}, 1 \mathrm{H}, 2^{\prime}-\mathrm{H}\right), 7.33-7.41$ (m, 3H, 3"''-H, 4'"'-H, 5'"'-H), 7.49-7.56 (m, $2 \mathrm{H}, 2^{\prime \prime \prime '-H, ~ 6 ' " '-H), ~} 7.65$ (dd, $\left.J_{3^{\prime}, 2^{\prime}}=15.4 \mathrm{~Hz}, J_{3^{\prime}, 4^{\prime}}=10.0 \mathrm{~Hz}, 1 \mathrm{H}, 3^{\prime}-\mathrm{H}\right), 8.11-8.15$ (m, 2H, 2"'-H, 6"'-H) ppm.

${ }^{13} \mathrm{C}-\mathrm{NMR}\left(125.75 \mathrm{MHz}, \mathrm{CDCl}_{3} / \mathrm{CHCl}_{3}\right.$, DsBrOk30-500804, 2.11.2020): $\delta=-4.3[1$ ["-O-Si$\left(\mathrm{CH}_{3}\right)_{2}$ ], 18.3 [1"-O-Si- $\mathrm{C}\left(\mathrm{CH}_{3}\right)_{3}$ ], 25.8 [1"-O-Si-C $\left(\mathrm{CH}_{3}\right)_{3}$ ], $36.0\left(5-\mathrm{CH}_{2}\right), 43.5\left(4\right.$ '"- $\left.\mathrm{CH}_{2}\right), 65.3$ (C-5), 99.9 (C-3), 120.5 (C-2", C-6"), 121.4 (C-2'), 124.1 (C-2"', C-6"'), 127.3 (C-4'), 127.7 (C2'"', C-6"''), 128.9 (C-3'", 5-H'"'), 129.1 (C-3'"', C-5'"'), 129.8 (C-4'"'), 130.3 (C-3", C-4", C-5"), 136.0 (C-1'"'), 143.3 (C-4'"), 143.3 (C-5'), 145.4 (C-3'), 147.7 (C-1'"), 155.0 (C-1"), 173.7 (C1'), 174.0 (C-2), 193.1 (C-4) ppm.

edHSQC [,short-range H,C-COSY“, $\left.500.42 \mathrm{MHz} / 125.75 \mathrm{MHz}, \mathrm{CDCl}_{3} ; \delta\left({ }^{1} \mathrm{H}\right) \leftrightarrow \delta\left({ }^{13} \mathrm{C}\right)\right]$ : 0.18 [s, 6H, 1"-O-Si- $\left.\left(\mathrm{CH}_{3}\right)_{2}\right] \leftrightarrow-4.3$ [1"-O-Si- $\left.\left(\mathrm{CH}_{3}\right)_{2}\right], 0.97$ [s, 1"-O-Si-C $\left.\left(\mathrm{CH}_{3}\right)_{3}\right] \leftrightarrow 25.8$ [1"O-Si- $\left.\mathrm{C}\left(\mathrm{CH}_{3}\right)_{3}\right], \mathrm{AB}$ signal $\left(\delta_{\mathrm{A}}=2.93, \delta_{\mathrm{B}}=3.21,5-\mathrm{CH}_{2}\right) \leftrightarrow 36.0\left(5-\mathrm{CH}_{2}\right), 3.80-3.85(\mathrm{~m}, 5-\mathrm{H})$

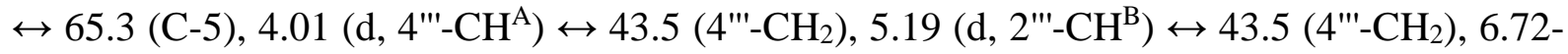
6.77 (m, 2"-H, 6"-H) ↔ 120.5 (C-2", C-6"), 6.93-6.98 (m, 3"-H, 5"-H) ↔ 130.3 (C-3", C-5"), 6.99-7.09 (m, 4'-H) $127.3\left(\mathrm{C}-4{ }^{\prime}\right), 6.99-7.09$ (m, 5'-H) 143.3 (C-5'), 7.13-7.17 (m, 3"'-H, 5"'-H) 128.9 (C-3"', 5-H'"), 7.30 (d, 2'-H) ↔ 121.4 (C-2'), 7.33-7.41 (m, 3'"'-H, 5"'-H) ↔ 129.1 (C-3"'', C-5"''), 7.33-7.41 (m, 4"''-H) ↔ 129.8 (C-4"'"), 7.49-7.56 (m, 2"'-H, 6"'--H) ↔ 127.7 (C-2"'", C-6"'"), 7.65 (dd, 3'-H) ↔ 145.4 (C-3'), 8.11-8.15 (m, 2"'-H, 6"'-H) 124.1 (C2"', C-6"').

Specific rotation $(S)-\mathbf{1 6 a}[\alpha]_{D}^{20}=-719.5^{\circ}\left(\mathrm{c}=0.38\right.$ in $\left.\mathrm{CHCl}_{3}\right)$

HRMS: (EI, $70 \mathrm{eV}): \mathrm{m} / \mathrm{z}=609.2427[\mathrm{M}-\mathrm{H}]^{+}$, corresponds to the molecular formula $\mathrm{C}_{35} \mathrm{H}_{37} \mathrm{O}_{6} \mathrm{~N}_{2} \mathrm{Si}(609.2426)\left\{[\mathrm{M}-\mathrm{H}]^{+}\right\}$with a deviation of $0.2 \mathrm{ppm}$.

IR (KBr): $\tilde{v}=3030,2955,2930,2860,1695,1645,1610,1595,1560,1525,1510,1450,1415$, $1345,1265,1170,1105,1000,955,915,840,780,750,690,630,610 \mathrm{~cm}^{-1}$. 


\section{Methyl (2S)-3-\{1'-[(tert-Butyldimethylsilyl)oxy]phenyl\}-2-[(4",5"-dimethoxy-1"- nitrobenzyl)amino]propanoate propanoate $[(S)-18 c]$}

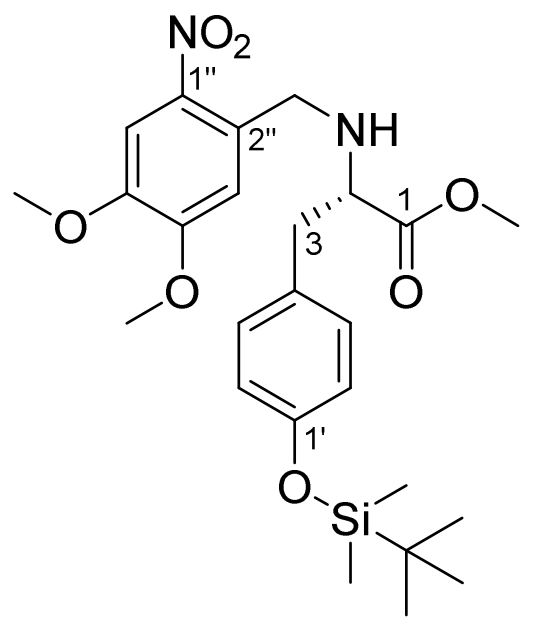

(S)-18c

The amine $(S)$-20 (300 mg, $0.96 \mathrm{mmol})$ was dissolved in $\mathrm{MeOH}(15 \mathrm{~mL})$. It was added HOAc (12 drops) and 4,5-dimethoxy-2-nitrobenzaldehyd (207 mg, $1.06 \mathrm{mmol}, 1.1$ eq.). After $1 \mathrm{~min}$ at $25^{\circ} \mathrm{C}$ it was added $\mathrm{NaBH}_{3} \mathrm{CN}(107 \mathrm{mg}, 1.44 \mathrm{mmol}, 1.5$ eq. $)$. The mixture was stirred at $25^{\circ} \mathrm{C}$ for $5 \mathrm{~h}$. The solvent was removed under reduced pressure. It was added $\mathrm{H}_{2} \mathrm{O}(10 \mathrm{~mL})$ and $\mathrm{Et}_{2} \mathrm{O}$ $(20 \mathrm{~mL})$. The layers were separated, and aq. layer was extracted with $\mathrm{Et}_{2} \mathrm{O}(3 \times 25 \mathrm{~mL})$. The combined organic layers were dried over $\mathrm{MgSO}_{4}$ and concentrated under reduced pressure. Purification by flash chromatography $\left(c \mathrm{C}_{6} \mathrm{H}_{12}: \mathrm{AcOEt}=4: 1,3.5 \mathrm{~cm}, 20 \mathrm{~cm}, 30 \mathrm{~mL}\right)$ afforded $(S)-18 c$ (327 mg, 67\%, $0.64 \mathrm{mmol})$ as a yellow oil.

$\mathbf{R}_{\mathbf{f}}\left(c \mathrm{C}_{6} \mathrm{H}_{12}: \mathrm{EtOAc}=4: 1\right): 0.30$

1H-NMR (500.42 MHz, CDCl $/ \mathrm{CHCl}_{3}$, DsBrOk23-500700, 26.10.2020): $\delta=0.17$ [s, 6H, 1'O-Si- $\left.\left(\mathrm{CH}_{3}\right)_{2}\right], 0.96$ [s, 9H, 1'-O-Si-C $\left.\left(\mathrm{CH}_{3}\right)_{3}\right], 2.10$ (br. s, $\left.1 \mathrm{H}, N \mathrm{H}\right), \mathrm{AB}$ signal $\left(\delta_{\mathrm{A}}=2.85\right.$, $\delta_{\mathrm{B}}=2.89, J_{\mathrm{AB}}=13.4 \mathrm{~Hz}$, in addition split by $\left.J_{3-\mathrm{H}^{\mathrm{A}}, 2}=6.1 \mathrm{~Hz}, J_{3-\mathrm{H}^{\mathrm{B}}, 2}=7.8 \mathrm{~Hz}, 2 \mathrm{H}, 3-\mathrm{H}_{2}\right), 3.48$ $\left(\mathrm{dd}, J_{2,3-\mathrm{H}^{\mathrm{A}}}=6.1 \mathrm{~Hz}, J_{2,3-\mathrm{H}^{\mathrm{B}}}=7.8 \mathrm{~Hz}, 1 \mathrm{H}, 2-\mathrm{H}\right), 3.64\left(\mathrm{~s}, 3 \mathrm{H}, 1-\mathrm{OCH}_{3}\right), 3.86\left(\mathrm{~s}, 3 \mathrm{H}, 5^{\prime \prime}-\mathrm{OCH}_{3}\right)$, $3.91\left(\mathrm{~s}, 3 \mathrm{H}, 4 "-\mathrm{OCH}_{3}\right), 3.93\left(\mathrm{~d},{ }^{2} J=15.4 \mathrm{~Hz}, 1 \mathrm{H}, 2^{2}-\mathrm{CH}^{\mathrm{A}}\right), 4.11\left(\mathrm{~d},{ }^{2} J=15.4 \mathrm{~Hz}, 1 \mathrm{H}, 2^{\prime \prime}-\mathrm{CH}^{\mathrm{B}}\right)$, 6.71-6.74 (m, 2H, 2'-H, 6'-H), 7.00-7.03 (m, 2H, 3'-H, 5'-H), 7.05 (s, 1H, 3"-H), 7.58 (s, 1H, 6"H) ppm.

${ }^{13} \mathrm{C}-\mathrm{NMR}$ (125.75 MHz, $\mathrm{CDCl}_{3} / \mathrm{CHCl}_{3}$, DsBrOk23-500704, 26.10.2020): $\delta=-4.4$ [1'- O-Si$\left(\mathrm{CH}_{3}\right)_{2}$ ], 18.2 [1'-O-Si- $C\left(\mathrm{CH}_{3}\right)_{3}$ ], 25.7 [1'-O-Si-C $\left(\mathrm{CH}_{3}\right)_{3}$ ], 39.1 (C-3), 49.3 (2"- $\left.\mathrm{CH}_{2}\right), 51.8$ (1$\left.\mathrm{OCH}_{3}\right), 56.4$ (5"--OCH $), 56.4$ (4"--OCH 3 ), 62.8 (C-2), 108.2 (C-6"), 112.1 (C-3"), 120.0 (C-2', 
Christian Drescher and Reinhard Brückner; 3D-Structure Clarifying Total Synthesis of the (Polyenoyl)tetramic Acid Militarinon B. A Highly Acid-Labile N-Protecting Group for Amides

C-6'), 129.9 (C-4'), 130.2 (C-3', C-5'), 130.9 (C-2"), 140.8 (C-1"), 147.6 (C-4"), 153.3 (C-5"), $154.6\left(\mathrm{C}-1{ }^{\prime}\right), 174.9$ (C-1) ppm.

edHSQC [,,short-range H,C-COSY“, 500.06 MHz/125.75 MHz, $\mathrm{CDCl}_{3} ; \delta\left({ }^{1} \mathrm{H}\right) \leftrightarrow \delta\left({ }^{13} \mathrm{C}\right)$ ]: 0.17 [s, 1'-O-Si- $\left.\left(\mathrm{CH}_{3}\right)_{2}\right] \leftrightarrow-4.4$ [1'- O-Si- $\left.\left(\mathrm{CH}_{3}\right)_{2}\right], 0.96$ [s, 1'-O-Si-C $\left.\left(\mathrm{CH}_{3}\right)_{3}\right] \leftrightarrow 25.7$ [1'-O-Si$\left.\mathrm{C}\left(\mathrm{CH}_{3}\right)_{3}\right]$, AB signal $\left(\delta_{\mathrm{A}}=2.85, \delta_{\mathrm{B}}=2.89,3-\mathrm{H}_{2}\right) \leftrightarrow 39.1(\mathrm{C}-3), 3.48(\mathrm{dd}, 2-\mathrm{H}) \leftrightarrow 62.8(\mathrm{C}-2)$, $3.64\left(\mathrm{~s}, 1-\mathrm{OCH}_{3}\right) \leftrightarrow 51.8\left(1-\mathrm{OCH}_{3}\right), 3.86\left(\mathrm{~s}, 5^{\prime \prime}-\mathrm{OCH}_{3}\right) \leftrightarrow 56.4\left(5^{\prime \prime}-\mathrm{OCH}_{3}\right), 3.91\left(\mathrm{~s}, 4 "-\mathrm{OCH}_{3}\right)$ $\leftrightarrow 56.4\left(4 "-\mathrm{OCH}_{3}\right), 3.93\left(\mathrm{~d}, 2 "-\mathrm{CH}^{\mathrm{A}}\right) \leftrightarrow 49.3\left(2 "-\mathrm{CH}_{2}\right), 4.11\left(\mathrm{~d}, 2^{\prime \prime}-\mathrm{CH}^{\mathrm{B}}\right) \leftrightarrow 49.3\left(2 "-\mathrm{CH}_{2}\right)$, 6.71-6.74 (m, 2'-H, 6'-H) $120.0\left(\mathrm{C}^{\prime} 2^{\prime}, \mathrm{C}^{\prime}-6^{\prime}\right), 7.00-7.03\left(\mathrm{~m}, 3^{\prime}-\mathrm{H}, 5^{\prime}-\mathrm{H}\right) \leftrightarrow 130.2\left(\mathrm{C}-3^{\prime}, \mathrm{C}^{\prime} 5^{\prime}\right)$, 7.05 (s, 3"-H) 112.1 (C-3"), 7.58 (s, 6"-H) 108.2 (C-6").

HRMS: $(\mathrm{EI}, 70 \mathrm{eV}): \mathrm{m} / \mathrm{z}=505.2371[\mathrm{M}+\mathrm{H}]^{+}$, corresponds to the molecular formula $\mathrm{C}_{25} \mathrm{H}_{37} \mathrm{O}_{7} \mathrm{~N}_{2} \mathrm{Si}(505.2365)\left\{[\mathrm{M}+\mathrm{H}]^{+}\right\}$with a deviation of $1.3 \mathrm{ppm}$.

Specific rotation $(S)-\mathbf{1 8 c}[\alpha]_{D}^{20}=-0.2^{\circ}\left(\mathrm{c}=0.95\right.$ in $\left.\mathrm{CHCl}_{3}\right)$

IR (KBr): $\tilde{v}=2950,2930,2855,1735,1610,1580,1510,1465,1440,1330,1270,1220,1170$, $1065,990,915,840,795,780 \mathrm{~cm}^{-1}$.

Methyl (2'S)-2'- $\{N-[(1 Z, 2 Z, 4 E)-5-B r o m o-3-h y d r o x y-1-0 x o y p e n t a-2,4-d i e n y l]-N-(4 ' ", 5 ' "$ dimethoxy-1"'-nitrobenzyl) $\}-3$ '- $\{1$ '-[(tert-butyldimethylsilyl)oxy $]$ phenyl $\}$ propanoate $[Z$ enol-(S)-21c]

in equilibration with

Methyl (2'S)-2'-\{ $N$-[(1E,2Z,4E)-5-Bromo-3-hydroxy-1-oxoypenta-2,4-dienyl]- $N$-(4"',5'"'dimethoxy-1"'-nitrobenzyl) $\}-3$ '- $\{1$ "-[(tert-butyldimethylsilyl)oxy $]$ phenyl $\}$ propanoate $[E-$ enol-(S)-21c]

in equilibration with

Methyl (2'S)-2'-\{N-[(1Z,4E)-5-Bromo-1,3-dioxopent-4-enyl]- $N-(4$ "',5'"-dimethoxy-1'"'nitrobenzyl) $\}-3$ '- $\left\{1^{\prime \prime}-[(\right.$ tert-butyldimethylsilyl)oxy $]$ phenyl $\}$ propanoate [Z-keto-(S)-21c]

in equilibration with

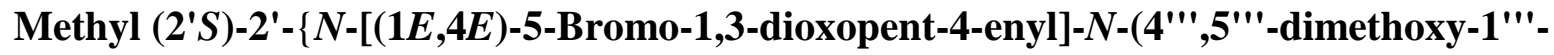
nitrobenzyl) $\}-3^{\prime}-\left\{1^{\prime \prime}-[(\right.$ tert-butyldimethylsilyl)oxy]phenyl $\}$ propanoate [E-keto-(S)- 21c] 


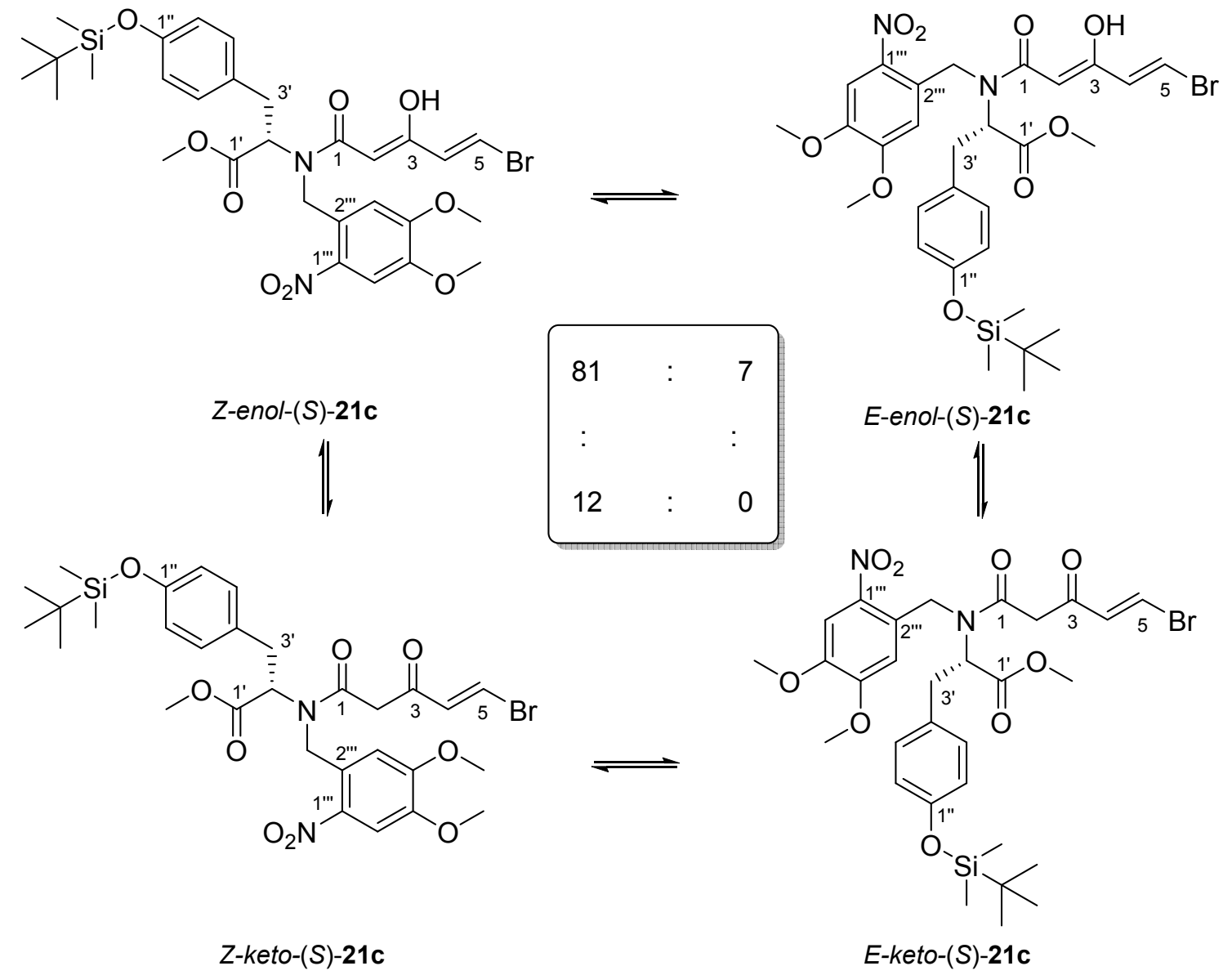

A solution of $N$-DMNB-tyrosine methyl ester $(S)$-18c $(164 \mathrm{mg}, 320 \mu \mathrm{mol})$ and the $\beta$ ketothioester 11 (95 mg, $480 \mu \mathrm{mol}, 1.5 \mathrm{eq}$.) in THF (5 mL) was treated with powdered molecular sieves $4 \AA$ ( $0.8 \mathrm{~g})$. The suspension stirred at $25^{\circ} \mathrm{C}$ for $30 \mathrm{~min}$. Then $\mathrm{AgO}_{2} \mathrm{CCF}_{3}$ (95 mg, $480 \mu \mathrm{mol}, 1.5$ eq.) was added and the mixture was stirred for $18 \mathrm{~h}$ at $25^{\circ} \mathrm{C}$. The solvent was removed under reduced pressure and $\mathrm{Et}_{2} \mathrm{O}(10 \mathrm{~mL})$ was added to the resulting suspension. The mixture was filtered, and the filtrate was concentrated under reduced pressure. Purification by flash chromatography $\left(c \mathrm{C}_{6} \mathrm{H}_{12}\right.$ :AcOEt $\left.=7: 1,2.0 \mathrm{~cm}, 20 \mathrm{~cm}, 20 \mathrm{~mL}\right)$ afforded $(S)$-21c $(152 \mathrm{mg}, 69 \%, 221 \mu \mathrm{mol})$ as a red oil. It represented a mixture of two rotamers in a keto and an enol form each [Z-enol-(S)-21c:E-enol- $(S)-21 \mathbf{c}: Z-k e t o-(S)-21 \mathbf{c}:$ E-keto- $(S)-21 \mathbf{c}=81: 7: 12: 0]{ }^{10}$ This ratio was determined by integrating the 4-H signals [at $\delta=6.33$ (Z-enol) und 6.45 (E-enol) ppm], by integrating the 2-H signal [at $\delta=3.58$ (Z-keto)] and by integrating the 3-OH signals [14.00 (Z-enol) and $14.08($ E-enol)].

$\mathbf{R}_{\mathbf{f}}\left(c \mathrm{C}_{6} \mathrm{H}_{12}: \mathrm{EtOAc}=8: 1\right): 0.35$

${ }^{1} \mathbf{H}$-NMR $\left(500.42 \mathrm{MHz}, \mathrm{CDCl}_{3} / \mathrm{CHCl}_{3}\right.$, sample contained 7 weight-\% of $c \mathrm{C}_{6} \mathrm{H}_{12}$, DsBrOk23500800, 26.10.2020): $\delta=0.17$ [s, 6H, 1"-O-Si- $\left.\left(\mathrm{CH}_{3}\right)_{2}\right], 0.98$ [s, 9H, 1"-O-Si-C( $\left.\left(\mathrm{CH}_{3}\right)_{3}\right], \mathrm{AB}$ signal $\left(\delta_{\mathrm{A}}=3.26, \delta_{\mathrm{B}}=3.36, J_{\mathrm{AB}}=14.2 \mathrm{~Hz}\right.$, in addition split by $J_{3^{\prime}-\mathrm{H}^{\mathrm{A}}, 2^{\prime}}=5.4 \mathrm{~Hz}, J_{3^{\prime}-}$ 
Christian Drescher and Reinhard Brückner; 3D-Structure Clarifying Total Synthesis of the (Polyenoyl)tetramic Acid Militarinon B. A Highly Acid-Labile N-Protecting Group for Amides

$\left.\mathrm{H}^{\mathrm{B}, 2^{\prime}}=9.9 \mathrm{~Hz}, 2 \mathrm{H}, 3{ }^{\prime}-\mathrm{H}_{2}\right), 3.73\left(\mathrm{~s}, 3 \mathrm{H}, 1^{\prime}-\mathrm{OCH}_{3}\right), 3.90\left(\mathrm{~s}, 3 \mathrm{H}, 5\right.$ ''- $\left.-\mathrm{OCH}_{3}\right), 3.94(\mathrm{~s}, 3 \mathrm{H}, 4$ '"--OCH 3$)$,

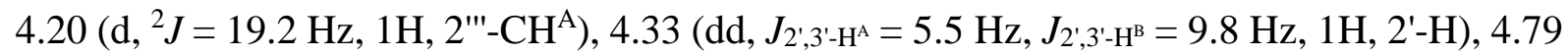
$\left(\mathrm{d},{ }^{2} J=19.2 \mathrm{~Hz}, 1 \mathrm{H}, 2 " '-\mathrm{CH}^{\mathrm{B}}\right), 4.79$ (s, 1H, 2-H), 6.33 (d, $\left.J_{4,5}=13.6 \mathrm{~Hz}, 1 \mathrm{H}, 4-\mathrm{H}\right), 6.76-6.80$ (m, 2H, 2"-H, 6"-H), 7.00-7.03 (m, 2H, 3"-H, 5'-H), 7.22 (d, J5,4 = 13.5 Hz, 1H, 5-H), 7.32 (s, 1H, 3"'-H), 7.71 (s, 1H, 6'"-H), 13.84 (s, 1-H, 3-OH) ppm.

${ }^{13}$-NMR (125.75 MHz, $\mathrm{CDCl}_{3} / \mathrm{CHCl}_{3}$, sample contained $c \mathrm{C}_{6} \mathrm{H}_{12}$, DsBrOk23-500804, 26.10.2020): $\delta=-4.4$ [1"-O-Si- $\left(\mathrm{CH}_{3}\right)_{2}$ ], 18.3 [1"-O-Si- $C\left(\mathrm{CH}_{3}\right)_{3}$ ], 25.8 [1"-O-Si-C $\left(\mathrm{CH}_{3}\right)_{3}$ ], 34.7 (C-3'), 50.9 (2"'-- $\left.\mathrm{CH}_{2}\right), 52.6\left(1^{\prime}-\mathrm{OCH}_{3}\right), 56.5\left(4\right.$ '"- $\left.-\mathrm{OCH}_{3}\right), 56.9\left(5^{\prime \prime}-\mathrm{OCH}_{3}\right), 62.7(\mathrm{C}-2$ '), $89.8(\mathrm{C}-$ 2), 108.7 (C-6"'), 110.8 (C-3"'), 117.8 (C-5), 120.6 (C-2", C-6"), 127.7 (C-2"'), 129.7 (C-4"), 130.1 (C-3", 5"), 132.3 (C-4), 139.4 (C-1"'), 148.0 (C-4"'), 154.1 (C-5"'), 154.9 (C-1"), 167.9 (C-3), 170.5 (C-1'), 173.1 (C-1) ppm.

edHSQC [,,short-range H,C-COSY“, 500.06 MHz/125.75 MHz, $\left.\mathrm{CDCl}_{3} ; \delta\left({ }^{1} \mathrm{H}\right) \leftrightarrow \delta\left({ }^{13} \mathrm{C}\right)\right]$ : 0.17 [s, 1"-O-Si- $\left.\left(\mathrm{CH}_{3}\right)_{2}\right] \leftrightarrow-4.4$ [1"-O-Si- $\left.\left(\mathrm{CH}_{3}\right)_{2}\right], 0.98$ [s, 1"-O-Si-C $\left.\left(\mathrm{CH}_{3}\right)_{3}\right] \leftrightarrow 25.8$ [1"-O-Si$\left.\mathrm{C}\left(\mathrm{CH}_{3}\right)_{3}\right]$, AB signal $\left(\delta_{\mathrm{A}}=3.26, \delta_{\mathrm{B}}=3.36,3^{\prime}-\mathrm{H}_{2}\right) \leftrightarrow 34.7\left(\mathrm{C}-33^{\prime}\right), 3.73\left(\mathrm{~s}, 1^{\prime}-\mathrm{OCH}_{3}\right) \leftrightarrow 52.6\left(1^{\prime}-\right.$ $\left.\mathrm{OCH}_{3}\right), 3.90(\mathrm{~s}, 5$ '"--OCH 3$) \leftrightarrow 56.9\left(5\right.$ "'- $\left.\mathrm{OCH}_{3}\right), 3.94(\mathrm{~s}, 4$ '"--OCH 3$) \leftrightarrow 56.5\left(4 " '-\mathrm{OCH}_{3}\right), 4.20(\mathrm{~d}$,

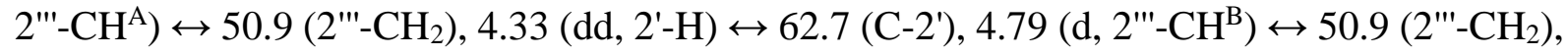
$4.79(\mathrm{~s}, 2-\mathrm{H}) \leftrightarrow 89.8(\mathrm{C}-2), 6.33(\mathrm{~d}, 4-\mathrm{H}) \leftrightarrow 132.3(\mathrm{C}-4), 6.76-6.80(\mathrm{~m}, 2$ "-H, 6"-H) 120.6 (C-2", C-6"), 7.00-7.03 (m, 3"-H, 5'-H) ↔ 130.1 (C-3", 5"), 7.22 (d, 5-H) ↔ 117.8 (C-5), 7.32 $\left(\mathrm{s}, 3\right.$ ''-H) $110.8\left(\mathrm{C}-3\right.$ '"), $7.71\left(\mathrm{~s}, 6^{\prime \prime \prime-H}\right) \leftrightarrow 108.7$ (C-6'").

HRMS: $($ EI, $70 \mathrm{eV}): \mathrm{m} / \mathrm{z}=681.1659[\mathrm{M}+\mathrm{H}]^{+}$, corresponds to the molecular formula $\mathrm{C}_{30} \mathrm{H}_{40} \mathrm{O}_{9} \mathrm{~N}_{2}{ }^{81} \mathrm{BrSi}(681.1660)\left\{[\mathrm{M}+\mathrm{H}]^{+}\right\}$with a deviation of $-0.2 \mathrm{ppm}$.

Specific rotation $(S)-21 \mathrm{c}[\alpha]_{D}^{20}=-140.1^{\circ}\left(\mathrm{c}=0.73\right.$ in $\left.\mathrm{CHCl}_{3}\right)$

IR (KBr): $\tilde{v}=3425,2955,2855,1740,1635,1585,1525,1510,1475,1440,1365,1330,1275$, $1220,1175,1105,1060,1015,945,915,840,780,755,550 \mathrm{~cm}^{-1}$. 
Christian Drescher and Reinhard Brückner; 3D-Structure Clarifying Total Synthesis of the (Polyenoyl)tetramic Acid Militarinon B. A Highly Acid-Labile N-Protecting Group for Amides

Methyl (2'S)-2'-\{N-[(1Z,2Z,4E,6E)-3-Hydroxy-7-phenylhepta-2,4,6-trienamido]- $N$ $(4 " ', 5$ '"'-dimethoxy-1'"'-nitrobenzyl $)\}-3$ '-\{1"'-[(tert-

butyldimethylsilyl)oxy]phenyl\}propanoate [Z-enol-(S)-22c]

in equilibration with

Methyl (2'S)-2' -\{N-[(1E,2Z,4E,6E)-3-Hydroxy-7-phenylhepta-2,4,6-trienamido]- $N$ (4'"',5"'-dimethoxy-1'"-nitrobenzyl) $\}-3$ '-\{1"-[(tertbutyldimethylsilyl)oxy]phenyl\}propanoate $[E-e n o l-(S)-22 c]$

in equilibration with

Methyl (2'S)-2'-\{N-[(1Z,4E,6E)-3-Oxo-7-phenylhepta-4,6-dienamido]- $N-\left(4{ }^{\prime \prime \prime}, 5\right.$ '"'dimethoxy-1"'-nitrobenzyl)\}-3'-\{1"'-[(tert-butyldimethylsilyl)oxy]phenyl\}propanoate [Zketo-(S)-22c]

in equilibration with

Methyl (2'S)-2'-\{N-[(1E,4E,6E)-3-Oxo-7-phenylhepta-4,6-dienamido]- $N$-(4'"',5'"'dimethoxy-1"'-nitrobenzyl)\}-3'-\{1'-[(tert-butyldimethylsilyl)oxy]phenyl $\}$ propanoate $[E$ keto-(S)-22c] 


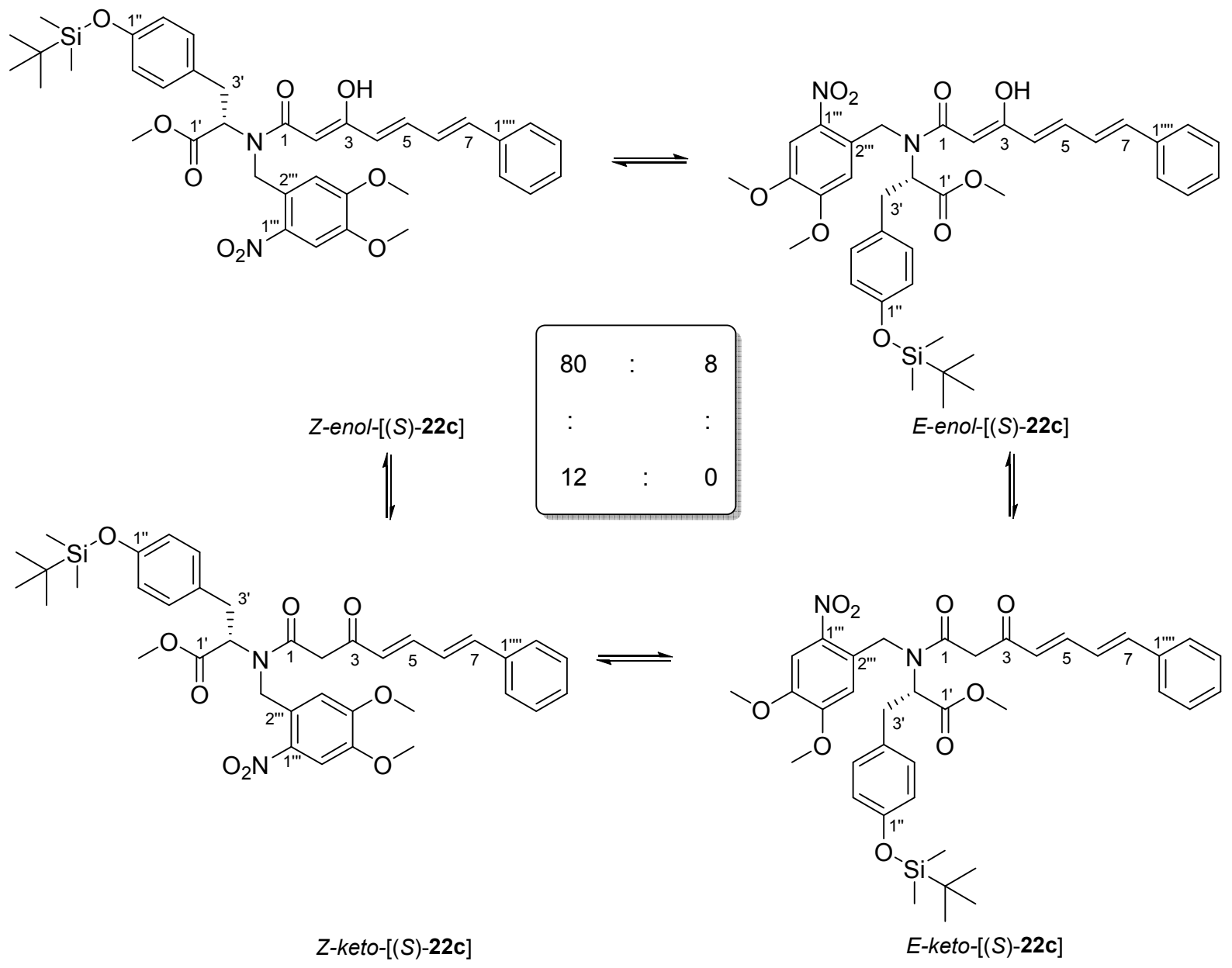

A solution of the western building block $(S)$-21c $(89 \mathrm{mg}, 132 \mu \mathrm{mol})$ and the stannane SI-7 (104 mg, $344 \mu \mathrm{mol}, 2.0$ eq.) in THF (5.0 mL) was deluded with $\operatorname{Pd}_{2}(\mathrm{dba})_{3}(6.2 \mathrm{mg}, 6.7 \mu \mathrm{mol}$, $5 \mathrm{~mol}-\%)$ and $\mathrm{AsPh}_{3}(10.4 \mathrm{mg}, 34.0 \mu \mathrm{mol}, 25 \mathrm{~mol}-\%)$. After $20 \mathrm{~h}$ at $25^{\circ} \mathrm{C}$ aq. $\mathrm{KF}$ solution $(8 \mathrm{M}$, $4.5 \mathrm{~mL}$ ) was added and it was sonicated in the ultrasonic bath for $1 \mathrm{~min}$. The layers were separated, and the organic layer was extracted with $\mathrm{Et}_{2} \mathrm{O}(3 \times 15 \mathrm{~mL})$. The combined organic layers were washed with brine $(10 \mathrm{~mL})$, tried over $\mathrm{Na}_{2} \mathrm{SO}_{4}$ and concentrated under reduced pressure. The residue was purified by flash chromatography $\left(c \mathrm{C}_{6} \mathrm{H}_{12}\right.$ :AcOEt $=7: 1,2.0 \mathrm{~cm}$, $20 \mathrm{~cm}, 10 \mathrm{~mL})$. The polyen $(S)-22 \mathrm{c}(65.7 \mathrm{mg}, 65 \%, 86 \mu \mathrm{mol})$ was obtained as a pale brown oil. It represented a mixture of two rotamers in a keto and an enol form each [Z-enol-(S)-22c:Eenol-(S)-22c:Z-keto- $(S)-22 c:$ E-keto- $(S)-22 c=80: 8: 12: 0] .{ }^{10}$ This ratio was determined by integrating 3-OH signals [13.90 (Z-enol) and 13.82 (E-enol)] and by integrating the $N$-CH ${ }^{\mathrm{B}}$ signal $[\delta=4.81($ Z-keto $)]$.

$\mathbf{R}_{\mathbf{f}}\left(c \mathrm{C}_{6} \mathrm{H}_{12}:\right.$ EtOAc $\left.=7: 1\right): 0.25$

${ }^{1} \mathbf{H}$-NMR $\left(500.42 \mathrm{MHz}, \mathrm{CDCl}_{3} / \mathrm{CHCl}_{3}\right.$, sample contained 1 weight-\% of $\left(\mathrm{Bu}_{3} \mathrm{Sn}\right)_{2} \mathrm{O}$, DsBrOk23-500900, 14.5.2020): $\delta=0.20$ [s, 6H, 1"-O-Si- $\left.\left(\mathrm{CH}_{3}\right)_{2}\right], 0.98$ [s, 9H, 1"-O-Si- 
Christian Drescher and Reinhard Brückner; 3D-Structure Clarifying Total Synthesis of the (Polyenoyl)tetramic Acid Militarinon B. A Highly Acid-Labile N-Protecting Group for Amides

$\left.\mathrm{C}\left(\mathrm{CH}_{3}\right)_{3}\right], \mathrm{AB}$ signal $\left(\delta_{\mathrm{A}}=3.27, \delta_{\mathrm{B}}=3.37, J_{\mathrm{AB}}=14.3 \mathrm{~Hz}\right.$, in addition split by $J_{3^{\prime}-\mathrm{H}^{\mathrm{A}}, 2^{\prime}}=5.4 \mathrm{~Hz}$, $\left.J_{3^{\prime}-\mathrm{H}^{\mathrm{B}}, 2^{\prime}}=9.7 \mathrm{~Hz}, 2 \mathrm{H}, 3{ }^{\prime}-\mathrm{H}_{2}\right), 3.74\left(\mathrm{~s}, 3 \mathrm{H}, 1^{\prime}-\mathrm{OCH}_{3}\right), 3.90\left(\mathrm{~s}, 3 \mathrm{H}, 5^{\prime \prime}-\mathrm{OCH}_{3}\right), 3.94\left(\mathrm{~s}, 3 \mathrm{H}, 4^{\prime \prime \prime}-\right.$

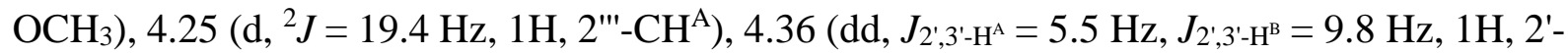
H), $4.81\left(\mathrm{~d},{ }^{2} J=19.4 \mathrm{~Hz}, 1 \mathrm{H}, 2^{2 '}-\mathrm{CH}^{\mathrm{B}}\right), 4.85(\mathrm{~s}, 1 \mathrm{H}, 2-\mathrm{H}), 5.86\left(\mathrm{dd}, J_{4,5}=15.1 \mathrm{~Hz}, J_{4,3-\mathrm{OH}}=1.4\right.$ Hz, 1H, 4-H), 6.76-6.81 (m, 4H, 6-H, 7-H, 2"-H, 6"-H), 7.00-7.06 (m, 2H, 3"-H, 5"-H), 7.21 $\left(\mathrm{dd}, J_{5,4}=14.8 \mathrm{~Hz}, J_{5,6}=10.3 \mathrm{~Hz}, 1 \mathrm{H}, 5-\mathrm{H}\right), 7.25-7.42$ (m, 6H, 3"'-H, 2"'--H, 3'"'-H, 4"'--H, 5'"'H, 6"'--H), 7.72 (s, 1H, 6"'-H), 13.90 (d, J3-OH,4 = 1.4 Hz, 1-H, 3-OH) ppm.

${ }^{13}$ C-NMR $\left(125.75 \mathrm{MHz}, \mathrm{CDCl}_{3} / \mathrm{CHCl}_{3}\right.$, sample contained $\left(\mathrm{Bu}_{3} \mathrm{Sn}\right)_{2} \mathrm{O}, \mathrm{DsBrOk} 23-500904$, 14.5.2020): $\delta=-4.4$ [1"-O-Si- $\left(\mathrm{CH}_{3}\right)_{2}$ ], 18.3 [1"-O-Si- $C\left(\mathrm{CH}_{3}\right)_{3}$ ], 25.8 [1"-O-Si-C $\left(\mathrm{CH}_{3}\right)_{3}$ ], 34.8

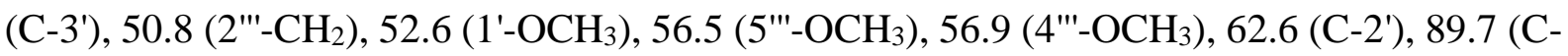
2), 108.6 (C-6"'), 110.9 (C-3"'), 120.5 (C-2", C-6"), 126.3 (C-4), 127.0 (C-2"'", C-6"'), 127.3 (C6), 127.5 (C-1"'), 128.2 (C-2'") 128.6 (C-4"'"), 129.8 (C-4"), 130.2 (C-3", C-5"), 137.1 (C-5), 138.1 (C-7), 139.4 (C-1"'), 147.9 (C-4'"), 154.1 (C-5"'), 154.9 (C-1"), 170.3 (C-3), 170.8 (C-1'), $173.3(\mathrm{C}-1) \mathrm{ppm}$.

edHSQC [,short-range H,C-COSY“, 500.06 MHz/125.75 MHz, $\left.\mathrm{CDCl}_{3} ; \delta\left({ }^{1} \mathrm{H}\right) \leftrightarrow \delta\left({ }^{13} \mathrm{C}\right)\right]$ : 0.20 [s, 1"-O-Si- $\left.\left(\mathrm{CH}_{3}\right)_{2}\right] \leftrightarrow-4.4$ [1"-O-Si- $\left.\left(\mathrm{CH}_{3}\right)_{2}\right], 0.98$ [s, 1"-O-Si-C $\left.\left(\mathrm{CH}_{3}\right)_{3}\right] \leftrightarrow 25.8$ [1"-O-Si$\left.\mathrm{C}\left(\mathrm{CH}_{3}\right)_{3}\right]$, AB signal $\left(\delta_{\mathrm{A}}=3.27, \delta_{\mathrm{B}}=3.37,3^{\prime}-\mathrm{H}_{2}\right) \leftrightarrow 35.8\left(\mathrm{C}-3{ }^{\prime}\right), 3.74\left(\mathrm{~s}, 1^{\prime}-\mathrm{OCH}_{3}\right) \leftrightarrow 52.6\left(1^{\prime}-\right.$

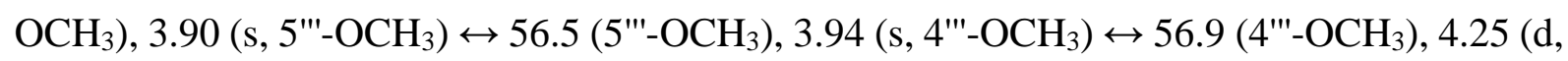

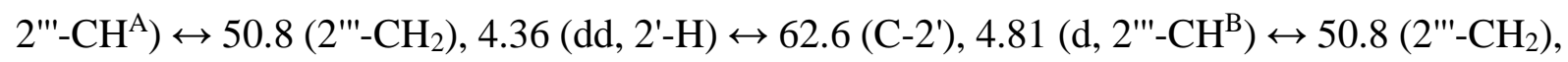
$4.85(\mathrm{~s}, 2-\mathrm{H}) \leftrightarrow 89.7(\mathrm{C}-2), 5.86(\mathrm{dd}, 4-\mathrm{H}) \leftrightarrow 126.3(\mathrm{C}-4), 6.76-6.81(\mathrm{~m}, 6-\mathrm{H}) \leftrightarrow 127.3$ (C-6), 6.76-6.81 (m, 7-H) 138.1 (C-7), 6.76-6.81 (m, 2"-H, 6"-H) 120.5 (C-2", C-6"), 7.00-7.06 (m, 3"-H, 5"-H) 130.2 (C-3", C-5"), 7.21 (dd, 5-H) ↔ 137.1 (C-5), 7.25-7.42 (m, 4"''-H) 128.6 (C-4"'"), 7.25-7.42 (m, 3'"'-H, 5"'-H) ↔ 128.8 (C-3"', C-5"'"), 7.25-7.42 (m, 2'"'-H, 6"'--H) $\leftrightarrow 127.0$ (C-2"'", C-6'"'), 7.25-7.42 (m, 3'"-H) 110.9 (C-3'"), 7.72 (s, 6"'-H) 108.6 (C-6"').

HRMS: $(E I, 70 \mathrm{eV}): \mathrm{m} / \mathrm{z}=703.3043[\mathrm{M}+\mathrm{H}]^{+}$, corresponds to the molecular formula $\mathrm{C}_{38} \mathrm{H}_{47} \mathrm{O}_{9} \mathrm{~N}_{2} \mathrm{Si}(703.3045)\left\{[\mathrm{M}+\mathrm{H}]^{+}\right\}$with a deviation of $-0.4 \mathrm{ppm}$.

Specific rotation $(S)-22 \mathrm{c}[\alpha]_{D}^{20}=-202.6^{\circ}\left(\mathrm{c}=0.95\right.$ in $\left.\mathrm{CHCl}_{3}\right)$

IR (KBr): $\tilde{v}=3435,2955,2855,1740,1630,1580,1570,1525,1510,1470,1360,1330,1275$, $1220,1185,1060,995,950,915,840,795,780,755,690 \mathrm{~cm}^{-1}$. 
Christian Drescher and Reinhard Brückner; 3D-Structure Clarifying Total Synthesis of the (Polyenoyl)tetramic Acid Militarinon B. A Highly Acid-Labile N-Protecting Group for Amides

(5S)-5-\{1"'-[(tert-Butyldimethylsilyl)oxy]benzyl\}-1-(4'"',5"'-dimethoxy-1'"'-nitrobenzyl)3-(1'Z,2' $\left.E, 4^{\prime} E\right)-1 '-h y d r o x y-5-p h e n y l p e n t a-2 ', 4{ }^{\prime}$-dien-1'-ylidene)pyrrolidine-2,4-dion [Zenol-(S)-16c]

in equilibration with

(5S)-5-\{1"'-[(tert-Butyldimethylsilyl)oxy]benzyl\}-1-(4'"',5'"'-dimethoxy-1"'-nitrobenzyl)3-(1'E,2' $\left.E, 4^{\prime} E\right)-1^{\prime}$-hydroxy-5-phenylpenta-2',4'-dien-1'-ylidene)pyrrolidine-2,4-dion [Eenol-(S)-16c]

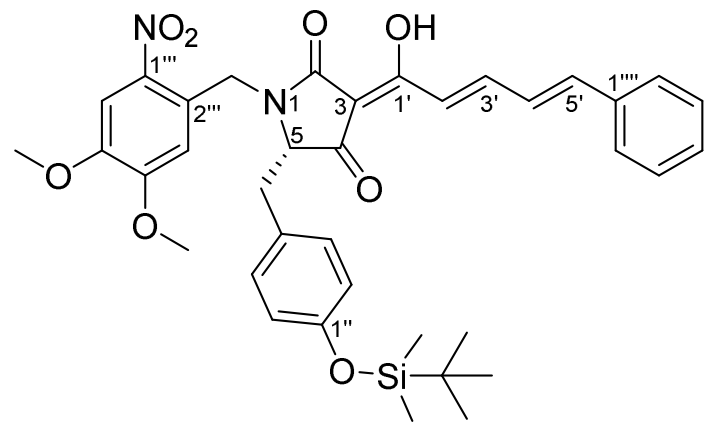

Z-enol-(S)-16c

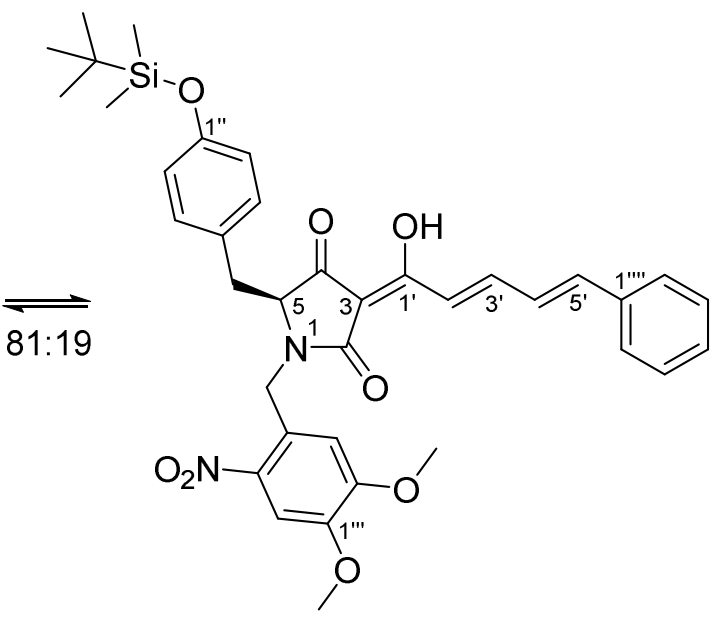

E-enot-(S)-16c

A solution of the polyen $(S)-22 c(39 \mathrm{mg}, 69 \mu \mathrm{mol})$ in $\mathrm{MeOH}(6.0 \mathrm{~mL})$ was deluded with $\mathrm{NaOMe}$ ( $18.8 \mathrm{mg}, 345 \mu \mathrm{mol}, 5.0$ eq.) at $25^{\circ} \mathrm{C}$. The mixture stirred at this temperature for $15 \mathrm{~min}$. It was added aq. citric acid (5 weight- $\%, 5 \mathrm{~mL})$ and $\mathrm{Et}_{2} \mathrm{O}(15 \mathrm{~mL})$. The organic layer was separated, and the aq. layer was extracted with $\mathrm{Et}_{2} \mathrm{O}(3 \times 15 \mathrm{~mL})$. The organic layers were tried over $\mathrm{Na}_{2} \mathrm{SO}_{4}$ and the solvent was removed under reduced pressure. The residue was purified reverse phase flash chromatography $\left(\mathrm{MeCN}: \mathrm{H}_{2} \mathrm{O}: \mathrm{TFA}=95: 5: 0.1,1.5 \mathrm{~cm}, 10 \mathrm{~cm}, 2 \mathrm{~mL}\right)$. The tetramic acid $(S)-16 c(29.4 \mathrm{mg}, 79 \%, 55 \mu \mathrm{mol})$ was obtained as a yellow powder. It was assigned as an 81:19 mixture of the $Z$ - and the $E$-enol of the title compounds $(S)-\mathbf{1 6 c}$. This ratio was determined by integrating the triplets of the respective 5-H [3.89 (Z-enol) and 4.01 (E-enol)].

$\mathbf{R}_{\mathbf{f}}\left(\mathrm{MeCN}: \mathrm{H}_{2} \mathrm{O}: \mathrm{TFA}=95: 5: 0.1\right): 0.40$

${ }^{1} \mathbf{H}-\mathrm{NMR}\left(500.42 \mathrm{MHz}, \mathrm{CDCl}_{3} / \mathrm{CHCl}_{3}\right.$, DsBrOk23-500100, 26.10.2020): $\delta=0.14$ [s, 6H, 1"O-Si- $\left.\left(\mathrm{CH}_{3}\right)_{2}\right], 0.94$ [s, 9H, 1"-O-Si-C $\left.\left(\mathrm{CH}_{3}\right)_{3}\right], \mathrm{AB}$ signal $\left(\delta_{\mathrm{A}}=3.12, \delta_{\mathrm{B}}=3.19, J_{\mathrm{AB}}=14.8 \mathrm{~Hz}\right.$, in addition split by $\left.J_{5-\mathrm{CH}^{\mathrm{A}}, 5}=4.7 \mathrm{~Hz}, J_{5-\mathrm{CH}^{\mathrm{B}}, 5}=8.3 \mathrm{~Hz}, 2 \mathrm{H}, 5-\mathrm{CH}_{2}\right), 3.90\left(\mathrm{~s}, 3 \mathrm{H}, 5^{\prime \prime}-\mathrm{OCH}_{3}\right), 3.94$ $\left(\mathrm{s}, 3 \mathrm{H}, 4 " '-\mathrm{OCH}_{3}\right), 4.03\left(\mathrm{t}, J_{5,5-\mathrm{CH}}=5.5 \mathrm{~Hz}, J_{5,5-\mathrm{CH}}=9.8 \mathrm{~Hz}, 1 \mathrm{H}, 5-\mathrm{H}\right), 4.75\left(\mathrm{~d},{ }^{2} J=16.2 \mathrm{~Hz}\right.$, 
Christian Drescher and Reinhard Brückner; 3D-Structure Clarifying Total Synthesis of the (Polyenoyl)tetramic Acid Militarinon B. A Highly Acid-Labile N-Protecting Group for Amides

1H, 2"'-CH ${ }^{\mathrm{A}}$ ), $5.02\left(\mathrm{~d},{ }^{2} J=16.2 \mathrm{~Hz}, 1 \mathrm{H}, 2^{2 "}-\mathrm{CH}^{\mathrm{B}}\right.$ ), 6.64-6.68 (m, 2H, 2"-H, 6"-H), 6.87-6.91 (m, 3H, 3'"-H, 3"-H, 5"-H), 6.95-7.10 (m, 2H, 4'-H, 5'-H), 7.26 (d, J J',3' = $\left.15.2 \mathrm{~Hz}, 1 \mathrm{H}, 2^{\prime}-\mathrm{H}\right)$, 7.31-7.40 (m, 3H, 3"'"-H, 4'"'-H, 5"'--H), 7.46-7.54 (m, 2H, 2"'"-H, 6'"'-H), 7.60 (dd, $\left.J_{3^{\prime}, 2^{\prime}}=15.3 \mathrm{~Hz}, J_{3^{\prime}, 4^{\prime}}=10.6 \mathrm{~Hz}, 1 \mathrm{H}, 3^{\prime}-\mathrm{H}\right), 7.26\left(\mathrm{~s}, 1 \mathrm{H}, 6^{\prime \prime \prime}-\mathrm{H}\right) \mathrm{ppm}$.

${ }^{13}$ C-NMR (125.75 MHz, $\mathrm{CDCl}_{3} / \mathrm{CHCl}_{3}$, sample contained $c \mathrm{C}_{6} \mathrm{H}_{12}$, DsBrOk23-501004, 26.10.2020): $\delta=-4.4$ [1"-O-Si- $\left(\mathrm{CH}_{3}\right)_{2}$ ], 25.8 [1"-O-Si-C $\left.\left(\mathrm{CH}_{3}\right)_{3}\right], 34.7\left(5-\mathrm{CH}_{2}\right), 41.1\left(2\right.$ '"-- $\left.\mathrm{CH}_{2}\right)$, 56.5 (4"'--OCH 3 ), 56.6 (5'"--OCH 3 ), 66.2 (C-2'), 100.4 (C-3), 108.3 (C-6"'), 112.0 (C-3"'), 120.1 (C-2", C-6"), 121.5 (C-2'), 126.8 (C-2'"), 127.3 (C-4'), 127.4 (C-4"), 127.7 (C-2'"', C-6"''), 129.0 (C-3"', C-5"'"), 129.8 (C-4"''), 130.5 (C-3", C-5"), 136.0 (C-1"'), 140.8 (C-1'"), 143.1 (C-5'), 145.1 (C-3'), 148.4 (C-4'"), 153.7 (C-5'"), 154.8 (C-1"), 173.2 (C-1'), 174.5 (C-2), 193.8 (C-4) ppm.

edHSQC [,,short-range H,C-COSY“, $\left.500.42 \mathrm{MHz} / 125.75 \mathrm{MHz}, \mathrm{CDCl}_{3} ; \delta\left({ }^{1} \mathrm{H}\right) \leftrightarrow \delta\left({ }^{13} \mathrm{C}\right)\right]$ : 0.14 [s, 1"-O-Si- $\left.\left(\mathrm{CH}_{3}\right)_{2}\right] \leftrightarrow-4.4$ [1"-O-Si- $\left.\left(\mathrm{CH}_{3}\right)_{2}\right], 0.94$ [s, 1"-O-Si-C $\left.\left(\mathrm{CH}_{3}\right)_{3}\right] \leftrightarrow 25.8$ [1"-O-Si$\left.\mathrm{C}\left(\mathrm{CH}_{3}\right)_{3}\right], \mathrm{AB}$ signal $\left(\delta_{\mathrm{A}}=3.12, \delta_{\mathrm{B}}=3.19,5-\mathrm{CH}_{2}\right) \leftrightarrow 34.7\left(5-\mathrm{CH}_{2}\right), 3.90\left(\mathrm{~s}, 5^{\prime \prime \prime}-\mathrm{OCH}_{3}\right) \leftrightarrow 56.6$

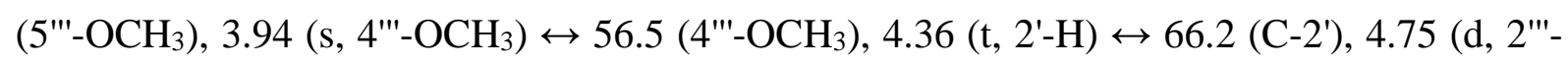
$\left.\mathrm{CH}^{\mathrm{A}}\right) \leftrightarrow 41.1\left(2 " '-\mathrm{CH}_{2}\right), 5.02\left(\mathrm{~d}, 2\right.$ "'-- $\left.\mathrm{CH}^{\mathrm{B}}\right) \leftrightarrow 41.1\left(2^{2 '-}-\mathrm{CH}_{2}\right), 6.64-6.68$ (m, 2"-H, 6"-H) 120.1 (C-2", C-6"), 6.87-6.91 (m, 3'"-H) ↔ 112.0 (C-3"'), 6.87-6.91 (m, 3"-H, 5"-H) 130.5 (C-3", C-5"), 6.95-7.10 (m, 4'-H) $127.3\left(\mathrm{C}-4^{\prime}\right), 6.95-7.10\left(\mathrm{~m}, 5^{\prime}-\mathrm{H}\right) \leftrightarrow 143.1\left(\mathrm{C}-5^{\prime}\right), 7.26(\mathrm{~d}$, 2'-H) 121.5 (C-2'), 7.31-7.40 (m, 4'"'-H) ↔ 129.8 (C-4'"'), 7.31-7.40 (m, 3'"'-H, 5"'--H) 129.0 (C-3'"', C-5'"'), 7.46-7.54 (m, 2"'--H, 6"'"-H) 127.7 (C-2"'", C-6'"'), 7.60 (dd, 3'-H) $145.1\left(\mathrm{C}-3\right.$ '), $7.26\left(\mathrm{~s}, 6^{\prime \prime \prime}-\mathrm{H}\right) \leftrightarrow 108.3\left(\mathrm{C}-6^{\prime \prime \prime}\right)$.

Specific rotation $(S)-16 \mathrm{c}[\alpha]_{D}^{20}=-569.7^{\circ}\left(\mathrm{c}=0.42\right.$ in $\left.\mathrm{CHCl}_{3}\right)$

HRMS: (EI, $70 \mathrm{eV}): \mathrm{m} / \mathrm{z}=669.2640[\mathrm{M}-\mathrm{H}]^{+}$, corresponds to the molecular formula $\mathrm{C}_{37} \mathrm{H}_{41} \mathrm{O}_{8} \mathrm{~N}_{2} \mathrm{Si}(669.2638)\left\{[\mathrm{M}-\mathrm{H}]^{+}\right\}$with a deviation of $0.3 \mathrm{ppm}$.

IR (KBr): $\tilde{v}=2930,2855,1700,1610,1590,1560,1520,1510,145,1360,1330,1275,1220$, $1170,1060,1000,915,840,795,780,750,690 \mathrm{~cm}^{-1}$. 


\section{Methyl (2S)-3-\{1'-[(tert-Butyldimethylsilyl)oxy]phenyl\}-2-[(1",3",5"'- trimethoxybenzyl)amino]propanoate $[(S)-18 \mathrm{e}]$}

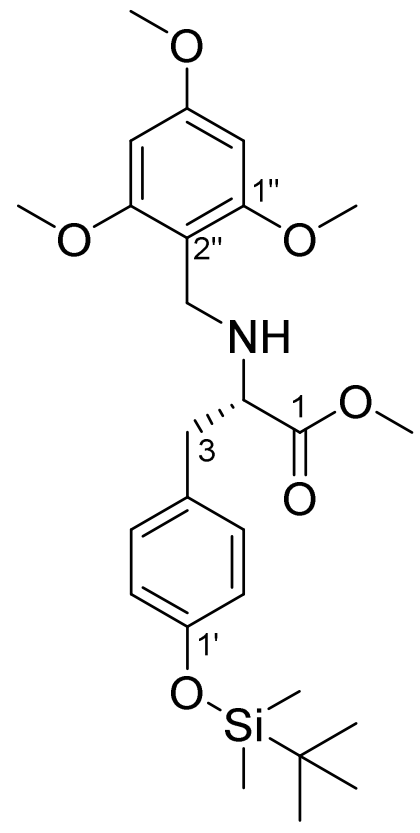

(S)-18e

The amine $(S)$-20 (235 mg, $0.75 \mathrm{mmol})$ was dissolved in $\mathrm{MeOH}(9 \mathrm{~mL})$. It was added HOAc (8 drops) and 2,4,6-trimethoxybenzaldehyde ( $150 \mathrm{mg}, 0.75 \mathrm{mmol}, 1.0$ eq.). The mixture stirred for $10 \mathrm{sec}$ at $25^{\circ} \mathrm{C}$. The mixture was diluted with $\mathrm{NaBH}_{3} \mathrm{CN}$ (90 mg, $1.37 \mathrm{mmol}, 1.8$ eq.) and stirred for $3 \mathrm{~h}$ at $25^{\circ} \mathrm{C}$. The solvent was removed under reduced pressure. It was added $\mathrm{H}_{2} \mathrm{O}$ $(5 \mathrm{ml})$ and $\mathrm{Et}_{2} \mathrm{O}(15 \mathrm{~mL})$. The layers were separated, and the aq. layer was extracted with $\mathrm{Et}_{2} \mathrm{O}$ $(3 \times 25 \mathrm{~mL})$. The combined organic layers were dried over $\mathrm{Na}_{2} \mathrm{SO}_{4}$ and the solvent was removed under reduced pressure. The residue was purified by flash chromatography $(2.5 \mathrm{~cm}$, $20 \mathrm{~cm}, c \mathrm{C}_{6} \mathrm{H}_{12}:$ EtOAc $\left.=1: 1,25 \mathrm{~mL}\right)$ to give the product $(S)-\mathbf{1 8 e}(317 \mathrm{mg}, 85 \%, 0.64 \mathrm{mmol})$ as a colorless oil.

$\mathbf{R}_{\mathbf{f}}\left(c \mathrm{C}_{6} \mathrm{H}_{12}: \mathrm{EtOAc}=4: 1\right): 0.30$

${ }^{\mathbf{1}} \mathbf{H}$-NMR $\left(500.06 \mathrm{MHz}, \mathrm{CDCl}_{3} / \mathrm{CHCl}_{3}\right.$, sample contained trace amounts of EtOAc, DsBrAp20500300, 20.4.2020): $\delta=0.17$ [s, 6H, 1'-O-Si- $\left(\mathrm{CH}_{3}\right)_{2}$ ], 0.97 [s, 9H, 1'-O-Si-C $\left(\mathrm{CH}_{3}\right)_{3}$ ], 2.02 (br. $\mathrm{s}, 1 \mathrm{H}, N \mathrm{H}), 2.84\left(\mathrm{~m}_{\mathrm{c}}, 2 \mathrm{H}, 3-\mathrm{H}_{2}\right), 3.42\left(\mathrm{dd}, J_{2,3-\mathrm{H}^{\mathrm{A}}}=6.6 \mathrm{~Hz}, J_{2,3-\mathrm{H}^{\mathrm{B}}}=7.6 \mathrm{~Hz}, 1 \mathrm{H}, 2-\mathrm{H}\right), 3.55(\mathrm{~s}$, $\left.3 \mathrm{H}, 1-\mathrm{OCH}_{3}\right), 3.69$ (s, 6H, 1"-- $\mathrm{OCH}_{3}, 3$ "- $\left.\mathrm{OCH}_{3}\right), 3.75$ (s, 2H, 2"- $\left.\mathrm{CH}_{2}\right), 3.78$ (s, 3H, 5"-OCH $)$, 6.05 (s, 2H, 4"-H, 6"-H), 6.69-6.72 (m, 2H, 2'-H, 6'-H), 6.94-6.97 (m, 2H, 3'-H, 5'-H) ppm.

${ }^{13}$ C-NMR (125.75 MHz, $\left.\mathrm{CDCl}_{3} / \mathrm{CHCl}_{3}, \mathrm{DsBrAp} 20-500304,20.4 .2020\right): \delta=-4.4$ [1'-O-Si$\left(\mathrm{CH}_{3}\right)_{2}$ ], 18.3 [1'-O-Si-C(CH$)_{3}$ ], 25.8 [1'-O-Si-C $\left(\mathrm{CH}_{3}\right)_{3}$ ], 38.9 (C-3), $39.8\left(2\right.$ "- $\left.\mathrm{CH}_{2}\right), 51.5$ (1- 
Christian Drescher and Reinhard Brückner; 3D-Structure Clarifying Total Synthesis of the (Polyenoyl)tetramic Acid Militarinon B. A Highly Acid-Labile N-Protecting Group for Amides

$\left.\mathrm{OCH}_{3}\right), 55.4\left(5 "-\mathrm{OCH}_{3}\right), 55.6\left(1 "-\mathrm{OCH}_{3}, 3 "-\mathrm{OCH}_{3}\right), 62.1(\mathrm{C}-2), 90.3(\mathrm{C}-4 ", \mathrm{C}-6 "), 108.3(\mathrm{C}-$ 2"), 119.9 (C-2', C-6'), 130.1 (C-3', C-5'), 130.3 (C-4'), 154.3 (C-1'), 159.4 (C-1", C-3"), 160.5 (C-5"), 175.2 (C-1) ppm.

edHSQC [,,short-range H,C-COSY“, $\left.500.06 \mathrm{MHz} / 125.75 \mathrm{MHz}, \mathrm{CDCl}_{3} ; \delta\left({ }^{1} \mathrm{H}\right) \leftrightarrow \delta\left({ }^{13} \mathrm{C}\right)\right]$ : 0.17 [s, 1'-O-Si- $\left.\left(\mathrm{CH}_{3}\right)_{2}\right] \leftrightarrow-4.4$ [1'-O-Si- $\left.\left(\mathrm{CH}_{3}\right)_{2}\right], 0.97$ [s, 1'-O-Si-C $\left.\left(\mathrm{CH}_{3}\right)_{3}\right] \leftrightarrow 25.8$ [1'-O-Si$\left.\mathrm{C}\left(\mathrm{CH}_{3}\right)_{3}\right], 2.84\left(\mathrm{~m}_{\mathrm{c}}, 3-\mathrm{H}_{2}\right) \leftrightarrow 38.9(\mathrm{C}-3), 3.42(\mathrm{dd}, 2-\mathrm{H}) \leftrightarrow 62.1(\mathrm{C}-2), 3.55\left(\mathrm{~s}, 1-\mathrm{OCH}_{3}\right) \leftrightarrow 51.5$ $\left(1-\mathrm{OCH}_{3}\right), 3.69\left(\mathrm{~s}, 1 "-\mathrm{OCH}_{3}, 3\right.$ "--OCH 3 ) $55.6\left(1 "-\mathrm{OCH}_{3}, 3\right.$ "- $\left.-\mathrm{OCH}_{3}\right), 3.75\left(\mathrm{~s}, 2\right.$ "- $\left.\mathrm{CH}_{2}\right) \leftrightarrow 39.8$

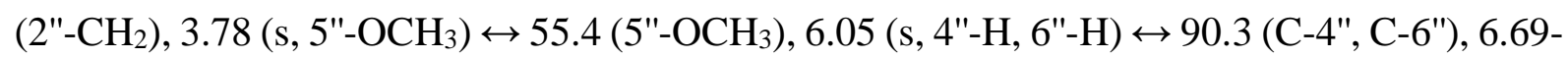
$6.72\left(\mathrm{~m}, 2^{\prime}-\mathrm{H}, 6^{\prime}-\mathrm{H}\right) \leftrightarrow 119.9\left(\mathrm{C}-2^{\prime}, \mathrm{C}^{\prime} \mathbf{6}^{\prime}\right), 6.94-6.97\left(\mathrm{~m}, 3^{\prime}-\mathrm{H}, 5^{\prime}-\mathrm{H}\right) \leftrightarrow 130.1$ (C-3', C-5').

Specific rotation $(S)-18 \mathrm{e}[\alpha]_{D}^{20}=+21.4^{\circ}\left(\mathrm{c}=1.12\right.$ in $\left.\mathrm{CHCl}_{3}\right)$

HRMS: (EI, $70 \mathrm{eV}): \mathrm{m} / \mathrm{z}=490.2617[\mathrm{M}+\mathrm{H}]^{+}$, corresponds to the molecular formula $\mathrm{C}_{26} \mathrm{H}_{40} \mathrm{O}_{6} \mathrm{NSi}(490.2619)\left\{[\mathrm{M}+\mathrm{H}]^{+}\right\}$with a deviation of $-0.5 \mathrm{ppm}$.

IR (KBr): $\tilde{v}=3000,2955,2935,2900,2860,2840,1735,1610,1510,1465,1435,1420,1255$, $1225,1205,1170,1150,1135,1060,1040,915,840,815,780 \mathrm{~cm}^{-1}$.

Methyl (2'S)-2'-\{ $N$-[(1T,2Z,4E)-5-Bromo-3-hydroxy-1-oxoypenta-2,4-dienyl]- $N$ $(1 " ', 3$ "',5"'-trimethoxybenzyl $)\}-3$ '- $\{1$ "-[(tert-butyldimethylsilyl)oxy $]$ phenyl $\}$ propanoate [Z-enol-(S)-21e] in equilibration with

Methyl (2'S)-2'-\{ $N$-[(1Z,4E)-5-Bromo-1,3-dioxopent-4-enyl]- $N-\left(1 '{ }^{\prime \prime}, 3\right.$ '",5"'trimethoxybenzyl) $\}-3$ '- $\{1$ "'-[(tert-butyldimethylsilyl)oxy $]$ phenyl $\}$ propanoate $[$ Z-keto-(S)21e]<smiles>COC(=O)[C@H](Cc1ccc(O[Si](C)(C)C(C)(C)C)cc1)N1CC(=O)N(Cc2c(OC)cc(OC)cc2OC)CC1=O</smiles>

Z-enol-[(S)-21e]

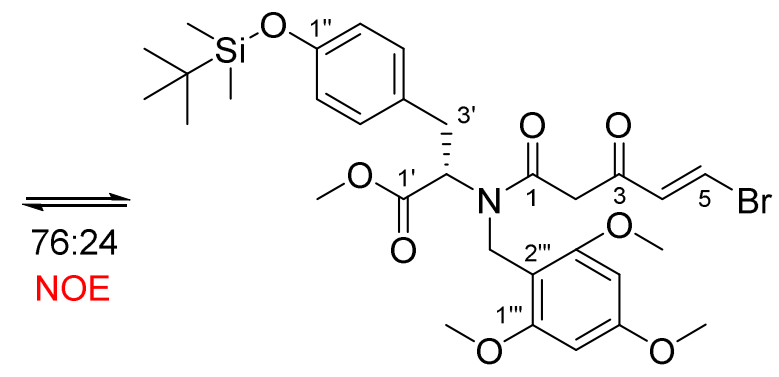

Z-keto-[(S)-21e] 
Christian Drescher and Reinhard Brückner; 3D-Structure Clarifying Total Synthesis of the (Polyenoyl)tetramic Acid Militarinon B. A Highly Acid-Labile N-Protecting Group for Amides

A solution of $N$-TMB-tyrosine methyl ester $(S)$-18e $(150 \mathrm{mg}, 391 \mu \mathrm{mol})$ and the $\beta$ ketothioester 11 (144 mg, $547 \mu \mathrm{mol}, 1.4$ eq.) in THF $(5 \mathrm{~mL})$ was treated with powdered molecular sieves $4 \AA(0.4 \mathrm{~g})$. The suspension stirred at $25^{\circ} \mathrm{C}$ for $30 \mathrm{~min}$. Then $\mathrm{AgO}_{2} \mathrm{CCF}_{3}$ (130 mg, $586 \mu \mathrm{mol}, 1.5$ eq.) was added and the mixture was stirred for $18 \mathrm{~h}$ at $25^{\circ} \mathrm{C}$. The solvent was removed under reduced pressure and $\mathrm{Et}_{2} \mathrm{O}(20 \mathrm{~mL})$ was added to the resulting suspension. The mixture was filtered, and the filtrate was concentrated under reduced pressure. Purification by flash chromatography $\left(c \mathrm{C}_{6} \mathrm{H}_{12}\right.$ :AcOEt $\left.=4: 1,2.0 \mathrm{~cm}, 20 \mathrm{~cm}, 15 \mathrm{~mL}\right)$ afforded $(S)-\mathbf{2 1 e}$ (183 mg, 84\%, $328 \mu \mathrm{mol}$, as an orange oil. It was assigned as a 76:24 mixture of the enol-and the keto-tautomer of the title compound $(S)$-21e. This ratio was determined by integrating the singlets of the respective 5- $\mathrm{H}[7.19$ (enol) and $7.70($ keto $)] .^{9}$

$\mathbf{R}_{\mathbf{f}}\left(c \mathrm{C}_{6} \mathrm{H}_{12}: \mathrm{EtOAc}=8: 1\right): 0.32$

${ }^{1} \mathbf{H}-\mathbf{N M R}\left(500.06 \mathrm{MHz}, \mathrm{CDCl}_{3} / \mathrm{CHCl}_{3}\right.$, sample contained 4 weight-\% of $c \mathrm{C}_{6} \mathrm{H}_{12}$, DsBrAp21500400, 22.4.2020): $\delta=0.16$ [s, 6H, 1"-O-Si- $\left.\left(\mathrm{CH}_{3}\right)_{2}\right], 0.97$ [s, 9H, 1"-O-Si-C $\left.\left(\mathrm{CH}_{3}\right)_{3}\right]$, AB signal $\left(\delta_{\mathrm{A}}=3.08, \delta_{\mathrm{B}}=3.31, J_{\mathrm{AB}}=13.9 \mathrm{~Hz}\right.$ in addition split by $J_{3^{\prime}-\mathrm{H}^{\mathrm{A}}, 2^{\prime}}=8.8 \mathrm{~Hz}, J_{3^{\prime}-\mathrm{H}^{\mathrm{B}}, 2^{\prime}}=5.9 \mathrm{~Hz}, 2 \mathrm{H}$,

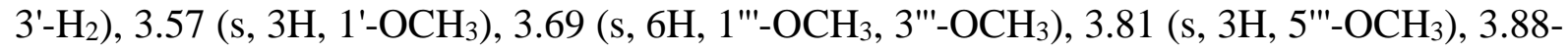
$3.99\left(\mathrm{~m}, 2 \mathrm{H}, 2^{\prime}-\mathrm{H}, 11^{\prime \prime}-\mathrm{CH}^{\mathrm{A}}\right), 4.31\left(\mathrm{~d},{ }^{2} J=14.7 \mathrm{~Hz}, 1 \mathrm{H}, 1^{\prime \prime}-\mathrm{CH}^{\mathrm{B}}\right), 5.62$ (s, 1H, 2-H), 6.02 (s, 2H, 4"'-H, 6"'-H), 6.54 (d, J4,5 = 13.6 Hz, 1H, 4-H), 6.63-6.67 (m, 2H, 2"-H, 6"-H), 6.84-6.88 (m, 2H, 3"-H, 5"-H), 7.19 (d, J5,4 = 13.6 Hz, 1H, 5-H), 14.08 (s, 1H, 3-OH) ppm.

${ }^{13}$ C-NMR $\left(125.75 \mathrm{MHz}, \mathrm{CDCl}_{3} / \mathrm{CHCl}_{3}\right.$, sample contained $c \mathrm{C}_{6} \mathrm{H}_{12}$, DsBrAp21-500405, 22.4.2020): $\delta=-4.4$ [1"-O-Si- $\left(\mathrm{CH}_{3}\right)_{2}$ ], 18.3 [1"--O-Si- $C\left(\mathrm{CH}_{3}\right)_{3}$ ], 25.8 [1"-O-Si-C $\left(\mathrm{CH}_{3}\right)_{3}$ ], 34.5

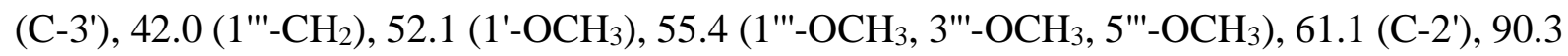
(C-4"', C-6"'), 91.6 (C-2), 104.1 (C-2"'), 115.6 (C-5), 119.8 (C-2", C-6"), 130.4 (C-3", C-5"), 131.5 (C-4"), 133.1 (C-4), 154.0 (C-1"), 159.8 (C-1"', C-3"'), 161.6 (C-5"'), 165.8 (C-3), 171.7 (C-1), $171.8\left(\mathrm{C}-1^{\prime}\right) \mathrm{ppm}$.

edHSQC [,short-range H,C-COSY“, $\left.500.06 \mathrm{MHz} / 125.75 \mathrm{MHz}, \mathrm{CDCl}_{3} ; \delta\left({ }^{1} \mathrm{H}\right) \leftrightarrow \delta\left({ }^{13} \mathrm{C}\right)\right]$ : 0.16 [s, 1"-O-Si- $\left.\left(\mathrm{CH}_{3}\right)_{2}\right] \leftrightarrow-4.4$ [1"-O-Si- $\left.\left(\mathrm{CH}_{3}\right)_{2}\right], 0.97$ [s, 1"-O-Si-C $\left.\left(\mathrm{CH}_{3}\right)_{3}\right] \leftrightarrow 25.8$ [1"-O-Si$\left.\mathrm{C}\left(\mathrm{CH}_{3}\right)_{3}\right]$, AB signal $\left(\delta_{\mathrm{A}}=3.08, \delta_{\mathrm{B}}=3.31,3^{\prime}-\mathrm{H}_{2}\right) \leftrightarrow 34.5\left(\mathrm{C}-3{ }^{\prime}\right), 3.57\left(\mathrm{~s}, 1^{\prime}-\mathrm{OCH}_{3}\right) \leftrightarrow 52.1\left(1^{\prime}-\right.$

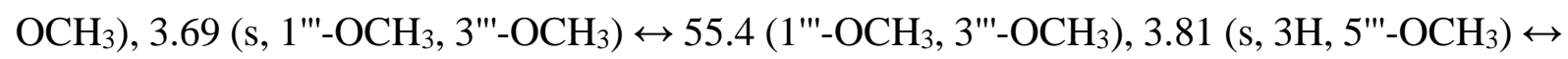

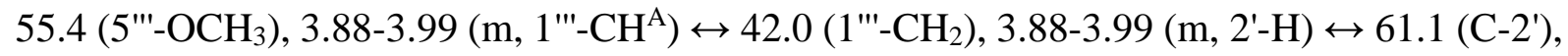
$4.31\left(\mathrm{~d}, 1\right.$ '"'-CH $\left.\mathrm{CH}^{\mathrm{B}}\right) \leftrightarrow 42.0\left(1\right.$ '"-- $\left.\mathrm{CH}_{2}\right), 5.62(\mathrm{~s}, 2-\mathrm{H}) \leftrightarrow 91.6(\mathrm{C}-2), 6.02(\mathrm{~s}, 4$ "'-H, 6"'-H) 90.3 (C-4"', C-6"'), 6.54 (d, 4-H) 133.1 (C-4), 6.63-6.67 (m, 2"-H, 6"-H) ↔ 119.8 (C-2", C-6"), 6.84-6.88 (m, 3"-H, 5"-H) ↔ 130.4 (C-3", C-5"), 7.19 (d, 5-H) 115.6 (C-5). 
Christian Drescher and Reinhard Brückner; 3D-Structure Clarifying Total Synthesis of the (Polyenoyl)tetramic Acid Militarinon B. A Highly Acid-Labile N-Protecting Group for Amides

Specific rotation $(S)-21 \mathrm{e}[\alpha]_{D}^{20}=-189.9^{\circ}\left(\mathrm{c}=0.89\right.$ in $\left.\mathrm{CHCl}_{3}\right)$

HRMS: (EI, $70 \mathrm{eV}): \mathrm{m} / \mathrm{z}=664.1932[\mathrm{M}+\mathrm{H}]^{+}$, corresponds to the molecular formula $\mathrm{C}_{31} \mathrm{H}_{43} \mathrm{O}_{8} \mathrm{NBrSi}(664.1932)\left\{[\mathrm{M}+\mathrm{H}]^{+}\right\}$with a deviation of $-0.6 \mathrm{ppm}$.

IR (KBr): $\tilde{v}=3080,3000,2955,2935,2895,2860,1740,1635,1610,1585,1510,1480,1470$, $1420,1390,1330,1250,1205,1150,1135,915,840,780 \mathrm{~cm}^{-1}$.

Methyl (2'S)-2'-\{ $N$-[(1Z,2Z,4E,6E)-3-Hydroxy-7-phenylhepta-2,4,6-trienamido]- $N$ $(1 " ', 3$ "',5'"-trimethoxybenzyl) $\}-3 '-\{1$ "'-[(tert-butyldimethylsilyl)oxy]phenyl $\}$ propanoate [Z-enol-(S)-22e]

in equilibration with

Methyl (2'S)-2'-\{N-[(1E,4E,6E)-3-Oxo-7-phenylhepta-4,6-dienamido]- $N$-(1"',3'"',5'"trimethoxybenzyl $)\}-3 '-\left\{1^{\prime \prime}-[(\right.$ tert-butyldimethylsilyl)oxy $]$ phenyl $\}$ propanoate $[$ E-keto-(S)22e]<smiles>COC(=O)[C@H](Cc1ccc(O[Si](C)(C)C(C)(C)C)cc1)N(Cc1c(OC)cc(OC)cc1OC)C(=O)/C=C(O)/C=C/C=C/c1ccccc1</smiles>

Z-enol-[(S)-22e]<smiles>COC(=O)[C@H](Cc1ccc(O[Si](C)(C)C(C)(C)C)cc1)N(Cc1c(OC)cc(OC)cc1OC)C(=O)CC(=O)/C=C/C=C/c1ccccc1</smiles>

Z-keto-[(S)-22e]

A solution of the western building block $(S)-21$ e $(150 \mathrm{mg}, 226 \mu \mathrm{mol})$ and the stannane SI-7 (177 mg, $452 \mu \mathrm{mol}, 2.0$ eq.) in THF (6.0 mL) was deluded with $\operatorname{Pd}_{2}(\mathrm{dba})_{3}(10.2 \mathrm{mg}, 11.3 \mu \mathrm{mol}$, $5 \mathrm{~mol}-\%)$ and $\mathrm{AsPh}_{3}(17.6 \mathrm{mg}, 56.6 \mu \mathrm{mol}, 25 \mathrm{~mol}-\%)$. After $24 \mathrm{~h}$ at $25^{\circ} \mathrm{C}$ aq. KF solution (8 M, $4.5 \mathrm{~mL}$ ) was added and it was sonicated in the ultrasonic bath for $1 \mathrm{~min}$. The layers were separated, and the organic layer was extracted with $\mathrm{Et}_{2} \mathrm{O}(3 \times 15 \mathrm{~mL})$. The combined organic layers were washed with brine $(10 \mathrm{~mL})$, tried over $\mathrm{Na}_{2} \mathrm{SO}_{4}$ and concentrated under reduced pressure. The residue was purified by flash chromatography $\left(c \mathrm{C}_{6} \mathrm{H}_{12}\right.$ : AcOEt $=7: 1,2.0 \mathrm{~cm}, 20$ $\mathrm{cm}, 10 \mathrm{~mL})$. The polyen $(S)-\mathbf{2 2 e}(106 \mathrm{mg}, 68 \%, 154 \mu \mathrm{mol})$ was obtained as a pale brown oil. It was assigned as a 56:44 mixture of the enol- and the keto-tautomer of the title compound $(S)$ 22e. This ratio was determined by integrating the singlets of the respective 4-H [6.11 (enol) and $6.48($ keto $)] .^{10}$ 
Christian Drescher and Reinhard Brückner; 3D-Structure Clarifying Total Synthesis of the (Polyenoyl)tetramic Acid Militarinon B. A Highly Acid-Labile N-Protecting Group for Amides

$\mathbf{R}_{\mathbf{f}}\left(c \mathrm{C}_{6} \mathrm{H}_{12}: \mathrm{EtOAc}=8: 1\right): 0.28$

${ }^{1} \mathrm{H}-\mathrm{NMR}\left(500.06 \mathrm{MHz}, \mathrm{CDCl}_{3} / \mathrm{CHCl}_{3}\right.$, sample contained trace amounts of $c \mathrm{C}_{6} \mathrm{H}_{12}$, sample contained 2 weight- $\%$ AcOEt and sample contained 9 weight- $\%$ of $\mathrm{O}\left(\mathrm{SnBu}_{3}\right)_{2}$ with $\mathrm{t}$ at 0.92 , $\mathrm{m}_{\mathrm{c}}$ at $1.30, \mathrm{~m}_{\mathrm{c}}$ at 1.35 and $\mathrm{m}_{\mathrm{c}}$ at 1.63, DsBrAp23-500300, 24.4.2020): $\delta=0.16$ [s, 6H, 1"-O-Si$\left.\left(\mathrm{CH}_{3}\right)_{2}\right], 0.97\left[\mathrm{~s}, 9 \mathrm{H}, 1 "-\mathrm{O}-\mathrm{Si}-\mathrm{C}\left(\mathrm{CH}_{3}\right)_{3}\right], \mathrm{AB}$ signal $\left(\delta_{\mathrm{A}}=3.10, \delta_{\mathrm{B}}=3.34, J_{\mathrm{AB}}=14.1 \mathrm{~Hz}\right.$ in addition split by $\left.J_{3^{\prime}-\mathrm{H}^{\mathrm{A}}, 2^{\prime}}=8.7 \mathrm{~Hz}, J_{3^{\prime}-\mathrm{H}^{\mathrm{B}}, 2^{\prime}}=6.1 \mathrm{~Hz}, 2 \mathrm{H}, 3^{\prime}-\mathrm{H}_{2}\right), 3.57\left(\mathrm{~s}, 3 \mathrm{H}, 1^{\prime}-\mathrm{OCH}_{3}\right), 3.71(\mathrm{~s}$,

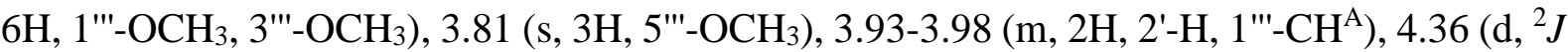
$=12.6 \mathrm{~Hz}, 1 \mathrm{H}, 1$ '"-CH $^{\mathrm{B}}$ ), 5.70 (s, 1H, 2-H), 6.03 (s, 2H, 4"'-H, 6"'-H), 6.11 (d, J4,5 = $15.4 \mathrm{~Hz}$, 1H, 4-H), 6.63-6.67 (m, 2H, 2"-H, 6"-H), 6.79 (d, J J,6 $=15.5 \mathrm{~Hz}, 1 \mathrm{H}, 7-\mathrm{H}), 6.87-6.91$ (m, 2H, 3"-H, 5"-H), 6.99 (dd, $\left.J_{6,7} \approx J_{6,5}=15.4 \mathrm{~Hz}, 1 \mathrm{H}, 6-\mathrm{H}\right), 7.23$ (dd, $J_{5,4}=15.2 \mathrm{~Hz}, J_{5,6}=11.0 \mathrm{~Hz}$, 1H, 5-H), 7.31-7.38 (m, 3H, 3'"'-H, 4"'--H, 5"'-H), 7.43-7.50 (m, 2H, 2'"'-H, 6"'-H), 14.12 (d, $\left.J_{3-\mathrm{OH}, 2}=1.2 \mathrm{~Hz}, 1 \mathrm{H}, 3-\mathrm{OH}\right) \mathrm{ppm}$.

${ }^{13} \mathrm{C}-\mathrm{NMR}\left(125.75 \mathrm{MHz}, \mathrm{CDCl}_{3} / \mathrm{CHCl}_{3}\right.$, sample contained $\mathrm{O}\left(\mathrm{SnBu}_{3}\right)_{2}$ at 13.6, 17.6, 26.9 and 27.9, DsBrAp23-500304, 23.4.2020): $\delta=-4.4$ [1"-O-Si- $\left(\mathrm{CH}_{3}\right)_{2}$ ], 18.3 [1"-O-Si-C( $\left.\left.\mathrm{CH}_{3}\right)_{3}\right], 25.8$

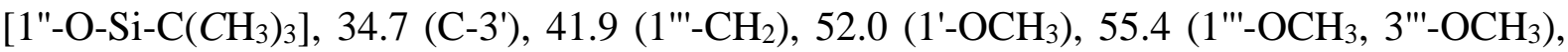
55.4 (5'"-OCH 3 ), 61.1 (C-2'), 90.3 (C-4"', C-6"'), 91.6 (C-2), 104.4 (C-2"'), 119.7 (C-2", C-6"), 126.9 (C-2'"', C-6"''), 127.5 (C-4), 127.8 (C-1'"'), 128.3 (C-4'"'), 128.8 (C-3'"', C-5'"'), 130.4 (C3", C-5"), 131.7 (C-4"), 135.3 (C-5), 136.8 (C-7), 142.0 (C-6), 153.9 (C-1"), 159.8 (C-1"', C3'"), 161.5 (C-5'"), 168.0 (C-3), 172.0 (C-1), 172.0 (C-1') ppm.

edHSQC [,,short-range H,C-COSY“, 500.06 MHz/125.75 MHz, $\left.\mathrm{CDCl}_{3} ; \delta\left({ }^{1} \mathrm{H}\right) \leftrightarrow \delta\left({ }^{13} \mathrm{C}\right)\right]$ : 0.16 [s, 1"-O-Si- $\left.\left(\mathrm{CH}_{3}\right)_{2}\right] \leftrightarrow-4.4$ [1"-O-Si- $\left.\left(\mathrm{CH}_{3}\right)_{2}\right], 0.97$ [s, 1"-O-Si-C $\left.\left(\mathrm{CH}_{3}\right)_{3}\right] \leftrightarrow 25.8$ [1"-O-Si$\left.\mathrm{C}\left(\mathrm{CH}_{3}\right)_{3}\right]$, AB signal $\left(\delta_{\mathrm{A}}=3.10, \delta_{\mathrm{B}}=3.34,3^{\prime}-\mathrm{H}_{2}\right) \leftrightarrow 34.7\left(\mathrm{C}-3{ }^{\prime}\right), 3.57\left(\mathrm{~s}, 1^{\prime}-\mathrm{OCH}_{3}\right) \leftrightarrow 52.0\left(1^{\prime}-\right.$

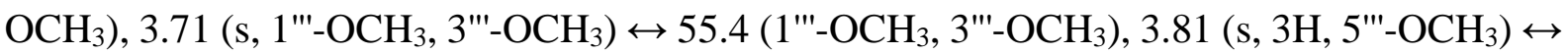
$55.4\left(5^{\prime \prime}-\mathrm{OCH}_{3}\right), 3.93-3.98\left(\mathrm{~m}, 1\right.$ '"--CH $\left.{ }^{\mathrm{A}}\right) \leftrightarrow 41.9\left(1{ }^{\prime \prime}-\mathrm{CH}_{2}\right), 3.93-3.98\left(\mathrm{~m}, 2^{\prime}-\mathrm{H}\right) \leftrightarrow 61.1\left(\mathrm{C}-2^{\prime}\right)$, $4.36\left(\mathrm{~d}, 1\right.$ 1"'-CH $\left.\mathrm{CH}^{\mathrm{B}}\right) \leftrightarrow 41.9\left(1\right.$ '"- $\left.\mathrm{CH}_{2}\right), 5.70(\mathrm{~s}, 2-\mathrm{H}) \leftrightarrow 91.6(\mathrm{C}-2), 6.03(\mathrm{~s}, 4$ "'-H, 6"'-H) ↔ 90.3 (C-4"', C-6"'), 6.11 (d, 4-H) ↔ 127.5 (C-4), 6.63-6.67 (m, 2"-H, 6"-H) ↔ 119.7 (C-2", C-6"), $6.79(\mathrm{~d}, 7-\mathrm{H}) \leftrightarrow 136.8(\mathrm{C}-7), 6.87-6.91$ (m, 3"-H, 5"-H) ↔ 130.4 (C-3", C-5"), 6.99 (dd, 6-H) $\leftrightarrow 142.0$ (C-6), 7.23 (dd, 5-H) 135.3 (C-5), 7.31-7.38 (m, 4'"'-H) 128.3 (C-4"'"), 7.31-7.38 (m, 3"''-H, 5'"'-H) 128.8 (C-3"'", C-5'"'), 7.43-7.50 (m, 2"'-H, 6"'-H) ↔ 126.9 (C-2"'", C-6"'').

Specific rotation $(S)-22 \mathrm{e}[\alpha]_{D}^{20}=-190.0^{\circ}\left(\mathrm{c}=1.06\right.$ in $\left.\mathrm{CHCl}_{3}\right)$

HRMS: $(\mathrm{EI}, 70 \mathrm{eV}): \mathrm{m} / \mathrm{z}=710.3115[\mathrm{M}+\mathrm{Na}]^{+}$, corresponds to the molecular formula $\mathrm{C}_{39} \mathrm{H}_{49} \mathrm{O}_{8} \mathrm{NNaSi}(710.3120)\left\{[\mathrm{M}+\mathrm{Na}]^{+}\right\}$with a deviation of $-0.6 \mathrm{ppm}$. 
Christian Drescher and Reinhard Brückner; 3D-Structure Clarifying Total Synthesis of the (Polyenoyl)tetramic Acid Militarinon B. A Highly Acid-Labile N-Protecting Group for Amides

IR (KBr): $\tilde{v}=3055,3025,3000,2930,2955,1740,1595,1510,1470,1420,1330,1250,1205$, $1150,1135,950,915,840,780 \mathrm{~cm}^{-1}$.

(5S)-5-\{1"'-[(tert-Butyldimethylsilyl)oxy]benzyl $\}-1-(1 " ', 3$ '", 5 "'-trimethoxybenzyl)-3$\left(1^{\prime} Z, 2^{\prime} E, 4^{\prime} E\right)-1 '$-hydroxy-5-phenylpenta-2',4' '-dien-1'-ylidene)pyrrolidine-2,4-dion [Zenol-(S)-SI-8]

in equilibration with

(5S)-5-\{1"-[(tert-Butyldimethylsilyl)oxy]benzyl $\}-1-(1 " ', 3$ '", 5'"'-trimethoxybenzyl)-3(1'E,2' $\left.E, 4^{\prime} E\right)-1$ '-hydroxy-5-phenylpenta-2',4' '-dien-1'-ylidene)pyrrolidine-2,4-dion [Eenol-(S)-SI-8]

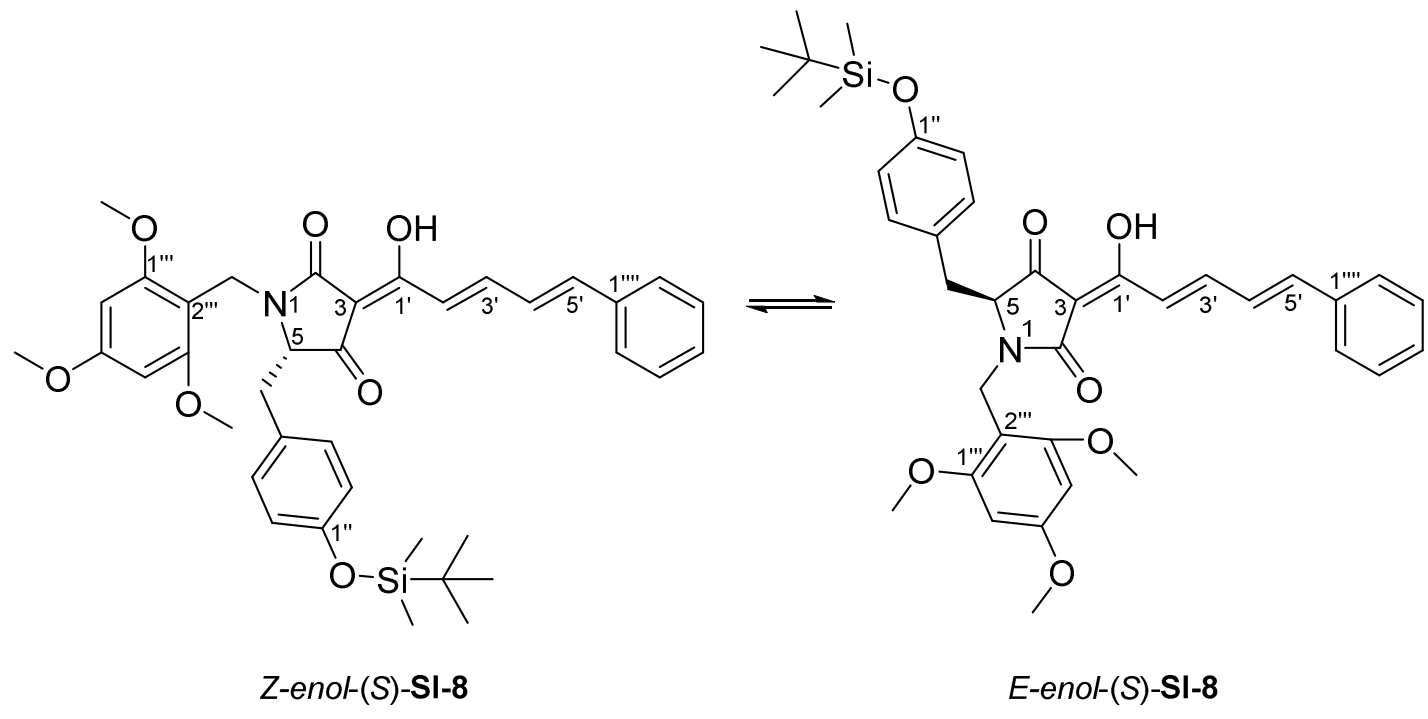

A solution of the polyen $(S)-22 \mathrm{e}(30.0 \mathrm{mg}, 44.1 \mu \mathrm{mol})$ in $\mathrm{MeOH}(5.0 \mathrm{~mL})$ was deluded with $\mathrm{NaOMe}\left(11.9 \mathrm{mg}, 220 \mu \mathrm{mol}, 5.0\right.$ eq.) at $25^{\circ} \mathrm{C}$. The mixture stirred at this temperature for $15 \mathrm{~min}$. It was added aq. citric acid (5 weight-\%, $3 \mathrm{~mL})$ and $\mathrm{Et}_{2} \mathrm{O}(10 \mathrm{~mL})$. The organic layer was separated, and the aq. layer was extracted with $\mathrm{Et}_{2} \mathrm{O}(3 \times 10 \mathrm{~mL})$. The organic layers were tried over $\mathrm{Na}_{2} \mathrm{SO}_{4}$ and the solvent was removed under reduced pressure. The residue was purified by reverse phase flash chromatography $\left(\mathrm{MeCN}: \mathrm{H}_{2} \mathrm{O}: \mathrm{TFA}=97: 3: 0.1,1.5 \mathrm{~cm}, 10 \mathrm{~cm}\right.$, $2 \mathrm{~mL})$. The tetramic acid $(S)-\mathbf{2 2 e}(22.0 \mathrm{mg}, 77 \%, 33.9 \mu \mathrm{mol})$ was obtained as a yellow powder. It was assigned as a 74:26 mixture of the $Z$ - and the $E$-enol of the title compounds $(S)$-277. This ratio was determined by integrating duplets of the respective 2'-H [7.04 (E-enol) and 7.18 (Zenol)]. 
Christian Drescher and Reinhard Brückner; 3D-Structure Clarifying Total Synthesis of the (Polyenoyl)tetramic Acid Militarinon B. A Highly Acid-Labile N-Protecting Group for Amides

$\mathbf{R}_{\mathbf{f}}\left(\mathrm{MeCN}: \mathrm{H}_{2} \mathrm{O}: \mathrm{TFA}=97: 3: 0.1\right): 0.22$

${ }^{1} \mathrm{H}-\mathrm{NMR}\left(500.06 \mathrm{MHz}, \mathrm{CDCl}_{3} / \mathrm{CHCl}_{3}\right.$, sample contained 1 weight-\% of $c \mathrm{C}_{6} \mathrm{H}_{12}$ and sample contained grease with $\mathrm{s}$ at 1.26, DsBrMi13-500300, 14.5.2020): $\delta=0.13$ [s, 6H, 1"-O-Si$\left.\left(\mathrm{CH}_{3}\right)_{2}\right], 0.93\left[\mathrm{~s}, 9 \mathrm{H}, 1\right.$ "-O-Si-C $\left.\left(\mathrm{CH}_{3}\right)_{3}\right], \mathrm{AB}$ signal $\left(\delta_{\mathrm{A}}=3.05, \delta_{\mathrm{B}}=3.08, J_{\mathrm{AB}}=14.3 \mathrm{~Hz}\right.$ in addition split by $\left.J_{5-\mathrm{CH}^{\mathrm{A}}, 2^{\prime}}=3.3 \mathrm{~Hz}, J_{5-\mathrm{CH}^{\mathrm{B}}, 2^{\prime}}=5.1 \mathrm{~Hz}, 2 \mathrm{H}, 5-\mathrm{CH}_{2}\right), 3.63\left(\mathrm{dd}, J_{5,5-\mathrm{CH}^{\mathrm{A}}}=3.4 \mathrm{~Hz}, J_{5,5-}\right.$ $\left.\mathrm{CH}^{\mathrm{B}}=5.0 \mathrm{~Hz}, 1 \mathrm{H}, 5-\mathrm{H}\right), 3.81\left(\mathrm{~s}, 6 \mathrm{H}, 1\right.$ '"'- $\mathrm{OCH}_{3}, 3$ '"- $\left.-\mathrm{OCH}_{3}\right), 3.83\left(\mathrm{~s}, 3 \mathrm{H}, 5\right.$ "'- $\left.\mathrm{OCH}_{3}\right), 4.41$ (d, $\left.{ }^{2} J=14.1 \mathrm{~Hz}, 1 \mathrm{H}, 2^{\prime \prime}-\mathrm{C}-\mathrm{H}^{\mathrm{A}}\right), 5.12$ (d, $\left.{ }^{2} J=14.1 \mathrm{~Hz}, 1 \mathrm{H}, 2^{\prime \prime}-\mathrm{C}-\mathrm{H}^{\mathrm{B}}\right), 6.14$ (s, 2H, 4"'-H, 6"'-H), 6.67-6.70 (m, 2H, 2"-H, 6"-H), 6.91-7.05 (m, 4H, 4'-H, 5'-H, 3"-H, 5"-H), 7.18 (d, J J',3' = 15.3 Hz, 1H, 2'-H), 7.30-7.34 (m, 1H, 4"'--H), 7.34-7.39 (m, 2H, 3'"'-H, 5"'-H), 7.46-7.53 (m, 3H, 3'H, 2"'--H, 6"'--H) ppm.

${ }^{13}$ C-NMR (125.75 MHz, $\mathrm{CDCl}_{3} / \mathrm{CHCl}_{3}$, DsBrMi13-500304, 14.5.2020): $\delta=-4.4$ [1"-O-Si$\left(\mathrm{CH}_{3}\right)_{2}$ ], 18.3 [1"-O-Si-C(CH$)_{3}$ ], 25.8 [1"-O-Si-C $\left(\mathrm{CH}_{3}\right)_{3}$ ], $32.6\left(2\right.$ "'- $\left.\mathrm{CH}_{2}\right), 33.8\left(5-\mathrm{CH}_{2}\right), 55.5$ (5"'-OCH 3 ), 55.8 (1"'-OCH 3 , 3"'-OCH $), 64.6$ (C-5), 90.5 (C-4"', C-6"'), 101.5 (C-3), 103.4 (C2"'), 120.0 (C-2", C-6"), 122.1 (C-2'), 127.5 (C-2"'", C-6"''), 127.6 (C-4'), 128.0 (C-4"), 129.0 (C-3"'", C-5"'"), 129.4 (C-4"'"), 130.8 (C-3", C-5"), 136.2 (C-1"'"), 141.8 (C-5'), 143.5 (C-3'), 154.5 (C-1"), 160.0 (C-1"', C-3"'), 161.6 (C-5"'), 172.1 (C-1'), 173.2 (C-2), 195.3 (C-4) ppm.

edHSQC [,,short-range H,C-COSY“, 500.06 MHz/125.75 MHz, $\mathrm{CDCl}_{3} ; \delta\left({ }^{1} \mathrm{H}\right) \leftrightarrow \delta\left({ }^{13} \mathrm{C}\right)$ ]: 0.13 [s, 1"-O-Si- $\left.\left(\mathrm{CH}_{3}\right)_{2}\right] \leftrightarrow-4.4\left[1 "-\mathrm{O}-\mathrm{Si}-\left(\mathrm{CH}_{3}\right)_{2}\right], 0.93$ [s, 1"-O-Si-C $\left.\left(\mathrm{CH}_{3}\right)_{3}\right] \leftrightarrow 25.8$ [1"-O-Si$\left.\mathrm{C}\left(\mathrm{CH}_{3}\right)_{3}\right], \mathrm{AB}$ signal $\left(\delta_{\mathrm{A}}=3.05, \delta_{\mathrm{B}}=3.08,5-\mathrm{CH}_{2}\right) \leftrightarrow 33.8\left(5-\mathrm{CH}_{2}\right), 3.63(\mathrm{dd}, 5-\mathrm{H}) \leftrightarrow 64.6(\mathrm{C}-$

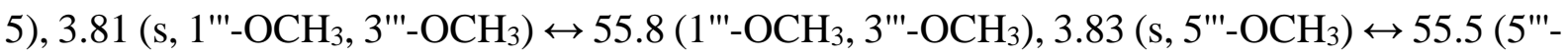

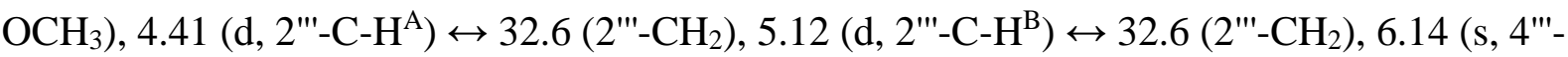
H, 6"'-H) ↔ 90.5 (C-4"', C-6"'), 6.67-6.70 (m, 2"-H, 6"-H) ↔ 120.0 (C-2", C-6"), 6.91-7.05 (m, 4'-H) $127.6(\mathrm{C}-4 '), 6.91-7.05\left(\mathrm{~m}, 5^{\prime}-\mathrm{H}\right) \leftrightarrow 141.8\left(\mathrm{C}-5\right.$ '), $6.91-7.05\left(\mathrm{~m}, 3 "-\mathrm{H}, 5^{\prime \prime}-\mathrm{H}\right) \leftrightarrow 130.8$ (C-3", C-5"), 7.18 (d, 2'-H) ↔ 122.1 (C-2'), 7.30-7.34 (m, 4"'--H) ↔ 129.4 (C-4'"'), 7.34-7.39 (m, 3"''-H, 5'"'-H) 129.0 (C-3"'", C-5'"'), 7.46-7.53 (m, 2"'"-H, 6"'"-H) ↔ 127.5 (C-2"'", C-6"''), 7.46-7.53 (m, 3'-H) $143.6\left(\mathrm{C}-3^{\prime}\right)$.

Specific rotation $(S)-22 \mathrm{e}[\alpha]_{D}^{20}=-798.2^{\circ}\left(\mathrm{c}=0.43\right.$ in $\left.\mathrm{CHCl}_{3}\right)$

HRMS: $(\mathrm{EI}, 70 \mathrm{eV}): \mathrm{m} / \mathrm{z}=656.3030[\mathrm{M}+\mathrm{H}]^{+}$, corresponds to the molecular formula $\mathrm{C}_{38} \mathrm{H}_{46} \mathrm{O}_{7} \mathrm{NSi}(656.3030)\left\{[\mathrm{M}+\mathrm{H}]^{+}\right\}$with a deviation of $-1.2 \mathrm{ppm}$.

IR (KBr): $\tilde{v}=2955,2930,2855,2902,1695,1610,1595,1560,1510,1440,14201360,1345$, $1255,1205,1185,1170,1151,1131,1110,1001,915,840,815,781,750 \mathrm{~cm}^{-1}$. 
(5S)-5-\{1'"-[(tert-Butyldimethylsilyl)oxy]benzyl $\}-3-\left(1 ' Z, 2^{\prime} E, 4^{\prime} E\right)-1^{\prime}-h y d r o x y-5-$ phenylpenta-2',4'-dien-1'-ylidene)pyrrolidine-2,4-dion [Z-enol-(S)-TBDMS-17]

in equilibration with

(5S)- 5-\{1'"-[(tert-Butyldimethylsilyl)oxy]benzyl\}-3-(1'E,2' $\left.E, 4^{\prime} E\right)-1^{\prime}-$ hydroxy-5phenylpenta-2',4'-dien-1'-ylidene)pyrrolidine-2,4-dion [E-enol-(S)-TBDMS-17]

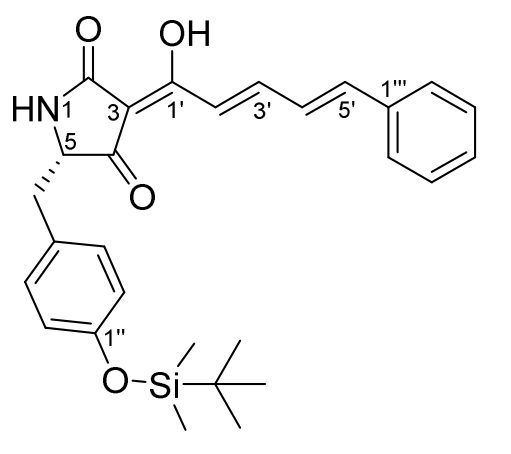

Z-enol-(S)-TBDMS-17

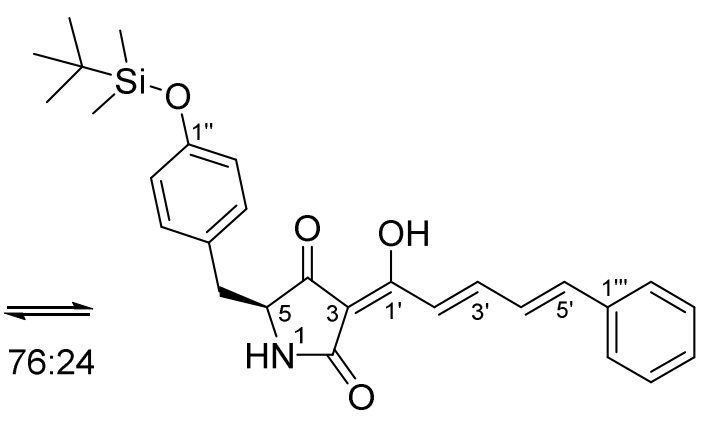

E-enol-(S)-TBDMS-17

Method A: The tetramic acid $(S)-\mathbf{S I - 8}(18.0 \mathrm{mg}, 27.5 \mu \mathrm{mol})$ was dissolved in $\mathrm{CH}_{2} \mathrm{Cl}_{2}(6.0 \mathrm{~mL})$. It was added $\mathrm{F}_{3} \mathrm{CCO}_{2} \mathrm{H}(60 \mu \mathrm{L})$ at $25^{\circ} \mathrm{C}$. After $4.5 \mathrm{~h}$ at this temperature the mixture was diluted with $\mathrm{H}_{2} \mathrm{O}(8 \mathrm{~mL})$. The layers were separated, and the aq. layer was extracted with $\mathrm{CH}_{2} \mathrm{Cl}_{2}$ $(3 \times 10 \mathrm{~mL})$. The combined organic layers were washed with brine $(5 \mathrm{~mL})$ and tried over $\mathrm{Na}_{2} \mathrm{SO}_{4}$. The solvent was removed under reduced pressure and the residue was purified by reverse phase flash chromatography $\left(\mathrm{MeCN}: \mathrm{H}_{2} \mathrm{O}: \mathrm{TFA}=90: 10: 0.1,1.5 \mathrm{~cm}, 10 \mathrm{~cm}, 2 \mathrm{~mL}\right)$. The tetramic acid $(S)$-TBMDS-17 $(11.7 \mathrm{mg}, 90 \%, 24.8 \mu \mathrm{mol})$ was obtained as a yellow powder. It was assigned as a 74:26 mixture of the $Z$ - and the $E$-enol of the title compounds $(S)$-TBMDS17. This ratio was determined by integrating singlets of the respective 1 "- $\mathrm{O}-\mathrm{Si}-\mathrm{C}\left(\mathrm{CH}_{3}\right)_{3}[0.91$ (E-enol) and 0.93 (Z-enol)].

Method B: A solution of the tetramic acid $(S)$-TBMDS-16a (44.0 mg, $72.0 \mu \mathrm{mol})$ in EtOH (3.2 $\mathrm{mL}$ ) was added to a solution of $\mathrm{Na}_{2} \mathrm{~S}_{2} \mathrm{O}_{4}\left(90 \mathrm{mg}, 432 \mu \mathrm{mol}, 6\right.$ eq.) and $\mathrm{NaHCO}_{3}$ (79 mg, 936 $\mu$ mol, 13 eq.) in $\mathrm{H}_{2} \mathrm{O}(2 \mathrm{~mL})$. The resulting mixture stirred for $20 \mathrm{~min}$ at $25^{\circ} \mathrm{C}$. The mixture was diluted with sat. $\mathrm{NH}_{4} \mathrm{Cl}$-solution $(10 \mathrm{~mL})$ and $\mathrm{Et}_{2} \mathrm{O}(20 \mathrm{~mL})$. The layers were separated, and the aq. layer was extracted with $\mathrm{Et}_{2} \mathrm{O}(3 \times 10 \mathrm{~mL})$. The combined organic layers were 
Christian Drescher and Reinhard Brückner; 3D-Structure Clarifying Total Synthesis of the (Polyenoyl)tetramic Acid Militarinon B. A Highly Acid-Labile N-Protecting Group for Amides

washed with brine $(5 \mathrm{~mL})$ and tried over $\mathrm{Na}_{2} \mathrm{SO}_{4}$. The solvent was removed under reduced pressure and crude $(S)-\mathbf{1 6 b}(41.0 \mathrm{mg}, 61.2 \mu \mathrm{mol})$ was dissolved in $\mathrm{CH}_{2} \mathrm{Cl}_{2}(550 \mu \mathrm{L})$. At $-20^{\circ} \mathrm{C}$ it was added $\mathrm{H}_{2} \mathrm{O}(30 \mu \mathrm{L})$ and DDQ $(18.5 \mathrm{mg}, 79 \mu \mathrm{mol}, 1.1$ eq. $)$. The mixture stirred at this temperature for $2 \mathrm{~h}$. The mixture was filtered and evaporated. The residue was purified by reverse phase flash chromatography $\left(\mathrm{MeCN}: \mathrm{H}_{2} \mathrm{O}: \mathrm{TFA}=90: 10: 0.1,1.5 \mathrm{~cm}, 10 \mathrm{~cm}, 2 \mathrm{~mL}\right)$. The tetramic acid $(S)$-TBMDS-17 $(6.4 \mathrm{mg}, 19 \%, 13.6 \mu \mathrm{mol})$ was obtained as a yellow powder. It was assigned as a 74:26 mixture of the $Z$ - and the $E$-enol of the title compounds ( $S$ )-TBMDS17. This ratio was determined by integrating singlets of the respective 1 "-O-Si- $\mathrm{C}\left(\mathrm{CH}_{3}\right)_{3}[0.91$ (E-enol) and 0.93 (Z-enol)].

$\mathbf{R}_{\mathbf{f}}\left(\mathrm{MeCN}: \mathrm{H}_{2} \mathrm{O}: \mathrm{TFA}=90: 10: 0.1\right): 0.25$

${ }^{1} \mathbf{H}$-NMR $(500.06 \mathrm{MHz}, \mathrm{MeOD} / \mathrm{MeOH}$, sample contained grease with singlet at 1.27 and sample contained unknown impurity with $\mathrm{m}$ at 3.61-3.82, DsBrMi13-500300, 15.5.2020): $\delta=$ 0.13 [s, 6H, 1"-O-Si- $\left(\mathrm{CH}_{3}\right)_{2}$ ], 0.93 [s, 9H, 1"-O-Si-C $\left.\left(\mathrm{CH}_{3}\right)_{3}\right], \mathrm{AB}$ signal $\left(\delta_{\mathrm{A}}=2.93, \delta_{\mathrm{B}}=3.01\right.$, $J_{\mathrm{AB}}=14.1 \mathrm{~Hz}$ in addition split by $J_{5-\mathrm{CH}^{\mathrm{A}}, 2^{\prime}}=4.3 \mathrm{~Hz}, J_{5-\mathrm{CH}^{\mathrm{B}}, 2^{\prime}}=5.4 \mathrm{~Hz}, 2 \mathrm{H}, 5-\mathrm{CH}_{2}$ ), 4.08 (br. s, 1H, 5-H), 6.68-6.72 (m, 2H, 2"-H, 6"-H), 7.00-7.15 (m, 4H, 4'-H, 5'-H, 3"-H, 5"-H), 7.21 (d, $\left.J_{2^{\prime}, 3^{\prime}}=15.3 \mathrm{~Hz}, 1 \mathrm{H}, 2^{\prime}-\mathrm{H}\right)$, 7.29-7.40 (m, 3H, 3'"-H, 4'"-H, 5"'-H), 7.50-7.59 (m, 2H, 2"'-H, 6'"$\mathrm{H}), 7.62\left(\mathrm{dd}, J_{3^{\prime}, 2^{\prime}}=15.1 \mathrm{~Hz}, J_{3^{\prime}, 4^{\prime}}=10.3 \mathrm{~Hz}, 1 \mathrm{H}, 3^{\prime}-\mathrm{H}\right) \mathrm{ppm}$.

${ }^{13}$ C-NMR (125.75 MHz, MeOD/MeOH, DsBrMi14-500306, 15.5.2020): $\delta=-4.3$ [1"-O-Si$\left(\mathrm{CH}_{3}\right)_{2}$ ], 18.3 [1"-O-Si-C $\left(\mathrm{CH}_{3}\right)_{3}$ ], 26.2 [1"-O-Si-C $\left(\mathrm{CH}_{3}\right)_{3}$ ], $37.7\left(5-\mathrm{CH}_{2}\right), 64.2(\mathrm{C}-5), 121.0(\mathrm{C}-$ 2", C-6"), 122.2 (C-2'), 128.3 (C-4'), 128.8 (C-2"', C-6"'), 128.8 (C-4"), 130.0 (C-3"', C-5'"), 130.6 (C-4"'), 131.9 (C-3", C-5"), 137.5 (C-1'"), 144.4 (C-5'), 146.2 (C-3'), 155.9 (C-1") ppm.* * C-2, C-4 and C-1' were not found.

edHSQC [,,short-range H,C-COSY“, 500.06 MHz/125.75 MHz, $\mathrm{CDCl}_{3} ; \delta\left({ }^{1} \mathrm{H}\right) \leftrightarrow \delta\left({ }^{13} \mathrm{C}\right)$ ]: 0.13 [s, 1"-O-Si- $\left.\left(\mathrm{CH}_{3}\right)_{2}\right] \leftrightarrow-4.3$ [1"-O-Si- $\left.\left(\mathrm{CH}_{3}\right)_{2}\right], 0.93$ [s, 1"-O-Si-C $\left.\left(\mathrm{CH}_{3}\right)_{3}\right] \leftrightarrow 26.2$ [1"-O-Si$\left.\mathrm{C}\left(\mathrm{CH}_{3}\right)_{3}\right], \mathrm{AB}$ signal $\left(\delta_{\mathrm{A}}=2.93, \delta_{\mathrm{B}}=3.01,5-\mathrm{CH}_{2}\right) \leftrightarrow 37.7\left(5-\mathrm{CH}_{2}\right), 4.08$ (br. s, 5-H) 64.2 (C-5), 6.68-6.72 (m, 2"-H, 6"-H) 121.0 (C-2", C-6"), 7.00-7.15 (m, 4'-H) 128.3 (C-4'), 7.00-7.15 (m, 5'-H) 144.4 (C-5'), 7.00-7.15 (m, 3"-H, 5"-H) 131.9 (C-3", C-5"), 7.21 (d, $\left.2^{\prime}-\mathrm{H}\right) \leftrightarrow 122.2(\mathrm{C}-2$ '), 7.29-7.40 (m, 4'"-H) 130.6 (C-4'"), 7.29-7.40 (m, 3'"-H, 5'"-H) 130.0 (C-3"', C-5"'), 7.50-7.59 (m, 2'"-H, 6"'-H) 128.8 (C-2"', C-6'"), 7.62 (dd, 3'-H) 146.2 (C-3').

Specific rotation $(S)$-TBMDS-17 $[\alpha]_{D}^{20}=-649.1^{\circ}\left(\mathrm{c}=0.19\right.$ in $\left.\mathrm{CHCl}_{3}\right)$ 
Christian Drescher and Reinhard Brückner; 3D-Structure Clarifying Total Synthesis of the (Polyenoyl)tetramic Acid Militarinon B. A Highly Acid-Labile N-Protecting Group for Amides

HRMS: (EI, $70 \mathrm{eV}): \mathrm{m} / \mathrm{z}=476.2259[\mathrm{M}+\mathrm{H}]^{+}$, corresponds to the molecular formula $\mathrm{C}_{28} \mathrm{H}_{34} \mathrm{O}_{4} \mathrm{NSi}(476.2252)\left\{[\mathrm{M}+\mathrm{H}]^{+}\right\}$with a deviation of $1.5 \mathrm{ppm}$.

IR (KBr): $\tilde{v}=2955,2930,2855,1655,1610,1595,1580,1560,1510,1470,1465,1450,1430$, $1360,1350,1260,1205,1170,1000,915,870,840,810,780,750,725 \mathrm{~cm}^{-1}$.

(5S)-5-(1'-Hydroxybenzyl)-1-(1'"',3'",5'"'-trimethoxybenzyl)-3-(1'Z,2'E,4'E)-1'-hydroxy5-phenylpenta-2',4"'-dien-1'-ylidene)pyrrolidine-2,4-dion [Z-enol-(S)-16e] in equilibration with

(5S)-5-(1'-Hydroxybenzyl)-1-(1'"',3'",5'"'-trimethoxybenzyl)-3-(1'E,2' E,4'E)-1'-hydroxy5-phenylpenta-2',4' '-dien-1'-ylidene)pyrrolidine-2,4-dion [E-enol-(S)-16e]
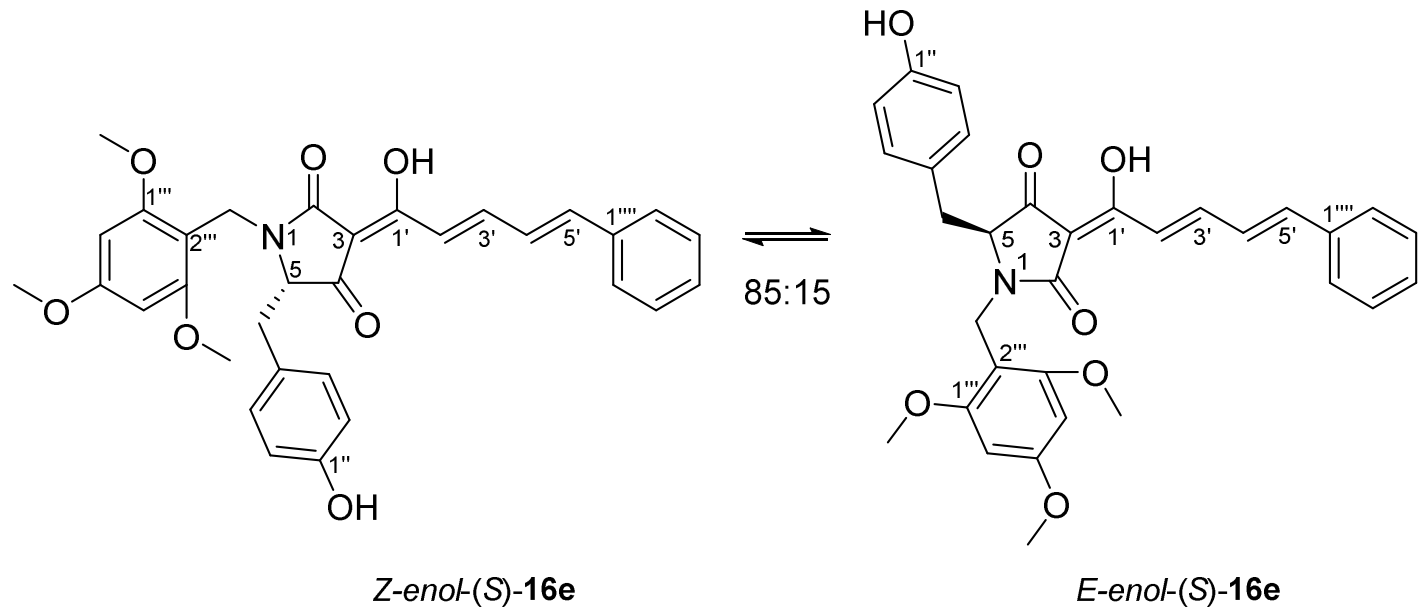

E-enol-(S)-16e

A solution of the polyen $(S)-\mathbf{2 2 e}(42.0 \mathrm{mg}, 61 \mu \mathrm{mol})$ in THF $(2.0 \mathrm{~mL})$ was deluded with TBAF ( $1 \mathrm{M}$ in THF, $305 \mu \mathrm{L}, 305 \mu \mathrm{mol}, 5.0$ eq.) at $25^{\circ} \mathrm{C}$. At this temperature the mixture was stirred for $1 \mathrm{~h}$. The solvent was removed under reduced pressure and the residue was obtained with sat. aq. $\mathrm{NH}_{4} \mathrm{Cl}(4.0 \mathrm{~mL}), \mathrm{HCl}(1 \mathrm{M}, 1.0 \mathrm{~mL})$ and $\mathrm{Et}_{2} \mathrm{O}(10 \mathrm{~mL})$. The layers were separated, and the organic layer was extracted with $\mathrm{Et}_{2} \mathrm{O}(3 \times 10 \mathrm{~mL})$. The combined organic layers were tried over $\mathrm{Na}_{2} \mathrm{SO}_{4}$ and concentrated under reduced pressure. The residue was purified by reverse phase flash chromatography ( $\left.\mathrm{MeCN}: \mathrm{H}_{2} \mathrm{O}: \mathrm{TFA}=80: 20: 0.1,1.5 \mathrm{~cm}, 10 \mathrm{~cm}, 2 \mathrm{~mL}\right)$. The tetramic acid $(S)-321(29.0 \mathrm{mg}, 85 \%, 51.9 \mu \mathrm{mol})$ was obtained as a yellow powder. It was assigned as a 85:15 mixture of the $Z$ - and the $E$-enol of the title compounds $(S)$-16e. This ratio was determined by integrating singlets of the respective 1"'- $\mathrm{OCH}_{3} / 3$ '"-OCH 3.77 (E-enol) and 3.79 (Z-enol)].

$\mathbf{R}_{\mathbf{f}}\left(\mathrm{MeCN}: \mathrm{H}_{2} \mathrm{O}: \mathrm{TFA}=80: 20: 0.1\right): 0.29$ 
Christian Drescher and Reinhard Brückner; 3D-Structure Clarifying Total Synthesis of the (Polyenoyl)tetramic Acid Militarinon B. A Highly Acid-Labile N-Protecting Group for Amides

${ }^{1} \mathrm{H}-\mathrm{NMR}\left(500.06 \mathrm{MHz}, \mathrm{CDCl}_{3} / \mathrm{CHCl}_{3}\right.$, sample contained 6 weight- $\%$ of $\mathrm{O}\left(\mathrm{SnBu}_{3}\right)_{2}$ with $\mathrm{t}$ at $0.92, \mathrm{~m}_{\mathrm{c}}$ at $1.30, \mathrm{~m}_{\mathrm{c}}$ at 1.35 and $\mathrm{m}_{\mathrm{c}}$ at $\left.1.63, \mathrm{DsBrAp} 27-500400,28.4 .2020\right): \delta=\mathrm{AB}$ signal $\left(\delta_{\mathrm{A}}=3.04, \delta_{\mathrm{B}}=3.09, J_{\mathrm{AB}}=14.4 \mathrm{~Hz}\right.$ in addition split by $J_{5-\mathrm{CH}^{\mathrm{A}}, 2^{\prime}}=5.2 \mathrm{~Hz}, J_{5-\mathrm{CH}^{\mathrm{A}}, 2^{\prime}}=3.2 \mathrm{~Hz}, 2 \mathrm{H}$, 5- $\left.\mathrm{CH}_{2}\right), 3.66\left(\mathrm{dd}, J_{5,5-\mathrm{CH}^{\wedge}}=5.1 \mathrm{~Hz}, J_{5,5-\mathrm{CH}^{\mathrm{B}}}=3.1 \mathrm{~Hz}, 1 \mathrm{H}, 5-\mathrm{H}\right), 3.79\left(\mathrm{~s}, 6 \mathrm{H}, 1\right.$ '"- $\mathrm{OCH}_{3}, 3$ "'$\left.\mathrm{OCH}_{3}\right), 3.82\left(\mathrm{~s}, 3 \mathrm{H}, 5^{\prime \prime}-\mathrm{OCH}_{3}\right), 4.43\left(\mathrm{~d},{ }^{2} J=14.1 \mathrm{~Hz}, 1 \mathrm{H}, 2\right.$ ''-C-H $\left.{ }^{\mathrm{A}}\right), 5.14\left(\mathrm{~d},{ }^{2} J=14.1 \mathrm{~Hz}, 1 \mathrm{H}\right.$, 2'"-C-H ${ }^{\mathrm{B}}$ ), 6.12 (s, 2H, 4'"-H, 6"'-H), 6.63-6.67 (m, 2H, 2"-H, 6"-H), 6.89-7.00 (m, 4H, 4'-H, 5'H, 3"-H, 5"-H), 7.20 (d, J', 3' = 15.2 Hz, 1H, 2'-H), 7.29-7.38 (m, 3H, 3"'"-H, 4"'--H, 5"'--H), 7.43$7.45\left(\mathrm{~m}, 2 \mathrm{H}, 2^{\prime \prime \prime '-H, ~ 6 ' " '-H), ~} 7.51\left(\mathrm{ddd}, J_{3^{\prime}, 2^{\prime}}=15.2 \mathrm{~Hz}, J_{3^{\prime}, 4^{\prime}}=9.5 \mathrm{~Hz}, J_{3^{\prime}, 1^{\prime}-\mathrm{OH}}=1.3 \mathrm{~Hz}, 1 \mathrm{H}, 3^{\prime}-\right.\right.$ H) ppm.

${ }^{13} \mathbf{C}$-NMR $\left(125.75 \mathrm{MHz}, \mathrm{CDCl}_{3} / \mathrm{CHCl}_{3}\right.$, sample contained $\mathrm{O}\left(\mathrm{SnBu}_{3}\right)_{2}$ at 13.6, 17.6, 26.9 and 27.9, DsBrAp27-500404, 28.4.2020): $\delta=32.8\left(2 " '-\mathrm{CH}_{2}\right), 33.5\left(5-\mathrm{CH}_{2}\right), 55.5\left(5^{\prime \prime}-\mathrm{OCH}_{3}\right), 55.8$ (1"'-OCH 3 , 3"'-OCH 3 ), 64.8 (C-5), 90.5 (C-4"', C-6"'), 101.5 (C-3), 103.2 (C-2"'), 126.8 (C-4"), 115.3 (C-2", C-6"), 121.9 (C-2'), 127.5 (C-4'), 127.6 (C-2"'", C-6"'"), 128.9 (C-3'"', C-5"'"), 129.5 (C-4"'"), 130.9 (C-3", C-5"), 136.2 (C-1'"'), 142.1 (C-5'), 144.0 (C-3'), 154.9 (C-1"), 159.9 (C1'", C-3"'), 161.6 (C-5"'), 172.6 (C-1'), 173.3 (C-2), 195.4 (C-4) ppm.

edHSQC [,,short-range $\left.\mathrm{H}, \mathrm{C}-\mathrm{COSY} “, 500.06 \mathrm{MHz} / 125.75 \mathrm{MHz}, \mathrm{CDCl}_{3} ; \delta\left({ }^{1} \mathrm{H}\right) \leftrightarrow \delta\left({ }^{13} \mathrm{C}\right)\right]$ : AB signal $\left(\delta_{\mathrm{A}}=3.04, \delta_{\mathrm{B}}=3.09,5-\mathrm{CH}_{2}\right) \leftrightarrow 33.5\left(5-\mathrm{CH}_{2}\right), 3.66(\mathrm{dd}, 5-\mathrm{H}) \leftrightarrow 64.8(\mathrm{C}-5), 3.79\left(\mathrm{~s}, 1{ }^{\prime \prime}-\right.$ $\mathrm{OCH}_{3}, 3$ "'- $\left.\mathrm{OCH}_{3}\right) \leftrightarrow 55.8\left(1\right.$ "'- $\mathrm{OCH}_{3}, 3$ "'- $\left.\mathrm{OCH}_{3}\right), 3.82\left(\mathrm{~s}, 5\right.$ "'- $\left.\mathrm{OCH}_{3}\right) \leftrightarrow 55.5\left(5^{\prime \prime}-\mathrm{OCH}_{3}\right), 4.43$ $\left(\mathrm{d}, 2\right.$ "'-C-H $\left.{ }^{\mathrm{A}}\right) \leftrightarrow 32.8\left(2^{\prime \prime \prime}-\mathrm{CH}_{2}\right), 5.14\left(\mathrm{~d}, 2\right.$ "'-C-H $\left.{ }^{\mathrm{B}}\right) \leftrightarrow 32.8\left(2^{\prime \prime}-\mathrm{CH}_{2}\right), 6.12(\mathrm{~s}, 4$ "'-H, 6'"-H) 90.5 (C-4"', C-6"'), 6.63-6.67 (m, 2"-H, 6"-H) ↔ 115.3 (C-2", C-6"), 6.89-7.00 (m, 4'-H) ↔ 127.5 (C-4'), 6.89-7.00 (m, 5'-H) 142.1 (C-5'), 6.89-7.00 (m, 3"-H, 5"-H) 130.9 (C-3", C5"), $7.20\left(\mathrm{~d}, 2^{\prime}-\mathrm{H}\right) \leftrightarrow 121.9$ (C-2'), 7.29-7.38 (m, 3'"'-H, 5"'-H) ↔ 128.9 (C-3'"', C-5'"'), 7.297.38 (m, 4'"'-H) ↔ 129.5 (C-4'"'), 7.43-7.45 (m, 2'"'-H, 6"'-H) ↔ 127.6 (C-2"'", C-6'"'), 7.51 (ddd, $\left.3^{\prime}-\mathrm{H}\right) \leftrightarrow 144.0\left(\mathrm{C}-3^{\prime}\right)$.

Specific rotation $(S)-16 \mathbf{e}[\alpha]_{D}^{20}=-758.0^{\circ}\left(\mathrm{c}=0.22\right.$ in $\left.\mathrm{CHCl}_{3}\right)$

HRMS: (EI, $70 \mathrm{eV}): \mathrm{m} / \mathrm{z}=540.2025[\mathrm{M}-\mathrm{H}]^{+}$, corresponds to the molecular formula $\mathrm{C}_{32} \mathrm{H}_{30} \mathrm{O}_{7} \mathrm{~N}$ (540.2028) $\left\{[\mathrm{M}-\mathrm{H}]^{+}\right\}$with a deviation of $-0.5 \mathrm{ppm}$.

IR (KBr): $\tilde{v}=2980,2935,2865,1680,1610,1590,1515,1500,1450,1380,1350,1280,1230$, $1205,1150,1125 \mathrm{~cm}^{-1}$. 


\subsection{Synthesis of the two Diastereomers of the Western Block 10e}

\section{4-(Benzyloxy)benzaldehyde (14)}<smiles></smiles>

14

4-Hydroxybenzaldehyde (1.52 g, $12.4 \mathrm{mmol})$ and $\mathrm{K}_{2} \mathrm{CO}_{3}(5.19 \mathrm{~g}, 37.6 \mathrm{mmol}, 3.0$ eq.) were dissolved in DMF ( $8 \mathrm{~mL}$ ) and benzyl bromide (2.28 mL, $3.28 \mathrm{~g}, 19.2 \mathrm{mmol}, 1.6$ eq.) was added at $25^{\circ} \mathrm{C}$. After $3 \mathrm{~h}$ at this temperature the mixture was diluted with $\mathrm{H}_{2} \mathrm{O}(20 \mathrm{~mL})$. The organic layer was separated, and the aq. layer was extracted with EtOAc $(3 \times 20 \mathrm{ml})$. The combined organic layers were washed with brine $(20 \mathrm{~mL})$ and dried over $\mathrm{Na}_{2} \mathrm{SO}_{4}$. The solvent was removed under reduced pressure and the residue was purified by flash chromatography $(4.0 \mathrm{~cm}$, $20 \mathrm{~cm}, c \mathrm{C}_{6} \mathrm{H}_{12}: \mathrm{EtOAc}=10: 1,40 \mathrm{~mL}$ ) to give the product 14 [2.20 g, 84\%, 10.4 mmol, (Lit. ${ }^{11}$ : $69 \%)]$ as a white solid.

$\mathbf{R}_{\mathbf{f}}\left(\mathrm{cC}_{6} \mathrm{H}_{12}: \mathrm{AcOEt}=10: 1\right)=0.30$

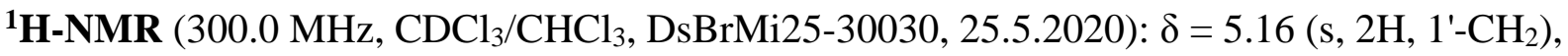
$7.08\left(\mathrm{~m}_{\mathrm{c}}, 2 \mathrm{H}, 3-\mathrm{H}, 5-\mathrm{H}\right), 7.33-7.45$ (m, 5H, 2'-H, 3'-H, 4'-H, 5'-H, 6'-H), 7.84 (mc , 2H, 2-H, 6H), 9.89 (s, 1H, 1-COH) ppm.

\footnotetext{
${ }^{11}$ J. Yi. G. Du, Y. Zhao, L. Zhang, B. Li, W. Zhu, C. Huang, Y. Li, F.Guo, Med. Chem. Res. 2018, 27, 1851-
} 1862 . 


\section{(R)-3-Isopropylpiperazine-2,5-dione [(R)-SI-9]}<smiles>CC(C)[C@H]1NC(=O)CNC1=O</smiles>

(R)-SI-9

Methyl $N$-(tert-Butoxycarbonyl)-D-valyl Glycinate (5.00 g, $17.3 \mathrm{mmol}$ ) was dissolved in 1,2dichlorobenzene $(45 \mathrm{~mL})$ and heated for $18 \mathrm{~h}$ to $185^{\circ} \mathrm{C}$. The resulting $\mathrm{MeOH}$ was distilled off. Then 1,2-dichlorobenzene $(30 \mathrm{~mL})$ was distilled off and the resulting mixture was cooled to $50^{\circ} \mathrm{C}$. It was added $t \mathrm{BuOMe}(25 \mathrm{~mL})$ and the resulting suspension was filtered. The white solid was dried in vacuum at $100^{\circ} \mathrm{C}$ for $24 \mathrm{~h}$. The product $(R)$-SI-9 [1.70 g, 63\%, 10.9 mmol (Lit. ${ }^{12}$ : $69 \%)$ ] was obtained as a white solid.

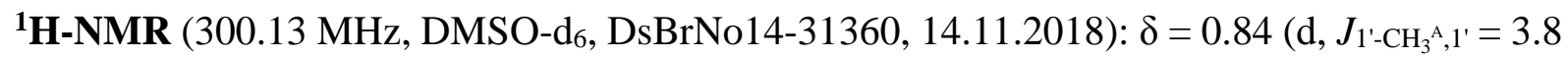

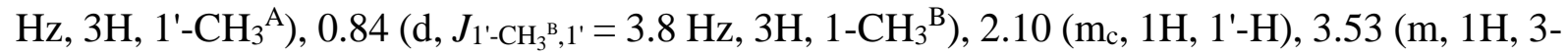
$\mathrm{H})$, AB signal $\left(\delta_{\mathrm{A}}=3.62, \delta_{\mathrm{B}}=3.82, J_{\mathrm{AB}}=17.6 \mathrm{~Hz}, 2 \mathrm{H}, 2-\mathrm{H}_{2}\right), 8.01$ (br. s, $\left.1 \mathrm{H}, 1-\mathrm{NH}\right), 8.20$ (br. $\mathrm{s}, 1 \mathrm{H}, 4-\mathrm{NH}) \mathrm{ppm}$.

(R)-3,6-Diethoxy-2-isopropyl-2,5-dihydropyrazine [(R)-15a]

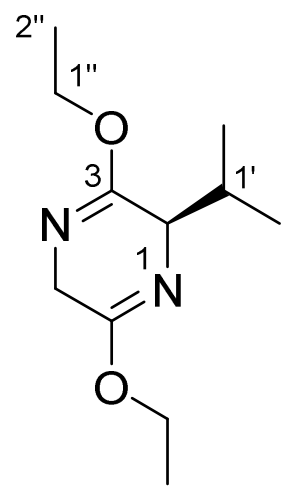

$(R)-15 a$

The bislactam $(R)$-SI-9 $(280 \mathrm{mg}, 1.79 \mathrm{mmol})$ was dissolved in $\mathrm{CH}_{2} \mathrm{Cl}_{2}(10 \mathrm{~mL}) . \mathrm{EtO}_{3} \cdot \mathrm{BF}_{4}$ $\left(1.32 \mathrm{~g}, 6.95 \mathrm{mmol}, 3.9\right.$ eq.) was added and the resulting mixture stirred for $4 \mathrm{~d}$ at $25^{\circ} \mathrm{C}$. The 
Christian Drescher and Reinhard Brückner; 3D-Structure Clarifying Total Synthesis of the (Polyenoyl)tetramic Acid Militarinon B. A Highly Acid-Labile N-Protecting Group for Amides

reaction mixture was added to mixture of aq. sat. $\mathrm{NaHCO}_{3}(10 \mathrm{~mL})$ and $\mathrm{CH}_{2} \mathrm{Cl}_{2}(10 \mathrm{~mL})$ which was cooled to $0^{\circ} \mathrm{C}$. It was added aq. $\mathrm{NaOH}(3 \mathrm{M}, 5 \mathrm{~mL})$. The organic layer was separated, and the aq. layer was extracted with $\mathrm{CH}_{2} \mathrm{Cl}_{2}(3 \times 10 \mathrm{ml})$. The combined organic layers were washed with brine $(10 \mathrm{~mL})$ and dried over $\mathrm{Na}_{2} \mathrm{SO}_{4}$. The solvent was removed under reduced pressure

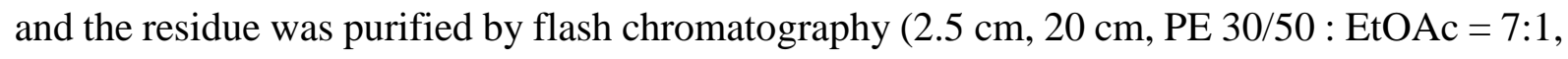
$15 \mathrm{~mL}$ ) to give the product $(R)-\mathbf{1 5 a}$ [262 $\mathrm{mg}, 77 \%, 1.23 \mathrm{mmol}$ (Lit. $\left.{ }^{13}: 90 \%\right)$ ] as a colorless liquid.

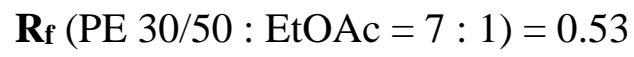

${ }^{1} \mathbf{H}$-NMR $\left(300.13 \mathrm{MHz}, \mathrm{CDCl}_{3} / \mathrm{CHCl}_{3}\right.$, sample contained 3 weight-\% of EtOAc, DsBrMi25-

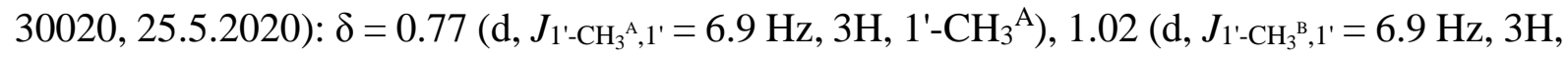

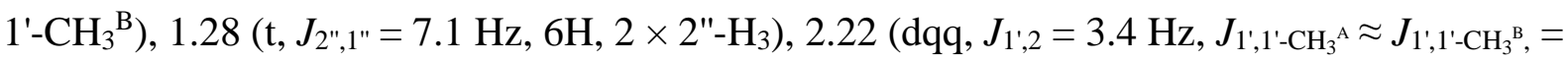
$6.9 \mathrm{~Hz}, 1 \mathrm{H}, 1 '-\mathrm{H}), 3.91-4.42\left(\mathrm{~m}, 7 \mathrm{H}, 5-\mathrm{H}_{2}, 2-\mathrm{H}, 2 \times 1 "-\mathrm{H}_{2}\right) \mathrm{ppm}$.

(S)-[4"'-(Benzyloxy)phenyl][(5S,2R)-3,6-diethoxy-2-isopropyl-1,4-dihydropyrazin-5yl]methanol $[(S, S, R)-13 a]$

separated from

$(R)$-[4'"'-(Benzyloxy)phenyl][(5S,2R)-3,6-diethoxy-2-isopropyl-1,4-dihydropyrazin-5yl]methanol $[(R, S, R)-13 a]$

${ }^{13}$ M. Sally, T. Marcello, D. Ludovic, S. Savade, V. Lewis, W. Isaac, G. Peter, V. M. Rob, H. Swen, Chem. Eur. J. 2017, 23, 9577-9584. 
<smiles></smiles>

$(S, S, R)-13 a$<smiles>[R16][R5]</smiles>

A solution of the Schöllkopf auxiliary $(R)-\mathbf{1 5 a}(1.03 \mathrm{~g}, 4.83 \mathrm{mmol}, 1.0$ eq.) in THF (20 mL) was treated with $n \mathrm{BuLi}\left(2.47 \mathrm{M}\right.$ in hexane, $2.20 \mathrm{~mL}, 5.43 \mathrm{mmol}, 1.1 \mathrm{eq}$.) at $-78^{\circ} \mathrm{C}$. After $1 \mathrm{~h}$ at this temperature it was added a solution of the benzaldehyde $\mathbf{1 4}(1.33 \mathrm{~g}, 6.28 \mathrm{mmol}, 1.3 \mathrm{eq}$.$) in$ THF $(11 \mathrm{~mL})$. The mixture was warmed to $-30^{\circ} \mathrm{C}$ and held this temperature for $2 \mathrm{~h}$. Phosphate buffer $\left(\mathrm{pH}=7.4,40 \mathrm{~mL}\right.$ ) was added and warmed to $25^{\circ} \mathrm{C}$. The layers were separated und the aq. layer was extracted with EtOAc $(3 \times 25 \mathrm{~mL})$. The combined organic layers were dried over $\mathrm{Na}_{2} \mathrm{SO}_{4}$ and the solvent was removed under reduced pressure. The residue was purified by flash chromatography $\left(6.0 \mathrm{~cm}, 20 \mathrm{~cm}, \mathrm{CH}_{2} \mathrm{Cl}_{2}:\right.$ Acetone $\left.=97: 3,100 \mathrm{~mL}\right)$ to give the product $(S, S, R)$-13a (950 mg, 47\%, $2.25 \mathrm{mmol})$ as a pale yellow oil and $(R, S, R)$-13a (953 mg, 47\%, $2.26 \mathrm{mmol})$ as a white solid (mp.: $\left.74-75^{\circ} \mathrm{C}\right)$.

$(S, S, R)-\mathbf{1 3 a}:$

$\mathbf{R}_{\mathbf{f}}\left(\mathrm{CH}_{2} \mathrm{Cl}_{2}:\right.$ Acetone $\left.=97: 3\right)=0.57$

${ }^{\mathbf{1}} \mathbf{H}$-NMR $\left(500.10 \mathrm{MHz}, \mathrm{CDCl}_{3} / \mathrm{CHCl}_{3}\right.$, sample contained 4 weight-\% of the benzaldehyde $\mathbf{1 4}$,

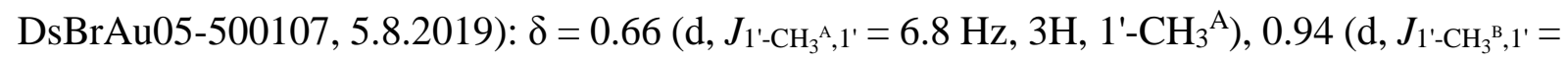
$\left.6.9 \mathrm{~Hz}, 3 \mathrm{H}, 1^{\prime}-\mathrm{CH}_{3}{ }^{\mathrm{B}}\right), 1.30\left(\mathrm{t}, J_{2^{\prime \prime}}{ }^{\prime \prime \prime}{ }^{\prime \mathrm{A}}=7.1 \mathrm{~Hz}, 3 \mathrm{H}, 2^{\prime \prime \mathrm{A}}-\mathrm{H}_{3}\right), 1.31\left(\mathrm{t}, J_{2^{\prime \prime}}\right.$, $1^{\prime \prime}$ в $=7.1 \mathrm{~Hz}, 3 \mathrm{H}, 2^{\prime \prime B}$

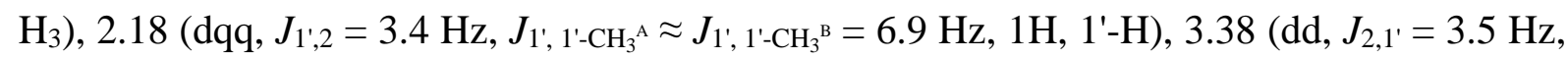

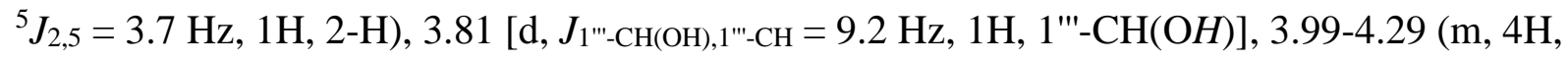
$\left.2 \times 1 "-\mathrm{H}_{2}\right), 4.44$ (dd, $\left.J_{5,1 " '-\mathrm{CH}}=4.6 \mathrm{~Hz}, J_{5,2}=3.7 \mathrm{~Hz}, 1 \mathrm{H}, 5-\mathrm{H}\right), 5.03$ (s, 2H, 1"'-- $\mathrm{CH}_{2}$ ), 5.15 (br. s, 1'"-CH), 6.82-6.70 (m, 2H, 3'"-H, 5'"-H), 7.06-7.12 (m, 2H, 2"'-H, 6"'-H), 7.28-7.33 (m, 1H, 4'"'-H), 7.35-7.44 (m, 4H, 2"'--H, 3"'--H, 5'"'-H, 6'"'-H) ppm. 
Christian Drescher and Reinhard Brückner; 3D-Structure Clarifying Total Synthesis of the (Polyenoyl)tetramic Acid Militarinon B. A Highly Acid-Labile N-Protecting Group for Amides

${ }^{13} \mathrm{C}$-NMR $\left(125.75 \mathrm{MHz}, \mathrm{CDCl}_{3} / \mathrm{CHCl}_{3}\right.$, sample contained the benzaldehyde 14, DsBrAu05500104): $\delta=14.4\left(\mathrm{C}-2^{\prime \prime}\right), 14.4\left(\mathrm{C}-2^{\prime \prime}\right), 16.7\left(1^{\prime}-\mathrm{CH}_{3}{ }^{\mathrm{A}}\right), 19.0\left(1^{\prime}-\mathrm{CH}_{3}{ }^{\mathrm{B}}\right), 31.6\left(\mathrm{C}-1^{\prime}\right), 60.6(\mathrm{C}-$ 2), 60.6 (2 × C-1"), 61.0 (C-5), 70.4 (1"'--CH2), 73.2 (1"'-CH), 114.1 (C-3"', C-5"'), 127.6 (C2'"', C-6"'"), 127.9 (C-2'", C-6'"), 128.6 (C-3"'", C-5"'"), 132.7 (C-1"'), 137.1 (C-1'"'), 158.2 (C4"'), 160.1 (C-3), 164.8 (C-6) ppm.

edHSQC [,,short-range H,C-COSY“, $\left.500.10 \mathrm{MHz} / 125.75 \mathrm{MHz}, \mathrm{CDCl}_{3} ; \delta\left({ }^{1} \mathrm{H}\right) \leftrightarrow \delta\left({ }^{13} \mathrm{C}\right)\right]$ : $0.66\left(\mathrm{~d}, 1^{\prime}-\mathrm{CH}_{3}{ }^{\mathrm{A}}\right) \leftrightarrow 16.7\left(1^{\prime}-\mathrm{CH}_{3}{ }^{\mathrm{A}}\right), 0.94\left(\mathrm{~d}, 1^{\prime}-\mathrm{CH}_{3}{ }^{\mathrm{B}}\right) \leftrightarrow 19.0\left(1^{\prime}-\mathrm{CH}_{3}{ }^{\mathrm{B}}\right), 1.29\left(\mathrm{t}, 2^{\prime \prime}{ }^{\mathrm{A}}-\mathrm{H}_{3}\right) \leftrightarrow$ $14.4\left(\mathrm{C}-2^{\prime \prime A}\right), 1.32\left(\mathrm{t}, 2^{\prime \prime B}-\mathrm{H}_{3}\right) \leftrightarrow 14.4\left(\mathrm{C}-2^{\prime \prime} \mathrm{B}\right), 2.18\left(\mathrm{dqq}, 1^{\prime}-\mathrm{H}\right) \leftrightarrow 31.6\left(\mathrm{C}-1^{\prime}\right), 3.38(\mathrm{dd}, 2-\mathrm{H})$ $\leftrightarrow 60.6$ (C-2), 3.99-4.29 (m, 4H, $\left.2 \times 1 "-\mathrm{H}_{2}\right) \leftrightarrow 60.6$ (2 × C-1"), 4.44 (dd, 5-H) 61.0 (C-5), 5.03 (s, 2H, 1"'--CH $\mathrm{CH}_{2} \leftrightarrow 70.4(1$ '"'-CH $), 5.15$ (br. s, 1"'-CH) 43.2 (1'"-CH), 6.82-6.70 (m, 3'"-H, 5"'-H) ↔ 114.1 (C-3'", C-5"'), 7.06-7.12 (m, 2"'-H, 6"'-H) ↔ 127.9 (C-2'", C-6"'), 7.28 $7.33(\mathrm{~m}, 4$ '"'-H) 128.0 (C-4'"'), 7.35-7.44 (m, 4H, 2"'"-H, 6'"'-H) 127.6 (C-2'"', C-6"'"), 7.357.44 (m, 3"'--H, 5'"'-H) 128.6 (C-3'"', C-5"'").

HRMS: (EI, $70 \mathrm{eV}): \mathrm{m} / \mathrm{z}=425.2440[\mathrm{M}+\mathrm{H}]^{+}$, corresponds to the molecular formula $\mathrm{C}_{25} \mathrm{H}_{33} \mathrm{O}_{4} \mathrm{~N}_{2}(425.2435)\left\{[\mathrm{M}+\mathrm{H}]^{+}\right\}$with a deviation of $1.3 \mathrm{ppm}$.

Specific rotation $(S, S, R)-\mathbf{1 3 a}[\alpha]_{D}^{20}=+51.1^{\circ}\left(\mathrm{c}=0.10\right.$ in $\left.\mathrm{CHCl}_{3}\right)$.

IR (KBr): $\tilde{v}=2975,2930,2900,2870,1695,1610,1585,1511,1455,1380,1365,1350,1330$, $1305,1240,1175,1160,1140,1115,1095,1035,985,905,860,940,810,735 \mathrm{~cm}^{-1}$.

$(R, S, R)-\mathbf{1 3 a}:$

$\mathbf{R}_{\mathbf{f}}\left(\mathrm{CH}_{2} \mathrm{Cl}_{2}:\right.$ Acetone $\left.=97: 3\right)=0.23$

${ }^{1} \mathrm{H}-\mathrm{NMR}\left(500.10 \mathrm{MHz}, \mathrm{CDCl}_{3} / \mathrm{CHCl}_{3}\right.$, sample contained 4 weight-\% of the Schöllkopf auxiliary $(R)$-15a, sample contained 3 weight-\% of the benzaldehyde 14, DsBrAu05-500200, 5.8.2019): $\delta=0.69\left(\mathrm{~d}, J_{1^{\prime}-\mathrm{CH}_{3}{ }^{\mathrm{A}}, 1^{\prime}}=6.8 \mathrm{~Hz}, 3 \mathrm{H}, 1^{\prime}-\mathrm{CH}_{3}{ }^{\mathrm{A}}\right), 0.99\left(\mathrm{~d}, J_{1^{\prime}-\mathrm{CH}_{3}{ }^{\mathrm{B}}, 1^{\prime}}=6.9 \mathrm{~Hz}, 3 \mathrm{H}, 1^{\prime}-\mathrm{CH}_{3}{ }^{\mathrm{B}}\right)$,

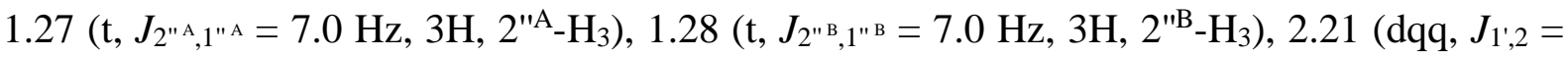

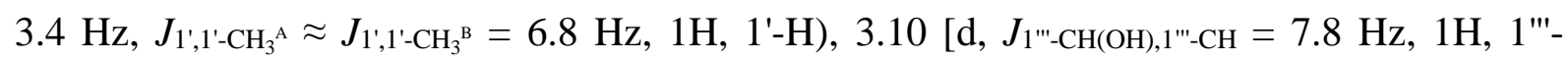
$\mathrm{CH}(\mathrm{OH})], 3.73\left(\mathrm{dd}, J_{2,1^{\prime}} \approx{ }^{5} J_{2,5}=3.5 \mathrm{~Hz}, 1 \mathrm{H}, 2-\mathrm{H}\right), 4.04-4.19\left(\mathrm{~m}, 4 \mathrm{H}, 2 \times 1 "-\mathrm{H}_{2}\right), 4.20\left(\mathrm{t}, J_{5,2} \approx\right.$ $\left.J_{5,1 " '-\mathrm{CH}}=3.6 \mathrm{~Hz}, 1 \mathrm{H}, 5-\mathrm{H}\right), 4.99\left(\mathrm{dd}, J_{1{ }^{\prime \prime}-\mathrm{CH}, 1{ }^{\prime \prime}-\mathrm{CH}(\mathrm{OH})}=7.6 \mathrm{~Hz}, J_{1}{ }^{\prime \prime-\mathrm{CH}, 5}=3.6 \mathrm{~Hz}, 1 \mathrm{H}, 1{ }^{\prime \prime}-\mathrm{CH}\right)$, 5.06 (s, 2H, 1"''-CH $)$, 6.91-6.95 (m, 2H, 3"'-H, 5'"-H), 7.27-7.30 (m, 2H, 2"'-H, 6"'-H), 7.287.34 (m, 1H, 4'"'-H), 7.35-7.40 (m, 2H, 3'"'-H, 5'"'-H), 7.41-7.45 (m, 2H, 2'"'-H, 6"'"-H) ppm. 
Christian Drescher and Reinhard Brückner; 3D-Structure Clarifying Total Synthesis of the (Polyenoyl)tetramic Acid Militarinon B. A Highly Acid-Labile N-Protecting Group for Amides

${ }^{13}$ C-NMR $\left(125.75 \mathrm{MHz}, \mathrm{CDCl}_{3} / \mathrm{CHCl}_{3}\right.$, sample contained the Schöllkopf auxiliary $(R)-\mathbf{1 5 a}$, sample contained the benzaldehyde 14, DsBrAu05-500204, 5.8.2019): $\delta=14.4\left(\mathrm{C}-2^{\mathrm{A}}\right), 14.4$ $\left(\mathrm{C}-2^{\prime \prime} \mathrm{B}\right), 16.9\left(1^{\prime}-\mathrm{CH}_{3}{ }^{\mathrm{A}}\right), 19.2\left(1^{\prime}-\mathrm{CH}_{3}{ }^{\mathrm{B}}\right), 31.9\left(\mathrm{C}-1^{\prime}\right), 61.0(\mathrm{C}-2), 61.1(2 \times \mathrm{C}-1 "), 61.1(\mathrm{C}-5)$, 70.1 (1'"'-CH2), 74.3 (1"'-CH), 114.3 (C-3"', C-5'"), 127.6 (C-2"'", C-6'"'), 128.0 (C-2'", C-6"'), 128.0 (C-4'"'), 128.6 (C-3'"', C-5'"'), 134.3 (C-1'"), 137.2 (C-1"''), 158.2 (C-4"'), 161.0 (C-3), 165.5 (C-6) ppm.

edHSQC [,short-range H,C-COSY“, 500.10 MHz/125.75 MHz, $\left.\mathrm{CDCl}_{3} ; \delta\left({ }^{1} \mathrm{H}\right) \leftrightarrow \delta\left({ }^{13} \mathrm{C}\right)\right]$ : $0.69\left(\mathrm{~d}, 1^{\prime}-\mathrm{CH}_{3}{ }^{\mathrm{A}}\right) \leftrightarrow 16.9\left(1^{\prime}-\mathrm{CH}_{3}{ }^{\mathrm{A}}\right), 0.99\left(\mathrm{~d}, 1^{\prime}-\mathrm{CH}_{3}{ }^{\mathrm{B}}\right) \leftrightarrow 19.2\left(1^{\prime}-\mathrm{CH}_{3}{ }^{\mathrm{B}}\right), 1.27\left(\mathrm{t}, 2^{\prime \prime} \mathrm{A}-\mathrm{H}_{3}\right) \leftrightarrow$ $14.4\left(\mathrm{C}-2^{\prime \prime} \mathrm{A}\right), 1.28\left(\mathrm{t}, 2^{\prime \prime} \mathrm{B}-\mathrm{H}_{3}\right) \leftrightarrow 14.4\left(\mathrm{C}-2^{\prime \prime} \mathrm{B}\right), 2.21\left(\mathrm{dqq}, 1^{\prime}-\mathrm{H}\right) \leftrightarrow 31.9(\mathrm{C}-1$ '), 3.73 (dd, 2-H) $\leftrightarrow 61.0$ (C-2), 4.04-4.19 (m, $\left.2 \times 1 "-\mathrm{H}_{2}\right) \leftrightarrow 61.1$ (2 × C-1"), 4.20 (t, 5-H) 61.1 (C-5), 4.99

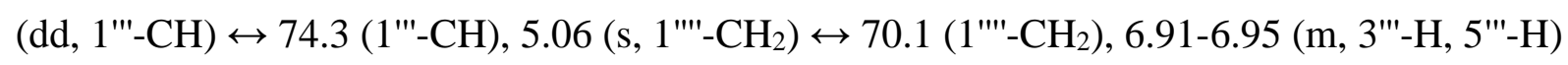
$\leftrightarrow 114.3$ (C-3"', C-5"'), 7.27-7.30 (m, 2"'-H, 6"'-H) ↔ 128.0 (C-2"', C-6"'), 7.28-7.34 (m, 4"'-$\mathrm{H}) \leftrightarrow 128.0$ (C-4"'"), 7.35-7.40 (m, 3"''-H, 5"'-H) 128.6 (C-3"'", C-5"'"), 7.41-7.45 (m, 2"''-H, 6"''-H) $127.6(\mathrm{C}-2$ "'", C-6'"').

HRMS: (EI, $70 \mathrm{eV}$ ): $\mathrm{m} / \mathrm{z}=425.2435[\mathrm{M}+\mathrm{H}]^{+}$, corresponds to the molecular formula $\mathrm{C}_{25} \mathrm{H}_{33} \mathrm{O}_{4} \mathrm{~N}_{2}(270.2435)\left\{[\mathrm{M}+\mathrm{H}]^{+}\right\}$with a deviation of $0.1 \mathrm{ppm}$.

Specific rotation $(R, S, R)-\mathbf{1 3 a}[\alpha]_{D}^{20}=-3.0^{\circ}\left(\mathrm{c}=0.10\right.$ in $\left.\mathrm{CHCl}_{3}\right)$

IR (KBr): $\tilde{v}=2975,2930,2900,2870,1695,1610,1510,1480,1455,1380,1365,1300,1240$, $1175,1160,1140,1115,1095,1135,985,920,860,835,740 \mathrm{~cm}^{-1}$.

Ethyl (2S,3S)-2-Amino-3-[4'-(benzyloxy)phenyl]-3-hydroxypropanoate [(S,S)-23]<smiles></smiles>

$(S, S)-23$ 
Christian Drescher and Reinhard Brückner; 3D-Structure Clarifying Total Synthesis of the (Polyenoyl)tetramic Acid Militarinon B. A Highly Acid-Labile N-Protecting Group for Amides

The pyrazine $(S, S, R)-\mathbf{1 3 a}(88 \mathrm{mg}, 206 \mathrm{mmol})$ was dissolved in $\mathrm{EtOH}(2.6 \mathrm{~mL})$ and aq. $\mathrm{HCl}$ ( $0.25 \mathrm{M}, 1.75 \mathrm{~mL}, 0.438 \mathrm{mmol}, 2.1$ eq.) was added. The mixture stirred at $25^{\circ} \mathrm{C}$ for $14 \mathrm{~h}$. It was added aq. sat $\mathrm{NaHCO}_{3}$-solution $(5 \mathrm{~mL})$ and aq. $\mathrm{NH}_{3}$-solution (25 weight- $\%, 2 \mathrm{~mL} \rightarrow \mathrm{pH}=9$ ). The mixture was extracted with $\mathrm{Et}_{2} \mathrm{O}(3 \times 15 \mathrm{~mL})$. The combined organic layers were dried over $\mathrm{Na}_{2} \mathrm{SO}_{4}$ and the solvent was removed under reduced pressure. The residue was purified by flash chromatography $\left(1.5 \mathrm{~cm}, 20 \mathrm{~cm}, \mathrm{CH}_{2} \mathrm{Cl}_{2}: \mathrm{MeOH}=25: 1,2 \mathrm{~mL}\right)$ to give the product $(S, S)-\mathbf{2 3}(39 \mathrm{mg}, 60 \%, 124 \mu \mathrm{mol})$ as a pale yellow oil.

$\boldsymbol{R f}_{\mathbf{f}}\left(\mathrm{CH}_{2} \mathrm{Cl}_{2}: \mathrm{MeOH}=20: 1\right): 0.17$

${ }^{1} \mathbf{H}-\mathbf{N M R}\left(400.13 \mathrm{MHz}, \mathrm{CDCl}_{3} / \mathrm{CHCl}_{3}, \mathrm{DsBrAu05-4010,5.8.2019):} \delta=1.23\right.$ (t, $J_{2 " ', 1 " '=}=7.0 \mathrm{~Hz}$, $3 \mathrm{H}, 2$ '"- $\left.\mathrm{H}_{3}\right), 3.76\left(\mathrm{~d}, J_{2,3}=5.8 \mathrm{~Hz}, 1 \mathrm{H}, 2-\mathrm{H}\right), 4.08-4.20\left(\mathrm{~m}, 2 \mathrm{H}, 1{ }^{\prime \prime}-\mathrm{H}_{2}\right), 4.90$ (d, $J_{3,2}=5.9 \mathrm{~Hz}$, 1H, 3-H), 5.05 (s, 2H, 1"'-CH $), 6.90-6.98$ (m, 2H, 3'-H, 5'-H), 7.18-7.22 (m, 2H, 2'-H, 6'-H), 7.28-7.34 (m, 1H, 4"-H), 7.35-7.44 (m, 4H, 2"-H, 3"-H, 5"-H, 6"-H) ppm.

${ }^{13} \mathrm{C}$-NMR (100.61 MHz, $\mathrm{CDCl}_{3} / \mathrm{CHCl}_{3}, \mathrm{DsBrAu05-4013,} \mathrm{5.8.2019):} \delta=14.2(\mathrm{C}-2$ "'), $60.0(\mathrm{C}-$ 2), 61.3 (C-1"'), 70.1 (1"-CH2), 74.2 (C-3), 114.8 (C-3', C-5'), 127.7 (C-2', C-6'), 128.0 (C-4"), 128.5 (C-2", C-6"), 128.7 (C-3", C-5"), 132.3 (C-1'), 137.0 (C-1"), 158.8 (C-4'), 173.3 (C-1) ppm.

edHSQC [,short-range H,C-COSY“, $\left.400.13 \mathrm{MHz} / 100.61 \mathrm{MHz}, \mathrm{CDCl}_{3} ; \delta\left({ }^{1} \mathrm{H}\right) \leftrightarrow \delta\left({ }^{13} \mathrm{C}\right)\right]$ : $1.23\left(\mathrm{t}, 2^{\prime \prime}-\mathrm{H}_{3}\right) \leftrightarrow 14.2\left(\mathrm{C}-2^{\prime \prime \prime}\right), 3.76(\mathrm{~d}, 2-\mathrm{H}) \leftrightarrow 60.0(\mathrm{C}-2), 4.08-4.20\left(\mathrm{~m}, 1^{\prime \prime \prime}-\mathrm{H}_{2}\right) \leftrightarrow 61.3(\mathrm{C}-$

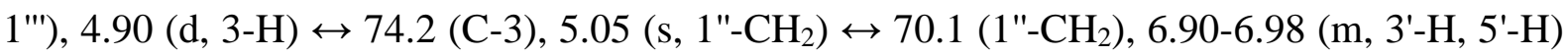
$\leftrightarrow 114.8$ (C-3', C-5'), 7.18-7.22 (m, 2'-H, 6'-H) 127.7 (C-2', C-6'), 7.28-7.34 (m, 4"-H) 128.0 (C-4"), 7.35-7.44 (m, 2"-H, 6"-H) ↔ 128.5 (C-2", C-6"), 7.35-7.44 (m, 3"-H, 5"-H) ↔ 128.7 (C-3", C-5").

HRMS: $($ EI, $70 \mathrm{eV}): \mathrm{m} / \mathrm{z}=316.1546[\mathrm{M}+\mathrm{H}]^{+}$, corresponds to the molecular formula $\mathrm{C}_{18} \mathrm{H}_{22} \mathrm{O}_{4} \mathrm{~N}$ (316.1543) $\left\{[\mathrm{M}+\mathrm{H}]^{+}\right\}$with a deviation of $0.9 \mathrm{ppm}$.

Specific rotation $(S, S)-23[\alpha]_{D}^{20}=+27.1^{\circ}\left(\mathrm{c}=0.10\right.$ in $\left.\mathrm{CHCl}_{3}\right)$.

IR (KBr): $\tilde{v}=3065,3035,2980,2960,2830,2905,2870,1890,1735,1610,1585,1510,1465$, $1455,1380,1300,1240,1175,1115,1025,915,860,835,815,790,740 \mathrm{~cm}^{-1}$. 


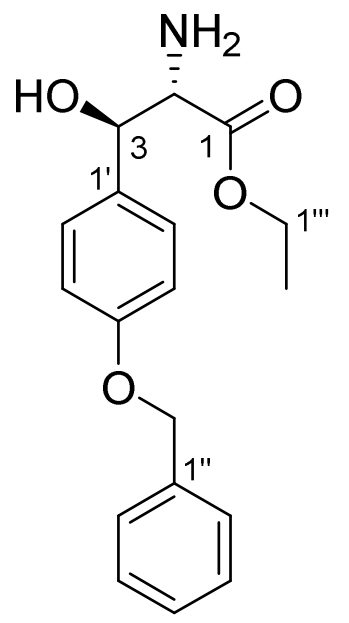

$(R, S)-23$

The pyrazine $(R, S, R)-13 \mathbf{a}(150 \mathrm{mg}, 352 \mu \mathrm{mol})$ was dissolved in $\mathrm{EtOH}(4.5 \mathrm{~mL})$ and aq. $\mathrm{HCl}$ (0.25 M, $3.0 \mathrm{~mL}, 740 \mu \mathrm{mol}, 2.1$ eq.) was added. The mixture stirred at $25^{\circ} \mathrm{C}$ for $14 \mathrm{~h}$. It was added aq. sat $\mathrm{NaHCO}_{3}$-solution $\left(10 \mathrm{~mL}\right.$ ) and aq. $\mathrm{NH}_{3}$-solution ( 25 weight- $\%, 3 \mathrm{~mL} \rightarrow \mathrm{pH}=9$ ). The mixture was extracted with $\mathrm{Et}_{2} \mathrm{O}(3 \times 25 \mathrm{~mL})$. The combined organic layers were dried over $\mathrm{Na}_{2} \mathrm{SO}_{4}$ and the solvent was removed under reduced pressure. The residue was purified by flash chromatography $\left(2.0 \mathrm{~cm}, 20 \mathrm{~cm}, \mathrm{CH}_{2} \mathrm{Cl}_{2}: \mathrm{MeOH}=30: 1,2 \mathrm{~mL}\right)$ to give the product $(R, S)-23(61 \mathrm{mg}, 55 \%, 194 \mu \mathrm{mol})$ as a clear oil.

$\mathbf{R}_{\mathbf{f}}\left(\mathrm{CH}_{2} \mathrm{Cl}_{2}: \mathrm{MeOH}=20: 1\right): 0.20$

${ }^{1} \mathbf{H}-\mathbf{N M R}\left(400.13 \mathrm{MHz}, \mathrm{CDCl}_{3} / \mathrm{CHCl}_{3}, \mathrm{DsBrJi} 30-4010,3.8 .2020\right): \delta=1.15$ (t, $J_{2 " ', 1^{\prime \prime}}=7.2 \mathrm{~Hz}$,

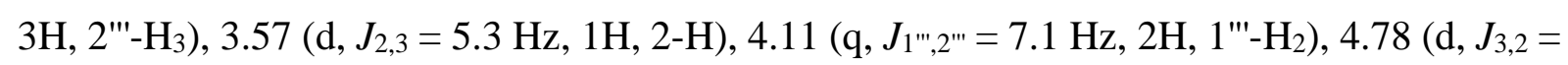
$5.2 \mathrm{~Hz}, 1 \mathrm{H}, 3-\mathrm{H}), 5.07$ (s, 2H, 1"-CH $)$, 6.93-6.98 (m, 2H, 3'-H, 5'-H), 7.26-7.29 (m, 2H, 2'-H, 6'-H), 7.29-7.34 (m, 1H, 4"-H), 7.35-7.44 (m, 4H, 2"-H, 3"-H, 5"-H, 6"-H) ppm.

${ }^{13}$ C-NMR (100.61 MHz, $\mathrm{CDCl}_{3} / \mathrm{CHCl}_{3}$, DsBrJi30-4014, 3.8.2020): $\delta=14.1$ (C-2'"), 60.9 (C2), 61.2 (C-1'"), 70.1 (1"-CH2), 74.1 (C-3), 114.9 (C-3', C-5'), 127.5 (C-2", C-6"), 127.5 (C-2', C-6'), 128.0 (C-4"), 128.7 (C-3", C-5"), 133.3 (C-1'), 137.1 (C-1"), 158.6 (C-4'), 173.5 (C-1) ppm.

edHSQC [,,short-range H,C-COSY“, $\left.400.13 \mathrm{MHz} / 100.61 \mathrm{MHz}, \mathrm{CDCl}_{3} ; \delta\left({ }^{1} \mathrm{H}\right) \leftrightarrow \delta\left({ }^{13} \mathrm{C}\right)\right]$ : $1.15\left(\mathrm{t}, 2^{\prime \prime \prime}-\mathrm{H}_{3}\right) \leftrightarrow 14.1\left(\mathrm{C}-2{ }^{\prime \prime}\right), 3.57(\mathrm{~d}, 2-\mathrm{H}) \leftrightarrow 60.9(\mathrm{C}-2), 4.11\left(\mathrm{q}, 1\right.$ '"'-H $\left.^{2}\right) \leftrightarrow 61.2\left(\mathrm{C}-1{ }^{\prime \prime \prime}\right)$, $4.78(\mathrm{~d}, 3-\mathrm{H}) \leftrightarrow 74.1(\mathrm{C}-3), 5.07\left(\mathrm{~s}, 1^{\prime \prime}-\mathrm{CH}_{2}\right) \leftrightarrow 70.1\left(1^{\prime \prime}-\mathrm{CH}_{2}\right), 6.93-6.98\left(\mathrm{~m}, 3 '-\mathrm{H}, 5^{\prime}-\mathrm{H}\right) \leftrightarrow$ 114.9 (C-3', C-5'), 7.26-7.29 (m, 2'-H, 6'-H) 127.5 (C-2', C-6'), 7.29-7.34 (m, 4"-H) 128.0 
Christian Drescher and Reinhard Brückner; 3D-Structure Clarifying Total Synthesis of the (Polyenoyl)tetramic Acid Militarinon B. A Highly Acid-Labile N-Protecting Group for Amides

(C-4"), 7.35-7.44 (m, 2"-H, 6"-H) ↔ 127.5 (C-2", C-6"), 7.35-7.44 (m, 3"-H, 5"-H) ↔ 128.7 (C-3", C-5").

HRMS: $($ EI, $70 \mathrm{eV}): \mathrm{m} / \mathrm{z}=316.1546[\mathrm{M}+\mathrm{H}]^{+}$, corresponds to the molecular formula $\mathrm{C}_{18} \mathrm{H}_{22} \mathrm{O}_{4} \mathrm{~N}$ (316.1543) $\left\{[\mathrm{M}+\mathrm{H}]^{+}\right\}$with a deviation of $0.7 \mathrm{ppm}$.

Specific rotation $(R, S)-23[\alpha]_{D}^{20}=+20.2^{\circ}\left(\mathrm{c}=1.85\right.$ in $\left.\mathrm{CHCl}_{3}\right)$.

IR (KBr): $\tilde{v}=3370,3065,3035,2980,2935,1730,1660,1610,1585,1510,1455,1380,1300$, $1240,1175,1110,1025,920,830,740,700,630,550 \mathrm{~cm}^{-1}$.

\section{Ethyl (2S,3S)-2-[(tert-Butyldimethylsilyl)amino]-3-[(tert-butyldimethylsilyl)oxy]-3-\{4'-}

[(tert-butyldimethylsilyl)oxy]phenyl $\}$ propanoate $[(S, S)-12 \mathrm{f}]$

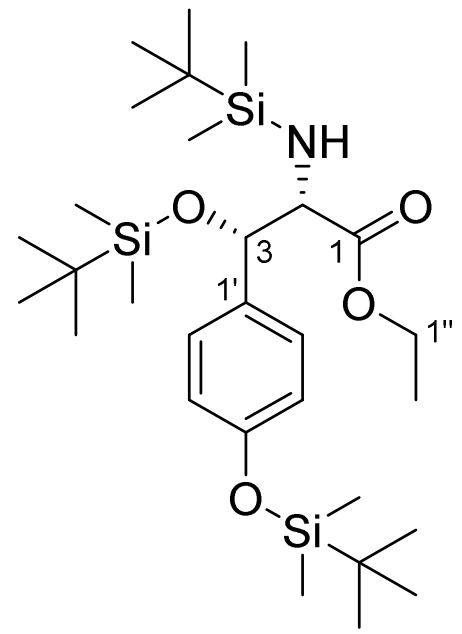

$(S, S)-\mathbf{1 2 f}$

The $\beta$-hydroxy tyrosine ester $(S, S)-23$ (202 mg, $641 \mu \mathrm{mol})$ was dissolved in $\mathrm{MeOH}(5 \mathrm{~mL})$. $\mathrm{Pd} / \mathrm{C}$ (10 weight- $\%, 22.5 \mathrm{mg}, 10 \mathrm{~mol}-\%$ ) was added and the mixture stirred under $\mathrm{H}_{2}$ atmosphere for $16 \mathrm{~h}$. The mixture was filtered over Celite ${ }^{\circledR}$ und washed with $\mathrm{CH}_{2} \mathrm{Cl}_{2}(150 \mathrm{~mL})$. The solvent was removed and the crude phenol $(S, S)-\mathbf{2 4}(111 \mathrm{mg}, 493 \mu \mathrm{mol})$ was dissolved in $\mathrm{CH}_{2} \mathrm{Cl}_{2}(10 \mathrm{~mL})$ was treated with $\mathrm{NEt}_{3}(478 \mu \mathrm{L}, 349 \mathrm{mg}, 3.45 \mathrm{mmol}, 7.0$ eq. $)$ and $t \mathrm{BuMe}_{2} \mathrm{SiOTf}$ (431 $\mu \mathrm{L}, 651 \mathrm{mg}, 2.46 \mathrm{mmol}, 5.0$ eq.) at $-15^{\circ} \mathrm{C}$. The mixture was warmed to $2^{\circ} \mathrm{C}$ and held this temperature for $19 \mathrm{~h}$. The mixture was diluted with aq. sat $\mathrm{NaHCO}_{3}$-solution $(10 \mathrm{~mL})$, the organic layer was separated, and the aq. layer was extracted with $\mathrm{CH}_{2} \mathrm{Cl}_{2}(3 \times 25 \mathrm{~mL})$. The combined organic layers were dried over $\mathrm{Na}_{2} \mathrm{SO}_{4}$ and the solvent was removed under reduced pressure. The residue was purified by flash chromatography $\left(2.5 \mathrm{~cm}, 20 \mathrm{~cm}, c \mathrm{C}_{6} \mathrm{H}_{12}:\right.$ EtOAc $=$ 
Christian Drescher and Reinhard Brückner; 3D-Structure Clarifying Total Synthesis of the (Polyenoyl)tetramic Acid Militarinon B. A Highly Acid-Labile N-Protecting Group for Amides

$5: 1,15 \mathrm{~mL})$ to give the product $(S, S)-\mathbf{1 2 f}(187 \mathrm{mg}, 52 \%$ over two steps, $331 \mu \mathrm{mol})$ as a colorless oil.

$\mathbf{R}_{\mathbf{f}}\left(c \mathrm{C}_{6} \mathrm{H}_{12}: \mathrm{EtOAc}=5: 1\right): 0.11$

${ }^{1} \mathrm{H}$-NMR $\left(500.10 \mathrm{MHz}\right.$, sample contained $\mathrm{H}_{2} \mathrm{O}$, sample contained 6 weight-\% of $\mathrm{O}\left(\mathrm{SiMe}_{2} t \mathrm{Bu}\right)_{2}$ with s at 0.02 and s at 0.87 and unknown impurity with s at $3.70, \mathrm{CDCl}_{3} / \mathrm{CHCl}_{3}, \mathrm{DsBrAu07-}$ 500500, 8.8.2019): $\delta=-0.38$ (s, 3H, 2-NH-Si- $\left.\mathrm{CH}_{3}{ }^{\mathrm{A}}\right),-0.37$ (s, 3H, 2-NH-Si-CH${ }^{\mathrm{B}}$ ), -0.27 (s, $\left.3 \mathrm{H}, 3-\mathrm{O}-\mathrm{Si}-\mathrm{CH}_{3}{ }^{\mathrm{A}}\right),-0.05$ (s, 3H, 3-O-Si- $\left.\mathrm{CH}_{3}{ }^{\mathrm{B}}\right), 0.16$ [s, 6H, 1'-O-Si- $\left(\mathrm{CH}_{3}\right)_{2}$ ], 0.70 [s, 9H, 2NH-Si-C( $\left(\mathrm{CH}_{3}\right)_{3}$ ], 0.80 [s, 9H, 3-O-Si-C( $\left.\left.\mathrm{CH}_{3}\right)_{3}\right], 0.98$ [s, 9H, 4'-O-Si-C(CH3) $)_{3}$, $1.28\left(\mathrm{t}, J_{2 "}, 1^{\prime \prime}=\right.$ $\left.7.1 \mathrm{~Hz}, 3 \mathrm{H}, 2 "-\mathrm{H}_{3}\right), 3.34\left(\mathrm{dd}, J_{2,3}=8.5 \mathrm{~Hz}, J_{2, \mathrm{NH}}=13.1 \mathrm{~Hz}, 1 \mathrm{H}, 2-\mathrm{H}\right), 4.16\left(\mathrm{~m}_{\mathrm{c}}, 2 \mathrm{H}, 1 "-\mathrm{H}_{2}\right)$, $4.42\left(\mathrm{~d}, J_{3,2}=8.4 \mathrm{~Hz}, 1 \mathrm{H}, 3-\mathrm{H}\right), 6.74-6.78$ (m, 2H, 3'-H, 5'-H), 7.11-7.19 (m, 2H, 2'-H, 6'-H) ppm.

${ }^{13} \mathrm{C}$-NMR (125.75 MHz, $\mathrm{CDCl}_{3} / \mathrm{CHCl}_{3}$, sample contained $\mathrm{O}\left(\mathrm{SiMe}_{2} t \mathrm{Bu}\right)_{2} \mathrm{DsBrAu07-500504):}$ $\delta=-5.6\left(2-\mathrm{NH}-\mathrm{Si}_{-} \mathrm{CH}_{3}{ }^{\mathrm{A}}\right),-5.3\left(2-\mathrm{NH}-\mathrm{Si}_{-}-\mathrm{CH}_{3}{ }^{\mathrm{B}}\right),-5.1\left(3-\mathrm{O}-\mathrm{Si}_{-} \mathrm{CH}_{3}{ }^{\mathrm{B}}\right),-4.6\left(3-\mathrm{O}-\mathrm{Si}-\mathrm{CH}_{3}{ }^{\mathrm{A}}\right),-$ 4.4 [1'-O-Si- $\left(\mathrm{CH}_{3}\right)_{2}$ ], 14.3 (C-2"), 17.9 [2-NH-Si-C $\left(\mathrm{CH}_{3}\right)_{3}$ ], 18.1 [3-O-Si-C( $\left.\mathrm{CH}_{3}\right)_{3}$ ], 18.3 [4'-O$\mathrm{Si}-\mathrm{C}\left(\mathrm{CH}_{3}\right)_{3}$ ], 25.7 [3-O-Si-C $\left(\mathrm{CH}_{3}\right)_{3}$ ], 25.8 [4'-O-Si-C $\left(\mathrm{CH}_{3}\right)_{3}$ ], 26.1 [2-NH-Si-C $\left(\mathrm{CH}_{3}\right)_{3}$ ], 60.4 (C-1"), 63.5 (C-2), 78.6 (C-3), 119.6 (C-3', C-5'), 128.7 (C-2', C-6'), 135.3 (C-1'), 155.2 (C-4'), $175.6(\mathrm{C}-1) \mathrm{ppm}$.

edHSQC [,,short-range H,C-COSY“, $\left.500.10 \mathrm{MHz} / 125.75 \mathrm{MHz}, \mathrm{CDCl}_{3} ; \delta\left({ }^{1} \mathrm{H}\right) \leftrightarrow \delta\left({ }^{13} \mathrm{C}\right)\right]$ : $-0.38\left(\mathrm{~s}, 2-\mathrm{NH}-\mathrm{Si}-\mathrm{CH}_{3}{ }^{\mathrm{A}}\right) \leftrightarrow-5.6\left(2-\mathrm{NH}-\mathrm{Si}_{-} \mathrm{CH}_{3}{ }^{\mathrm{A}}\right),-0.37\left(\mathrm{~s}, 2-\mathrm{NH}-\mathrm{Si}_{-} \mathrm{CH}_{3}{ }^{\mathrm{B}}\right) \leftrightarrow-5.3(2-\mathrm{NH}-$ $\left.\mathrm{Si}-\mathrm{CH}_{3}{ }^{\mathrm{B}}\right),-0.27\left(\mathrm{~s}, 3-\mathrm{O}-\mathrm{Si}-\mathrm{CH}_{3}{ }^{\mathrm{A}}\right) \leftrightarrow-4.6\left(3-\mathrm{O}-\mathrm{Si}_{-} \mathrm{CH}_{3}{ }^{\mathrm{A}}\right),-0.05\left(\mathrm{~s}, 3-\mathrm{O}-\mathrm{Si}_{-}-\mathrm{CH}_{3}{ }^{\mathrm{B}}\right) \leftrightarrow-5.1$ (3$\left.\mathrm{O}-\mathrm{Si}_{-}-\mathrm{CH}_{3}{ }^{\mathrm{B}}\right), 0.16$ [s, 1'-O-Si- $\left.\left(\mathrm{CH}_{3}\right)_{2}\right] \leftrightarrow-4.4$ [1'-O-Si- $\left.\left(\mathrm{CH}_{3}\right)_{2}\right], 0.70$ [s, 2-NH-Si-C $\left.\left(\mathrm{CH}_{3}\right)_{3}\right] \leftrightarrow$ 26.1 [2-NH-Si-C $\left(\mathrm{CH}_{3}\right)_{3}$ ], 0.80 [s, 3-O-Si-C $\left.\left(\mathrm{CH}_{3}\right)_{3}\right] \leftrightarrow 25.7$ [3-O-Si-C $\left(\mathrm{CH}_{3}\right)_{3}$ ], 0.98 [s, 1'-O-Si$\left.\mathrm{C}\left(\mathrm{CH}_{3}\right)_{3}\right] \leftrightarrow 25.8$ [4'-O-Si-C $\left.\left(\mathrm{CH}_{3}\right)_{3}\right], 1.28\left(\mathrm{t}, 2^{\prime \prime}-\mathrm{H}_{3}\right) \leftrightarrow 14.3(\mathrm{C}-2 "), 3.34$ (dd, 2-H) $63.5(\mathrm{C}-$ 2), $4.16\left(\mathrm{~m}_{\mathrm{c}}, 1^{\prime \prime}-\mathrm{H}_{2}\right) \leftrightarrow 60.4(\mathrm{C}-1 "), 4.42(\mathrm{~d}, 3-\mathrm{H}) \leftrightarrow 78.6(\mathrm{C}-3), 6.74-6.78\left(\mathrm{~m}, 3 '-\mathrm{H}, 5^{\prime}-\mathrm{H}\right) \leftrightarrow$ $119.6\left(\mathrm{C}-3^{\prime}, \mathrm{C}^{-5}\right)$, 7.11-7.19 (m, 2'-H, 6'-H) 128.7 (C-2', C-6').

HRMS: (EI, $70 \mathrm{eV}): \mathrm{m} / \mathrm{z}=568.3673[\mathrm{M}+\mathrm{H}]^{+}$, corresponds to the molecular formula $\mathrm{C}_{29} \mathrm{H}_{58} \mathrm{O}_{4} \mathrm{NSi}_{3}(568.33668)\left\{[\mathrm{M}+\mathrm{H}]^{+}\right\}$with a deviation of $0.9 \mathrm{ppm}$.

Specific rotation $(S, S)-\mathbf{1 2 f}[\alpha]_{D}^{20}=+43.0^{\circ}\left(\mathrm{c}=0.23\right.$ in $\left.\mathrm{CHCl}_{3}\right)$

IR (KBr): $\tilde{v}=2955,2930,2885,2860,1735,1610,1510,1470,1465,1415,1390,1360,1310$, $1255,1175,1165,1135,1100,1085,1005,935,915,875,835,810,780 \mathrm{~cm}^{-1}$. 


\section{Ethyl (2S,3R)-2-[(tert-Butyldimethylsilyl)amino]-3-[(tert-butyldimethylsilyl)oxy]-3-\{4'-} $[($ tert-butyldimethylsilyl)oxy]phenyl $\}$ propanoate $[(R, S)-12 f]$

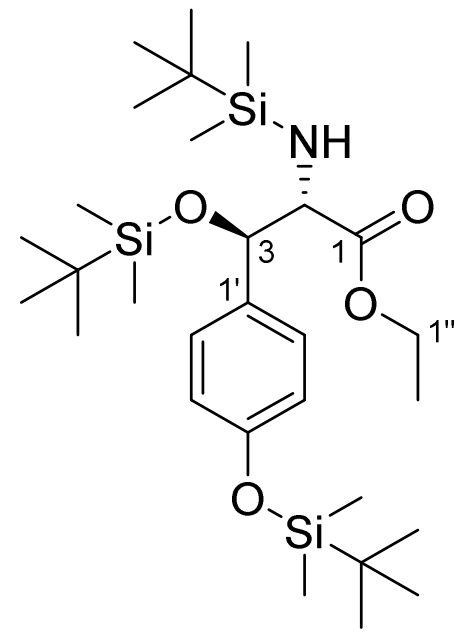

$(R, S)-12 f$

The $\beta$-hydroxy tyrosine ester $(R, S)$-23 (95 mg, $302 \mu \mathrm{mol})$ was dissolved in $\mathrm{MeOH}(3 \mathrm{~mL}) . \mathrm{Pd} / \mathrm{C}$ (10 weight- $\%, 10.6 \mathrm{mg}, 10 \mathrm{~mol}-\%$ ) was added and the mixture stirred under $\mathrm{H}_{2}$ atmosphere for 16 h. The mixture was filtered over Celite $\AA$ und washed with $\mathrm{CH}_{2} \mathrm{Cl}_{2}(100 \mathrm{~mL})$. The solvent was removed and the crude phenol $(R, S)-\mathbf{2 4}(49.7 \mathrm{mg}, 221 \mu \mathrm{mol})$ was dissolved in $\mathrm{CH}_{2} \mathrm{Cl}_{2}(5$ $\mathrm{mL})$ was treated with $\mathrm{NEt}_{3}\left(215 \mu \mathrm{L}, 157 \mathrm{mg}, 1.55 \mathrm{mmol}, 7.0\right.$ eq.) and $t \mathrm{BuMe}_{2} \operatorname{SiOTf}(194 \mu \mathrm{L}$, $294 \mathrm{mg}, 1.11 \mathrm{mmol}, 5.0$ eq.) at $-15^{\circ} \mathrm{C}$. The mixture was warmed to $2^{\circ} \mathrm{C}$ and held this temperature for $19 \mathrm{~h}$. The mixture was diluted with aq. sat $\mathrm{NaHCO}_{3}$-solution $(5 \mathrm{~mL})$, the organic layer was separated, and the aq. layer was extracted with $\mathrm{CH}_{2} \mathrm{Cl}_{2}(3 \times 15 \mathrm{~mL})$. The combined organic layers were dried over $\mathrm{Na}_{2} \mathrm{SO}_{4}$ and the solvent was removed under reduced pressure. The residue was purified by flash chromatography $\left(2.0 \mathrm{~cm}, 20 \mathrm{~cm}, c \mathrm{C}_{6} \mathrm{H}_{12}:\right.$ EtOAc $\left.=5: 1,15 \mathrm{~mL}\right)$ to give the product $(R, S)-\mathbf{1 2 f}(85.5 \mathrm{mg}, 50 \%$ over two steps, $151 \mu \mathrm{mol})$ as a colorless oil.

$\mathbf{R}_{\mathbf{f}}\left(c \mathrm{C}_{6} \mathrm{H}_{12}: \mathrm{EtOAc}=5: 1\right): 0.13$

${ }^{1} \mathbf{H}$-NMR $\left(400.13 \mathrm{MHz}, \mathrm{CDCl}_{3} / \mathrm{CHCl}_{3}\right.$, sample contained 17 weight-\% of $\mathrm{O}\left(\mathrm{SiMe}_{2} t \mathrm{Bu}\right)_{2}$ with $\mathrm{s}$ at 0.02 and $\mathrm{s}$ at 0.87 and unknown impurity with $\mathrm{s}$ at 3.68, DsBrJn02-4010, 2.6.2020): $\delta=-$ $0.41\left(\mathrm{~s}, 3 \mathrm{H}, 2-\mathrm{NH}-\mathrm{Si}-\mathrm{CH}_{3}{ }^{\mathrm{A}}\right),-0.29\left(\mathrm{~s}, 3 \mathrm{H}, 2-\mathrm{NH}-\mathrm{Si}_{-} \mathrm{CH}_{3}{ }^{\mathrm{B}}\right),-0.21\left(\mathrm{~s}, 3 \mathrm{H}, 3-\mathrm{O}-\mathrm{Si}_{-} \mathrm{CH}_{3}{ }^{\mathrm{A}}\right),-0.05$ (s, 3H, 3-O-Si- $\mathrm{CH}_{3}{ }^{\mathrm{B}}$ ), 0.16 (s, 3H, 1'-O-Si- $\mathrm{CH}_{3}{ }^{\mathrm{A}}$ ), 0.17 (s, 3H, 1'-O-Si-CH${ }_{3}^{\mathrm{B}}$ ), 0.74 [s, 9H, 2$\left.\mathrm{NH}-\mathrm{Si}-\mathrm{C}\left(\mathrm{CH}_{3}\right)_{3}\right], 0.86$ [s, 9H, 3-O-Si-C(CH$\left.)_{3}\right], 0.98$ [s, 9H, 4'-O-Si-C(CH3) $\left.)_{3}\right], 1.28\left(\mathrm{t}, J_{2 "}, 1^{\prime \prime}=\right.$ $\left.7.2 \mathrm{~Hz}, 3 \mathrm{H}, 2 "-\mathrm{H}_{3}\right), 3.38\left(\mathrm{dd}, J_{2,3}=2.6 \mathrm{~Hz}, J_{2, \mathrm{NH}}=13.0 \mathrm{~Hz}, 1 \mathrm{H}, 2-\mathrm{H}\right), 4.13\left(\mathrm{~m}_{\mathrm{c}}, 2 \mathrm{H}, 1 "-\mathrm{H}_{2}\right)$, $5.00\left(\mathrm{~d}, J_{3,2}=2.6 \mathrm{~Hz}, 1 \mathrm{H}, 3-\mathrm{H}\right), 6.74-6.78\left(\mathrm{~m}, 2 \mathrm{H}, 3^{\prime}-\mathrm{H}, 5^{\prime}-\mathrm{H}\right), 7.13-7.19$ (m, 2H, 2'-H, 6'-H) ppm. 
Christian Drescher and Reinhard Brückner; 3D-Structure Clarifying Total Synthesis of the (Polyenoyl)tetramic Acid Militarinon B. A Highly Acid-Labile N-Protecting Group for Amides

${ }^{13}$ C-NMR (100.61 MHz, $\mathrm{CDCl}_{3} / \mathrm{CHCl}_{3}$, DsBrJn02-4014, 2.6.2020): $\delta=-5.9\left(2-\mathrm{NH}-\mathrm{Si}_{-} \mathrm{CH}_{3}{ }^{\mathrm{A}}\right)$, $-5.3\left(3-\mathrm{O}-\mathrm{Si}-\mathrm{CH}_{3}{ }^{\mathrm{B}}\right),-5.2\left(2-\mathrm{NH}-\mathrm{Si}-\mathrm{CH}_{3}{ }^{\mathrm{B}}\right),-4.6\left(3-\mathrm{O}-\mathrm{Si}^{-} \mathrm{CH}_{3}{ }^{\mathrm{A}}\right),-4.4\left(1^{\prime}-\mathrm{O}-\mathrm{Si}^{-} \mathrm{CH}_{3}{ }^{\mathrm{A}}\right),-4.4\left(1^{\prime}-\right.$ $\left.\mathrm{O}-\mathrm{Si}_{-} \mathrm{CH}_{3}{ }^{\mathrm{B}}\right), 14.3(\mathrm{C}-2 "), 18.2$ [3-O-Si- $\left.\mathrm{C}\left(\mathrm{CH}_{3}\right)_{3}\right], 18.3$ [4'-O-Si-C(CH$\left.)_{3}\right], 18.4$ [2-NH-Si$C\left(\mathrm{CH}_{3}\right)_{3}$ ], 25.8 [4'-O-Si-C $\left(\mathrm{CH}_{3}\right)_{3}$ ], 25.9 [3-O-Si-C $\left(\mathrm{CH}_{3}\right)_{3}$ ], 26.2 [2-NH-Si-C $\left(\mathrm{CH}_{3}\right)_{3}$ ], $60.7(\mathrm{C}-$ 1"), 63.4 (C-2), 76.5 (C-3), 119.4 (C-3', C-5'), 128.1 (C-2', C-6'), 135.4 (C-1'), 154.9 (C-4'), $174.6(\mathrm{C}-1) \mathrm{ppm}$.

edHSQC [,,short-range H,C-COSY“, $\left.400.13 \mathrm{MHz} / 100.62 \mathrm{MHz}, \mathrm{CDCl}_{3} ; \delta\left({ }^{1} \mathrm{H}\right) \leftrightarrow \delta\left({ }^{13} \mathrm{C}\right)\right]$ : $0.41\left(\mathrm{~s}, 2-\mathrm{NH}-\mathrm{Si}-\mathrm{CH}_{3}{ }^{\mathrm{A}}\right) \leftrightarrow-5.9\left(2-\mathrm{NH}-\mathrm{Si}_{-}-\mathrm{CH}_{3}{ }^{\mathrm{A}}\right),-0.29$ (s, 2-NH-Si-CH$\left.{ }_{3}^{\mathrm{B}}\right) \leftrightarrow-5.2(2-\mathrm{NH}-\mathrm{Si}-$ $\mathrm{CH}_{3}{ }^{\mathrm{B}}$ ), $-0.21\left(\mathrm{~s}, 3-\mathrm{O}-\mathrm{Si}-\mathrm{CH}_{3}{ }^{\mathrm{A}}\right) \leftrightarrow-4.6\left(3-\mathrm{O}-\mathrm{Si}_{-} \mathrm{CH}_{3}{ }^{\mathrm{A}}\right),-0.05$ (s, 3-O-Si-CH$\left.{ }^{\mathrm{B}}\right) \leftrightarrow-5.3$ (3-O$\left.\mathrm{Si}-\mathrm{CH}_{3}{ }^{\mathrm{B}}\right), 0.16\left(\mathrm{~s}, 1^{\prime}-\mathrm{O}-\mathrm{Si}_{-}-\mathrm{CH}_{3}{ }^{\mathrm{A}}\right) \leftrightarrow-4.4\left(1^{\prime}-\mathrm{O}-\mathrm{Si}^{-} \mathrm{CH}_{3}{ }^{\mathrm{A}}\right), 0.17\left(\mathrm{~s}, 1^{\prime}-\mathrm{O}-\mathrm{Si}^{-} \mathrm{CH}_{3}{ }^{\mathrm{B}}\right) \leftrightarrow-4.4\left(1^{\prime}-\mathrm{O}-\right.$ $\left.\mathrm{Si}-\mathrm{CH}_{3}{ }^{\mathrm{B}}\right), 0.74\left[\mathrm{~s}, 2-\mathrm{NH}-\mathrm{Si}-\mathrm{C}\left(\mathrm{CH}_{3}\right)_{3}\right] \leftrightarrow 26.2$ [2-NH-Si-C $\left.\left(\mathrm{CH}_{3}\right)_{3}\right], 0.86\left[\mathrm{~s}, 3-\mathrm{O}-\mathrm{Si}-\mathrm{C}\left(\mathrm{CH}_{3}\right)_{3}\right] \leftrightarrow$ 25.9 [3-O-Si-C $\left(\mathrm{CH}_{3}\right)_{3}$ ], 0.98 [s, 4'-O-Si-C $\left.\left(\mathrm{CH}_{3}\right)_{3}\right] \leftrightarrow 25.8$ [4'-O-Si-C $\left.\left(\mathrm{CH}_{3}\right)_{3}\right], 1.28\left(\mathrm{t}, 2^{\prime \prime}-\mathrm{H}_{3}\right) \leftrightarrow$ 14.3 (C-2"), 3.38 (dd, 2-H) 63.4 (C-2), 4.13 (mc, 1"--H2) 60.7 (C-1"), 5.00 (d, 3-H) 76.5 (C-3), 6.74-6.78 (m, 3'-H, 5'-H) 119.4 (C-3', C-5'), 7.13-7.19 (m, 2'-H, 6'-H) 128.1 (C-2', C-6').

HRMS: (EI, $70 \mathrm{eV}): \mathrm{m} / \mathrm{z}=568.3673[\mathrm{M}+\mathrm{H}]^{+}$, corresponds to the molecular formula $\mathrm{C}_{29} \mathrm{H}_{58} \mathrm{O}_{4} \mathrm{NSi}_{3}(568.33668)\left\{[\mathrm{M}+\mathrm{H}]^{+}\right\}$with a deviation of $0.9 \mathrm{ppm}$.

Specific rotation $(R, S)-\mathbf{1 2 f}[\alpha]_{D}^{20}=-28.9^{\circ}\left(\mathrm{c}=0.51\right.$ in $\left.\mathrm{CHCl}_{3}\right)$.

IR (KBr): $\tilde{v}=2955,2930,2885,2860,1750,1725,1610,1510,1472,1465,1415,1390,1360$, $1295,1255,1180,1165,1150,1105,1080,1005,930,915,835,810,780 \mathrm{~cm}^{-1}$. 
Ethyl (2S,3S)-3-[(tert-Butyldimethylsilyl)oxy]-3-\{4'-[(tert-butyldimethylsilyl)oxy]phenyl\}$2-\left[\left(1^{\prime \prime}, 3^{\prime \prime}, 5^{\prime \prime}\right.\right.$-trimethoxybenzyl)amino]propanoate $[(S, S)-12 \mathrm{e}]$

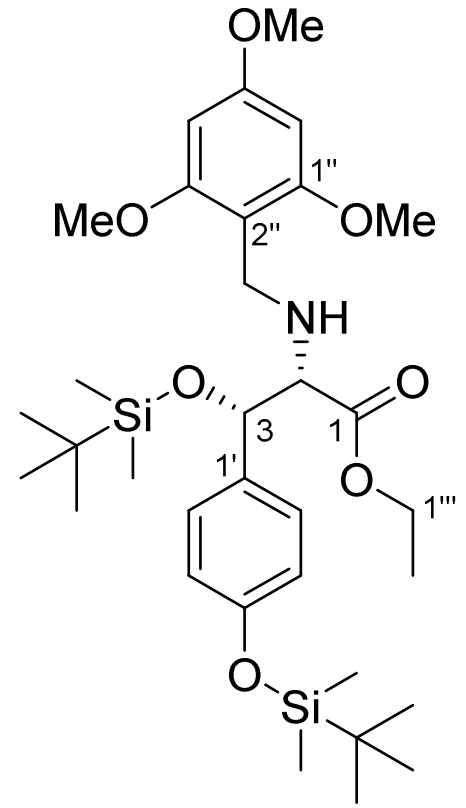

$(S, S)-12 e$

The amine $(S, S)-\mathbf{1 2 f}(25.0 \mathrm{mg}, 44 \mu \mathrm{mol})$ was dissolved in $\mathrm{MeOH}(1 \mathrm{~mL})$. It was added HOAc (1 drop) and 2,4,6-trimethoxybenzaldehyde (9.5 mg, $48 \mu \mathrm{mol}, 1.1$ eq.). The mixture stirred for $10 \mathrm{sec}$ at $25^{\circ} \mathrm{C}$. The mixture was diluted with $\mathrm{NaBH}_{3} \mathrm{CN}(5.5 \mathrm{mg}, 88 \mu \mathrm{mol}, 2.0$ eq.) and stirred for $3 \mathrm{~h}$ at $25^{\circ} \mathrm{C}$. The solvent was removed under reduced pressure. It was added $\mathrm{H}_{2} \mathrm{O}(2 \mathrm{ml})$ and $\mathrm{Et}_{2} \mathrm{O}(5 \mathrm{~mL})$. The layers were separated, and the aq. layer was extracted with $\mathrm{Et}_{2} \mathrm{O}(3 \times 5 \mathrm{~mL})$. The combined organic layers were dried over $\mathrm{Na}_{2} \mathrm{SO}_{4}$ and the solvent was removed under reduced pressure. The residue was purified by flash chromatography $\left(1.0 \mathrm{~cm}, 20 \mathrm{~cm}, c \mathrm{C}_{6} \mathrm{H}_{12}\right.$ : EtOAc $=4: 1,25 \mathrm{~mL})$ to give the product $(S, S)-\mathbf{1 2 e}(24.6 \mathrm{mg}, 88 \%, 39 \mu \mathrm{mol})$ as a colorless oil.

$\mathbf{R}_{\mathbf{f}}\left(c \mathrm{C}_{6} \mathrm{H}_{12}: \mathrm{EtOAc}=4: 1\right)=0.36$

${ }^{1} \mathbf{H}$-NMR $\left(500.06 \mathrm{MHz}, \mathrm{CDCl}_{3} / \mathrm{CHCl}_{3}\right.$, sample contained trace amounts of $c \mathrm{C}_{6} \mathrm{H}_{12}$, DsBrMi05500100, 6.5.2020): $\delta=-0.32$ (s, 3H, 3-O-Si-CH${ }^{\mathrm{A}}$ ), -0.09 (s, 3H, 3-O-Si-CH${ }^{\mathrm{B}}$ ), 0.18 [s, 6H, 4'-O-Si- $\left(\mathrm{CH}_{3}\right)_{2}$ ], 0.76 [s, 9H, 3-O-Si-C $\left.\left(\mathrm{CH}_{3}\right)_{3}\right], 0.97$ [s, 9H, 4'-O-Si-C $\left.\left(\mathrm{CH}_{3}\right)_{3}\right], 1.25$ (t, J'"',1"' = $7.1 \mathrm{~Hz}, 3 \mathrm{H}, 2$ '"-H 3 ), 1.92 (br. s, $1 \mathrm{H}, N \mathrm{H}), 3.33$ (d, J2,3 = 8.0 Hz, 1H, 2-H), 3.59-3.68 (m, 8H, 2"-

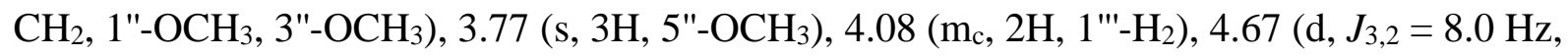
1H, 3-H), 6.01 (s, 2H, 4"-H, 6"-H), 6.74-6.77 (m, 2H, 3'-H, 5'-H), 7.12-7.17 (m, 2H, 2'-H, 6'H) ppm. 
Christian Drescher and Reinhard Brückner; 3D-Structure Clarifying Total Synthesis of the (Polyenoyl)tetramic Acid Militarinon B. A Highly Acid-Labile N-Protecting Group for Amides

${ }^{13}$ C-NMR (125.75 MHz, $\mathrm{CDCl}_{3} / \mathrm{CHCl}_{3}$, DsBrMi05-500106, 6.5.2020): $\delta=-5.3$ (3-O-Si$\left.\mathrm{CH}_{3}{ }^{\mathrm{B}}\right),-4.7$ (3-O-Si-CH$\left.{ }^{\mathrm{A}}\right),-4.3$ [4'-O-Si- $\left(\mathrm{CH}_{3}\right)_{2}$ ], 14.3 (C-2"'), 18.0 [3-O-Si-C $\left(\mathrm{CH}_{3}\right)_{3}$ ], 18.3 [4'-O-Si- $C\left(\mathrm{CH}_{3}\right)_{3}$ ], 25.7 [3-O-Si-C $\left(\mathrm{CH}_{3}\right)_{3}$ ], 25.8 [4'-O-Si-C $\left(\mathrm{CH}_{3}\right)_{3}$ ], 39.9 (2"- $\left.\mathrm{CH}_{2}\right), 55.4$ (5"$\mathrm{OCH}_{3}$ ), 55.5 (1"--OCH 3,3 "-OCH 3 ), 60.4 (C-1"'), 68.2 (C-2), 76.3 (C-3), 90.3 (C-4", C-6"), 108.4 (C-2"), 119.6 (C-3', C-5'), 128.6 (C-2', C-6'), 134.7 (C-1'), 155.3 (C-4'), 159.3 (C-1", C-3"), 160.4 (C-5"), 173.5 (C-1) ppm.

edHSQC [,,short-range H,C-COSY“, $\left.500.06 \mathrm{MHz} / 125.75 \mathrm{MHz}, \mathrm{CDCl}_{3} ; \delta\left({ }^{1} \mathrm{H}\right) \leftrightarrow \delta\left({ }^{13} \mathrm{C}\right)\right]$ : $0.32\left(\mathrm{~s}, 3-\mathrm{O}-\mathrm{Si}-\mathrm{CH}_{3}{ }^{\mathrm{A}}\right) \leftrightarrow-4.7\left(3-\mathrm{O}-\mathrm{Si}_{-} \mathrm{CH}_{3}{ }^{\mathrm{A}}\right),-0.09\left(\mathrm{~s}, 3-\mathrm{O}-\mathrm{Si}-\mathrm{CH}_{3}{ }^{\mathrm{B}}\right) \leftrightarrow-5.3\left(3-\mathrm{O}-\mathrm{Si}-\mathrm{CH}_{3}{ }^{\mathrm{B}}\right)$, 0.18 [s, 4'-O-Si- $\left.\left(\mathrm{CH}_{3}\right)_{2}\right] \leftrightarrow-4.3$ [4'-O-Si- $\left.\left(\mathrm{CH}_{3}\right)_{2}\right], 0.76$ [s, 3-O-Si-C $\left.\left(\mathrm{CH}_{3}\right)_{3}\right] \leftrightarrow 25.7$ [3-O-Si-

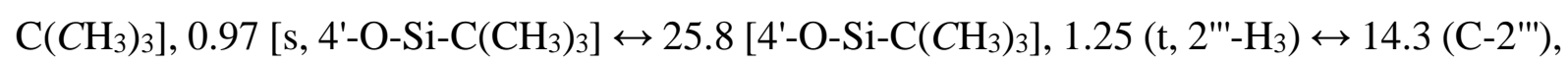
$3.33(\mathrm{~d}, 2-\mathrm{H}) \leftrightarrow 68.2(\mathrm{C}-2), 3.59-3.68\left(\mathrm{~m}, 2 "-\mathrm{CH}_{2}\right) \leftrightarrow 39.9\left(2 "-\mathrm{CH}_{2}\right), 3.59-3.68\left(\mathrm{~m}, 1 "-\mathrm{OCH}_{3}\right.$, 3"-- $\left.\mathrm{OCH}_{3}\right) \leftrightarrow 55.5\left(1 "-\mathrm{OCH}_{3}, 3\right.$ "--OCH 3$), 3.77\left(\mathrm{~s}, 5 "-\mathrm{OCH}_{3}\right) \leftrightarrow 55.4\left(5 "-\mathrm{OCH}_{3}\right), 4.08\left(\mathrm{~m}_{\mathrm{c}}, 1\right.$ "'$\left.\mathrm{H}_{2}\right) \leftrightarrow 60.4$ (C-1"'), 4.67 (d, 3-H) 76.3 (C-3), 6.01 (s, 4"-H, 6"-H) 90.3 (C-4", C-6"), 6.74$6.77\left(\mathrm{~m}, 3^{\prime}-\mathrm{H}, 5^{\prime}-\mathrm{H}\right) \leftrightarrow 119.6\left(\mathrm{C}-3^{\prime}, \mathrm{C}^{\prime} 5^{\prime}\right), 7.12-7.17\left(\mathrm{~m}, 2^{\prime}-\mathrm{H}, 6^{\prime}-\mathrm{H}\right) \leftrightarrow 128.6\left(\mathrm{C}-2^{\prime}, \mathrm{C}^{\prime} 6^{\prime}\right)$.

Specific rotation $(S, S)-12 \mathrm{e}[\alpha]_{D}^{20}=+27.6^{\circ}\left(\mathrm{c}=0.87\right.$ in $\left.\mathrm{CHCl}_{3}\right)$

HRMS: (EI, $70 \mathrm{eV}$ ): $\mathrm{m} / \mathrm{z}=634.3585[\mathrm{M}+\mathrm{H}]^{+}$, corresponds to the molecular formula $\mathrm{C}_{33} \mathrm{H}_{56} \mathrm{O}_{7} \mathrm{NSi}_{2}(634.3590)\left\{[\mathrm{M}+\mathrm{H}]^{+}\right\}$with a deviation of $-0.8 \mathrm{ppm}$.

IR (KBr): $\tilde{v}=2955,2930,2895,2860,1740,1610,1510,1470,1465,1440,1420,1255,1220$, 1180, 1165, 1150, 1140. 1095, 1060, 1040, 915, 860, 840, 810, $780 \mathrm{~cm}^{-1}$. 


\section{Ethyl (2S,3R)-3-[(tert-Butyldimethylsilyl)oxy]-3-\{4'-[(tert-}

butyldimethylsilyl)oxy]phenyl $\}-2-\left[\left(1^{\prime \prime}, 3^{\prime \prime}, 5 "-\right.\right.$ trimethoxybenzyl)amino]propanoate $[(R, S)$ -

12e]

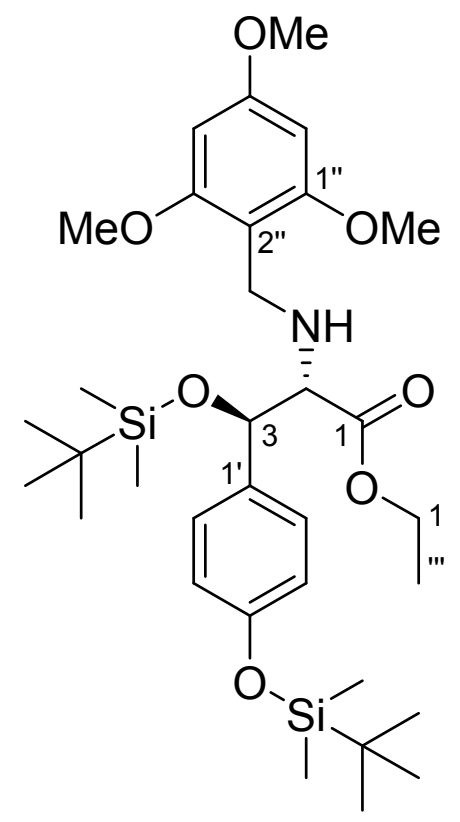

$(R, S)-12 \mathrm{e}$

The amine $(R, S)-\mathbf{1 2 f}(50 \mathrm{mg}, 88 \mu \mathrm{mol})$ was dissolved in $\mathrm{MeOH}(2 \mathrm{~mL})$. It was added HOAc (2 drops) and 2,4,6-trimethoxybenzaldehyde (19 mg, $96 \mu \mathrm{mol}, 1.1$ eq.). The mixture stirred for 10 $\mathrm{sec}$ at $25^{\circ} \mathrm{C}$. The mixture was diluted with $\mathrm{NaBH}_{3} \mathrm{CN}(11 \mathrm{mg}, 164 \mu \mathrm{mol}, 2.0$ eq. $)$ and stirred for $3 \mathrm{~h}$ at $25^{\circ} \mathrm{C}$. The solvent was removed under reduced pressure. It was added $\mathrm{H}_{2} \mathrm{O}(4 \mathrm{ml})$ and $\mathrm{Et}_{2} \mathrm{O}(10 \mathrm{~mL})$. The layers were separated, and the aq. layer was extracted with $\mathrm{Et}_{2} \mathrm{O}(3 \times 5 \mathrm{~mL})$. The combined organic layers were dried over $\mathrm{Na}_{2} \mathrm{SO}_{4}$ and the solvent was removed under reduced pressure. The residue was purified by flash chromatography $\left(1.0 \mathrm{~cm}, 20 \mathrm{~cm}, c \mathrm{C}_{6} \mathrm{H}_{12}\right.$ : EtOAc $=4: 1,25 \mathrm{~mL})$ to give the product $(R, S)-\mathbf{1 2} \mathbf{e}(47.5 \mathrm{mg}, 85 \%, 75 \mu \mathrm{mol})$ as a colorless oil.

$\mathbf{R}_{\mathbf{f}}\left(c \mathrm{C}_{6} \mathrm{H}_{12}: \mathrm{EtOAc}=4: 1\right)=0.35$

${ }^{1} \mathbf{H}$-NMR $\left(400.13 \mathrm{MHz}, \mathrm{CDCl}_{3} / \mathrm{CHCl}_{3}\right.$, sample contained trace amounts of $c \mathrm{C}_{6} \mathrm{H}_{12}$ and unknown impurity with s at 3.73, DsBrJn04-4020, 4.6.2020): $\delta=-0.27$ (s, 3H, 3-O-Si- $\left.\mathrm{CH}_{3}{ }^{\mathrm{A}}\right)$, -0.01 (s, 3H, 3-O-Si- $\mathrm{CH}_{3}{ }^{\mathrm{B}}$ ), 0.16 [s, 6H, 4'-O-Si- $\left(\mathrm{CH}_{3}\right)_{2}$ ], 0.82 [s, 9H, 3-O-Si-C $\left(\mathrm{CH}_{3}\right)_{3}$ ], 0.96

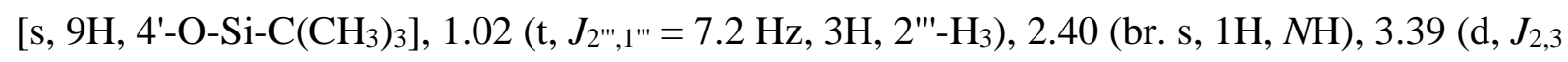
$=7.1 \mathrm{~Hz}, 1 \mathrm{H}, 2-\mathrm{H}), 3.70\left(\mathrm{~d},{ }^{2} J=13.4 \mathrm{~Hz}, 1 \mathrm{H}, 2^{2}-\mathrm{C}-\mathrm{H}^{\mathrm{A}}\right), 3.72\left(\mathrm{~s}, 6 \mathrm{H}, 1\right.$ "- $\mathrm{OCH}_{3}, 3$ "-OCH $), 3.76$ $\left(\mathrm{d},{ }^{2} J=13.2 \mathrm{~Hz}, 1 \mathrm{H}, 2^{\prime \prime}-\mathrm{C}-\mathrm{H}^{\mathrm{B}}\right), 3.79\left(\mathrm{~s}, 3 \mathrm{H}, 5 "-\mathrm{OCH}_{3}\right), 3.90\left(\mathrm{~m}_{\mathrm{c}}, 2 \mathrm{H}, 1^{\prime \prime \prime}-\mathrm{H}_{2}\right), 4.73\left(\mathrm{~d}, J_{3,2}=7.1\right.$ 
Christian Drescher and Reinhard Brückner; 3D-Structure Clarifying Total Synthesis of the (Polyenoyl)tetramic Acid Militarinon B. A Highly Acid-Labile N-Protecting Group for Amides

Hz, 1H, 3-H), 6.07 (s, 2H, 4"-H, 6"-H), 6.69-6.73 (m, 2H, 3'-H, 5'-H), 7.08-7.13 (m, 2H, 2'-H, 6'-H) ppm.

${ }^{13}$ C-NMR (100.61 MHz, $\mathrm{CDCl}_{3} / \mathrm{CHCl}_{3}$, DsBrMi05-500106, 6.5.2020): $\delta=-5.0$ (3-O-Si$\left.\mathrm{CH}_{3}{ }^{\mathrm{B}}\right),-4.5\left(3-\mathrm{O}-\mathrm{Si}_{-}-\mathrm{CH}_{3}{ }^{\mathrm{A}}\right),-4.4\left(4^{\prime}-\mathrm{O}-\mathrm{Si}_{-} \mathrm{CH}_{3}{ }^{\mathrm{B}}\right),-4.4\left(4^{\prime}-\mathrm{O}-\mathrm{Si}-\mathrm{CH}_{3}{ }^{\mathrm{A}}\right), 14.1\left(\mathrm{C}-2^{2}\right.$ ') 18.2 [3-O$\mathrm{Si}-\mathrm{C}\left(\mathrm{CH}_{3}\right)_{3}$ ], 18.3 [4'-O-Si- $\mathrm{C}\left(\mathrm{CH}_{3}\right)_{3}$ ], 25.8 [4'-O-Si-C $\left(\mathrm{CH}_{3}\right)_{3}$ ], 25.8 [3-O-Si-C $\left(\mathrm{CH}_{3}\right)_{3}$ ], $39.8\left(2^{\prime \prime}-\right.$ $\mathrm{CH}_{2}$ ), 55.4 (5"-OCH 3$), 55.6$ (1"--OCH 3,3 "-OCH $), 60.2$ (C-1"'), 68.8 (C-2), 76.7 (C-3), 90.4 (C4", C-6"), 108.9 (C-2"), 119.4 (C-3', C-5'), 128.5 (C-2', C-6'), 134.3 (C-1'), 155.2 (C-4'), 159.5 (C-1", C-3"), 160.4 (C-5"), 173.6 (C-1) ppm.

edHSQC [,,short-range H,C-COSY“, $\left.400.13 \mathrm{MHz} / 100.61 \mathrm{MHz}, \mathrm{CDCl}_{3} ; \delta\left({ }^{1} \mathrm{H}\right) \leftrightarrow \delta\left({ }^{13} \mathrm{C}\right)\right]$ : $0.27\left(\mathrm{~s}, 3-\mathrm{O}-\mathrm{Si}-\mathrm{CH}_{3}{ }^{\mathrm{A}}\right) \leftrightarrow-4.5\left(3-\mathrm{O}-\mathrm{Si}-\mathrm{CH}_{3}{ }^{\mathrm{A}}\right),-0.01\left(\mathrm{~s}, 3-\mathrm{O}-\mathrm{Si}_{-} \mathrm{CH}_{3}{ }^{\mathrm{B}}\right) \leftrightarrow-5.0\left(3-\mathrm{O}-\mathrm{Si}-\mathrm{CH}_{3}{ }^{\mathrm{B}}\right)$, $0.16\left(\mathrm{~s}, 4^{\prime}-\mathrm{O}-\mathrm{Si}-\mathrm{CH}_{3}{ }^{\mathrm{A}}\right) \leftrightarrow-4.4\left(4^{\prime}-\mathrm{O}-\mathrm{Si}-\mathrm{CH}_{3}{ }^{\mathrm{A}}\right), 0.16\left(\mathrm{~s}, 4^{\prime}-\mathrm{O}-\mathrm{Si}-\mathrm{CH}_{3}{ }^{\mathrm{B}}\right) \leftrightarrow-4.4\left(4^{\prime}-\mathrm{O}-\mathrm{Si}-\mathrm{CH}_{3}{ }^{\mathrm{B}}\right)$, 0.82 [s, 3-O-Si-C $\left.\left(\mathrm{CH}_{3}\right)_{3}\right] \leftrightarrow 25.8$ [3-O-Si-C $\left.\left(\mathrm{CH}_{3}\right)_{3}\right], 0.96$ [s, 4'-O-Si-C $\left.\left(\mathrm{CH}_{3}\right)_{3}\right] \leftrightarrow 25.8$ [4'-O$\left.\mathrm{Si}-\mathrm{C}\left(\mathrm{CH}_{3}\right)_{3}\right], 1.02\left(\mathrm{t}, 2^{\prime \prime \prime}-\mathrm{H}_{3}\right) \leftrightarrow 14.1\left(\mathrm{C}-2{ }^{\prime \prime \prime}\right), 3.39(\mathrm{~d}, 2-\mathrm{H}) \leftrightarrow 68.8(\mathrm{C}-2), 3.70\left(\mathrm{~d}, 2\right.$ "-C-H $\left.{ }^{\mathrm{A}}\right) \leftrightarrow$ $39.8\left(2^{\prime \prime}-\mathrm{CH}_{2}\right), 3.72\left(\mathrm{~s}, 1^{2}-\mathrm{OCH}_{3}, 3\right.$ "- $\left.\mathrm{OCH}_{3}\right) \leftrightarrow 55.6\left(1 "-\mathrm{OCH}_{3}, 3\right.$ "'-OCH 3$), 3.76\left(\mathrm{~d}, 2^{\prime \prime}-\mathrm{C}-\mathrm{H}^{\mathrm{B}}\right) \leftrightarrow$ $39.8\left(2 "-\mathrm{CH}_{2}\right), 3.79\left(\mathrm{~s}, 3 \mathrm{H}, 5 "-\mathrm{OCH}_{3}\right) \leftrightarrow 55.4\left(5 "-\mathrm{OCH}_{3}\right), 3.90\left(\mathrm{~m}_{\mathrm{c}}, 1\right.$ "'-- $\left.\mathrm{2}\right) \leftrightarrow 60.2\left(\mathrm{C}-1{ }^{\prime \prime \prime}\right), 4.73$ (d, 3-H) ↔ 76.7 (C-3), 6.07 (s, 4"-H, 6"-H) ↔ 90.4 (C-4", C-6"), 6.69-6.73 (m, 3'-H, 5'-H) ↔ 119.4 (C-3', C-5'), 7.08-7.13 (m, 2'-H, 6'-H) 128.5 (C-2', C-6').

Specific rotation $(R, S)-12 \mathrm{e}[\alpha]_{D}^{20}=-51.0^{\circ}\left(\mathrm{c}=0.75\right.$ in $\left.\mathrm{CHCl}_{3}\right)$

HRMS: (EI, $70 \mathrm{eV}): \mathrm{m} / \mathrm{z}=634.3585[\mathrm{M}+\mathrm{H}]^{+}$, corresponds to the molecular formula $\mathrm{C}_{33} \mathrm{H}_{56} \mathrm{O}_{7} \mathrm{NSi}_{2}(634.3590)\left\{[\mathrm{M}+\mathrm{H}]^{+}\right\}$with a deviation of $-0.8 \mathrm{ppm}$.

IR (KBr): $\tilde{v}=3435,2955,2935,2855,1645,1510,1465,1520,1465,1415,1315,1255,1225$, $1205,1165,1150,1140,1105,1060,1005,950,915,835,805,780 \mathrm{~cm}^{-1}$.

Ethyl (2'S,3'S)-2'-\{N-[(1Z,2Z,4E)-5-Bromo-3-hydroxy-1-oxoypenta-2,4-dienyl]- $N$ $(1 " ', 3$ "', 5'"-trimethoxybenzyl) $\}-3$ '-[(tert-butyldimethylsilyl)oxy]-3'-\{1"-[(tertbutyldimethylsilyl)oxy]phenyl $\}$ propanoate $[Z$-enol-(S,S)-10e]

in equilibration with

Ethyl (2'S,3'S)-2'-\{N-[(1Z,4E)-5-Bromo-1,3-dioxopent-4-enyl]- $N-\left(1{ }^{\prime \prime \prime}, 3\right.$ '", 5 "'trimethoxybenzyl $)\}-3 '-\left[(\right.$ tert-butyldimethylsilyl)oxy $]-3 '-\left\{1^{\prime \prime}-[(\right.$ tertbutyldimethylsilyl)oxy]phenyl $\}$ propanoate $[Z-k e t o-(S, S)-10 \mathrm{e}]$ 


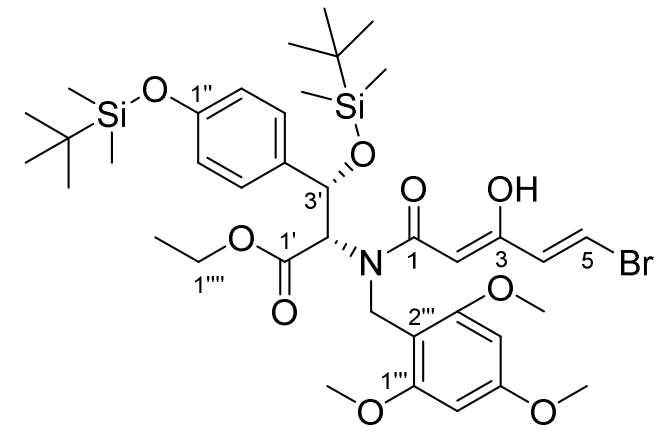

Z-enol-[(S, S)-10e]

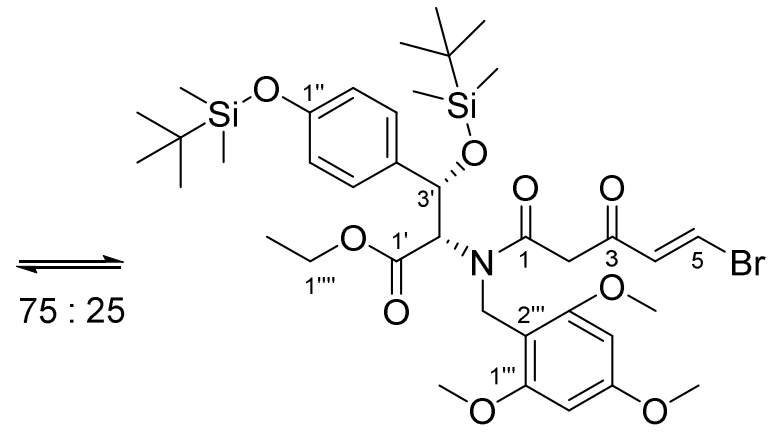

Z-keto-[(S, S)-10e]

A solution of amine $(S, S)-\mathbf{1 2 e}(100 \mathrm{mg}, 158 \mu \mathrm{mol})$ and the $\beta$-ketothioester $11(63 \mathrm{mg}, 237 \mu \mathrm{mol}$, 1.5 eq. $)$ in THF ( $8 \mathrm{~mL})$ was treated with powdered molecular sieves $4 \AA(0.6 \mathrm{~g})$. The suspension stirred at $25^{\circ} \mathrm{C}$ for $30 \mathrm{~min}$. Then $\mathrm{AgO}_{2} \mathrm{CCF}_{3}(65 \mathrm{mg}, 237 \mu \mathrm{mol}, 1.5$ eq.) was added and the mixture was stirred for $21 \mathrm{~h}$ at $25^{\circ} \mathrm{C}$. The solvent was removed under reduced pressure and $\mathrm{Et}_{2} \mathrm{O}(20 \mathrm{~mL})$ was added to the resulting suspension. The mixture was filtered, and the filtrate was concentrated under reduced pressure. Purification by flash chromatography $\left(c \mathrm{C}_{6} \mathrm{H}_{12}: \mathrm{AcOEt}=8: 1,2.0 \mathrm{~cm}, 20 \mathrm{~cm}, 15 \mathrm{~mL}\right)$ afforded $(S, S)-\mathbf{1 0 e}(107 \mathrm{mg}, 84 \%, 199 \mu \mathrm{mol}$, as an orange oil. It was assigned as an 75:25 mixture of the keto- and the enol-tautomer of the title compound $(S, S)$-10e. This ratio was determined by integrating the singlets of the respective 4 "'$\mathrm{H}$ and 6"'-H [6.09 (enol) and 6.13 (keto).$^{10}$

$\mathbf{R}_{\mathbf{f}}\left(c \mathrm{C}_{6} \mathrm{H}_{12}: \mathrm{EtOAc}=8: 1\right)=0.41$

${ }^{1} \mathbf{H}$-NMR (500.06 MHz, CDCl $3 / \mathrm{CHCl}_{3}$, DsBrMi08-500200, 11.5.2020): $\delta=-0.24$ (s, 3H, 3'-O$\left.\mathrm{Si}-\mathrm{CH}_{3}{ }^{\mathrm{A}}\right), 0.11\left(\mathrm{~s}, 3 \mathrm{H}, 3^{\prime}-\mathrm{O}-\mathrm{Si}-\mathrm{CH}_{3}{ }^{\mathrm{B}}\right), 0.15$ [s, 6H, 1"-O-Si- $\left.\left(\mathrm{CH}_{3}\right)_{2}\right], 0.81$ [s, 9H, 3'-O-Si$\left.\mathrm{C}\left(\mathrm{CH}_{3}\right)_{3}\right], 0.96$ [s, 9H, 4"-O-Si-C $\left.\left(\mathrm{CH}_{3}\right)_{3}\right], 1.02$ (t, $J_{2^{\prime \prime \prime}, 1^{\prime \prime \prime}}=7.1 \mathrm{~Hz}, 3 \mathrm{H}, 2$ '"'-H3), 3.71-3.77 (m,

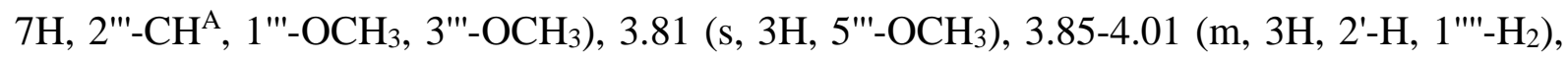
$4.06\left(\mathrm{~d},{ }^{2} J=14.8 \mathrm{~Hz}, 1 \mathrm{H}, 2^{\prime \prime}-\mathrm{CH}^{\mathrm{B}}\right), 5.34(\mathrm{~s}, 1 \mathrm{H}, 2-\mathrm{H}), 5.51\left(\mathrm{~d}, J_{3^{\prime}, 2^{\prime}}=7.7 \mathrm{~Hz}, 1 \mathrm{H}, 3^{\prime}-\mathrm{H}\right), 6.07$ (s, 2H, 4"'-H, 6"'-H), 6.42 (dd, $\left.J_{4,5}=13.6 \mathrm{~Hz}, J_{4,3-\mathrm{OH}}=1.2 \mathrm{~Hz}, 1 \mathrm{H}, 4-\mathrm{H}\right), 6.67\left(\mathrm{~d}, J_{2 ", 3 "}=J_{6 ", 5 "}\right.$ 8.6 Hz, 2H, 2"-H, 6"-H), 7.09-7.16 (m, 3H, 5-H, 3"-H, 5"-H), 14.01 (d, J3-OH,4 = 1.4 Hz, 1H, 3$\mathrm{OH}) \mathrm{ppm}$.

${ }^{13}$ C-NMR (125.75 MHz, $\mathrm{CDCl}_{3} / \mathrm{CHCl}_{3}$, DsBrMi08-500205, 11.5.2020): $\delta=-4.9$ (3'-O-Si$\left.\mathrm{CH}_{3}{ }^{\mathrm{B}}\right),-4.6\left(3^{\prime}-\mathrm{O}-\mathrm{Si}-\mathrm{CH}_{3}{ }^{\mathrm{A}}\right),-4.4$ [1"-O-Si- $\left(\mathrm{CH}_{3}\right)_{2}$ ], $14.1\left(\mathrm{C}-2^{\prime \prime \prime \prime}\right), 18.1$ [3'-O-Si-C $\left(\mathrm{CH}_{3}\right)_{3}$ ], 18.4 [4"-O-Si- $C\left(\mathrm{CH}_{3}\right)_{3}$ ], 25.8 [4"-O-Si-C $\left(\mathrm{CH}_{3}\right)_{3}$ ], 25.9 [3'-O-Si-C $\left(\mathrm{CH}_{3}\right)_{3}$ ], $41.6\left(2^{\prime \prime}-\mathrm{CH}_{2}\right), 55.3$ (1'"$\mathrm{OCH}_{3}, 3$ '"--OCH 3 ), 55.4 (5"'-OCH 3 ), 60.3 (C-1"'"), 65.5 (C-2'), 72.5 (C-3'), 90.3 (C-4"', C-6"'), 91.4 (C-2), 104.4 (C-2"') 115.3 (C-5), 119.4 (C-2", C-6"), 128.3 (C-3", C-5"), 133.0 (C-4), 134.6 
Christian Drescher and Reinhard Brückner; 3D-Structure Clarifying Total Synthesis of the (Polyenoyl)tetramic Acid Militarinon B. A Highly Acid-Labile N-Protecting Group for Amides

(C-4"), 154.9 (C-1"), 159.9 (C-1"', C-3"'), 161.5 (C-5"'), 165.5 (C-3), 170.0 (C-1'), 171.5 (C-1) ppm.

edHSQC [,,short-range H,C-COSY“, $\left.500.06 \mathrm{MHz} / 125.75 \mathrm{MHz}, \mathrm{CDCl}_{3} ; \delta\left({ }^{1} \mathrm{H}\right) \leftrightarrow \delta\left({ }^{13} \mathrm{C}\right)\right]$ : $0.24\left(\mathrm{~s}, 3^{\prime}-\mathrm{O}-\mathrm{Si}-\mathrm{CH}_{3}{ }^{\mathrm{A}}\right) \leftrightarrow-4.6\left(3^{\prime}-\mathrm{O}-\mathrm{Si}-\mathrm{CH}_{3}{ }^{\mathrm{A}}\right), 0.11\left(\mathrm{~s}, 3^{\prime}-\mathrm{O}-\mathrm{Si}_{-}-\mathrm{CH}_{3}{ }^{\mathrm{B}}\right) \leftrightarrow-4.9\left(3^{\prime}-\mathrm{O}-\mathrm{Si}-\mathrm{CH}_{3}{ }^{\mathrm{B}}\right)$, 0.15 [s, 1"-O-Si- $\left.\left(\mathrm{CH}_{3}\right)_{2}\right] \leftrightarrow-4.4$ [1"-O-Si- $\left.\left(\mathrm{CH}_{3}\right)_{2}\right], 0.81$ [s, 3'-O-Si-C $\left.\left(\mathrm{CH}_{3}\right)_{3}\right] \leftrightarrow 25.9$ [3'-O-Si$\left.\mathrm{C}\left(\mathrm{CH}_{3}\right)_{3}\right], 0.96$ [s, 4"-O-Si-C $\left.\left(\mathrm{CH}_{3}\right)_{3}\right] \leftrightarrow 25.8$ [4"-O-Si-C $\left.\left(\mathrm{CH}_{3}\right)_{3}\right], 1.02$ (t, 2'"'-H $\left.\mathrm{H}_{3}\right) 14.1(\mathrm{C}-$

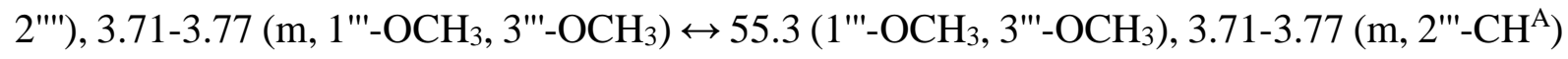
$\leftrightarrow 41.6\left(2^{\prime \prime}-\mathrm{CH}_{2}\right), 3.81\left(\mathrm{~s}, 5^{\prime \prime}-\mathrm{OCH}_{3}\right) \leftrightarrow 55.4\left(5^{\prime \prime}-\mathrm{OCH}_{3}\right), 3.85-4.01\left(\mathrm{~m}, 2\right.$ '-H) $65.5\left(\mathrm{C}-2^{\prime}\right)$,

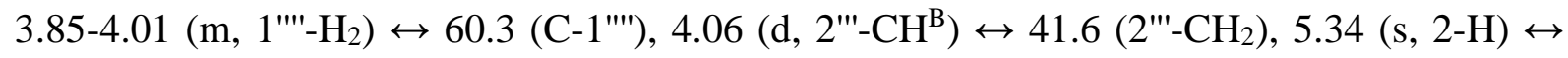
91.4 (C-2), 5.51 (d, 3'-H) 72.5 (C-3'), 6.07 (s, 4"'-H, 6"'-H) ↔ 90.3 (C-4"', C-6"'), 6.42 (dd, 4-H) 133.0 (C-4), 6.67 (d, 2"-H, 6"-H) ↔ 119.4 (C-2", C-6"), 7.09-7.16 (m, 5-H) 115.3 (C-5), 7.09-7.16 (m, 3"-H, 5"-H) 128.3 (C-3", C-5").

Specific rotation $(S, S)-\mathbf{1 0 e}[\alpha]_{D}^{20}=-23.8^{\circ}\left(\mathrm{c}=0.34\right.$ in $\left.\mathrm{CHCl}_{3}\right)$

HRMS: $(\mathrm{EI}, 70 \mathrm{eV}): \mathrm{m} / \mathrm{z}=832.2698[\mathrm{M}+\mathrm{Na}]^{+}$, corresponds to the molecular formula $\mathrm{C}_{38} \mathrm{H}_{58} \mathrm{O}_{9} \mathrm{NBrSi}_{2} \mathrm{Na}(832.2705)\left\{[\mathrm{M}+\mathrm{Na}]^{+}\right\}$with a deviation of $-0.8 \mathrm{ppm}$.

IR (KBr): $\tilde{v}=2955,2930,2855,1750,1650,1605,1595,1585,1510,1470,1465,1440,1400$, $1335,1255,1205,1190,1165,1150,1115,1105,910,860,840,805,780,760 \mathrm{~cm}^{-1}$.

Ethyl (2'S,3'R)-2'-\{N-[(1Z,2Z,4E)-5-Bromo-3-hydroxy-1-oxoypenta-2,4-dienyl]- $N$ $(1 " ', 3$ '", 5'"-trimethoxybenzyl) $\}-3$ '-[(tert-butyldimethylsilyl)oxy $]-3$ '- $\{1 "$ "-[(tertbutyldimethylsilyl)oxy]phenyl $\}$ propanoate $[Z-e n o l-(R, S)-10 \mathrm{e}]$ in equilibration with

Ethyl $(2 ' S, 3 ' R)-2 '-\left\{N-[(1 Z, 4 E)-5-B r o m o-1,3-d i o x o p e n t-4-e n y l]-N-\left(1^{\prime \prime \prime}, 3{ }^{\prime \prime \prime}, 5\right.\right.$ '"'trimethoxybenzyl) $\}-3^{\prime}-\left[(\right.$ tert-butyldimethylsilyl)oxy $]-3 '-\left\{1^{\prime \prime}-[(\right.$ tertbutyldimethylsilyl)oxy]phenyl $\}$ propanoate $[Z-k e t o-(R, S)-10 \mathrm{e}]$ 


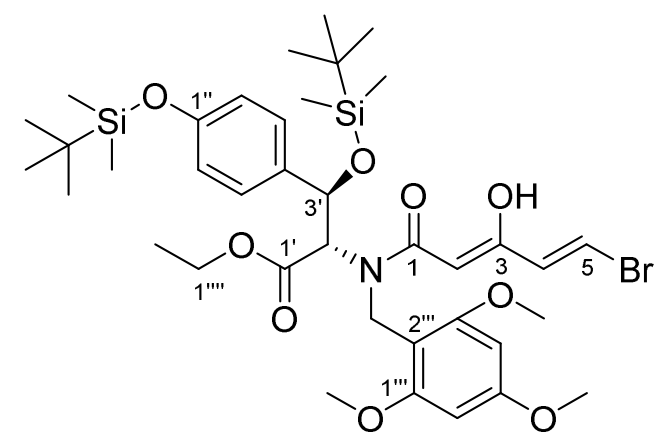

Z-enol-[(R,S)-10e]

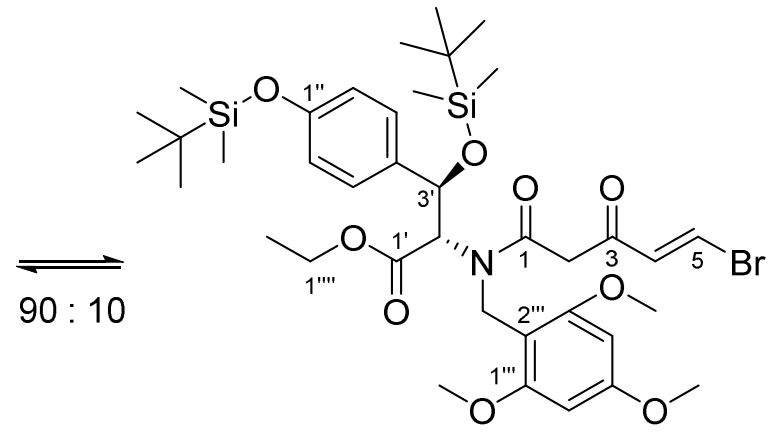

Z-keto-[(R,S)-10e]

A solution of amine $(R, S)-\mathbf{1 2 e}(75 \mathrm{mg}, 119 \mu \mathrm{mol})$ and the $\beta$-ketothioester 11 (47 mg, $178 \mu \mathrm{mol}$, 1.5 eq.) in THF ( $5 \mathrm{~mL})$ was treated with powdered molecular sieves $4 \AA(0.5 \mathrm{~g})$. The suspension stirred at $25^{\circ} \mathrm{C}$ for $30 \mathrm{~min}$. Then $\mathrm{AgO}_{2} \mathrm{CCF}_{3}$ (49 mg, $179 \mu \mathrm{mol}, 1.5$ eq.) was added and the mixture was stirred for $20 \mathrm{~h}$ at $25^{\circ} \mathrm{C}$. The solvent was removed under reduced pressure and $\mathrm{Et}_{2} \mathrm{O}(20 \mathrm{~mL})$ was added to the resulting suspension. The mixture was filtered, and the filtrate was concentrated under reduced pressure. Purification by flash chromatography $\left(c \mathrm{C}_{6} \mathrm{H}_{12}: \mathrm{AcOEt}=8: 1,2.0 \mathrm{~cm}, 20 \mathrm{~cm}, 15 \mathrm{~mL}\right)$ afforded $(R, S)-\mathbf{1 0 e}(76 \mathrm{mg}, 80 \%, 95 \mu \mathrm{mol}$, as an white solid (mp.: $147-149^{\circ} \mathrm{C}$ ). It was assigned as a 90:10 mixture of the keto- and the enoltautomer of the title compound $(R, S)$-10e. This ratio was determined by integrating the singlets of the respective 5-H $[7.18$ (enol) and 7.63 (keto) $] .{ }^{10}$

$\mathbf{R f}_{\mathbf{f}}\left(c \mathrm{C}_{6} \mathrm{H}_{12}:\right.$ EtOAc $\left.=8: 1\right): 0.40$

${ }^{1} \mathbf{H}$-NMR $\left(500.06 \mathrm{MHz}, \mathrm{CDCl}_{3} / \mathrm{CHCl}_{3}\right.$, sample contained 1 weight-\% of $c \mathrm{C}_{6} \mathrm{H}_{12}$, and unknown impurity with s at 3.33, DsBrJn08-500600, 8.6.2020): $\delta=-0.32\left(\mathrm{~s}, 3 \mathrm{H}, 3^{\prime}-\mathrm{O}-\mathrm{Si}_{-}-\mathrm{CH}_{3}{ }^{\mathrm{A}}\right),-0.05$ (s, 3H, 3'-O-Si- $\left.\mathrm{CH}_{3}{ }^{\mathrm{B}}\right), 0.17$ [s, 6H, 1"-O-Si- $\left.\left(\mathrm{CH}_{3}\right)_{2}\right], 0.77$ [s, 9H, 3'-O-Si-C(CH$\left.)_{3}\right], 0.90$ (t,

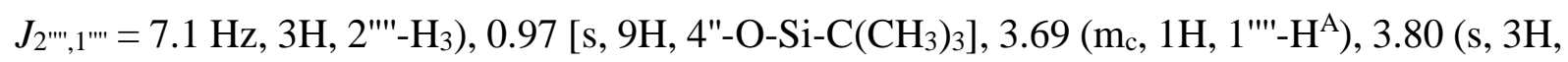

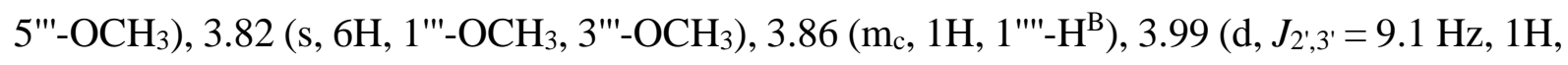
$\left.2^{\prime}-\mathrm{H}\right), \mathrm{AB}$ signal $\left(\delta_{\mathrm{A}}=4.67, \delta_{\mathrm{B}}=4.77, J_{\mathrm{AB}}=14.6 \mathrm{~Hz}, 2 \mathrm{H}, 2^{\prime \prime}-\mathrm{CH}_{2}\right), 5.42\left(\mathrm{~d}, J_{3^{\prime}, 2^{\prime}}=9.1 \mathrm{~Hz}, 1 \mathrm{H}\right.$, 3'-H), 5.75 (s, 1H, 2-H), 6.11 (s, 2H, 4'"-H, 6'"-H), 6.58 (dd, $J_{4,5}=13.5 \mathrm{~Hz}, J_{4,3-\mathrm{OH}}=0.9 \mathrm{~Hz}, 1 \mathrm{H}$, 4-H), 6.72-6.77 (m, 2H, 2"-H, 6"-H), 7.18 (d, J5,4 = 13.5 Hz, 1H, 5-H), 7.37-7.41 (m, 2H, 3"-H, 5"-H), $14.01\left(\mathrm{~d}, J_{3-\mathrm{OH}, 4}=1.2 \mathrm{~Hz}, 1 \mathrm{H}, 3-\mathrm{OH}\right) \mathrm{ppm}$.

${ }^{13}$ C-NMR (125.75 MHz, $\left.\mathrm{CDCl}_{3} / \mathrm{CHCl}_{3}, \mathrm{DsBrJn08}-500604,8.6 .2020\right)$ : -5.2 (3'-O-Si-CH $\left.3{ }^{\mathrm{B}}\right)$, $4.9\left(3^{\prime}-\mathrm{O}-\mathrm{Si}_{-}-\mathrm{CH}_{3}{ }^{\mathrm{A}}\right),-4.3$ [1"-O-Si- $\left(\mathrm{CH}_{3}\right)_{2}$ ], $13.9\left(\mathrm{C}-2\right.$ '"'), 17.9 [3'-O-Si-C $\left(\mathrm{CH}_{3}\right)_{3}$ ], 18.3 [4"-O$\mathrm{Si}-\mathrm{C}\left(\mathrm{CH}_{3}\right)_{3}$ ], 25.8 [3'-O-Si-C $\left(\mathrm{CH}_{3}\right)_{3}, 4$ "'-O-Si-C $\left(\mathrm{CH}_{3}\right)_{3}$ ], 42.9 (2"'- $\left.\mathrm{CH}_{2}\right), 55.4$ (1"'-- $\mathrm{OCH}_{3}, 3$ '"$\left.\mathrm{OCH}_{3}, 5^{\prime \prime \prime}-\mathrm{OCH}_{3}\right), 60.2$ (C-1'"'), 64.7 (C-2'), 72.1 (C-3'), 90.2 (C-4'", C-5"'), 91.7 (C-2), 104.6 
Christian Drescher and Reinhard Brückner; 3D-Structure Clarifying Total Synthesis of the (Polyenoyl)tetramic Acid Militarinon B. A Highly Acid-Labile N-Protecting Group for Amides

(C-2"'), 115.3 (C-5), 119.3 (C-2", C-6"), 129.6 (C-3", C-5"), 133.1 (C-4), 137.3 (C-4"), 154.9 (C-1"), 160.1 (C-1"', C-3"'), 161.5 (C-5"'), 165.5 (C-3), 169.4 (C-1'), 171.2 (C-1) ppm.

edHSQC [,,short-range H,C-COSY“, $\left.500.06 \mathrm{MHz} / 125.75 \mathrm{MHz}, \mathrm{CDCl}_{3} ; \delta\left({ }^{1} \mathrm{H}\right) \leftrightarrow \delta\left({ }^{13} \mathrm{C}\right)\right]$ : $0.32\left(\mathrm{~s}, 3^{\prime}-\mathrm{O}-\mathrm{Si}-\mathrm{CH}_{3}{ }^{\mathrm{A}}\right) \leftrightarrow-4.9\left(3^{\prime}-\mathrm{O}-\mathrm{Si}-\mathrm{CH}_{3}{ }^{\mathrm{A}}\right),-0.05\left(\mathrm{~s}, 3^{\prime}-\mathrm{O}-\mathrm{Si}_{-} \mathrm{CH}_{3}{ }^{\mathrm{B}}\right) \leftrightarrow-5.2\left(3^{\prime}-\mathrm{O}-\mathrm{Si}-\mathrm{CH}_{3}{ }^{\mathrm{B}}\right)$, 0.17 [s, 1"-O-Si- $\left.\left(\mathrm{CH}_{3}\right)_{2}\right] \leftrightarrow-4.3$ [1"-O-Si- $\left.\left(\mathrm{CH}_{3}\right)_{2}\right], 0.77$ [s, 9H, 3'-O-Si-C(CH3) 3 ] 25.8 [3'$\mathrm{O}-\mathrm{Si}-\mathrm{C}\left(\mathrm{CH}_{3}\right)_{3}$ ], 0.90 (t, 2"'--H$\left.{ }_{3}\right) \leftrightarrow 13.9$ (C-2'"'), 0.97 [s, 4"-O-Si-C $\left.\left(\mathrm{CH}_{3}\right)_{3}\right] \leftrightarrow 25.8$ [4"-O-Si-

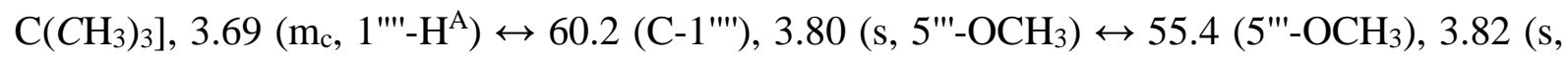

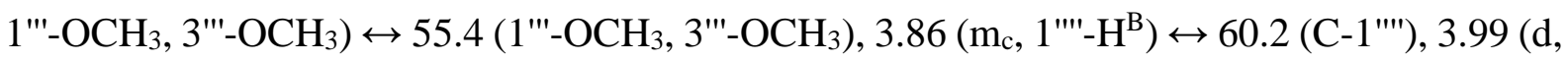
$\left.2^{\prime}-\mathrm{H}\right) \leftrightarrow 64.7\left(\mathrm{C}-2^{\prime}\right), \mathrm{AB}$ signal $\left(\delta_{\mathrm{A}}=4.67, \delta_{\mathrm{B}}=3.77,2^{\prime \prime}-\mathrm{CH}_{2}\right) \leftrightarrow 42.9\left(2^{\prime \prime \prime}-\mathrm{CH}_{2}\right), 5.42\left(\mathrm{~d}, 3^{\prime}-\mathrm{H}\right)$ $\leftrightarrow 72.1$ (C-3'), 5.75 (s, 2-H) ↔91.7 (C-2), 6.11 (s, 4'"-H, 6"'-H) ↔90.2 (C-4"', C-5'"), 6.58 $(\mathrm{dd}, 4-\mathrm{H}) \leftrightarrow 133.1$ (C-4), 6.72-6.77 (m, 2"-H, 6"-H) ↔ 119.3 (C-2", C-6"), 7.18 (d, 5-H) 115.3 (C-5), 7.37-7.41 (m, 3"-H, 5"-H) ↔ 129.6 (C-3", C-5").

Specific rotation $(R, S)-10 \mathbf{e}[\alpha]_{D}^{20}=-122.8^{\circ}\left(\mathrm{c}=0.44\right.$ in $\left.\mathrm{CHCl}_{3}\right)$

HRMS: $(\mathrm{EI}, 70 \mathrm{eV}): \mathrm{m} / \mathrm{z}=832.2706[\mathrm{M}+\mathrm{Na}]^{+}$, corresponds to the molecular formula $\mathrm{C}_{38} \mathrm{H}_{58} \mathrm{O}_{9} \mathrm{~N}^{81} \mathrm{BrNaSi}_{2}(832.2705)\left\{[\mathrm{M}+\mathrm{Na}]^{+}\right\}$with a deviation of $0.1 \mathrm{ppm}$.

IR (KBr): $\tilde{v}=2955,2885,2895,2855,1725,1635,1615,1605,1588,1505,1490,1465,1455$, $1330,1250,1225,1205,1155,1135,1085,1045,940,910,860,835,780 \mathrm{~cm}^{-1}$. 


\subsection{Synthesis of the Tetramic Acid Militarinone $\mathrm{B}[(S, S, R, R)-3]$ and} its Epimer $(R, S, R, R)-3$

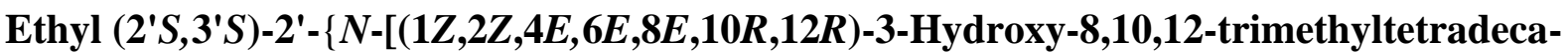
2,4,6,8-tetraenamido]- $N-(1 " ', 3$ '",5'"'-trimethoxybenzyl $)\}-3$ '-[(tertbutyldimethylsilyl)oxy]-3'-\{1"'-[(tert-butyldimethylsilyl)oxy]phenyl $\}$ propanoate [Z-enol$(S, S, R, R)-26]$

in equilibration with

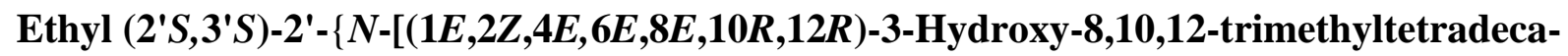
2,4,6,8-tetraenamido]- $N-(1 " ', 3$ '",5'"'-trimethoxybenzyl $)\}-3$ '-[(tertbutyldimethylsilyl)oxy $]-3 '-\left\{1^{\prime \prime}-[(\right.$ tert-butyldimethylsilyl)oxy $]$ phenyl $\}$ propanoate $[$ E-enol$(S, S, R, R)-26]$

in equilibration with

Ethyl (2'S,3' $S)-2 '-\{N-[(1 Z, 4 E, 6 E, 8 E, 10 R, 12 R)-3-0 x 0-8,10,12-t r i m e t h y l t e t r a d e c a-4,6,8-$ trienamido]- $N$-(1"',3'",5'"'-trimethoxybenzyl) $\}-3$ '-[(tert-butyldimethylsilyl)oxy $]-3$ '- $\left\{1^{\prime \prime}-\right.$ [(tert-butyldimethylsilyl)oxy]phenyl $\}$ propanoate $[Z-k e t o-(S, S, R, R)-26]$ in equilibration with

Ethyl $(2 ' S, 3 ' S)-2 '-\{N-[(1 E, 4 E, 6 E, 8 E, 10 R, 12 R)-3-0 x 0-8,10,12-t r i m e t h y l t e t r a d e c a-4,6,8-$ trienamido]- $N$-(1"',3'",5'"'-trimethoxybenzyl $)\}-3$ '-[(tert-butyldimethylsilyl)oxy $]-3$ '-\{1"[(tert-butyldimethylsilyl)oxy]phenyl $\}$ propanoate $[$ E-keto- $(S, S, R, R)-26]$ 
Christian Drescher and Reinhard Brückner; 3D-Structure Clarifying Total Synthesis of the (Polyenoyl)tetramic Acid Militarinon B. A Highly Acid-Labile N-Protecting Group for Amides

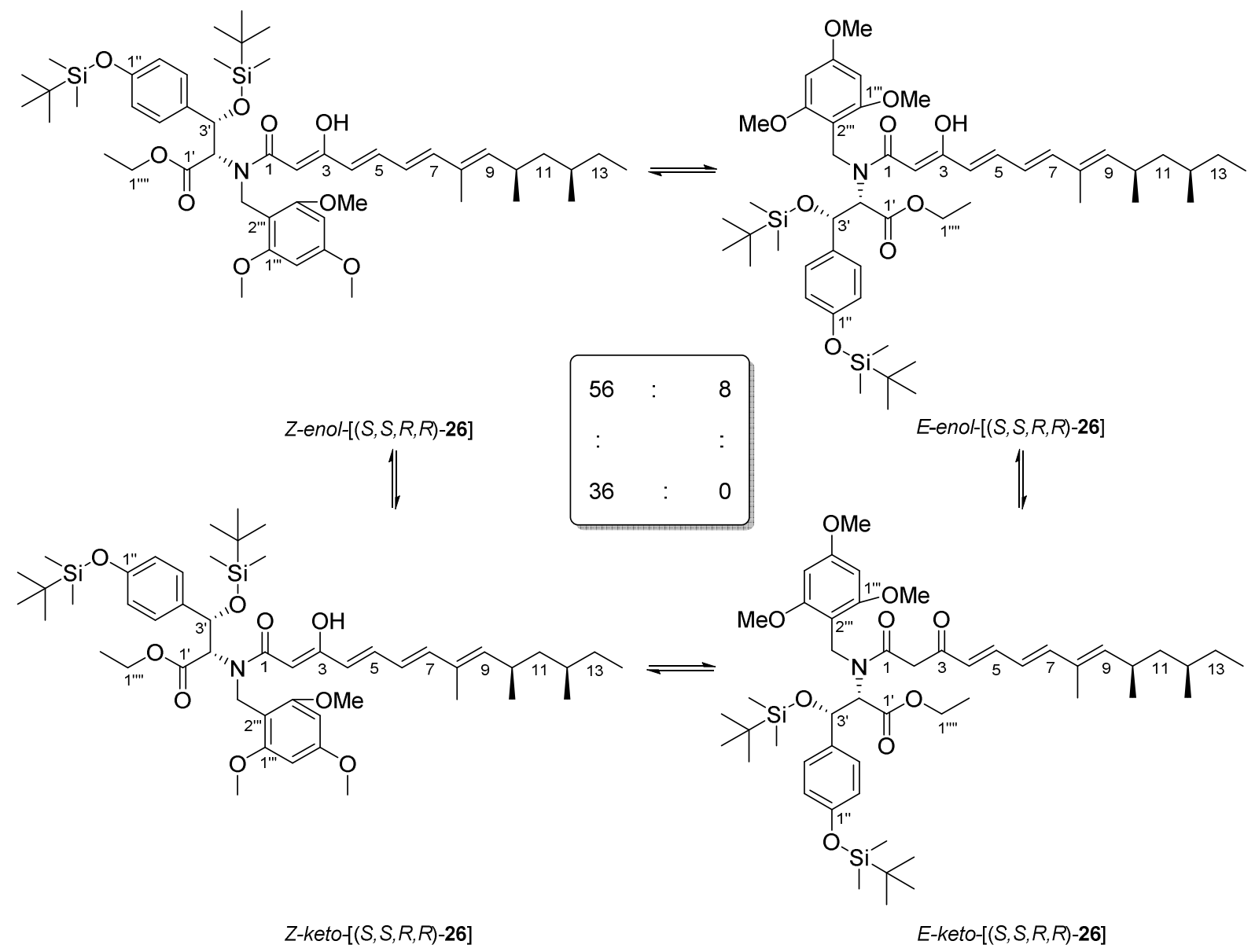

The alkyne $(R, R)-\mathbf{2 5}^{14}(15.0 \mathrm{mg}, 82 \mu \mathrm{mol})$ was dissolved in THF $(1.0 \mathrm{~mL})$. It was added $\mathrm{Pd}\left(\mathrm{PPh}_{3}\right)_{2} \mathrm{Cl}_{2}(2.9 \mathrm{mg}, 4.1 \mu \mathrm{mol}, 5 \mathrm{~mol}-\%)$ and a solution of $\mathrm{Bu}_{3} \mathrm{SnH}$ (35 mg, $123 \mu \mathrm{mol}, 1.5$ eq.) in THF $(0.5 \mathrm{~mL})$. The mixture was stirred at $25^{\circ} \mathrm{C}$ for $30 \mathrm{~min}$. Then a solution of $(S, S)-\mathbf{1 0 e}(51$ $\mathrm{mg}, 65 \mu \mathrm{mol}, 0.8$ eq.) in THF (1.5 mL) was added. Immediately it was added $\mathrm{Pd}_{2}(\mathrm{dba})_{3}(4.3 \mathrm{mg}$, $4.7 \mu \mathrm{mol}, 6 \mathrm{~mol}-\%)$ and $\mathrm{AsPh}_{3}(7.2 \mathrm{mg}, 20.5 \mu \mathrm{mol}, 25 \mathrm{~mol}-\%)$. The mixture was stirred at $25^{\circ} \mathrm{C}$ for $24 \mathrm{~h}$. The mixture was diluted with $\mathrm{H}_{2} \mathrm{O}(4.5 \mathrm{~mL})$ und it was. The layers were separated, and the organic layer was extracted with $\mathrm{Et}_{2} \mathrm{O}(3 \times 20 \mathrm{~mL})$. The combined organic layers were washed with brine $(10 \mathrm{~mL})$, tried over $\mathrm{Na}_{2} \mathrm{SO}_{4}$ and concentrated under reduced pressure. The residue was purified by flash chromatography $\left(c \mathrm{C}_{6} \mathrm{H}_{12}\right.$ : AcOEt $\left.=8: 1,1.5 \mathrm{~cm}, 20 \mathrm{~cm}, 10 \mathrm{~mL}\right)$. The polyen $(S, S, S, R)-26(33.8 \mathrm{mg}, 60 \%, 39.0 \mu \mathrm{mol})$ was obtained as a pale brown oil. It represented a mixture of two rotamers in a keto and an enol form each [Z-enol-(S,S,R,R)-26:Eenol-(S,S,R,R)-26:Z-keto- $(S, S, R, R)-26: E-k e t o-(S, S, R, R)-\mathbf{2 6}=56: 8: 36: 0] .{ }^{10}$ This ratio was determined by integrating the 5-H signals [at $\delta=7.06$ (Z-enol) and 7.28 (Z-keto) ppm] and compared with die 3-OH signals [14.04 (Z-enol) and 14.42 (E-enol)].

${ }^{14}$ C. Drescher, M. Keller, O. Potterat, M. Hamburger, R. Brückner, Org. Lett. 2020, 22, 2559-2563. 
Christian Drescher and Reinhard Brückner; 3D-Structure Clarifying Total Synthesis of the (Polyenoyl)tetramic Acid Militarinon B. A Highly Acid-Labile N-Protecting Group for Amides

$\mathbf{R}_{\mathbf{f}}\left(c \mathrm{C}_{6} \mathrm{H}_{12}: \mathrm{EtOAc}=8: 1\right)=0.30$

Z-enol:

${ }^{1} \mathbf{H}-\mathbf{N M R}\left(500.06 \mathrm{MHz}, \mathrm{CDCl}_{3} / \mathrm{CHCl}_{3}\right.$, sample contained 4 weight-\% dba with $\mathrm{d}$ at 7.09 , $\mathrm{m}$ at 7.40-7.44, $\mathrm{m}$ at 7.61-7.66 and $\mathrm{d}$ at 7.75 and 7 weight-\% of $\mathrm{O}\left(\mathrm{SnBu}_{3}\right)_{2}$ with $\mathrm{t}$ at $0.92, \mathrm{~m}_{\mathrm{c}}$ at 1.30, $\mathrm{m}_{\mathrm{c}}$ at 1.35 and $\mathrm{m}_{\mathrm{c}}$ at 1.63, DsBrMi13-500100, 14.5.2020): $\delta=-0.24\left(\mathrm{~s}, 3 \mathrm{H}, 3^{\prime}-\mathrm{O}-\mathrm{Si}-\mathrm{CH}_{3}{ }^{\mathrm{A}}\right)$, 0.12 (s, 3H, 3'-O-Si-CH$\left.{ }^{\mathrm{B}}\right), 0.14$ [s, 6H, 1"-O-Si- $\left(\mathrm{CH}_{3}\right)_{2}$ ], 0.81 [s, 9H, 3'-O-Si-C( $\left.\mathrm{CH}_{3}\right)_{3}$ ], 0.81$0.87\left(\mathrm{~m}, 6 \mathrm{H}, 14-\mathrm{H}_{3}, 12-\mathrm{CH}_{3}\right), 0.95-0.99\left(\mathrm{~m}, 3 \mathrm{H}, 10-\mathrm{CH}_{3}\right), 0.95$ [s, 9H, 1"-O-Si-C(CH$\left.)_{3}\right], 1.01$ $\left(\mathrm{t}, J_{2}^{\prime \prime \prime}, 1^{\prime \prime \prime}=7.1 \mathrm{~Hz}, 3 \mathrm{H}, 2{ }^{\prime \prime \prime}-\mathrm{H}_{3}\right), 1.06-1.16\left(\mathrm{~m}, 1 \mathrm{H}, 11-\mathrm{H}^{\mathrm{A}}\right), 1.06-1.16\left(\mathrm{~m}, 1 \mathrm{H}, 13-\mathrm{H}^{\mathrm{A}}\right), 1.19-1.32$ $(\mathrm{m}, 1 \mathrm{H}, 12-\mathrm{H}), 1.19-1.32\left(\mathrm{~m}, 1 \mathrm{H}, 13-\mathrm{H}^{\mathrm{B}}\right), 1.19-1.32\left(\mathrm{~m}, 1 \mathrm{H}, 11-\mathrm{H}^{\mathrm{B}}\right), 1.79\left(\mathrm{~d},{ }^{4} J_{8-\mathrm{CH}_{3}, 9}=1.0 \mathrm{~Hz}\right.$, $\left.3 \mathrm{H}, 8-\mathrm{CH}_{3}\right), 2.62\left(\mathrm{~m}_{\mathrm{c}}, 1 \mathrm{H}, 10-\mathrm{H}\right), 3.73\left(\mathrm{~d},{ }^{2} J=14.6 \mathrm{~Hz}, 1 \mathrm{H}, 2^{2} "-\mathrm{C}-\mathrm{H}^{\mathrm{A}}\right), 3.74\left(\mathrm{~s}, 6 \mathrm{H}, 1{ }^{\prime \prime}-\mathrm{OCH}_{3}\right.$,

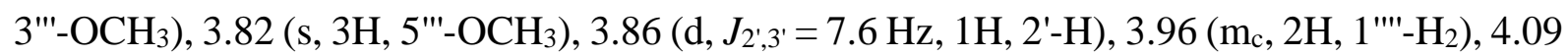
$\left(\mathrm{d},{ }^{2} J=14.6 \mathrm{~Hz}, 1 \mathrm{H}, 2^{\prime \prime}-\mathrm{C}-\mathrm{H}^{\mathrm{B}}\right), 5.36(\mathrm{~s}, 1 \mathrm{H}, 2-\mathrm{H}), 5.36\left(\mathrm{~d}, J_{9,10}=9.6 \mathrm{~Hz}, 1 \mathrm{H}, 9-\mathrm{H}\right), 5.53\left(\mathrm{~d}, J_{3^{\prime}, 2^{\prime}}\right.$ = $\left.7.7 \mathrm{~Hz}, 1 \mathrm{H}, 3^{\prime}-\mathrm{H}\right), 5.86$ (d, J4,5 = 15.1 Hz, 1H, 4-H), 6.06 (s, 2H, 4"'-H, 6'"-H), 6.21 (dd, $\left.J_{6,5}=11.0 \mathrm{~Hz}, J_{6,7}=15.1 \mathrm{~Hz}, 1 \mathrm{H}, 6-\mathrm{H}\right), 6.45\left(\mathrm{~d}, J_{7,6}=15.3 \mathrm{~Hz}, 1 \mathrm{H}, 7-\mathrm{H}\right), 6.64-6.70\left(\mathrm{~m}, 2 \mathrm{H}, 2^{\prime \prime}-\right.$ H, 6"-H), 7.06 (dd, J5,6 $\left.=11.0 \mathrm{~Hz}, J_{5,4}=15.0 \mathrm{~Hz}, 1 \mathrm{H}, 5-\mathrm{H}\right), 7.10-7.15$ (m, 2H, 3"-H, 5"-H), $14.04\left(\mathrm{~d},{ }^{4} \mathrm{~J}_{3-\mathrm{OH}, 4}=1.3 \mathrm{~Hz}, 1 \mathrm{H}, 3-\mathrm{OH}\right) \mathrm{ppm}$.

${ }^{13}$ C-NMR $\left(125.75 \mathrm{MHz}, \mathrm{CDCl}_{3} / \mathrm{CHCl}_{3}\right.$, sample contained dba at $125.5,128.5,129.1,130.6$ and 143.4 and sample contained and $\mathrm{O}\left(\mathrm{SnBu}_{3}\right)_{2}$ at 13.7, 17.6, 26.9 and 28.1, DsBrMi13-500100, 14.5.2020): $\delta=-4.9$ (3'-O-Si-CH$\left.{ }_{3}^{\mathrm{B}}\right),-4.5$ (3'-O-Si-CH$\left.{ }^{\mathrm{A}}\right),-4.4$ [1"-O-Si- $\left.\left(\mathrm{CH}_{3}\right)_{2}\right], 11.4(\mathrm{C}-14)$, $12.6\left(8-\mathrm{CH}_{3}\right), 14.2$ (C-2"'), 18.1 [3'-O-Si- $\left.C\left(\mathrm{CH}_{3}\right)_{3}\right], 18.4$ [4"-O-Si-C $\left.\left(\mathrm{CH}_{3}\right)_{3}\right], 19.2\left(12-\mathrm{CH}_{3}\right)$, 21.5 (10- $\left.\mathrm{CH}_{3}\right), 25.8$ [4"-O-Si-C $\left(\mathrm{CH}_{3}\right)_{3}$ ], 25.9 [3'-O-Si-C $\left(\mathrm{CH}_{3}\right)_{3}$ ], 30.2 (C-13), 30.7 (C-10), 32.4

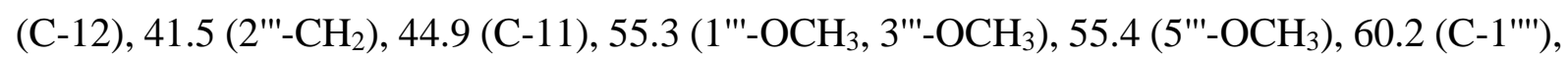
65.4 (C-2'), 72.6 (C-3'), 90.3 (C-4"', C-6"'), 90.8 (C-2), 104.9 (C-2"'), 119.3 (C-2", C-6"), 125.1 (C-6), 125.9 (C-4), 128.5 (C-3", C-5"), 132.4 (C-8), 134.8 (C-4"), 135.8 (C-5), 142.6 (C-7), 143.4 (C-9), 154.8 (C-1"), 159.9 (C-1'", C-3"'), 161.3 (C-5"'), 168.1 (C-3), 170.3 (C-1), 171.9 (C-1') ppm.

edHSQC [,,short-range H,C-COSY“, $\left.500.06 \mathrm{MHz} / 125.75 \mathrm{MHz}, \mathrm{CDCl}_{3}\right] ; \delta\left({ }^{1} \mathrm{H}\right) \leftrightarrow \delta\left({ }^{13} \mathrm{C}\right)$ : $0.24\left(\mathrm{~s}, 3^{\prime}-\mathrm{O}-\mathrm{Si}-\mathrm{CH}_{3}{ }^{\mathrm{A}}\right) \leftrightarrow-4.5\left(3^{\prime}-\mathrm{O}-\mathrm{Si}-\mathrm{CH}_{3}{ }^{\mathrm{A}}\right), 0.12\left(\mathrm{~s}, 3^{\prime}-\mathrm{O}-\mathrm{Si}-\mathrm{CH}_{3}{ }^{\mathrm{B}}\right) \leftrightarrow-4.9\left(3^{\prime}-\mathrm{O}-\mathrm{Si}-\mathrm{CH}_{3}{ }^{\mathrm{B}}\right)$, 0.14 [s, 1"-O-Si- $\left.\left(\mathrm{CH}_{3}\right)_{2}\right] \leftrightarrow-4.4$ [1"-O-Si- $\left.\left(\mathrm{CH}_{3}\right)_{2}\right], 0.81$ [s, 3'-O-Si-C $\left.\left(\mathrm{CH}_{3}\right)_{3}\right] \leftrightarrow 25.9$ [3'-O-Si$\left.\mathrm{C}\left(\mathrm{CH}_{3}\right)_{3}\right], 0.81-0.87\left(\mathrm{~m}, 14-\mathrm{H}_{3}\right) \leftrightarrow 11.4(\mathrm{C}-14), 0.81-0.87\left(\mathrm{~m}, 12-\mathrm{CH}_{3}\right) \leftrightarrow 19.2\left(12-\mathrm{CH}_{3}\right), 0.95-$ $0.99\left(\mathrm{~m}, 10-\mathrm{CH}_{3}\right) \leftrightarrow 21.5\left(10-\mathrm{CH}_{3}\right), 0.95$ [s, 4"-O-Si-C $\left.\left(\mathrm{CH}_{3}\right)_{3}\right] \leftrightarrow 25.8$ [4"-O-Si-C $\left.\left(\mathrm{CH}_{3}\right)_{3}\right], 1.01$ $\left(\mathrm{t}, 2^{\prime \prime \prime}-\mathrm{H}_{3}\right) \leftrightarrow 14.2\left(\mathrm{C}-2^{\prime \prime \prime \prime}\right), 1.06-1.16\left(\mathrm{~m}, 11-\mathrm{H}^{\mathrm{A}}\right) \leftrightarrow 44.9(\mathrm{C}-11), 1.06-1.16\left(\mathrm{~m}, 13-\mathrm{H}^{\mathrm{A}}\right) \leftrightarrow 30.2$ 
Christian Drescher and Reinhard Brückner; 3D-Structure Clarifying Total Synthesis of the (Polyenoyl)tetramic Acid Militarinon B. A Highly Acid-Labile N-Protecting Group for Amides

(C-13), 1.19-1.32 (m, 12-H) $32.4(\mathrm{C}-12), 1.19-1.32\left(\mathrm{~m}, 13-\mathrm{H}^{\mathrm{B}}\right) \leftrightarrow 30.2(\mathrm{C}-13), 1.19-1.32$ $\left(\mathrm{m}, 11-\mathrm{H}^{\mathrm{B}}\right) \leftrightarrow 44.9(\mathrm{C}-11), 1.79\left(\mathrm{~d}, 8-\mathrm{CH}_{3}\right) \leftrightarrow 12.6\left(8-\mathrm{CH}_{3}\right), 2.62\left(\mathrm{~m}_{\mathrm{c}}, 10-\mathrm{H}\right) \leftrightarrow 30.7(\mathrm{C}-10)$,

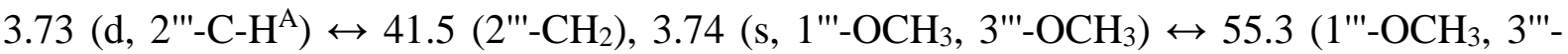
$\left.\mathrm{OCH}_{3}\right), 3.82\left(\mathrm{~s}, 5^{\prime \prime}-\mathrm{OCH}_{3}\right) \leftrightarrow 55.4\left(5^{\prime \prime}-\mathrm{OCH}_{3}\right), 3.86\left(\mathrm{~d}, 2^{\prime}-\mathrm{H}\right) \leftrightarrow 65.4(\mathrm{C}-2 '), 3.96\left(\mathrm{~m}_{\mathrm{c}}, 1^{\prime \prime " '-H_{2}}\right)$ $\leftrightarrow 60.2\left(\mathrm{C}-1\right.$ "'"), 4.09 (d, 2"'-C-H $\left.{ }^{\mathrm{B}}\right) \leftrightarrow 41.5\left(2 " '-\mathrm{CH}_{2}\right), 5.36(\mathrm{~s}, 2-\mathrm{H}) \leftrightarrow 90.8(\mathrm{C}-2), 5.36(\mathrm{~d}, 9-\mathrm{H})$ $\leftrightarrow 143.3$ (C-9), $5.53\left(\mathrm{~d}, 3^{\prime}-\mathrm{H}\right) \leftrightarrow 72.6$ (C-3'), 5.86 (d, 4-H) ↔ 125.6 (C-4), 6.06 (s, 4'"-H, 6'"H) 90.3 (C-4"', C-6"'), $6.21(\mathrm{dd}, 6-\mathrm{H}) \leftrightarrow 125.1$ (C-6), 6.45 (d, 7-H) ↔ 142.6 (C-7), 6.646.70 (m, 2"-H, 6"-H) ↔ 119.3 (C-2", C-6"), 7.06 (dd, 5-H) ↔ 135.8 (C-5), 7.10-7.15 (m, 3"-H, $5 "-H) \leftrightarrow 128.3(\mathrm{C}-3 ", \mathrm{C}-5 ")$.

Specific rotation $(S, S, R, R)-26[\alpha]_{D}^{20}=-183.9^{\circ}\left(\mathrm{c}=0.77\right.$ in $\left.\mathrm{CHCl}_{3}\right)$

HRMS: (EI, $70 \mathrm{eV}): \mathrm{m} / \mathrm{z}=928.4987[\mathrm{M}+\mathrm{Cl}]^{-}$, corresponds to the molecular formula $\mathrm{C}_{50} \mathrm{H}_{79} \mathrm{O}_{9} \mathrm{NClSi}_{2}(928.4987)\left\{[\mathrm{M}+\mathrm{Cl}]^{-}\right\}$with a deviation of $0.0 \mathrm{ppm}$.

IR (KBr): $\tilde{v}=2955,2930,2905,2855,1745,1635,1610,1575,1510,1500,1470,1465,1420$, $1335,1255,1205,1185,1165,1150,1135,1100,1075,915,860,840,815,780 \mathrm{~cm}^{-1}$.

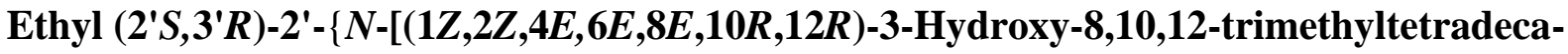
2,4,6,8-tetraenamido]- $N$-(1"',3'",5'"'-trimethoxybenzyl $)\}-3$ '-[(tertbutyldimethylsilyl)oxy $]-3$ '-\{1"'-[(tert-butyldimethylsilyl)oxy]phenyl $\}$ propanoate [Z-enol$(R, S, R, R)-26]$

in equilibration with

Ethyl $(2 ' S, 3 ' R)-2 '-\{N-[(1 E, 2 Z, 4 E, 6 E, 8 E, 10 R, 12 R)-3-H y d r o x y-8,10,12-$ trimethyltetradeca-2,4,6,8-tetraenamido]- $N-(1$ '",3"',5'"'-trimethoxybenzyl) $\}-3$ '-[(tertbutyldimethylsilyl)oxy]-3'-\{1'-[(tert-butyldimethylsilyl)oxy]phenyl $\}$ propanoate [E-enol$(R, S, R, R)-26]$

in equilibration with

Ethyl $(2 ' S, 3 ' R)-2 '-\{N-[(1 Z, 4 E, 6 E, 8 E, 10 R, 12 R)-3-0 x 0-8,10,12-t r i m e t h y l t e t r a d e c a-4,6,8-$ trienamido]- $N$-(1"',3'",5'"'-trimethoxybenzyl) $\}-3$ '-[(tert-butyldimethylsilyl)oxy]-3'-\{1"'[(tert-butyldimethylsilyl)oxy]phenyl $\}$ propanoate $[Z$-keto-(2'S,3'R,10R,12R)-26] 


\section{Ethyl $(2 ' S, 3 ' R)-2 '-\{N-[(1 E, 4 E, 6 E, 8 E, 10 R, 12 R)-3-0 x 0-8,10,12-t r i m e t h y l t e t r a d e c a-4,6,8-$} trienamido]- $N-(1 " ', 3$ '",5'"'-trimethoxybenzyl $)\}-3$ '-[(tert-butyldimethylsilyl)oxy]-3'-\{1"$[($ tert-butyldimethylsilyl)oxy $]$ phenyl $\}$ propanoate $[$ E-keto- $(R, S, R, R)-26]$

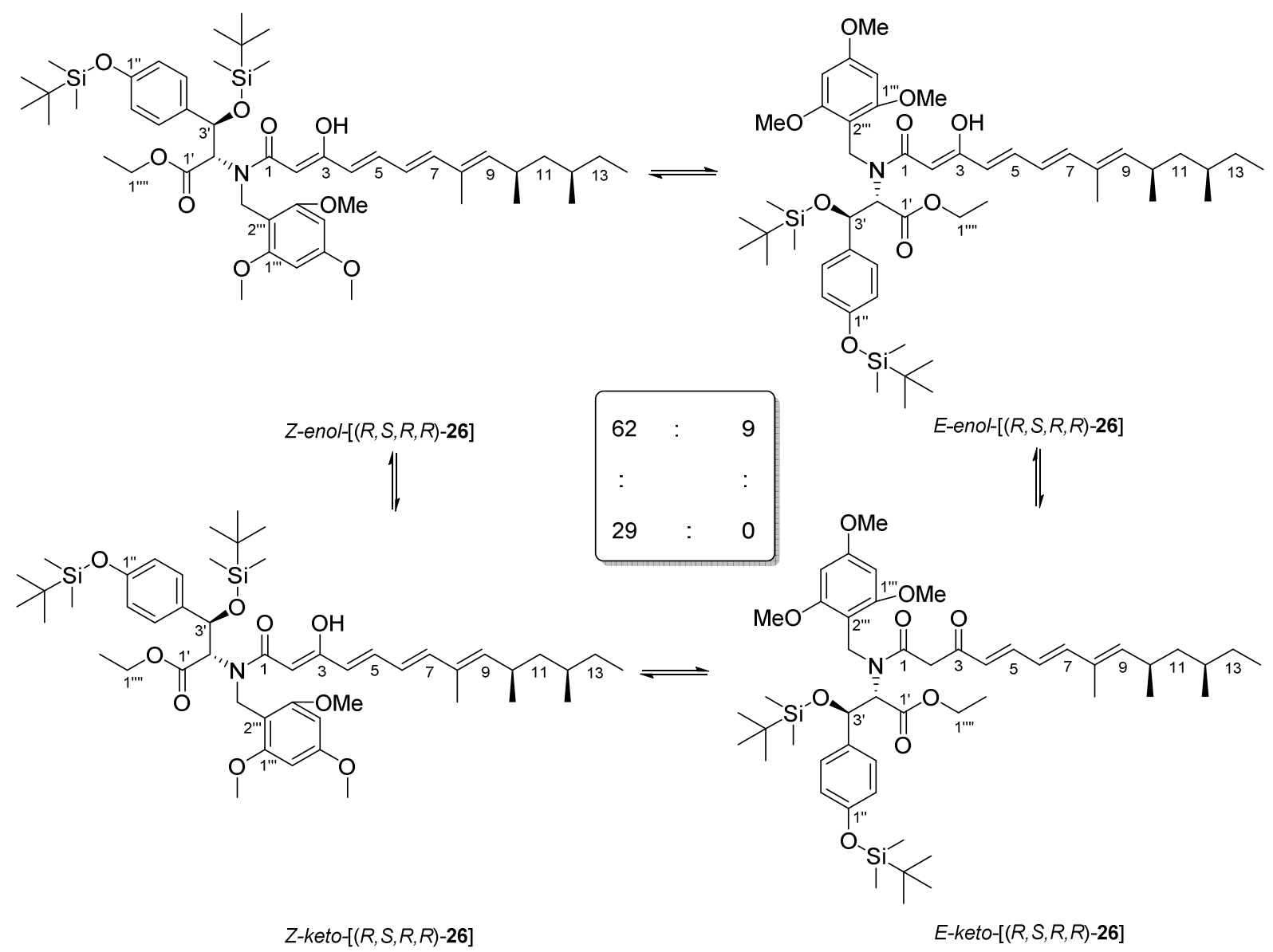

The alkyne $(R, R)-25(30.0 \mathrm{mg}, 164 \mu \mathrm{mol})$ was dissolved in THF $(2.5 \mathrm{~mL})$. It was added $\mathrm{Pd}\left(\mathrm{PPh}_{3}\right)_{2} \mathrm{Cl}_{2}(5.8 \mathrm{mg}, 8.2 \mu \mathrm{mol}, 5 \mathrm{~mol}-\%)$ and a solution of $\mathrm{Bu}_{3} \mathrm{SnH}$ (70 mg, $246 \mu \mathrm{mol}, 1.5$ eq.) in THF $(1.5 \mathrm{~mL})$. The mixture was stirred at $25^{\circ} \mathrm{C}$ for $30 \mathrm{~min}$. Then a solution of $(R, S)-\mathbf{1 0 e}(102$ $\mathrm{mg}, 130 \mu \mathrm{mol}, 0.8$ eq.) in THF $(2.5 \mathrm{~mL})$ was added. Immediately it was added $\mathrm{Pd}_{2}(\mathrm{dba})_{3}$ (8.4 mg, $9.4 \mu \mathrm{mol}, 6 \mathrm{~mol}-\%)$ and $\mathrm{AsPh}_{3}(14.5 \mathrm{mg}, 41.2 \mu \mathrm{mol}, 25 \mathrm{~mol}-\%)$. The mixture was stirred at $25^{\circ} \mathrm{C}$ for $24 \mathrm{~h}$. The mixture was diluted with $\mathrm{H}_{2} \mathrm{O}(10 \mathrm{~mL})$ und it was. The layers were separated, and the organic layer was extracted with $\mathrm{Et}_{2} \mathrm{O}(3 \times 20 \mathrm{~mL})$. The combined organic layers were washed with brine $(10 \mathrm{~mL})$, tried over $\mathrm{Na}_{2} \mathrm{SO}_{4}$ and concentrated under reduced pressure. The residue was purified by flash chromatography $\left(c \mathrm{C}_{6} \mathrm{H}_{12}: \mathrm{AcOEt}=8: 1,2.0 \mathrm{~cm}, 20\right.$ $\mathrm{cm}, 10 \mathrm{~mL})$. The polyen $(R, S, R, R)-\mathbf{2 6}(70.0 \mathrm{mg}, 62 \%, 80.6 \mu \mathrm{mol})$ was obtained as a pale brown oil. It represented a mixture of two rotamers in a keto and an enol form each [Z-enol- $(R, S, R, R)$ 26:E-enol- $(R, S, R, R)-26: Z$-keto- $(R, S, R, R)-26: E-k e t o-(R, S, R, R)-26=62: 9: 29: 0] .{ }^{10} \quad$ This ratio was determined by integrating the $3^{\prime}-\mathrm{O}-\mathrm{Si}^{-} \mathrm{CH}_{3}{ }^{\mathrm{A}}$ signals [at $\delta=-0.37$ (Z-keto), -0.31 (Z-enol) 
Christian Drescher and Reinhard Brückner; 3D-Structure Clarifying Total Synthesis of the (Polyenoyl)tetramic Acid Militarinon B. A Highly Acid-Labile N-Protecting Group for Amides

and -0.30 (E-enol) ppm] and compared with die 3-OH signals [14.10 (Z-enol) and 14.42 (Eenol)].

$\mathbf{R}_{\mathbf{f}}\left(c \mathrm{C}_{6} \mathrm{H}_{12}: \mathrm{EtOAc}=8: 1\right)=0.30$

Z-enol:

${ }^{1} \mathbf{H}-\mathbf{N M R}\left(500.06 \mathrm{MHz}, \mathrm{CDCl}_{3} / \mathrm{CHCl}_{3}\right.$, sample contained $\mathrm{H}_{2} \mathrm{O}$, sample contained 1 weight-\% of $c \mathrm{C}_{6} \mathrm{H}_{12}$ and 6 weight- $\%$ of $\mathrm{O}\left(\mathrm{SnBu}_{3}\right)_{2}$ with $\mathrm{t}$ at $0.92, \mathrm{~m}_{\mathrm{c}}$ at $1.30, \mathrm{~m}_{\mathrm{c}}$ at 1.35 and $\mathrm{m}_{\mathrm{c}}$ at 1.63 , DsBrJn10-500200, 12.6.2020): $\delta=-0.31$ (s, 3H, 3'-O-Si-CH$\left.{ }_{3}{ }^{\mathrm{A}}\right),-0.04$ (s, 3H, 3'-O-Si-CH${ }^{\mathrm{B}}$ ), 0.17 [s, 6H, 1"-O-Si- $\left(\mathrm{CH}_{3}\right)_{2}$ ], 0.77 [s, 9H, 3'-O-Si-C(CH3) 3 ], 0.80-0.86 (m, 6H, 14- $\left.\mathrm{H}_{3}, 12-\mathrm{CH}_{3}\right)$,

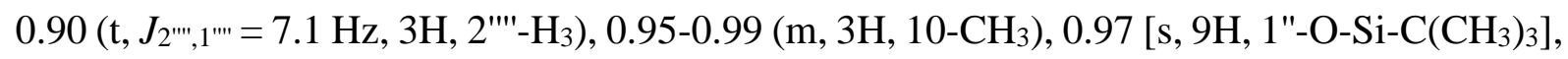
1.06-1.16 (m, $\left.1 \mathrm{H}, 11-\mathrm{H}^{\mathrm{A}}\right), 1.06-1.16\left(\mathrm{~m}, 1 \mathrm{H}, 13-\mathrm{H}^{\mathrm{A}}\right), 1.19-1.32(\mathrm{~m}, 1 \mathrm{H}, 12-\mathrm{H}), 1.19-1.32(\mathrm{~m}$, $\left.1 \mathrm{H}, 13-\mathrm{H}^{\mathrm{B}}\right), 1.19-1.32\left(\mathrm{~m}, 1 \mathrm{H}, 11-\mathrm{H}^{\mathrm{B}}\right), 1.81\left(\mathrm{~d},{ }^{4} \mathrm{~J}_{8-\mathrm{CH}_{3}, 9}=1.0 \mathrm{~Hz}, 3 \mathrm{H}, 8-\mathrm{CH}_{3}\right), 2.62\left(\mathrm{~m}_{\mathrm{c}}, 1 \mathrm{H}\right.$,

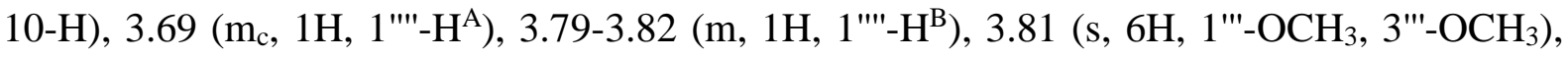
$3.87\left(\mathrm{~s}, 3 \mathrm{H}, 5^{\prime \prime}-\mathrm{OCH}_{3}\right), 3.96\left(\mathrm{~d}, J_{2^{\prime}, 3^{\prime}}=8.9 \mathrm{~Hz}, 1 \mathrm{H}, 2^{\prime}-\mathrm{H}\right), 4.62-4.81\left(\mathrm{~m}, 2 \mathrm{H}, 2{ }^{\prime \prime}-\mathrm{CH}_{2}\right), 5.36(\mathrm{~d}$, $\left.J_{9,10}=9.6 \mathrm{~Hz}, 1 \mathrm{H}, 9-\mathrm{H}\right), 5.44\left(\mathrm{~d}, J_{3^{\prime}, 2^{\prime}}=8.9 \mathrm{~Hz}, 1 \mathrm{H}, 3^{\prime}-\mathrm{H}\right), 5.76(\mathrm{~s}, 1 \mathrm{H}, 2-\mathrm{H}), 6.02(\mathrm{~d}$, $\left.J_{4,5}=15.1 \mathrm{~Hz}, 1 \mathrm{H}, 4-\mathrm{H}\right), 6.10$ (s, 2H, 4"'-H, 6"'-H), 6.25 (dd, $J_{6,5}=11.0 \mathrm{~Hz}, J_{6,7}=15.3 \mathrm{~Hz}, 1 \mathrm{H}$, 6-H), 6.47 (d, $\left.J_{7,6}=15.3 \mathrm{~Hz}, 1 \mathrm{H}, 7-\mathrm{H}\right), 6.74-6.76(\mathrm{~m}, 2 \mathrm{H}, 2 "-\mathrm{H}, 6 "-\mathrm{H}), 7.10$ (dd, $J_{5,6}=10.9 \mathrm{~Hz}$, $\left.J_{5,4}=15.0 \mathrm{~Hz}, 1 \mathrm{H}, 5-\mathrm{H}\right), 7.38-7.43(\mathrm{~m}, 2 \mathrm{H}, 3 "-\mathrm{H}, 5 "-\mathrm{H}), 14.09\left(\mathrm{~d},{ }^{4} J_{3-\mathrm{OH}, 4}=1.3 \mathrm{~Hz}, 1 \mathrm{H}, 3-\mathrm{OH}\right)$ ppm.

${ }^{13} \mathrm{C}-\mathrm{NMR}\left(125.75 \mathrm{MHz}, \mathrm{CDCl}_{3} / \mathrm{CHCl}_{3}\right.$, sample contained and $\mathrm{O}\left(\mathrm{SnBu}_{3}\right)_{2}$ at $13.7,17.6,26.9$ and 28.1, DsBrJn10-500204, 12.6.2020): $\delta=-5.2\left(3^{\prime}-\mathrm{O}-\mathrm{Si}_{-}-\mathrm{CH}_{3}{ }^{\mathrm{A}}\right),-4.9$ (3'-O-Si-CH$\left.{ }^{\mathrm{B}}\right),-4.3$ [1"-O-Si- $\left(\mathrm{CH}_{3}\right)_{2}$ ], 11.4 (C-14), $12.6\left(8-\mathrm{CH}_{3}\right), 13.7$ (C-2'"'), 18.0 [3'-O-Si-C $\left(\mathrm{CH}_{3}\right)_{3}$ ], 18.3 [1"-O$\mathrm{Si}-\mathrm{C}\left(\mathrm{CH}_{3}\right)_{3}$ ], $19.2\left(12-\mathrm{CH}_{3}\right), 21.5\left(10-\mathrm{CH}_{3}\right), 25.8$ [3'-O-Si-C $\left.\left(\mathrm{CH}_{3}\right)_{3}, 1^{\prime \prime}-\mathrm{O}-\mathrm{Si}-\mathrm{C}\left(\mathrm{CH}_{3}\right)_{3}\right], 30.2(\mathrm{C}-$ 13), 30.7 (C-10), 32.4 (C-12), 42.8 (2'"-- $\left.\mathrm{CH}_{2}\right), 44.9$ (C-11), 55.4 (1"'--OCH 3,3 '"--OCH $), 55.5$ (5"'-OCH 3 ), 60.1 (C-1"'), 64.6 (C-2'), 72.2 (C-3'), 90.2 (C-4'", C-6"'), 91.1 (C-2), 105.1 (C-2"'), 119.3 (C-2", C-6"), 125.2 (C-6), 126.0 (C-4), 129.6 (C-3", C-5"), 132.4 (C-8), 135.7 (C-5), 137.6 (C-4"), 142.5 (C-7), 143.2 (C-9), 154.8 (C-1"), 160.1 (C-1'", C-3"'), 161.4 (C-5'"), 168.1 (C-3), 169.7 (C-1), $171.6\left(\mathrm{C}-1^{\prime}\right) \mathrm{ppm}$.

edHSQC [,short-range H,C-COSY“, 500.10 MHz/125.75 MHz, $\mathrm{CDCl}_{3}$ ]; $\delta\left({ }^{1} \mathrm{H}\right) \leftrightarrow \delta\left({ }^{13} \mathrm{C}\right)$ : $0.31\left(\mathrm{~s}, 3^{\prime}-\mathrm{O}-\mathrm{Si}-\mathrm{CH}_{3}{ }^{\mathrm{A}}\right) \leftrightarrow-5.1\left(3^{\prime}-\mathrm{O}-\mathrm{Si}^{-} \mathrm{CH}_{3}{ }^{\mathrm{A}}\right),-0.04\left(\mathrm{~s}, 3^{\prime}-\mathrm{O}-\mathrm{Si}^{-} \mathrm{CH}_{3}{ }^{\mathrm{B}}\right) \leftrightarrow-4.9\left(3^{\prime}-\mathrm{O}-\mathrm{Si}-\mathrm{CH}_{3}{ }^{\mathrm{B}}\right)$, 0.17 [s, 1"-O-Si- $\left.\left(\mathrm{CH}_{3}\right)_{2}\right] \leftrightarrow-4.3$ [1"-O-Si- $\left.\left(\mathrm{CH}_{3}\right)_{2}\right], 0.77$ [s, 3'-O-Si-C $\left.\left(\mathrm{CH}_{3}\right)_{3}\right] \leftrightarrow 25.8$ [3'-O-Si$\left.\mathrm{C}\left(\mathrm{CH}_{3}\right)_{3}\right], 0.80-0.86\left(\mathrm{~m}, 12-\mathrm{CH}_{3}\right) \leftrightarrow 19.2\left(12-\mathrm{CH}_{3}\right), 0.80-0.86\left(\mathrm{~m}, 14-\mathrm{H}_{3}\right) \leftrightarrow 11.4(\mathrm{C}-14), 0.90$ $(\mathrm{t}, 2$ "'"-H 3$) \leftrightarrow 13.7\left(\mathrm{C}-2\right.$ '"'), 0.95-0.99 (m, 10- $\left.\mathrm{CH}_{3}\right) \leftrightarrow 21.5\left(10-\mathrm{CH}_{3}\right), 0.97$ [s, 1"-O-Si-C $\left.\left(\mathrm{CH}_{3}\right)_{3}\right]$ 
Christian Drescher and Reinhard Brückner; 3D-Structure Clarifying Total Synthesis of the (Polyenoyl)tetramic Acid Militarinon B. A Highly Acid-Labile N-Protecting Group for Amides

$\leftrightarrow 25.8\left[1 "-\mathrm{O}-\mathrm{Si}-\mathrm{C}\left(\mathrm{CH}_{3}\right)_{3}\right], 1.06-1.16\left(\mathrm{~m}, 11-\mathrm{H}^{\mathrm{A}}\right) \leftrightarrow 44.9(\mathrm{C}-11), 1.06-1.16\left(\mathrm{~m}, 13-\mathrm{H}^{\mathrm{A}}\right) \leftrightarrow 30.2$ (C-13), 1.19-1.32 (m, 12-H) $32.4(\mathrm{C}-12), 1.19-1.32\left(\mathrm{~m}, 13-\mathrm{H}^{\mathrm{B}}\right) \leftrightarrow 30.2(\mathrm{C}-13), 1.19-1.32$ $\left(\mathrm{m}, 11-\mathrm{H}^{\mathrm{B}}\right) \leftrightarrow 44.9(\mathrm{C}-11), 1.81\left(\mathrm{~d}, 8-\mathrm{CH}_{3}\right) \leftrightarrow 12.6\left(8-\mathrm{CH}_{3}\right), 2.62\left(\mathrm{~m}_{\mathrm{c}}, 10-\mathrm{H}\right) \leftrightarrow 30.7(\mathrm{C}-10)$,

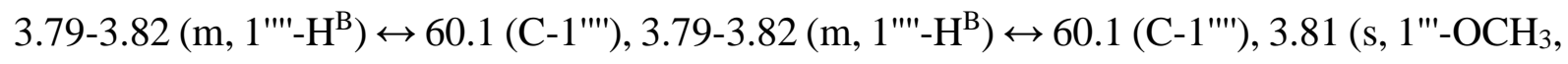

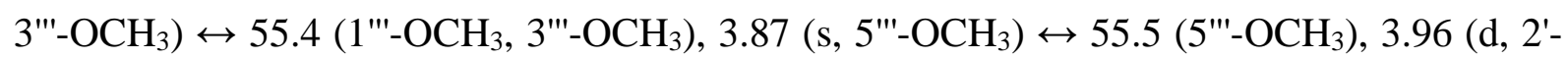
$\mathrm{H}) \leftrightarrow 64.6(\mathrm{C}-2$ '), 4.62-4.81 (m, 2"'-CH 2$) \leftrightarrow 42.8\left(2^{2 '}-\mathrm{CH}_{2}\right), 5.36(\mathrm{~d}, 9-\mathrm{H}) \leftrightarrow 143.2(\mathrm{C}-9), 5.44$ $\left(\mathrm{d}, 3^{\prime}-\mathrm{H}\right) \leftrightarrow 72.2\left(\mathrm{C}-3^{\prime}\right), 5.76(\mathrm{~s}, 2-\mathrm{H}) \leftrightarrow 91.1(\mathrm{C}-2), 6.02(\mathrm{~d}, 4-\mathrm{H}) \leftrightarrow 126.0(\mathrm{C}-4), 6.10$ (s, 4'"H, 6"'-H) ↔ 90.2 (C-4'", C-6"'), 6.25 (dd, 6-H) ↔ 125.2 (C-6), 6.47 (d, 7-H) ↔ 142.5 (C-7), 6.74-6.76 (m, 2"-H, 6"-H) ↔119.3 (C-2", C-6"), 7.10 (dd, 5-H) 135.7 (C-5), 7.38-7.43 (m, 3"-H, 5"-H) 129.6 (C-3", C-5").

Specific rotation $(R, S, R, R)-26[\alpha]_{D}^{20}=-106.3^{\circ}\left(\mathrm{c}=0.71\right.$ in $\left.\mathrm{CHCl}_{3}\right)$

HRMS: (EI, $70 \mathrm{eV}): \mathrm{m} / \mathrm{z}=894.5361[\mathrm{M}+\mathrm{H}]^{+}$, corresponds to the molecular formula $\mathrm{C}_{50} \mathrm{H}_{80} \mathrm{O}_{9} \mathrm{NSi}_{2}(894.5366)\left\{[\mathrm{M}+\mathrm{H}]^{+}\right\}$with a deviation of $-0.5 \mathrm{ppm}$.

IR (KBr): $\tilde{v}=2955,2930,2905,2855,1740,1635,1610,1595,1575,1510,1500,1480,1470$, $1465,1255,1240,1205,1185,1170,1150,1135,1080,1055,915,855,835,780 \mathrm{~cm}^{-1}$.

$(5 S)-5-\{(S)-[($ tert-Butyldimethylsilyl)oxy $]\{1 "-[($ tertbutyldimethylsilyl)oxy)phenyl]methyl $\}-3-\left[\left(1^{\prime} Z, 2^{\prime} E, 4^{\prime} E, 6^{\prime} E, 8^{\prime} R, 10^{\prime} R\right)-1^{\prime}-h y d r o x y-\right.$ 6',8',10'-trimethyldodeca-2',4',6'-trien-1'-ylidene]-1-(1'",3'"',5'"'trimethoxybenzyl)pyrrolidine-2,4-dione [Z-enol-(S,S,R,R)-27] in a $77: 23$ mixture with (5S)-5-\{(S)-[(tert-Butyldimethylsilyl)oxy] $]\{1 "-[($ tertbutyldimethylsilyl)oxy)phenyl]methyl\}-3-[(1'E,2' $\left.E, 4^{\prime} E, 6^{\prime} E, 8^{\prime} R, 10 ' R\right)-1^{\prime}-h y d r o x y-$ 6',8',10'-trimethyldodeca-2',4',6'-trien-1'-ylidene]-1-(1'",,3'",5'"'trimethoxybenzyl)pyrrolidine-2,4-dione [E-enol-(S,S,R,R)-27] 


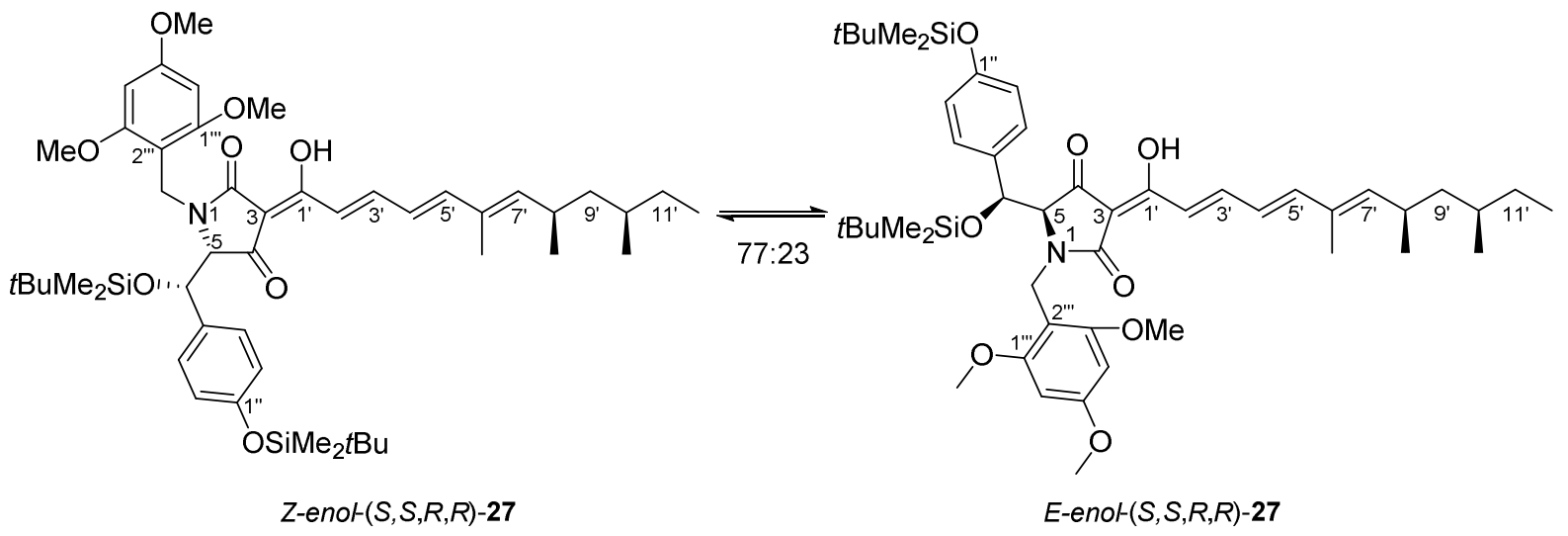

A solution of the polyen $(S, S, R, R)-\mathbf{2 6}(35.0 \mathrm{mg}, 39.2 \mu \mathrm{mol})$ in $\mathrm{MeOH}(4.0 \mathrm{~mL})$ was deluded with $\mathrm{NaOMe}\left(17.0 \mathrm{mg}, 314 \mu \mathrm{mol}, 8.0\right.$ eq.) at $25^{\circ} \mathrm{C}$. The mixture stirred at this temperature for $1.5 \mathrm{~h}$. It was added aq. citric acid (5 weight- $\%, 3 \mathrm{~mL})$ and $\mathrm{Et}_{2} \mathrm{O}(10 \mathrm{~mL})$. The organic layer was separated, and the aq. layer was extracted with $\mathrm{Et}_{2} \mathrm{O}(3 \times 10 \mathrm{~mL})$. The organic layers were tried over $\mathrm{Na}_{2} \mathrm{SO}_{4}$ and the solvent was removed under reduced pressure. The residue was purified reverse phase flash chromatography $\left(\mathrm{MeCN}: \mathrm{HCO}_{2} \mathrm{H}=100: 0.1,1.5 \mathrm{~cm}, 10 \mathrm{~cm}, 2 \mathrm{~mL}\right)$. The tetramic acid $(S)-27(20.3 \mathrm{mg}, 61 \%, 23.9 \mu \mathrm{mol})$ was obtained as a yellow oil. It was assigned as a 77:23 mixture of the $Z$ - and the $E$-enol of the title compounds $(S, S, R, R)-27$. This ratio was determined by integrating duplets of the respective 2'-H [6.52 (E-enol) and 7.11 (Z-enol)].

$\mathbf{R}_{\mathbf{f}}\left(\mathrm{MeCN}: \mathrm{HCO}_{2} \mathrm{H}=100: 0.1\right)=0.15$

${ }^{1} \mathbf{H}$-NMR $\left(500.10 \mathrm{MHz}, \mathrm{CDCl}_{3} / \mathrm{CHCl}_{3}\right.$, sample contained grease with s at 1.26 , DsBrJn05500300, 8.6.2020): $\delta=-0.13\left(\mathrm{~s}, 3 \mathrm{H}, 5-\mathrm{CH}-\mathrm{O}-\mathrm{Si}_{-} \mathrm{CH}_{3}{ }^{\mathrm{A}}\right), 0.08$ (s, 3H, 5-CH-O-Si-CH$\left.{ }_{3}{ }^{\mathrm{B}}\right), 0.16$ $\left(\mathrm{s}, 3 \mathrm{H}, 1\right.$ "-O-Si- $\left.\mathrm{CH}_{3}{ }^{\mathrm{A}}\right), 0.17$ (s, 3H, 1"-O-Si- $\left.\mathrm{CH}_{3}{ }^{\mathrm{B}}\right), 0.81$ [s, 9H, 5-CH-Si-C $\left(\mathrm{CH}_{3}\right)_{3}$ ], 0.81-0.87 $\left(\mathrm{m}, 6 \mathrm{H}, 122^{\prime}-\mathrm{H}_{3}, 10 '-\mathrm{CH}_{3}\right), 0.95-0.97$ (m, 3H, 8'- $\left.\mathrm{CH}_{3}\right), 0.96$ [s, 9H, 1"-O-Si-C( $\left.\left.\mathrm{CH}_{3}\right)_{3}\right], 1.07-1.17$ $\left(\mathrm{m}, 1 \mathrm{H}, 9^{\prime}-\mathrm{H}^{\mathrm{A}}\right), 1.07-1.17\left(\mathrm{~m}, 1 \mathrm{H}, 11^{\prime}-\mathrm{H}^{\mathrm{A}}\right), 1.20-1.34\left(\mathrm{~m}, 1 \mathrm{H}, 10^{\prime}-\mathrm{H}\right), 1.20-1.34\left(\mathrm{~m}, 1 \mathrm{H}, 11^{\prime}-\right.$ $\left.\mathrm{H}^{\mathrm{B}}\right), 1.20-1.34\left(\mathrm{~m}, 1 \mathrm{H}, 9^{\prime}-\mathrm{H}^{\mathrm{B}}\right), 1.80\left(\mathrm{~d},{ }^{4} J_{8-\mathrm{CH}_{3}, 9}=1.1 \mathrm{~Hz}, 3 \mathrm{H}, 66^{\prime}-\mathrm{CH}_{3}\right), 2.64\left(\mathrm{~m}_{\mathrm{c}}, 1 \mathrm{H}, 8^{\prime}-\mathrm{H}\right), 3.59-$ 3.62 (m, 1H, 5-H), 3.80 (s, 6H, 1'"--OCH 3,3 "'--OCH 3 ), 3.83 (s, 3H, 5"'-OCH 3 ), 4.49 (d, ${ }^{2} J=14.3$

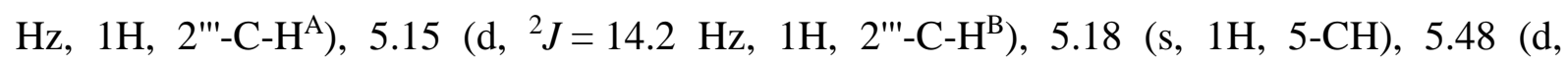
$\left.J_{7^{\prime}, 8^{\prime}}=9.6 \mathrm{~Hz}, 1 \mathrm{H}, 7^{\prime}-\mathrm{H}\right), 6.15$ (s, 2H, 4"'-H, 6'"-H), 6.39 (dd, $J_{4^{\prime}, 3^{\prime}}=11.1 \mathrm{~Hz}, J_{4^{\prime}, 5^{\prime}}=15.1 \mathrm{~Hz}, 1 \mathrm{H}$, 4'-H), 6.60 (d, J5',4' = 15.1 Hz, 1H, 5'-H), 6.69-6.74 (m, 2H, 2"-H, 6"-H), 6.93-6.97 (m, 2H, 3"$\left.\mathrm{H}, 5^{\prime \prime}-\mathrm{H}\right), 7.11\left(\mathrm{~d}, J_{2^{\prime}, 3^{\prime}}=15.1 \mathrm{~Hz}, 1 \mathrm{H}, 2^{\prime}-\mathrm{H}\right), 7.42\left(\mathrm{dd}, J_{3^{\prime}, 4^{\prime}}=11.0 \mathrm{~Hz}, J_{3^{\prime}, 2^{\prime}}=14.7 \mathrm{~Hz}, 1 \mathrm{H}, 3^{\prime}-\mathrm{H}\right)$ ppm.

${ }^{13}$ C-NMR (125.75 MHz, sample contained grease at 14.20, DsBrMi13-500100, 14.5.2020): $\delta$ $=-5.1\left(5-\mathrm{CH}-\mathrm{O}-\mathrm{Si}_{-} \mathrm{CH}_{3}{ }^{\mathrm{B}}\right),-4.6\left(5-\mathrm{CH}-\mathrm{O}-\mathrm{Si}_{-} \mathrm{CH}_{3}{ }^{\mathrm{A}}\right),-4.3\left(1 "-\mathrm{O}-\mathrm{Si}-\mathrm{CH}_{3}{ }^{\mathrm{A}}\right),-4.3(1 "-\mathrm{O}-\mathrm{Si}-$ 
Christian Drescher and Reinhard Brückner; 3D-Structure Clarifying Total Synthesis of the (Polyenoyl)tetramic Acid Militarinon B. A Highly Acid-Labile N-Protecting Group for Amides

$\left.\mathrm{CH}_{3}{ }^{\mathrm{B}}\right), 11.4\left(\mathrm{C}-12^{\prime}\right), 12.5\left(6\right.$ '- $\left.\mathrm{CH}_{3}\right), 18.1$ [5-CH-Si- $\mathrm{C}\left(\mathrm{CH}_{3}\right)_{3}$ ], 18.2 [1"-O-Si- $\mathrm{C}\left(\mathrm{CH}_{3}\right)_{3}$ ], 19.2 (10'$\left.\mathrm{CH}_{3}\right), 21.4\left(\mathrm{C}-8\right.$ '), 25.7 [5-CH-Si-C $\left(\mathrm{CH}_{3}\right)_{3}$ ], 25.7 [1"-O-Si-C $\left(\mathrm{CH}_{3}\right)_{3}$ ], $30.2(\mathrm{C}-11$ '), 30.9 (C-8'),

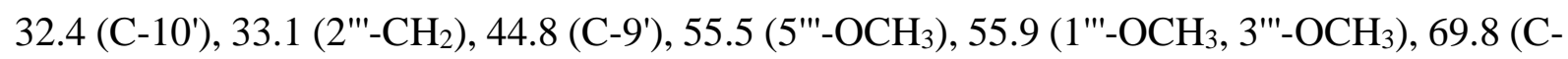
5), 72.7 (5-CH), 90.6 (C-4"', C-6"'), 102.0 (C-3), 103.7 (C-2'"), 119.3 (C-2", C-6"), 120.8 (C2'), 125.5 (C-4'), 127.5 (C-3", C-5"), 132.8 (C-6'), 133.2 (C-4"), 143.9 (C-3'), 146.3 (C-7'), 147.3 (C-5'), 154.8 (C-1"), 159.9 (C-1"', C-3'"), 161.5 (C-5"'), 171.5 (C-1'), 173.8 (C-2), 192.5 (C-3) ppm.

edHSQC [,,short-range H,C-COSY“, $\left.500.06 \mathrm{MHz} / 125.75 \mathrm{MHz}, \mathrm{CDCl}_{3}\right] ; \delta\left({ }^{1} \mathrm{H}\right) \leftrightarrow \delta\left({ }^{13} \mathrm{C}\right)$ : $0.13\left(\mathrm{~s}, 5-\mathrm{CH}-\mathrm{O}-\mathrm{Si}_{-} \mathrm{CH}_{3}{ }^{\mathrm{A}}\right) \leftrightarrow-4.6\left(5-\mathrm{CH}-\mathrm{O}-\mathrm{Si}_{-}-\mathrm{CH}_{3}{ }^{\mathrm{A}}\right), 0.08\left(\mathrm{~s}, 5-\mathrm{CH}-\mathrm{O}-\mathrm{Si}_{-} \mathrm{CH}_{3}{ }^{\mathrm{B}}\right) \leftrightarrow-5.1(5-$ $\left.\mathrm{CH}-\mathrm{O}-\mathrm{Si}-\mathrm{CH}_{3}{ }^{\mathrm{B}}\right), 0.16\left(\mathrm{~s}, 1^{1 "}-\mathrm{O}-\mathrm{Si}_{-}-\mathrm{CH}_{3}{ }^{\mathrm{A}}\right) \leftrightarrow-4.3\left(1 "-\mathrm{O}-\mathrm{Si}_{-} \mathrm{CH}_{3}{ }^{\mathrm{A}}\right), 0.17\left(\mathrm{~s}, 1\right.$ "-O-Si-CH$\left.{ }^{\mathrm{B}}\right) \leftrightarrow-$ $4.3\left(1 "-\mathrm{O}-\mathrm{Si}_{-} \mathrm{CH}_{3}{ }^{\mathrm{B}}\right), 0.81-0.87\left(\mathrm{~m}, 12^{\prime}-\mathrm{H}_{3}\right) \leftrightarrow 11.4\left(\mathrm{C}-12{ }^{\prime}\right), 0.81-0.87\left(\mathrm{~m}, 10^{\prime}-\mathrm{CH}_{3}\right) \leftrightarrow 19.2\left(10^{\prime}-\right.$ $\left.\mathrm{CH}_{3}\right), 0.81$ [s, 5-CH-Si-C $\left.\left(\mathrm{CH}_{3}\right)_{3}\right] \leftrightarrow 25.7$ [5-CH-Si-C $\left.\left(\mathrm{CH}_{3}\right)_{3}\right], 0.95-0.97\left(\mathrm{~m}, 8^{\prime}-\mathrm{CH}_{3}\right) \leftrightarrow 21.4$ $\left(\mathrm{C}-8^{\prime}\right), 0.96$ [s, 1"-O-Si-C $\left.\left(\mathrm{CH}_{3}\right)_{3}\right] \leftrightarrow 25.7$ [1"-O-Si-C $\left.\left(\mathrm{CH}_{3}\right)_{3}\right], 1.07-1.17\left(\mathrm{~m}, 9^{\prime}-\mathrm{H}^{\mathrm{A}}\right) \leftrightarrow 44.8(\mathrm{C}-$ 9'), 1.07-1.17 (m, 11'- $\left.\mathrm{H}^{\mathrm{A}}\right) \leftrightarrow 30.2\left(\mathrm{C}-11^{\prime}\right), 1.20-1.34\left(\mathrm{~m}, 10^{\prime}-\mathrm{H}\right) \leftrightarrow 32.4(\mathrm{C}-10 '), 1.20-1.34(\mathrm{~m}$, $\left.11^{\prime}-\mathrm{H}^{\mathrm{B}}\right) \leftrightarrow 30.2\left(\mathrm{C}-11^{\prime}\right), 1.20-1.34\left(\mathrm{~m}, 9^{\prime}-\mathrm{H}^{\mathrm{B}}\right) \leftrightarrow 44.8\left(\mathrm{C}-9^{\prime}\right), 1.80\left(\mathrm{~d}, 6^{\prime}-\mathrm{CH}_{3}\right) \leftrightarrow 12.5\left(6^{\prime}-\mathrm{CH}_{3}\right)$,

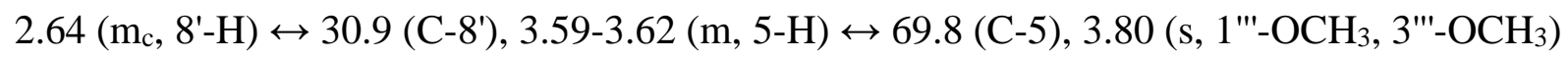
$\leftrightarrow 55.9\left(1\right.$ '"- $-\mathrm{OCH}_{3}, 3$ '"--OCH$), 3.83\left(\mathrm{~s}, 5\right.$ "'- $\left.-\mathrm{OCH}_{3}\right) \leftrightarrow 55.5\left(5^{\prime \prime}-\mathrm{OCH}_{3}\right), 4.49\left(\mathrm{~d}, 2\right.$ '"-C-H $\left.{ }^{\mathrm{A}}\right) \leftrightarrow$ $33.1\left(2 " '-\mathrm{CH}_{2}\right), 5.15\left(\mathrm{~d}, 2\right.$ "'-C-H $\left.{ }^{\mathrm{B}}\right) \leftrightarrow 33.1\left(2 " '-\mathrm{CH}_{2}\right), 5.18(\mathrm{~s}, 5-\mathrm{CH}) \leftrightarrow 72.7(5-\mathrm{CH}), 5.48(\mathrm{~d}$, 7'-H) 146.3 (C-7'), 6.15 (s, 4'"-H, 6"'-H) ↔ 90.6 (C-4'", C-6'"), 6.39 (dd, 4'-H) 125.5 (C4'), 6.60 (d, 5'-H) 147.3 (C-5'), 6.69-6.74 (m, 2"-H, 6"-H) 119.3 (C-2", C-6"), 6.93-6.97 (m, 3"-H, 5"-H) 127.5 (C-3", C-5"), 7.11 (d, 2'-H) 120.8 (C-2'), 7.42 (dd, 3'-H) 143.9 (C-3').

HRMS: (EI, $70 \mathrm{eV}): \mathrm{m} / \mathrm{z}=848.4951[\mathrm{M}+\mathrm{H}]^{+}$, corresponds to the molecular formula $\mathrm{C}_{48} \mathrm{H}_{74} \mathrm{O}_{8} \mathrm{NSi}_{2}(848.4947)\left\{[\mathrm{M}+\mathrm{H}]^{+}\right\}$with a deviation of $0.4 \mathrm{ppm}$.

Specific rotation $(S, S, R, R)-27[\alpha]_{D}^{20}=-42.4^{\circ}\left(\mathrm{c}=0.29\right.$ in $\left.\mathrm{CHCl}_{3}\right)$

IR (KBr): $\tilde{v}=2955,2930,2905,2855,1745,1705,1610,1595,1565,1510,1455,1420,1390$, $1360,1325,1255,1230,1205,1185,1165,1150,1135,1105,1090,1005,915,840 \mathrm{~cm}^{-1}$. 
Christian Drescher and Reinhard Brückner; 3D-Structure Clarifying Total Synthesis of the (Polyenoyl)tetramic Acid Militarinon B. A Highly Acid-Labile N-Protecting Group for Amides

$(5 S)-5-\{(R)-[($ tert-Butyldimethylsilyl)oxy $]\{1 "$ '-[(tert-

butyldimethylsilyl)oxy)phenyl]methyl $\}-3-\left[\left(1^{\prime} Z, 2^{\prime} E, 4^{\prime} E, 6^{\prime} E, 8^{\prime} R, 10^{\prime} R\right)-1^{\prime}-h y d r o x y-\right.$

6',8',10'-trimethyldodeca-2',4',6'-trien-1'-ylidene]-1-(1'"',3'",5'"-

trimethoxybenzyl)pyrrolidine-2,4-dione [Z-enol-(R,S,R,R)-27]

in a 83:17 mixture with

$(5 S)-5-\left\{(R)-[(\right.$ tert-Butyldimethylsilyl $)$ oxy $]\left\{1^{\prime \prime}-[(\right.$ tert-

butyldimethylsilyl)oxy)phenyl]methyl\}-3-[(1'E,2' $\left.E, 4^{\prime} E, 6^{\prime} E, 8^{\prime} R, 10^{\prime} R\right)-1^{\prime}$-hydroxy-

6',8',10'-trimethyldodeca-2',4',6'-trien-1'-ylidene]-1-(1'"',3'",5'"'-

trimethoxybenzyl)pyrrolidine-2,4-dione [E-enol-(R,S,R,R)-27]

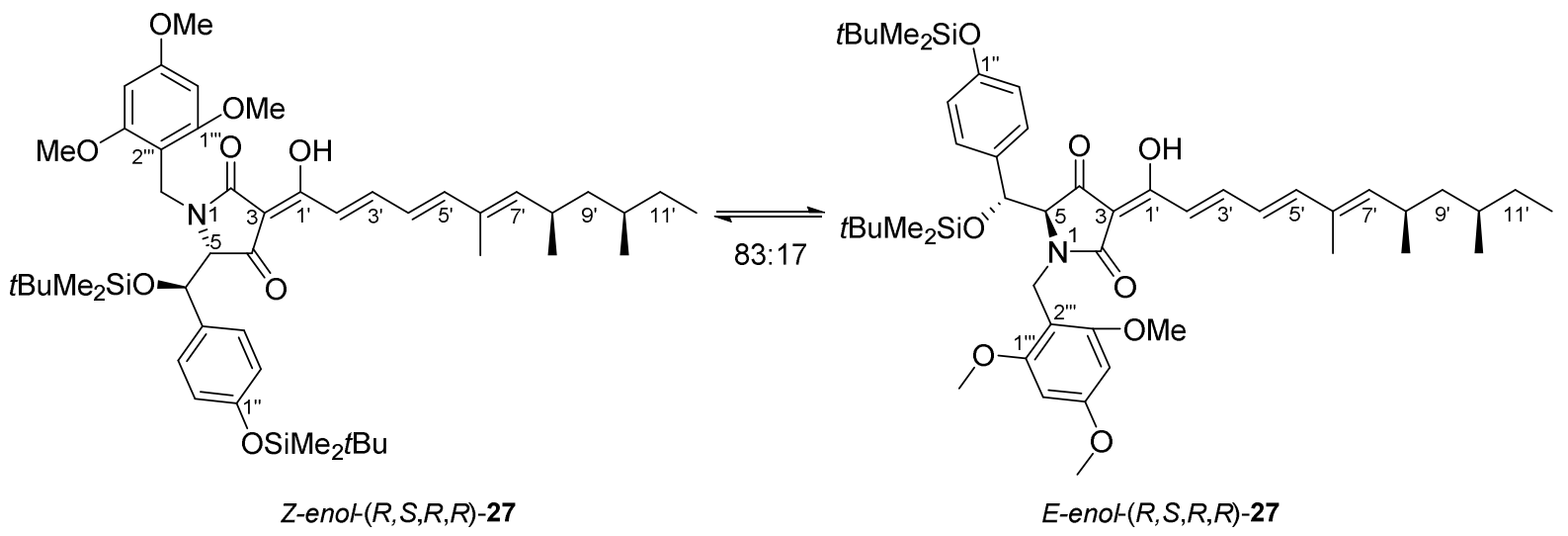

A solution of the polyen $(R, S, R, R)-27(20.0 \mathrm{mg}, 26.1 \mu \mathrm{mol})$ in $\mathrm{MeOH}(2.5 \mathrm{~mL})$ was deluded with $\mathrm{NaOMe}(11.3 \mathrm{mg}, 209 \mu \mathrm{mol}, 8.0 \mathrm{eq}$.$) at 25^{\circ} \mathrm{C}$. The mixture stirred at this temperature for $1.5 \mathrm{~h}$. It was added aq. citric acid (5 weight- $\%, 3 \mathrm{~mL})$ and $\mathrm{Et}_{2} \mathrm{O}(10 \mathrm{~mL})$. The organic layer was separated, and the aq. layer was extracted with $\mathrm{Et}_{2} \mathrm{O}(3 \times 10 \mathrm{~mL})$. The organic layers were tried over $\mathrm{Na}_{2} \mathrm{SO}_{4}$ and the solvent was removed under reduced pressure. The residue was purified reverse phase flash chromatography $\left(\mathrm{MeCN}: \mathrm{HCO}_{2} \mathrm{H}=100: 0.1,1.5 \mathrm{~cm}, 10 \mathrm{~cm}, 2 \mathrm{~mL}\right)$. The tetramic acid $(R, S, R, R)-27(13.1 \mathrm{mg}, 63 \%, 16.4 \mu \mathrm{mol})$ was obtained as a yellow oil. It was assigned as an 83:17 mixture of the $Z$ - and the $E$-enol of the title compounds $(R, S, R, R)-27$. This ratio was determined by integrating duplets of the respective 2'-H [6.52 (E-enol) and 7.09 (Zenol)].

$\mathbf{R}_{\mathbf{f}}\left(\mathrm{MeCN}: \mathrm{HCO}_{2} \mathrm{H}=100: 0.1\right)=0.15$

${ }^{1} \mathbf{H}-\mathbf{N M R}\left(500.06 \mathrm{MHz}, \mathrm{CDCl}_{3} / \mathrm{CHCl}_{3}\right.$, sample contained grease with s at 1.26 , DsBrJn10500300, 12.6.2020): $\delta=-0.20\left(\mathrm{~s}, 3 \mathrm{H}, 5-\mathrm{CH}-\mathrm{O}-\mathrm{Si}_{-} \mathrm{CH}_{3}{ }^{\mathrm{A}}\right),-0.11\left(\mathrm{~s}, 3 \mathrm{H}, 5-\mathrm{CH}-\mathrm{O}-\mathrm{Si}_{-} \mathrm{CH}_{3}{ }^{\mathrm{B}}\right), 0.14$ $\left(\mathrm{s}, 3 \mathrm{H}, 1 "-\mathrm{O}-\mathrm{Si}_{-} \mathrm{CH}_{3}{ }^{\mathrm{A}}\right), 0.15$ (s, 3H, 1"-O-Si-CH$\left.{ }^{\mathrm{B}}\right), 0.81-0.87$ (m, 3H, 12'- $\left.\mathrm{H}_{3}\right), 0.81-0.87$ (m, 
Christian Drescher and Reinhard Brückner; 3D-Structure Clarifying Total Synthesis of the (Polyenoyl)tetramic Acid Militarinon B. A Highly Acid-Labile N-Protecting Group for Amides

$\left.3 \mathrm{H}, 10 '-\mathrm{CH}_{3}\right), 0.83$ [s, 9H, 5-CH-Si-C( $\left.\left(\mathrm{CH}_{3}\right)_{3}\right], 0.94$ [s, 9H, 1"-O-Si-C $\left.\left(\mathrm{CH}_{3}\right)_{3}\right], 0.97$ (d, J J'$\left.\mathrm{CH}_{3}, 8^{\prime}=6.7 \mathrm{~Hz}, 3 \mathrm{H}, 8^{\prime}-\mathrm{CH}_{3}\right), 1.07-1.17\left(\mathrm{~m}, 1 \mathrm{H}, 9^{\prime}-\mathrm{H}^{\mathrm{A}}\right), 1.07-1.17\left(\mathrm{~m}, 1 \mathrm{H}, 11^{\prime}-\mathrm{H}^{\mathrm{A}}\right), 1.21-1.34(\mathrm{~m}$, $\left.1 \mathrm{H}, 10^{\prime}-\mathrm{H}\right), 1.21-1.34\left(\mathrm{~m}, 1 \mathrm{H}, 11^{\prime}-\mathrm{H}^{\mathrm{B}}\right), 1.21-1.34\left(\mathrm{~m}, 1 \mathrm{H}, 9^{\prime}-\mathrm{H}^{\mathrm{B}}\right), 1.80\left(\mathrm{~d},{ }^{4} J_{8-\mathrm{CH}_{3}, 9}=1.0 \mathrm{~Hz}, 3 \mathrm{H}\right.$, 6'- $\left.\mathrm{CH}_{3}\right), 2.64\left(\mathrm{~m}_{\mathrm{c}}, 1 \mathrm{H}, 8^{\prime}-\mathrm{H}\right), 3.67\left(\mathrm{~s}, 6 \mathrm{H}, 1\right.$ '"'- $\mathrm{OCH}_{3}, 3$ ''-- $\left.\mathrm{OCH}_{3}\right), 3.75\left(\mathrm{~d}, J_{5,5-\mathrm{CH}}=2.7 \mathrm{~Hz}, 1 \mathrm{H}\right.$, 5-H), 3.76 (s, 3H, 5"'-OCH $), 3.87$ (d, $\left.{ }^{2} J=14.7 \mathrm{~Hz}, 1 \mathrm{H}, 2^{2} "-\mathrm{C}-\mathrm{H}^{\mathrm{A}}\right), 4.88\left(\mathrm{~d},{ }^{2} J=14.7 \mathrm{~Hz}, 1 \mathrm{H}\right.$, $\left.2^{\prime \prime}-\mathrm{C}-\mathrm{H}^{\mathrm{B}}\right), 5.06\left(\mathrm{~d}, J_{5-\mathrm{CH}, 5}=2.7 \mathrm{~Hz}, 1 \mathrm{H}, 5-\mathrm{CH}\right), 5.49$ (d, $\left.J_{7^{\prime}, 8^{\prime}}=9.6 \mathrm{~Hz}, 1 \mathrm{H}, 7^{\prime}-\mathrm{H}\right), 6.02$ (s, 2H, 4'"-H, 6"'-H), 6.40 (dd, $\left.J_{4^{\prime}, 3^{\prime}}=11.2 \mathrm{~Hz}, J_{4^{\prime}, 5^{\prime}}=15.2 \mathrm{~Hz}, 1 \mathrm{H}, 4^{\prime}-\mathrm{H}\right), 6.61$ (d, J $5_{5^{\prime}, 4^{\prime}}=15.1 \mathrm{~Hz}, 1 \mathrm{H}$, 5'-H), 6.73-6.77 (m, 2H, 2"-H, 6"-H), 7.09 (d, J J',3' = 15.3 Hz, 1H, 2'-H), 7.19-7.23 (m, 2H, 3"H, 5"-H), 7.41 (dd, $\left.J_{3^{\prime}, 4^{\prime}}=11.2 \mathrm{~Hz}, J_{3^{\prime}, 2^{\prime}}=15.2 \mathrm{~Hz}, 1 \mathrm{H}, 3^{\prime}-\mathrm{H}\right) \mathrm{ppm}$.

${ }^{13}$ C-NMR (125.75 MHz, DsBrJn10-500104, 12.6.2020): $\delta=-5.5$ (5-CH-O-Si-CH $\left.{ }_{3}^{\mathrm{A}}\right),-4.7$ (5$\left.\mathrm{CH}-\mathrm{O}-\mathrm{Si}-\mathrm{CH}_{3}{ }^{\mathrm{B}}\right),-4.4\left(1^{\prime \prime}-\mathrm{O}-\mathrm{Si}_{-}-\mathrm{CH}_{3}{ }^{\mathrm{A}}\right),-4.4$ (1"-O-Si- $\left.\mathrm{CH}_{3}{ }^{\mathrm{B}}\right), 11.4\left(\mathrm{C}-12\right.$ '), $12.5\left(6^{\prime}-\mathrm{CH}_{3}\right), 18.2$ [5-CH-Si- $\left.C\left(\mathrm{CH}_{3}\right)_{3}\right], 18.3$ [1"-O-Si- $\left.C\left(\mathrm{CH}_{3}\right)_{3}\right], 19.2\left(10^{\prime}-\mathrm{CH}_{3}\right), 21.4\left(8^{\prime}-\mathrm{CH}_{3}\right), 25.8$ [1"-O-Si$\mathrm{C}\left(\mathrm{CH}_{3}\right)_{3}$ ], 25.8 [5-CH-Si-C $\left(\mathrm{CH}_{3}\right)_{3}$ ], 30.2 (C-11'), $31.0\left(\mathrm{C}-8^{\prime}\right), 32.4\left(\mathrm{C}-10^{\prime}\right), 35.1\left(2^{\prime \prime}-\mathrm{CH}_{2}\right), 44.8$ (C-9'), 55.4 (5"'--OCH 3$), 55.8$ (1"'--OCH 3 , 3'"--OCH $), 70.6$ (C-5), 73.2 (5-CH), 90.4 (C-4'", C6"'), 101.8 (C-3), 104.2 (C-2'"), 119.7 (C-2", C-6"), 120.6 (C-2'), 125.4 (C-4'), 127.6 (C-3", C5"), 132.8 (C-6'), 133.7 (C-4"), 144.2 (C-3'), 146.4 (C-7'), 147.6 (C-5'), 155.1 (C-1"), 159.7 (C1"', C-3"'), 161.0 (C-5"'), 171.9 (C-1'), 175.1 (C-2), 194.1 (C-3) ppm.

edHSQC [,,short-range H,C-COSY“, 500.06 MHz/125.75 MHz, $\mathrm{CDCl}_{3}$ ]; $\delta\left({ }^{1} \mathrm{H}\right) \leftrightarrow \delta\left({ }^{13} \mathrm{C}\right)$ : $0.20\left(\mathrm{~s}, 5-\mathrm{CH}-\mathrm{O}-\mathrm{Si}_{-} \mathrm{CH}_{3}{ }^{\mathrm{A}}\right) \leftrightarrow-5.5\left(5-\mathrm{CH}-\mathrm{O}-\mathrm{Si}_{-} \mathrm{CH}_{3}{ }^{\mathrm{A}}\right),-0.11\left(\mathrm{~s}, 5-\mathrm{CH}-\mathrm{O}-\mathrm{Si}_{-}-\mathrm{CH}_{3}{ }^{\mathrm{B}}\right) \leftrightarrow-4.7(5-$

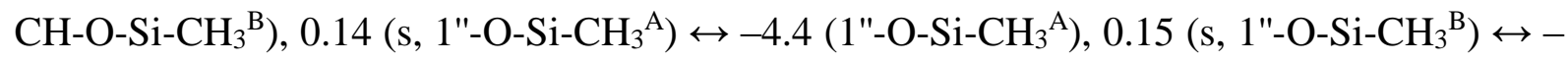
$4.4\left(1 "-\mathrm{O}-\mathrm{Si}_{-} \mathrm{CH}_{3}{ }^{\mathrm{B}}\right), 0.81-0.87\left(\mathrm{~m}, 12^{\prime}-\mathrm{H}_{3}\right) \leftrightarrow 11.4(\mathrm{C}-12 '), 0.81-0.87\left(\mathrm{~m}, 10^{\prime}-\mathrm{CH}_{3}\right) \leftrightarrow 19.2\left(10^{\prime}-\right.$ $\left.\mathrm{CH}_{3}\right), 0.83$ [s, 5-CH-Si-C $\left.\left(\mathrm{CH}_{3}\right)_{3}\right] \leftrightarrow 25.8$ [5-CH-Si-C $\left.\left(\mathrm{CH}_{3}\right)_{3}\right], 0.94$ [s, 1"-O-Si-C $\left.\left(\mathrm{CH}_{3}\right)_{3}\right] \leftrightarrow$ 25.8 [1"-O-Si-C $\left.\left(\mathrm{CH}_{3}\right)_{3}\right], 0.97\left(\mathrm{~d}, 8^{\prime}-\mathrm{CH}_{3}\right) \leftrightarrow 21.4\left(8^{\prime}-\mathrm{CH}_{3}\right), 1.07-1.17\left(\mathrm{~m}, 9^{\prime}-\mathrm{H}^{\mathrm{A}}\right) \leftrightarrow 44.8\left(\mathrm{C}-9^{\prime}\right)$,

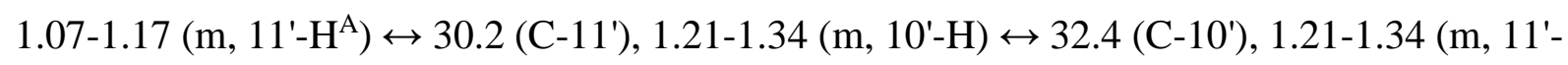
$\left.\mathrm{H}^{\mathrm{B}}\right) \leftrightarrow 30.2\left(\mathrm{C}-11^{\prime}\right), 1.21-1.34\left(\mathrm{~m}, 9^{\prime}-\mathrm{H}^{\mathrm{B}}\right) \leftrightarrow 44.8\left(\mathrm{C}-9^{\prime}\right), 1.80\left(\mathrm{~d}, 6^{\prime}-\mathrm{CH}_{3}\right) \leftrightarrow 12.5\left(6^{\prime}-\mathrm{CH}_{3}\right), 2.64$

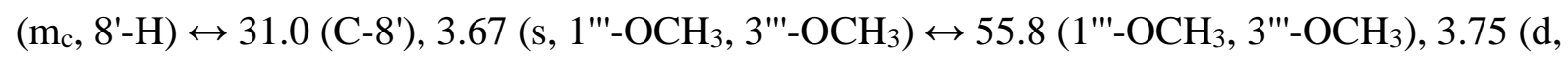
$5-\mathrm{H}) \leftrightarrow 70.6(\mathrm{C}-5), 3.76\left(\mathrm{~s}, 5{ }^{\prime \prime}-\mathrm{OCH}_{3}\right) \leftrightarrow 55.4\left(5^{\prime \prime}-\mathrm{OCH}_{3}\right), 3.87\left(\mathrm{~d}, 2\right.$ "'-C-H $\left.{ }^{\mathrm{A}}\right) \leftrightarrow 35.1\left(2^{\prime \prime}-\right.$ $\left.\mathrm{CH}_{2}\right), 4.88\left(\mathrm{~d}, 2\right.$ '"-C-H $\left.{ }^{\mathrm{B}}\right) \leftrightarrow 35.1\left(2^{\prime \prime}-\mathrm{CH}_{2}\right), 5.06(\mathrm{~d}, 5-\mathrm{CH}) \leftrightarrow 73.2(5-\mathrm{CH}), 5.49\left(\mathrm{~d}, 7^{\prime}-\mathrm{H}\right) \leftrightarrow$ 146.4 (C-7'), 6.02 (s, 4"'-H, 6'"-H) ↔90.4 (C-4"', C-6"'), 6.40 (dd, 4'-H) 125.4 (C-4'), 6.61 $\left(\mathrm{d}, 5^{\prime}-\mathrm{H}\right) \leftrightarrow 147.6\left(\mathrm{C}-5^{\prime}\right), 6.73-6.77$ (m, 2"-H, 6"-H) 119.7 (C-2", C-6"), 7.09 (d, 2'-H) 120.6 (C-2'), 7.19-7.23 (m, 3"-H, 5"-H) 127.6 (C-3", C-5"), 7.41 (dd, 3'-H) 144.2 (C-3').

Specific rotation $(S, R, R, R)-27[\alpha]_{D}^{20}=-325.9^{\circ}\left(\mathrm{c}=0.61\right.$ in $\left.\mathrm{CHCl}_{3}\right)$ 
Christian Drescher and Reinhard Brückner; 3D-Structure Clarifying Total Synthesis of the (Polyenoyl)tetramic Acid Militarinon B. A Highly Acid-Labile N-Protecting Group for Amides

HRMS: $(\mathrm{EI}, 70 \mathrm{eV}): \mathrm{m} / \mathrm{z}=870.4760[\mathrm{M}+\mathrm{Na}]^{+}$, corresponds to the molecular formula $\mathrm{C}_{48} \mathrm{H}_{73} \mathrm{O}_{8} \mathrm{NNaSi}_{2}(870.4767)\left\{[\mathrm{M}+\mathrm{Na}]^{+}\right\}$with a deviation of $-0.8 \mathrm{ppm}$.

IR (KBr): $\tilde{v}=2955,2930,2900,2855,1700,1625,1610,1595,1560,1510,1455,1420,1390$, $1360,1320,1255,1230,1205,1165,1150,1130,1090,1000,915,840,810,780 \mathrm{~cm}^{-1}$.

(5S)-5-\{(S)-[(tert-Butyldimethylsilyl)oxy]\{1"-[(tertbutyldimethylsilyl)oxy)phenyl]methyl $\}-3-\left[\left(1^{\prime} Z, 2^{\prime} E, 4^{\prime} E, 6^{\prime} E, 8^{\prime} R, 10^{\prime} R\right)-1^{\prime}-h y d r o x y-\right.$ $6^{\prime}, 8^{\prime}, 10$ '-trimethyldodeca-2',4',6' -trien-1'-ylidene]pyrrolidine-2,4-dione [Z-enol$(S, S, R, R)-28]$

in a $72: 28$ mixture with

(5S)-5-\{(S)-[(tert-Butyldimethylsilyl)oxy] $]\{1 "-[($ tert-

butyldimethylsilyl)oxy)phenyl]methyl $\}-3-\left[\left(1^{\prime} E, 2^{\prime} E, 4^{\prime} E, 6^{\prime} E, 8^{\prime} R, 10^{\prime} R\right)-1^{\prime}-h y d r o x y-\right.$ $6^{\prime}, 8^{\prime}, 10$ '-trimethyldodeca-2',4',6' -trien-1'-ylidene]pyrrolidine-2,4-dione [E-enol$(S, S, R, R)-28]$

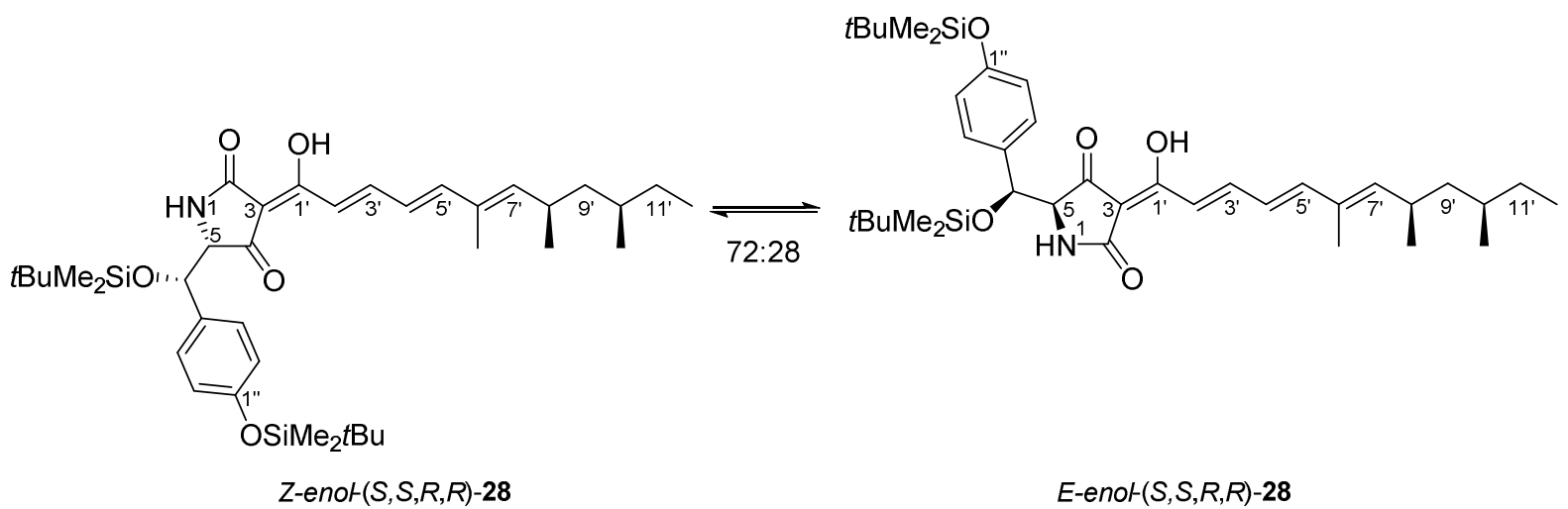

The tetramic acid $(S, S, R, R)-27(13.0 \mathrm{mg}, 15.3 \mu \mathrm{mol})$ was dissolved in $\mathrm{CH}_{2} \mathrm{Cl}_{2}(6.0 \mathrm{~mL})$. It was added $\mathrm{F}_{3} \mathrm{CCO}_{2} \mathrm{H}(60 \mu \mathrm{L})$ at $25^{\circ} \mathrm{C}$. After $4.5 \mathrm{~h}$ at this temperature the mixture was diluted with $\mathrm{H}_{2} \mathrm{O}(8 \mathrm{~mL})$. The layers were separated, and the aq. layer was extracted with $\mathrm{CH}_{2} \mathrm{Cl}_{2}(3 \times 10$ $\mathrm{mL})$. The combined organic layers were washed with brine $(5 \mathrm{~mL})$ and tried over $\mathrm{Na}_{2} \mathrm{SO}_{4}$. The solvent was removed under reduced pressure and the residue was purified by reverse phase flash chromatography $\left(\mathrm{MeCN}: \mathrm{HCO}_{2} \mathrm{H}=100: 0.1,1.5 \mathrm{~cm}, 10 \mathrm{~cm}, 2 \mathrm{~mL}\right)$. The tetramic acid $(S, S, R, R)-\mathbf{2 8}(7.2 \mathrm{mg}, 70 \%, 10.7 \mu \mathrm{mol})$ was obtained as a yellow powder (mp.: $\left.95-97^{\circ} \mathrm{C}\right)$. It was assigned as a 72:28 mixture of the $Z$ - and the $E$-enol of the title compounds $(S, S, R, R)-28$. This 
Christian Drescher and Reinhard Brückner; 3D-Structure Clarifying Total Synthesis of the (Polyenoyl)tetramic Acid Militarinon B. A Highly Acid-Labile N-Protecting Group for Amides

ratio was determined by integrating dd's of the respective 3'-H [7.33 (E-enol) and 7.46 (Zenol)].

\section{$\mathbf{R}_{\mathbf{f}}\left(\mathrm{MeCN}: \mathrm{HCO}_{2} \mathrm{H}=100: 0.1\right)=0.18$}

${ }^{1}$ H-NMR $(500.06 \mathrm{MHz}, \mathrm{MeOD} / \mathrm{MeOH}$, sample contained grease with s at 1.26 and unknown impurity with $m$ at 3.91-3.98, DsBrSe29-500600, 2.6.2020): -0.05 (s, 3H, 3'-O-Si-CH ${ }^{\mathrm{A}}$ ), 0.08 (s, 3H, 3'-O-Si- $\left.\mathrm{CH}_{3}{ }^{\mathrm{B}}\right), 0.11$ [s, 6H, 1"-O-Si- $\left.\left(\mathrm{CH}_{3}\right)_{2}\right], 0.83-0.87$ (m, 3H, 10'-- $\left.\mathrm{CH}_{3}\right), 0.86$ (t, $\left.J_{12}, 11^{\prime}=7.4 \mathrm{~Hz}, 3 \mathrm{H}, 12^{\prime}-\mathrm{H}_{3}\right), 0.89$ [s, 9H, 5-CH-O-Si-C $\left.\left(\mathrm{CH}_{3}\right)_{3}\right], 0.92$ [s, 9H, 1"-O-Si-C(CH 3$\left.)_{3}\right]$, $0.97\left(\mathrm{~d}, J_{8^{\prime}-\mathrm{CH}_{3}, 8^{\prime}}=6.6 \mathrm{~Hz}, 3 \mathrm{H}, 8^{\prime}-\mathrm{CH}_{3}\right), 1.10-1.19\left(\mathrm{~m}, 1 \mathrm{H}, 9^{\prime}-\mathrm{H}^{\mathrm{A}}\right), 1.10-1.19\left(\mathrm{~m}, 1 \mathrm{H}, 11^{\prime}-\mathrm{H}^{\mathrm{A}}\right)$, 1.23-1.37 (m, 1H, 10'-H), 1.23-1.37 (m, 1H, 9'- $\left.\mathrm{H}^{\mathrm{B}}\right), 1.23-1.37\left(\mathrm{~m}, 1 \mathrm{H}, 11^{\prime}-\mathrm{H}^{\mathrm{B}}\right), 1.85\left(\mathrm{~s}, 3 \mathrm{H}, 66^{\prime}-\right.$ $\left.\mathrm{CH}_{3}\right), 2.70\left(\mathrm{~m}_{\mathrm{c}}, 1 \mathrm{H}, 8^{\prime}-\mathrm{H}\right), 4.14$ (br. s, $\left.1 \mathrm{H}, 5-\mathrm{H}\right), 5.09$ (d, J5- $\left.\mathrm{CH}, 5=3.2 \mathrm{~Hz}, 1 \mathrm{H}, 5-\mathrm{CH}\right), 5.58$ (d, $\left.J_{7^{\prime}, 8^{\prime}}=9.8 \mathrm{~Hz}, 1 \mathrm{H}, 7^{\prime}-\mathrm{H}\right), 6.42\left(\mathrm{dd}, J_{4^{\prime}, 3^{\prime}}=11.3 \mathrm{~Hz}, J_{4^{\prime}, 5^{\prime}}=15.0 \mathrm{~Hz}, 1 \mathrm{H}, 4^{\prime}-\mathrm{H}\right), 6.67-6.72(\mathrm{~m}, 2 \mathrm{H}$, 2"-H, 6"-H), 6.74 (d, J $\left.J_{5^{\prime}, 4^{\prime}}=15.1 \mathrm{~Hz}, 1 \mathrm{H}, 5^{\prime}-\mathrm{H}\right), 6.99$ (d, $\left.J_{2^{\prime}, 3^{\prime}}=15.2 \mathrm{~Hz}, 1 \mathrm{H}, 2^{\prime}-\mathrm{H}\right), 7.10-7.16$ (m, $\left.2 \mathrm{H}, 3^{\prime \prime}-\mathrm{H}, 5^{\prime \prime}-\mathrm{H}\right), 7.46\left(\mathrm{dd}, J_{3^{\prime}, 4^{\prime}}=11.4 \mathrm{~Hz}, J_{3^{\prime}, 2^{\prime}}=14.9 \mathrm{~Hz}, 1 \mathrm{H}, 3^{\prime}-\mathrm{H}\right) \mathrm{ppm}$.

${ }^{13} \mathrm{C}-\mathrm{NMR}$ (125.75 MHz, MeOD/MeOH, sample contained grease at 14.43, DsBr29-500602, 2.06.2020): $\delta=-4.9\left(3^{\prime}-\mathrm{O}-\mathrm{Si}_{-}-\mathrm{CH}_{3}{ }^{\mathrm{B}}\right),-4.6\left(3^{\prime}-\mathrm{O}-\mathrm{Si}^{-} \mathrm{CH}_{3}{ }^{\mathrm{A}}\right),-4.3$ [1"-O-Si- $\left.\left(\mathrm{CH}_{3}\right)_{2}\right], 11.7\left(\mathrm{C}-12^{\prime}\right)$, $12.6\left(6 '-\mathrm{CH}_{3}\right), 19.1$ [5-CH-O-Si- $\left.C\left(\mathrm{CH}_{3}\right)_{3}\right], 19.1$ [1"-O-Si- $\left.C\left(\mathrm{CH}_{3}\right)_{3}\right], 19.5\left(10\right.$ '- $\left.\mathrm{CH}_{3}\right), 21.5(8$ '$\left.\mathrm{CH}_{3}\right), 26.2$ [1"-O-Si-C $\left(\mathrm{CH}_{3}\right)_{3}$ ], 26.3 [5-CH-O-Si-C $\left(\mathrm{CH}_{3}\right)_{3}$ ], $31.3\left(\mathrm{C}-11^{\prime}\right), 32.1\left(\mathrm{C}-8^{\prime}\right), 33.7$ (C10'), 45.8 (C-9'), 69.8 (C-5), 76.4 (5-CH), 120.4 (C-2", C-6"), 120.7 (C-2'), 126.3 (C-4'), 129.6 (C-3", C-5"), 132.8 (C-4"), 134.3 (C-6'), 147.1 (C-3'), 148.3 (C-7'), 150.2 (C-5'), 156.8 (C-1") ppm.*

${ }^{*} \mathrm{C}-2, \mathrm{C}-3, \mathrm{C}-4$ and $\mathrm{C}-1$ ' were not found.

edHSQC [,,short-range H,C-COSY“, 500.06 MHz/125.75 MHz, MeOD; $\delta\left({ }^{1} \mathrm{H}\right) \leftrightarrow \delta\left({ }^{13} \mathrm{C}\right)$ : $0.05\left(\mathrm{~s}, 3^{\prime}-\mathrm{O}-\mathrm{Si}-\mathrm{CH}_{3}{ }^{\mathrm{A}}\right) \leftrightarrow-4.6\left(3^{\prime}-\mathrm{O}-\mathrm{Si}-\mathrm{CH}_{3}{ }^{\mathrm{A}}\right), 0.08\left(3^{\prime}-\mathrm{O}-\mathrm{Si}-\mathrm{CH}_{3}{ }^{\mathrm{B}}\right) \leftrightarrow-4.9\left(3^{\prime}-\mathrm{O}-\mathrm{Si}-\mathrm{CH}_{3}{ }^{\mathrm{B}}\right)$, 0.11 [s, 1"-O-Si- $\left.\left(\mathrm{CH}_{3}\right)_{2}\right] \leftrightarrow-4.3$ [1"-O-Si- $\left.\left(\mathrm{CH}_{3}\right)_{2}\right], 0.83-0.87\left(\mathrm{~m}, 10 '-\mathrm{CH}_{3}\right) \leftrightarrow 19.5\left(10 '-\mathrm{CH}_{3}\right)$, $0.86\left(\mathrm{t}, 12^{\prime}-\mathrm{H}_{3}\right) \leftrightarrow 11.7\left(\mathrm{C}-12^{\prime}\right), 0.89$ [s, 5-CH-O-Si-C $\left.\left(\mathrm{CH}_{3}\right)_{3}\right] \leftrightarrow 26.3$ [5-CH-O-Si-C $\left.\left(\mathrm{CH}_{3}\right)_{3}\right]$, 0.92 [s, 1"-O-Si-C $\left.\left(\mathrm{CH}_{3}\right)_{3}\right] \leftrightarrow 26.2\left[1 "-\mathrm{O}-\mathrm{Si}-\mathrm{C}\left(\mathrm{CH}_{3}\right)_{3}\right], 0.97\left(\mathrm{~d}, 8^{\prime}-\mathrm{CH}_{3}\right) \leftrightarrow 21.5\left(8^{\prime}-\mathrm{CH}_{3}\right), 1.10-$ $1.19\left(\mathrm{~m}, 9^{\prime}-\mathrm{H}^{\mathrm{A}}\right) \leftrightarrow 45.8\left(\mathrm{C}-9^{\prime}\right), 1.10-1.19\left(\mathrm{~m}, 11^{\prime}-\mathrm{H}^{\mathrm{A}}\right) \leftrightarrow 31.3\left(\mathrm{C}-11^{\prime}\right), 1.23-1.37\left(\mathrm{~m}, 10^{\prime}-\mathrm{H}\right) \leftrightarrow$ $33.7\left(\mathrm{C}-10^{\prime}\right), 1.23-1.37\left(\mathrm{~m}, 9^{\prime}-\mathrm{H}^{\mathrm{B}}\right) \leftrightarrow 45.8\left(\mathrm{C}-9^{\prime}\right), 1.23-1.37\left(\mathrm{~m}, 11^{\prime}-\mathrm{H}^{\mathrm{B}}\right) \leftrightarrow 31.3\left(\mathrm{C}-11^{\prime}\right), 1.85$ $\left(\mathrm{s}, 6^{\prime}-\mathrm{CH}_{3}\right) \leftrightarrow 12.6\left(6 '-\mathrm{CH}_{3}\right), 2.70\left(\mathrm{~m}_{\mathrm{c}}, 8^{\prime}-\mathrm{H}\right) \leftrightarrow 32.1\left(\mathrm{C}-8^{\prime}\right), 4.14$ (br. s, 5-H) 69.8 (C-5), 5.09 $(\mathrm{d}, 5-\mathrm{CH}) \leftrightarrow 76.4(5-\mathrm{CH}), 5.58\left(\mathrm{~d}, 7^{\prime}-\mathrm{H}\right) \leftrightarrow 148.3\left(\mathrm{C}-7^{\prime}\right), 6.42\left(\mathrm{dd}, 4^{\prime}-\mathrm{H}\right) \leftrightarrow 126.3\left(\mathrm{C}-4^{\prime}\right), 6.67-$ $6.72(\mathrm{~m}, 2 "-\mathrm{H}, 6 "-\mathrm{H}) \leftrightarrow 120.4$ (C-2", C-6"), 6.74 (d, 5'-H) ↔ 150.2 (C-5'), 6.99 (d, 2'-H) 120.7 (C-2'), 7.10-7.16 (m, 3"-H, 5"-H) ↔ 129.6 (C-3", C-5"), 7.46 (dd, 3'-H) 147.1 (C-3'). 
Christian Drescher and Reinhard Brückner; 3D-Structure Clarifying Total Synthesis of the (Polyenoyl)tetramic Acid Militarinon B. A Highly Acid-Labile N-Protecting Group for Amides

HRMS: $(\mathrm{EI}, 70 \mathrm{eV}): \mathrm{m} / \mathrm{z}=668.4163[\mathrm{M}+\mathrm{H}]^{+}$, corresponds to the molecular formula $\mathrm{C}_{38} \mathrm{H}_{62} \mathrm{O}_{5} \mathrm{NSi}_{2}(668.4161)\left\{[\mathrm{M}+\mathrm{H}]^{+}\right\}$with a deviation of $0.3 \mathrm{ppm}$.

Specific rotation $(S, S, R, R)-28[\alpha]_{D}^{20}=-404.1^{\circ}\left(\mathrm{c}=0.58\right.$ in $\left.\mathrm{CHCl}_{3}\right)$.

IR (KBr): $\tilde{v}=2960,2930,2860,1655,1630,1565,1510,1470,1465,1430,1390,1360,1280$, $1255,1215,1170,1110,1085,1000,910,875,835,780 \mathrm{~cm}^{-1}$.

$(5 S)-5-\{(R)-[($ tert-Butyldimethylsilyl)oxy $]\{1 "-[($ tert-

butyldimethylsilyl)oxy)phenyl]methyl $\}-3-\left[\left(1^{\prime} Z, 2^{\prime} E, 4^{\prime} E, 6^{\prime} E, 8^{\prime} R, 10^{\prime} R\right)-1^{\prime}-h y d r o x y-\right.$ $6^{\prime}, 8^{\prime}, 10$ '-trimethyldodeca-2',4',6'-trien-1'-ylidene]pyrrolidine-2,4-dione [Z-enol-

$(R, S, R, R)-28]$

in a 67:33 mixture with

$(5 S)-5-\left\{(R)-[(\right.$ tert-Butyldimethylsilyl $)$ oxy $]\left\{1^{\prime \prime}-[(\right.$ tert-

butyldimethylsilyl)oxy)phenyl]methyl\}-3-[(1'E,2' $\left.E, 4^{\prime} E, 6^{\prime} E, 8^{\prime} R, 10 ' R\right)-1^{\prime}$-hydroxy-

6',8',10'-trimethyldodeca-2',4',6' -trien-1'-ylidene]pyrrolidine-2,4-dione [E-enol-

$(R, S, R, R)-28]$

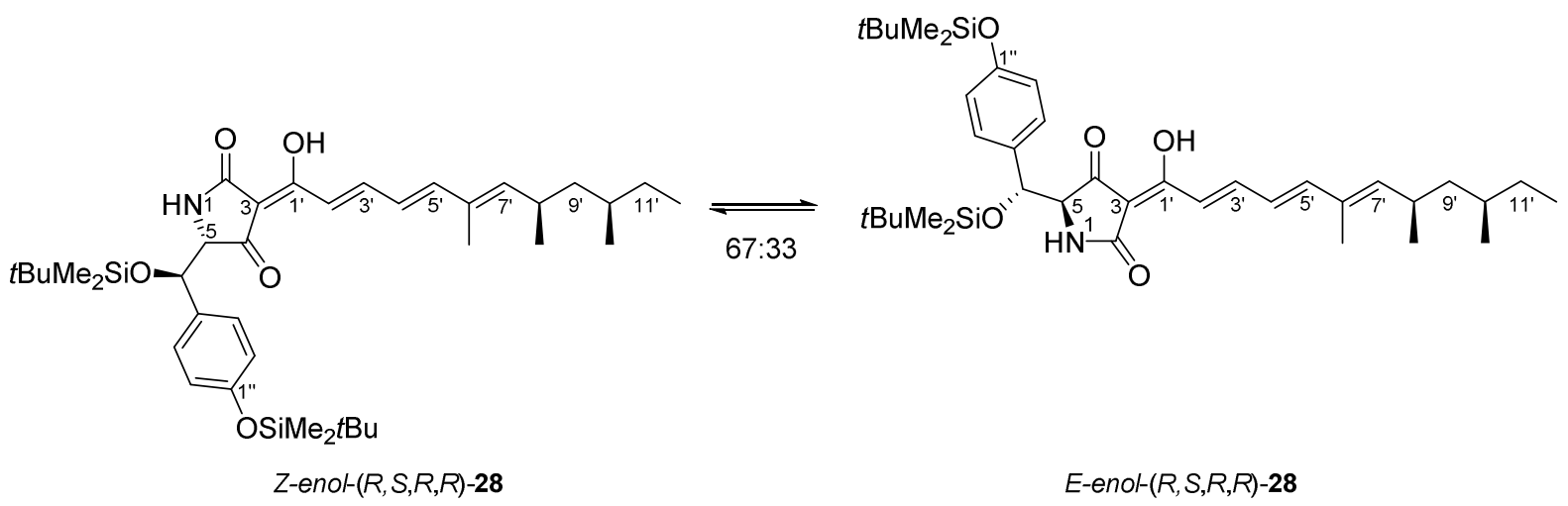

The tetramic acid $(R, S, R, R)-27(11.0 \mathrm{mg}, 13.0 \mu \mathrm{mol})$ was dissolved in $\mathrm{CH}_{2} \mathrm{Cl}_{2}(5.0 \mathrm{~mL})$. It was added $\mathrm{F}_{3} \mathrm{CCO}_{2} \mathrm{H}(50 \mu \mathrm{L})$ at $25^{\circ} \mathrm{C}$. After $4.5 \mathrm{~h}$ at this temperature the mixture was diluted with $\mathrm{H}_{2} \mathrm{O}(6 \mathrm{~mL})$. The layers were separated, and the aq. layer was extracted with $\mathrm{CH}_{2} \mathrm{Cl}_{2}(3 \times 10$ $\mathrm{mL})$. The combined organic layers were washed with brine $(5 \mathrm{~mL})$ and tried over $\mathrm{Na}_{2} \mathrm{SO}_{4}$. The solvent was removed under reduced pressure and the residue was purified by reverse phase flash chromatography $\left(\mathrm{MeCN}: \mathrm{HCO}_{2} \mathrm{H}=100: 0.1,1.5 \mathrm{~cm}, 10 \mathrm{~cm}, 2 \mathrm{~mL}\right)$. The tetramic acid $(R, S, R, R)-28(6.1 \mathrm{mg}, 68 \%, 8.8 \mu \mathrm{mol})$ was obtained as a yellow powder (mp.: $\left.77-80^{\circ} \mathrm{C}\right)$. It was assigned as a 67:33 mixture of the $Z$ - and the $E$-enol of the title compounds $(R, S, R, R)-\mathbf{2 8}$. This 
Christian Drescher and Reinhard Brückner; 3D-Structure Clarifying Total Synthesis of the (Polyenoyl)tetramic Acid Militarinon B. A Highly Acid-Labile N-Protecting Group for Amides

ratio was determined by integrating dd's of the respective 3'-H [7.44 (E-enol) and 7.56 (Zenol)].

\section{$\mathbf{R}_{\mathbf{f}}\left(\mathrm{MeCN}: \mathrm{HCO}_{2} \mathrm{H}=100: 0.1\right)=0.17$}

${ }^{1}$ H-NMR $(500.06 \mathrm{MHz}, \mathrm{MeOD} / \mathrm{MeOH}$, sample contained grease with s at 1.26 , DsBrJn16500700, 16.6.2020): -0.22 (s, 3H, 3'-O-Si- $\mathrm{CH}_{3}{ }^{\mathrm{A}}$ ), -0.07 (s, 3H, 3'-O-Si-CH ${ }_{3}^{\mathrm{B}}$ ), 0.19 [s, 6H, 1"O-Si- $\left.\left(\mathrm{CH}_{3}\right)_{2}\right], 0.78$ [s, 9H, 5-CH-O-Si-C( $\left.\left.\mathrm{CH}_{3}\right)_{3}\right], 0.85\left(\mathrm{~d}, J_{10}{ }^{\prime}-\mathrm{CH}_{3}, 10^{\prime}=6.4 \mathrm{~Hz}, 3 \mathrm{H}, 10^{\prime}-\mathrm{CH}_{3}\right), 0.86$ $\left(\mathrm{t}, J_{12^{\prime}, 11^{\prime}}=7.4 \mathrm{~Hz}, 3 \mathrm{H}, 12^{\prime}-\mathrm{H}_{3}\right), 0.98\left(\mathrm{~d}, J_{8^{\prime}-\mathrm{CH}_{3}, 8^{\prime}}=6.4 \mathrm{~Hz}, 3 \mathrm{H}, 8^{\prime}-\mathrm{CH}_{3}\right), 0.99$ [s, 9H, 1"-O-Si$\left.\mathrm{C}\left(\mathrm{CH}_{3}\right)_{3}\right], 1.10-1.20\left(\mathrm{~m}, 1 \mathrm{H}, 9^{\prime}-\mathrm{H}^{\mathrm{A}}\right), 1.10-1.20\left(\mathrm{~m}, 1 \mathrm{H}, 11^{\prime}-\mathrm{H}^{\mathrm{A}}\right), 1.23-1.39\left(\mathrm{~m}, 1 \mathrm{H}, 10^{\prime}-\mathrm{H}\right), 1.23-$ $1.39\left(\mathrm{~m}, 1 \mathrm{H}, 9^{\prime}-\mathrm{H}^{\mathrm{B}}\right), 1.23-1.39\left(\mathrm{~m}, 1 \mathrm{H}, 11^{\prime}-\mathrm{H}^{\mathrm{B}}\right), 1.87\left(\mathrm{~s}, 3 \mathrm{H}, 6^{\prime}-\mathrm{CH}_{3}\right), 2.71\left(\mathrm{~m}_{\mathrm{c}}, 1 \mathrm{H}, 8^{\prime}-\mathrm{H}\right), 3.87$ (br. s, 1H, 5-H), 5.12 (s, 1H, 5-CH), 5.60 (d, $\left.J_{7^{\prime}, 8^{\prime}}=9.7 \mathrm{~Hz}, 1 \mathrm{H}, 7^{\prime}-\mathrm{H}\right), 6.48$ (dd, $J_{4^{\prime}, 3^{\prime}}=11.2 \mathrm{~Hz}$, $\left.J_{4^{\prime}, 5^{\prime}}=15.0 \mathrm{~Hz}, 1 \mathrm{H}, 4^{\prime}-\mathrm{H}\right), 6.79$ (d, $\left.J_{5^{\prime}, 4^{\prime}}=15.2 \mathrm{~Hz}, 1 \mathrm{H}, 5^{\prime}-\mathrm{H}\right), 6.82-6.87$ (m, 2H, 2"-H, 6"-H), $7.19\left(\mathrm{~d}, J_{2^{\prime}, 3^{\prime}}=15.3 \mathrm{~Hz}, 1 \mathrm{H}, 2^{\prime}-\mathrm{H}\right), 7.25-7.30\left(\mathrm{~m}, 2 \mathrm{H}, 3^{\prime \prime}-\mathrm{H}, 5^{\prime \prime}-\mathrm{H}\right), 7.59$ (dd, $J_{3^{\prime}, 4^{\prime}}=11.1 \mathrm{~Hz}$, $\left.J_{3^{\prime}, 2^{\prime}}=15.1 \mathrm{~Hz}, 1 \mathrm{H}, 3^{\prime}-\mathrm{H}\right) \mathrm{ppm}$.

${ }^{13}$ C-NMR (125.75 MHz, MeOD/MeOH, DsBrJn16-500704, 16.6.2020): $\delta=-5.4$ (3'-O-Si$\left.\mathrm{CH}_{3}{ }^{\mathrm{B}}\right),-4.4\left(3^{\prime}-\mathrm{O}-\mathrm{Si}_{-}-\mathrm{CH}_{3}{ }^{\mathrm{A}}\right),-4.3$ [1"-O-Si- $\left.\left(\mathrm{CH}_{3}\right)_{2}\right], 11.7(\mathrm{C}-12 '), 12.6\left(6\right.$ '- $\left.\mathrm{CH}_{3}\right), 18.9$ [5-CH-O$\left.\mathrm{Si}-\mathrm{C}\left(\mathrm{CH}_{3}\right)_{3}\right], 19.1$ [1"-O-Si- $\left.\mathrm{C}\left(\mathrm{CH}_{3}\right)_{3}\right], 19.5\left(10^{\prime}-\mathrm{CH}_{3}\right), 21.5\left(8^{\prime}-\mathrm{CH}_{3}\right), 26.1$ [5-CH-O-Si$\mathrm{C}\left(\mathrm{CH}_{3}\right)_{3}$ ], 26.2 [1"-O-Si-C $\left(\mathrm{CH}_{3}\right)_{3}$ ], 31.2 (C-11'), 32.1 (C-8'), 33.7 (C-10'), $45.8(\mathrm{C}-9$ '), 70.3 (C5), 74.5 (5-CH), 121.1 (C-2", C-6", C-2'), 126.2 (C-4'), 128.6 (C-3", C-5"), 134.3 (C-6'), 135.4 (C-4"), 147.1 (C-3'), 148.2 (C-7'), 150.2 (C-5'), 156.7 (C-1") ppm.*

${ }^{*} \mathrm{C}-2, \mathrm{C}-3, \mathrm{C}-4$ and $\mathrm{C}-1$ ' were not found.

edHSQC [,,short-range H,C-COSY“, 500.06 MHz/125.75 MHz, MeOD; $\delta\left({ }^{1} \mathrm{H}\right) \leftrightarrow \delta\left({ }^{13} \mathrm{C}\right)$ : $0.22\left(\mathrm{~s}, 3^{\prime}-\mathrm{O}-\mathrm{Si}-\mathrm{CH}_{3}{ }^{\mathrm{A}}\right) \leftrightarrow-4.4\left(3^{\prime}-\mathrm{O}-\mathrm{Si}^{-} \mathrm{CH}_{3}{ }^{\mathrm{A}}\right),-0.07\left(\mathrm{~s}, 3^{\prime}-\mathrm{O}-\mathrm{Si}^{-} \mathrm{CH}_{3}{ }^{\mathrm{B}}\right), \leftrightarrow-5.4$ (3'-O-Si$\left.\mathrm{CH}_{3}{ }^{\mathrm{B}}\right), 0.19$ [s, 1"-O-Si- $\left.\left(\mathrm{CH}_{3}\right)_{2}\right] \leftrightarrow-4.3$ [1"-O-Si- $\left.\left(\mathrm{CH}_{3}\right)_{2}\right], 0.78\left[\mathrm{~s}, 5-\mathrm{CH}-\mathrm{O}-\mathrm{Si}-\mathrm{C}\left(\mathrm{CH}_{3}\right)_{3}\right] \leftrightarrow 26.1$ [5-CH-O-Si-C $\left.\left(\mathrm{CH}_{3}\right)_{3}\right], 0.85\left(\mathrm{~d}, 10^{\prime}-\mathrm{CH}_{3}\right) \leftrightarrow 19.5\left(10^{\prime}-\mathrm{CH}_{3}\right), 0.86\left(\mathrm{t}, 12^{\prime}-\mathrm{H}_{3}\right) \leftrightarrow 11.7\left(\mathrm{C}-12^{\prime}\right)$, $0.98\left(\mathrm{~d}, 8^{\prime}-\mathrm{CH}_{3}\right) \leftrightarrow 21.5\left(8^{\prime}-\mathrm{CH}_{3}\right), 0.99$ [s, 1"-O-Si-C $\left.\left(\mathrm{CH}_{3}\right)_{3}\right] \leftrightarrow 26.2$ [1"-O-Si-C $\left.\left(\mathrm{CH}_{3}\right)_{3}\right], 1.10-$ $1.20\left(\mathrm{~m}, 9^{\prime}-\mathrm{H}^{\mathrm{A}}\right) \leftrightarrow 45.8\left(\mathrm{C}-9^{\prime}\right), 1.10-1.20\left(\mathrm{~m}, 11^{\prime}-\mathrm{H}^{\mathrm{A}}\right) \leftrightarrow 31.2\left(\mathrm{C}-11^{\prime}\right), 1.23-1.39\left(\mathrm{~m}, 10^{\prime}-\mathrm{H}\right) \leftrightarrow$ $33.7\left(\mathrm{C}-10^{\prime}\right), 1.23-1.39\left(\mathrm{~m}, 9^{\prime}-\mathrm{H}^{\mathrm{B}}\right) \leftrightarrow 45.8\left(\mathrm{C}-9^{\prime}\right), 1.23-1.39\left(\mathrm{~m}, 11^{\prime}-\mathrm{H}^{\mathrm{B}}\right) \leftrightarrow 31.2\left(\mathrm{C}-11^{\prime}\right), 1.87$ $\left(\mathrm{s}, 6^{\prime}-\mathrm{CH}_{3}\right) \leftrightarrow 12.6\left(6^{\prime}-\mathrm{CH}_{3}\right), 2.71\left(\mathrm{~m}_{\mathrm{c}}, 8^{\prime}-\mathrm{H}\right) \leftrightarrow 32.1\left(\mathrm{C}-8^{\prime}\right), 3.87$ (br. s, 5-H) $(\mathrm{s}, 5-\mathrm{CH}) \leftrightarrow 74.5(5-\mathrm{CH}), 5.60\left(\mathrm{~d}, 7^{\prime}-\mathrm{H}\right) \leftrightarrow 148.2\left(\mathrm{C}-7^{\prime}\right), 6.48\left(\mathrm{dd}, 4^{\prime}-\mathrm{H}\right) \leftrightarrow 126.2\left(\mathrm{C}-4^{\prime}\right), 6.79$ $\left(\mathrm{d}, 5^{\prime}-\mathrm{H}\right) \leftrightarrow 150.2\left(\mathrm{C}-5^{\prime}\right), 6.82-6.87$ (m, 2"-H, 6"-H) 121.1 (C-2", C-6"), 7.19 (d, 2'-H) $121.1\left(\mathrm{C}-2^{\prime}\right), 7.25-7.30\left(\mathrm{~m}, 3^{\prime \prime}-\mathrm{H}, 5^{\prime \prime}-\mathrm{H}\right) \leftrightarrow 128.6$ (C-3", C-5"), 7.59 (dd, 3'-H) 147.0 (C-3'). 
Christian Drescher and Reinhard Brückner; 3D-Structure Clarifying Total Synthesis of the (Polyenoyl)tetramic Acid Militarinon B. A Highly Acid-Labile N-Protecting Group for Amides

Specific rotation $(R, S, R, R)-28[\alpha]_{D}^{20}=-280.0^{\circ}\left(\mathrm{c}=0.21\right.$ in $\left.\mathrm{CHCl}_{3}\right)$

HRMS: (EI, $70 \mathrm{eV}): \mathrm{m} / \mathrm{z}=668.4154[\mathrm{M}+\mathrm{H}]^{+}$, corresponds to the molecular formula $\mathrm{C}_{38} \mathrm{H}_{62} \mathrm{O}_{5} \mathrm{NSi}_{2}(668.4154)\left\{[\mathrm{M}+\mathrm{H}]^{+}\right\}$with a deviation of $-1.0 \mathrm{ppm}$.

IR (KBr): $\tilde{v}=2960,2930,2900,2855,1695,1660,1625,1610,1595,1560,1510,1470,1460$, $1435,1390,1375,1360,1260,1200,1165,1110,1075,1000,915,840,810,780 \mathrm{~cm}^{-1}$.

(5S)-5-[(S)-Hydroxy(1'-hydroxyphenyl)methyl]-3-[(1'Z,2' $\left.E, 4^{\prime} E, 6^{\prime} E, 8^{\prime} R, 10 ' R\right)-1^{\prime}-$ hydroxy-6',8',10'-trimethyldodeca-2',4',6' -trien-1'-ylidene]pyrrolidine-2,4-dione [Z-enol$(S, S, R, R)-3]$

in a $77: 23$ mixture with

(5S)-5-[(S)-Hydroxy(1'-hydroxyphenyl)methyl]-3-[(1'E,2' $\left.E, 4^{\prime} E, 6^{\prime} E, 8^{\prime} R, 10 ' R\right)-1^{\prime}-$ hydroxy-6',8',10'-trimethyldodeca-2',4',6' -trien-1'-ylidene]pyrrolidine-2,4-dione [E-enol$(S, S, R, R)-3]$

separated from

(5S)-5-\{(S)-[(tert-Butyldimethylsilyl)oxy](1"'-hydroxyphenyl)methyl $\}-3-$ [(1'Z,2' $\left.E, 4^{\prime} E, 6^{\prime} E, 8^{\prime} R, 10^{\prime} R\right)-1^{\prime}-$ hydroxy-6',8',10'-trimethyldodeca-2',4',6'-trien-1'ylidene]pyrrolidine-2,4-dione [Z-enol-(S,S,R,R)-29] in a 67:33 mixture with

(5S)-5-\{(S)-[(tert-Butyldimethylsilyl)oxy](1"-hydroxyphenyl)methyl $\}-3-$ [(1'E,2' $\left.E, 4^{\prime} E, 6^{\prime} E, 8^{\prime} R, 10^{\prime} R\right)-1^{\prime}$-hydroxy-6' $, 8^{\prime}, 10^{\prime}$-trimethyldodeca-2',4',6'-trien-1'ylidene]pyrrolidine-2,4-dione [E-enol-(S,S,R,R)-29] 

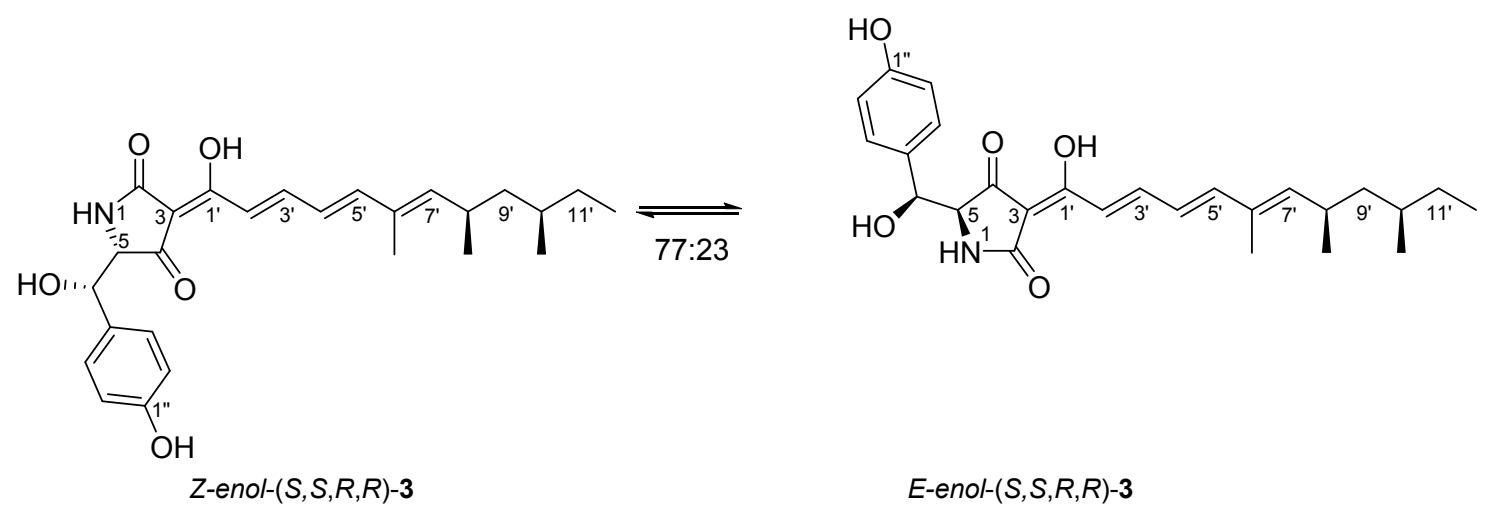

separated from

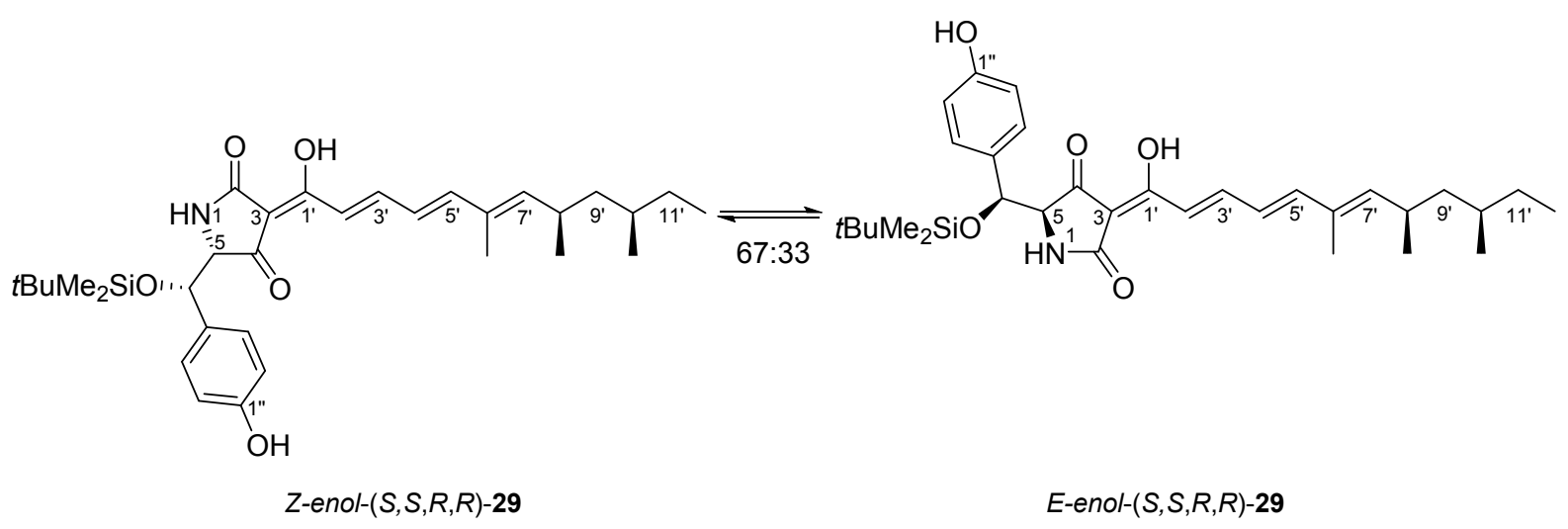

Method A: A solution of the tetramic acid $(S, S, R, R)-28(4.0 \mathrm{mg}, 6.0 \mu \mathrm{mol})$ in THF $(2.0 \mathrm{~mL})$ was treated with HOAc $(62 \mu \mathrm{L}, 216 \mu \mathrm{mol}, 36$ eq. $)$ and TBAF (1 M in THF, $72 \mu \mathrm{L}, 72 \mu \mathrm{mol}, 12$ eq.) at $0^{\circ} \mathrm{C}$. The mixture was warmed to $10^{\circ} \mathrm{C}$ and held this temperature for $3 \mathrm{~d}$. It was added aq. citric acid ( 5 weight- $\%, 3 \mathrm{~mL}$ ) and $\mathrm{Et}_{2} \mathrm{O}(10 \mathrm{~mL})$. The organic layer was separated, and the aq. layer was extracted with $\mathrm{Et}_{2} \mathrm{O}(3 \times 10 \mathrm{~mL})$. The organic layers were tried over $\mathrm{Na}_{2} \mathrm{SO}_{4}$ and the solvent was removed under reduced pressure. The residue was purified reverse phase flash chromatography (MeCN: $\left.\mathrm{H}_{2} \mathrm{O}: \mathrm{HCO}_{2} \mathrm{H}=85: 15: 0.1,1.5 \mathrm{~cm}, 10 \mathrm{~cm}, 2 \mathrm{~mL}\right)$. The product $(S, S, R, R)-3$ [0.9 mg, 35\%, $2.1 \mu \mathrm{mol}, d r=98: 2$ (analyzed by HPLC)] was obtained as a yellow powder (mp.: $\left.129-133^{\circ} \mathrm{C}\right)$. The byproduct $(S, S, R, R)-29(1.5 \mathrm{mg}, 30 \%, 1.8 \mu \mathrm{mol})$ was also obtained as a yellow solid. $(S, S, R, R)-\mathbf{3}$ was assigned as a 77:23 mixture of the $Z$ - and the $E$ enol- form. This ratio was determined by integrating dd's of the respective 3'-H [7.36 (E-enol) and $7.49(Z$-enol)]. (S,S,R,R)-29 was assigned as a 67:33 mixture of the $Z$ - and the $E$-enol- form. This ratio was determined by integrating dd's of the respective 3'-H [7.37 (E-enol) and 7.50 (Zenol)].

Method B : A solution of tetramic acid $(S, S, R, R)-29(1.5 \mathrm{mg}, 1.8 \mu \mathrm{mol})$ in THF $(0.7 \mathrm{~mL})$ was treated with HOAc (19 $\mu \mathrm{L}, 65 \mu \mathrm{mol}, 36$ eq.) and TBAF ( $1 \mathrm{M}$ in THF, $22 \mu \mathrm{L}, 22 \mu \mathrm{mol}, 12$ eq.) at $0^{\circ} \mathrm{C}$. The mixture was warmed to $10^{\circ} \mathrm{C}$ and held this temperature for $3 \mathrm{~d}$. It was added aq. 
Christian Drescher and Reinhard Brückner; 3D-Structure Clarifying Total Synthesis of the (Polyenoyl)tetramic Acid Militarinon B. A Highly Acid-Labile N-Protecting Group for Amides

citric acid (5 weight-\%, $2 \mathrm{~mL}$ ) and $\mathrm{Et}_{2} \mathrm{O}(5 \mathrm{~mL})$. The organic layer was separated, and the aq. layer was extracted with $\mathrm{Et}_{2} \mathrm{O}(3 \times 5 \mathrm{~mL})$. The organic layers were tried over $\mathrm{Na}_{2} \mathrm{SO}_{4}$ and the solvent was removed under reduced pressure. The residue was purified reverse phase flash chromatography (MeCN: $\left.\mathrm{H}_{2} \mathrm{O}: \mathrm{HCO}_{2} \mathrm{H}=85: 15: 0.1,1.5 \mathrm{~cm}, 10 \mathrm{~cm}, 2 \mathrm{~mL}\right)$. The product $(S, S, R, R)-3$ [0.3 mg, 25\%, $2.1 \mu \mathrm{mol}, d r=98: 2$ (analyzed by HPLC)] was obtained as a yellow powder. $(S, S, R, R)-\mathbf{3}$ was assigned as a 77:23 mixture of the $Z$ - and the $E$-enol-form. This ratio was determined by integrating dd's of the respective 3'-H [7.36 (E-enol) and 7.49 (Z-enol)].

$(S, S, R, R)-3:^{15}$

$\mathbf{R}_{\mathbf{f}}\left(\mathrm{MeCN}: \mathrm{H}_{2} \mathrm{O}: \mathrm{HCO}_{2} \mathrm{H}=85: 15: 0.1\right)=0.43$

${ }^{1} \mathbf{H}$-NMR $\left(500.06 \mathrm{MHz}, \mathrm{MeOD} / \mathrm{MeOH}\right.$, sample contained 2 weight-\% of $\mathrm{CH}_{2} \mathrm{Cl}_{2}, \mathrm{DsBrJ} 110$ 500700, 13.7.2020): $\delta=0.83-0.88\left(\mathrm{~m}, 3 \mathrm{H}, 10^{\prime}-\mathrm{CH}_{3}\right), 0.80-0.87\left(\mathrm{~m}, 3 \mathrm{H}, 12^{\prime}-\mathrm{H}_{3}\right), 0.97\left(\mathrm{~d}, J_{8^{\prime}-}\right.$ $\left.\mathrm{CH}_{3}, 8^{\prime}=6.6 \mathrm{~Hz}, 3 \mathrm{H}, 8^{\prime}-\mathrm{CH}_{3}\right), 1.10-1.18\left(\mathrm{~m}, 1 \mathrm{H}, 9^{\prime}-\mathrm{H}^{\mathrm{A}}\right), 1.10-1.18\left(\mathrm{~m}, 1 \mathrm{H}, 11^{\prime}-\mathrm{H}^{\mathrm{A}}\right), 1.22-1.37(\mathrm{~m}$, $\left.1 \mathrm{H}, 10^{\prime}-\mathrm{H}\right), 1.22-1.37\left(\mathrm{~m}, 1 \mathrm{H}, 9^{\prime}-\mathrm{H}^{\mathrm{B}}\right), 1.22-1.37\left(\mathrm{~m}, 1 \mathrm{H}, 11^{\prime}-\mathrm{H}^{\mathrm{B}}\right), 1.85\left(\mathrm{~d}, J_{6^{\prime}-\mathrm{CH}_{3}, 7^{\prime}}=1.0 \mathrm{~Hz}, 3 \mathrm{H}\right.$, 6'- $\left.\mathrm{CH}_{3}\right), 2.70\left(\mathrm{~m}_{\mathrm{c}}, 1 \mathrm{H}, 8^{\prime}-\mathrm{H}\right), 4.21$ (br. s, 1H, 5-H), 4.98 (d, J5- $\left.{ }_{5 \mathrm{H}, 5}=3.8 \mathrm{~Hz}, 1 \mathrm{H}, 5-\mathrm{CH}\right), 5.58$ $\left(\mathrm{d}, J_{7^{\prime}, 8^{\prime}}=9.9 \mathrm{~Hz}, 1 \mathrm{H}, 7^{\prime}-\mathrm{H}\right), 6.43\left(\mathrm{dd}, J_{4^{\prime}, 3^{\prime}}=11.2 \mathrm{~Hz}, J_{4^{\prime}, 5^{\prime}}=15.1 \mathrm{~Hz}, 1 \mathrm{H}, 4^{\prime}-\mathrm{H}\right), 6.64-6.68(\mathrm{~m}$,

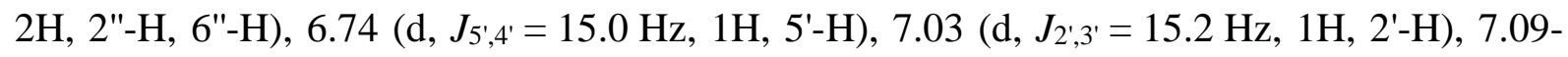
$7.13\left(\mathrm{~m}, 2 \mathrm{H}, 3^{\prime \prime}-\mathrm{H}, 5^{\prime \prime}-\mathrm{H}\right), 7.49$ (dd, $J_{3^{\prime}, 4^{\prime}}=11.3 \mathrm{~Hz}, J_{3^{\prime}, 2^{\prime}}=15.0 \mathrm{~Hz}, 1 \mathrm{H}, 3$ '-H) ppm.

${ }^{13}$ C-NMR (125.75 MHz, MeOD/MeOH, DsBrJl10-500704, 13.7.2020): $\delta=11.7$ (C-12'), 12.6 (6'- $\left.\mathrm{CH}_{3}\right), 19.5\left(10^{\prime}-\mathrm{CH}_{3}\right), 21.5\left(8^{\prime}-\mathrm{CH}_{3}\right), 31.2\left(\mathrm{C}-11^{\prime}\right), 32.1$ (C-8'), $33.7(\mathrm{C}-10 '), 45.9\left(\mathrm{C}-9^{\prime}\right), 74.9$ (5-CH), 115.6 (C-2", C-6"), 126.3 (C-4'), 129.6 (C-3", C-5"), 130.7 (C-4"), 134.3 (C-6'), 158.2 (C-1") ppm.*

${ }^{*} \mathrm{C}-2, \mathrm{C}-3, \mathrm{C}-4, \mathrm{C}-5, \mathrm{C}-1$ ', C-2', C-3', C-5' and C-7' were not found.

edHSQC [,,short-range H,C-COSY“, 500.10 MHz/125.75 MHz, MeOD; $\delta\left({ }^{1} \mathrm{H}\right) \leftrightarrow \delta\left({ }^{13} \mathrm{C}\right)$ : 0.80-0.87 (m, 12'- $\left.\mathrm{H}_{3}\right) \leftrightarrow 11.7\left(\mathrm{C}-12^{\prime}\right), 0.83-0.88\left(\mathrm{~m}, 10^{\prime}-\mathrm{CH}_{3}\right) \leftrightarrow 19.5\left(10^{\prime}-\mathrm{CH}_{3}\right), 0.97$ (d, 8'$\left.\mathrm{CH}_{3}\right) \leftrightarrow 21.5\left(8^{\prime}-\mathrm{CH}_{3}\right), 1.10-1.18\left(\mathrm{~m}, 9^{\prime}-\mathrm{H}^{\mathrm{A}}\right) \leftrightarrow 45.9\left(\mathrm{C}-9^{\prime}\right), 1.10-1.18\left(\mathrm{~m}, 11^{\prime}-\mathrm{H}^{\mathrm{A}}\right) \leftrightarrow 31.2(\mathrm{C}-$ $\left.11^{\prime}\right), 1.22-1.37\left(\mathrm{~m}, 10^{\prime}-\mathrm{H}\right) \leftrightarrow 33.7\left(\mathrm{C}-10^{\prime}\right), 1.22-1.37\left(\mathrm{~m}, 9^{\prime}-\mathrm{H}^{\mathrm{B}}\right) \leftrightarrow 45.9\left(\mathrm{C}-9^{\prime}\right), 1.22-1.37(\mathrm{~m}$, $\left.11^{\prime}-\mathrm{H}^{\mathrm{B}}\right) \leftrightarrow 31.2\left(\mathrm{C}-11^{\prime}\right), 1.85\left(\mathrm{~d}, 6^{\prime}-\mathrm{CH}_{3}\right) \leftrightarrow 12.6\left(6^{\prime}-\mathrm{CH}_{3}\right), 2.70\left(\mathrm{~m}_{\mathrm{c}}, 8^{\prime}-\mathrm{H}\right) \leftrightarrow 32.1\left(\mathrm{C}-8^{\prime}\right), 4.98$ $(\mathrm{d}, 5-\mathrm{CH}) \leftrightarrow 74.9(5-\mathrm{CH}), 6.43\left(\mathrm{dd}, 4^{\prime}-\mathrm{H}\right) \leftrightarrow 126.3\left(\mathrm{C}-44^{\prime}\right), 6.64-6.68(\mathrm{~m}, 2 "-\mathrm{H}, 6$ "'-H) (C-2", C-6"), 7.09-7.13 (m, 3"-H, 5"-H) ↔ 129.6 (C-3", C-5").

\footnotetext{
${ }^{15}$ The analytical data are consistent with the literature. ${ }^{14}$
} 
Christian Drescher and Reinhard Brückner; 3D-Structure Clarifying Total Synthesis of the (Polyenoyl)tetramic Acid Militarinon B. A Highly Acid-Labile N-Protecting Group for Amides

Specific rotation $(S, S, R, R)-3[\alpha]_{D}^{20}=-271.0^{\circ}\left(\mathrm{c}=0.05\right.$ in $\left.\mathrm{CHCl}_{3}\right)$

HRMS: $(\mathrm{EI}, 70 \mathrm{eV}): \mathrm{m} / \mathrm{z}=438.2285[\mathrm{M}-\mathrm{H}]^{+}$, corresponds to the molecular formula $\mathrm{C}_{26} \mathrm{H}_{32} \mathrm{O}_{5} \mathrm{~N}$ (438.2286) $\left\{[\mathrm{M}-\mathrm{H}]^{+}\right\}$with a deviation of $-0.3 \mathrm{ppm}$.

IR (KBr): $\tilde{v}=2960,2925,2875,1650,1595,1550,1520,1430,1375,1315,1280,1230,1170$, $1110,1080,1045,1020,1000,955,865,835,795,730 \mathrm{~cm}^{-1}$.

$(S, S, R, R)-29:$

$\mathbf{R}_{\mathbf{f}}\left(\mathrm{MeCN}: \mathrm{H}_{2} \mathrm{O}: \mathrm{HCO}_{2} \mathrm{H}=85: 15: 0.1\right)=0.12$

${ }^{1}$ H-NMR (500.06 MHz, MeOD/MeOH, DsBrFe08-500500, 10.2.2021): $\delta=-0.07$ (s, 3H, 5$\mathrm{CH}-\mathrm{O}-\mathrm{Si}_{-} \mathrm{CH}_{3}{ }^{\mathrm{A}}$ ), 0.07 (s, 3H, 5-CH-O-Si-CH${ }_{3}{ }^{\mathrm{A}}$ ), 0.83-0.87 (m, 6H, 10'- $\mathrm{CH}_{3}, 12$ '- $-\mathrm{H}_{3}$ ), 0.88 [s, 9H, 5-CH-O-Si-C $\left.\left(\mathrm{CH}_{3}\right)_{3}\right], 0.97\left(\mathrm{~d}, J_{8^{\prime}-\mathrm{CH}_{3}, 8^{\prime}}=6.6 \mathrm{~Hz}, 3 \mathrm{H}, 8^{\prime}-\mathrm{CH}_{3}\right), 1.10-1.19\left(\mathrm{~m}, 1 \mathrm{H}, 9^{\prime}-\mathrm{H}^{\mathrm{A}}\right)$, 1.10-1.19 (m, 1H, 11'- $\left.\mathrm{H}^{\mathrm{A}}\right), 1.22-1.37\left(\mathrm{~m}, 1 \mathrm{H}, 10^{\prime}-\mathrm{H}\right), 1.22-1.37\left(\mathrm{~m}, 1 \mathrm{H}, 9^{\prime}-\mathrm{H}^{\mathrm{B}}\right), 1.22-1.37(\mathrm{~m}$, $\left.1 \mathrm{H}, 11^{\prime}-\mathrm{H}^{\mathrm{B}}\right), 1.85\left(\mathrm{~d}, J_{6^{\prime}}-\mathrm{CH}_{3}, 7^{\prime}=6.6 \mathrm{~Hz}, 3 \mathrm{H}, 6^{\prime}-\mathrm{CH}_{3}\right), 2.69\left(\mathrm{~m}_{\mathrm{c}}, 1 \mathrm{H}, 8^{\prime}-\mathrm{H}\right), 4.13$ (br. s, 1H, 5-H), $5.07\left(\mathrm{~d}, J_{5-\mathrm{CH}, 5}=3.0 \mathrm{~Hz}, 1 \mathrm{H}, 5-\mathrm{CH}\right), 5.58\left(\mathrm{~d}, J_{7^{\prime}, 8^{\prime}}=9.8 \mathrm{~Hz}, 1 \mathrm{H}, 7^{\prime}-\mathrm{H}\right), 6.43\left(\mathrm{dd}, J_{4^{\prime}, 3^{\prime}}=11.3 \mathrm{~Hz}\right.$, $\left.J_{4^{\prime}, 5^{\prime}}=15.1 \mathrm{~Hz}, 1 \mathrm{H}, 4^{\prime}-\mathrm{H}\right), 6.63-6.68\left(\mathrm{~m}, 2 \mathrm{H}, 2^{\prime \prime}-\mathrm{H}, 66^{\prime \prime}-\mathrm{H}\right), 6.75\left(\mathrm{~d}, J_{5^{\prime}, 4^{\prime}}=15.2 \mathrm{~Hz}, 1 \mathrm{H}, 5^{\prime}-\mathrm{H}\right)$, $7.03\left(\mathrm{~d}, J_{2^{\prime}, 3^{\prime}}=15.3 \mathrm{~Hz}, 1 \mathrm{H}, 2^{\prime}-\mathrm{H}\right), 7.06-7.11\left(\mathrm{~m}, 2 \mathrm{H}, 3^{\prime \prime}-\mathrm{H}, 5^{\prime \prime}-\mathrm{H}\right), 7.49$ (dd, $J_{3^{\prime}, 4^{\prime}}=11.4 \mathrm{~Hz}$, $\left.J_{3^{\prime}, 2^{\prime}}=15.1 \mathrm{~Hz}, 1 \mathrm{H}, 3^{\prime}-\mathrm{H}\right) \mathrm{ppm}$.

${ }^{13}$ C-NMR (125.75 MHz, MeOD/MeOH, DsBrFe08-500504, 10.2.2021): $\delta=-4.6$ (5-CH-O-Si$\left.\mathrm{CH}_{3}{ }^{\mathrm{A}}\right),-5.0\left(5-\mathrm{CH}-\mathrm{O}-\mathrm{Si}-\mathrm{CH}_{3}{ }^{\mathrm{B}}\right), 11.6\left(\mathrm{C}-12^{\prime}\right), 12.6\left(6{ }^{\prime}-\mathrm{CH}_{3}\right), 19.1$ [5-CH-O-Si-C $\left(\mathrm{CH}_{3}\right)_{3}$ ], 19.5 $\left(10^{\prime}-\mathrm{CH}_{3}\right), 21.5\left(8^{\prime}-\mathrm{CH}_{3}\right), 26.3$ [5-CH-O-Si-C $\left(\mathrm{CH}_{3}\right)_{3}$ ], $31.2\left(\mathrm{C}-11\right.$ '), $32.1\left(\mathrm{C}-8^{\prime}\right), 33.7\left(\mathrm{C}-10^{\prime}\right)$, 45.8 (C-9'), 70.8 (C-5), 76.4 (5-CH), 115.5 (C-2", C-6"), 120.9 (C-2'), 126.3 (C-4'), 129.6 (C3", C-5"), 134.3 (C-6'), 158.2 (C-1") ppm.*

${ }^{*} \mathrm{C}-2, \mathrm{C}-3, \mathrm{C}-4, \mathrm{C}-1^{\prime}, \mathrm{C}-3$ ', C-5' and C-7' were not found.

edHSQC [,,short-range H,C-COSY“, 500.10 MHz/125.75 MHz, MeOD; $\delta\left({ }^{1} \mathrm{H}\right) \leftrightarrow \delta\left({ }^{13} \mathrm{C}\right)$ : $0.07\left(\mathrm{~s}, 5-\mathrm{CH}-\mathrm{O}-\mathrm{Si}-\mathrm{CH}_{3}{ }^{\mathrm{A}}\right) \leftrightarrow-4.6\left(5-\mathrm{CH}-\mathrm{O}-\mathrm{Si}_{-} \mathrm{CH}_{3}{ }^{\mathrm{A}}\right), 0.07$ (s, 5-CH-O-Si-CH$\left.{ }^{\mathrm{A}}\right) \leftrightarrow-5.0(5-$ CH-O-Si-CH $\left.{ }_{3}{ }^{\mathrm{B}}\right), 0.83-0.87\left(\mathrm{~m}, 12^{\prime}-\mathrm{H}_{3}\right) \leftrightarrow 11.6\left(\mathrm{C}-12^{\prime}\right), 0.83-0.87\left(\mathrm{~m}, 10^{\prime}-\mathrm{CH}_{3}\right) \leftrightarrow 19.5\left(10^{\prime}-\right.$ $\left.\mathrm{CH}_{3}\right), 0.88$ [s, 5-CH-O-Si-C $\left(\mathrm{CH}_{3}\right)_{3}$ ] $\left.\mathrm{CH}_{3}\right), 1.10-1.19\left(\mathrm{~m}, 9^{\prime}-\mathrm{H}^{\mathrm{A}}\right) \leftrightarrow 45.8\left(\mathrm{C}-9^{\prime}\right), 1.10-1.19\left(\mathrm{~m}, 11^{\prime}-\mathrm{H}^{\mathrm{A}}\right) \leftrightarrow 31.2\left(\mathrm{C}-11^{\prime}\right), 1.22-1.37(\mathrm{~m}$, $\left.10^{\prime}-\mathrm{H}\right) \leftrightarrow 33.7\left(\mathrm{C}-10^{\prime}\right), 1.22-1.37\left(\mathrm{~m}, 9^{\prime}-\mathrm{H}^{\mathrm{B}}\right) \leftrightarrow 45.8\left(\mathrm{C}-9^{\prime}\right), 1.22-1.37\left(\mathrm{~m}, 11^{\prime}-\mathrm{H}^{\mathrm{B}}\right) \leftrightarrow 31.2(\mathrm{C}-$ $\left.11^{\prime}\right), 1.85\left(\mathrm{~d}, 6^{\prime}-\mathrm{CH}_{3}\right) \leftrightarrow 12.6\left(6^{\prime}-\mathrm{CH}_{3}\right), 2.69\left(\mathrm{~m}_{\mathrm{c}}, 8^{\prime}-\mathrm{H}\right) \leftrightarrow 32.1\left(\mathrm{C}-8^{\prime}\right), 4.13$ (br. s, 5-H) 70.8 (C-5), $5.07(\mathrm{~d}, 5-\mathrm{CH}) \leftrightarrow 76.4(5-\mathrm{CH}), 6.43\left(\mathrm{dd}, 4^{\prime}-\mathrm{H}\right) \leftrightarrow 126.3(\mathrm{C}-4$ '), 6.63-6.68 (m, 2"-H, 6"- 
Christian Drescher and Reinhard Brückner; 3D-Structure Clarifying Total Synthesis of the (Polyenoyl)tetramic Acid Militarinon B. A Highly Acid-Labile N-Protecting Group for Amides

H) 115.5 (C-2", C-6"), $7.03\left(\mathrm{~d}, 2^{\prime}-\mathrm{H}\right) \leftrightarrow 120.9$ (C-2'), 7.06-7.11 (m, 3"-H, 5"-H) ↔ 129.6 (C-3", C-5").

HRMS: (EI, $70 \mathrm{eV}): \mathrm{m} / \mathrm{z}=552.3154[\mathrm{M}-\mathrm{H}]^{+}$, corresponds to the molecular formula $\mathrm{C}_{32} \mathrm{H}_{46} \mathrm{O}_{5} \mathrm{NSi}(552.3151)\left\{[\mathrm{M}-\mathrm{H}]^{+}\right\}$with a deviation of $0.5 \mathrm{ppm}$.

Specific rotation $(S, S, R, R)-\mathbf{2 9}[\alpha]_{D}^{20}=-571.0^{\circ}\left(\mathrm{c}=0.04\right.$ in $\left.\mathrm{CHCl}_{3}\right)$

IR (KBr): $\tilde{v}=3240,2960,2925,2855,1655,1595,1555,1515,1430,1250,1170,1085,1000$, $875,835,780,660 \mathrm{~cm}^{-1}$.

(5S)-5-[(R)-Hydroxy(1'-hydroxyphenyl)methyl]-3-[(1'Z,2' $\left.E, 4^{\prime} E, 6^{\prime} E, 8^{\prime} R, 10 ' R\right)-1^{\prime}-$ hydroxy-6',8',10'-trimethyldodeca-2',4',6' -trien-1'-ylidene]pyrrolidine-2,4-dione [Z-enol-

$$
(R, S, R, R)-3]
$$

in an unknown mixture with

(5S)-5-[(R)-Hydroxy(1'-hydroxyphenyl)methyl]-3-[(1'E,2' $\left.E, 4^{\prime} E, 6^{\prime} E, 8^{\prime} R, 10^{\prime} R\right)-1^{\prime}-$ hydroxy-6',8',10'-trimethyldodeca-2',4',6'-trien-1'-ylidene]pyrrolidine-2,4-dione [E-enol$(R, S, R, R)-3]$

separated from

(5S)-5-\{(R)-[(tert-Butyldimethylsilyl)oxy](1"'-hydroxyphenyl)methyl $\}-3-$ [(1'Z,2' $\left.E, 4^{\prime} E, 6^{\prime} E, 8^{\prime} R, 10^{\prime} R\right)-1^{\prime}$-hydroxy-6',8',10'-trimethyldodeca-2',4',6'-trien-1'ylidene]pyrrolidine-2,4-dione [Z-enol-(R,S,R,R)-29] in a 69:31 mixture with

(5S)-5-\{(R)-[(tert-Butyldimethylsilyl)oxy](1"'-hydroxyphenyl)methyl $\}-3-$ [(1'E,2' $\left.E, 4^{\prime} E, 6^{\prime} E, 8^{\prime} R, 10^{\prime} R\right)-1^{\prime}$-hydroxy-6',8',10'-trimethyldodeca-2',4',6'-trien-1'ylidene]pyrrolidine-2,4-dione $[E$-enol-(R,S,R,R)-29] 


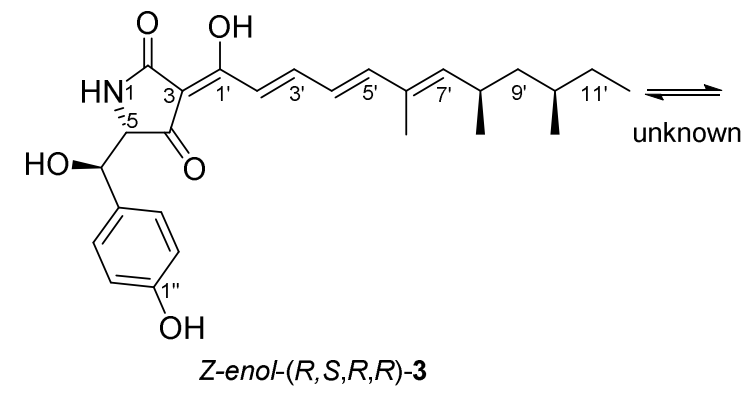

separated from<smiles>CCC(C)CC(C)/C=C(C)/C=C/C=C/C(O)=C1C(=O)NC([C@H](O)c2ccc(O)cc2)C1=O</smiles>

E-enol-(R,S,R,R)-3

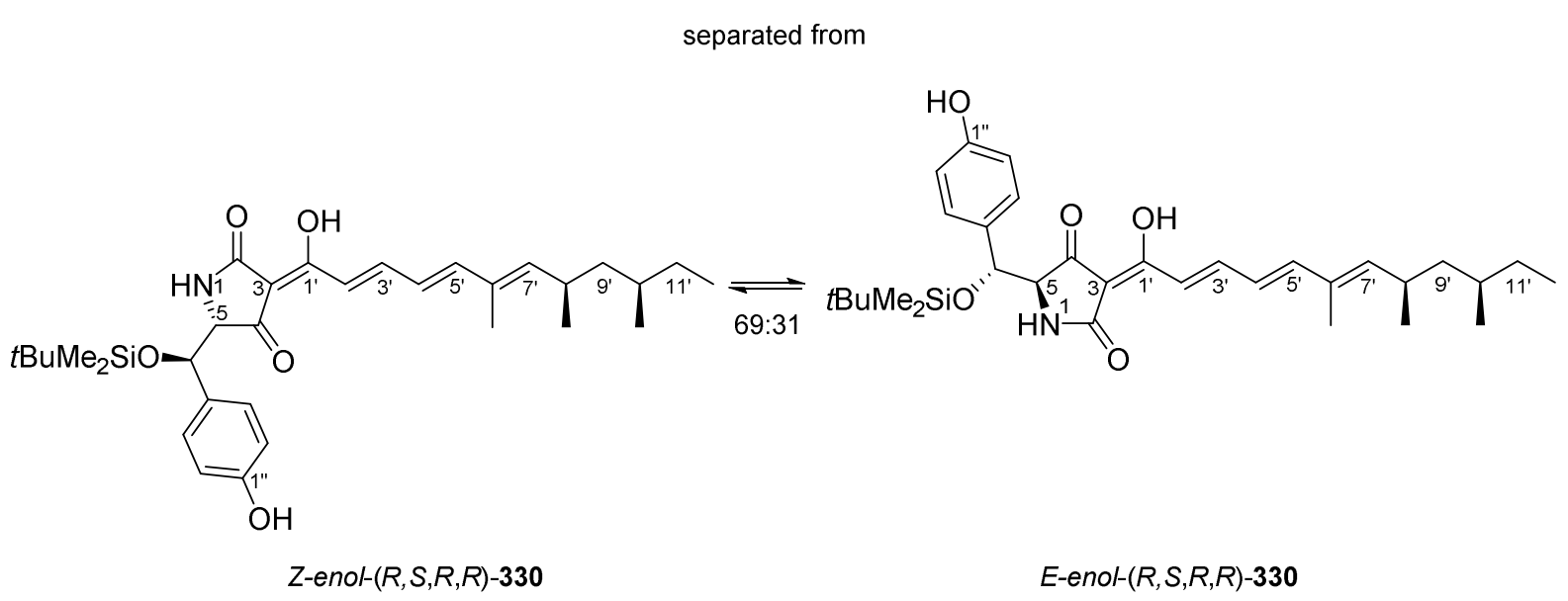

Method A: A solution of the tetramic acid $(R, S, R, R)-28(8.0 \mathrm{mg}, 12.0 \mu \mathrm{mol})$ in THF $(4.0 \mathrm{~mL})$ was treated with HOAc $(124 \mu \mathrm{L}, 216 \mu \mathrm{mol}, 36$ eq.) and TBAF (1 M in THF, $144 \mu \mathrm{L}, 72 \mu \mathrm{mol}$, 12 eq.) at $0^{\circ} \mathrm{C}$. The mixture was warmed to $25^{\circ} \mathrm{C}$ and held this temperature for $40 \mathrm{~h}$. It was added aq. citric acid (5 weight- $\%, 5 \mathrm{~mL})$ and $\mathrm{Et}_{2} \mathrm{O}(10 \mathrm{~mL})$. The organic layer was separated, and the aq. layer was extracted with $\mathrm{Et}_{2} \mathrm{O}(3 \times 10 \mathrm{~mL})$. The organic layers were tried over $\mathrm{Na}_{2} \mathrm{SO}_{4}$ and the solvent was removed under reduced pressure. The residue was purified reverse phase flash chromatography ( $\left.\mathrm{MeCN}: \mathrm{H}_{2} \mathrm{O}: \mathrm{HCO}_{2} \mathrm{H}=85: 15: 0.1,1.5 \mathrm{~cm}, 10 \mathrm{~cm}, 2 \mathrm{~mL}\right)$. The product $(R, S, R, R)-3$ [0.25 mg, 5\%, $0.6 \mu \mathrm{mol}, d r=61: 39$ (analyzed by HPLC)] was obtained as a yellow oil. The byproduct $(R, S, R, R)-29(5.4 \mathrm{mg}, 54 \%, 6.5 \mu \mathrm{mol})$ was also obtained as a yellow solid. $(R, S, R, R)-3$ was assigned as an unknown mixture of the $Z$ - and the $E$-enol-form. $(R, S, R, R)-29$ was assigned as a 69:31 mixture of the $Z$ - and the $E$-enol-form. This ratio was determined by integrating dd's of the respective 3'-H [7.37 (E-enol) and 7.50 (Z-enol)].

$(R, S, R, R)-3:$

Rf $\left(\mathrm{MeCN}: \mathrm{H}_{2} \mathrm{O}: \mathrm{HCO}_{2} \mathrm{H}=85: 15: 0.1\right)=0.43$

${ }^{\mathbf{1}} \mathbf{H}-\mathrm{NMR}$ (500.06 MHz, MeOD/MeOH, DsBrJ108-500300, 8.7.2020): $\delta$ = to less material for an analysis. 
Christian Drescher and Reinhard Brückner; 3D-Structure Clarifying Total Synthesis of the (Polyenoyl)tetramic Acid Militarinon B. A Highly Acid-Labile N-Protecting Group for Amides

$(R, S, R, R)-29:$

$\mathbf{R}_{\mathbf{f}}\left(\mathrm{MeCN}: \mathrm{H}_{2} \mathrm{O}: \mathrm{HCO}_{2} \mathrm{H}=85: 15: 0.1\right)=0.12$

${ }^{1}$ H-NMR (500.06 MHz, MeOD/MeOH, DsBrJl10-500600, 13.7.2020): $\delta=-0.22$ (s, 3H, 5$\left.\mathrm{CHO}-\mathrm{Si}_{-} \mathrm{CH}_{3}{ }^{\mathrm{A}}\right),-0.07$ (s, 3H, 5-CHO-Si-CH$\left.{ }^{\mathrm{B}}\right), 0.78$ [s, 9H, 5-CHO-Si-C $\left.\left(\mathrm{CH}_{3}\right)_{3}\right], 0.86\left(\mathrm{~d}, J_{10}\right.$ $\left.\mathrm{CH}_{3}, 10^{\prime}=6.3 \mathrm{~Hz}, 3 \mathrm{H}, 10^{\prime}-\mathrm{CH}_{3}\right), 0.87\left(\mathrm{t}, J_{12^{\prime}, 11^{\prime}}=7.3 \mathrm{~Hz}, 3 \mathrm{H}, 12^{\prime}-\mathrm{H}_{3}\right), 0.99\left(\mathrm{~d}, J_{8^{\prime}-\mathrm{CH}_{3}, 8^{\prime}}=6.3 \mathrm{~Hz}\right.$, $\left.3 \mathrm{H}, 8^{\prime}-\mathrm{CH}_{3}\right), 1.11-1.20\left(\mathrm{~m}, 1 \mathrm{H}, 9^{\prime}-\mathrm{H}^{\mathrm{A}}\right), 1.11-1.20\left(\mathrm{~m}, 1 \mathrm{H}, 11^{\prime}-\mathrm{H}^{\mathrm{A}}\right), 1.24-1.39\left(\mathrm{~m}, 1 \mathrm{H}, 10^{\prime}-\mathrm{H}\right)$, 1.24-1.39 (m, 1H, 9'- $\left.\mathrm{H}^{\mathrm{B}}\right), 1.24-1.39\left(\mathrm{~m}, 1 \mathrm{H}, 11^{\prime}-\mathrm{H}^{\mathrm{B}}\right), 1.86-1.87\left(\mathrm{~m}, 3 \mathrm{H}, 6^{\prime}-\mathrm{CH}_{3}\right), 2.71\left(\mathrm{~m}_{\mathrm{c}}, 1 \mathrm{H}\right.$, $\left.8^{\prime}-\mathrm{H}\right), 3.85$ (br. s, $\left.1 \mathrm{H}, 5-\mathrm{H}\right), 5.09-5.11\left(\mathrm{~d}, J_{5-\mathrm{CH}, 5}=3.8 \mathrm{~Hz}, 1 \mathrm{H}, 5-\mathrm{CH}\right), 5.60\left(\mathrm{~d}, J_{7^{\prime}, 8^{\prime}}=9.8 \mathrm{~Hz}\right.$, $\left.1 \mathrm{H}, 7^{\prime}-\mathrm{H}\right), 6.48\left(\mathrm{dd}, J_{4^{\prime}, 3^{\prime}}=11.2 \mathrm{~Hz}, J_{4^{\prime}, 5^{\prime}}=15.1 \mathrm{~Hz}, 1 \mathrm{H}, 4^{\prime}-\mathrm{H}\right), 6.76-6.82$ (m, 3H, 5'-H, 2"-H, 6"H), 7.16-7.24 (m, 3H, 2'-H, 3"-H, 5"-H), $7.59\left(\mathrm{dd}, J_{3^{\prime}, 4^{\prime}}=11.3 \mathrm{~Hz}, J_{3^{\prime}, 2^{\prime}}=15.3 \mathrm{~Hz}, 1 \mathrm{H}, 3^{\prime}-\mathrm{H}\right)$ ppm.

${ }^{13}$ C-NMR (125.75 MHz, MeOD/MeOH, DsBrJ110-500604, 13.7.2020): $\delta=-5.4$ (5-CHO-Si$\left.\mathrm{CH}_{3}{ }^{\mathrm{B}}\right),-4.4\left(5-\mathrm{CHO}-\mathrm{Si}-\mathrm{CH}_{3}{ }^{\mathrm{A}}\right), 11.7\left(\mathrm{C}-12^{\prime}\right), 12.6\left(6^{\prime}-\mathrm{CH}_{3}\right), 18.9$ [5-CHO-Si-C $\left(\mathrm{CH}_{3}\right)_{3}$ ], 19.5 $\left(10^{\prime}-\mathrm{CH}_{3}\right), 21.5\left(8^{\prime}-\mathrm{CH}_{3}\right), 26.1$ [5-CHO-Si-C $\left(\mathrm{CH}_{3}\right)_{3}$ ], $31.2\left(\mathrm{C}-11^{\prime}\right), 32.1\left(\mathrm{C}-8^{\prime}\right), 33.7\left(\mathrm{C}-10^{\prime}\right)$, 45.9 (C-9'), 70.4 (C-5), 74.5 (5-CH), 116.2 (C-2", C-6"), 121.0 (C-2'), 126.2 (C-4'), 128.4 (C3", C-6"), 133.3 (C-4"), 134.3 (C-6'), 147.0 (C-3'), 148.2 (C-7'), 150.1 (C-5'), 158.3 (C-1") ppm.*

${ }^{*} \mathrm{C}-2, \mathrm{C}-3, \mathrm{C}-4$ and $\mathrm{C}-1$ ' were not found.

edHSQC [,,short-range H,C-COSY“, 500.06 MHz/125.75 MHz, MeOD; $\delta\left({ }^{1} \mathrm{H}\right) \leftrightarrow \delta\left({ }^{13} \mathrm{C}\right):-$ $0.22\left(\mathrm{~s}, 5-\mathrm{CHO}-\mathrm{Si}-\mathrm{CH}_{3}{ }^{\mathrm{A}}\right) \leftrightarrow-4.4\left(5-\mathrm{CHO}-\mathrm{Si}_{-}-\mathrm{CH}_{3}{ }^{\mathrm{A}}\right),-0.07\left(\mathrm{~s}, 5-\mathrm{CHO}-\mathrm{Si}_{-}-\mathrm{CH}_{3}{ }^{\mathrm{B}}\right) \leftrightarrow-5.4(5-$ $\left.\mathrm{CHO}-\mathrm{Si}_{-} \mathrm{CH}_{3}{ }^{\mathrm{B}}\right), 0.78$ [s, 5-CHO-Si-C $\left(\mathrm{CH}_{3}\right)_{3}$ ] $\leftrightarrow 19.5\left(10^{\prime}-\mathrm{CH}_{3}\right), 0.87\left(\mathrm{t}, 12^{\prime}-\mathrm{H}_{3}\right) \leftrightarrow 11.7\left(\mathrm{C}-12^{\prime}\right), 0.99\left(\mathrm{~d}, 8^{\prime}-\mathrm{CH}_{3}\right) \leftrightarrow 21.5\left(8^{\prime}-\mathrm{CH}_{3}\right), 1.11-1.20$ $\left(\mathrm{m}, 9^{\prime}-\mathrm{H}^{\mathrm{A}}\right) \leftrightarrow 45.9\left(\mathrm{C}-9^{\prime}\right), 1.11-1.20\left(\mathrm{~m}, 11^{\prime}-\mathrm{H}^{\mathrm{A}}\right) \leftrightarrow 31.2\left(\mathrm{C}-11^{\prime}\right), 1.24-1.39\left(\mathrm{~m}, 10^{\prime}-\mathrm{H}\right) \leftrightarrow 33.7$ $(\mathrm{C}-10 '), 1.24-1.39\left(\mathrm{~m}, 9^{\prime}-\mathrm{H}^{\mathrm{B}}\right) \leftrightarrow 45.9\left(\mathrm{C}-9^{\prime}\right), 1.24-1.39\left(\mathrm{~m}, 1^{\prime}-\mathrm{H}^{\mathrm{B}}\right) \leftrightarrow 31.2\left(\mathrm{C}-11^{\prime}\right), 1.86-1.87$ $\left(\mathrm{m}, 66^{\prime}-\mathrm{CH}_{3}\right) \leftrightarrow 12.6\left(6 '-\mathrm{CH}_{3}\right), 2.71\left(\mathrm{~m}_{\mathrm{c}}, 1 \mathrm{H}, 8^{\prime}-\mathrm{H}\right) \leftrightarrow 32.1\left(\mathrm{C}-8^{\prime}\right), 3.85$ (br. s, 5-H) 5), 5.09-5.11 (d, 5-CH) $74.5(5-\mathrm{CH}), 5.60\left(\mathrm{~d}, 7^{\prime}-\mathrm{H}\right) \leftrightarrow 148.2\left(\mathrm{C}^{\prime} 7^{\prime}\right), 6.48\left(\mathrm{dd}, 4^{\prime}-\mathrm{H}\right) \leftrightarrow 126.2$ (C-4'), 6.76-6.82 (m, 5'-H) ↔ 150.1 (C-5'), 6.76-6.82 (m, 2"-H, 6"-H) ↔ 116.2 (C-2", C-6"), 7.16-7.24 (m, 2'-H) 121.0 (C-2'), 7.16-7.24 (m, 3"-H, 5"-H) 128.4 (C-3", C-6"), 7.59 (dd, $\left.3^{\prime}-\mathrm{H}\right) \leftrightarrow 147.0\left(\mathrm{C}-3^{\prime}\right)$.

HRMS: (EI, $70 \mathrm{eV}): \mathrm{m} / \mathrm{z}=552.3149[\mathrm{M}-\mathrm{H}]^{+}$, corresponds to the molecular formula $\mathrm{C}_{32} \mathrm{H}_{46} \mathrm{O}_{5} \mathrm{NSi}(552.3151)\left\{[\mathrm{M}-\mathrm{H}]^{+}\right\}$with a deviation of $-0.2 \mathrm{ppm}$. 
Christian Drescher and Reinhard Brückner; 3D-Structure Clarifying Total Synthesis of the (Polyenoyl)tetramic Acid Militarinon B. A Highly Acid-Labile N-Protecting Group for Amides

Specific rotation $(R, S, R, R)-\mathbf{2 9}[\alpha]_{D}^{20}=-326.2^{\circ}(\mathrm{c}=0.07$ in $\mathrm{MeOH})$

IR (KBr): $\tilde{v}=2960,2930,2855,1890,1650,1595,1555,1515,1430,1360,1310,1280,1255$, $1230,1205,1165,1115,1080,1000,955,930,895,865,835,815,780,735 \mathrm{~cm}^{-1}$.

$(4 R, 6 R, \mathrm{E})-2,4,6-T r i m e t h y l o c t-2-e n-1-o l ~[(R, R)-8]$

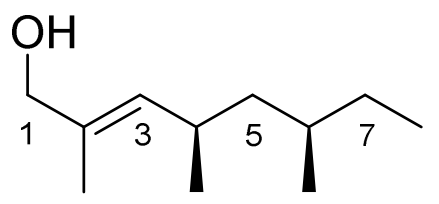

$(R, R)-8$

Natural militarinone A $[(R, R)-6]^{14}(12.0 \mathrm{mg}, 26.4 \mu \mathrm{mol})$ in THF: $\mathrm{H}_{2} \mathrm{O}(1: 1,3.8 \mathrm{~mL})$ was treated with $\mathrm{OsO}_{4} \cdot 2 \mathrm{H}_{2} \mathrm{O}$ (3.0 mg, $10.6 \mu \mathrm{mol}, 0.40$ eq.) and $\mathrm{NaIO}_{4}(86.4 \mathrm{mg}, 403 \mu \mathrm{mol}, 15.3$ eq.). The suspension stirred at $25^{\circ} \mathrm{C}$ for $1.5 \mathrm{~h}$. Then $\mathrm{OsO}_{4} \cdot 2 \mathrm{H}_{2} \mathrm{O}(1.5 \mathrm{mg}, 5.4 \mu \mathrm{mol}, 0.20$ eq. $)$ and $\mathrm{NaIO}_{4}$ (61.1 mg, $288 \mu \mathrm{mol}, 10.9$ eq.) was added. After $1 \mathrm{~h}$ at $25^{\circ} \mathrm{C}$ it was added again $\mathrm{OsO}_{4} \cdot 2 \mathrm{H}_{2} \mathrm{O}(1.5$ mg, $5.4 \mu \mathrm{mol}, 0.20$ eq.) and $\mathrm{NaIO}_{4}(61.1 \mathrm{mg}, 288 \mu \mathrm{mol}, 10.9$ eq.). The mixture stirred for $1 \mathrm{~h}$. The mixture cooled to $0^{\circ} \mathrm{C}$ and it was added $\mathrm{NaBH}_{4}(130 \mathrm{mg}, 3.46 \mathrm{mmol}, 131$ eq.). The mixture warmed to $25^{\circ} \mathrm{C}$ and held this temperature for $1.5 \mathrm{~h}$. The mixture was diluted with brine $(2 \mathrm{~mL})$ and $\mathrm{Et}_{2} \mathrm{O}(5 \mathrm{~mL})$. The layers were separated and the aq. layer was extracted with $\mathrm{Et}_{2} \mathrm{O}$ $(3 \times 10 \mathrm{~mL})$. The combined organic layers were dried over $\mathrm{Na}_{2} \mathrm{SO}_{4}$ and the solvent was removed under reduced pressure. The residue was purified by flash chromatography (PE 30/50: $\left.\mathrm{Et}_{2} \mathrm{O}=3: 1,0.5 \mathrm{~cm}, 12 \mathrm{~cm}, 0.5 \mathrm{~mL}\right)$. The alcohol $(R, R)-8(1.0 \mathrm{mg}, 22 \%, 5.8 \mu \mathrm{mol})$ was obtained as a colorless oil.

$\mathbf{R}_{\mathbf{f}}\left(\mathrm{PE} 30 / 50: \mathrm{Et}_{2} \mathrm{O}=3: 1\right)=0.44$

${ }^{1} \mathbf{H}-\mathbf{N M R}\left(300.13 \mathrm{MHz}, \mathrm{CDCl}_{3} / \mathrm{CHCl}_{3}, \mathrm{DsBrOc} 15-30120,15.10 .2018\right): \delta=0.82\left(\mathrm{~d}, J_{6-}\right.$ $\left.\mathrm{CH}_{3,6}=6.3 \mathrm{~Hz}, 3 \mathrm{H}, 6-\mathrm{CH}_{3}\right), 0.85\left(\mathrm{t}, J_{8,7}=7.1 \mathrm{~Hz}, 3 \mathrm{H}, 8-\mathrm{H}_{3}\right), 0.92\left(\mathrm{~d}, J_{4-\mathrm{CH}_{3}, 4}=6.7 \mathrm{~Hz}, 3 \mathrm{H}, 4-\right.$ $\left.\mathrm{CH}_{3}\right), 1.02-1.16\left(\mathrm{~m}, 1 \mathrm{H}, 7-\mathrm{H}^{\mathrm{A}}\right), 1.02-1.16\left(\mathrm{~m}, 1 \mathrm{H}, 5-\mathrm{H}^{\mathrm{A}}\right), 1.19-1.32(\mathrm{~m}, 1 \mathrm{H}, 6-\mathrm{H}), 1.19-1.32$ $\left(\mathrm{m}, 1 \mathrm{H}, 7-\mathrm{H}^{\mathrm{B}}\right), 1.19-1.32\left(\mathrm{~m}, 1 \mathrm{H}, 5-\mathrm{H}^{\mathrm{B}}\right), 1.68\left(\mathrm{~d}, J_{2-\mathrm{CH}_{3}, 3}=1.4 \mathrm{~Hz}, 3 \mathrm{H}, 2-\mathrm{CH}_{3}\right), 2.49\left(\mathrm{~m}_{\mathrm{c}}, 1 \mathrm{H}\right.$, $4-\mathrm{H}), 3.99\left(\mathrm{~d}, J_{1,1-\mathrm{OH}}=1.2 \mathrm{~Hz}, 2 \mathrm{H}, 1-\mathrm{H}_{2}\right), 5.13\left(\mathrm{dq}, J_{3,4}=9.6 \mathrm{~Hz},{ }^{4} J_{3,2-\mathrm{CH}_{3}}=1.3 \mathrm{~Hz}, 1 \mathrm{H}, 3-\mathrm{H}\right)$ ppm. 
Christian Drescher and Reinhard Brückner; 3D-Structure Clarifying Total Synthesis of the (Polyenoyl)tetramic Acid Militarinon B. A Highly Acid-Labile N-Protecting Group for Amides

\subsection{Chiral GLC Studies of the Enantiomeric Alcohol 8 and HPLC- Reports of the Tetramic Acid Militarinone B $[(S, S, R, R)-3]$ and its Epimer $(R, S, R, R)-3$}

Data File C: \CHEM32\1\DATA \DEF_GC 2021-04-19 17-06-40\CD-204_205.D

Sample Name: cd-204

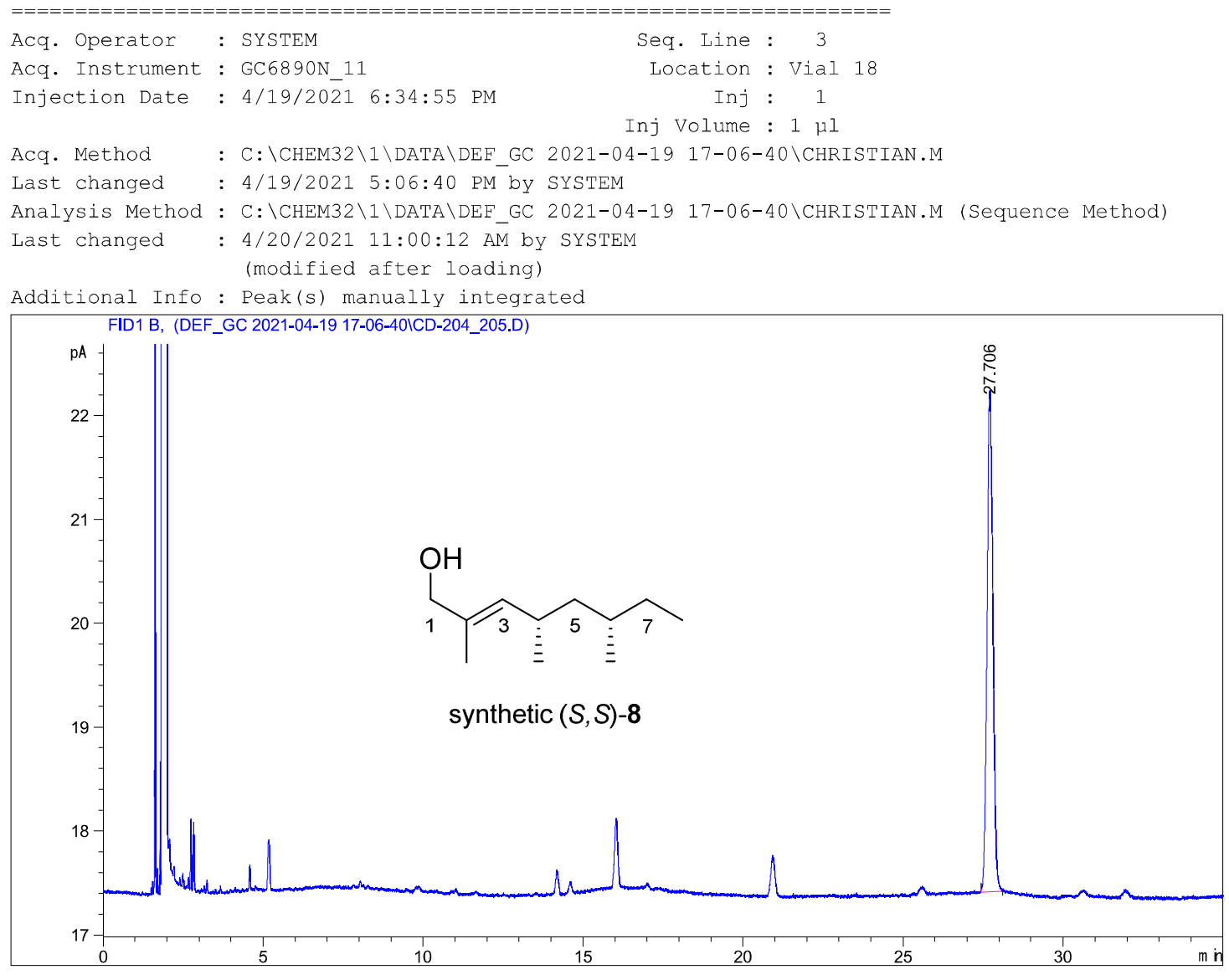

Area Percent Report

$\begin{array}{llc}\text { Sorted By } & : & \text { Signal } \\ \text { Multiplier } & : & 1.0000 \\ \text { Dilution } & : & 1.0000 \\ \text { Do not use Multiplier } & \& & \text { Dilution Factor with }\end{array}$

Signal 1: FID1 B,

\begin{tabular}{|c|c|c|c|c|c|c|}
\hline $\begin{array}{c}\text { Peak } \\
\text { \# }\end{array}$ & $\begin{array}{c}\text { RetTime } \\
\text { [min] }\end{array}$ & Type & $\begin{array}{l}\text { Width } \\
\text { [min] }\end{array}$ & $\begin{array}{r}\text { Area } \\
{\left[\mathrm{pA}^{*} \mathrm{~s}\right]}\end{array}$ & $\begin{array}{l}\text { Height } \\
{[\mathrm{pA}]}\end{array}$ & $\begin{array}{c}\text { Area } \\
\frac{\circ}{0}\end{array}$ \\
\hline--- & -------- & 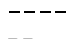 & ------1 & ---------- & $-------\mid$ & $--------\mid$ \\
\hline 1 & 27.706 & $\mathrm{BB}$ & 0.2008 & 61.89531 & 4.78968 & $1.000 \mathrm{e} 2$ \\
\hline Total & $S:$ & & & 61.89531 & 4.78968 & \\
\hline
\end{tabular}


Christian Drescher and Reinhard Brückner; 3D-Structure Clarifying Total Synthesis of the (Polyenoyl)tetramic Acid Militarinon B. A Highly Acid-Labile N-Protecting Group for Amides

Data File C:\CHEM32\1\DATA \DEF_GC 2021-04-19 17-06-40\CD-205.D Sample Name: cd-205
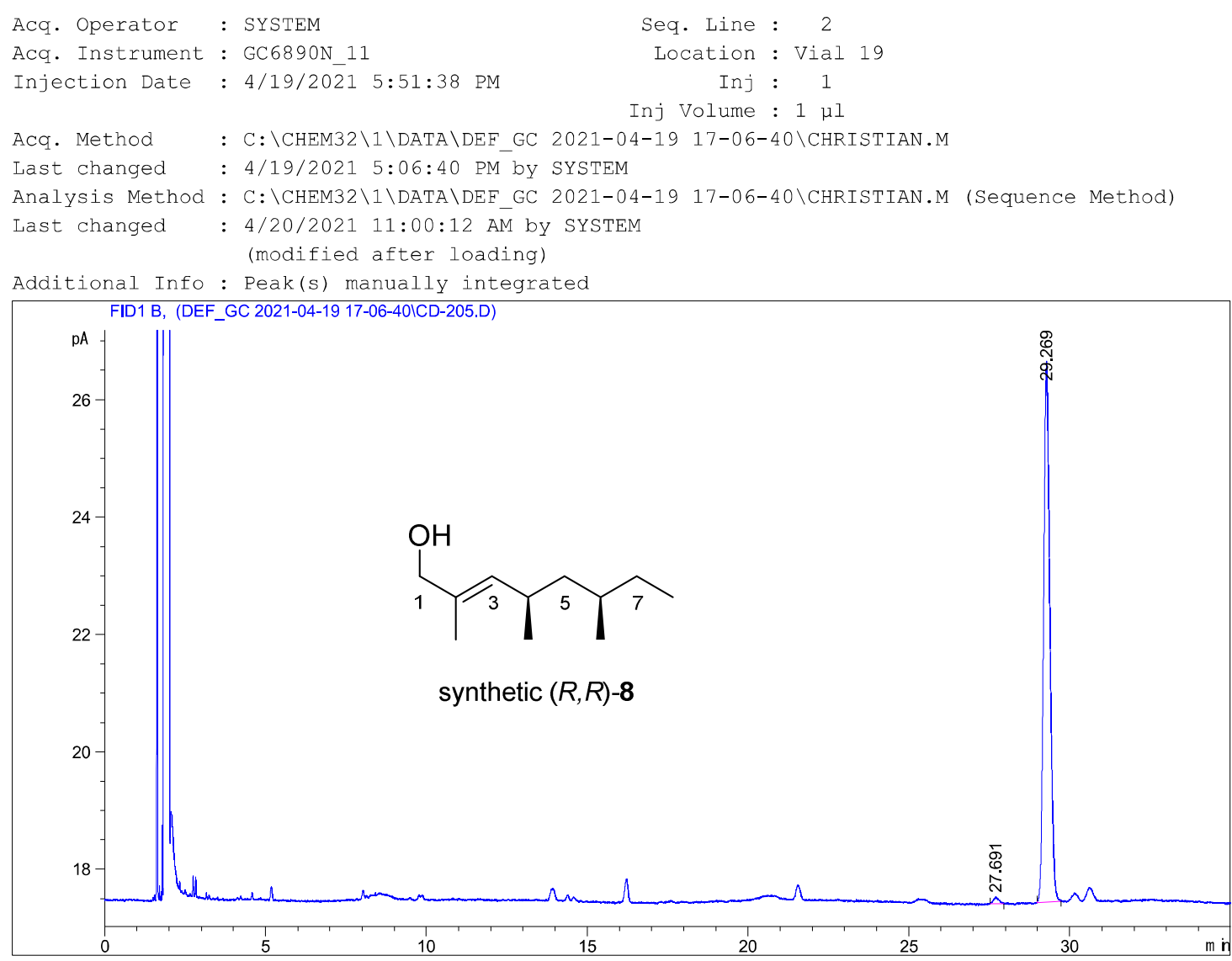

Area Percent Report

$\begin{array}{lll}\text { Sorted By } & : & \text { Signal } \\ \text { Multiplier } & : & 1.0000 \\ \text { Dilution } & : & 1.0000\end{array}$

Do not use Multiplier \& Dilution Factor with ISTDs

Signal 1: FID1 B,

\begin{tabular}{|c|c|c|c|c|c|c|}
\hline $\begin{array}{c}\text { Peak } \\
\quad \#\end{array}$ & $\begin{array}{c}\text { RetTime } \\
\text { [min] }\end{array}$ & Type & $\begin{array}{l}\text { Width } \\
\text { [min] }\end{array}$ & $\begin{array}{r}\text { Area } \\
{\left[\mathrm{pA}^{\star} \mathrm{s}\right]}\end{array}$ & $\begin{array}{l}\text { Height } \\
{[\mathrm{pA}]}\end{array}$ & $\begin{array}{c}\text { Area } \\
\%\end{array}$ \\
\hline \multicolumn{7}{|c|}{---- |------- | } \\
\hline 1 & 27.691 & BB & 0.1601 & 1.45984 & $1.18206 \mathrm{e}-1$ & 1.16884 \\
\hline 2 & 29.269 & $\mathrm{BB}$ & 0.2122 & 123.43648 & 9.10082 & 98.83116 \\
\hline Total & Is: & & & 124.89632 & 9.21902 & \\
\hline
\end{tabular}


Christian Drescher and Reinhard Brückner; 3D-Structure Clarifying Total Synthesis of the (Polyenoyl)tetramic Acid Militarinon B. A Highly Acid-Labile N-Protecting Group for Amides

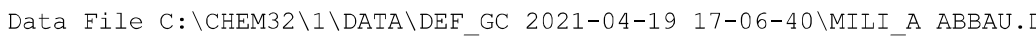

Sample Name: Mili_A Abbau

\begin{tabular}{|c|c|c|c|}
\hline Acq. Operator & : SYSTEM & Seq. Line & 1 \\
\hline Acq. Instrument & : GC6890N_11 & Location & : Vial 20 \\
\hline Injection Date & $: 4 / 19 / 2021 \quad 5: 08: 27 \mathrm{PM}$ & $\begin{array}{r}\text { Inj } \\
\text { Inj Volume }\end{array}$ & $\begin{array}{ll}: & 1 \\
: & 1\end{array}$ \\
\hline
\end{tabular}

Acq. Method : C: \CHEM32\1\DATA \DEF_GC 2021-04-19 17-06-40\CHRISTIAN.M

Last changed : 4/19/2021 5:06:40 PM by SYSTEM

Analysis Method : C: \CHEM32\1\DATA \DEF_GC 2021-04-19 17-06-40\CHRISTIAN.M (Sequence Method)

Last changed : 4/20/2021 10:57:58 AM by SYSTEM

(modified after loading)

Additional Info : Peak(s) manually integrated

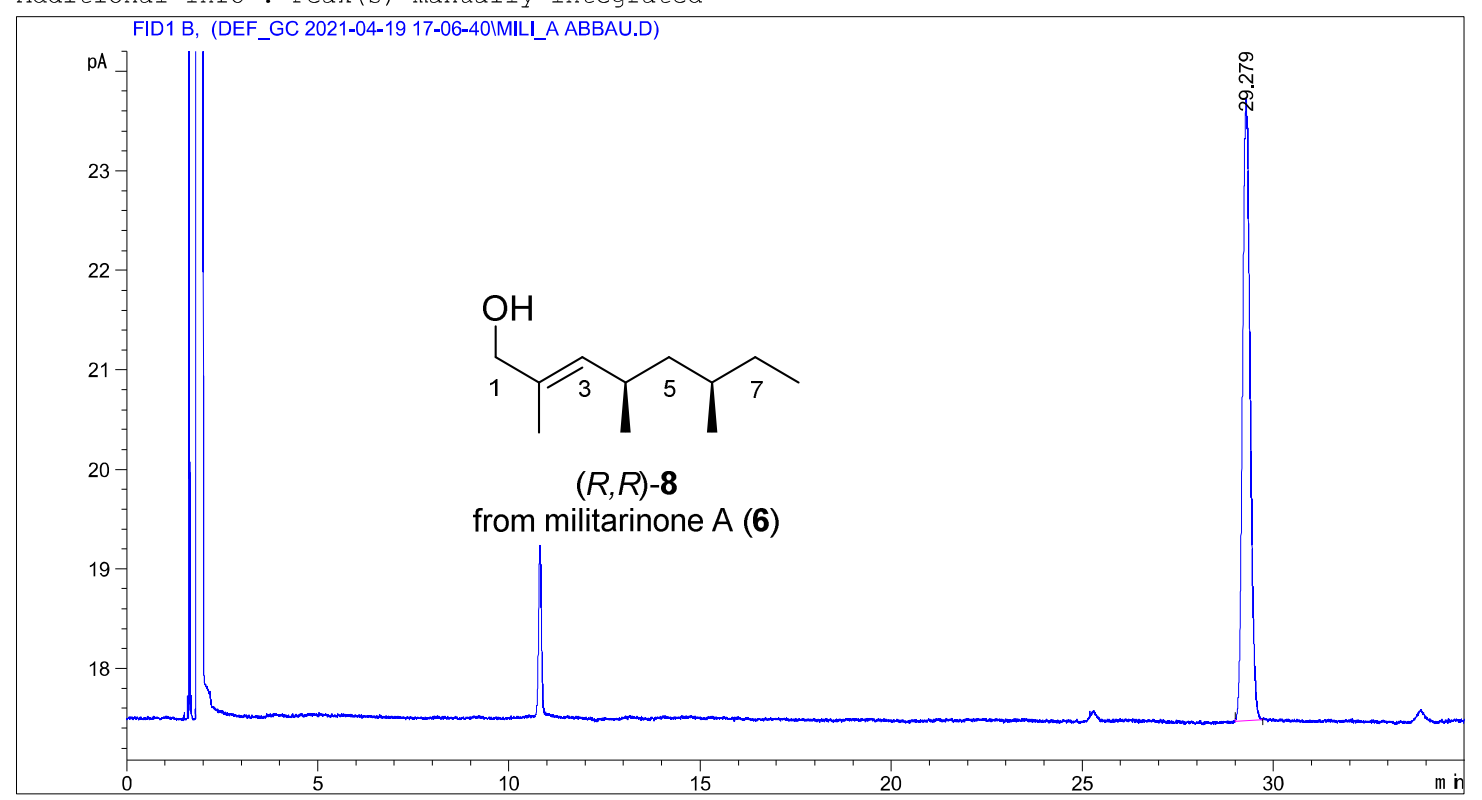

Area Percent Report

$\begin{array}{lll}\text { Sorted By } & : & \text { Signal } \\ \text { Multiplier } & : & 1.0000 \\ \text { Dilution } & : & 1.0000\end{array}$

Do not use Multiplier \& Dilution Factor with ISTDs

Signal 1: FID1 B,

\begin{tabular}{|c|c|c|c|c|c|c|}
\hline $\begin{array}{c}\text { Peak } \\
\#\end{array}$ & $\begin{array}{c}\text { RetTime } \\
\text { [min] }\end{array}$ & Type & $\begin{array}{l}\text { Width } \\
\text { [min] }\end{array}$ & $\begin{array}{r}\text { Area } \\
{\left[\mathrm{pA}^{*} \mathrm{~s}\right]}\end{array}$ & $\begin{array}{l}\text { Height } \\
{[\mathrm{pA}]}\end{array}$ & $\begin{array}{c}\text { Area } \\
\frac{\circ}{0}\end{array}$ \\
\hline--1 & $-------\mid$ & $\mid----$ & ------ & --------- & --------1 & $\mid-------1$ \\
\hline 1 & 29.279 & $\mathrm{BB}$ & 0.2109 & 85.54961 & 6.20448 & $1.000 \mathrm{e} 2$ \\
\hline Total & ls : & & & 85.54961 & 6.20448 & \\
\hline
\end{tabular}


Christian Drescher and Reinhard Brückner; 3D-Structure Clarifying Total Synthesis of the (Polyenoyl)tetramic Acid Militarinon B. A Highly Acid-Labile N-Protecting Group for Amides

D-7000 HSM: Kreibich

Series:0011

Report: original

System: 4C1-LC1

\section{D-7000 HPLC System Manager Report}

Analyzed: 07.07.20 15:02

Reported: 07.07 .20 15:22

Data Path: F: \Kreibich \DATA $\backslash 0011 \backslash$

System(acquisition): 4C1-LC1

Application: Kreibich

Sample Name: cd-M-B

Injection from this vial: 1 of 1

Processed: $07.07 .20 \quad 15: 22$

Series : 0011

Vial Number: 3

Vial Type: UNK

Chrom Type: Fixed wL Chromatogram, $400 \mathrm{~nm}$

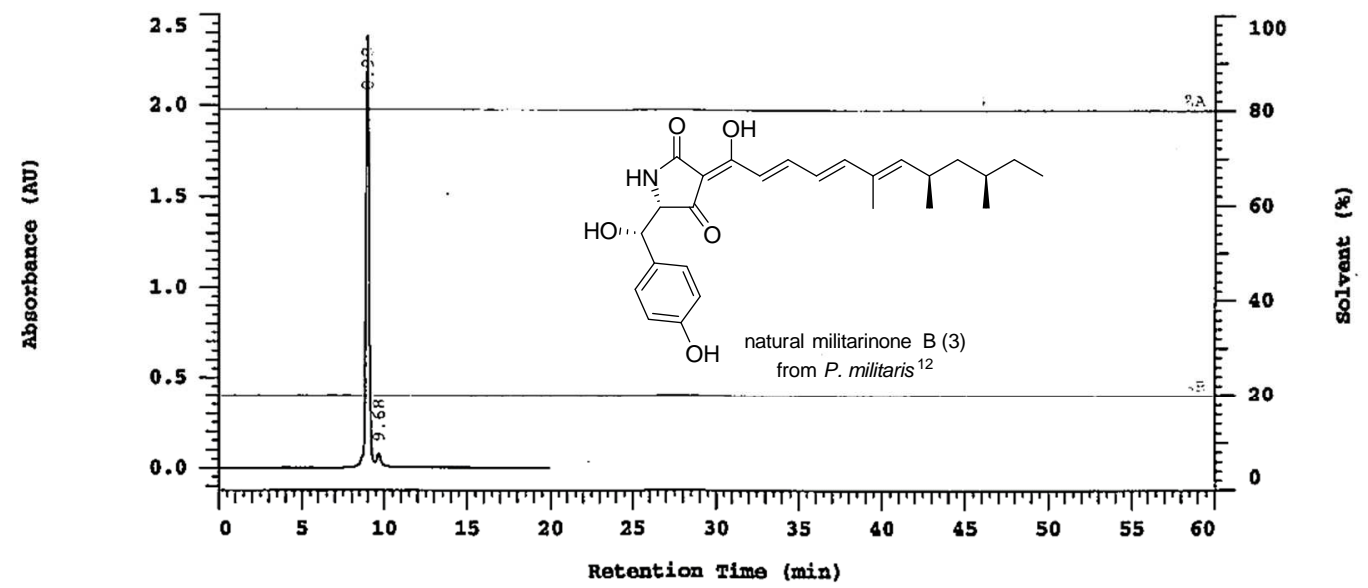

Acquisition Method: Phenmex_LunaC18_standard

Column Type:

Pump A Type: L-6200

Solvent A: Acetonitril

Developed by: Braukmüller

Solvent B: Wasser

enomenex LUNA C18 5 mici

A, $4.6 \times 250 \mathrm{~mm}$ mit $4 \times 3 \mathrm{~mm}$ Vorsäule, $5 \mathrm{microm}$; MeCN $/ \mathrm{H} 2 \mathrm{O}$

$80: 20,1.0 \mathrm{~mL} / \mathrm{min}, 254 \mathrm{~nm}$

Peak Quantitation: AREA

Calculation Method: AREA\&

No.

RT

Area

\begin{tabular}{rrr}
1 & 8.98 & 14462640 \\
2 & 9.68 & 276170 \\
\hline
\end{tabular}

14738810

Peak rejection level: 0

$9\{1: 1,9$

Page Indicator $1 / 1$ 
Christian Drescher and Reinhard Brückner; 3D-Structure Clarifying Total Synthesis of the (Polyenoyl)tetramic Acid Militarinon B. A Highly Acid-Labile N-Protecting Group for Amides

D-7000 HSM: Kreibich

Series: 0010

Report: original

System: 4C1-LC1

\section{D-7000 HPLC System Manager Report}

Analyzed: $07.07 .20 \quad 14: 40$

Reported: 07.07 .20 15:00

Data Path: F: \Kreibich \DATA $\backslash 0010 \backslash$

System(acquisition): 4C1-LC1

Processed: $07.07 .2015: 00$

Application: Kreibich

Sample Name: CD-HOAC

Series: 0010

Injection from this vial: 1 of 1

Vial Number: 2

Vial Type: UNK

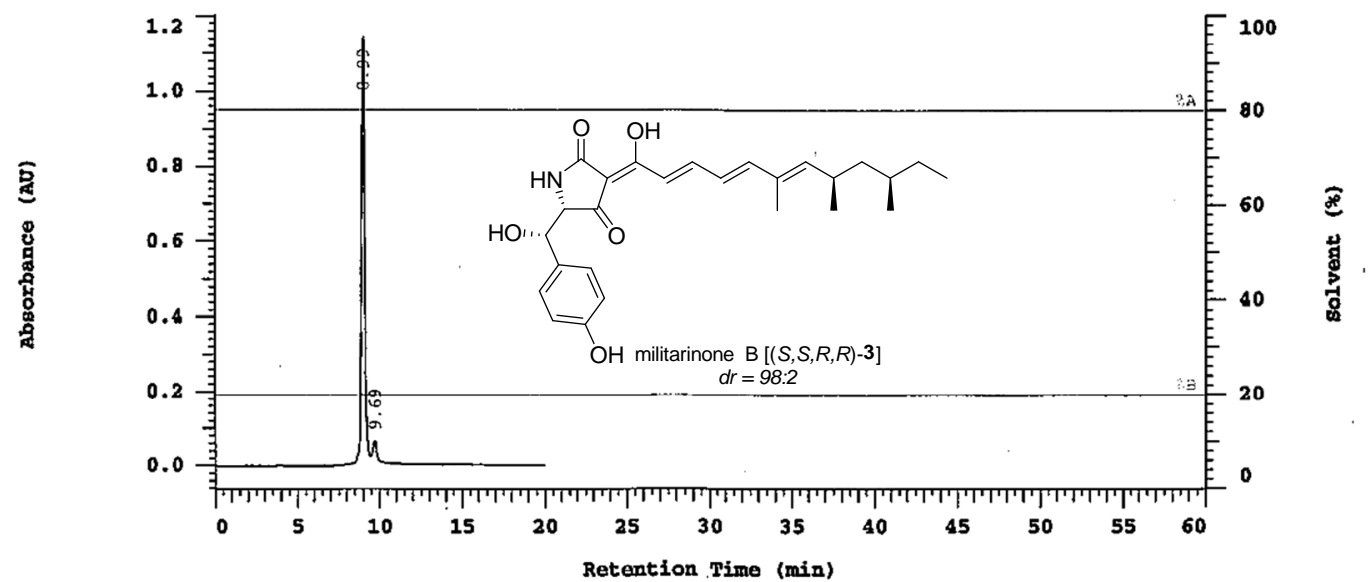

Acquisition Method: Phenmex_Lunac18_Standard

Column Type:

Developed by: Braukmüller

Pump A Type: L-6200

Solvent A: Acetonitril

Solvent B: Wasser

Method Description: Testbedingungen für Phenomenex LUNA C18 5 microm, 100

A, $4.6 \times 250 \mathrm{~mm}$ mit $4 \times 3 \mathrm{~mm}$ Vorsäule, $5 \mathrm{microm} ; \mathrm{MeCN} / \mathrm{H} 2 \mathrm{O}$

$80: 20,1.0 \mathrm{~mL} / \mathrm{min}, 254 \mathrm{~nm}$

Peak Quantitation: AREA

Calculation Method: AREA\&

No.

RT

Area

\begin{tabular}{rrr}
\hline 1 & 8.99 & 6838529 \\
2 & 9.69 & 162502 \\
\hline
\end{tabular}

7001031

Peak rejection level: 0

$97,6: 2,4$

Page Indicator $1 / 1$ 
Christian Drescher and Reinhard Brückner; 3D-Structure Clarifying Total Synthesis of the (Polyenoyl)tetramic Acid Militarinon B. A Highly Acid-Labile N-Protecting Group for Amides

D-7000 HSM: Kreibich

Series: 0009

Report: original

System: 4C1-LC1

\section{D-7000 HPLC System Manager Report}

Analyzed: $07.07 .2014: 18$

Reported: $07.07 .2014: 39$

Data Path: F:\Kreibich $\backslash$ DATA $\backslash 0009 \backslash$

System(acquisition): 4C1-LC1

Processed: $07.07 .2014: 39$

Application: Kreibich

Sample Name: $c d-b$

Series: 0009

Injection from this vial: 1 of 1

Vial Number: 1

Vial Type: UNK

Volume: $10.0 \mathrm{ul}$

Chrom Type: Fixed WL Chromatogram, 400 nm

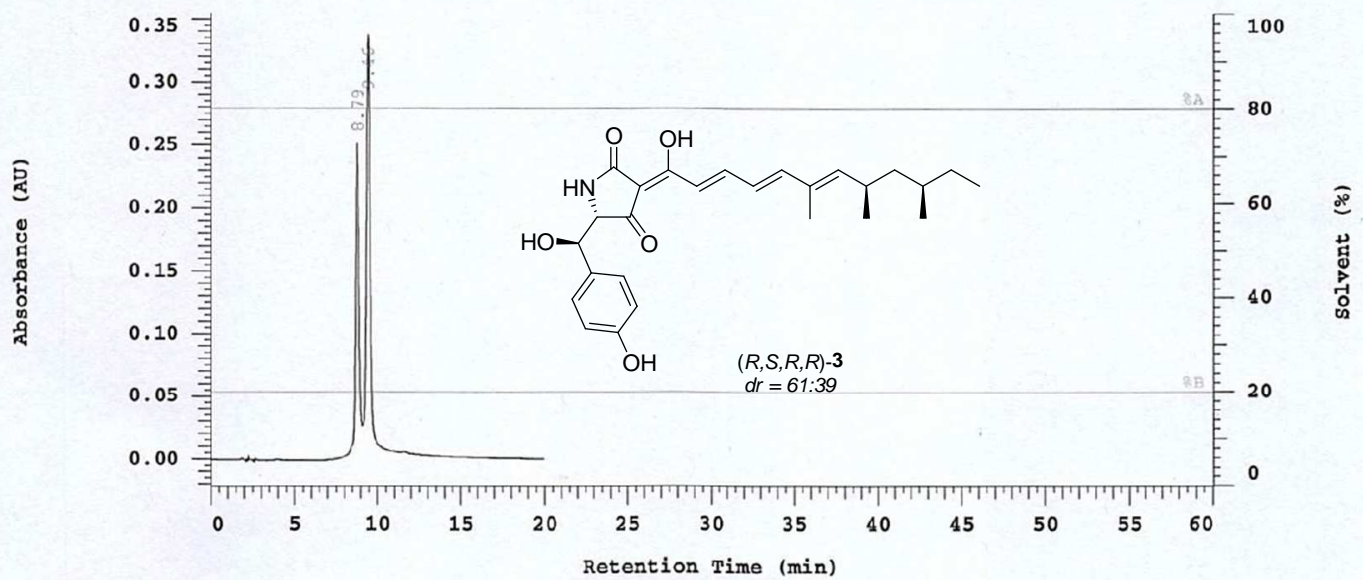

Acquisition Method: Phenmex_LunaC18_Standard

Column Type:

Developed by: Braukmüller

Pump A Type: L-6200

Solvent A: Acetonitril

Solvent B: Wasser

Method Description: Testbedingungen für Phenomenex LUNA C18 5 microm, 100

A, $4.6 \times 250 \mathrm{~mm}$ mit $4 \times 3 \mathrm{~mm}$ Vorsäule, $5 \mathrm{microm}$; MeCN/H2O

$80: 20,1.0 \mathrm{~mL} / \mathrm{min}, 254 \mathrm{~nm}$

Peak Quantitation: AREA

Calculation Method: AREA\%

No.

RT

Area

\begin{tabular}{lll}
\hline 1 & 8.79 & 1384987 \\
2 & 9.46 & 1987272 \\
\hline & & 3372259 \\
\hline
\end{tabular}

Peak rejection level: 0

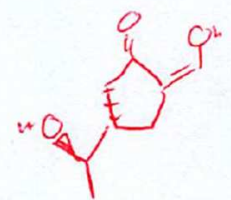

Page Indicator $1 / 1$ 
Christian Drescher and Reinhard Brückner; 3D-Structure Clarifying Total Synthesis of the (Polyenoyl)tetramic Acid Militarinon B. A Highly Acid-Labile N-Protecting Group for Amides

\section{3. ${ }^{1} \mathrm{H}$ and ${ }^{13} \mathrm{C}$ NMR Spectra in Numerical Order}

3.1 ${ }^{1} \mathrm{H}$ and ${ }^{13} \mathrm{C}$ NMR to the Synthesis of the Tetramic Acid $(S)$-17 Using die DMB-, $p$ NB-, DMNB- and TMB-Protecting Group. 
Christian Drescher and Reinhard Brückner; 3D-Structure Clarifying Total Synthesis of the (Polyenoyl)tetramic Acid Militarinon B. A Highly Acid-Labile N-Protecting Group for Amides

trans-3-Bromacrylic acid (SI-1)

${ }^{1} \mathbf{H}$ NMR (300.13 MHz, $\left.\mathrm{CDCl}_{3}\right)$ :

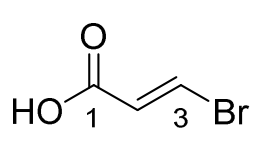

SI-1

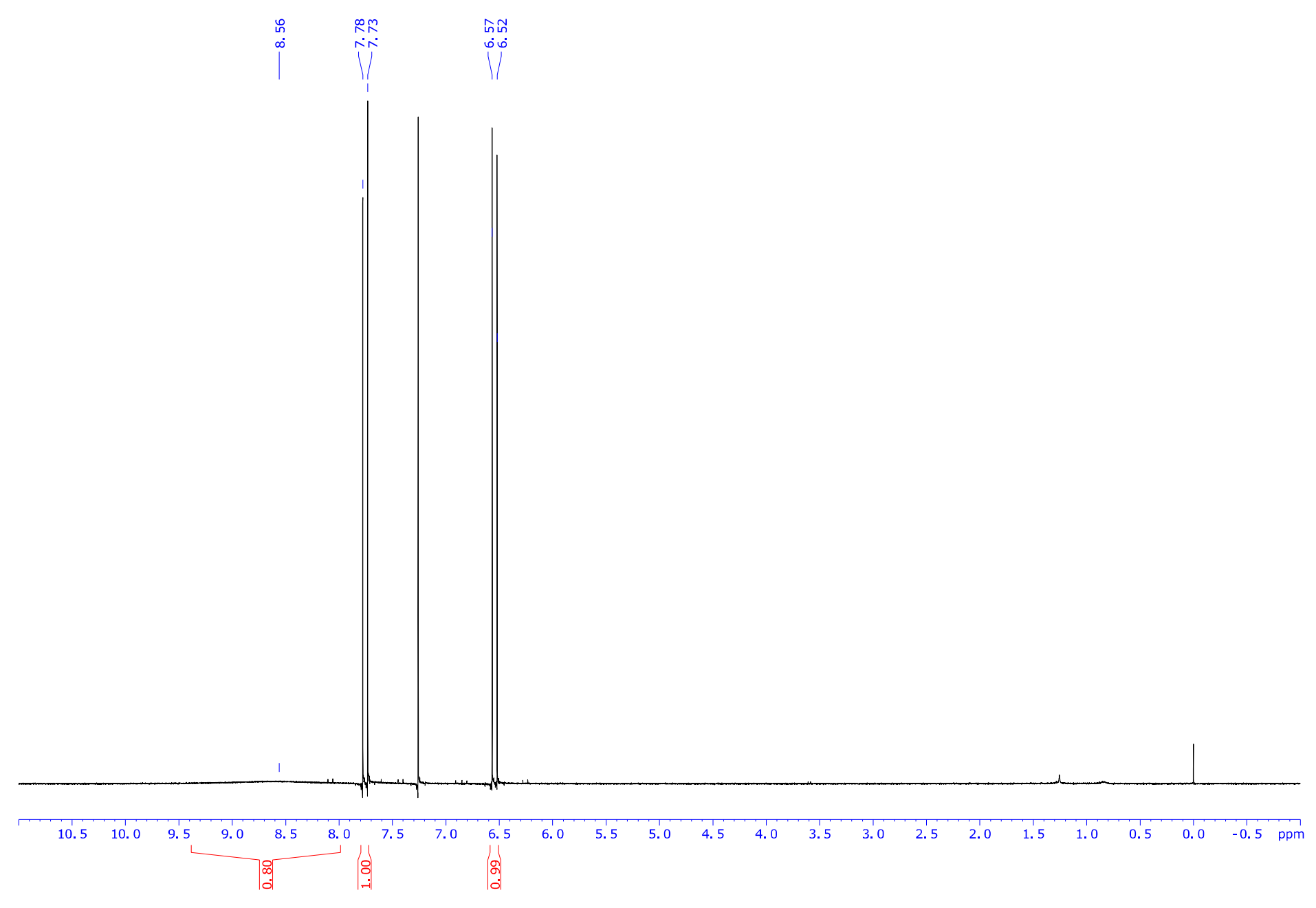


Christian Drescher and Reinhard Brückner; 3D-Structure Clarifying Total Synthesis of the (Polyenoyl)tetramic Acid Militarinon B. A Highly Acid-Labile N-Protecting Group for Amides

\section{$\mathrm{N}$-Methylmethoxyamine (SI-2)}

${ }^{1} \mathbf{H}$ NMR $\left(300.13 \mathrm{MHz}, \mathrm{CDCl}_{3}\right)$ :

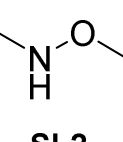

SI-2

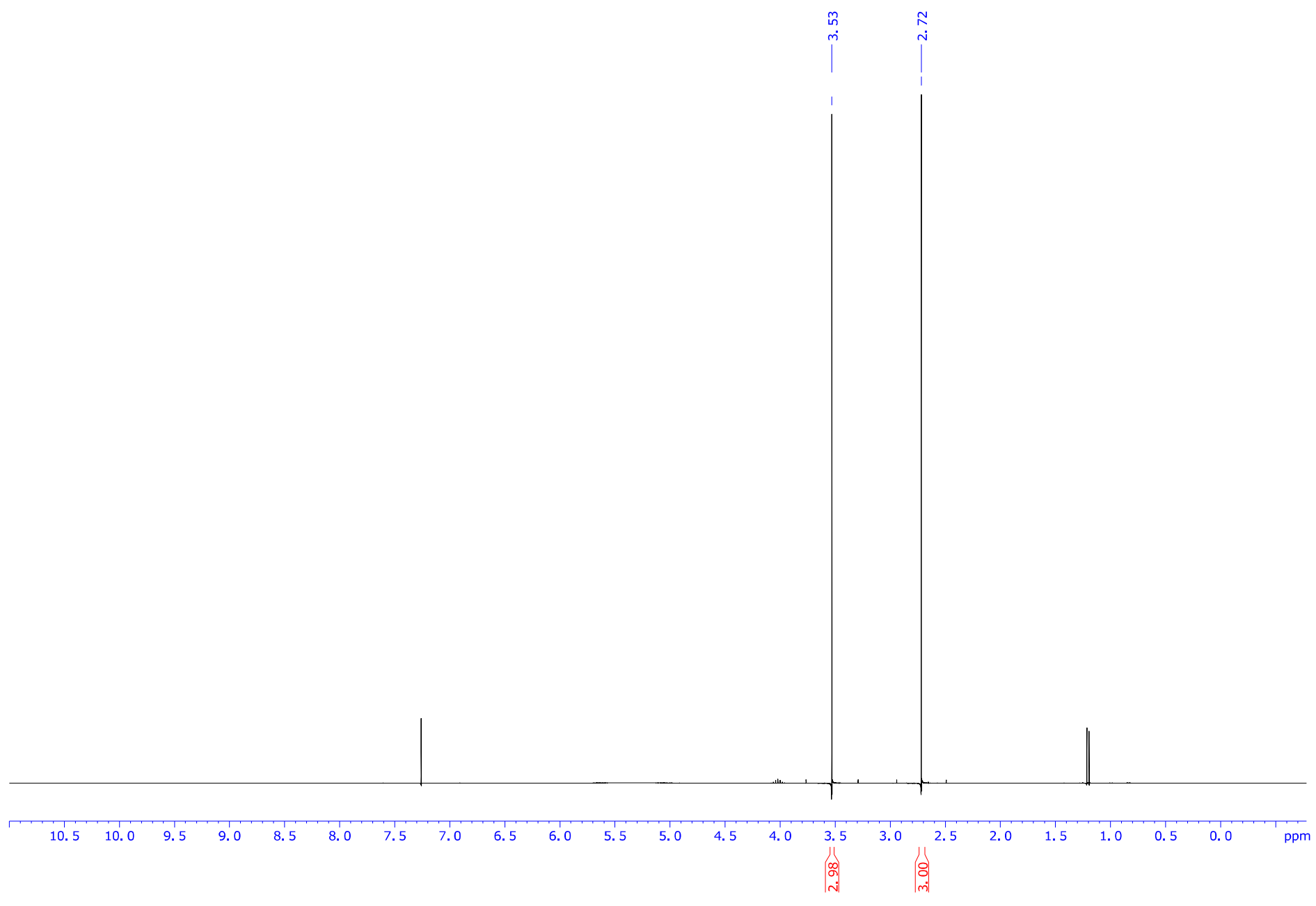


Christian Drescher and Reinhard Brückner; 3D-Structure Clarifying Total Synthesis of the (Polyenoyl)tetramic Acid Militarinon B. A Highly Acid-Labile N-Protecting Group for Amides

trans-3-Bromo- $N$-methoxy- $N$-methylacrylamide (SI-3)

${ }^{1} \mathbf{H}$ NMR (300.13 MHz, $\left.\mathrm{CDCl}_{3}\right)$ :

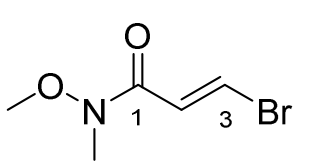

SI-3

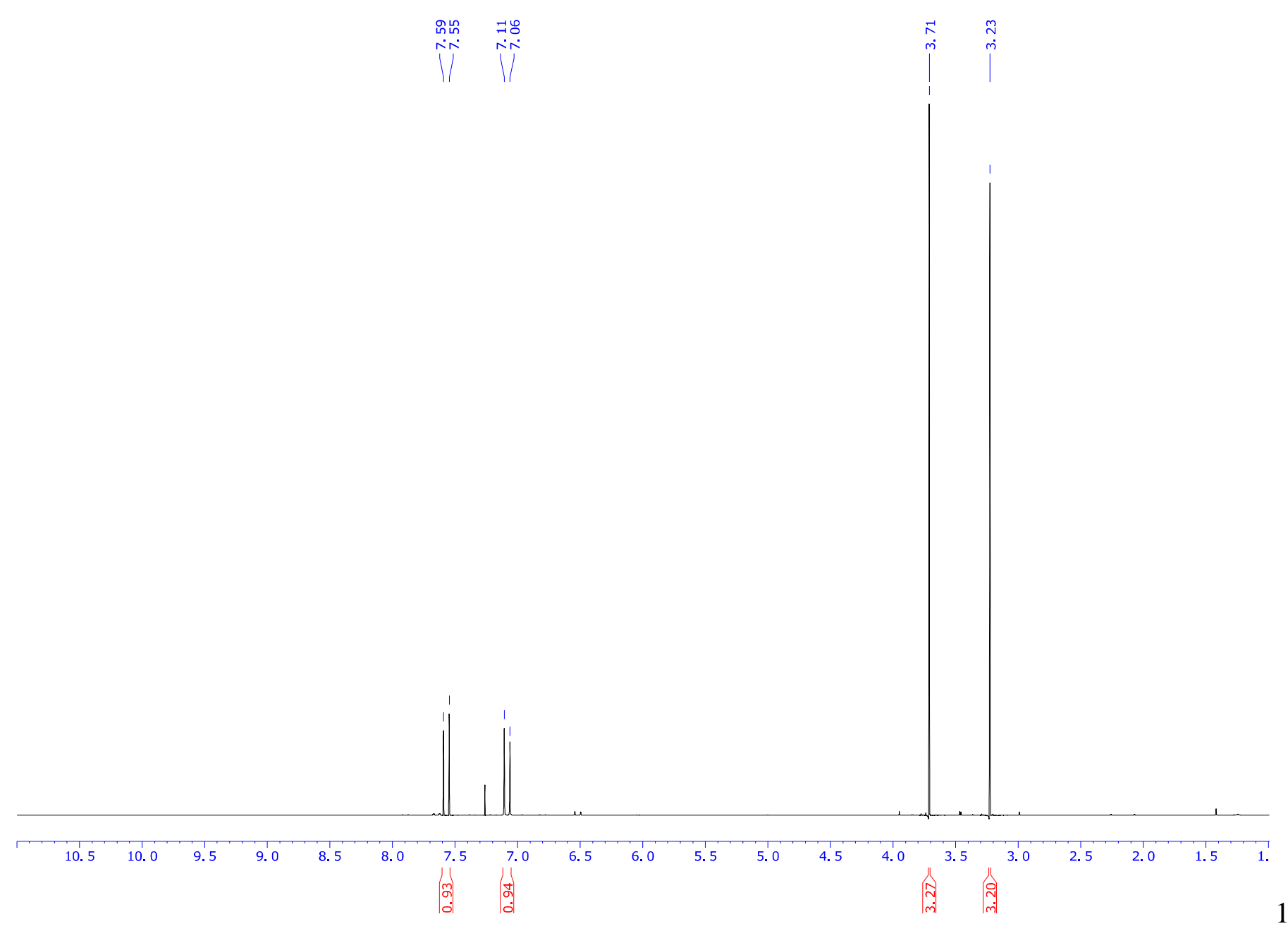




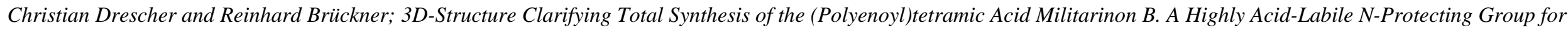
Amides

\section{$t$-Butylthioacetate (SI-4)}

${ }^{1} \mathbf{H}$ NMR (300.13 MHz, $\mathrm{CDCl}_{3}$ ):

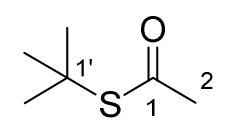

SI-4

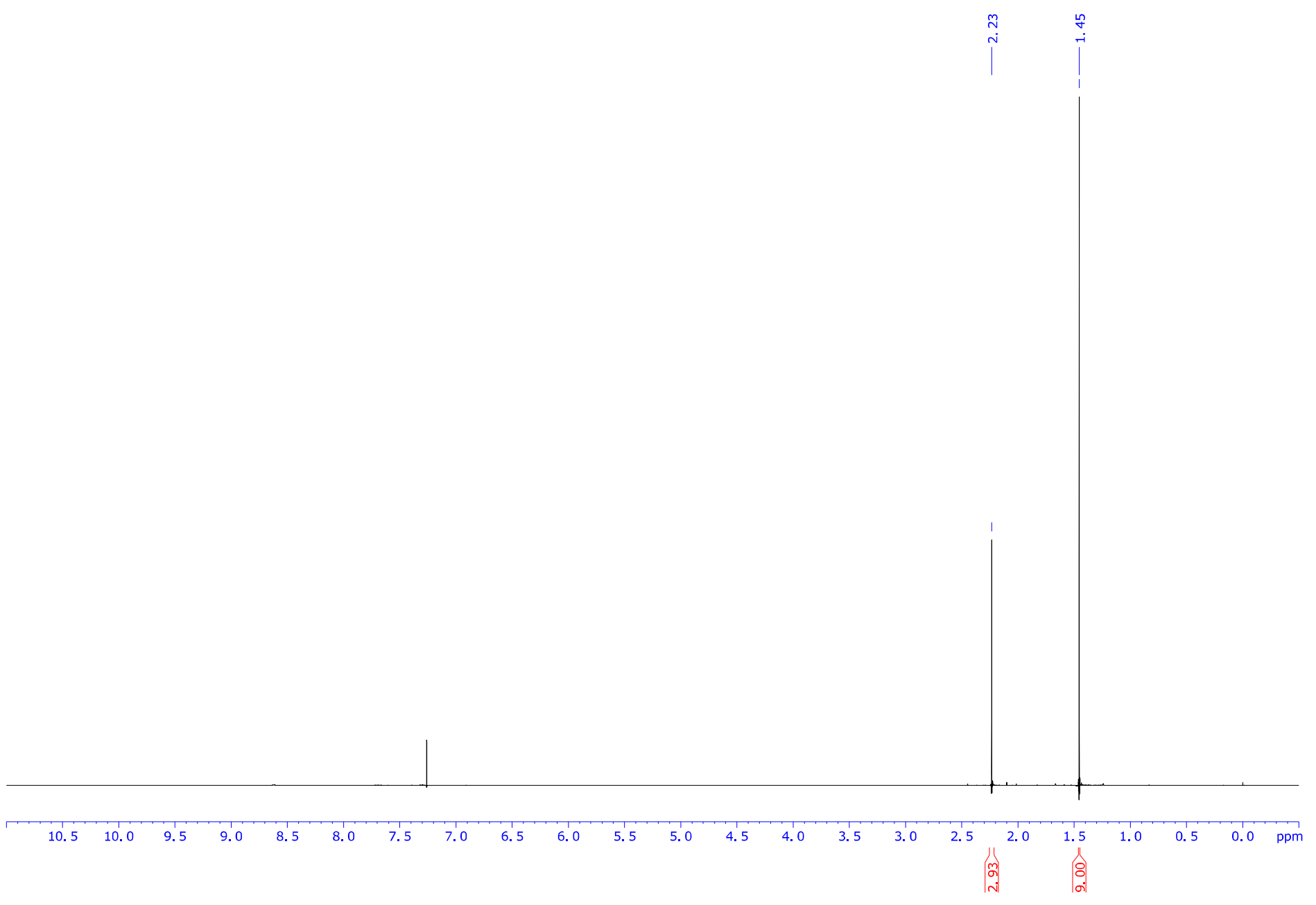


Christian Drescher and Reinhard Brückner; 3D-Structure Clarifying Total Synthesis of the (Polyenoyl)tetramic Acid Militarinon B. A Highly Acid-Labile N-Protecting Group for Amides

\section{S-tert-Butyl (E,E)-5-Bromo-3-hydroxypenta-2,4-dienethioate [enol-(11)]}

${ }^{1} \mathbf{H}$ NMR (300.13 MHz, $\left.\mathrm{CDCl}_{3}\right)$ :
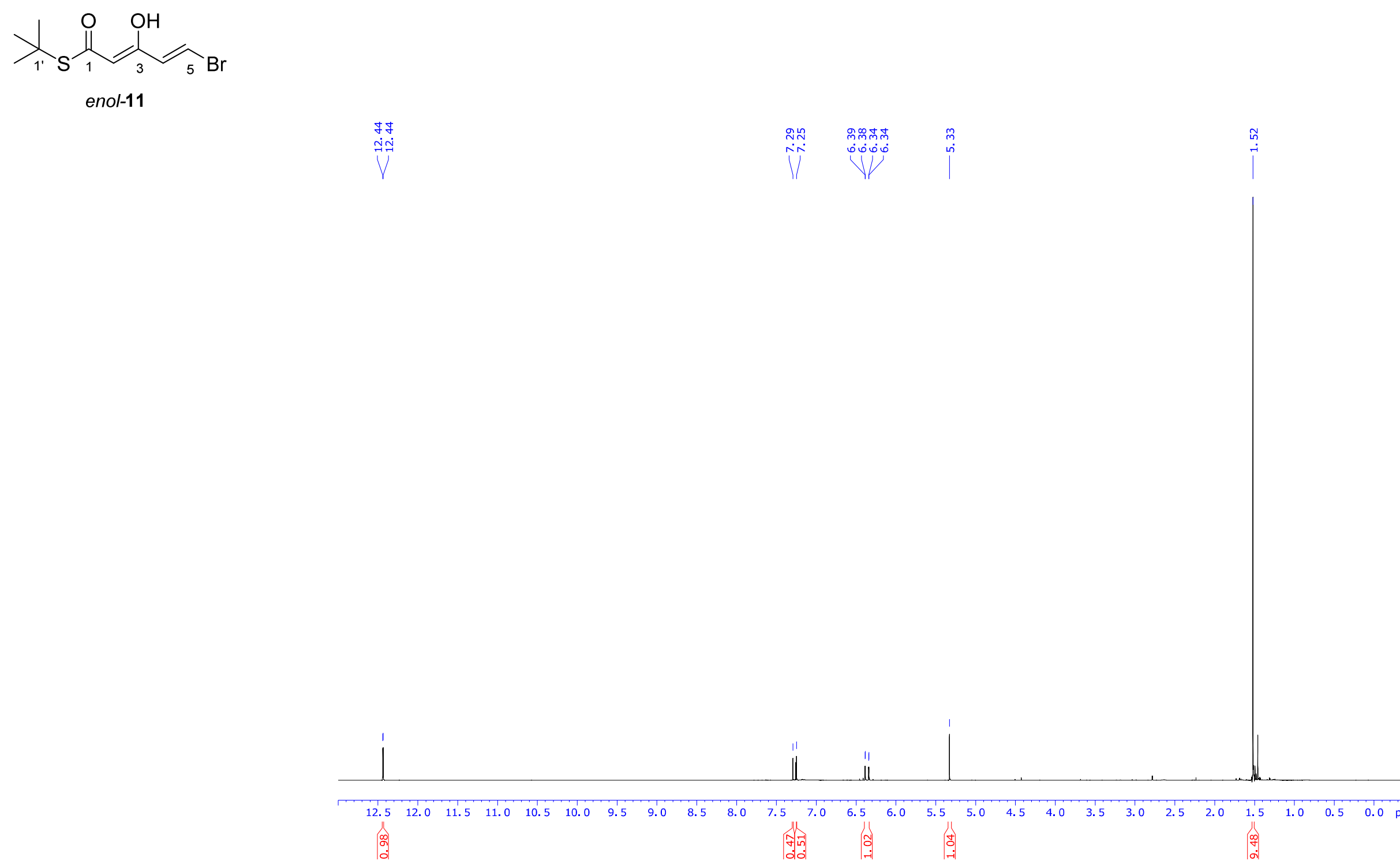
Christian Drescher and Reinhard Brückner; 3D-Structure Clarifying Total Synthesis of the (Polyenoyl)tetramic Acid Militarinon B. A Highly Acid-Labile N-Protecting Group for Amides

\section{Methyl (2S)-Amino-3-(4'-hydroxyphenyl)propanoate [(S)-SI-5]}

${ }^{1} \mathbf{H}$ NMR (300.13 MHz, $\left.\mathrm{D}_{2} \mathrm{O}\right)$ :

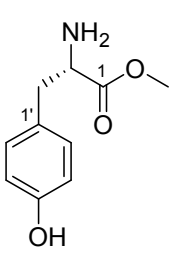

S)

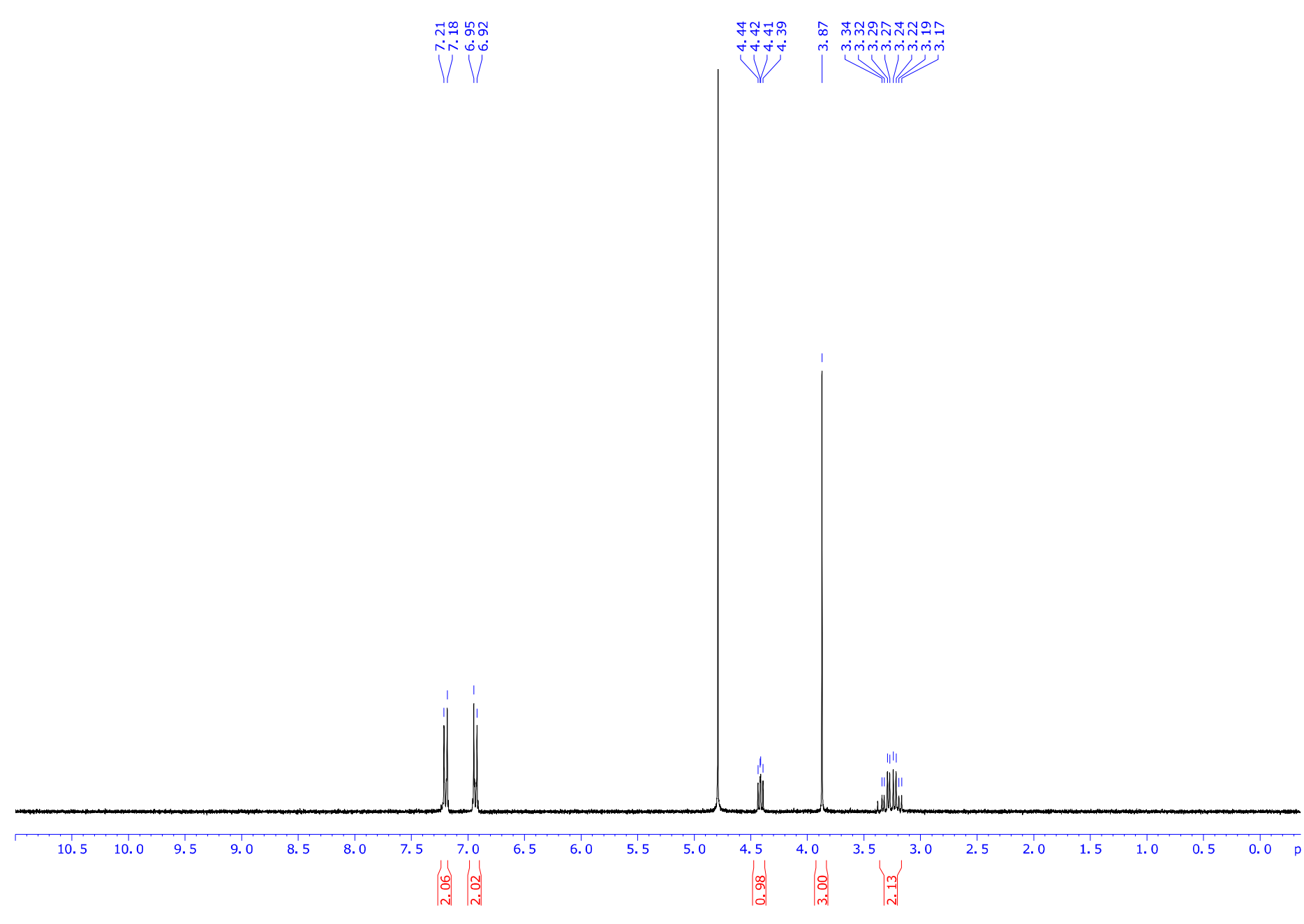


Christian Drescher and Reinhard Brückner; 3D-Structure Clarifying Total Synthesis of the (Polyenoyl)tetramic Acid Militarinon B. A Highly Acid-Labile N-Protecting Group for Amides

Methyl (2S)-2-Amino-3-\{4'-[(tert-butyldimethylsilyl)oxy]phenyl $\}$ propanoate $[(S)-20]$

${ }^{1} \mathbf{H}$ NMR (300.13 MHz, $\left.\mathrm{CDCl}_{3}\right)$ :

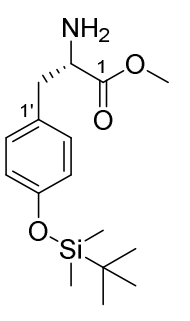

(S)-20

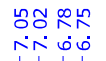

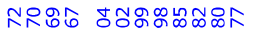

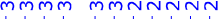

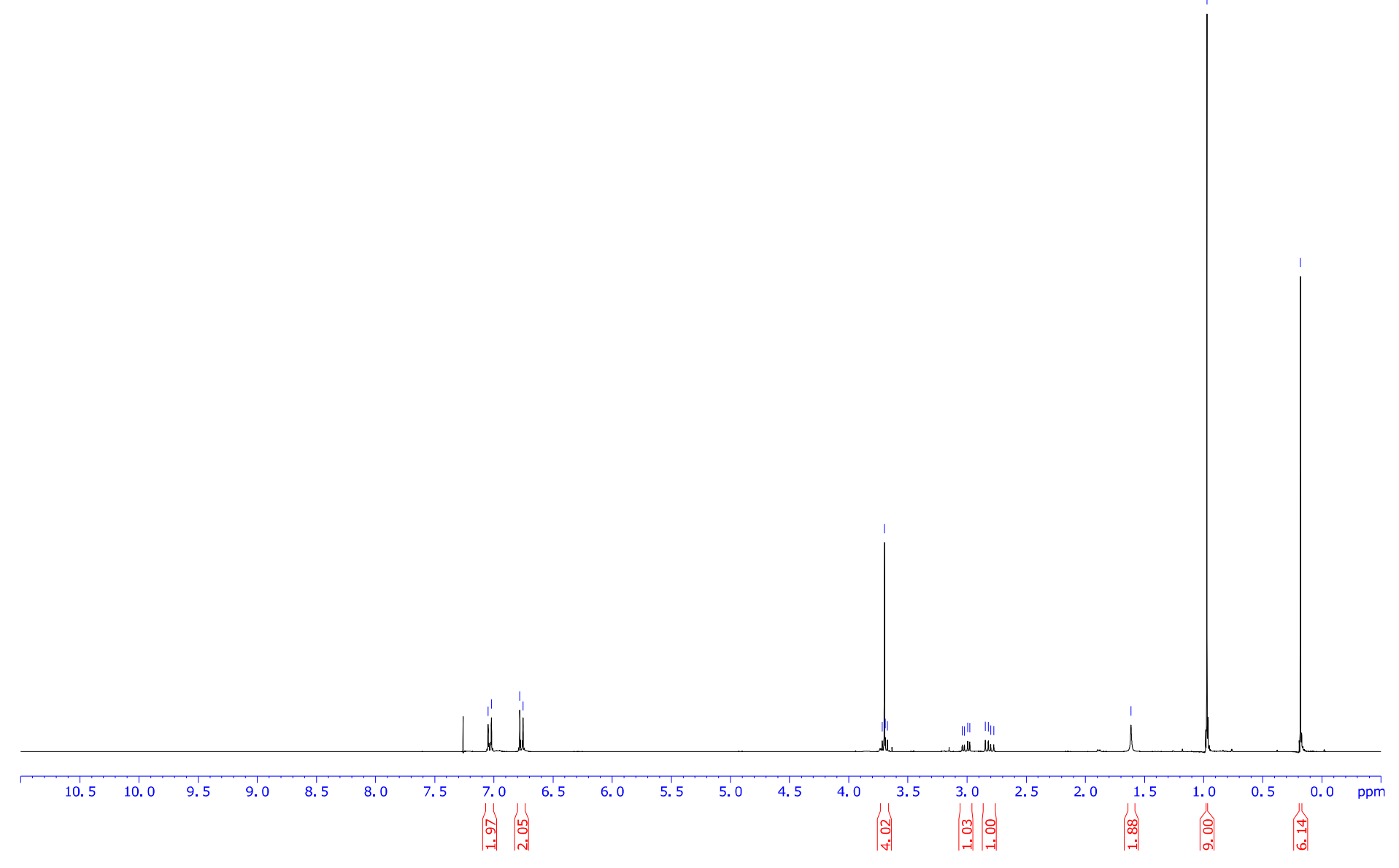


Christian Drescher and Reinhard Brückner; 3D-Structure Clarifying Total Synthesis of the (Polyenoyl)tetramic Acid Militarinon B. A Highly Acid-Labile N-Protecting Group for Amides

Methyl (2S)-3-\{4'-[(tert-Butyldimethylsilyl)oxy]phenyl\}-2-[(1",5"-dimethoxybenzyl)amino]propanoate [(S)-18d]

${ }^{1} \mathbf{H}$ NMR (500.10 MHz, $\left.\mathrm{CDCl}_{3}\right)$ :

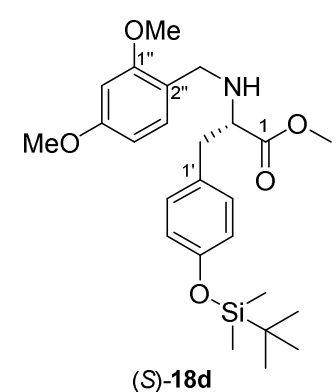

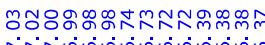

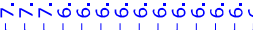

(S)-18d

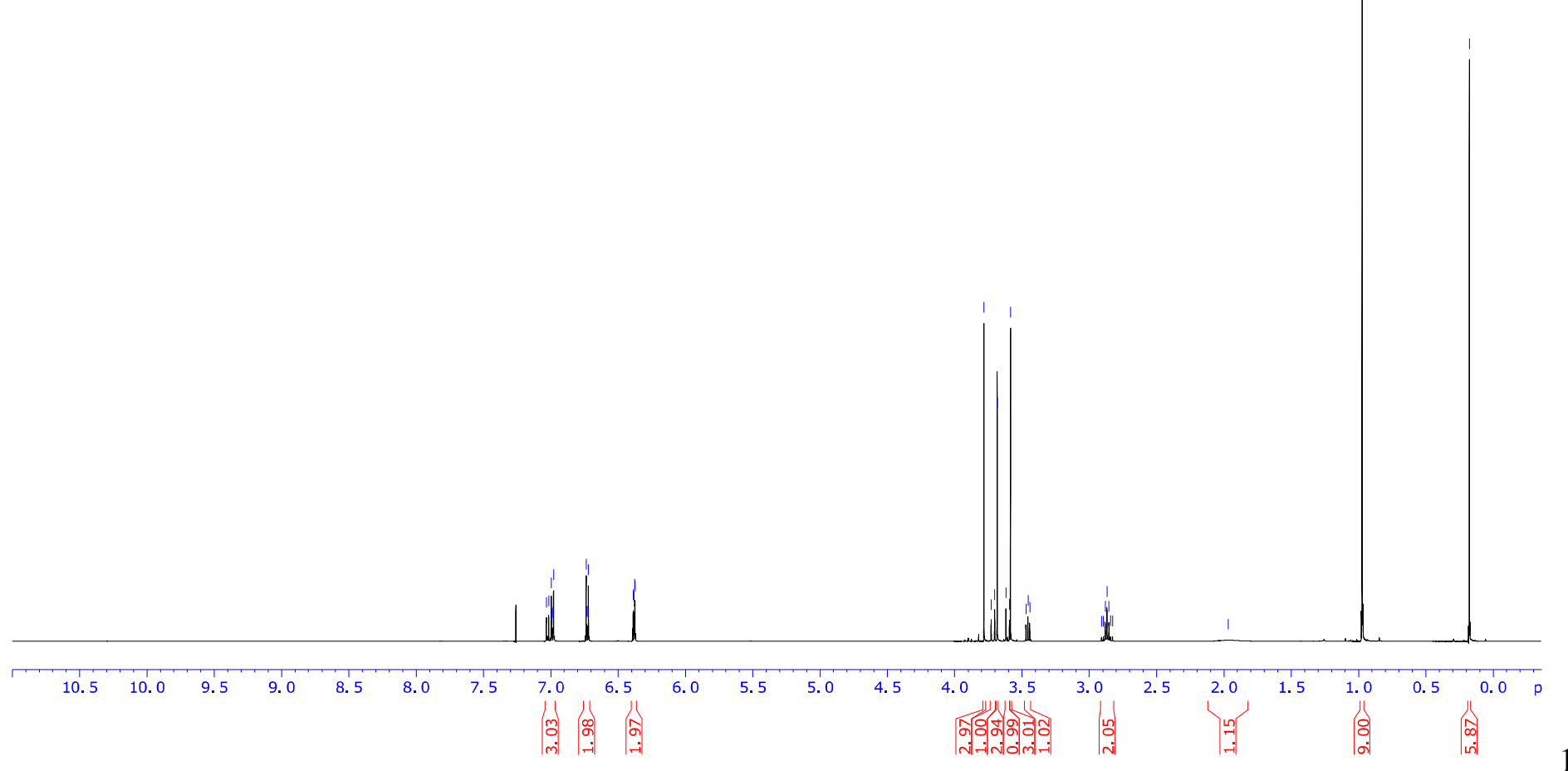


Christian Drescher and Reinhard Brückner; 3D-Structure Clarifying Total Synthesis of the (Polyenoyl)tetramic Acid Militarinon B. A Highly Acid-Labile N-Protecting Group for Amides

Methyl (2S)-3-\{4'-[(tert-Butyldimethylsilyl)oxy]phenyl\}-2-[(1",5"-dimethoxybenzyl)amino]propanoate [(S)-18d]

${ }^{13} \mathrm{C}$ NMR (125.75 MHz, $\left.\mathrm{CDCl}_{3}\right)$ :

OMe

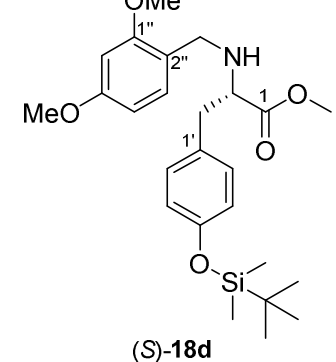

(S)-18d

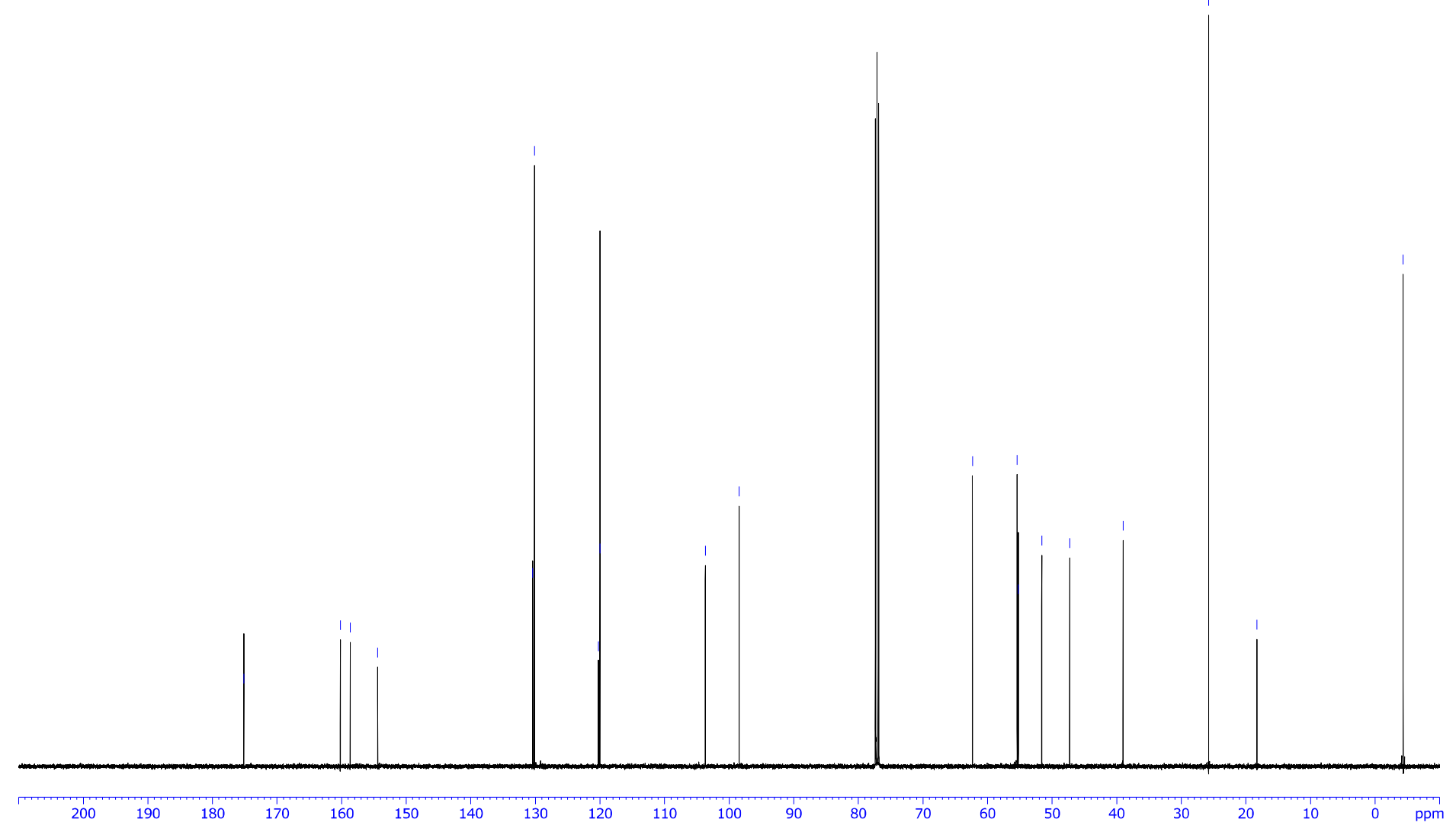


Christian Drescher and Reinhard Brückner; 3D-Structure Clarifying Total Synthesis of the (Polyenoyl)tetramic Acid Militarinon B. A Highly Acid-Labile N-Protecting Group for Amides

Methyl (2'S)-2'-\{N-[(1Z,2Z,4E)-5-Bromo-3-hydroxy-1-oxoypenta-2,4-dienyl]- $N$-(1'",5'"'-dimethoxybenzyl) $\}-3$ '- $\{1$ "-[(tertbutyldimethylsilyl)oxy]phenyl $\}$ propanoate $[Z-e n o l-(S)-21 d]$

${ }^{1} \mathbf{H}$ NMR (500.10 MHz, $\left.\mathrm{CDCl}_{3}\right)$ :

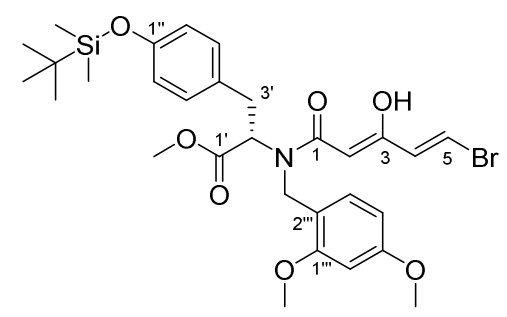

Z-enol-(S)-21d 
Christian Drescher and Reinhard Brückner; 3D-Structure Clarifying Total Synthesis of the (Polyenoyl)tetramic Acid Militarinon B. A Highly Acid-Labile N-Protecting Group for Amides

Methyl (2'S)-2'-\{N-[(1Z,2Z,4E)-5-Bromo-3-hydroxy-1-oxoypenta-2,4-dienyl]- $N$-(1'",5'"'-dimethoxybenzyl) $\}-3$ '- $\{1$ "-[(tertbutyldimethylsilyl)oxy]phenyl $\}$ propanoate $[Z-e n o l-(S)-21 d]$

${ }^{13} \mathrm{C}$ NMR (125.75 MHz, $\left.\mathrm{CDCl}_{3}\right)$ :
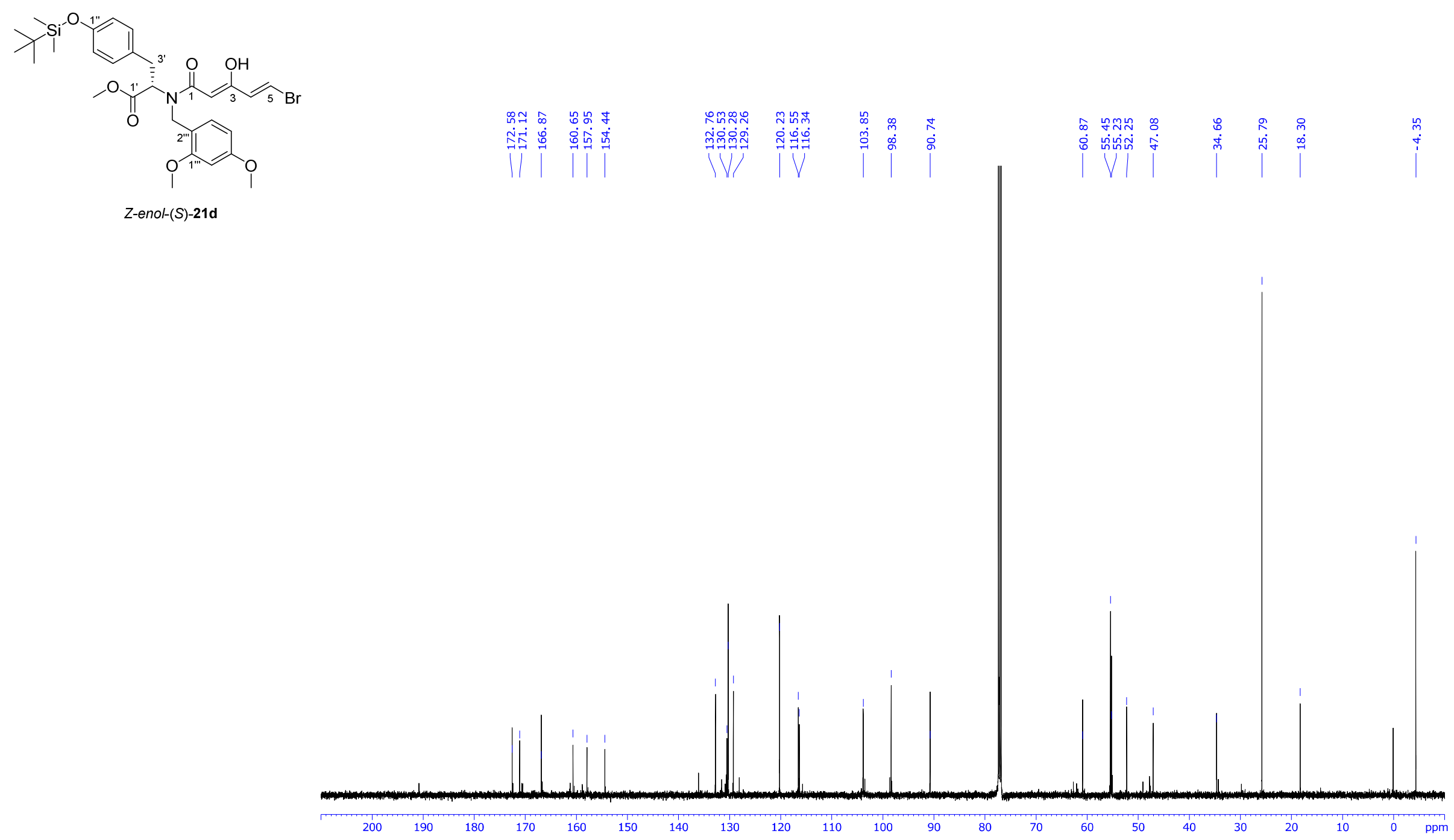

Z-enol-(S)-21d 
Christian Drescher and Reinhard Brückner; 3D-Structure Clarifying Total Synthesis of the (Polyenoyl)tetramic Acid Militarinon B. A Highly Acid-Labile N-Protecting Group for Amides

\section{(E)-(2-Iodovinyl)benzene (SI-6)}

${ }^{1} \mathbf{H}$ NMR $\left(300.06 \mathrm{MHz}, \mathrm{CDCl}_{3}\right)$ :

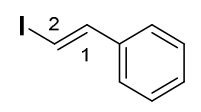

SI-6

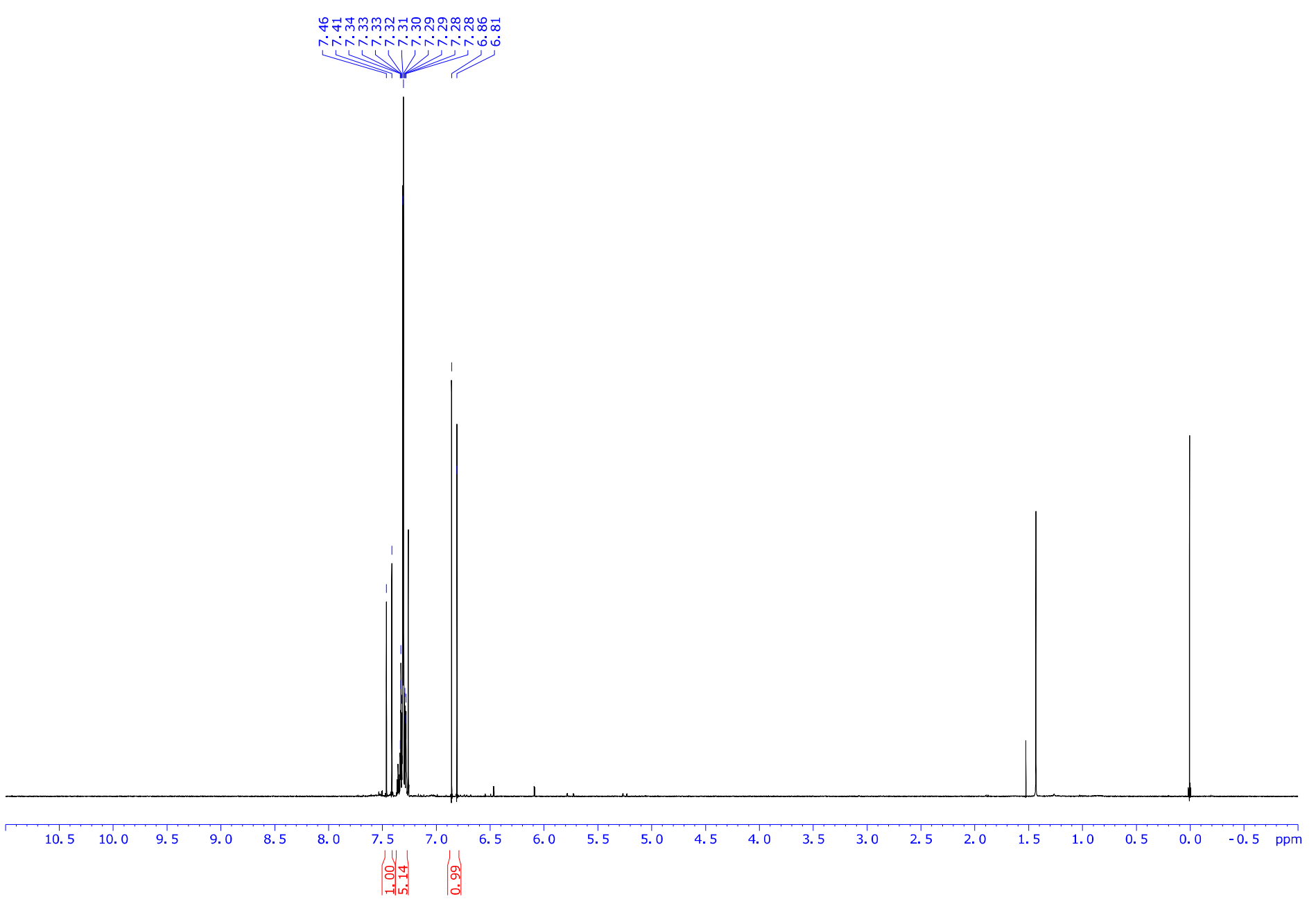


Christian Drescher and Reinhard Brückner; 3D-Structure Clarifying Total Synthesis of the (Polyenoyl)tetramic Acid Militarinon B. A Highly Acid-Labile N-Protecting Group for Amides

\section{(E)-Tributyl(styryl)stannane (SI-7)}

${ }^{1} \mathbf{H}$ NMR (300.06 MHz, $\left.\mathrm{CDCl}_{3}\right)$ :

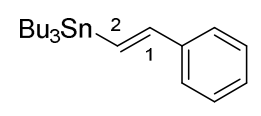

SI-7

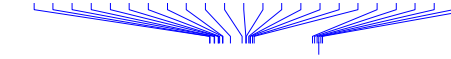


Christian Drescher and Reinhard Brückner; 3D-Structure Clarifying Total Synthesis of the (Polyenoyl)tetramic Acid Militarinon B. A Highly Acid-Labile N-Protecting Group for Amides

Methyl (2'S)-2'-\{N-[(1Z,2Z,4E,6E)-3-Hydroxy-7-phenylhepta-2,4,6-trienamido]- $N$-(1'",5"'-dimethoxybenzyl)\}-3'-\{1"'-[(tertbutyldimethylsilyl)oxy]phenyl\}propanoate [Z-enol-(S)-22d]

${ }^{1} \mathbf{H}$ NMR (500.10 MHz, $\left.\mathrm{CDCl}_{3}\right)$ :

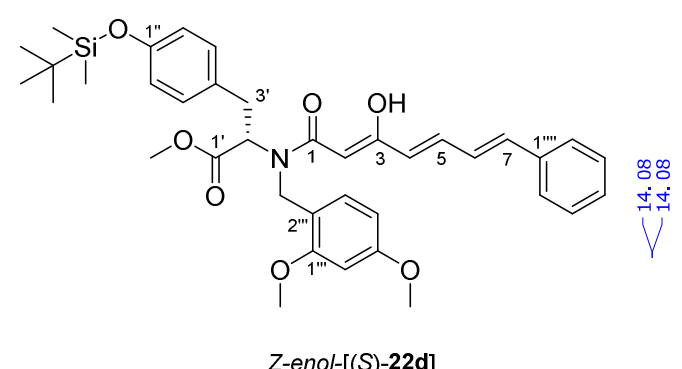

Z-enol-[(S)-22d] 
Christian Drescher and Reinhard Brückner; 3D-Structure Clarifying Total Synthesis of the (Polyenoyl)tetramic Acid Militarinon B. A Highly Acid-Labile N-Protecting Group for Amides

Methyl (2'S)-2'-\{N-[(1Z,2Z,4E,6E)-3-Hydroxy-7-phenylhepta-2,4,6-trienamido]- $N$-(1'",5'"'-dimethoxybenzyl)\}-3'-\{1"'-[(tertbutyldimethylsilyl)oxy]phenyl\}propanoate [Z-enol-(S)-22d]

${ }^{13} \mathrm{C}$ NMR (125.75 MHz, $\left.\mathrm{CDCl}_{3}\right)$ :
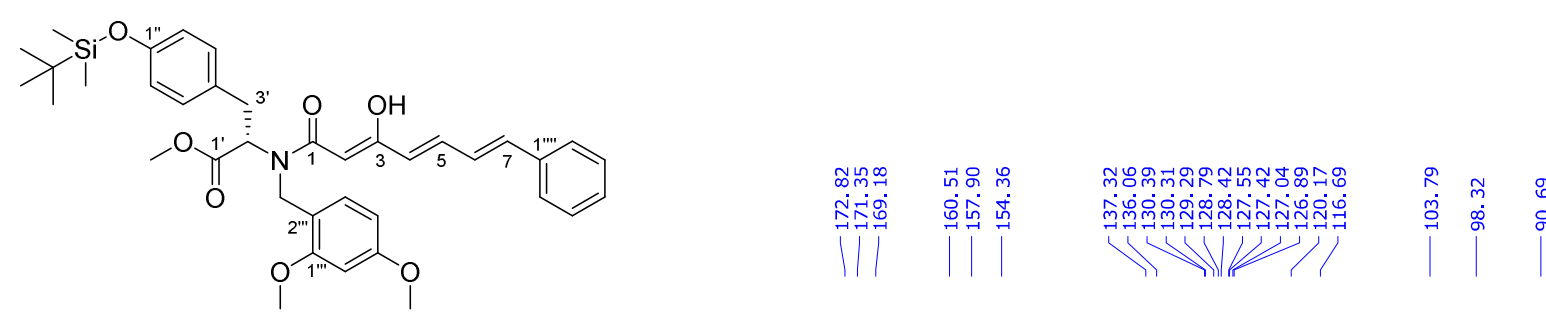

Z-enol-[(S)-22d] 
Christian Drescher and Reinhard Brückner; 3D-Structure Clarifying Total Synthesis of the (Polyenoyl)tetramic Acid Militarinon B. A Highly Acid-Labile N-Protecting Group for Amides

(5S)-5-(1"-Hydroxybenzyl)-1-(1'",5'"'-dimethoxybenzyl)-3-(1'Z,2'E,4'E)-1'-hydroxy-5-phenylpenta-2',4"'-dien-1'-ylidene)pyrrolidine-2,4dion [Z-enol-(S)-16d]

${ }^{1} \mathbf{H}$ NMR $\left(500.10 \mathrm{MHz}, \mathrm{CDCl}_{3}\right)$ :

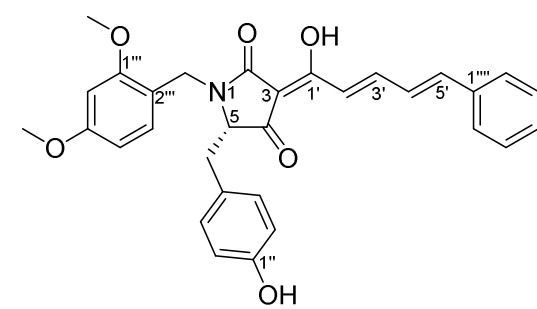

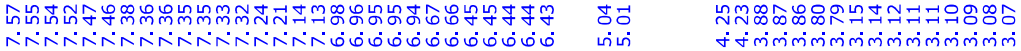

Z-enol-(S)-16d

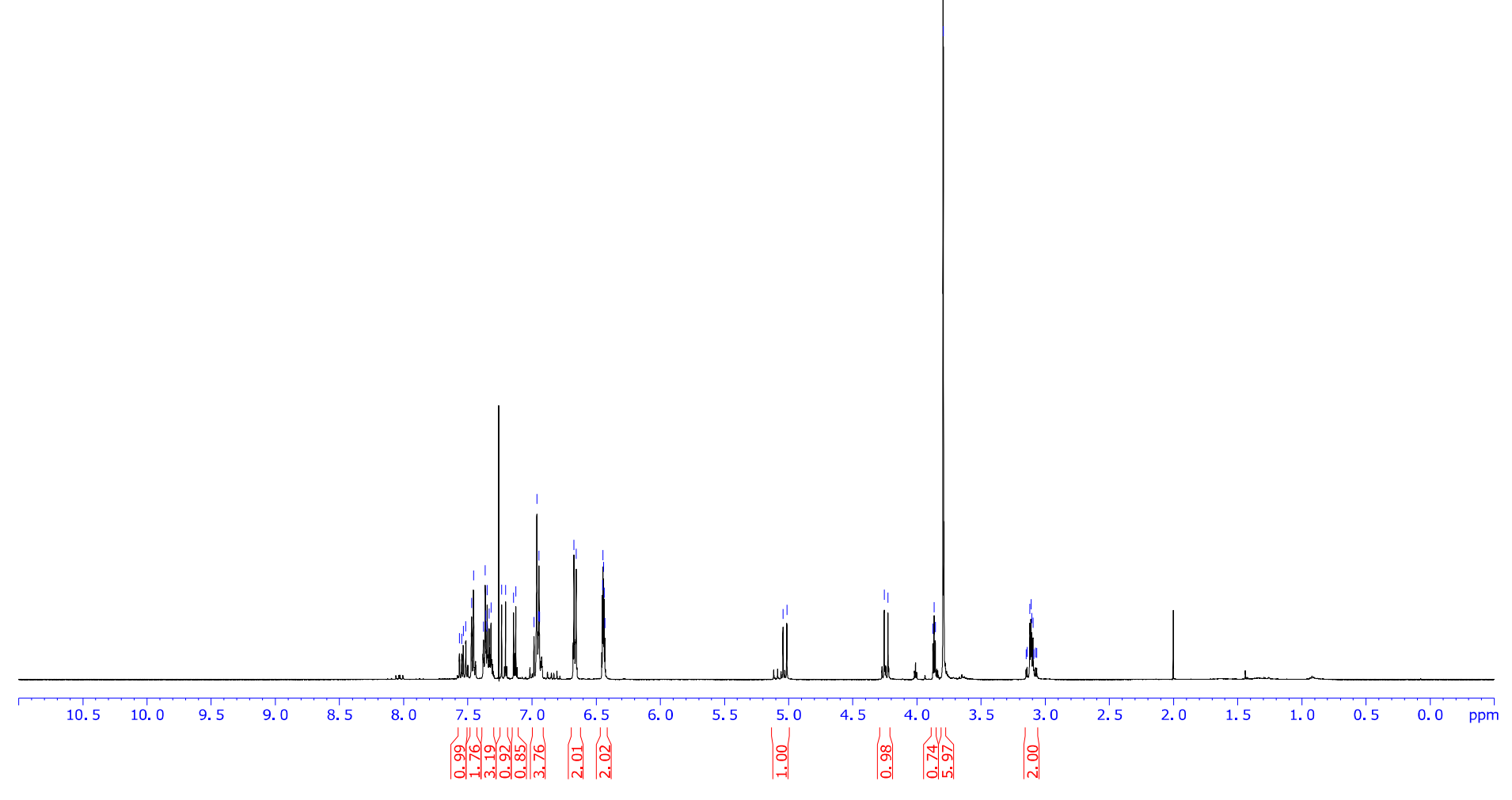


Christian Drescher and Reinhard Brückner; 3D-Structure Clarifying Total Synthesis of the (Polyenoyl)tetramic Acid Militarinon B. A Highly Acid-Labile N-Protecting Group for Amides

(5S)-5-(1"-Hydroxybenzyl)-1-(1"',5'"'-dimethoxybenzyl)-3-(1'Z,2' E,4'E)-1'-hydroxy-5-phenylpenta-2',4''-dien-1'-ylidene)pyrrolidine-2,4dion [Z-enol-(S)-16d]

${ }^{13} \mathrm{C}$ NMR (125.75 MHz, $\left.\mathrm{CDCl}_{3}\right)$ :

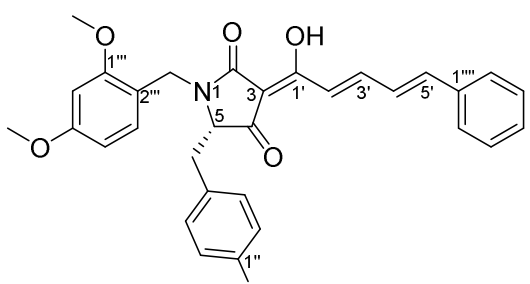

$\mathrm{OH}$

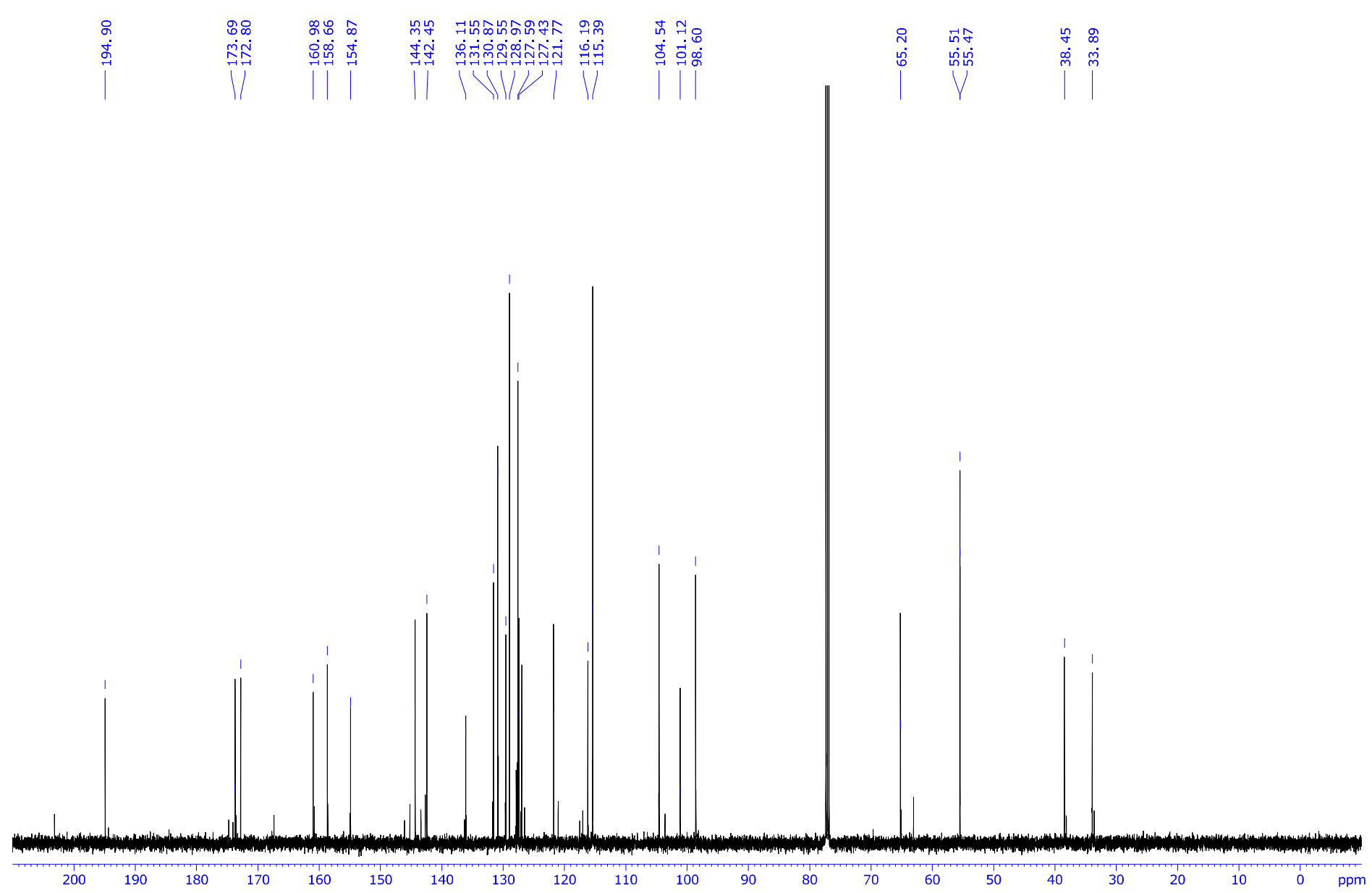

Z-enol-(S)-16d 
Christian Drescher and Reinhard Brückner; 3D-Structure Clarifying Total Synthesis of the (Polyenoyl)tetramic Acid Militarinon B. A Highly Acid-Labile N-Protecting Group for Amides

(5S)-5-(1'-Hydroxybenzyl)-3-(1'Z,2' E,4' E)-1'-hydroxy-5-phenylpenta-2',4'-dien-1'-ylidene)pyrrolidine-2,4-dion [Z-enol-(S)-17]

${ }^{1}$ H NMR (500.10 MHz, MeOD):
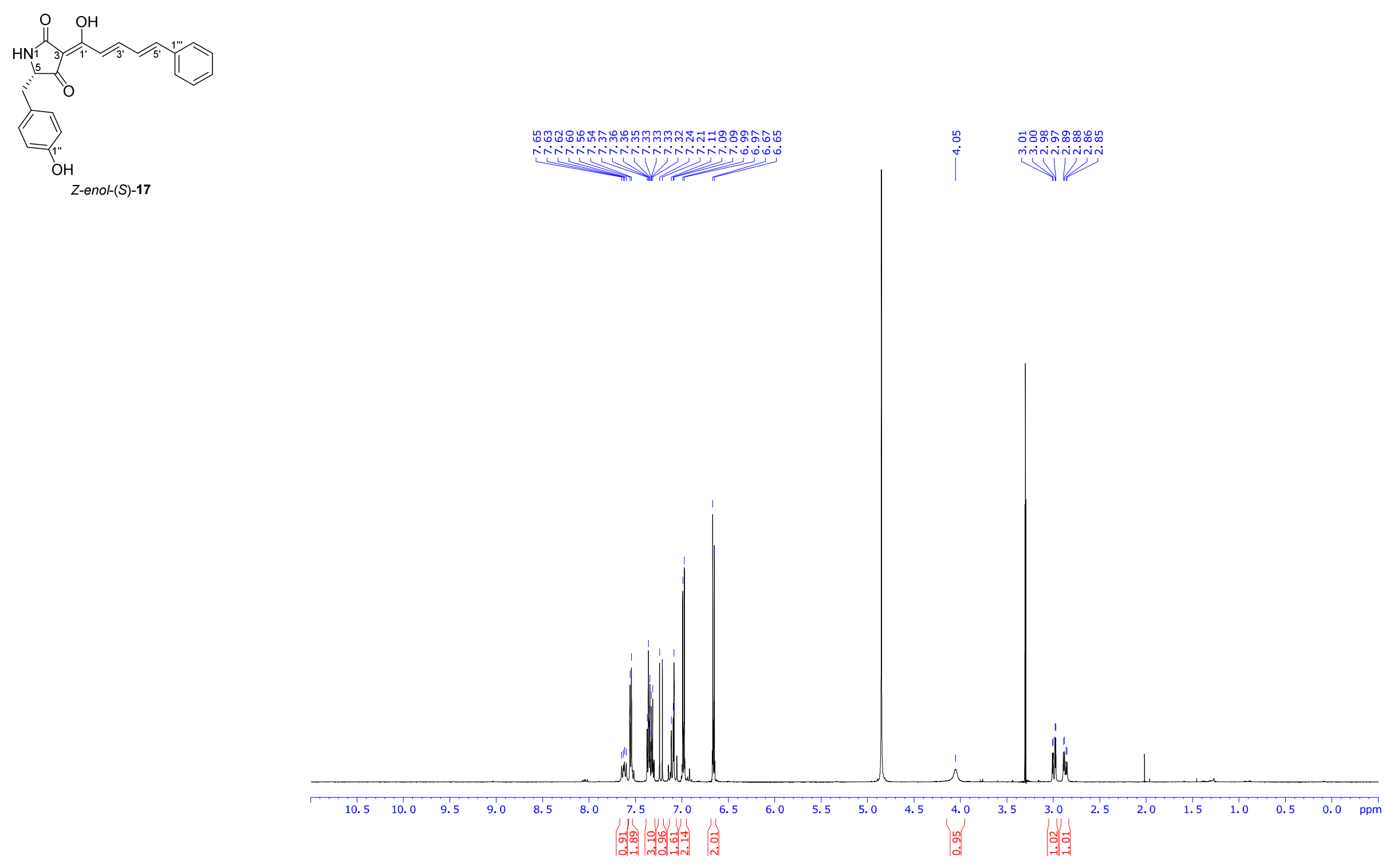
Christian Drescher and Reinhard Brückner; 3D-Structure Clarifying Total Synthesis of the (Polyenoyl)tetramic Acid Militarinon B. A Highly Acid-Labile N-Protecting Group for Amides

(5S)-5-(1'-Hydroxybenzyl)-3-(1'Z,2' E,4' E)-1'-hydroxy-5-phenylpenta-2',4'-dien-1'-ylidene)pyrrolidine-2,4-dion [Z-enol-(S)-17] ${ }^{13}$ C NMR (125.75 MHz, MeOD):
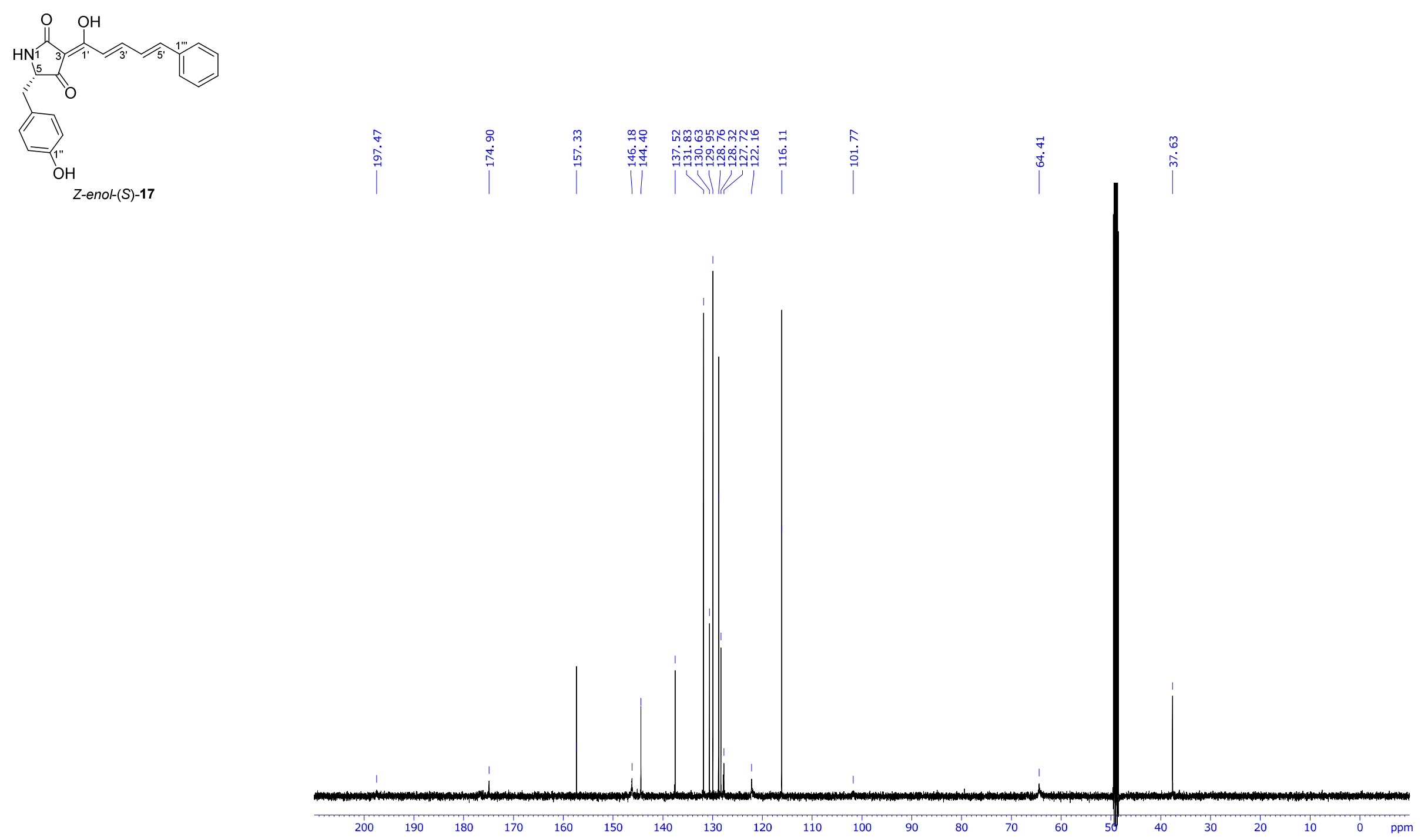
Christian Drescher and Reinhard Brückner; 3D-Structure Clarifying Total Synthesis of the (Polyenoyl)tetramic Acid Militarinon B. A Highly Acid-Labile N-Protecting Group for Amides

\section{Methyl (2S)-3-\{1'-[(tert-Butyldimethylsilyl)oxy]phenyl\}-2-[(1"'-nitrobenzyl)amino]propanoate [(S)-18a]}

${ }^{1} \mathbf{H}$ NMR (500.10 MHz, $\left.\mathrm{CDCl}_{3}\right)$ :

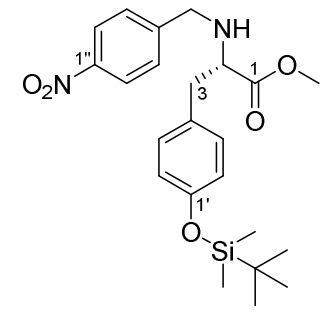

$(S)-\mathbf{1 8 a}$
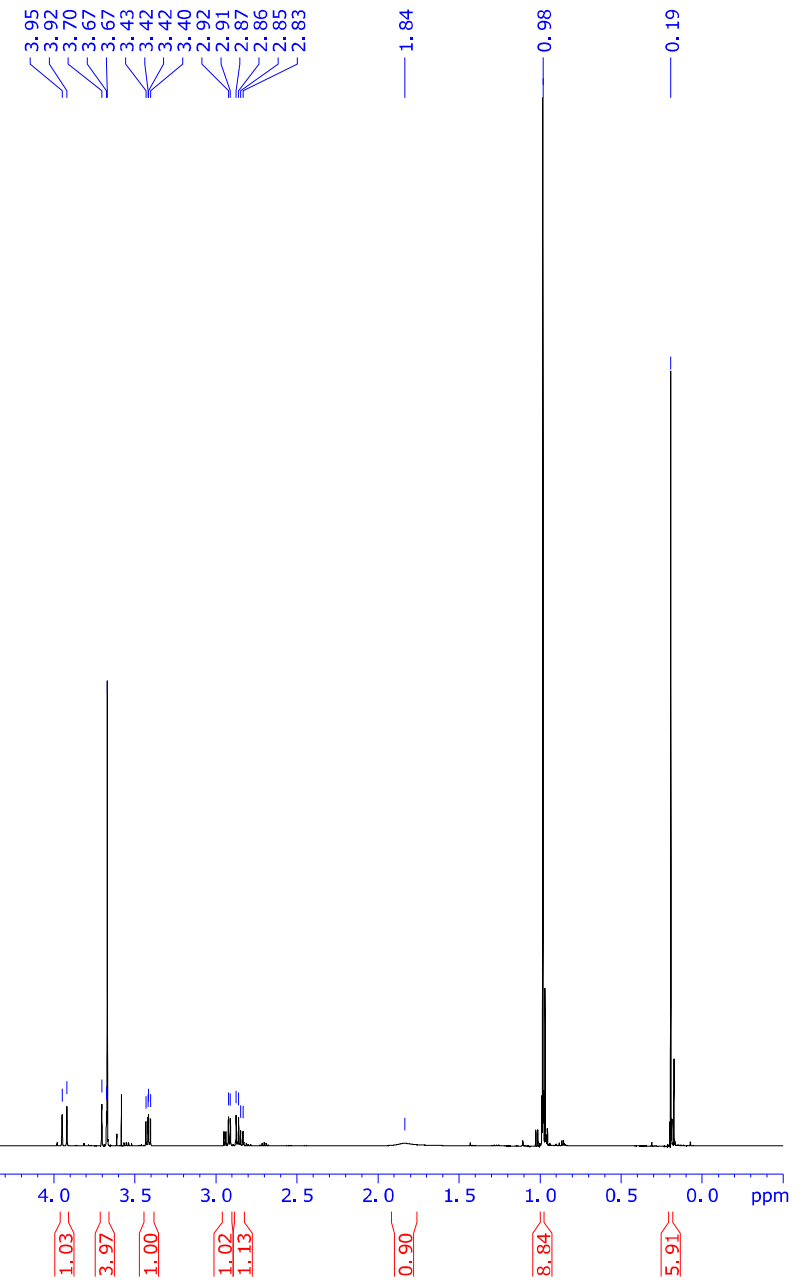
Christian Drescher and Reinhard Brückner; 3D-Structure Clarifying Total Synthesis of the (Polyenoyl)tetramic Acid Militarinon B. A Highly Acid-Labile N-Protecting Group for Amides

\section{Methyl (2S)-3-\{1'-[(tert-Butyldimethylsilyl)oxy]phenyl\}-2-[(1"'-nitrobenzyl)amino]propanoate [(S)-18a]}

${ }^{13} \mathrm{C}$ NMR (125.75 MHz, $\left.\mathrm{CDCl}_{3}\right)$ :

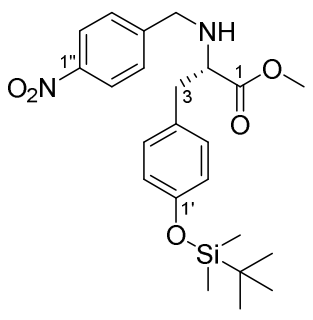

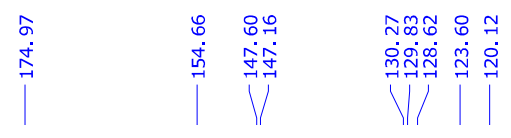

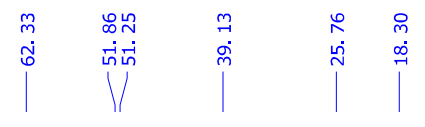

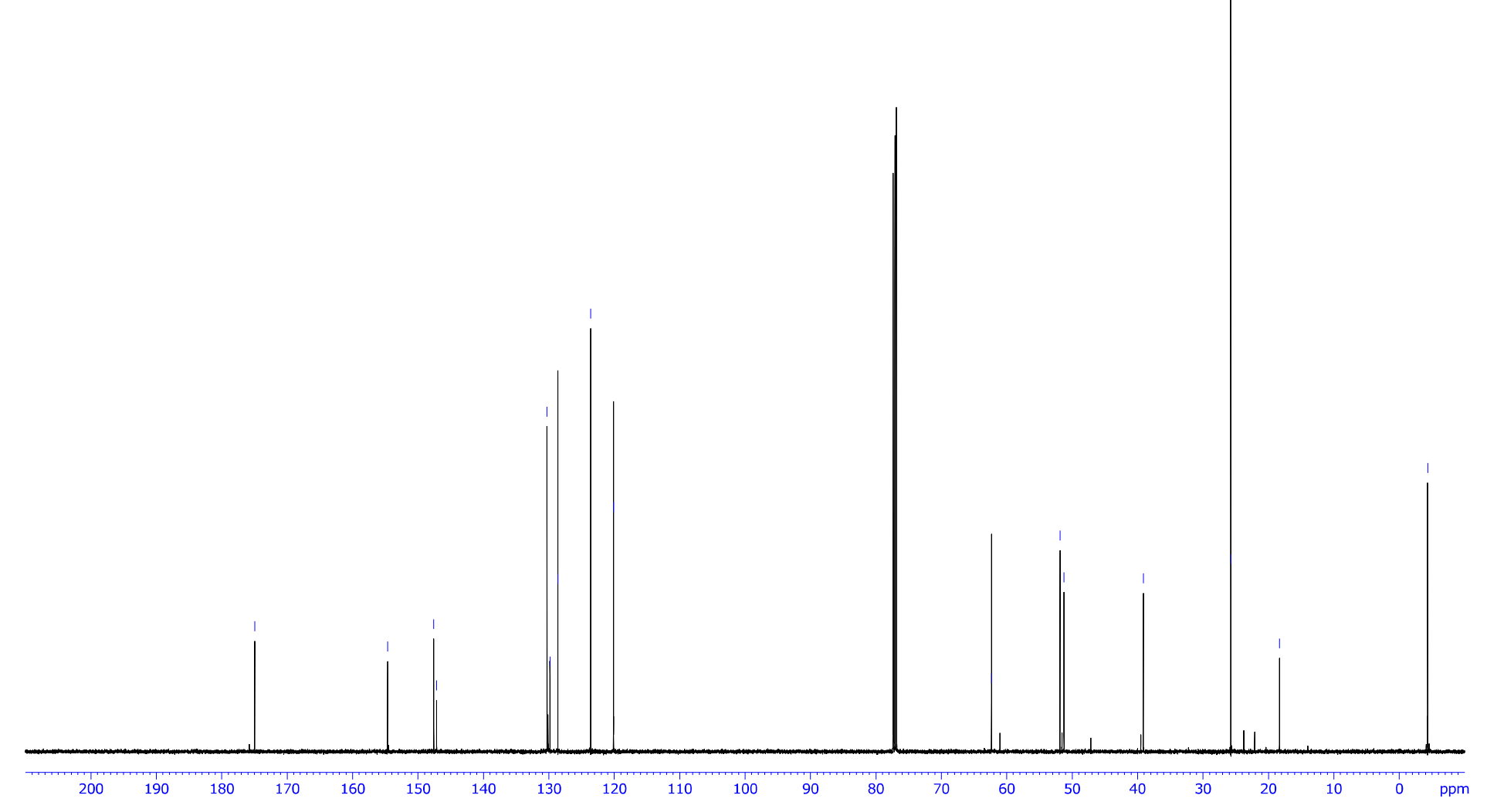


Christian Drescher and Reinhard Brückner; 3D-Structure Clarifying Total Synthesis of the (Polyenoyl)tetramic Acid Militarinon B. A Highly Acid-Labile N-Protecting Group for Amides

Methyl (2'S)-2'-\{N-[(1Z,2Z,4E)-5-Bromo-3-hydroxy-1-oxoypenta-2,4-dienyl]- $N$-(1'"'-nitrobenzyl) $\}-3$ '- $\{1$ "-[(tertbutyldimethylsilyl)oxy]phenyl $\}$ propanoate $[Z-e n o l-(S)-21 a]$

${ }^{1} \mathbf{H}$ NMR (500.10 MHz, $\left.\mathrm{CDCl}_{3}\right)$ :
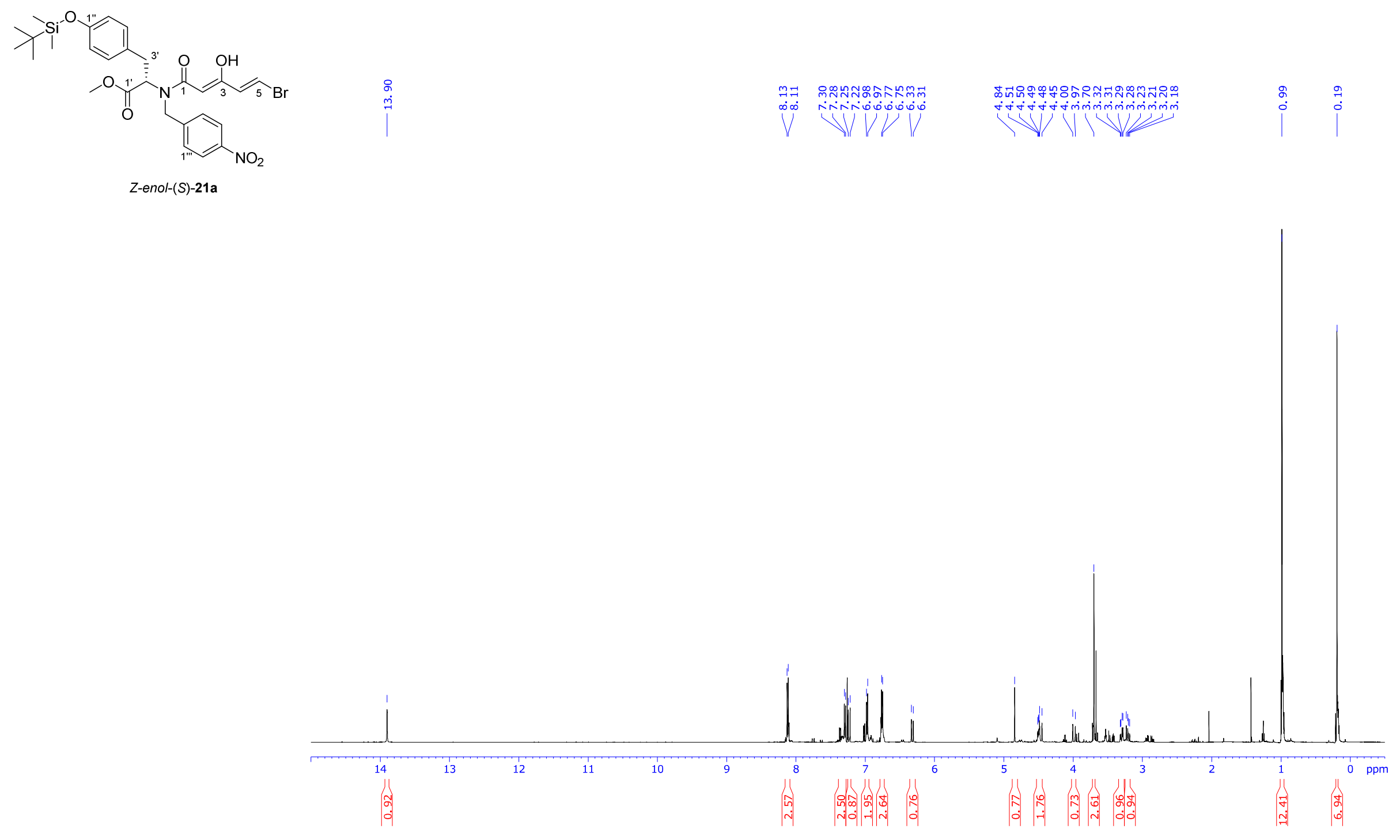
Christian Drescher and Reinhard Brückner; 3D-Structure Clarifying Total Synthesis of the (Polyenoyl)tetramic Acid Militarinon B. A Highly Acid-Labile N-Protecting Group for Amides

Methyl (2'S)-2'-\{N-[(1Z,2Z,4E)-5-Bromo-3-hydroxy-1-oxoypenta-2,4-dienyl]-N-(1'"'-nitrobenzyl) $\}-3$ '- $\{1$ '-[(tertbutyldimethylsilyl)oxy]phenyl $\}$ propanoate $[Z-e n o l-(S)-21 a]$

${ }^{13} \mathrm{C}$ NMR (125.75 MHz, $\left.\mathrm{CDCl}_{3}\right)$ :
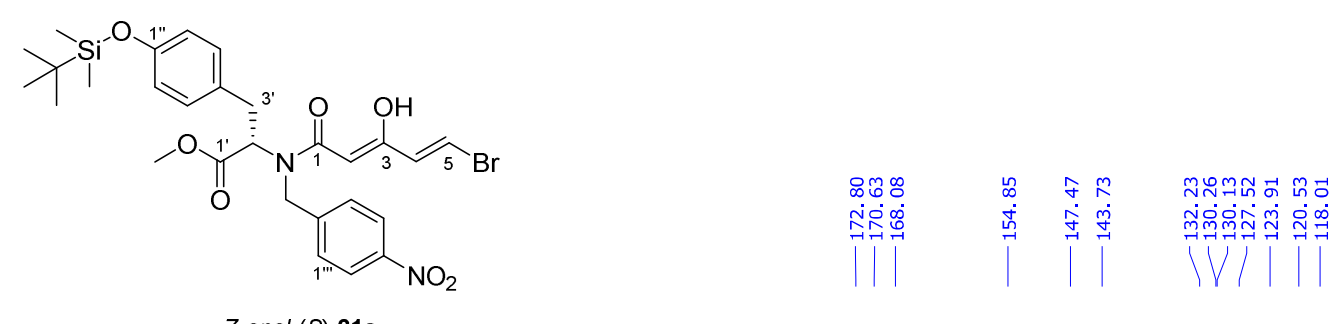

Z-enol-(S)-21a

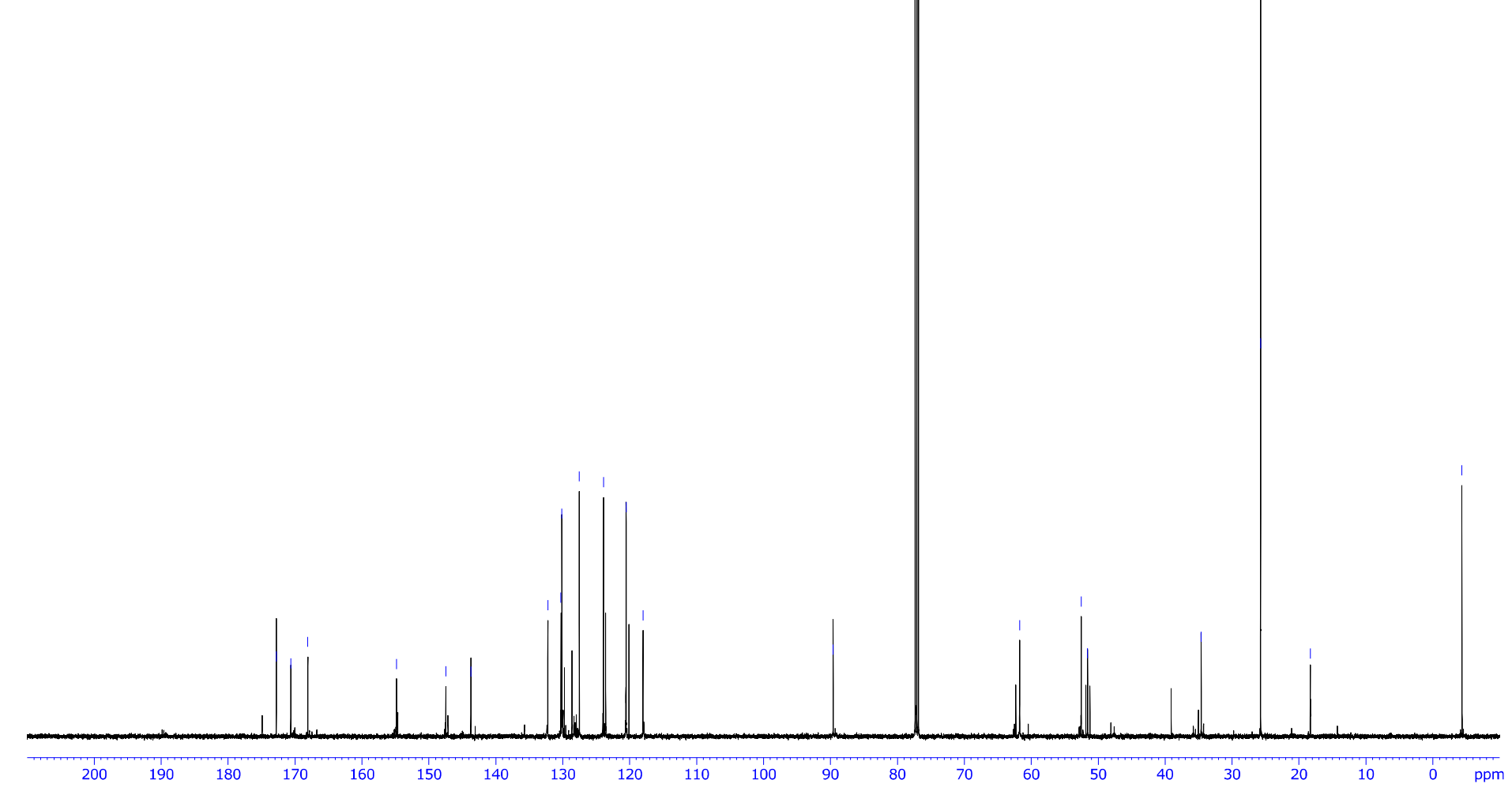


Christian Drescher and Reinhard Brückner; 3D-Structure Clarifying Total Synthesis of the (Polyenoyl)tetramic Acid Militarinon B. A Highly Acid-Labile N-Protecting Group for Amides

Methyl (2'S)-2'-\{N-[(1Z,2Z,4E,6E)-3-Hydroxy-7-phenylhepta-2,4,6-trienamido]- $N$-(1'"'-nitrobenzyl) $\}-3$ '-\{1"'-[(tertbutyldimethylsilyl)oxy]phenyl\}propanoate [Z-enol-(S)-22a]

${ }^{1} \mathbf{H}$ NMR (500.10 MHz, $\left.\mathrm{CDCl}_{3}\right)$ :

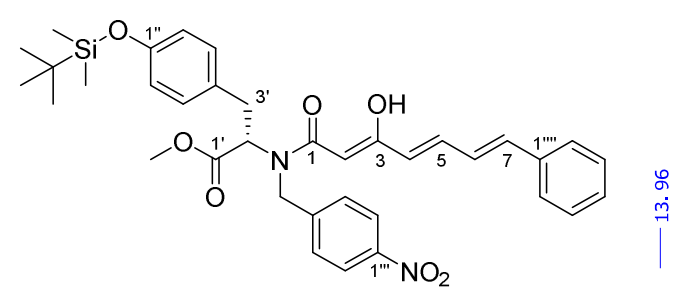

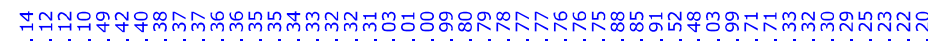

Z-enol-[(S)-22a]

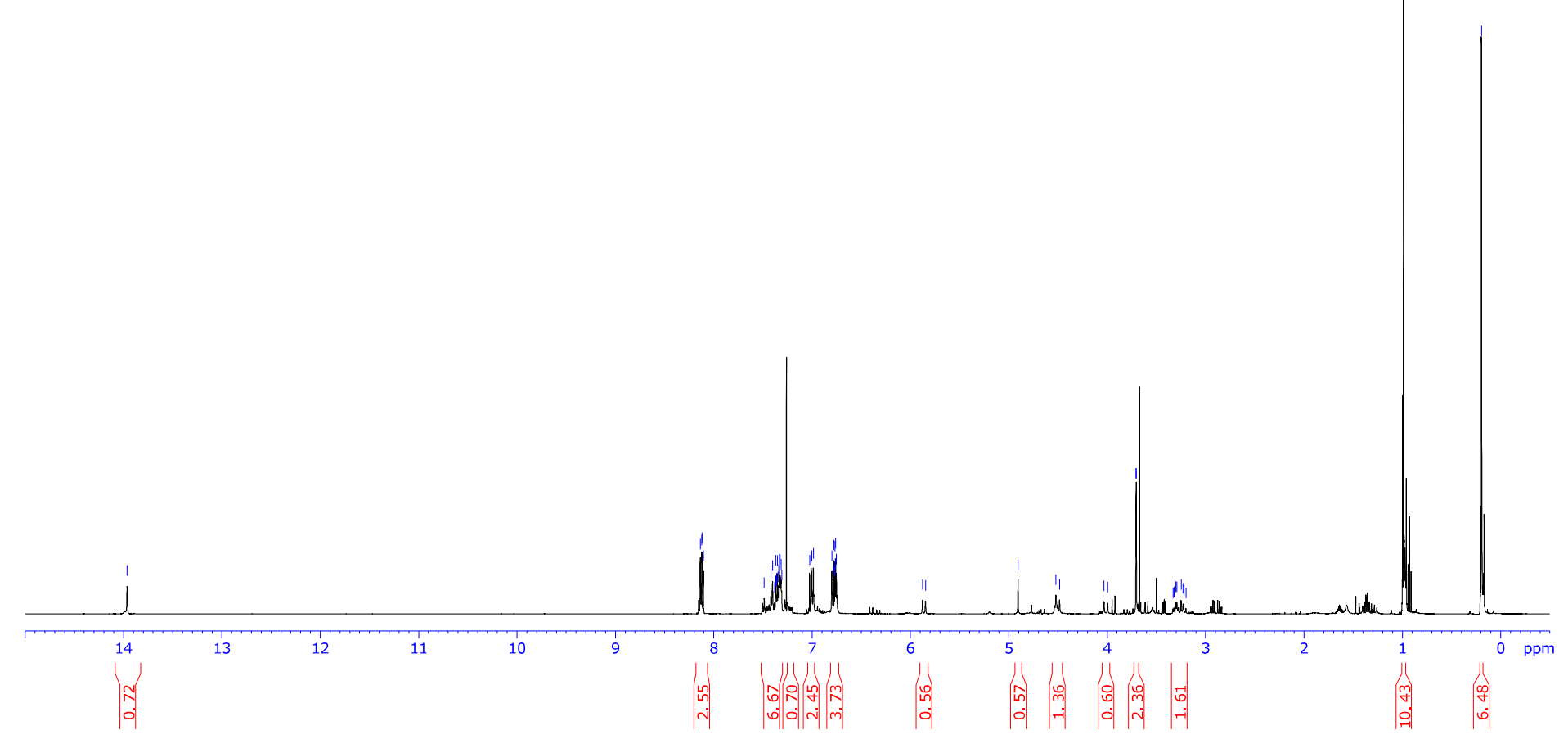


Christian Drescher and Reinhard Brückner; 3D-Structure Clarifying Total Synthesis of the (Polyenoyl)tetramic Acid Militarinon B. A Highly Acid-Labile N-Protecting Group for Amides

Methyl (2'S)-2'-\{N-[(1Z,2Z,4E,6E)-3-Hydroxy-7-phenylhepta-2,4,6-trienamido]- $N$-(1'"'-nitrobenzyl) $\}-3$ '-\{1"'-[(tertbutyldimethylsilyl)oxy]phenyl\}propanoate [Z-enol-(S)-22a]

${ }^{13} \mathrm{C}$ NMR (125.75 MHz, $\left.\mathrm{CDCl}_{3}\right)$ :
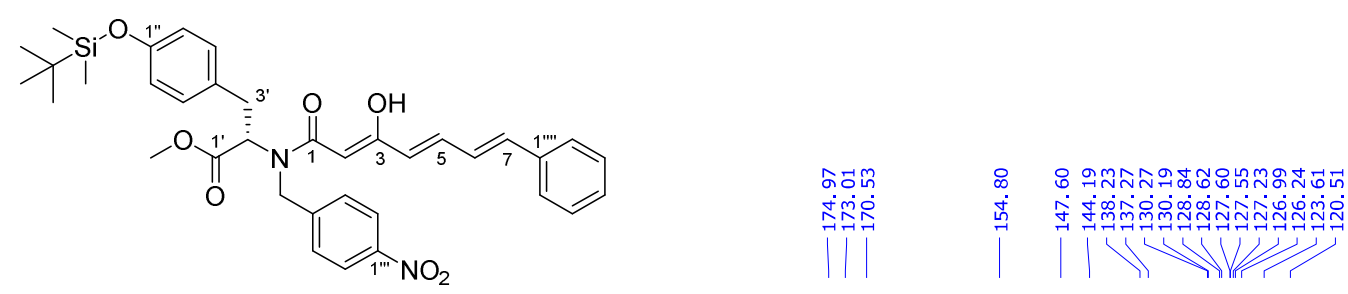

Z-enol-[(S)-22a]

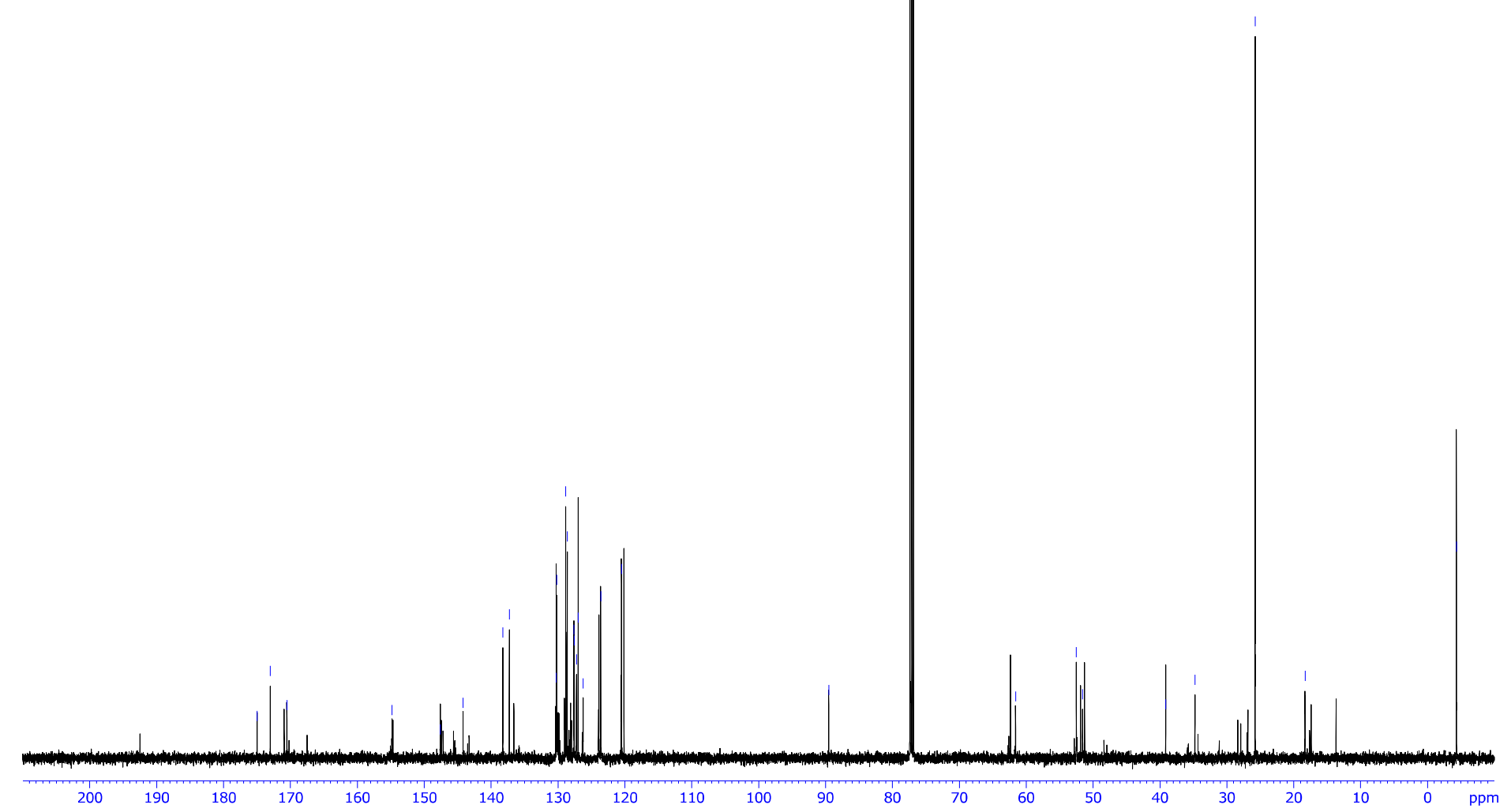


Christian Drescher and Reinhard Brückner; 3D-Structure Clarifying Total Synthesis of the (Polyenoyl)tetramic Acid Militarinon B. A Highly Acid-Labile N-Protecting Group for Amides

(5S)-5-\{1"'-[(tert-Butyldimethylsilyl)oxy]benzyl\}-1-(1'"'-nitrobenzyl)-3-(1'Z,2'E,4'E)-1'-hydroxy-5-phenylpenta-2',4''-dien-1'ylidene)pyrrolidine-2,4-dion [Z-enol-(S)-16a]

${ }^{1} \mathbf{H}$ NMR (500.10 MHz, $\left.\mathrm{CDCl}_{3}\right)$ :

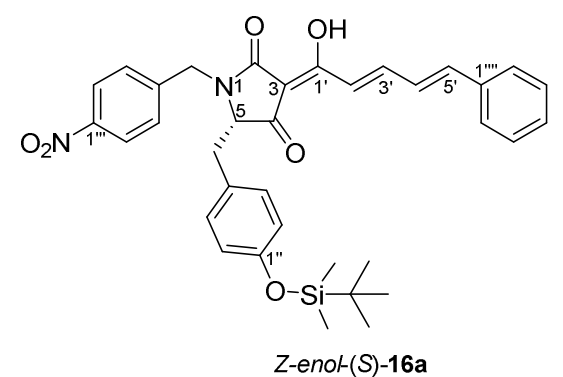

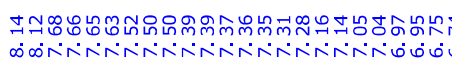

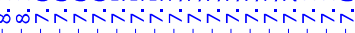

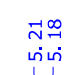

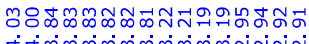
Z-enol-(S)-16a

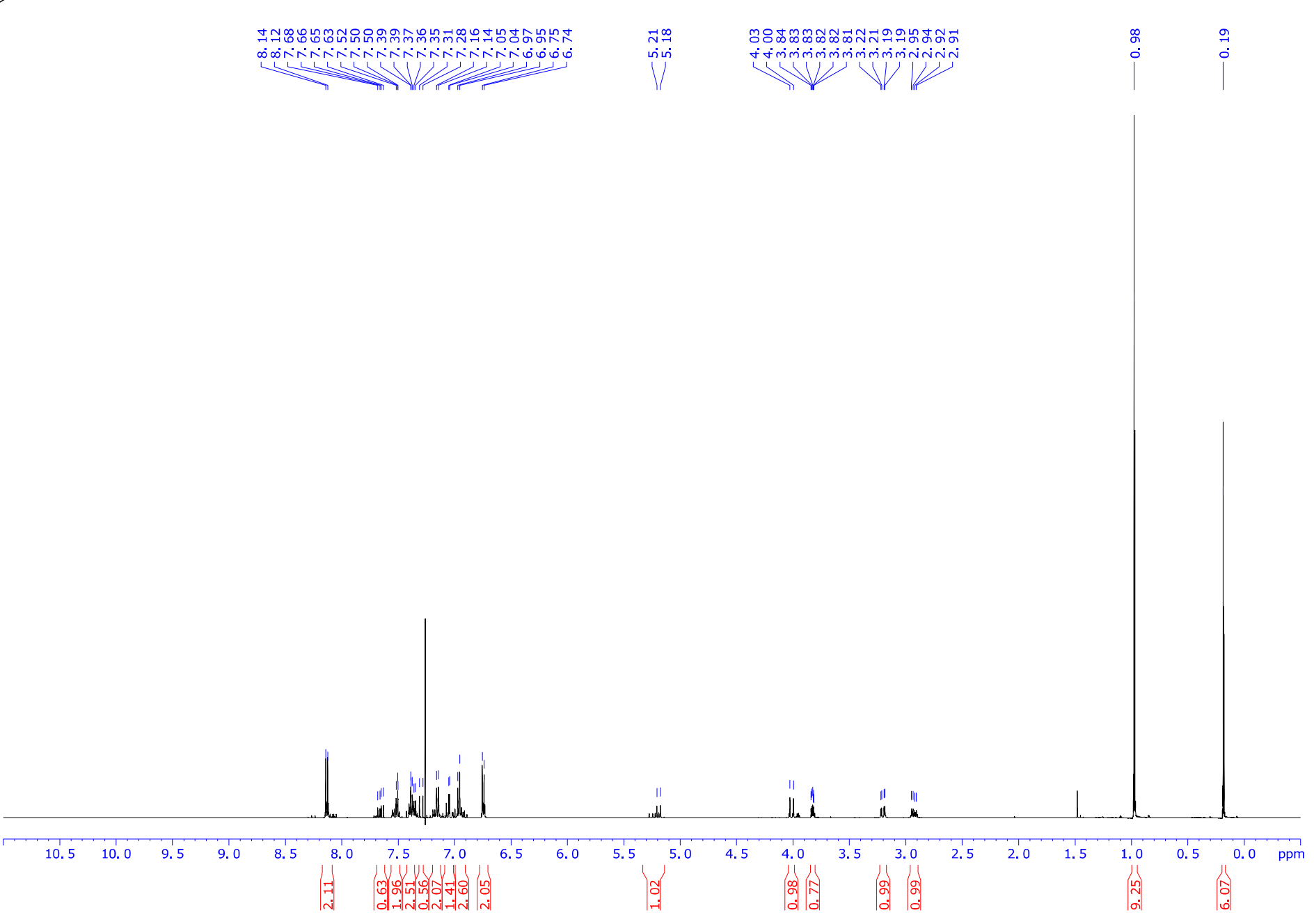


Christian Drescher and Reinhard Brückner; 3D-Structure Clarifying Total Synthesis of the (Polyenoyl)tetramic Acid Militarinon B. A Highly Acid-Labile N-Protecting Group for Amides

(5S)-5-\{1"'-[(tert-Butyldimethylsilyl)oxy]benzyl\}-1-(1'"'-nitrobenzyl)-3-(1'Z,2'E,4'E)-1'-hydroxy-5-phenylpenta-2',4' '-dien-1'ylidene)pyrrolidine-2,4-dion [Z-enol-(S)-16a]

${ }^{13} \mathrm{C}$ NMR (125.75 MHz, $\left.\mathrm{CDCl}_{3}\right)$ :
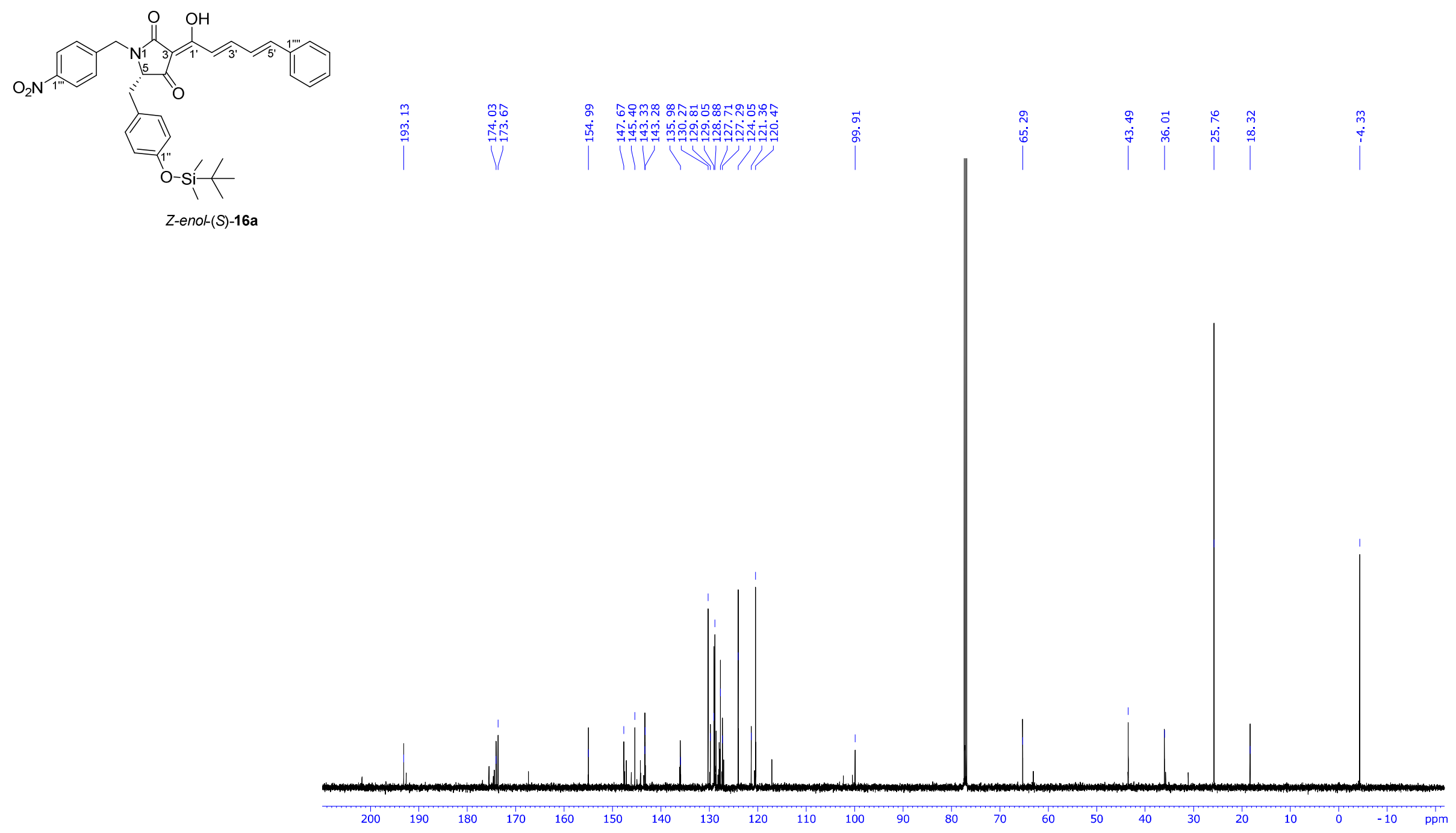
Christian Drescher and Reinhard Brückner; 3D-Structure Clarifying Total Synthesis of the (Polyenoyl)tetramic Acid Militarinon B. A Highly Acid-Labile N-Protecting Group for Amides

Methyl (2S)-3-\{1'-[(tert-Butyldimethylsilyl)oxy]phenyl\}-2-[(4",5"-dimethoxy-1"-nitrobenzyl)amino]propanoate propanoate [(S)-18c] ${ }^{1} \mathbf{H}$ NMR (500.10 MHz, $\left.\mathrm{CDCl}_{3}\right)$ :
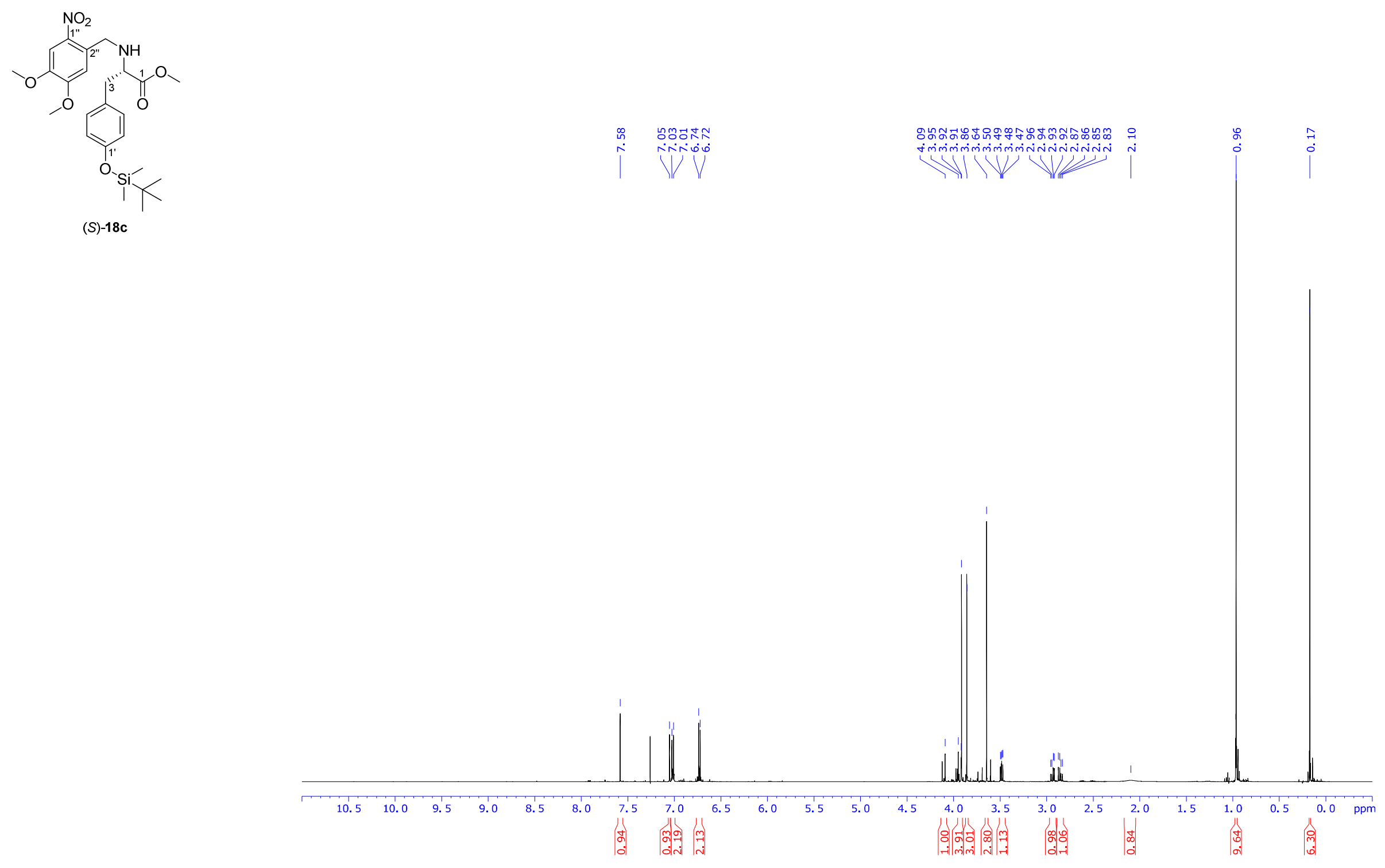

(S)-18c 
Christian Drescher and Reinhard Brückner; 3D-Structure Clarifying Total Synthesis of the (Polyenoyl)tetramic Acid Militarinon B. A Highly Acid-Labile N-Protecting Group for Amides

Methyl (2S)-3-\{1'-[(tert-Butyldimethylsilyl)oxy]phenyl\}-2-[(4",5"-dimethoxy-1"-nitrobenzyl)amino]propanoate propanoate [(S)-18c] ${ }^{13}$ C NMR (125.75 MHz, $\left.\mathrm{CDCl}_{3}\right)$ :

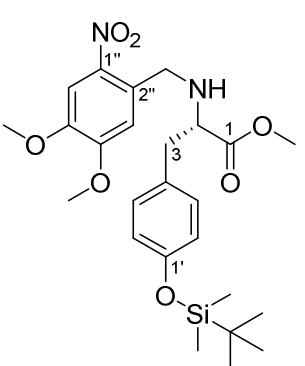

(S)-18c

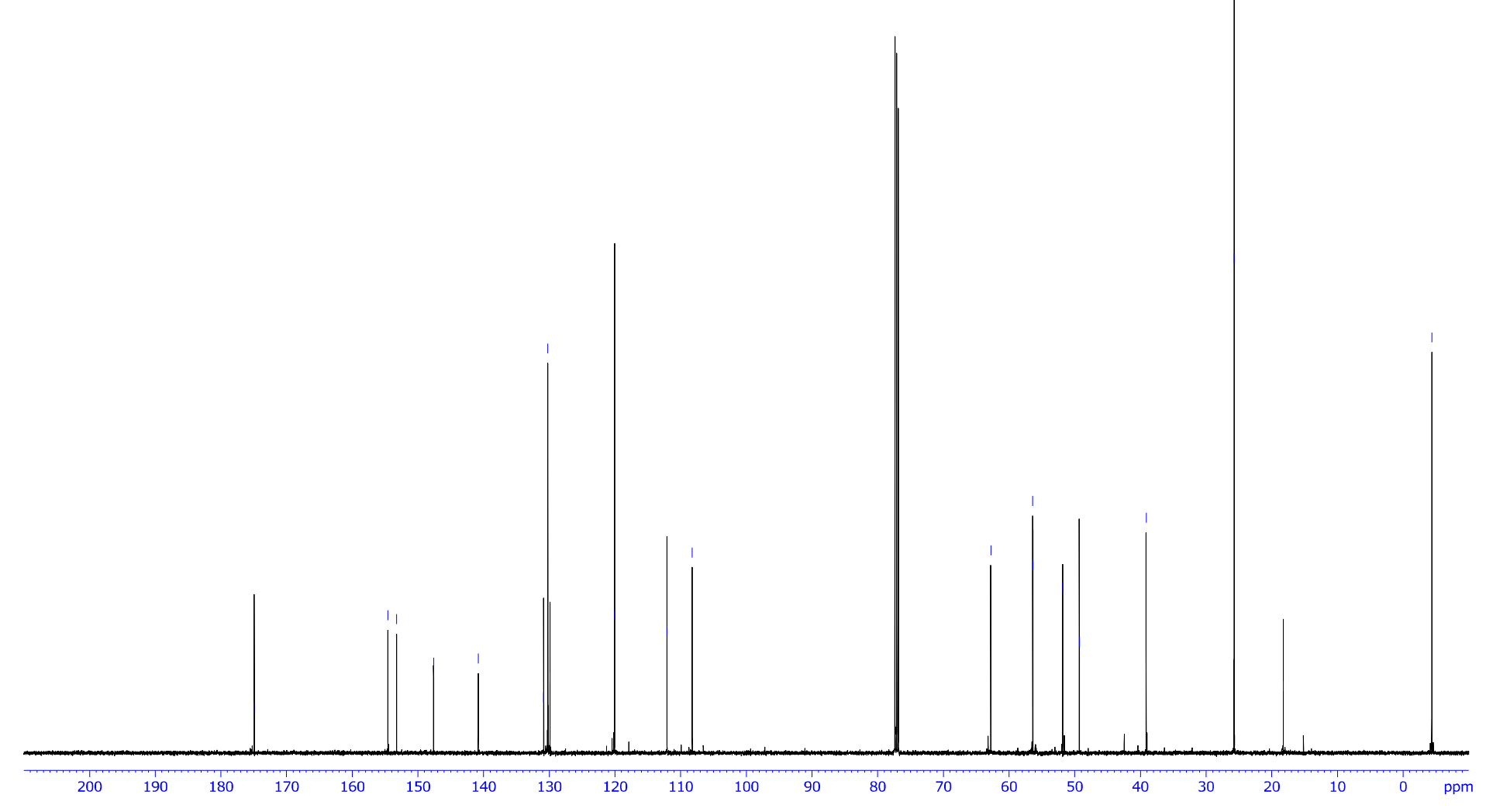


Christian Drescher and Reinhard Brückner; 3D-Structure Clarifying Total Synthesis of the (Polyenoyl)tetramic Acid Militarinon B. A Highly Acid-Labile N-Protecting Group for Amides

Methyl (2'S)-2'-\{N-[(1Z,2Z,4E)-5-Bromo-3-hydroxy-1-oxoypenta-2,4-dienyl]- $N$-(4'"',5'"'-dimethoxy-1"'-nitrobenzyl) $\}-3$ '- $\{1$ '"-[(tertbutyldimethylsilyl)oxy]phenyl $\}$ propanoate $[Z-e n o l-(S)-21 \mathrm{c}]$

${ }^{1} \mathbf{H}$ NMR (500.10 MHz, $\left.\mathrm{CDCl}_{3}\right)$ :

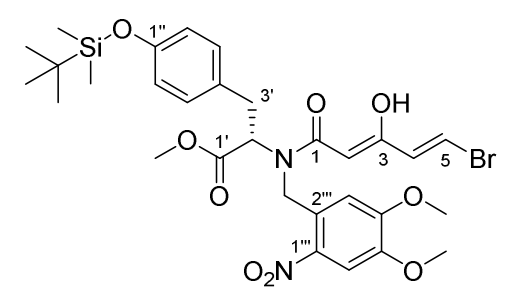

Z-enot-(S)-21c

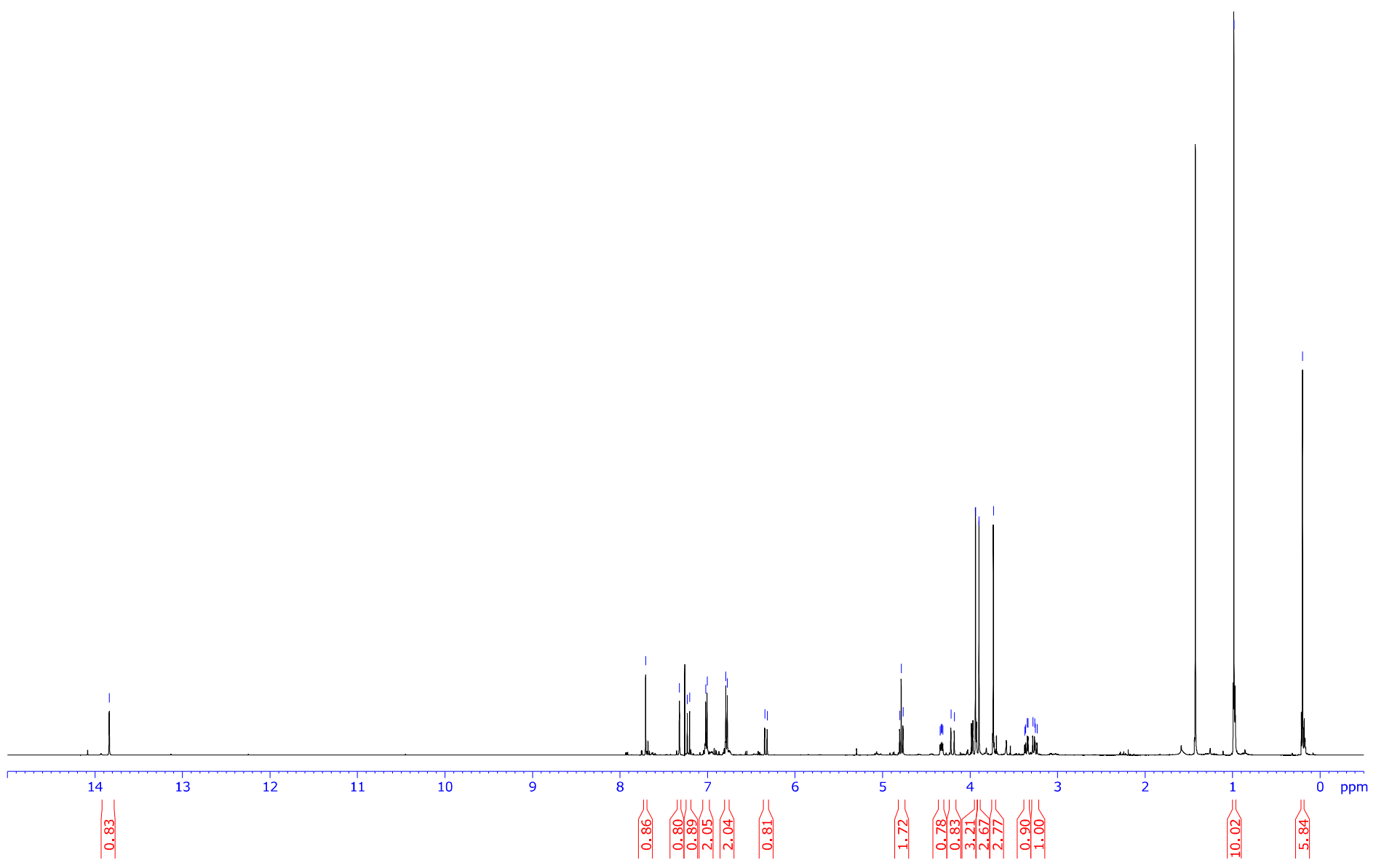


Christian Drescher and Reinhard Brückner; 3D-Structure Clarifying Total Synthesis of the (Polyenoyl)tetramic Acid Militarinon B. A Highly Acid-Labile N-Protecting Group for Amides

Methyl (2'S)-2'-\{N-[(1Z,2Z,4E)-5-Bromo-3-hydroxy-1-oxoypenta-2,4-dienyl]- $N$-(4'",5'"'-dimethoxy-1"'-nitrobenzyl) $\}-3$ '- $\{1$ "'-[(tertbutyldimethylsilyl)oxy]phenyl $\}$ propanoate $[Z-e n o l-(S)-21 \mathrm{c}]$

${ }^{13} \mathrm{C}$ NMR (125.75 MHz, $\left.\mathrm{CDCl}_{3}\right)$ :
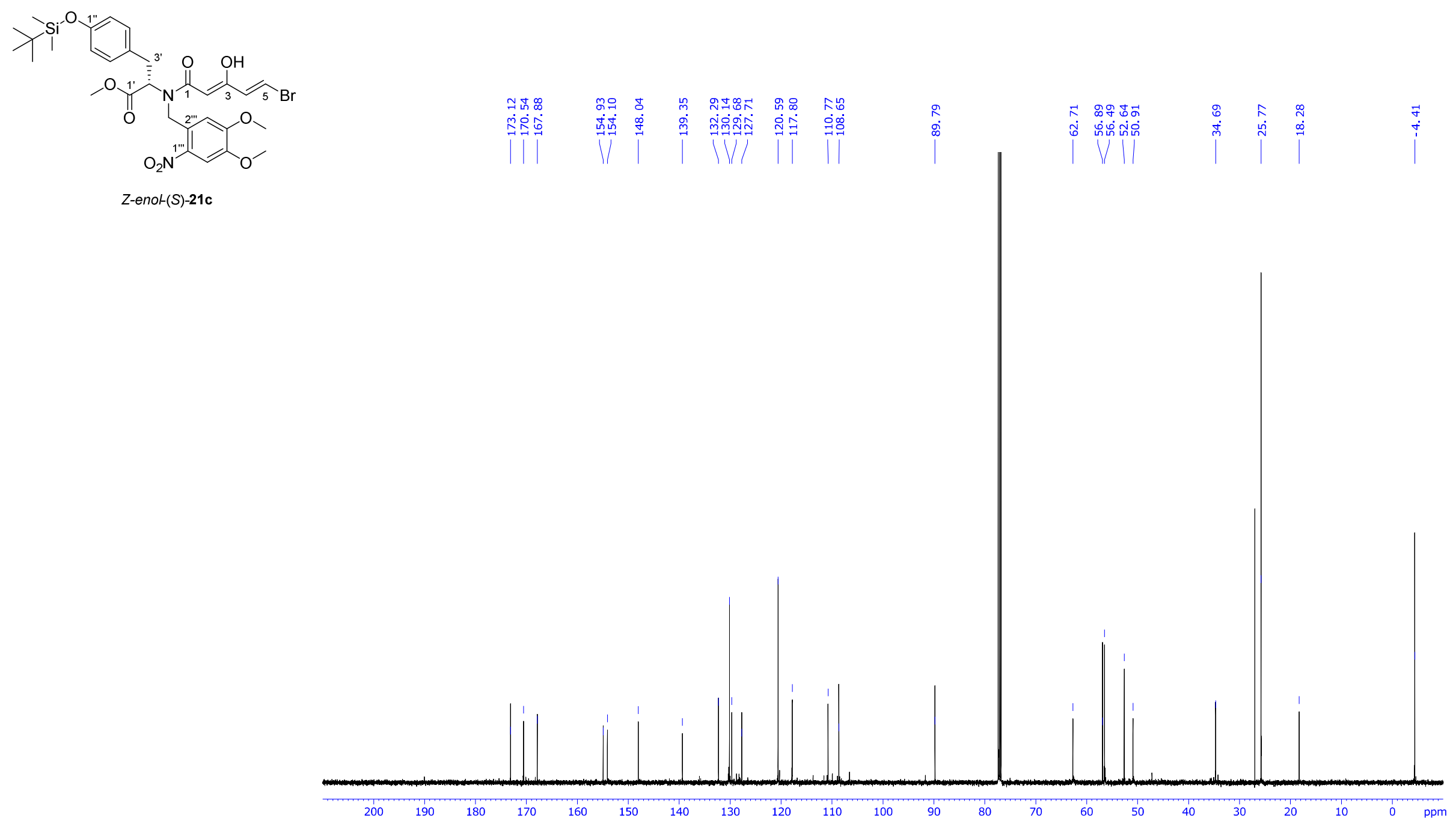

Z-enot-(S)-21c 
Christian Drescher and Reinhard Brückner; 3D-Structure Clarifying Total Synthesis of the (Polyenoyl)tetramic Acid Militarinon B. A Highly Acid-Labile N-Protecting Group for Amides

Methyl (2'S)-2'-\{N-[(1Z,2Z,4E,6E)-3-Hydroxy-7-phenylhepta-2,4,6-trienamido]- $N$-(4'"',5"'-dimethoxy-1"'-nitrobenzyl)\}-3'-\{1"'-[(tertbutyldimethylsilyl)oxy]phenyl\}propanoate [Z-enol-(S)-22c]

${ }^{1} \mathbf{H}$ NMR (500.10 MHz, $\left.\mathrm{CDCl}_{3}\right)$ :

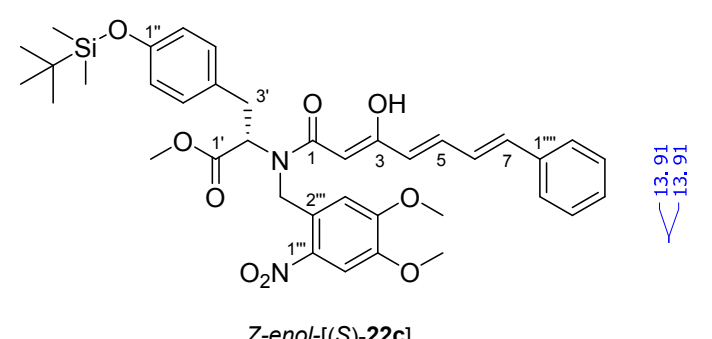

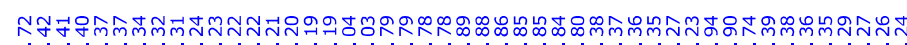

Z-enol-[(S)-22c]

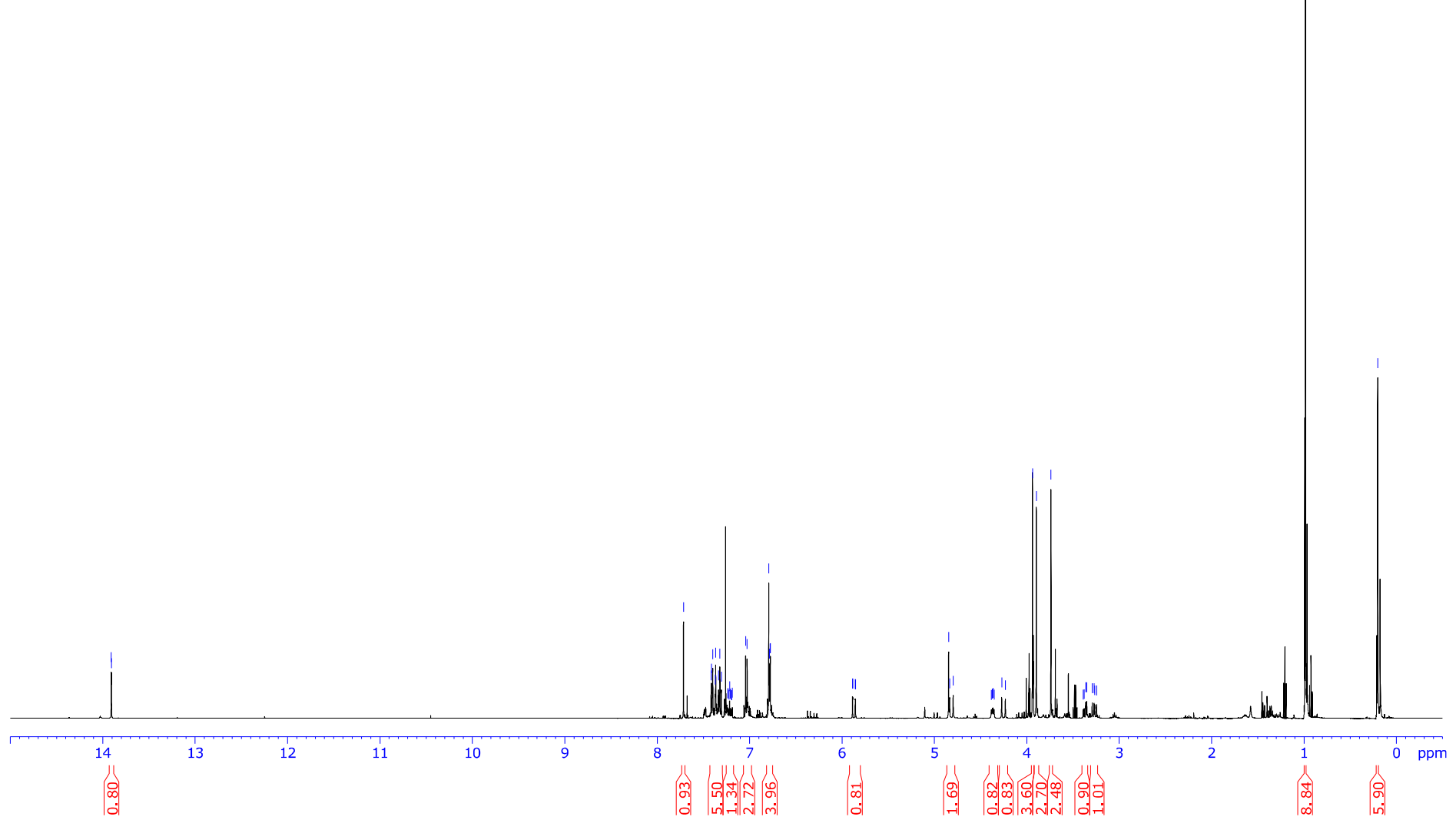


Christian Drescher and Reinhard Brückner; 3D-Structure Clarifying Total Synthesis of the (Polyenoyl)tetramic Acid Militarinon B. A Highly Acid-Labile N-Protecting Group for Amides

Methyl (2'S)-2'-\{N-[(1Z,2Z,4E,6E)-3-Hydroxy-7-phenylhepta-2,4,6-trienamido]- $N$-(4'"',5"'-dimethoxy-1"'-nitrobenzyl)\}-3'-\{1"'-[(tertbutyldimethylsilyl)oxy]phenyl\}propanoate [Z-enol-(S)-22c]

${ }^{13}$ C NMR (125.75 MHz, $\left.\mathrm{CDCl}_{3}\right)$ :
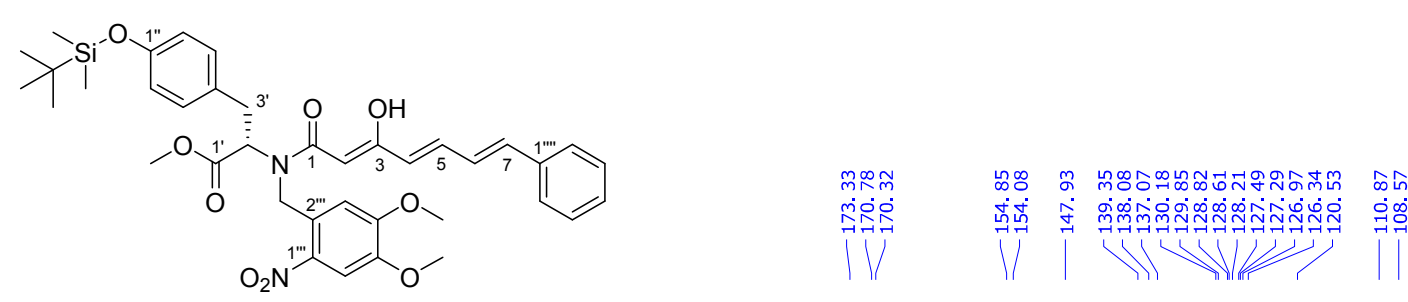

Z-enol-[(S)-22c]

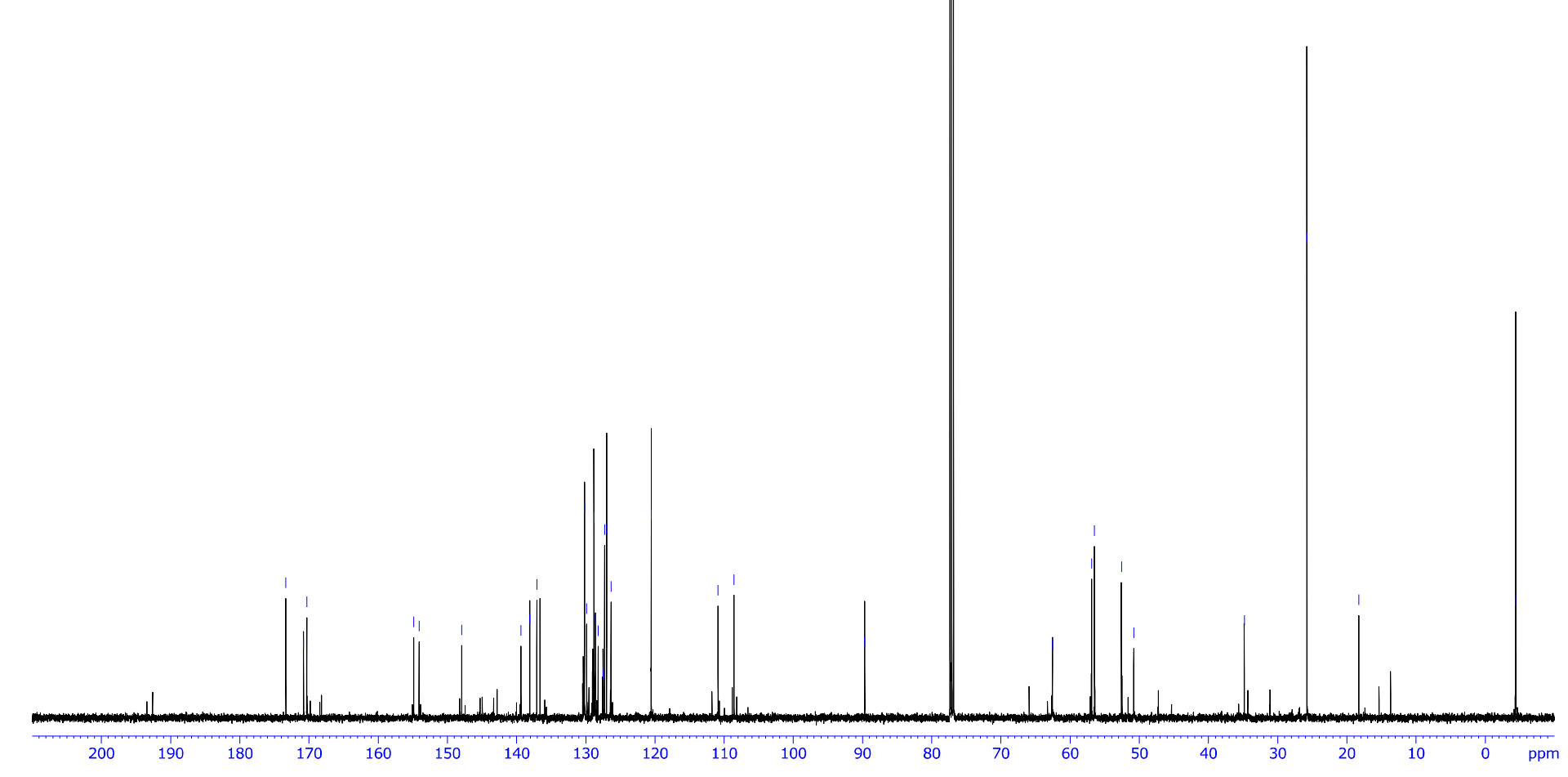


Christian Drescher and Reinhard Brückner; 3D-Structure Clarifying Total Synthesis of the (Polyenoyl)tetramic Acid Militarinon B. A Highly Acid-Labile N-Protecting Group for Amides

(5S)-5-\{1'-[(tert-Butyldimethylsilyl)oxy]benzyl\}-1-(4'",5"'-dimethoxy-1'"'-nitrobenzyl)-3-(1'Z,2'E,4'E)-1'-hydroxy-5-phenylpenta-2',4"'dien-1'-ylidene)pyrrolidine-2,4-dion [Z-enol-(S)-16c]

${ }^{1} \mathbf{H}$ NMR (500.10 MHz, $\left.\mathrm{CDCl}_{3}\right)$ :

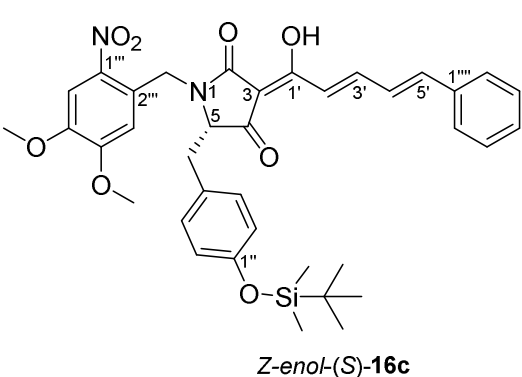

Z-enol-(S)-16c

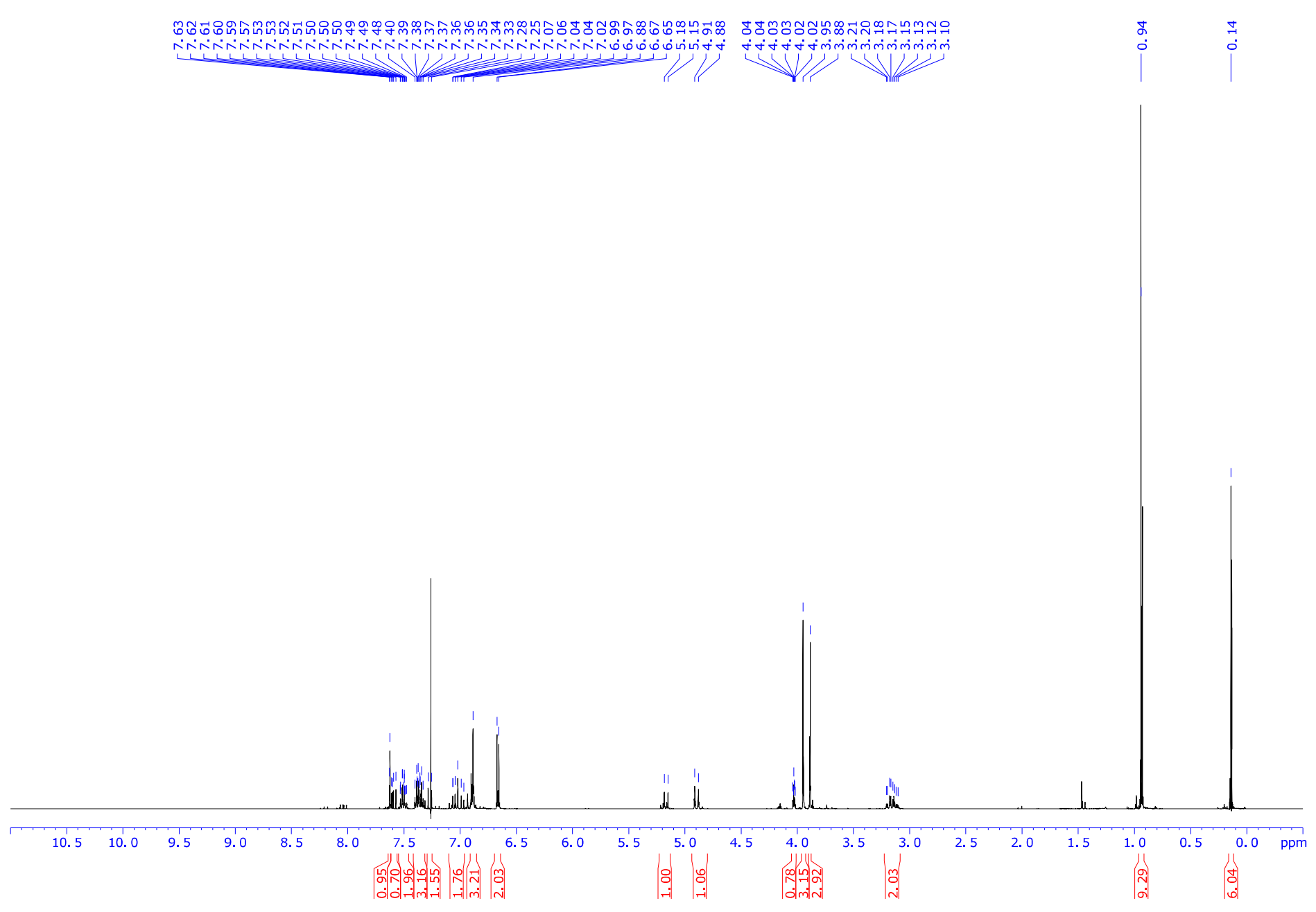


Christian Drescher and Reinhard Brückner; 3D-Structure Clarifying Total Synthesis of the (Polyenoyl)tetramic Acid Militarinon B. A Highly Acid-Labile N-Protecting Group for Amides

(5S)-5-\{1'-[(tert-Butyldimethylsilyl)oxy]benzyl\}-1-(4'",5"'-dimethoxy-1'"'-nitrobenzyl)-3-(1'Z,2'E,4'E)-1'-hydroxy-5-phenylpenta-2',4"'dien-1'-ylidene)pyrrolidine-2,4-dion [Z-enol-(S)-16c]

${ }^{13}$ C NMR (125.75 MHz, $\left.\mathrm{CDCl}_{3}\right)$ :
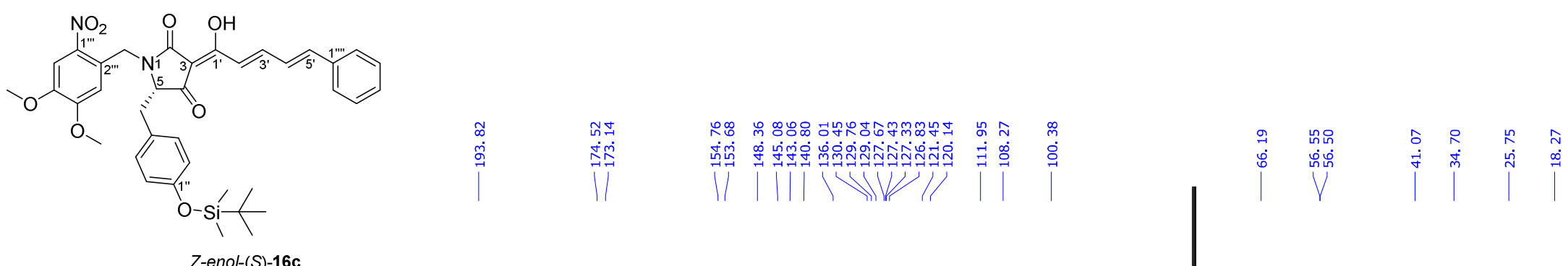

$\underset{d}{4}$

Z-enol-(S)-16c

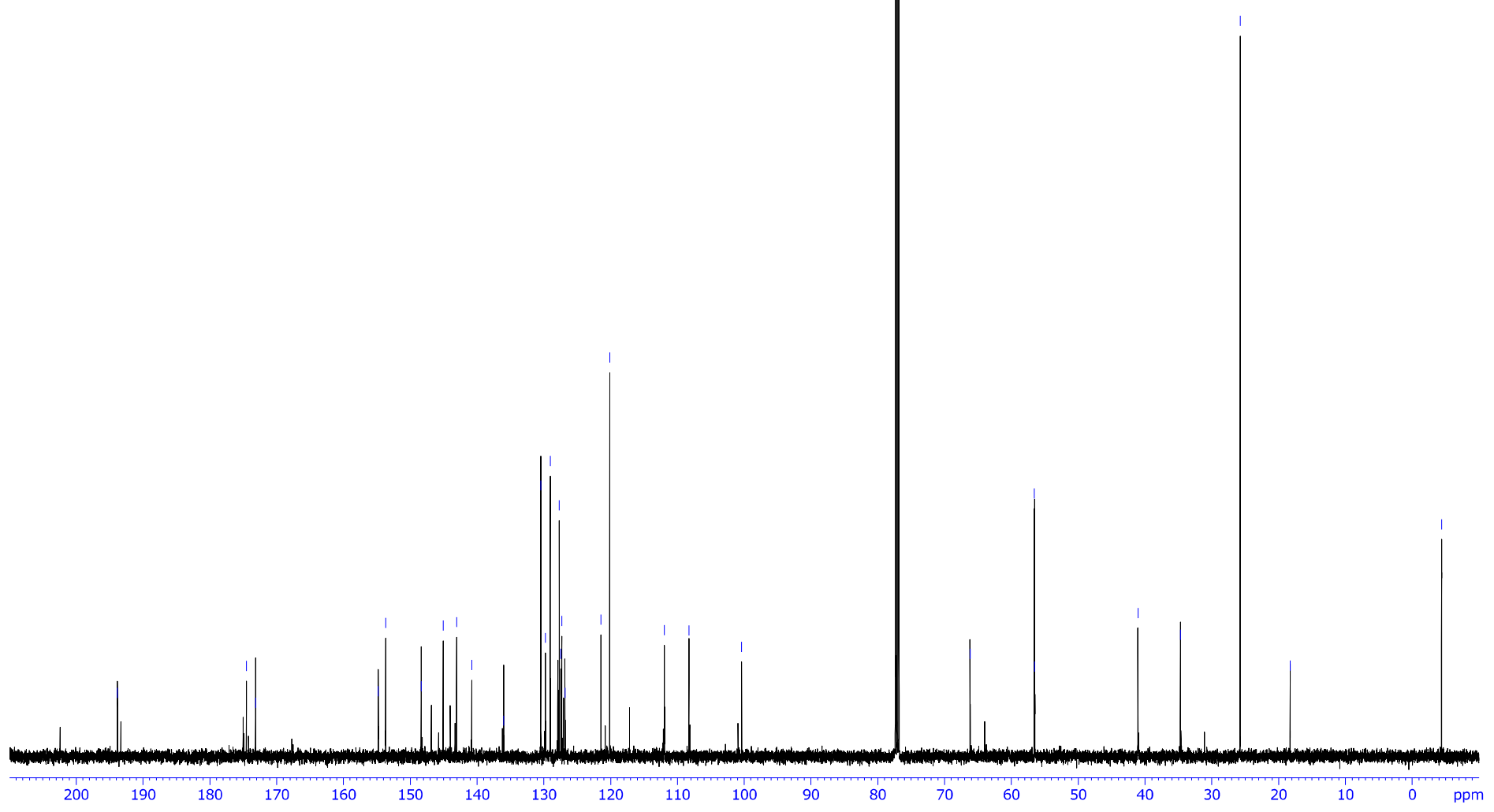


Christian Drescher and Reinhard Brückner; 3D-Structure Clarifying Total Synthesis of the (Polyenoyl)tetramic Acid Militarinon B. A Highly Acid-Labile N-Protecting Group for Amides

\section{Methyl (2S)-3-\{1'-[(tert-Butyldimethylsilyl)oxy]phenyl\}-2-[(1",3",5"-trimethoxybenzyl)amino]propanoate $[(S)-18 \mathrm{e}]$}

${ }^{1} \mathbf{H}$ NMR (500.10 MHz, $\left.\mathrm{CDCl}_{3}\right)$ :
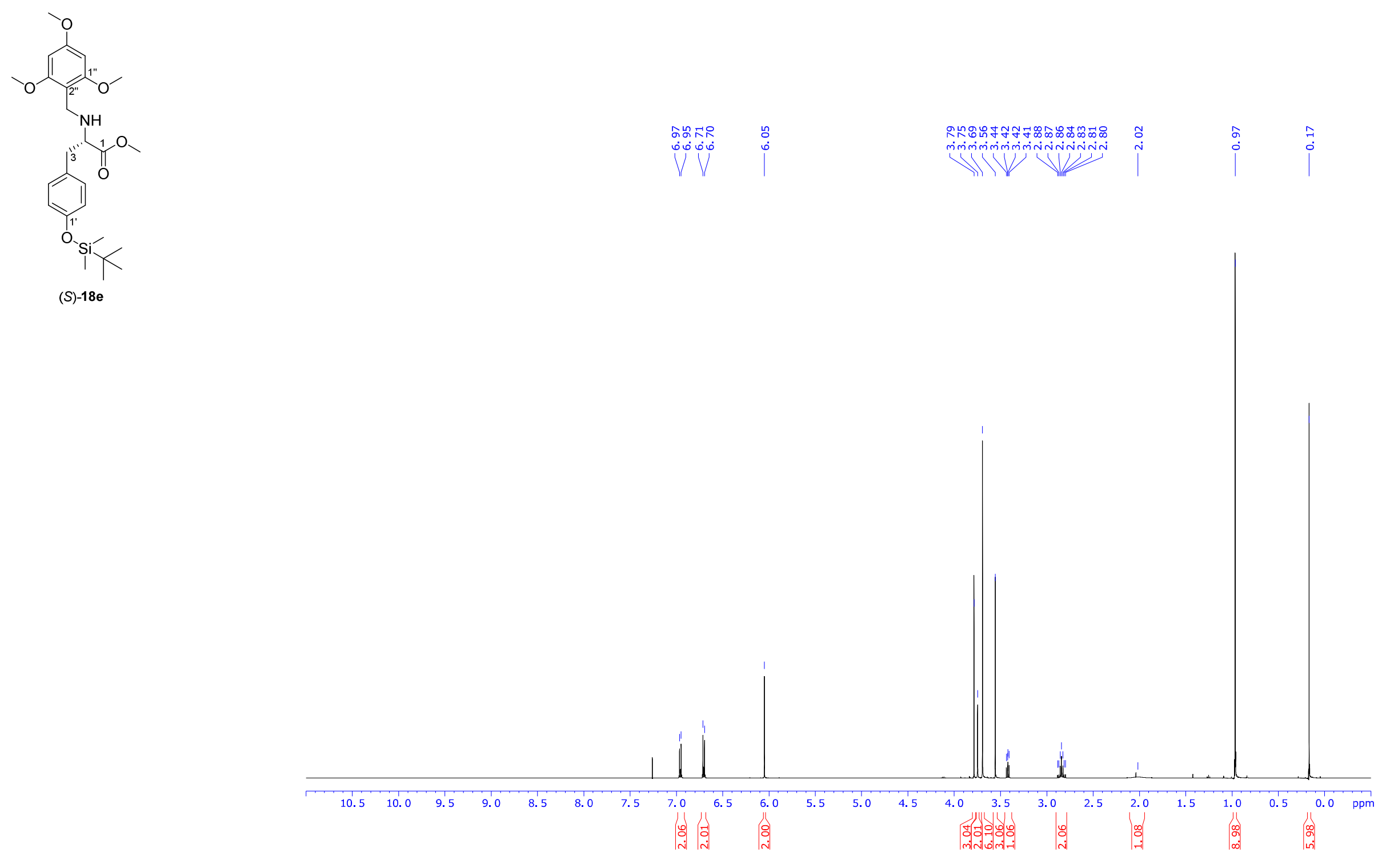

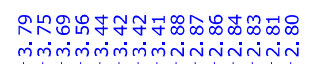


Christian Drescher and Reinhard Brückner; 3D-Structure Clarifying Total Synthesis of the (Polyenoyl)tetramic Acid Militarinon B. A Highly Acid-Labile N-Protecting Group for Amides

Methyl (2S)-3-\{1'-[(tert-Butyldimethylsilyl)oxy]phenyl\}-2-[(1",3",5"-trimethoxybenzyl)amino]propanoate [(S)-18e]

${ }^{13} \mathrm{C}$ NMR (125.75 MHz, $\left.\mathrm{CDCl}_{3}\right)$ :

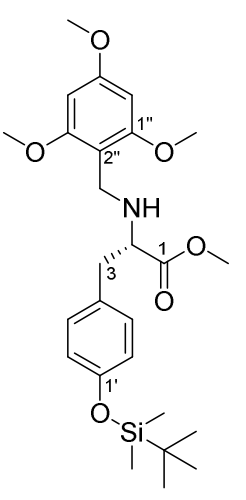

(S)-18e

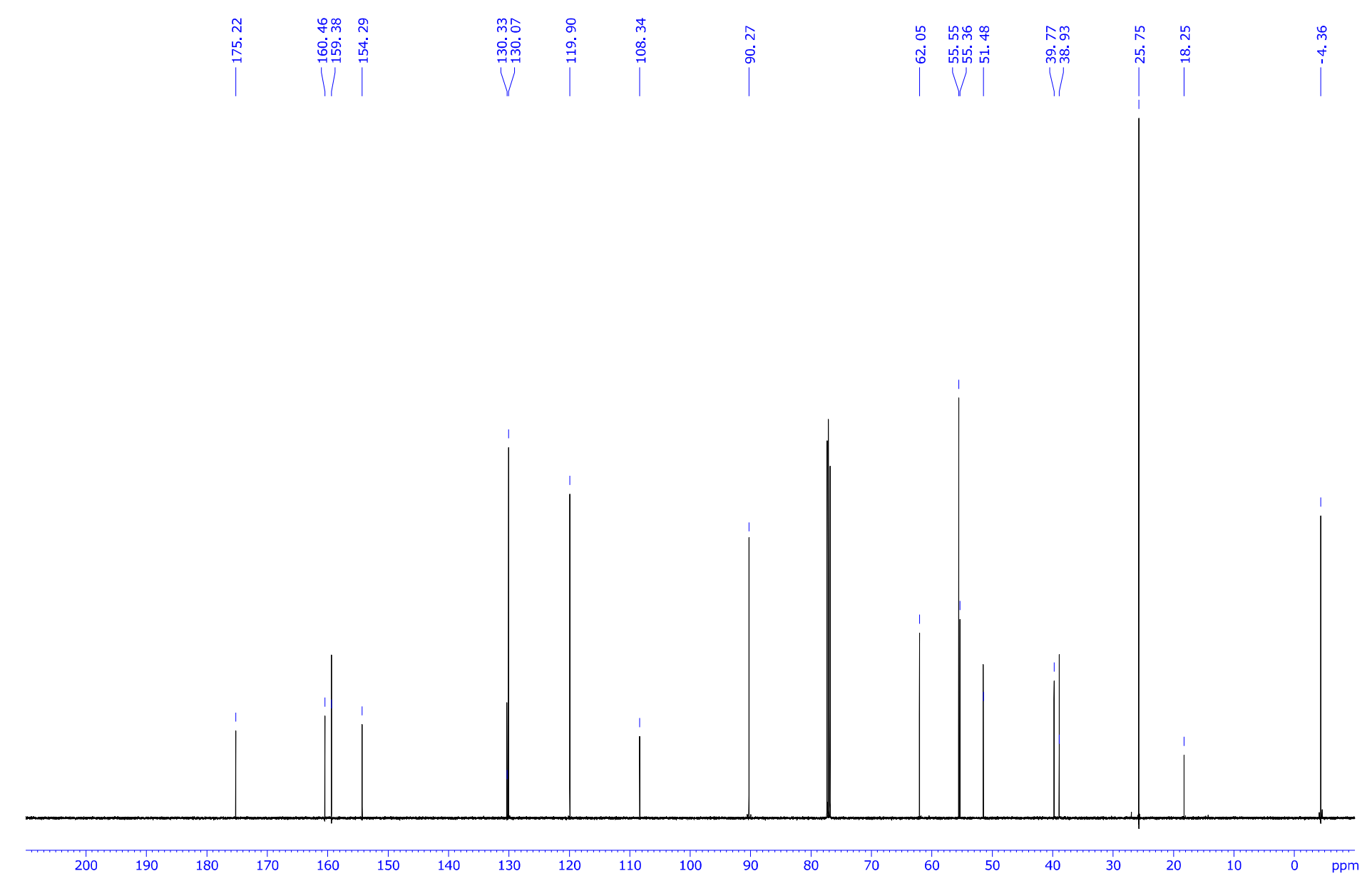


Christian Drescher and Reinhard Brückner; 3D-Structure Clarifying Total Synthesis of the (Polyenoyl)tetramic Acid Militarinon B. A Highly Acid-Labile N-Protecting Group for Amides

Methyl (2'S)-2'-\{N-[(1T,2Z,4E)-5-Bromo-3-hydroxy-1-oxoypenta-2,4-dienyl]- $N$-(1"',3'",5"'-trimethoxybenzyl) $\}-3$ '- $\{1$ "'-[(tertbutyldimethylsilyl)oxy]phenyl $\}$ propanoate $[Z$-enol-(S)-21e]

${ }^{1} \mathbf{H}$ NMR (500.10 MHz, $\left.\mathrm{CDCl}_{3}\right)$ :

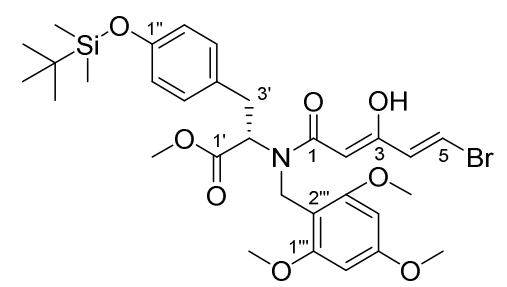

Z-enol[(S)-21e] 
Christian Drescher and Reinhard Brückner; 3D-Structure Clarifying Total Synthesis of the (Polyenoyl)tetramic Acid Militarinon B. A Highly Acid-Labile N-Protecting Group for Amides

Methyl (2'S)-2'-\{N-[(1T,2Z,4E)-5-Bromo-3-hydroxy-1-oxoypenta-2,4-dienyl]- $N$-(1"',3'",5"'-trimethoxybenzyl) $\}-3$ '- $\{1$ "'-[(tertbutyldimethylsilyl)oxy]phenyl $\}$ propanoate $[Z-e n o l-(S)-21 \mathrm{e}]$

${ }^{13} \mathrm{C}$ NMR (125.75 MHz, $\left.\mathrm{CDCl}_{3}\right)$ :
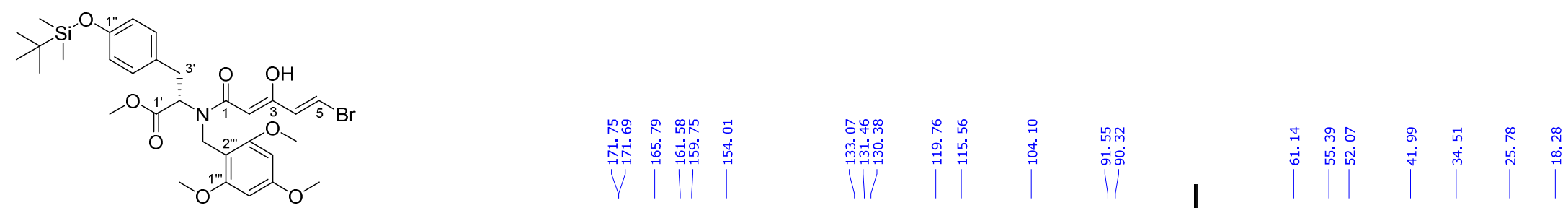

Z-enol[(S)-21e]

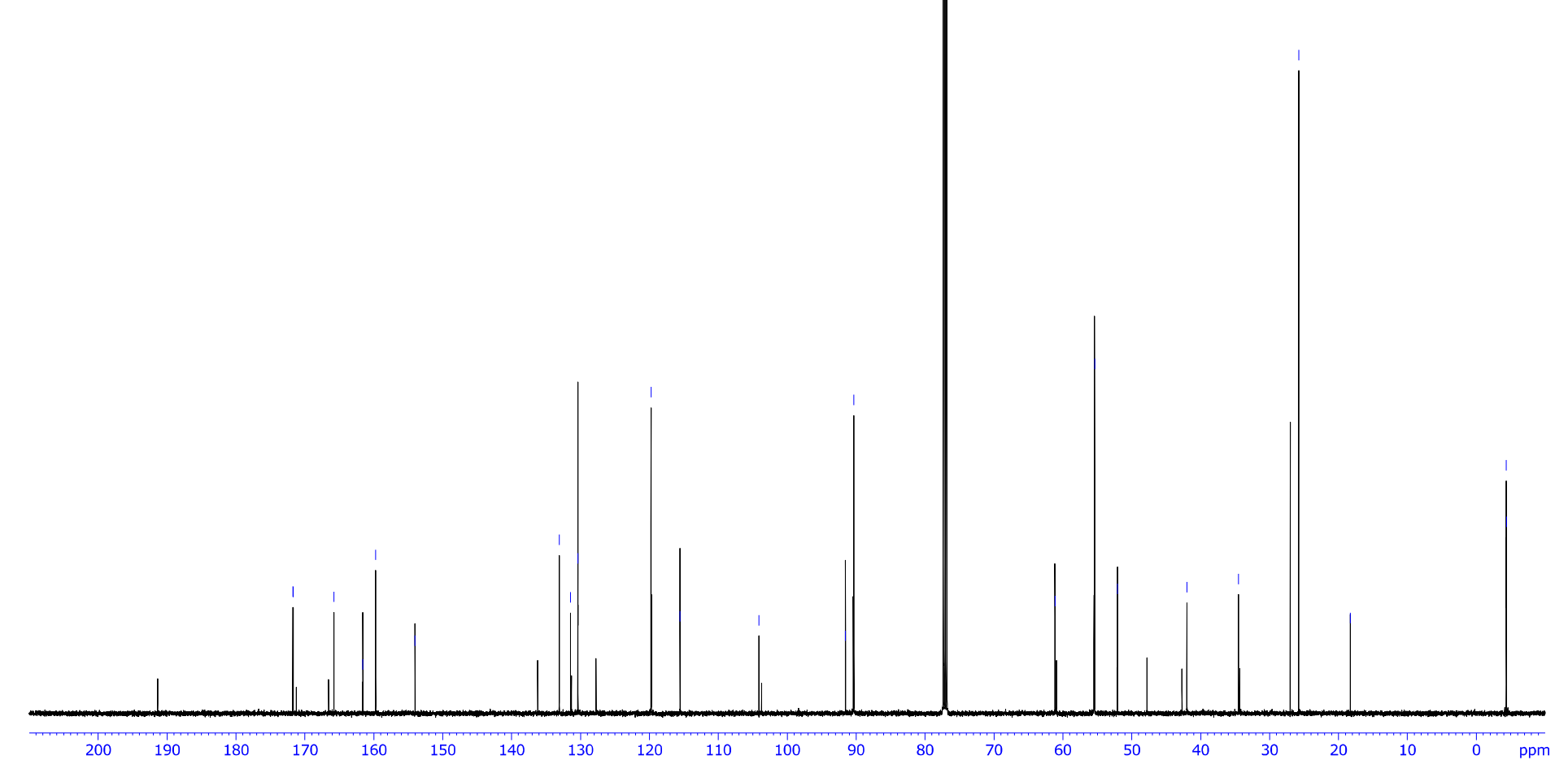


Christian Drescher and Reinhard Brückner; 3D-Structure Clarifying Total Synthesis of the (Polyenoyl)tetramic Acid Militarinon B. A Highly Acid-Labile N-Protecting Group for Amides

Methyl (2'S)-2'-\{N-[(1Z,2Z,4E,6E)-3-Hydroxy-7-phenylhepta-2,4,6-trienamido]- $N$-(1'",,3"',5'"'-trimethoxybenzyl) $\}-3$ '-\{1"'-[(tertbutyldimethylsilyl)oxy]phenyl\}propanoate [Z-enol-(S)-22e]

${ }^{1} \mathbf{H}$ NMR (500.10 MHz, $\left.\mathrm{CDCl}_{3}\right)$ :

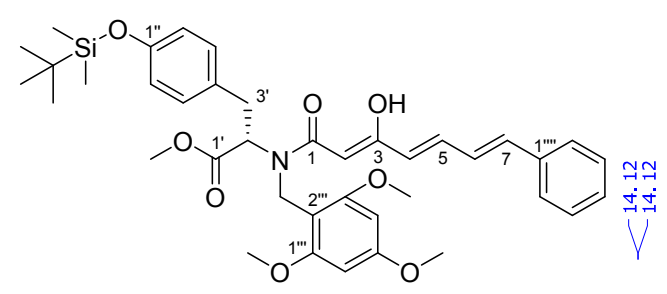

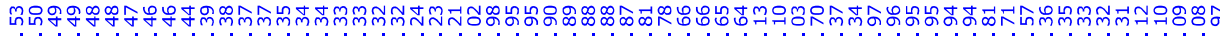
- ${ }^{1 m}=$

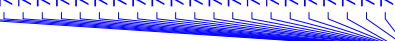

Z-enol-[(S)-22e] 
Christian Drescher and Reinhard Brückner; 3D-Structure Clarifying Total Synthesis of the (Polyenoyl)tetramic Acid Militarinon B. A Highly Acid-Labile N-Protecting Group for Amides

Methyl (2'S)-2'-\{N-[(1Z,2Z,4E,6E)-3-Hydroxy-7-phenylhepta-2,4,6-trienamido]- $N$-(1'"',3"',5'"'-trimethoxybenzyl)\}-3'-\{1"'-[(tertbutyldimethylsilyl)oxy]phenyl\}propanoate [Z-enol-(S)-22e]

${ }^{13}$ C NMR (125.75 MHz, $\left.\mathrm{CDCl}_{3}\right)$ :
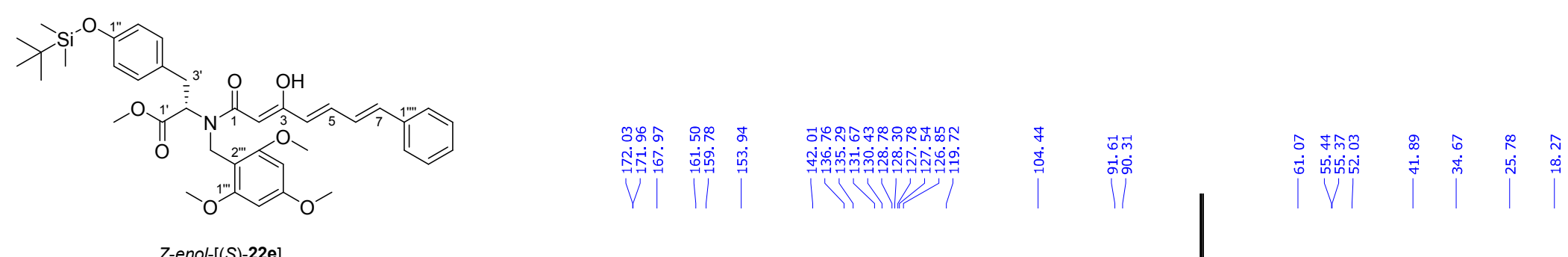

Z-enol-[(S)-22e]

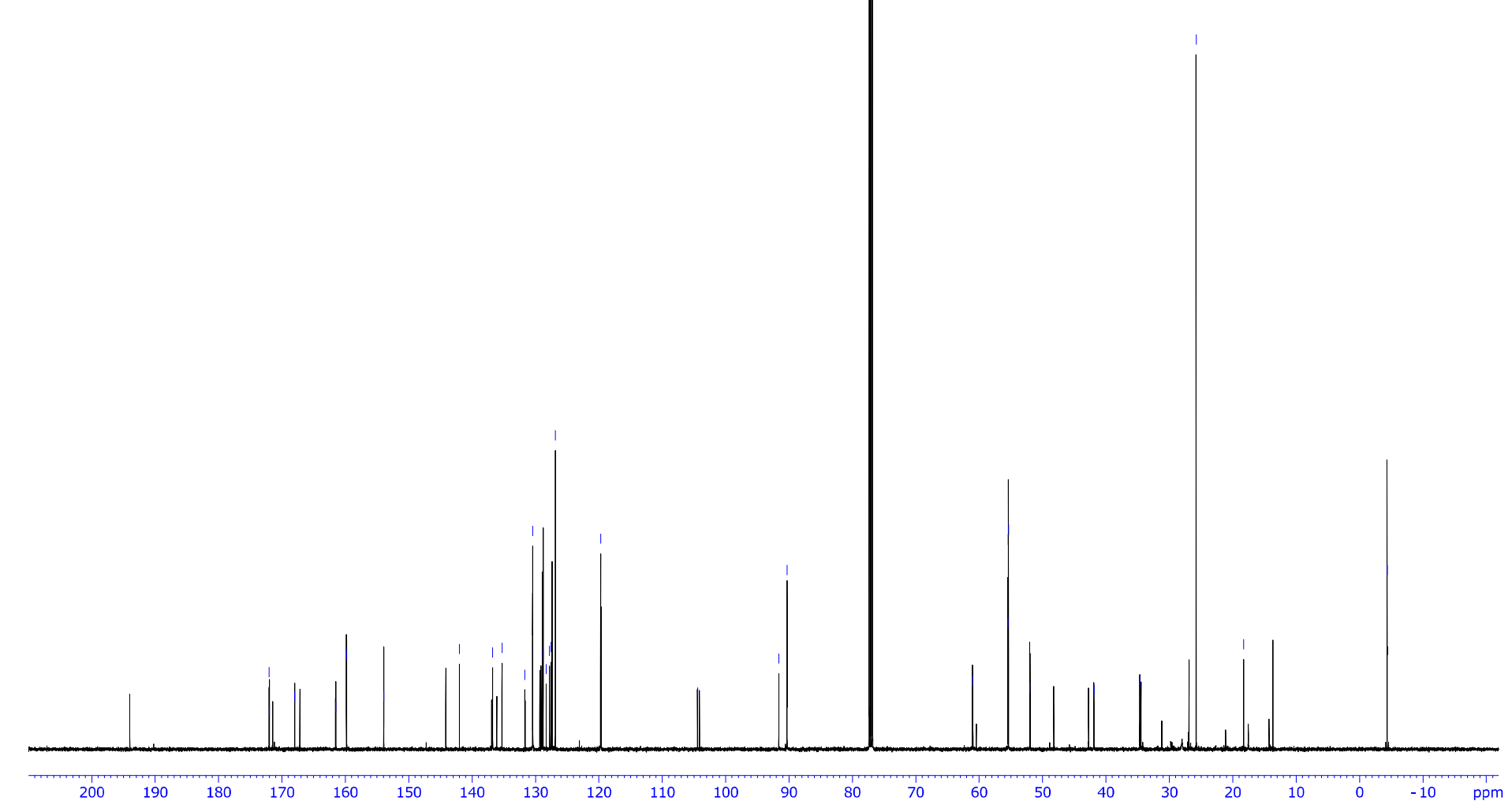


Christian Drescher and Reinhard Brückner; 3D-Structure Clarifying Total Synthesis of the (Polyenoyl)tetramic Acid Militarinon B. A Highly Acid-Labile N-Protecting Group for Amides

(5S)-5-\{1"'-[(tert-Butyldimethylsilyl)oxy]benzyl\}-1-(1'"',3'", 5'"-trimethoxybenzyl)-3-(1'Z,2'E,4'E)-1'-hydroxy-5-phenylpenta-2',4"'-dien-1'ylidene)pyrrolidine-2,4-dion [Z-enol-(S)-SI-8]

${ }^{1} \mathbf{H}$ NMR (500.10 MHz, $\left.\mathrm{CDCl}_{3}\right)$ :

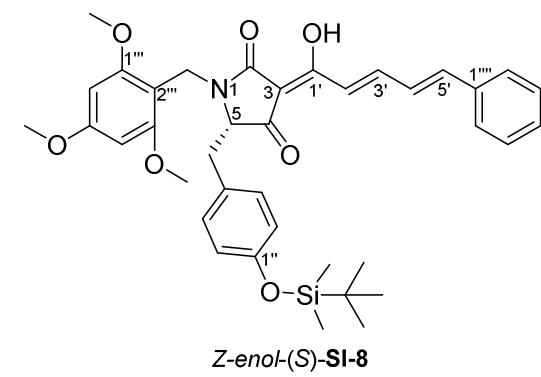

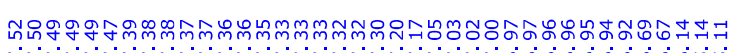

Z-enol-(S)-SI-8

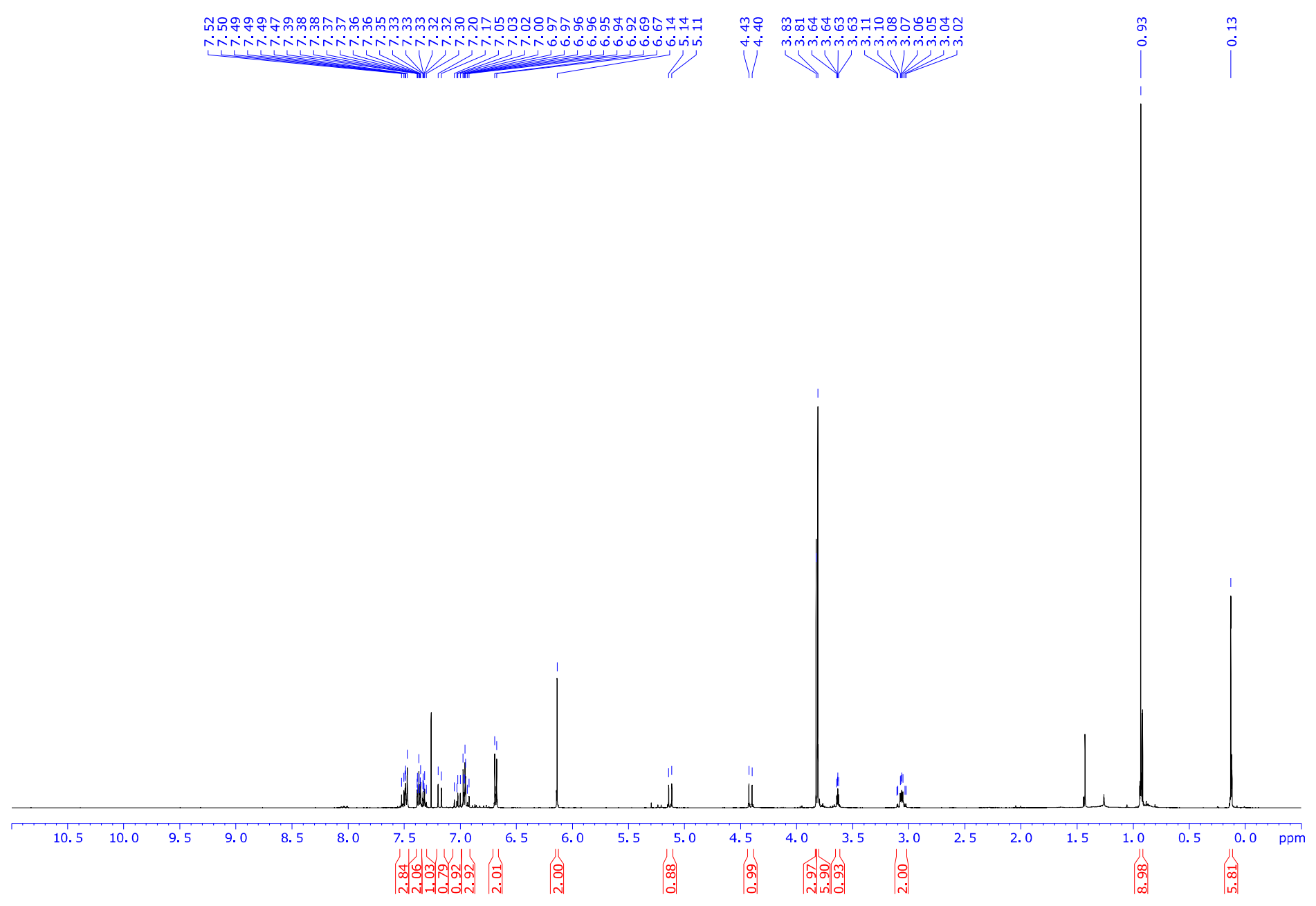


Christian Drescher and Reinhard Brückner; 3D-Structure Clarifying Total Synthesis of the (Polyenoyl)tetramic Acid Militarinon B. A Highly Acid-Labile N-Protecting Group for Amides

(5S)-5-\{1"'-[(tert-Butyldimethylsilyl)oxy]benzyl\}-1-(1'"',3'", 5'"-trimethoxybenzyl)-3-(1'Z,2'E,4'E)-1'-hydroxy-5-phenylpenta-2',4"'-dien-1'ylidene)pyrrolidine-2,4-dion [Z-enol-(S)-SI-8]

${ }^{13} \mathrm{C}$ NMR (125.75 MHz, $\left.\mathrm{CDCl}_{3}\right)$ :

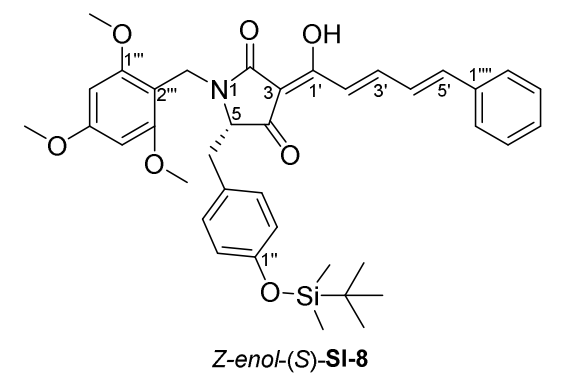

Z-enol-(S)-SI-8

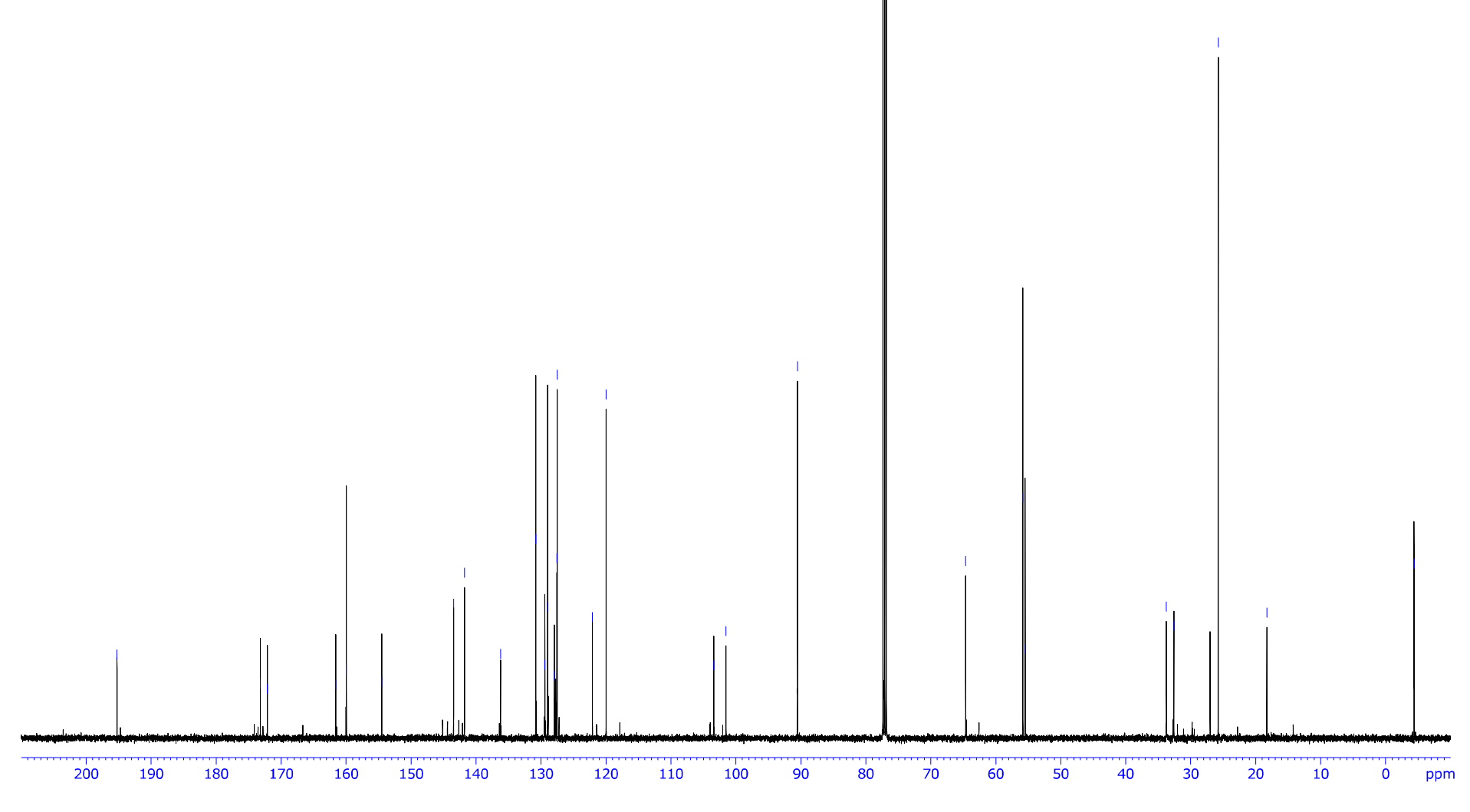


Christian Drescher and Reinhard Brückner; 3D-Structure Clarifying Total Synthesis of the (Polyenoyl)tetramic Acid Militarinon B. A Highly Acid-Labile N-Protecting Group for Amides

(5S)-5-\{1'-[(tert-Butyldimethylsilyl)oxy]benzyl\}-3-(1'Z,2'E,4'E)-1'-hydroxy-5-phenylpenta-2',4'-dien-1'-ylidene)pyrrolidine-2,4-dion [Zenol-(S)-TBDMS-17]

${ }^{1}$ H NMR (500.10 MHz, $\left.\mathrm{CDCl}_{3}\right)$ :
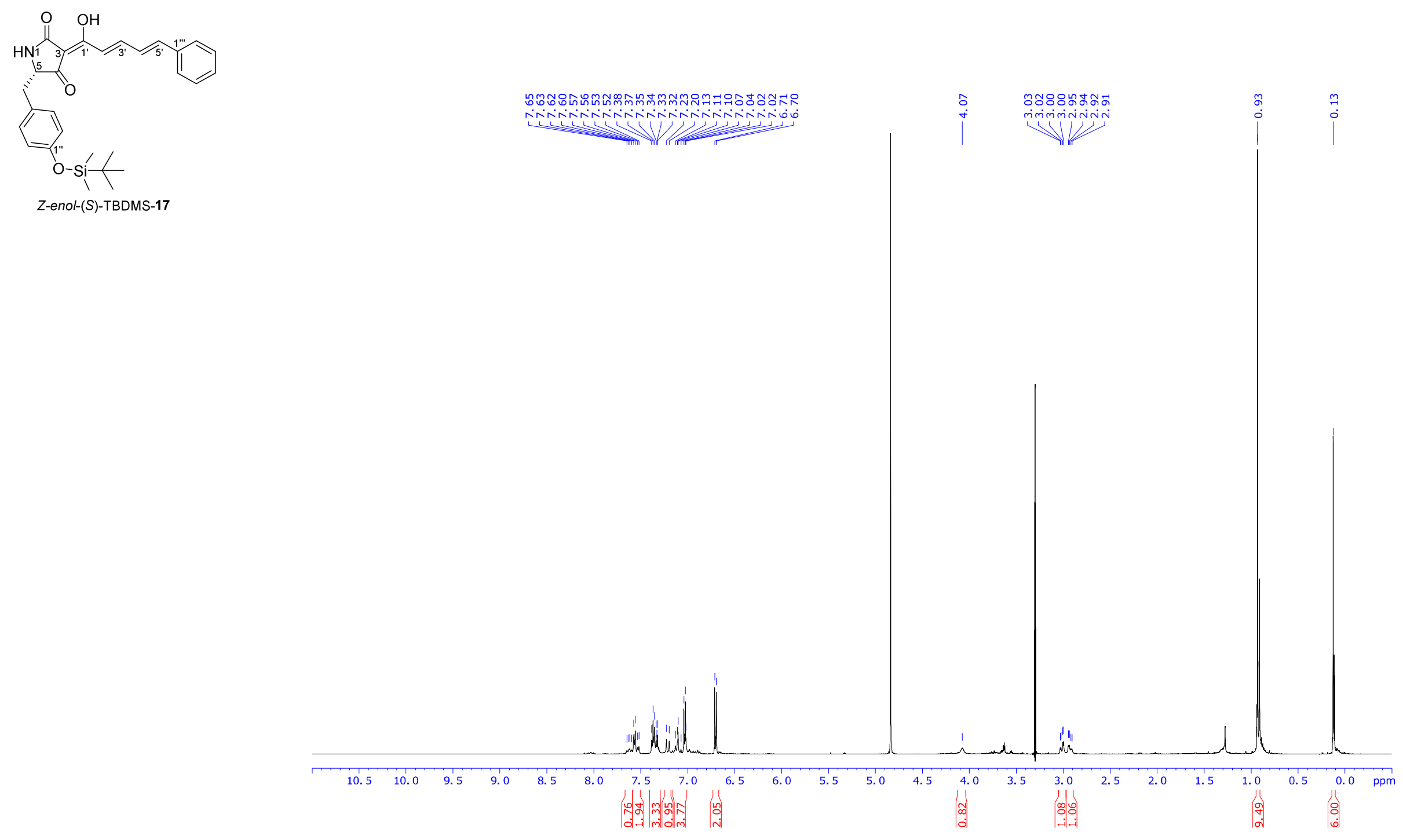
Christian Drescher and Reinhard Brückner; 3D-Structure Clarifying Total Synthesis of the (Polyenoyl)tetramic Acid Militarinon B. A Highly Acid-Labile N-Protecting Group for Amides

(5S)-5-\{1'-[(tert-Butyldimethylsilyl)oxy]benzyl\}-3-(1'Z,2'E,4'E)-1'-hydroxy-5-phenylpenta-2',4'-dien-1'-ylidene)pyrrolidine-2,4-dion [Zenol-(S)-TBDMS-17]

${ }^{13} \mathbf{C}$ NMR $\left(125.75 \mathrm{MHz}, \mathrm{CDCl}_{3}\right)$ :

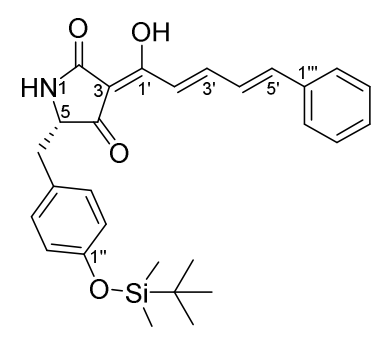

Z-enol-(S)-TBDMS-17

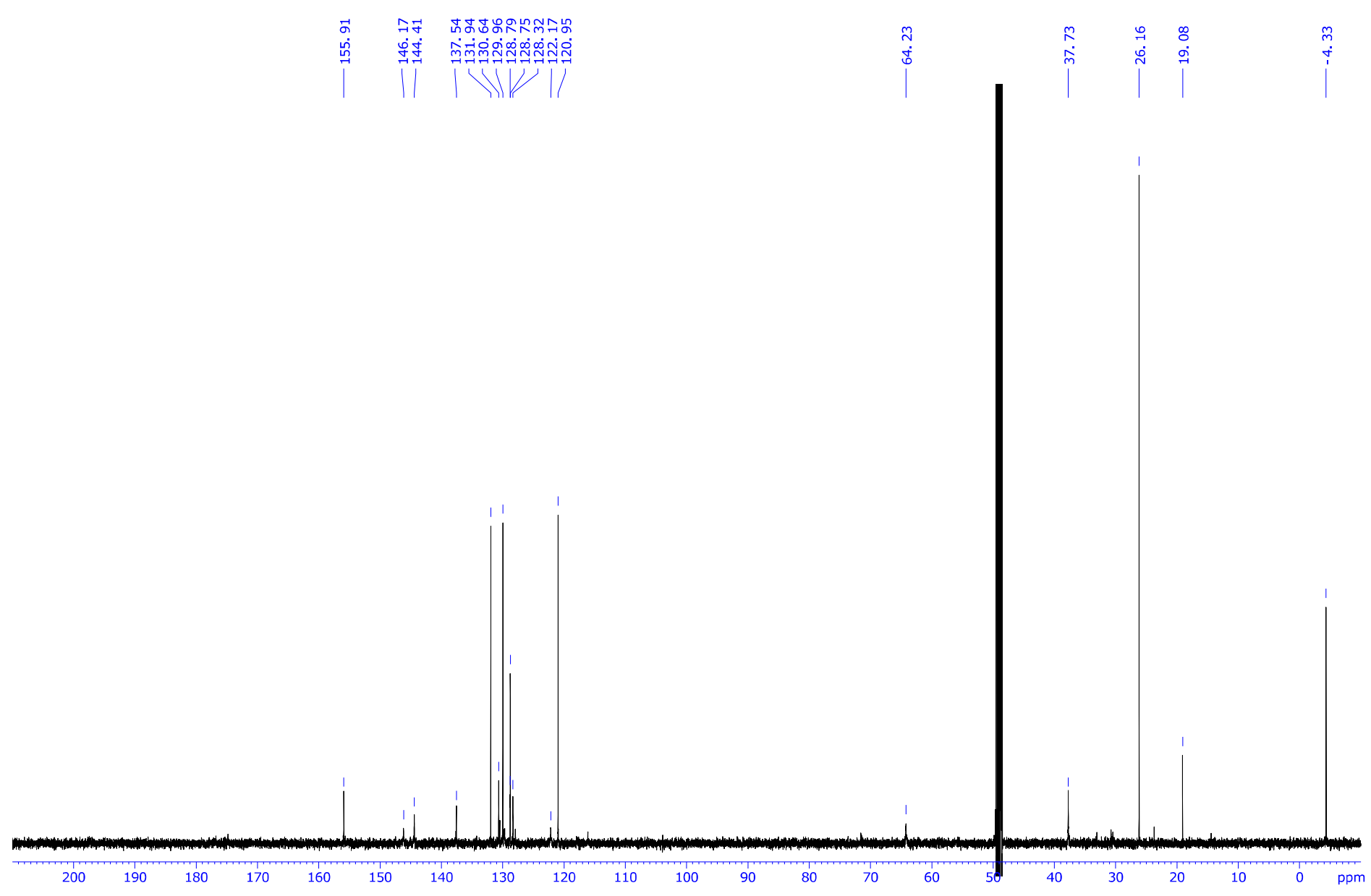


Christian Drescher and Reinhard Brückner; 3D-Structure Clarifying Total Synthesis of the (Polyenoyl)tetramic Acid Militarinon B. A Highly Acid-Labile N-Protecting Group for Amides

(5S)-5-(1"'-Hydroxybenzyl)-1-(1"',3'",5'"'-trimethoxybenzyl)-3-(1'Z,2'E,4'E)-1'-hydroxy-5-phenylpenta-2',4''-dien-1'-ylidene)pyrrolidine2,4-dion [Z-enol-(S)-16e]

${ }^{1} \mathbf{H}$ NMR $\left(500.10 \mathrm{MHz}, \mathrm{CDCl}_{3}\right)$ :

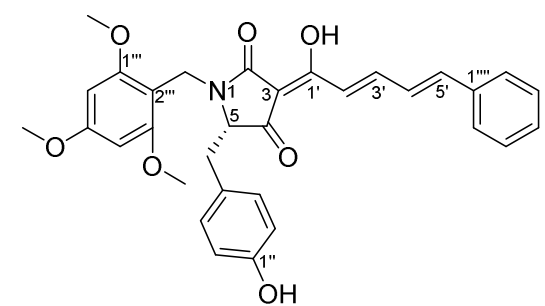

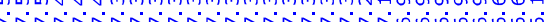

Z-enol-(S)-16e
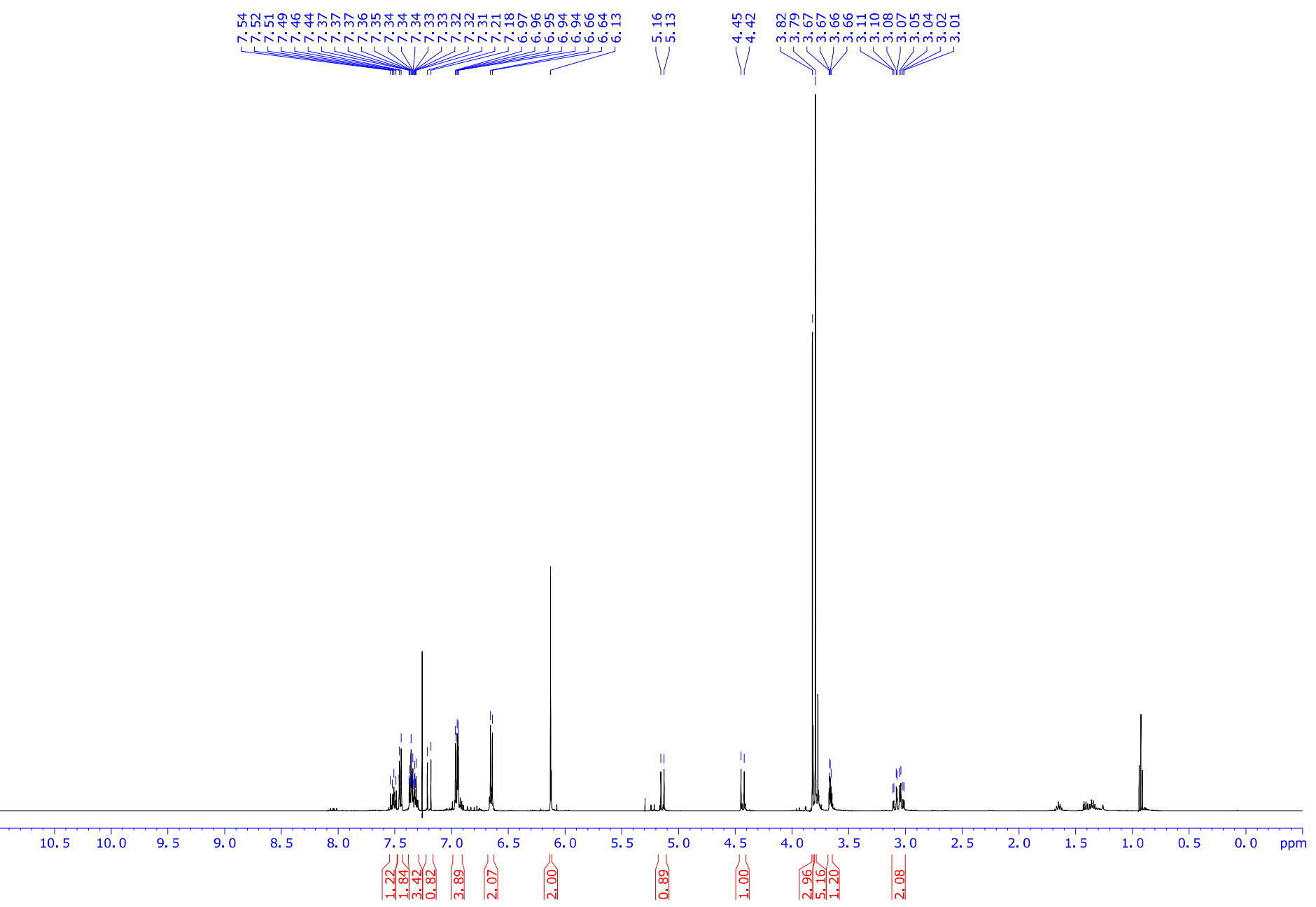
Christian Drescher and Reinhard Brückner; 3D-Structure Clarifying Total Synthesis of the (Polyenoyl)tetramic Acid Militarinon B. A Highly Acid-Labile N-Protecting Group for Amides

(5S)-5-(1"'-Hydroxybenzyl)-1-(1"',3'",5'"'-trimethoxybenzyl)-3-(1'Z,2'E,4'E)-1'-hydroxy-5-phenylpenta-2',4''-dien-1'-ylidene)pyrrolidine2,4-dion [Z-enol-(S)-16e]

${ }^{13}$ C NMR (125.75 MHz, $\left.\mathrm{CDCl}_{3}\right)$ :
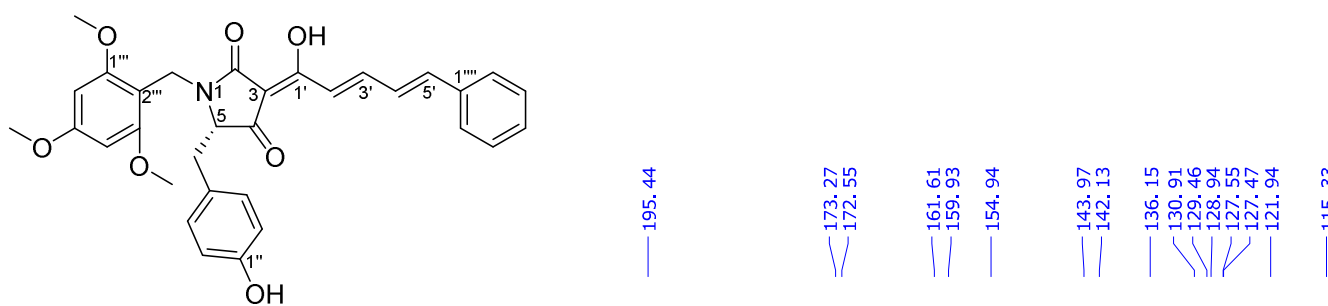

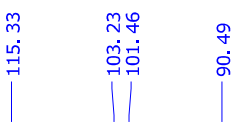

Z-enol-(S)-16e

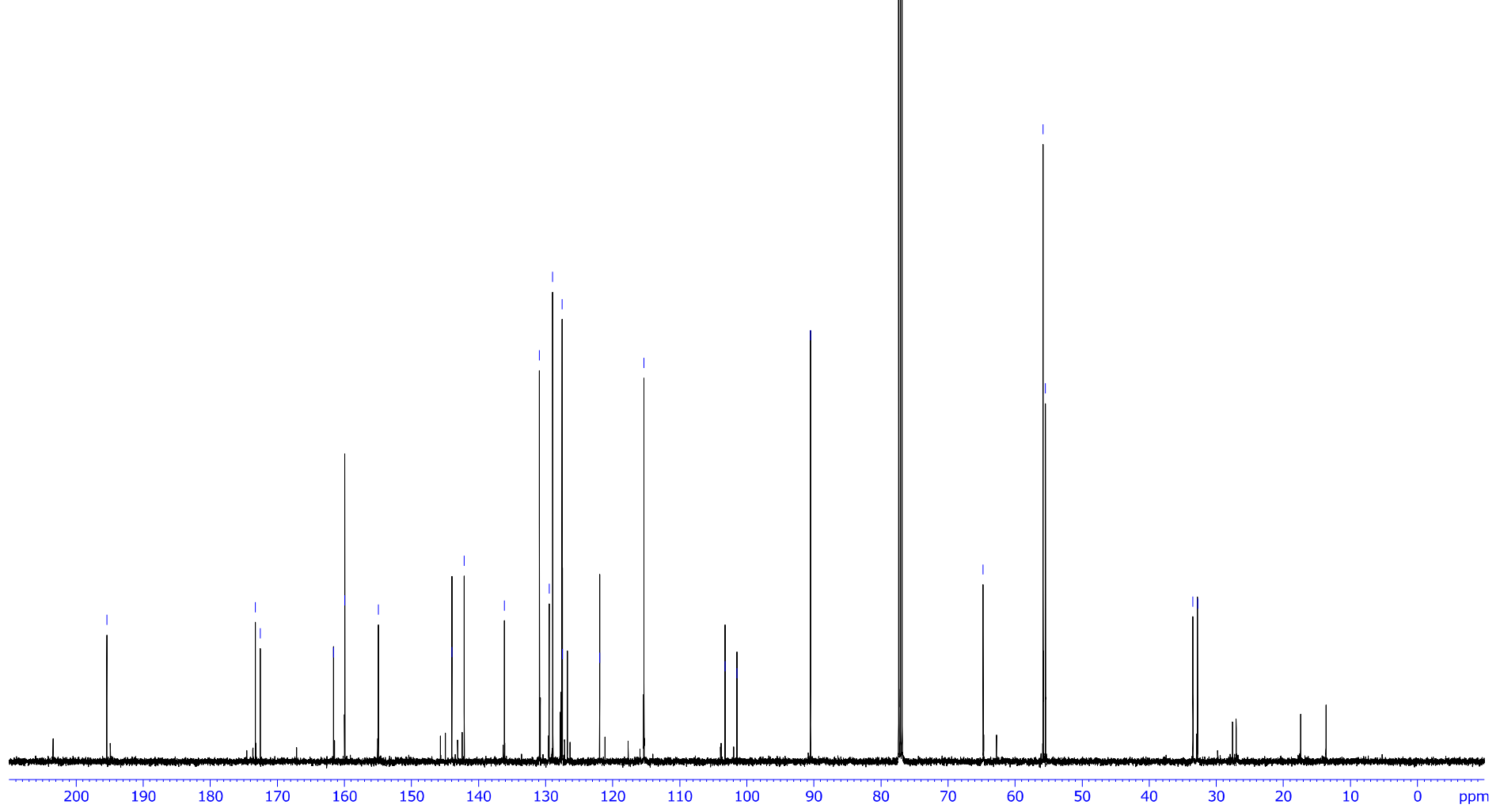


Christian Drescher and Reinhard Brückner; 3D-Structure Clarifying Total Synthesis of the (Polyenoyl)tetramic Acid Militarinon B. A Highly Acid-Labile N-Protecting Group for Amides

$3.2{ }^{1} \mathrm{H}$ and ${ }^{13} \mathrm{C}$ NMR Spectra to the Synthesis of the two Western Blocks $(S, S)-10 \mathrm{e}$ and $(R, S)-10 \mathrm{e}$ 
Christian Drescher and Reinhard Brückner; 3D-Structure Clarifying Total Synthesis of the (Polyenoyl)tetramic Acid Militarinon B. A Highly Acid-Labile N-Protecting Group for Amides

\section{4-(Benzyloxy)benzaldehyde (14)}

${ }^{1} \mathbf{H}$ NMR (300.13 MHz, $\mathrm{CDCl}_{3}$ ):
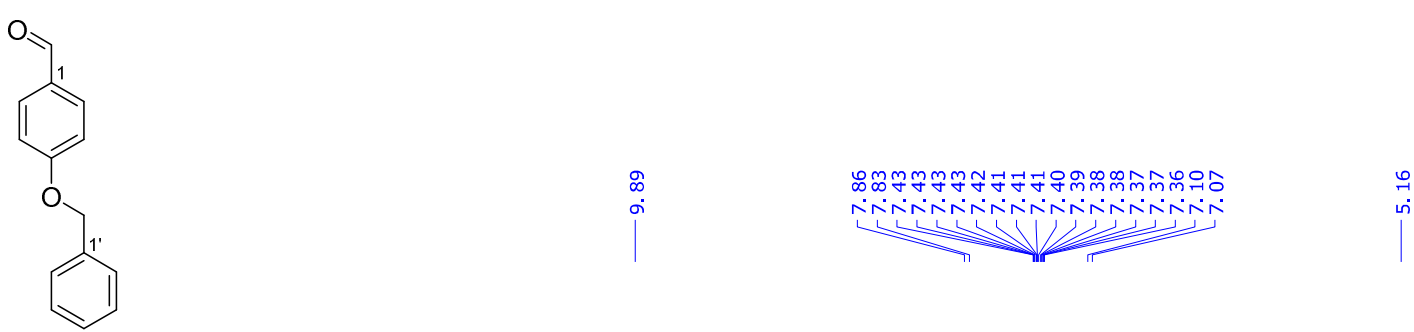

14

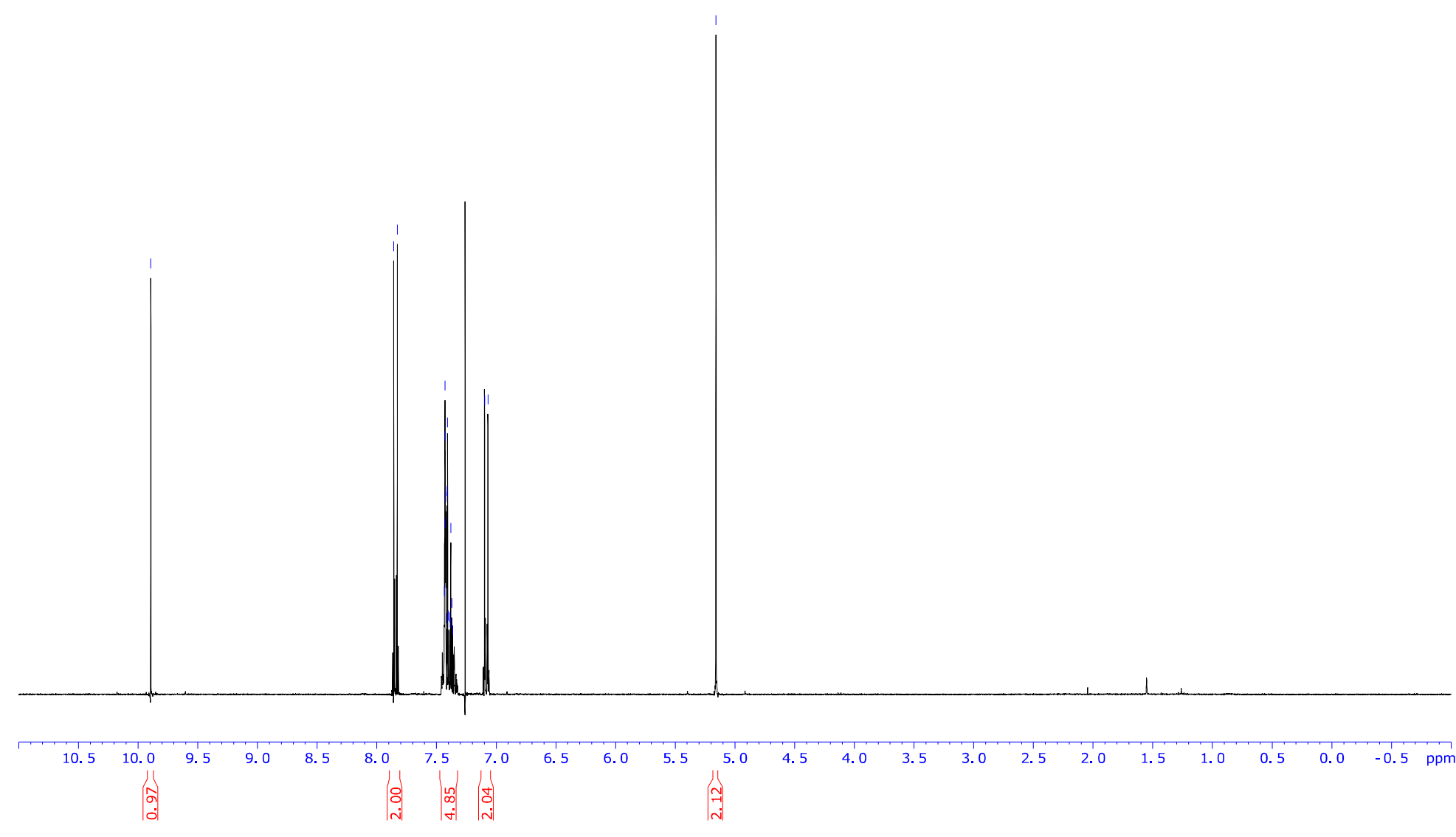


Christian Drescher and Reinhard Brückner; 3D-Structure Clarifying Total Synthesis of the (Polyenoyl)tetramic Acid Militarinon B. A Highly Acid-Labile N-Protecting Group for Amides

\section{(R)-3-Isopropylpiperazine-2,5-dione [(R)-SI-9]}

${ }^{1}$ H NMR (300.13 MHz, DMSO):
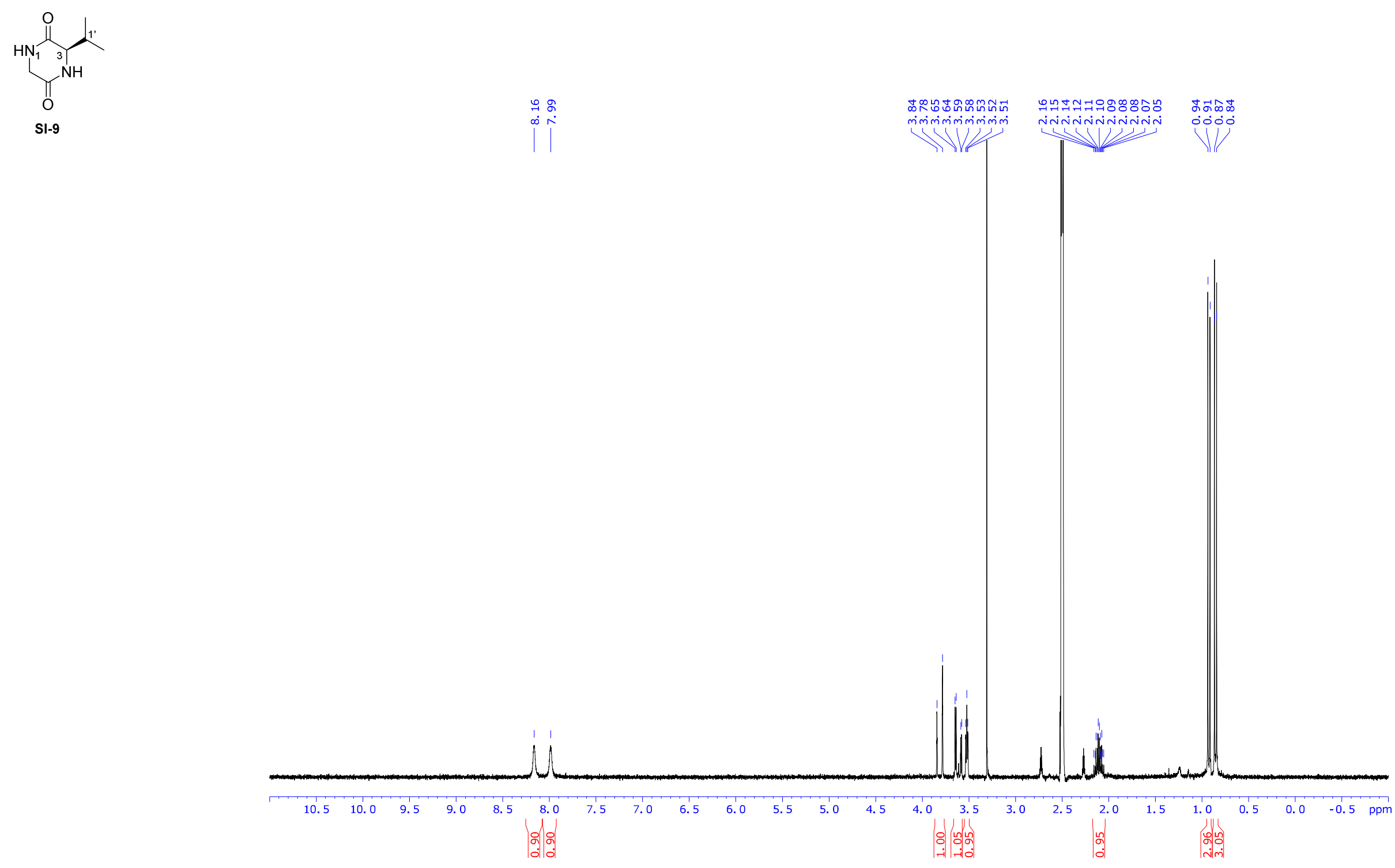
Christian Drescher and Reinhard Brückner; 3D-Structure Clarifying Total Synthesis of the (Polyenoyl)tetramic Acid Militarinon B. A Highly Acid-Labile N-Protecting Group for Amides

\section{(R)-3,6-Diethoxy-2-isopropyl-2,5-dihydropyrazine [(R)-15a]}

${ }^{1} \mathbf{H}$ NMR (300.13 MHz, $\left.\mathrm{CDCl}_{3}\right)$ :

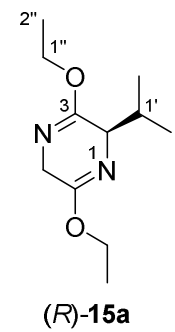

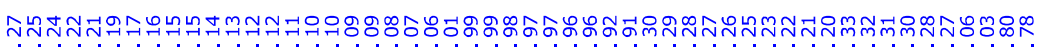

$(R)-15 \mathbf{a}$

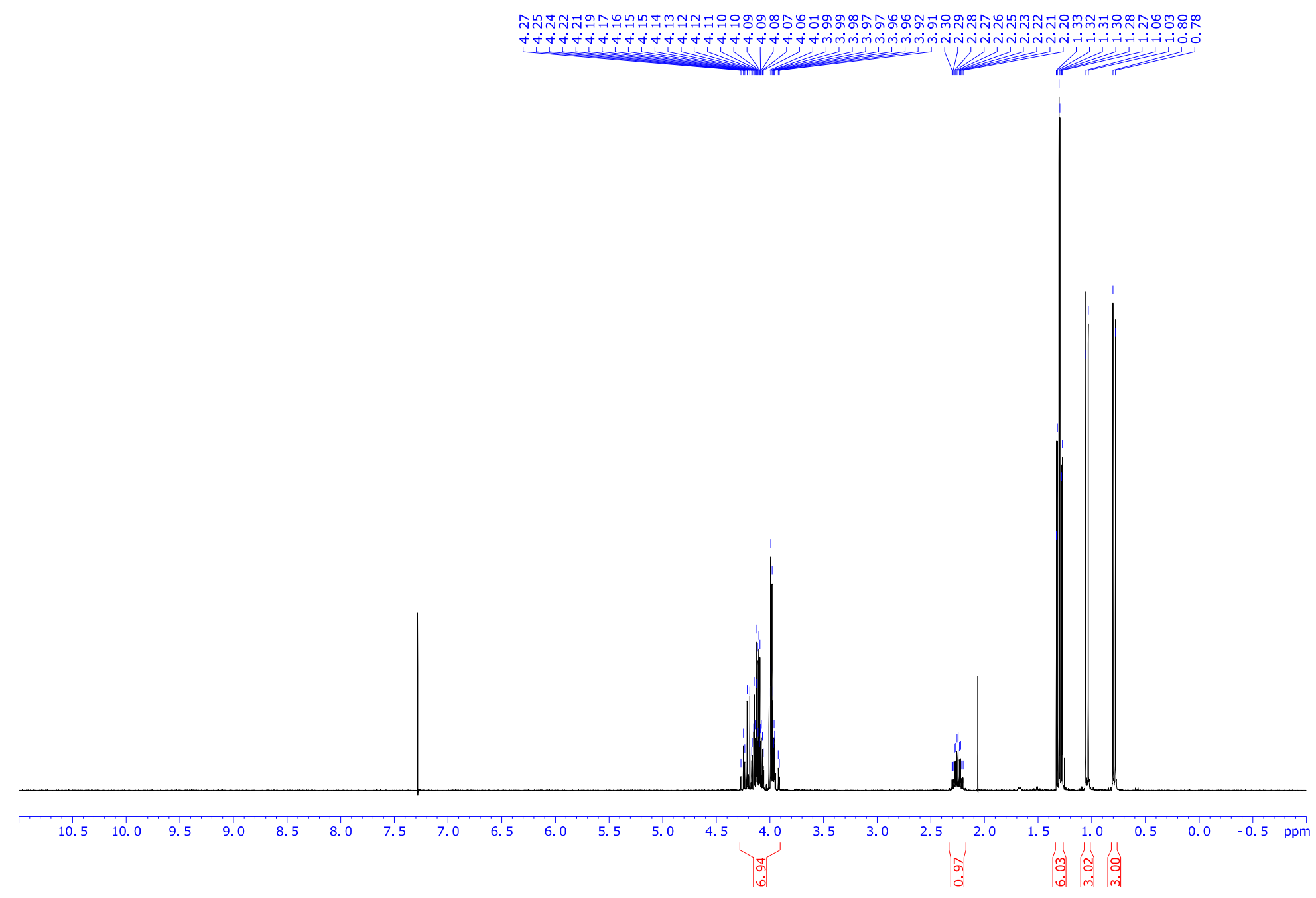


Christian Drescher and Reinhard Brückner; 3D-Structure Clarifying Total Synthesis of the (Polyenoyl)tetramic Acid Militarinon B. A Highly Acid-Labile N-Protecting Group for Amides

\section{(S)-[4'"'-(Benzyloxy)phenyl][(5S,2R)-3,6-diethoxy-2-isopropyl-1,4-dihydropyrazin-5-yl]methanol $[(S, S, R)-13 a]$}

${ }^{1} \mathbf{H}$ NMR (500.06 MHz, $\left.\mathrm{CDCl}_{3}\right)$ :

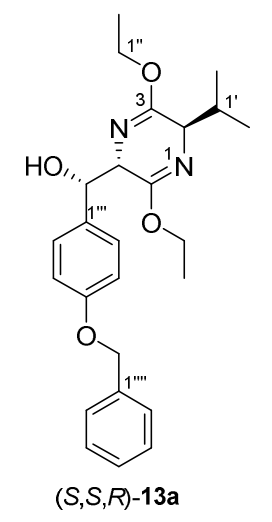

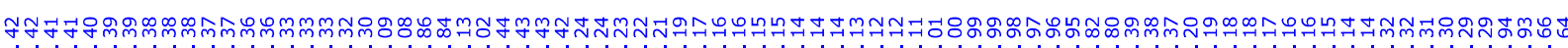
A

$(S, S, R)-13 a$

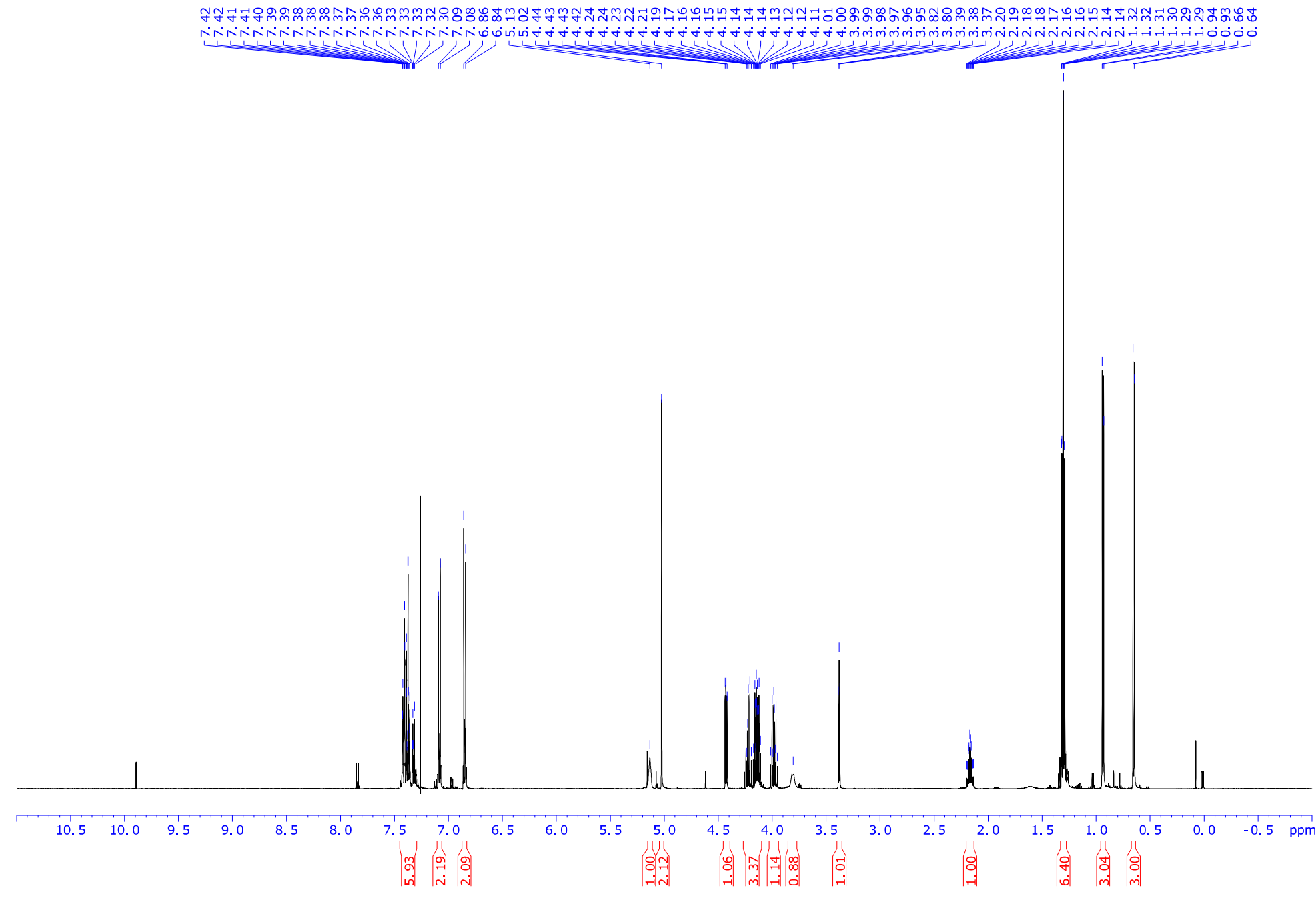


Christian Drescher and Reinhard Brückner; 3D-Structure Clarifying Total Synthesis of the (Polyenoyl)tetramic Acid Militarinon B. A Highly Acid-Labile N-Protecting Group for Amides

\section{(S)-[4'"'-(Benzyloxy)phenyl][(5S,2R)-3,6-diethoxy-2-isopropyl-1,4-dihydropyrazin-5-yl]methanol $[(S, S, R)-13 \mathrm{a}]$}

${ }^{13} \mathrm{C}$ NMR $\left(125.75 \mathrm{MHz}, \mathrm{CDCl}_{3}\right)$ :
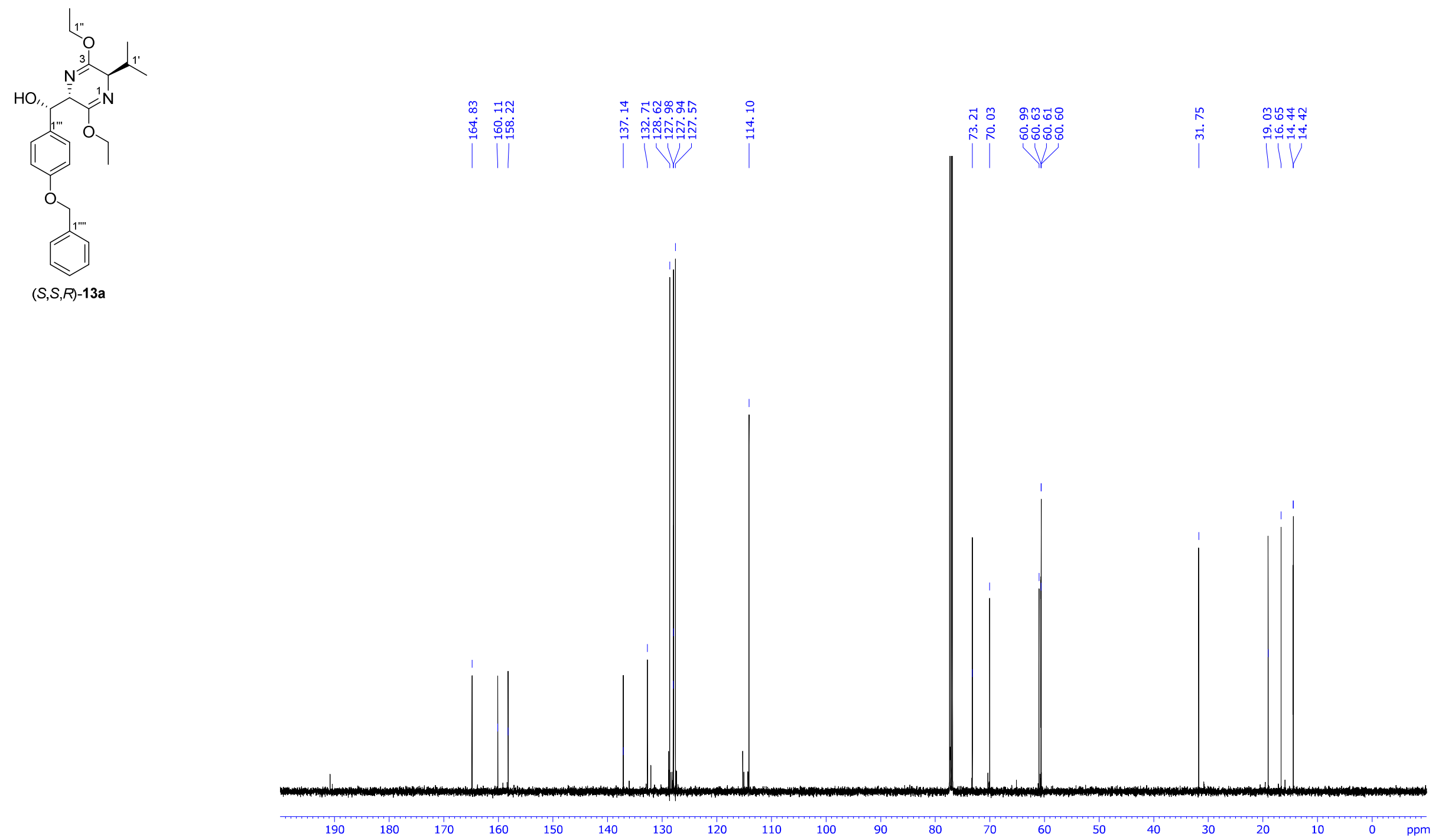
Christian Drescher and Reinhard Brückner; 3D-Structure Clarifying Total Synthesis of the (Polyenoyl)tetramic Acid Militarinon B. A Highly Acid-Labile N-Protecting Group for Amides

\section{$(R)$-[4'"-(Benzyloxy)phenyl][(5S,2R)-3,6-diethoxy-2-isopropyl-1,4-dihydropyrazin-5-yl]methanol [(R,S,R)-13a]}

${ }^{1} \mathbf{H}$ NMR $\left(500.06 \mathrm{MHz}, \mathrm{CDCl}_{3}\right)$ :

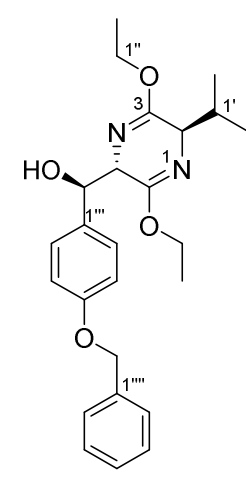

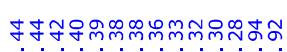

$$
\begin{aligned}
& \text { riviniviriviniviogo }
\end{aligned}
$$

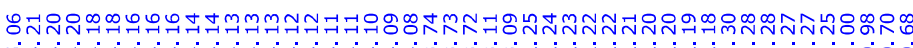

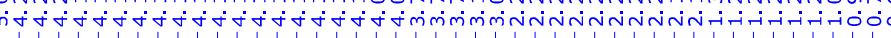

$(R, S, R)-\mathbf{1 3 a}$

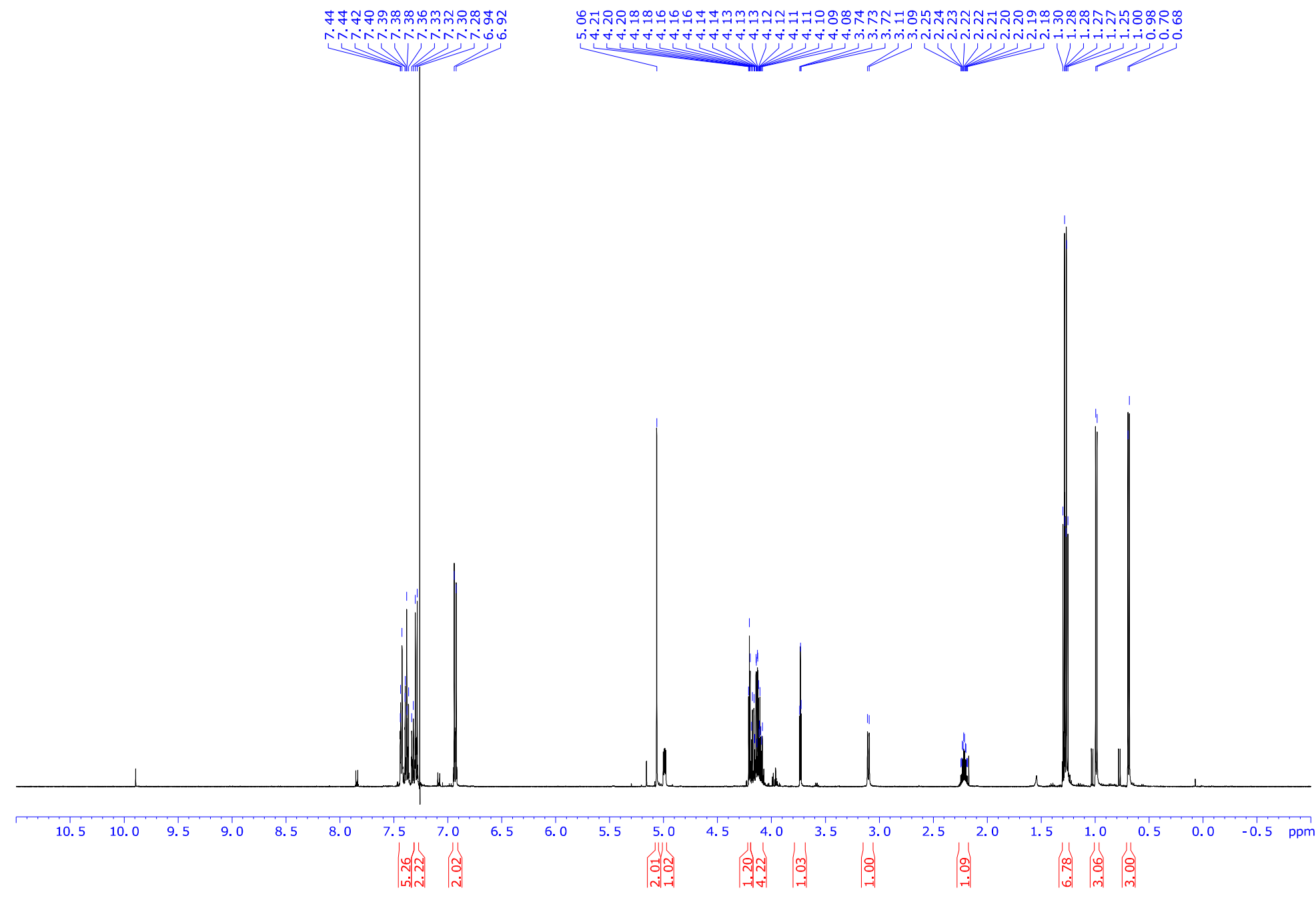


Christian Drescher and Reinhard Brückner; 3D-Structure Clarifying Total Synthesis of the (Polyenoyl)tetramic Acid Militarinon B. A Highly Acid-Labile N-Protecting Group for Amides

(R)-[4'"'-(Benzyloxy)phenyl][(5S,2R)-3,6-diethoxy-2-isopropyl-1,4-dihydropyrazin-5-yl]methanol [(R,S,R)-13a]

${ }^{13} \mathrm{C}$ NMR $\left(125.75 \mathrm{MHz}, \mathrm{CDCl}_{3}\right)$ :
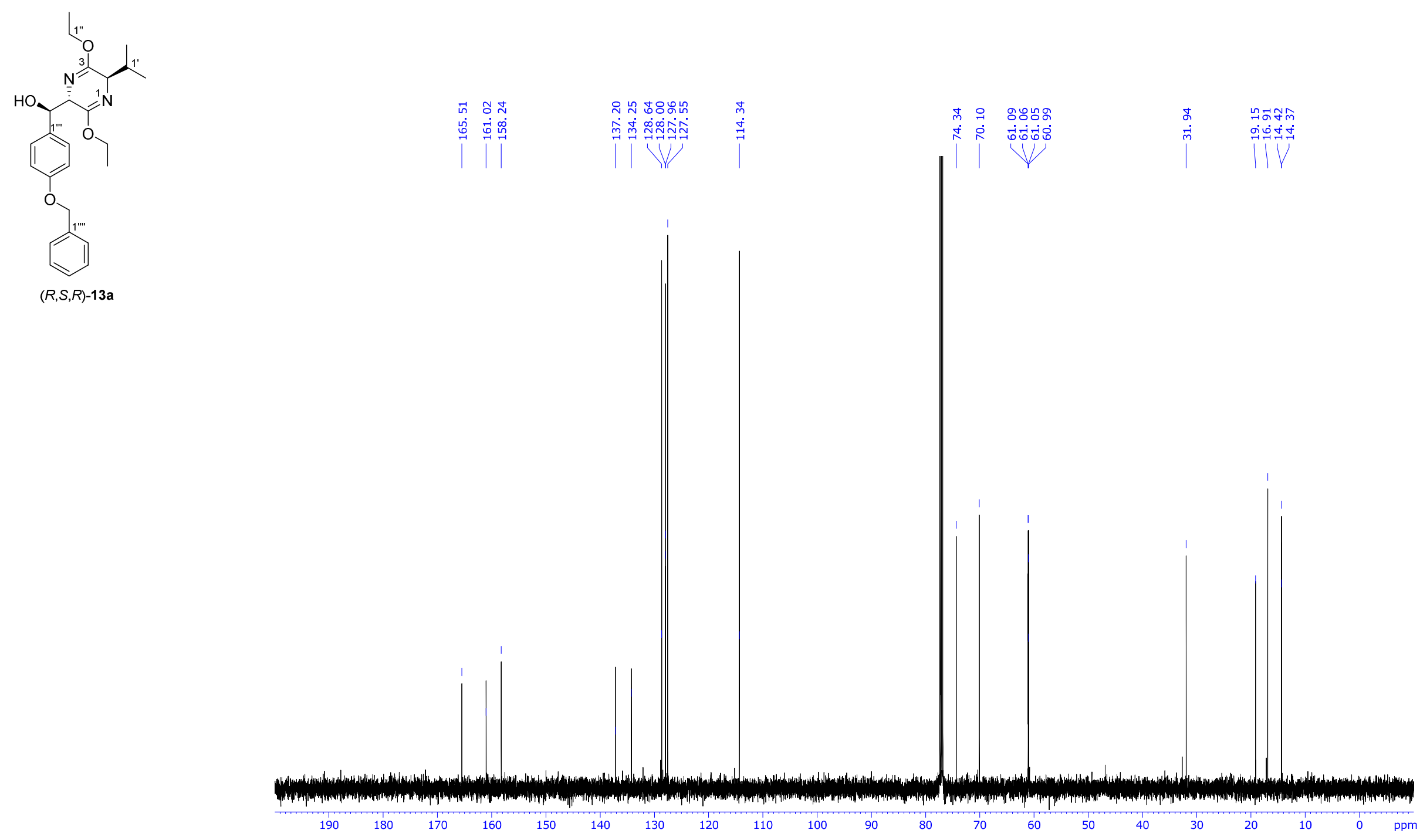
Christian Drescher and Reinhard Brückner; 3D-Structure Clarifying Total Synthesis of the (Polyenoyl)tetramic Acid Militarinon B. A Highly Acid-Labile N-Protecting Group for Amides

\section{Ethyl (2S,3S)-2-Amino-3-[4'-(benzyloxy)phenyl]-3-hydroxypropanoate [(S,S)-23]}

${ }^{1} \mathbf{H}$ NMR $\left(500.06 \mathrm{MHz}, \mathrm{CDCl}_{3}\right)$ :
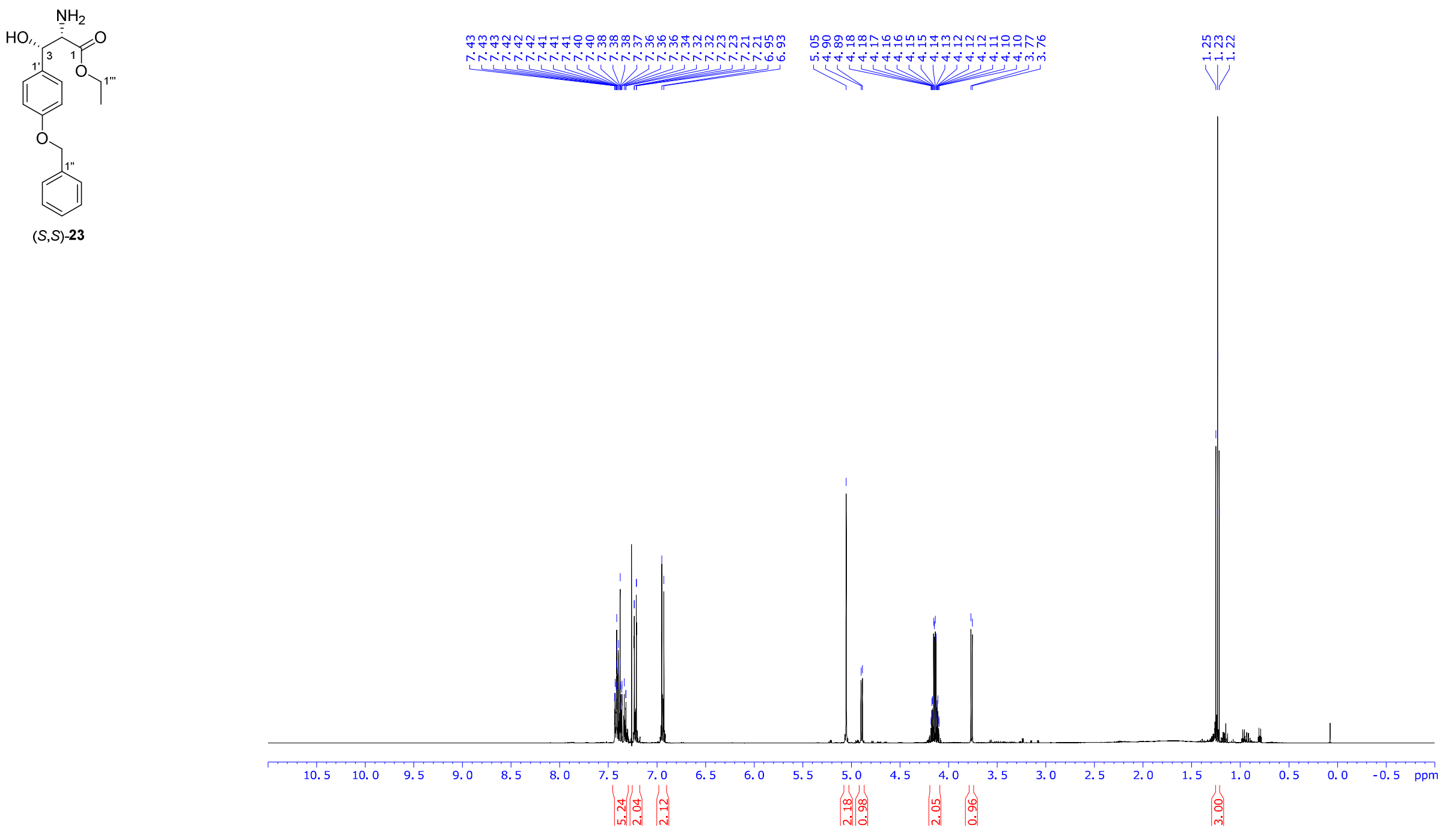
Christian Drescher and Reinhard Brückner; 3D-Structure Clarifying Total Synthesis of the (Polyenoyl)tetramic Acid Militarinon B. A Highly Acid-Labile N-Protecting Group for Amides

Ethyl (2S,3S)-2-Amino-3-[4' -(benzyloxy)phenyl]-3-hydroxypropanoate [(S,S)-23]

${ }^{13} \mathrm{C}$ NMR (125.75 MHz, $\left.\mathrm{CDCl}_{3}\right)$ :

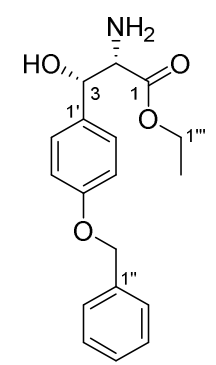

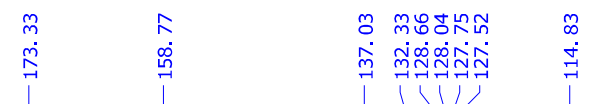

$(S, S)-23$

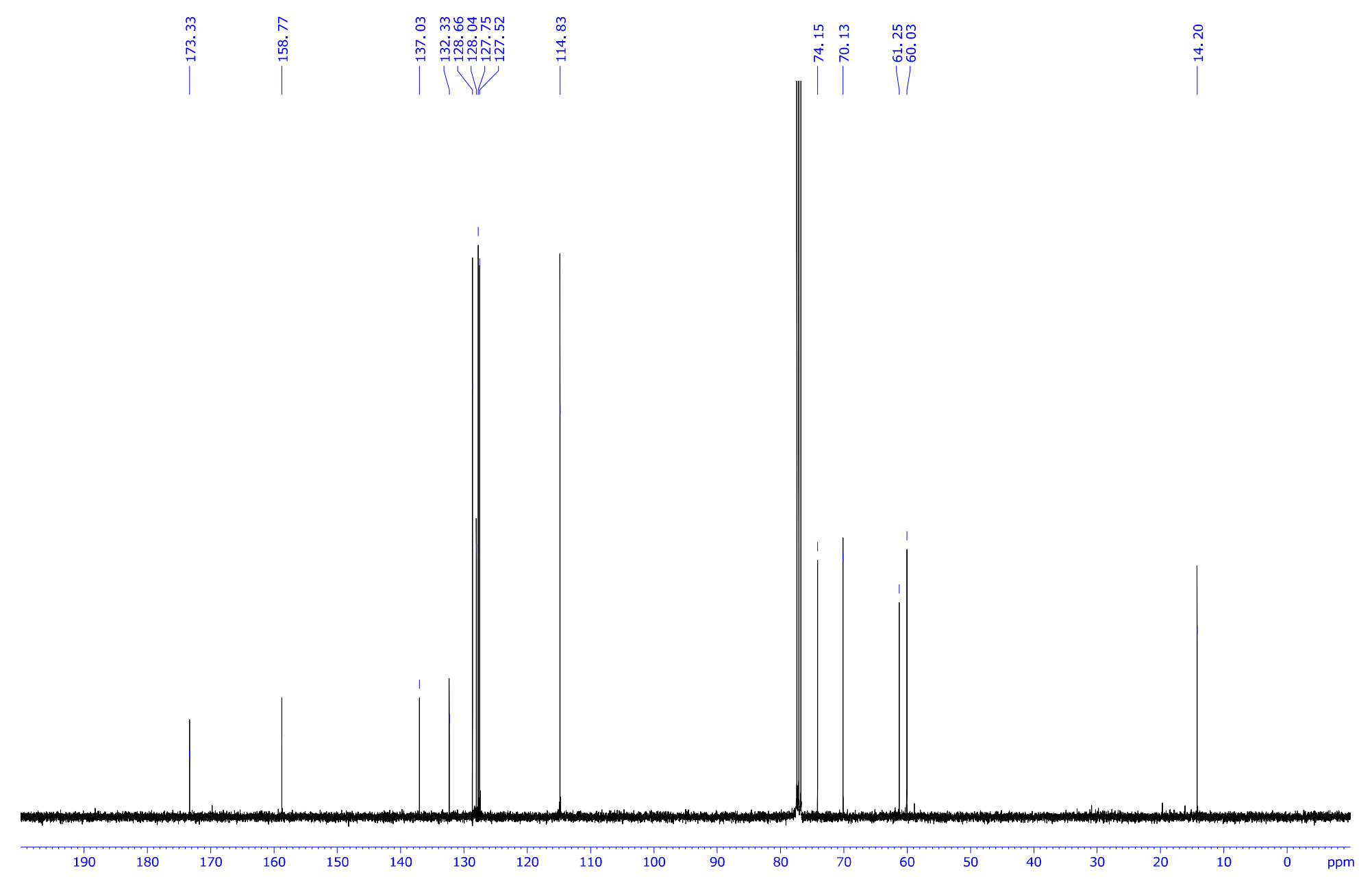


Christian Drescher and Reinhard Brückner; 3D-Structure Clarifying Total Synthesis of the (Polyenoyl)tetramic Acid Militarinon B. A Highly Acid-Labile N-Protecting Group for Amides

\section{Ethyl (2S,3R)-2-Amino-3-[4'-(benzyloxy)phenyl]-3-hydroxypropanoate [(R,S)-23]}

${ }^{1} \mathbf{H}$ NMR $\left(500.06 \mathrm{MHz}, \mathrm{CDCl}_{3}\right)$ :
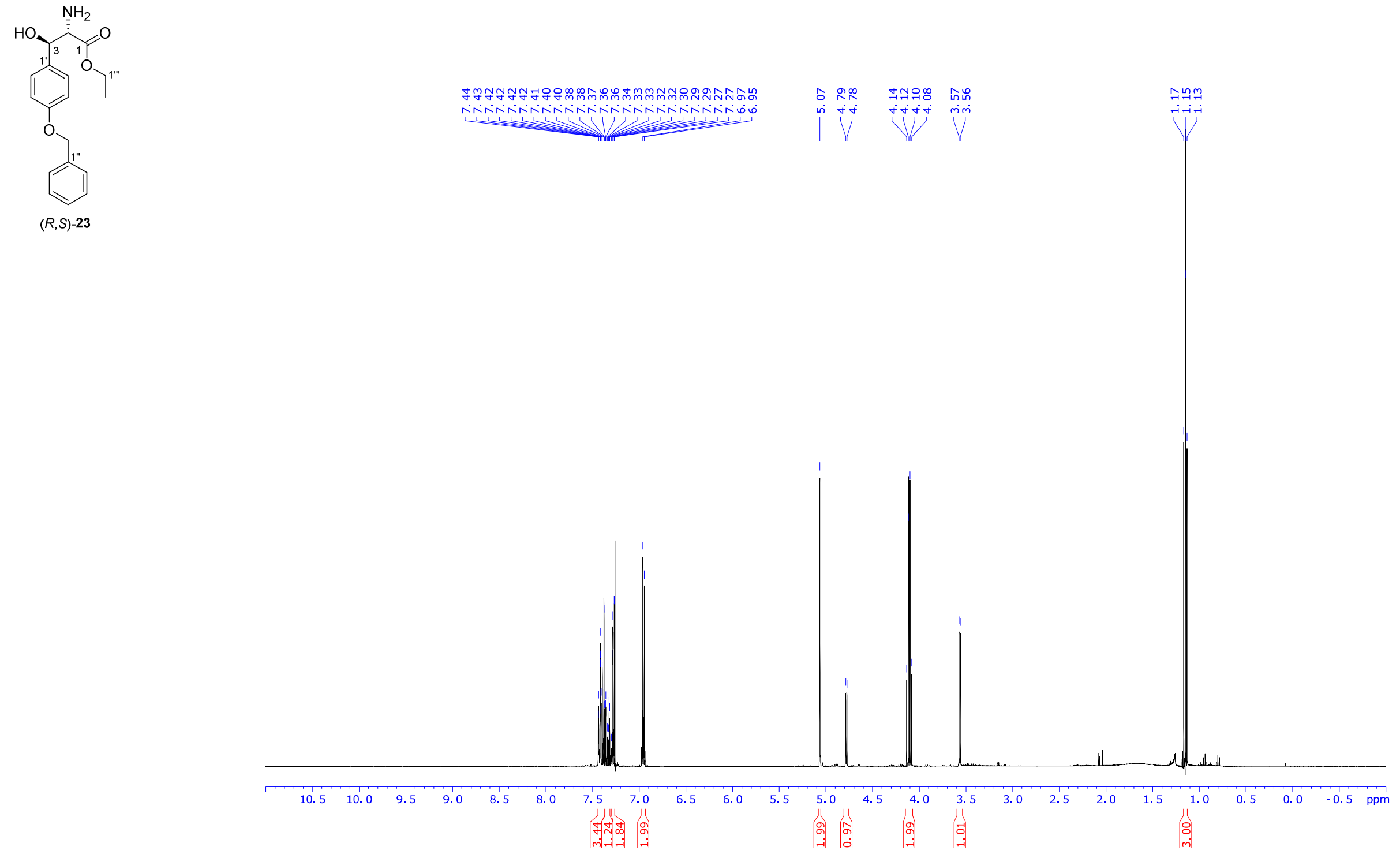
Christian Drescher and Reinhard Brückner; 3D-Structure Clarifying Total Synthesis of the (Polyenoyl)tetramic Acid Militarinon B. A Highly Acid-Labile N-Protecting Group for Amides

\section{Ethyl (2S,3R)-2-Amino-3-[4'-(benzyloxy)phenyl]-3-hydroxypropanoate [(R,S)-23]}

${ }^{13}$ C NMR (125.75 MHz, $\left.\mathrm{CDCl}_{3}\right)$ :
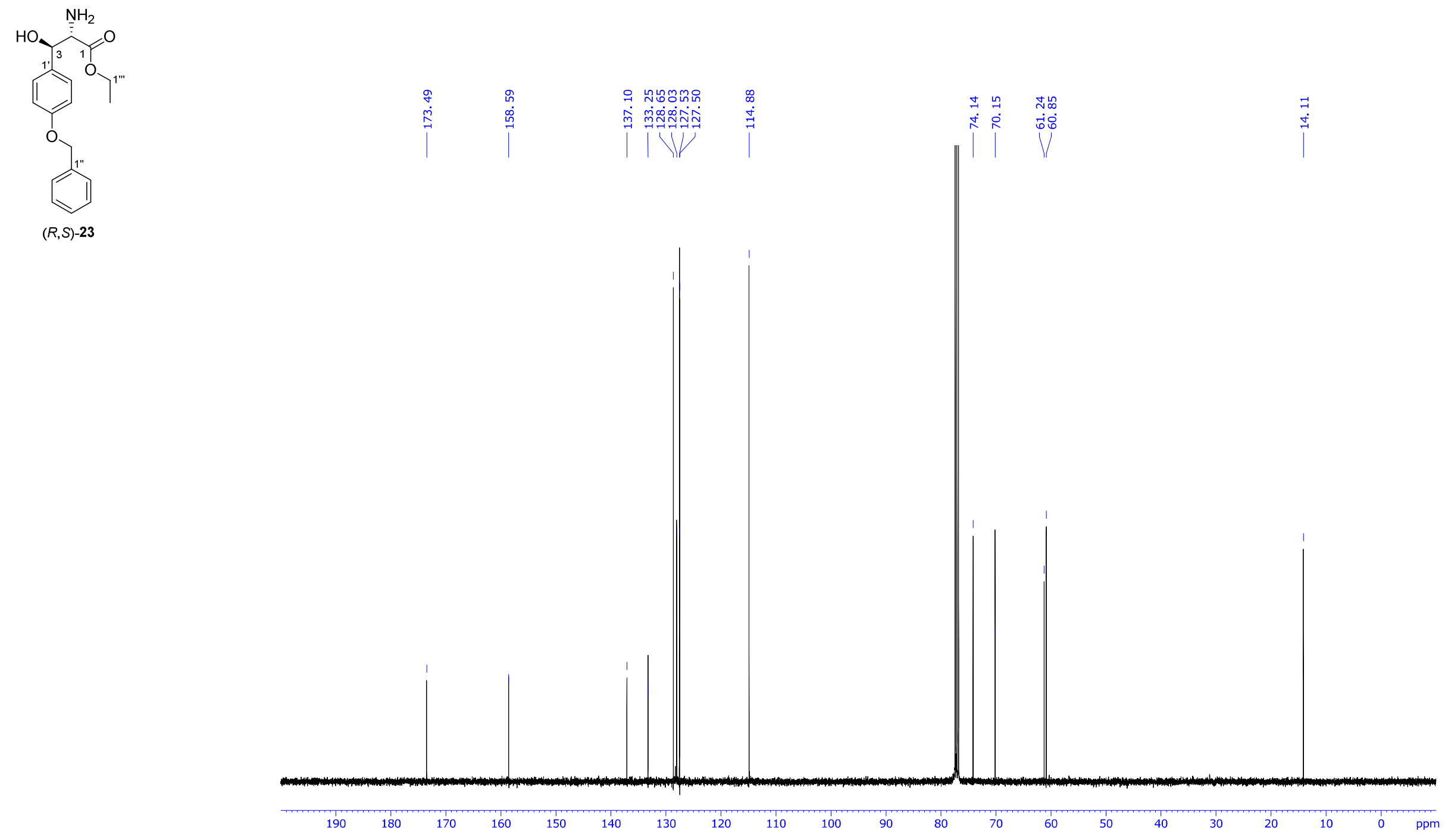
Christian Drescher and Reinhard Brückner; 3D-Structure Clarifying Total Synthesis of the (Polyenoyl)tetramic Acid Militarinon B. A Highly Acid-Labile N-Protecting Group for Amides

Ethyl (2S,3S)-2-[(tert-Butyldimethylsilyl)amino]-3-[(tert-butyldimethylsilyl)oxy]-3-\{4'-[(tert-butyldimethylsilyl)oxy]phenyl $\}$ propanoate [(S,S)-12f]

${ }^{1} \mathbf{H}$ NMR $\left(500.06 \mathrm{MHz}, \mathrm{CDCl}_{3}\right)$ :

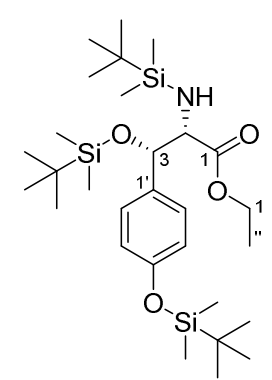

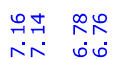

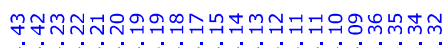

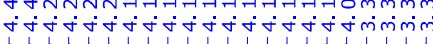

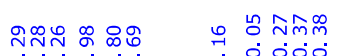

$\checkmark$

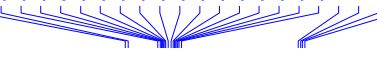

$(S, S)-12 f$

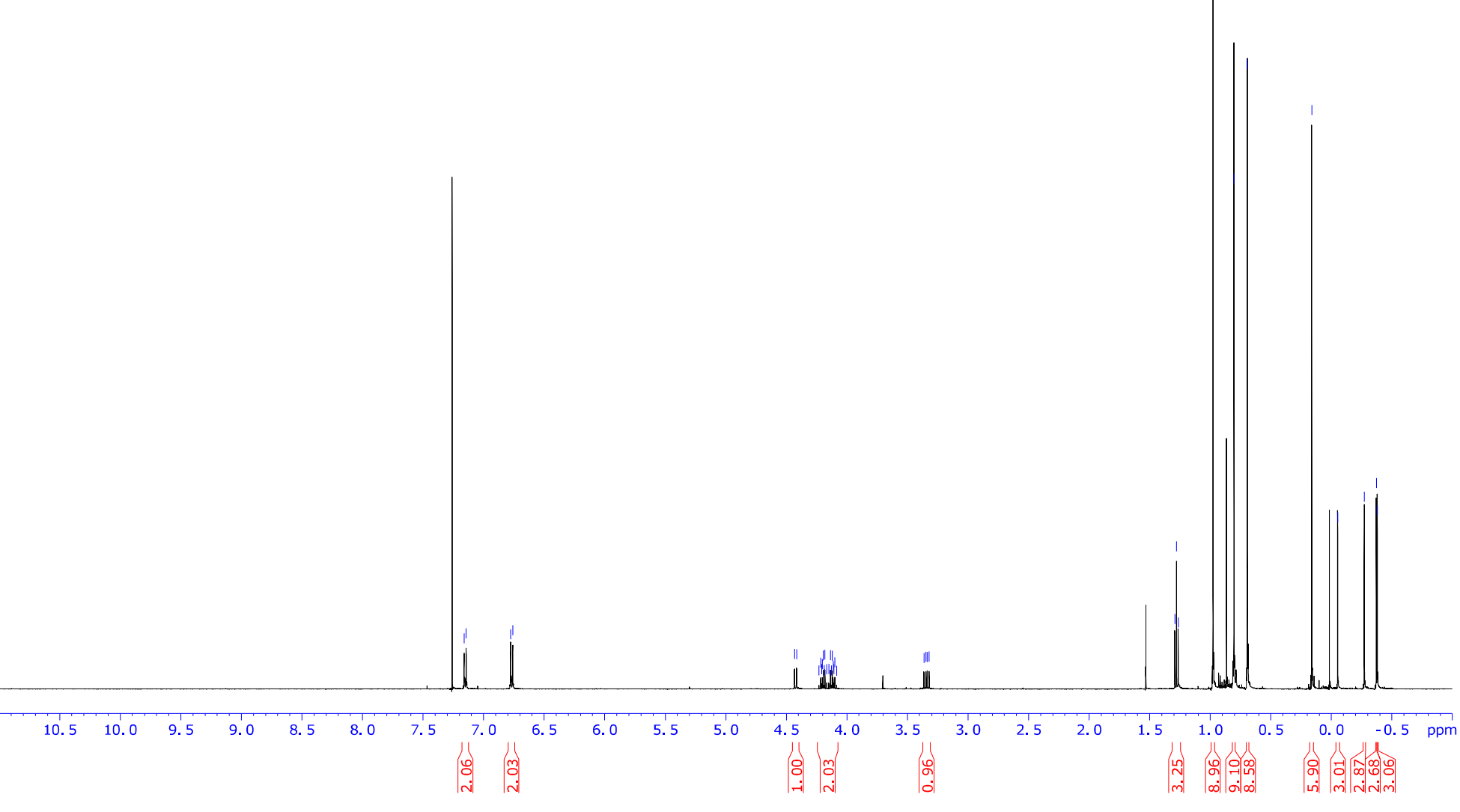


Christian Drescher and Reinhard Brückner; 3D-Structure Clarifying Total Synthesis of the (Polyenoyl)tetramic Acid Militarinon B. A Highly Acid-Labile N-Protecting Group for Amides

Ethyl (2S,3S)-2-[(tert-Butyldimethylsilyl)amino]-3-[(tert-butyldimethylsilyl)oxy]-3-\{4'-[(tert-butyldimethylsilyl)oxy]phenyl $\}$ propanoate [(S,S)-12f]

${ }^{13}$ C NMR (125.75 MHz, $\left.\mathrm{CDCl}_{3}\right)$ :
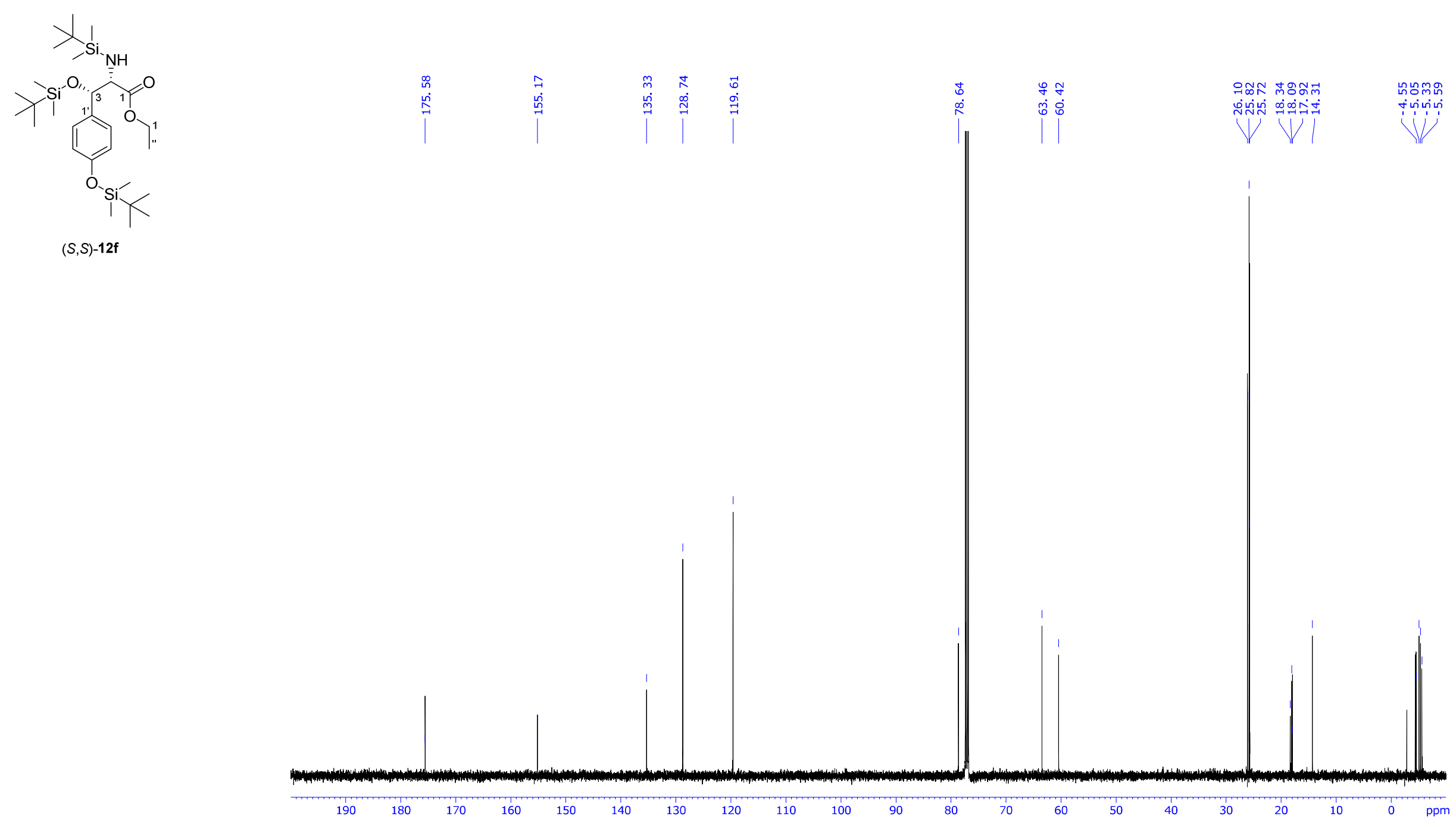
Christian Drescher and Reinhard Brückner; 3D-Structure Clarifying Total Synthesis of the (Polyenoyl)tetramic Acid Militarinon B. A Highly Acid-Labile N-Protecting Group for Amides

Ethyl (2S,3R)-2-[(tert-Butyldimethylsilyl)amino]-3-[(tert-butyldimethylsilyl)oxy]-3-\{4'-[(tert-butyldimethylsilyl)oxy]phenyl\}propanoate [(R,S)-12f]

${ }^{1}$ H NMR $\left(500.06 \mathrm{MHz}, \mathrm{CDCl}_{3}\right)$ :

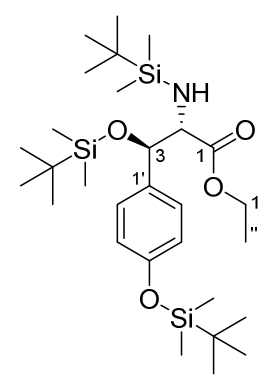

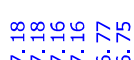

inisiog

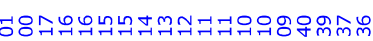

$\sin$

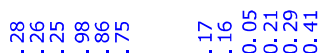


Christian Drescher and Reinhard Brückner; 3D-Structure Clarifying Total Synthesis of the (Polyenoyl)tetramic Acid Militarinon B. A Highly Acid-Labile N-Protecting Group for Amides

Ethyl (2S,3R)-2-[(tert-Butyldimethylsilyl)amino]-3-[(tert-butyldimethylsilyl)oxy]-3-\{4'-[(tert-butyldimethylsilyl)oxy]phenyl\}propanoate [(R,S)-12f]

${ }^{13}$ C NMR (125.75 MHz, $\left.\mathrm{CDCl}_{3}\right)$ :
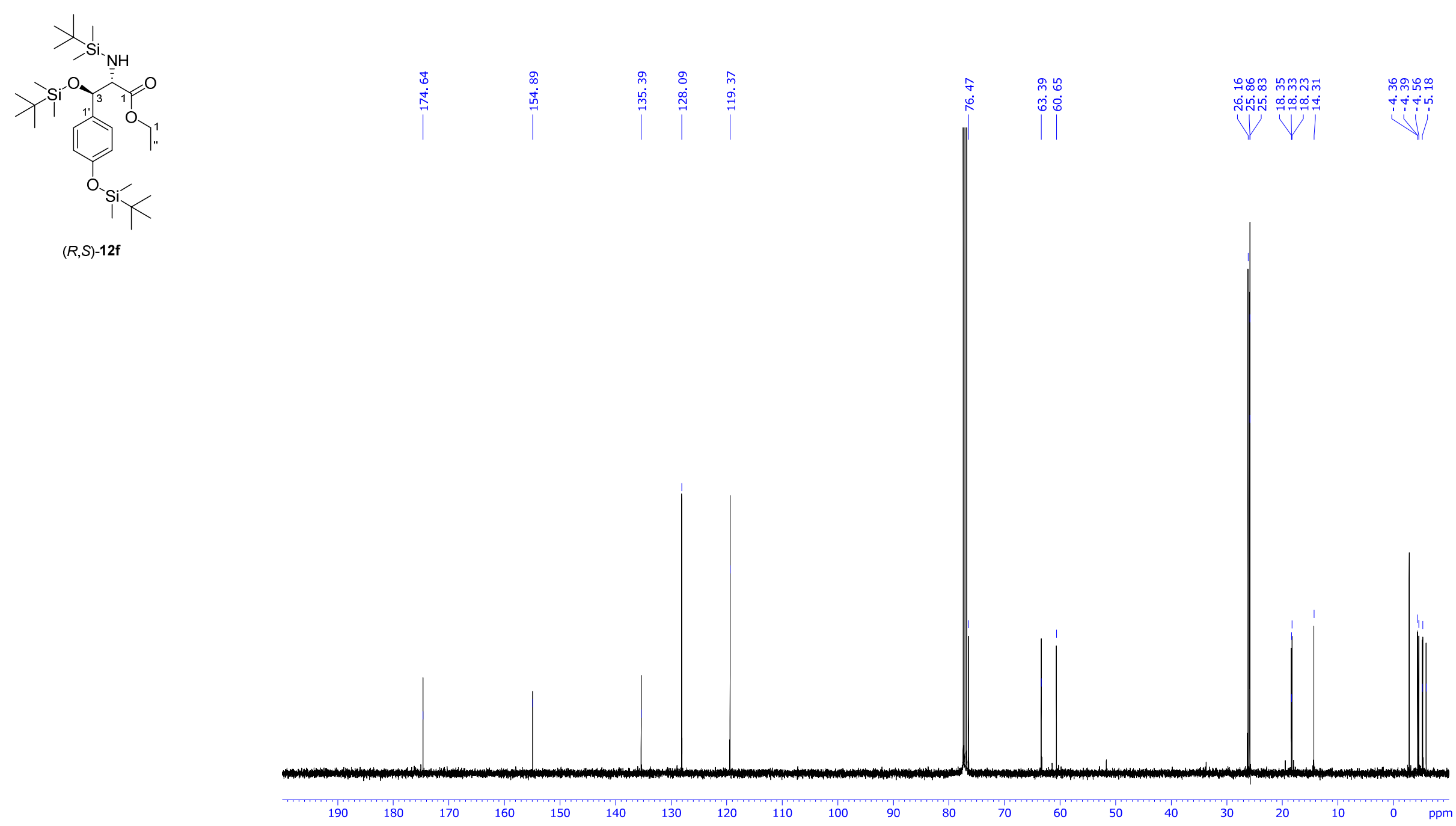
Christian Drescher and Reinhard Brückner; 3D-Structure Clarifying Total Synthesis of the (Polyenoyl)tetramic Acid Militarinon B. A Highly Acid-Labile N-Protecting Group for Amides

Ethyl (2S,3S)-3-[(tert-Butyldimethylsilyl)oxy]-3-\{4'-[(tert-butyldimethylsilyl)oxy]phenyl\}-2-[(1",3",5"'-trimethoxybenzyl)amino]propanoate $[(S, S)-12 f]$

${ }^{1} \mathbf{H}$ NMR $\left(500.06 \mathrm{MHz}, \mathrm{CDCl}_{3}\right)$ :

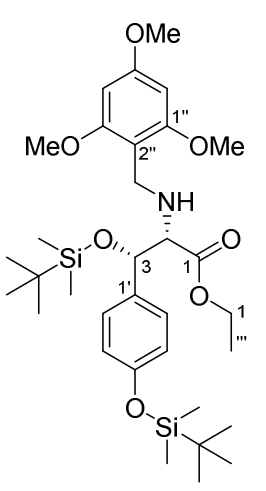

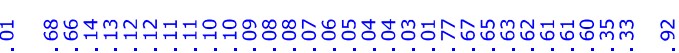

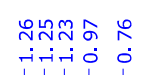

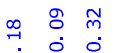

$\mathrm{H}$

$(S, S)-12 f$

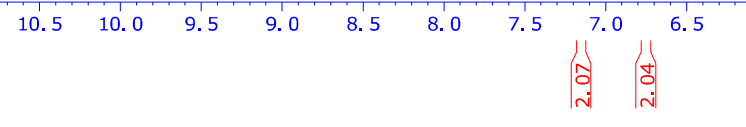

6.0
ले
i

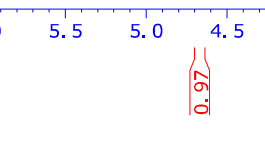

mi 
Christian Drescher and Reinhard Brückner; 3D-Structure Clarifying Total Synthesis of the (Polyenoyl)tetramic Acid Militarinon B. A Highly Acid-Labile N-Protecting Group for Amides

Ethyl (2S,3S)-3-[(tert-Butyldimethylsilyl)oxy]-3-\{4'-[(tert-butyldimethylsilyl)oxy]phenyl\}-2-[(1",3",5"-trimethoxybenzyl)amino]propanoate [(S,S)-12f]

${ }^{13}$ C NMR (125.75 MHz, $\left.\mathrm{CDCl}_{3}\right)$ :

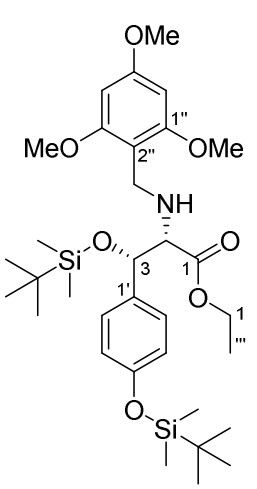

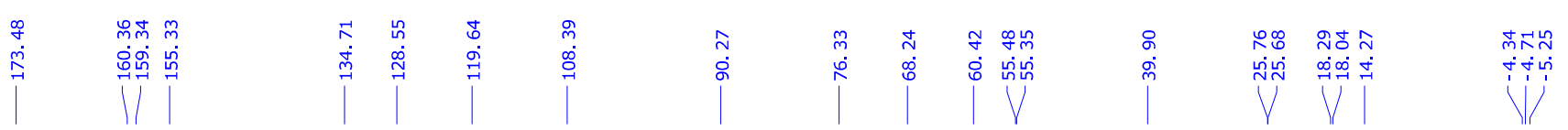

$(S, S)-12 f$

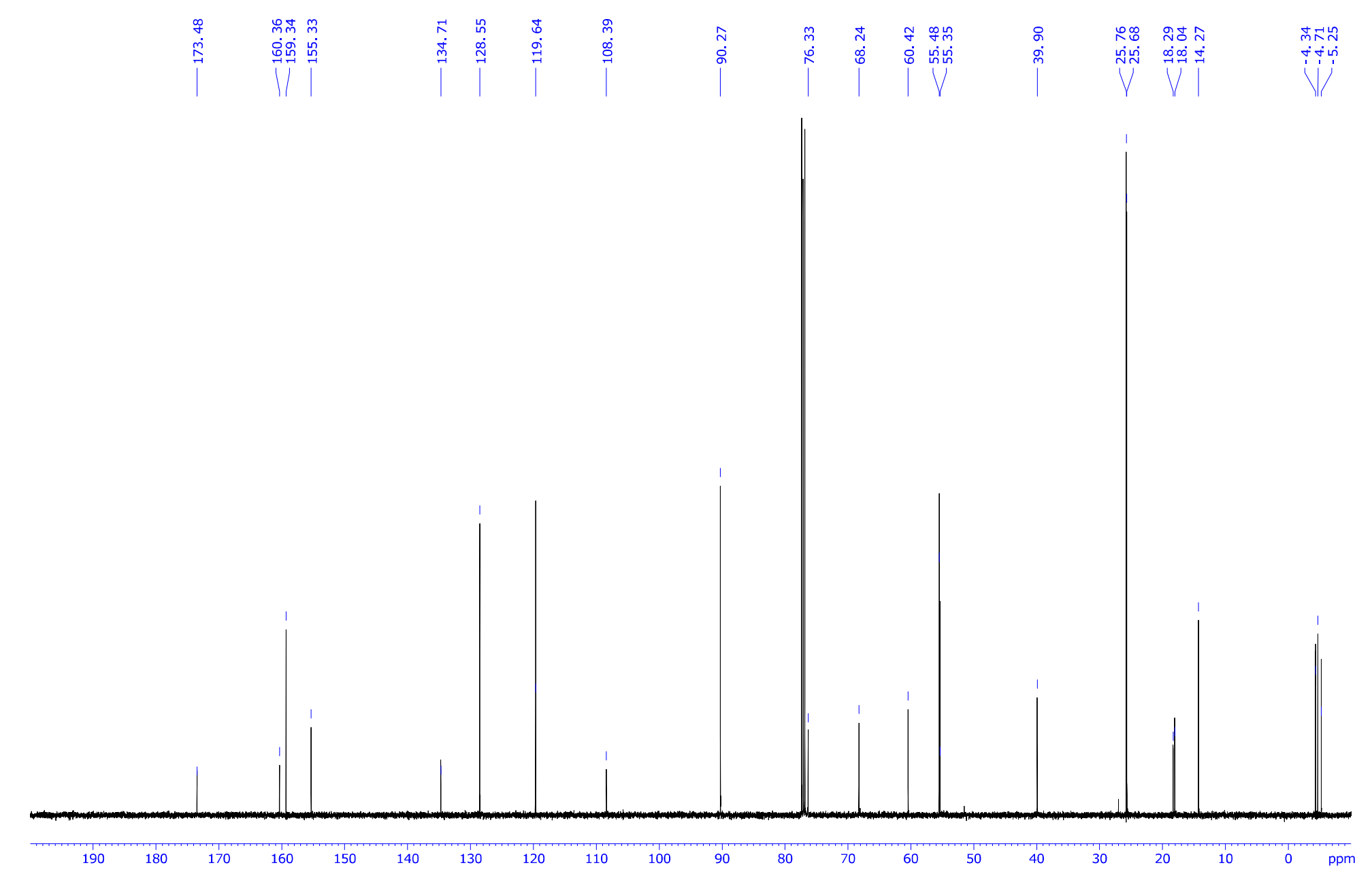


Christian Drescher and Reinhard Brückner; 3D-Structure Clarifying Total Synthesis of the (Polyenoyl)tetramic Acid Militarinon B. A Highly Acid-Labile N-Protecting Group for Amides

Ethyl (2S,3R)-3-[(tert-Butyldimethylsilyl)oxy]-3-\{4'-[(tert-butyldimethylsilyl)oxy]phenyl\}-2-[(1",3",5"'-trimethoxybenzyl)amino]propanoate [(R,S)-12f]

${ }^{1}$ H NMR $\left(500.06 \mathrm{MHz}, \mathrm{CDCl}_{3}\right)$ :
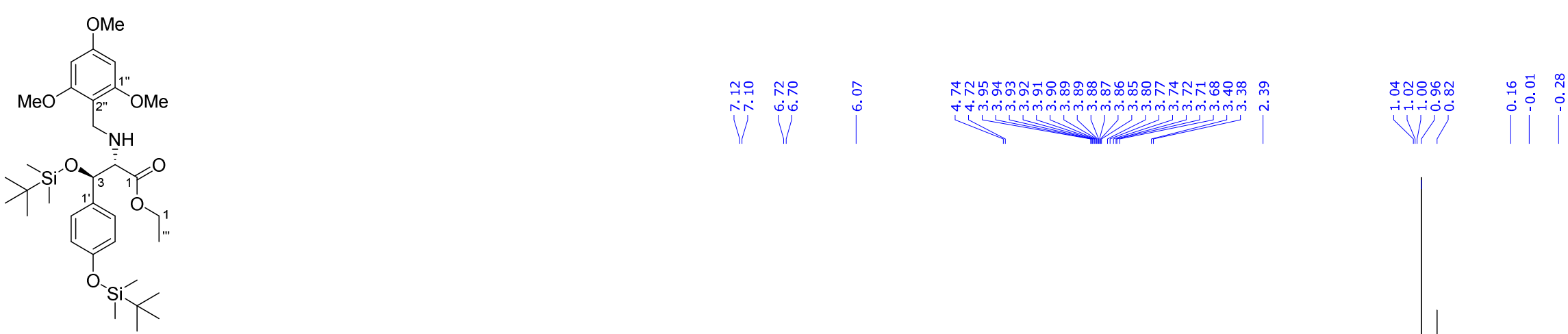

$(R, S)-12 f$ 
Christian Drescher and Reinhard Brückner; 3D-Structure Clarifying Total Synthesis of the (Polyenoyl)tetramic Acid Militarinon B. A Highly Acid-Labile N-Protecting Group for Amides

Ethyl (2S,3R)-3-[(tert-Butyldimethylsilyl)oxy]-3-\{4'-[(tert-butyldimethylsilyl)oxy]phenyl\}-2-[(1",3",5"'-trimethoxybenzyl)amino]propanoate [(S,R)-12f]

${ }^{13}$ C NMR (125.75 MHz, $\left.\mathrm{CDCl}_{3}\right)$ :

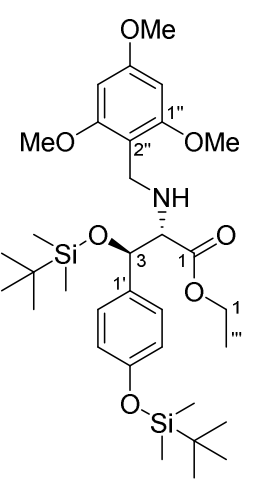

$(R, S)-12 f$

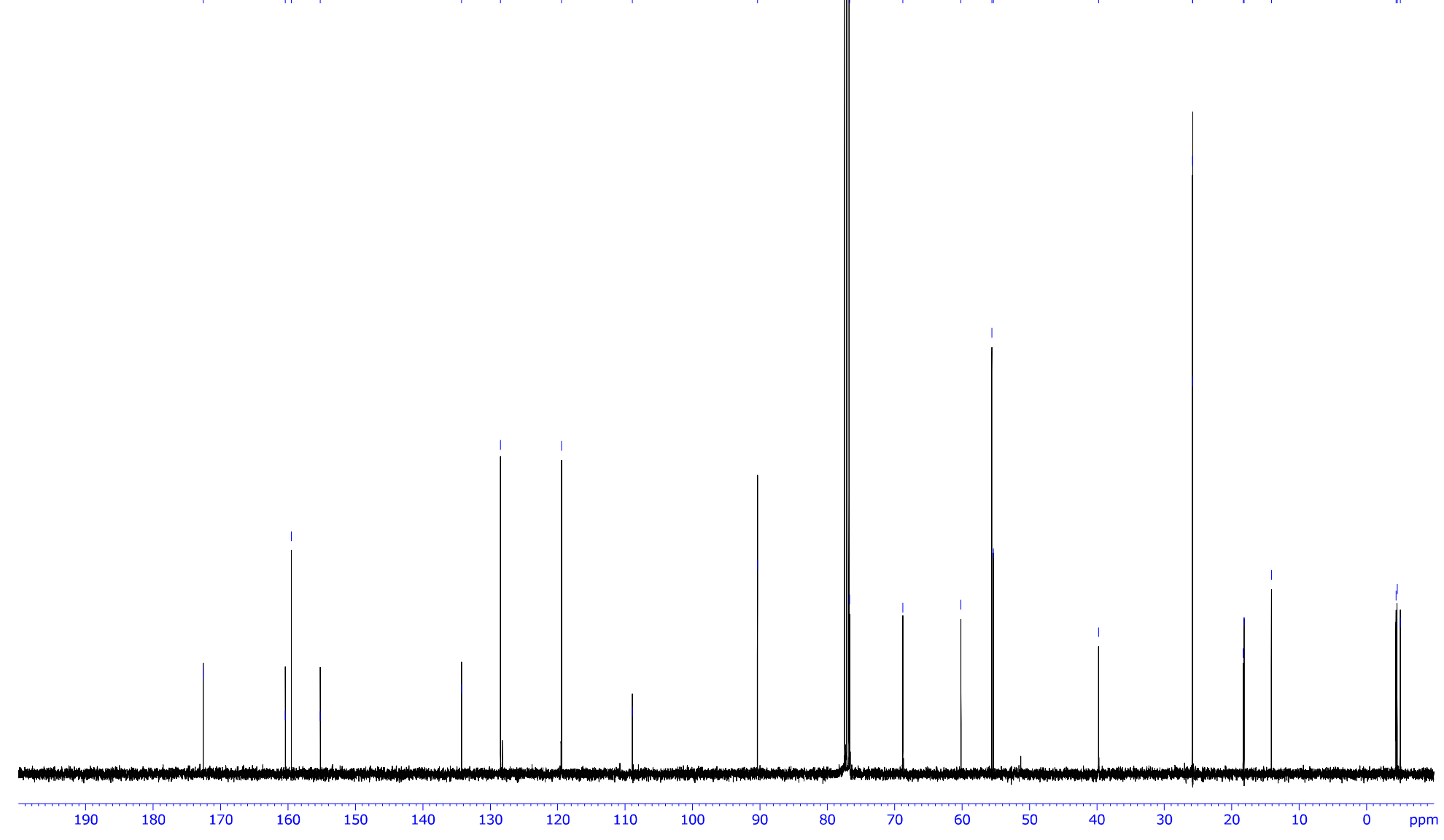


Christian Drescher and Reinhard Brückner; 3D-Structure Clarifying Total Synthesis of the (Polyenoyl)tetramic Acid Militarinon B. A Highly Acid-Labile N-Protecting Group for Amides

Ethyl $(2 ' S, 3 ' S)-2$ '- $\left\{N-[(1 Z, 2 Z, 4 E)-5-B r o m o-3-h y d r o x y-1-0 x o y p e n t a-2,4-d i e n y l]-N-\left(1^{\prime \prime \prime}, 3^{\prime \prime \prime}, 5^{\prime \prime \prime}-\right.\right.$ trimethoxybenzyl $\left.)\right\}-3 '-[(t e r t-$ butyldimethylsilyl)oxy]-3'-\{1'-[(tert-butyldimethylsilyl)oxy]phenyl $\}$ propanoate $[Z-e n o l-(S, S)-10 \mathrm{e}]$

${ }^{1} \mathbf{H}$ NMR $\left(500.06 \mathrm{MHz}, \mathrm{CDCl}_{3}\right)$ :

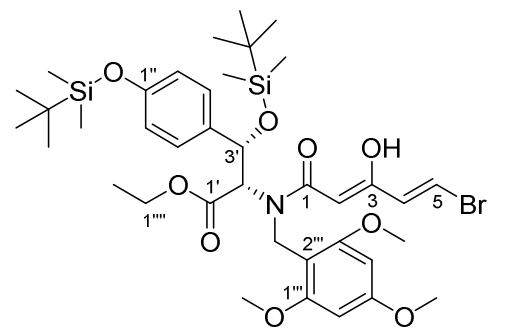

$Z$-enof $[(S, S)-10 \mathrm{e}]$

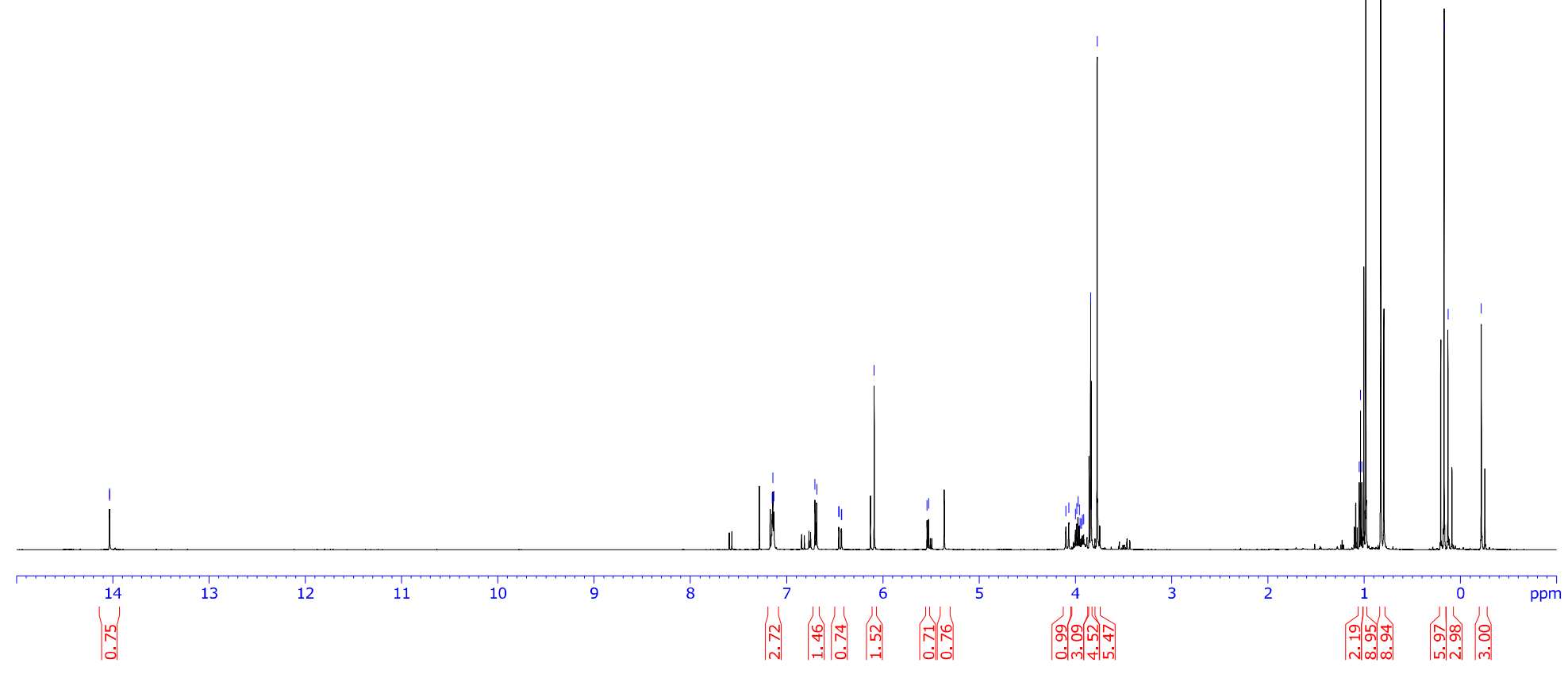


Christian Drescher and Reinhard Brückner; 3D-Structure Clarifying Total Synthesis of the (Polyenoyl)tetramic Acid Militarinon B. A Highly Acid-Labile N-Protecting Group for Amides

Ethyl $(2 ' S, 3 ' S)-2$ '- $\{N-[(1 Z, 2 Z, 4 E)-5-B r o m o-3-h y d r o x y-1-o x o y p e n t a-2,4-d i e n y l]-N-(1 " ', 3$ '", 5 '"'-trimethoxybenzyl) $\}-3$ '-[(tertbutyldimethylsilyl)oxy]-3'-\{1'-[(tert-butyldimethylsilyl)oxy]phenyl $\}$ propanoate $[Z-e n o l-(S, S)-10 \mathrm{e}]$

${ }^{13} \mathrm{C}$ NMR (125.75 MHz, $\left.\mathrm{CDCl}_{3}\right)$ :
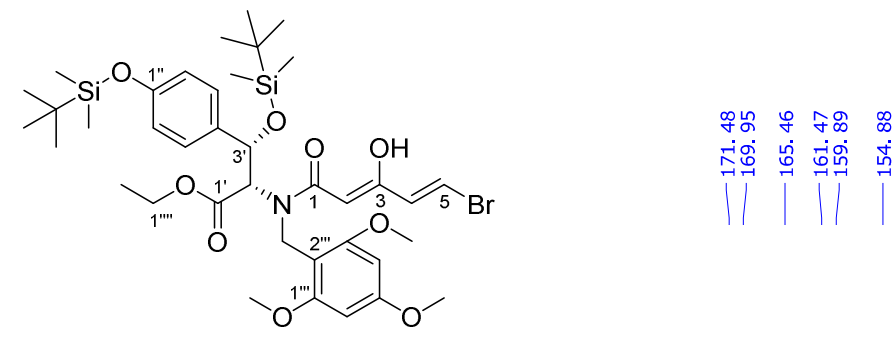

Z-enol-[(S,S)-10e]

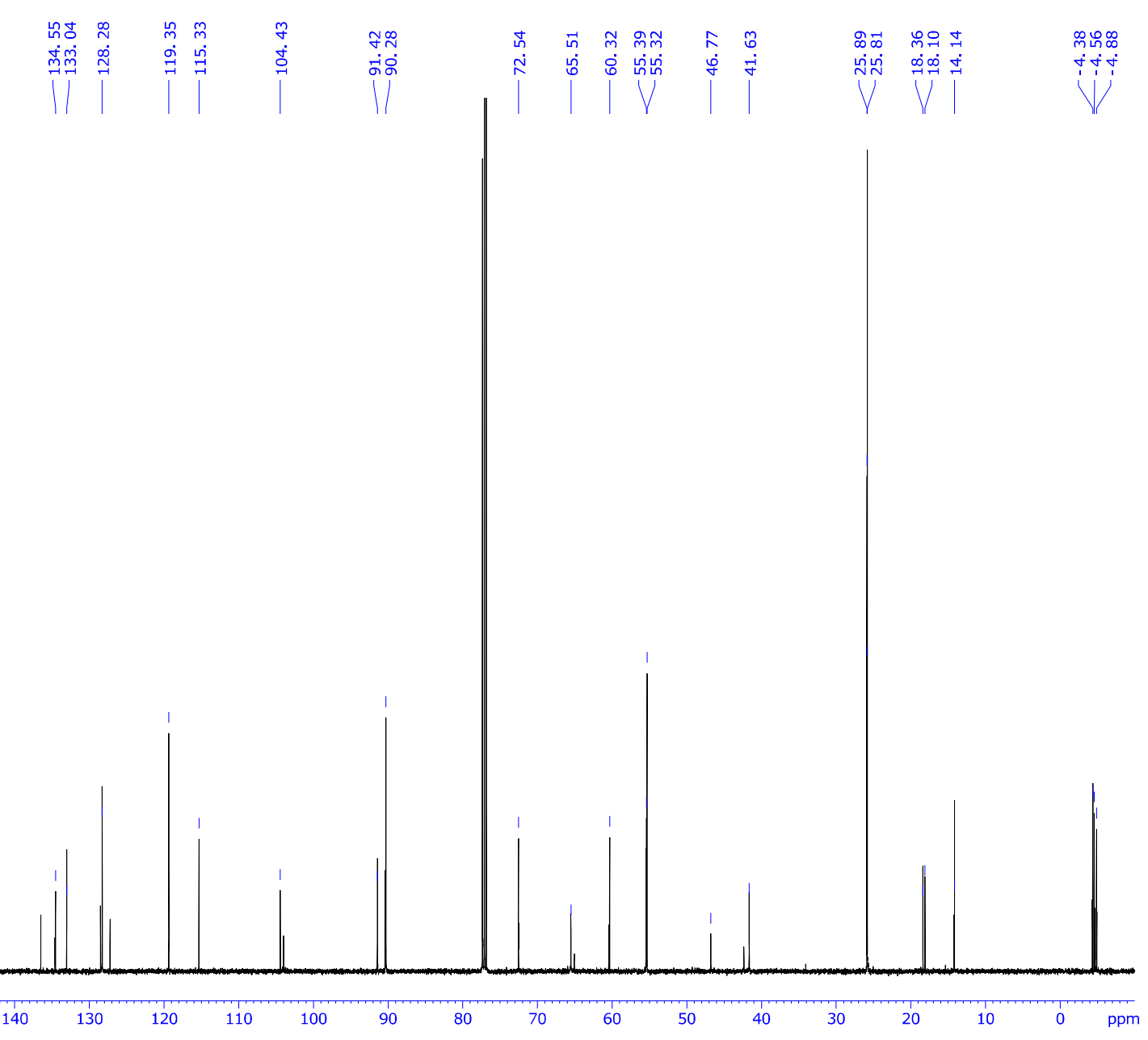


Christian Drescher and Reinhard Brückner; 3D-Structure Clarifying Total Synthesis of the (Polyenoyl)tetramic Acid Militarinon B. A Highly Acid-Labile N-Protecting Group for Amides

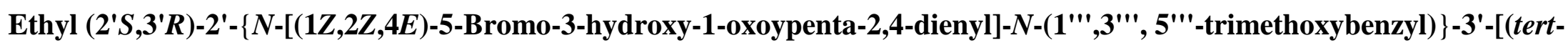
butyldimethylsilyl)oxy]-3'-\{1'-[(tert-butyldimethylsilyl)oxy]phenyl $\}$ propanoate $[Z$-enol- $(R, S)-10 \mathrm{e}]$

${ }^{1} \mathbf{H}$ NMR $\left(500.06 \mathrm{MHz}, \mathrm{CDCl}_{3}\right)$ :

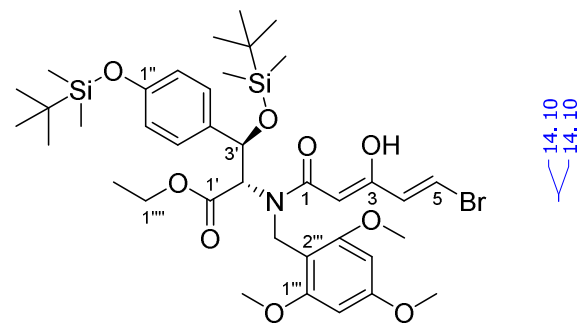

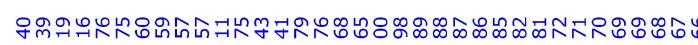

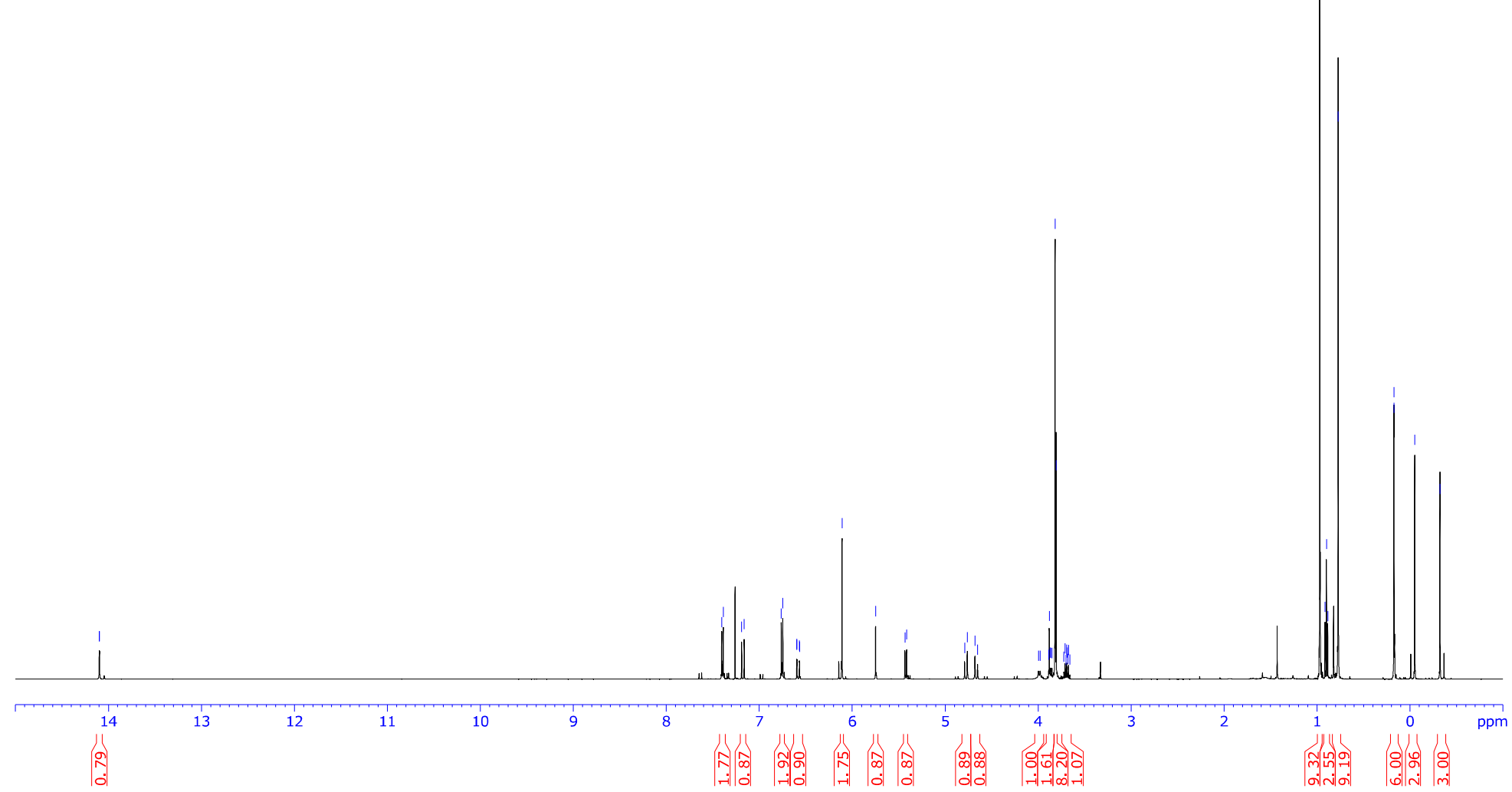


Christian Drescher and Reinhard Brückner; 3D-Structure Clarifying Total Synthesis of the (Polyenoyl)tetramic Acid Militarinon B. A Highly Acid-Labile N-Protecting Group for Amides

Ethyl $(2 ' S, 3 ' R)-2$ '- $\left\{N-[(1 Z, 2 Z, 4 E)-5-B r o m o-3-h y d r o x y-1-o x o y p e n t a-2,4-d i e n y l]-N-\left(1 '{ }^{\prime \prime}, 3 '{ }^{\prime \prime}, 5^{\prime \prime}\right.\right.$-trimethoxybenzyl $\left.)\right\}-3 '-[(t e r t-$ butyldimethylsilyl)oxy]-3'-\{1'-[(tert-butyldimethylsilyl)oxy]phenyl $\}$ propanoate $[Z$-enol- $(R, S)-10 \mathrm{e}]$

${ }^{13}$ C NMR (125.75 MHz, $\left.\mathrm{CDCl}_{3}\right)$ :
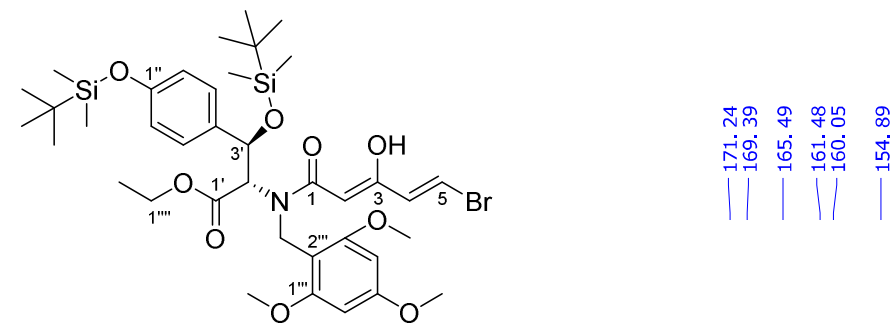

Z-enol-[(R,S)-10e]
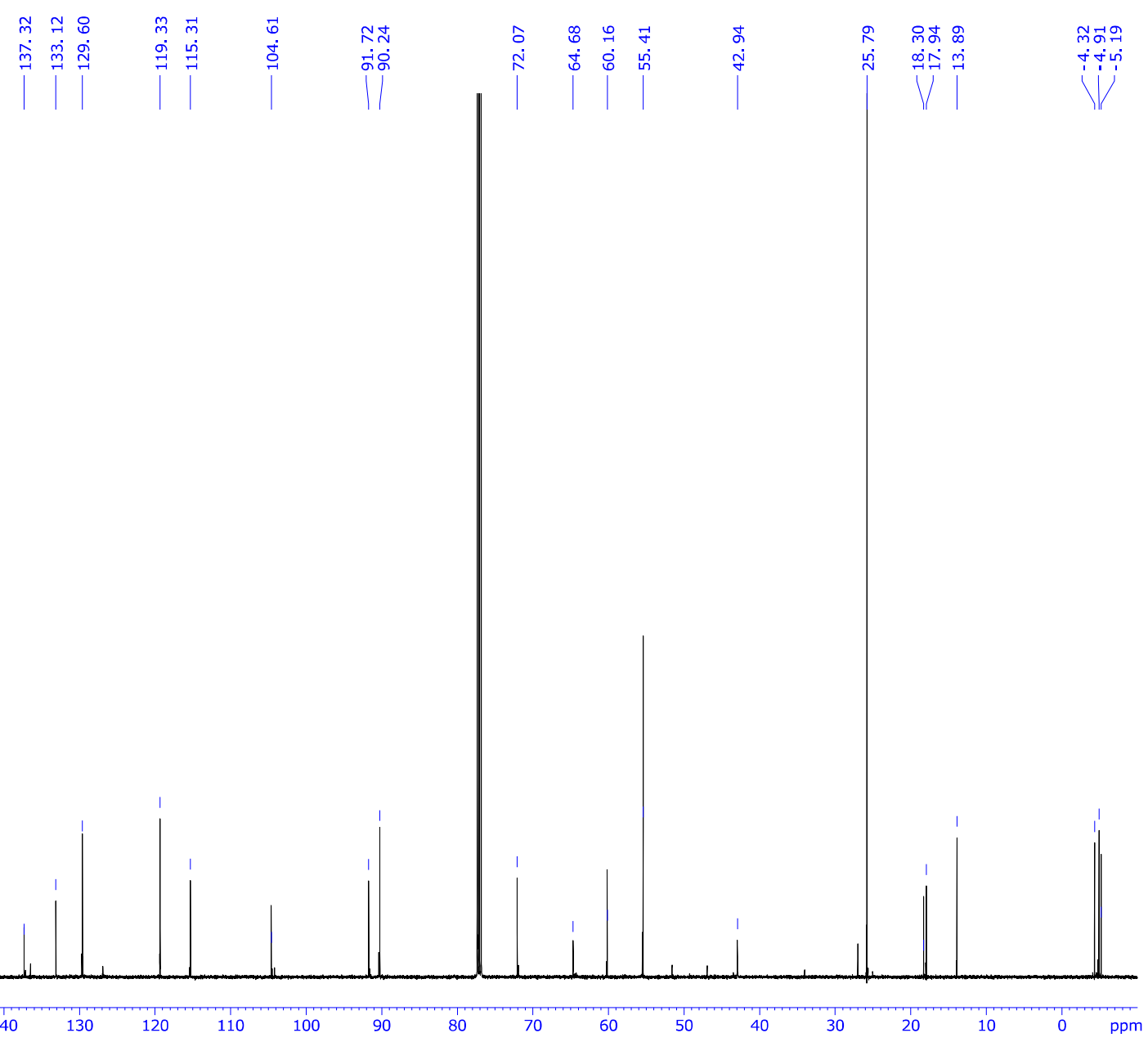
Christian Drescher and Reinhard Brückner; 3D-Structure Clarifying Total Synthesis of the (Polyenoyl)tetramic Acid Militarinon B. A Highly Acid-Labile N-Protecting Group for Amides

3.3 ${ }^{1} \mathrm{H}$ and ${ }^{13} \mathrm{C}$ NMR Spectra to the Synthesis of the Tetramic Acid Militarinone B $[(S, S, R, R)-3]$ and its Epimer $(R, S, R, R)-3$ 
Christian Drescher and Reinhard Brückner; 3D-Structure Clarifying Total Synthesis of the (Polyenoyl)tetramic Acid Militarinon B. A Highly Acid-Labile N-Protecting Group for Amides

Ethyl $(2 ' S, 3 ' S)-2$ '- $\{N-[(1 Z, 2 Z, 4 E, 6 E, 8 E, 10 R, 12 R)-3-H y d r o x y-8,10,12-t r i m e t h y l t e t r a d e c a-2,4,6,8$-tetraenamido]- $N-(1 ' ", 3$ '"',5'"'trimethoxybenzyl $)\}-3$ '-[(tert-butyldimethylsilyl)oxy]-3'- $\left\{1^{\prime \prime}-[(\right.$ tert-butyldimethylsilyl)oxy $]$ phenyl $\}$ propanoate $[Z$-enol-(S,S,R,R)-26]

${ }^{1} \mathbf{H}$ NMR $\left(500.06 \mathrm{MHz}, \mathrm{CDCl}_{3}\right)$ :

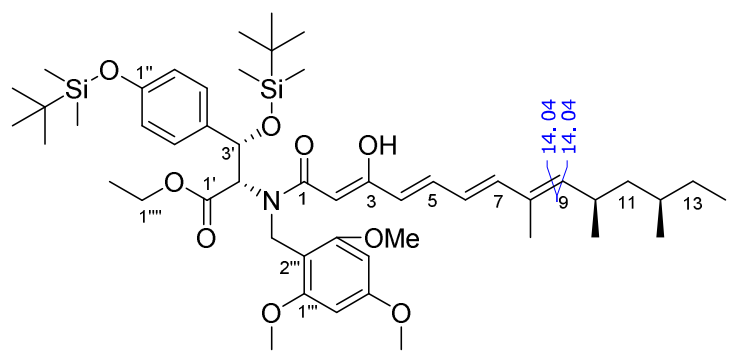

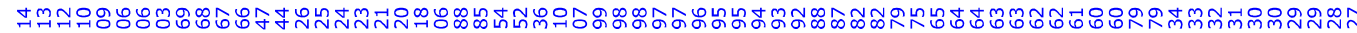

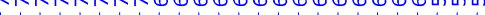

Z-enol-[(S,S,R,R)-26]

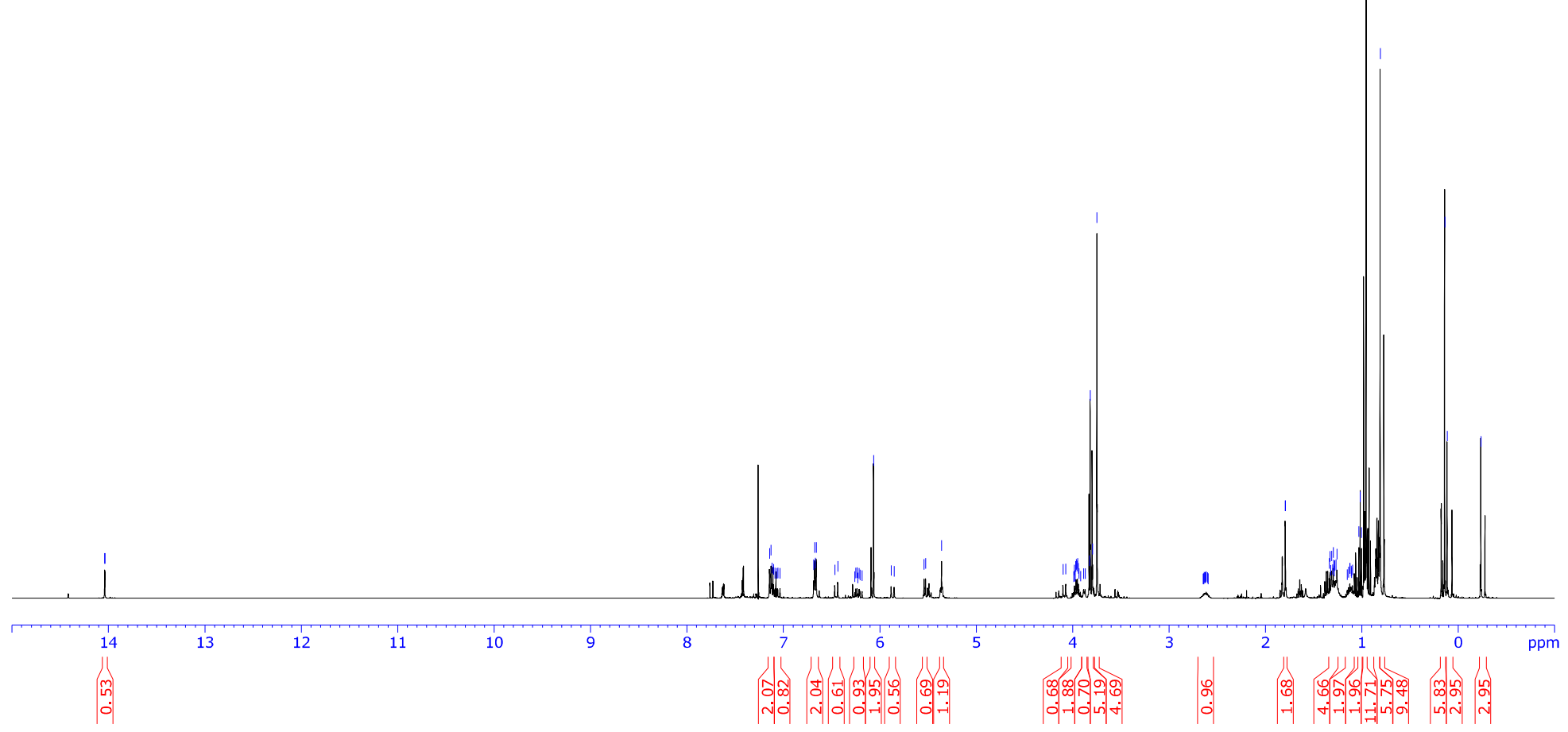


Christian Drescher and Reinhard Brückner; 3D-Structure Clarifying Total Synthesis of the (Polyenoyl)tetramic Acid Militarinon B. A Highly Acid-Labile N-Protecting Group for Amides

Ethyl $(2 ' S, 3 ' S)-2$ '- $\{N-[(1 Z, 2 Z, 4 E, 6 E, 8 E, 10 R, 12 R)-3-H y d r o x y-8,10,12-t r i m e t h y l t e t r a d e c a-2,4,6,8$-tetraenamido]- $N-(1 ' ", 3$ '"',5'"'trimethoxybenzyl $)\}-3$ '-[(tert-butyldimethylsilyl)oxy]-3'- $\left\{1^{\prime \prime}-[(\right.$ tert-butyldimethylsilyl)oxy $]$ phenyl $\}$ propanoate $[Z$-enol-(S,S,R,R)-26]

${ }^{13} \mathrm{C}$ NMR (125.75 MHz, $\left.\mathrm{CDCl}_{3}\right)$ :
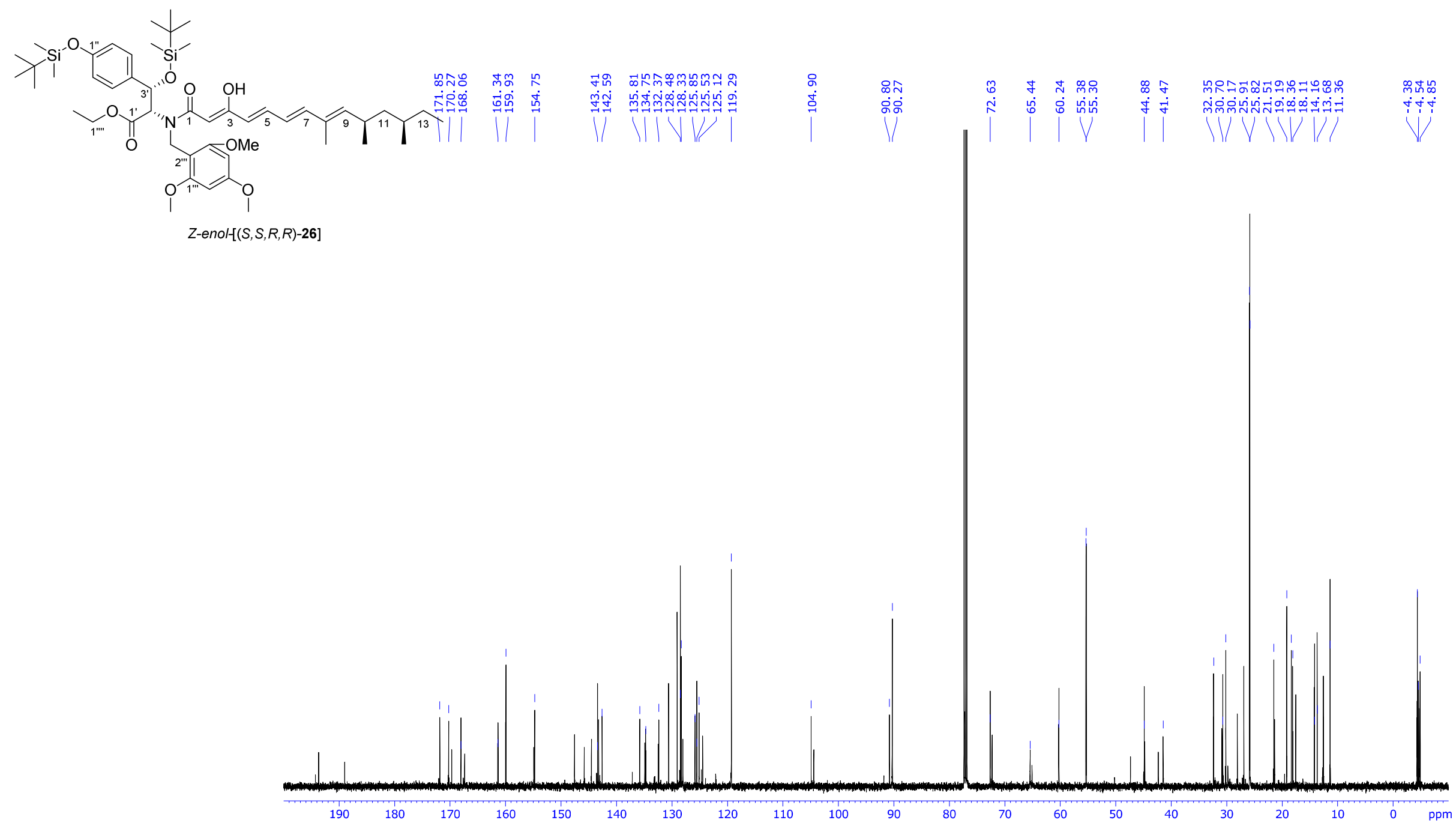
Christian Drescher and Reinhard Brückner; 3D-Structure Clarifying Total Synthesis of the (Polyenoyl)tetramic Acid Militarinon B. A Highly Acid-Labile N-Protecting Group for Amides

Ethyl $(2 ' S, 3 ' R)-2 '-\{N-[(1 Z, 2 Z, 4 E, 6 E, 8 E, 10 R, 12 R)-3-H y d r o x y-8,10,12-t r i m e t h y l t e t r a d e c a-2,4,6,8-t e t r a e n a m i d o]-N-(1 " ',, 3$ '",5'"'trimethoxybenzyl) $\}-3$ '-[(tert-butyldimethylsilyl)oxy]-3'-\{1''-[(tert-butyldimethylsilyl)oxy]phenyl $\}$ propanoate $[Z$-enol-(R,S,R,R)-26]

${ }^{1} \mathbf{H}$ NMR $\left(500.06 \mathrm{MHz}, \mathrm{CDCl}_{3}\right)$ :

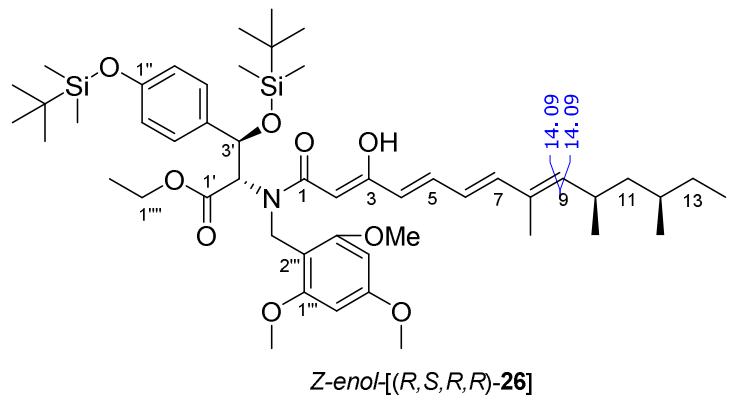

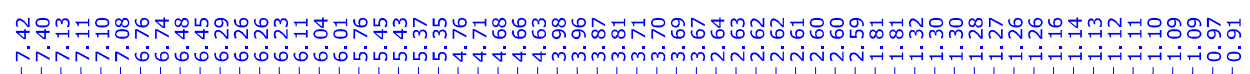

$Z$-enol-[(R,S,R,R)-26] 
Christian Drescher and Reinhard Brückner; 3D-Structure Clarifying Total Synthesis of the (Polyenoyl)tetramic Acid Militarinon B. A Highly Acid-Labile N-Protecting Group for Amides

Ethyl $(2 ' S, 3 ' R)-2 '-\{N-[(1 Z, 2 Z, 4 E, 6 E, 8 E, 10 R, 12 R)-3-H y d r o x y-8,10,12-t r i m e t h y l t e t r a d e c a-2,4,6,8-t e t r a e n a m i d o]-N-(1 " ',, 3$ '",5'"'trimethoxybenzyl) $\}-3$ '-[(tert-butyldimethylsilyl)oxy]-3'-\{1''-[(tert-butyldimethylsilyl)oxy]phenyl $\}$ propanoate $[Z$-enol-(R,S,R,R)-26]

${ }^{13} \mathrm{C}$ NMR (125.75 MHz, $\left.\mathrm{CDCl}_{3}\right)$ :
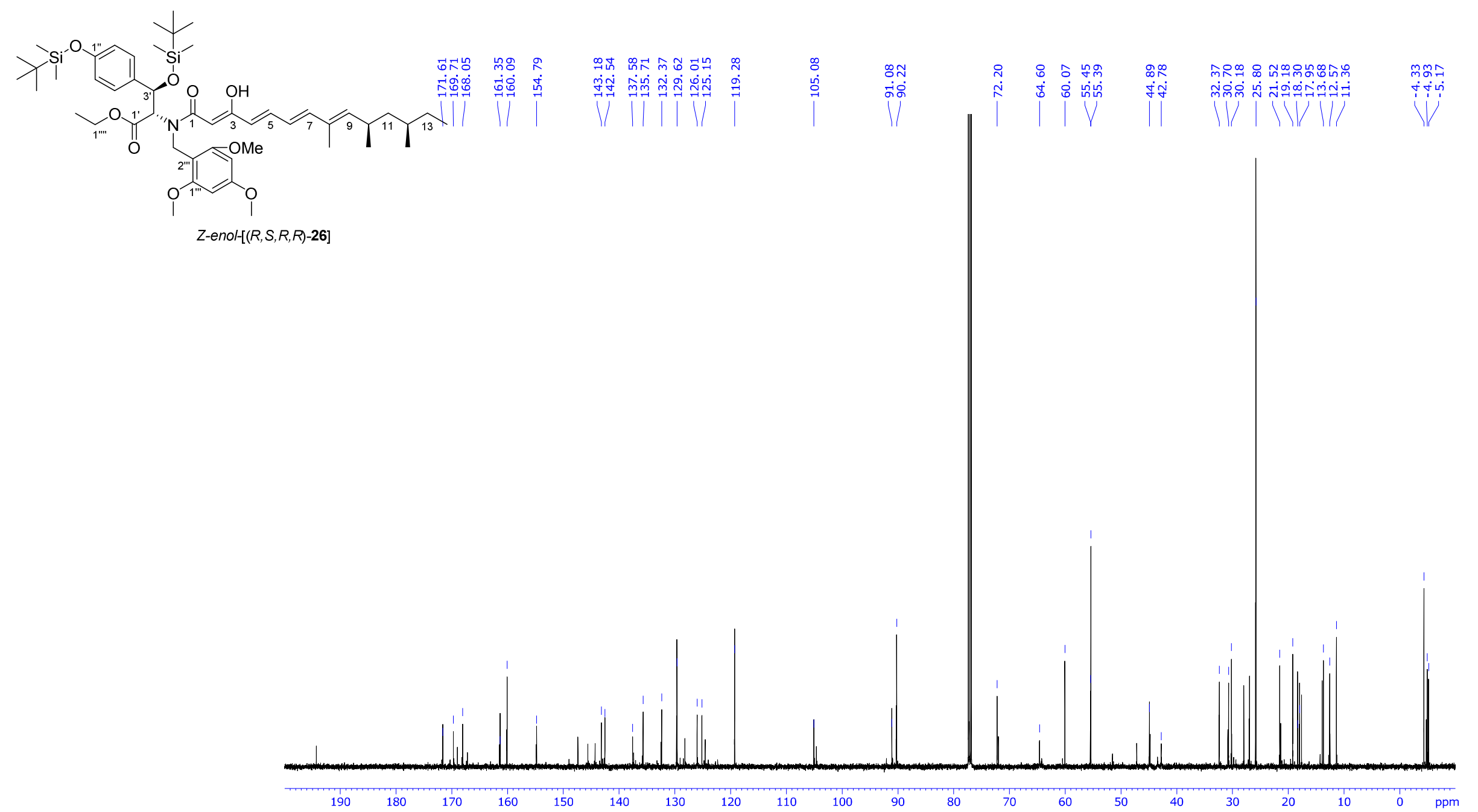
Christian Drescher and Reinhard Brückner; 3D-Structure Clarifying Total Synthesis of the (Polyenoyl)tetramic Acid Militarinon B. A Highly Acid-Labile N-Protecting Group for Amides

(5S)-5-\{(S)-[(tert-Butyldimethylsilyl)oxy] $\left\{1^{\prime \prime}-\left[(\right.\right.$ tert-butyldimethylsilyl)oxy)phenyl]methyl $\}-3-\left[\left(1^{\prime} Z, 2^{\prime} E, 4^{\prime} E, 6^{\prime} E, 8^{\prime} R, 10 ' R\right)-1^{\prime}-h y d r o x y-6 ', 8^{\prime}, 10 '-\right.$ trimethyldodeca-2',4',6'-trien-1'-ylidene]-1-(1"',3'",5'"-trimethoxybenzyl)pyrrolidine-2,4-dione [Z-enol-(S,S,R,R)-27]

${ }^{1} \mathbf{H}$ NMR $\left(500.06 \mathrm{MHz}, \mathrm{CDCl}_{3}\right)$ :

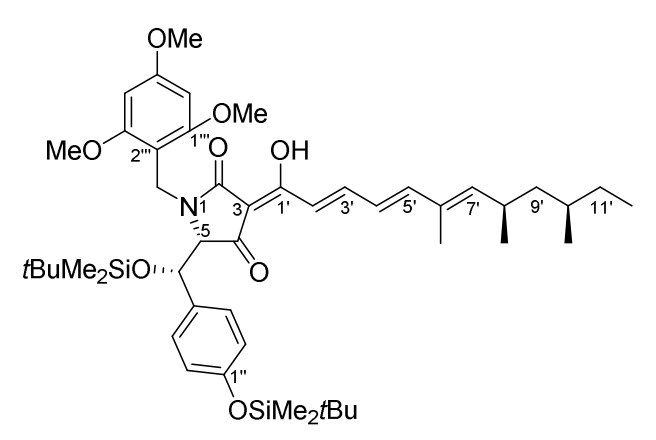

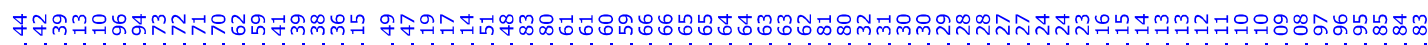

Z-enol-(S,S,R,R)-27

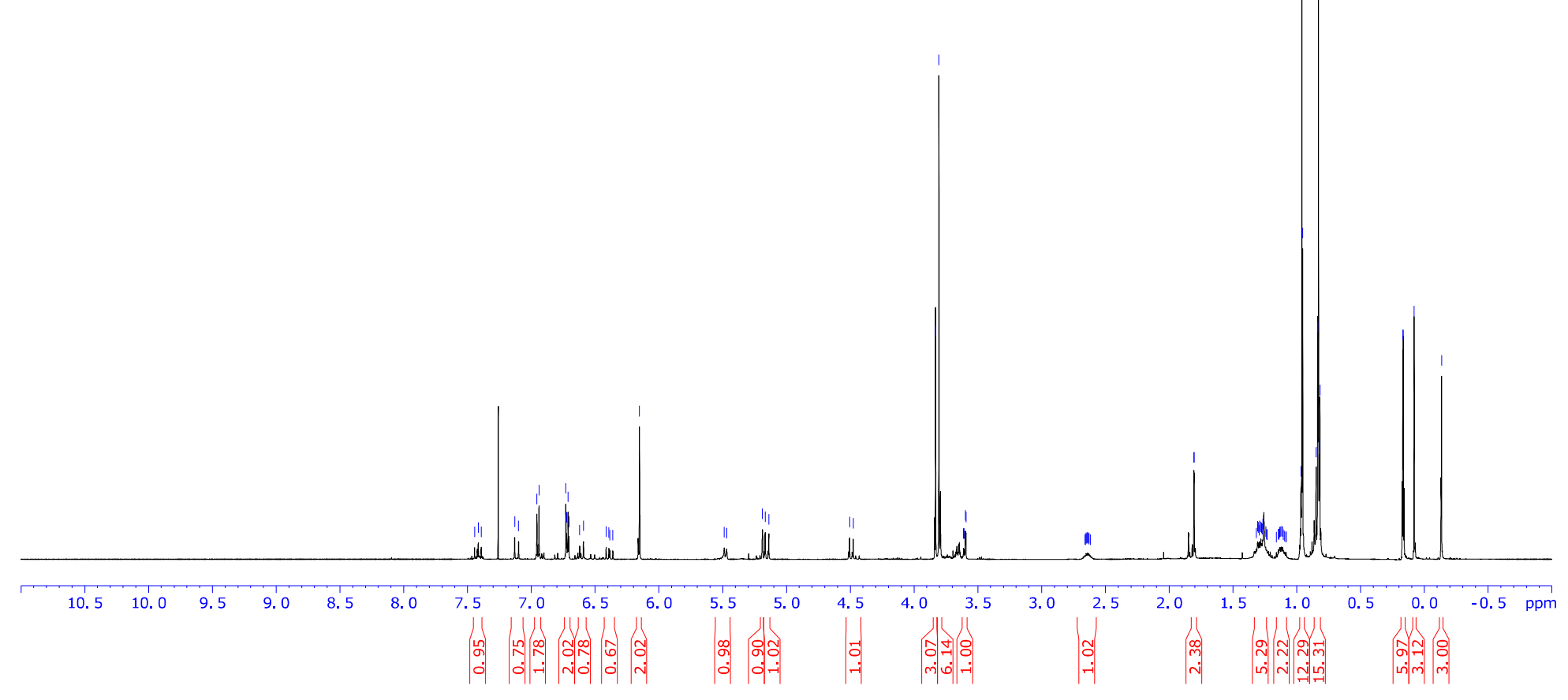


Christian Drescher and Reinhard Brückner; 3D-Structure Clarifying Total Synthesis of the (Polyenoyl)tetramic Acid Militarinon B. A Highly Acid-Labile N-Protecting Group for Amides

(5S)-5-\{(S)-[(tert-Butyldimethylsilyl)oxy] $\left\{1^{\prime \prime}-\left[(\right.\right.$ tert-butyldimethylsilyl)oxy)phenyl]methyl $\}-3-\left[\left(1^{\prime} Z, 2^{\prime} E, 4^{\prime} E, 6^{\prime} E, 8^{\prime} R, 10 ' R\right)-1^{\prime}-h y d r o x y-6 ', 8^{\prime}, 10 '-\right.$ trimethyldodeca-2',4',6'-trien-1'-ylidene]-1-(1"',3'",5'"'-trimethoxybenzyl)pyrrolidine-2,4-dione [Z-enol-(S,S,R,R)-27]

${ }^{13}$ C NMR (125.75 MHz, $\left.\mathrm{CDCl}_{3}\right)$ :

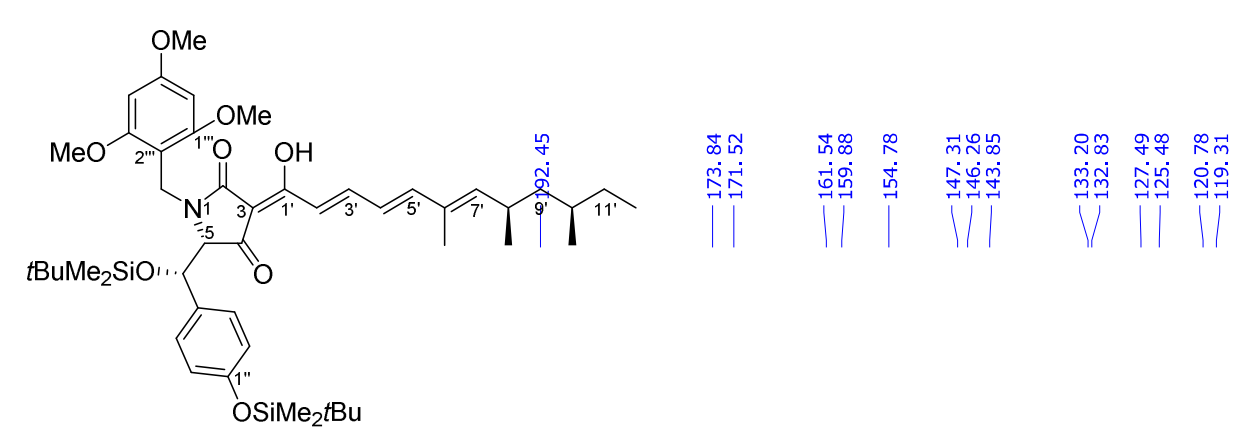

Z-enol-(S,S,R,R)-27

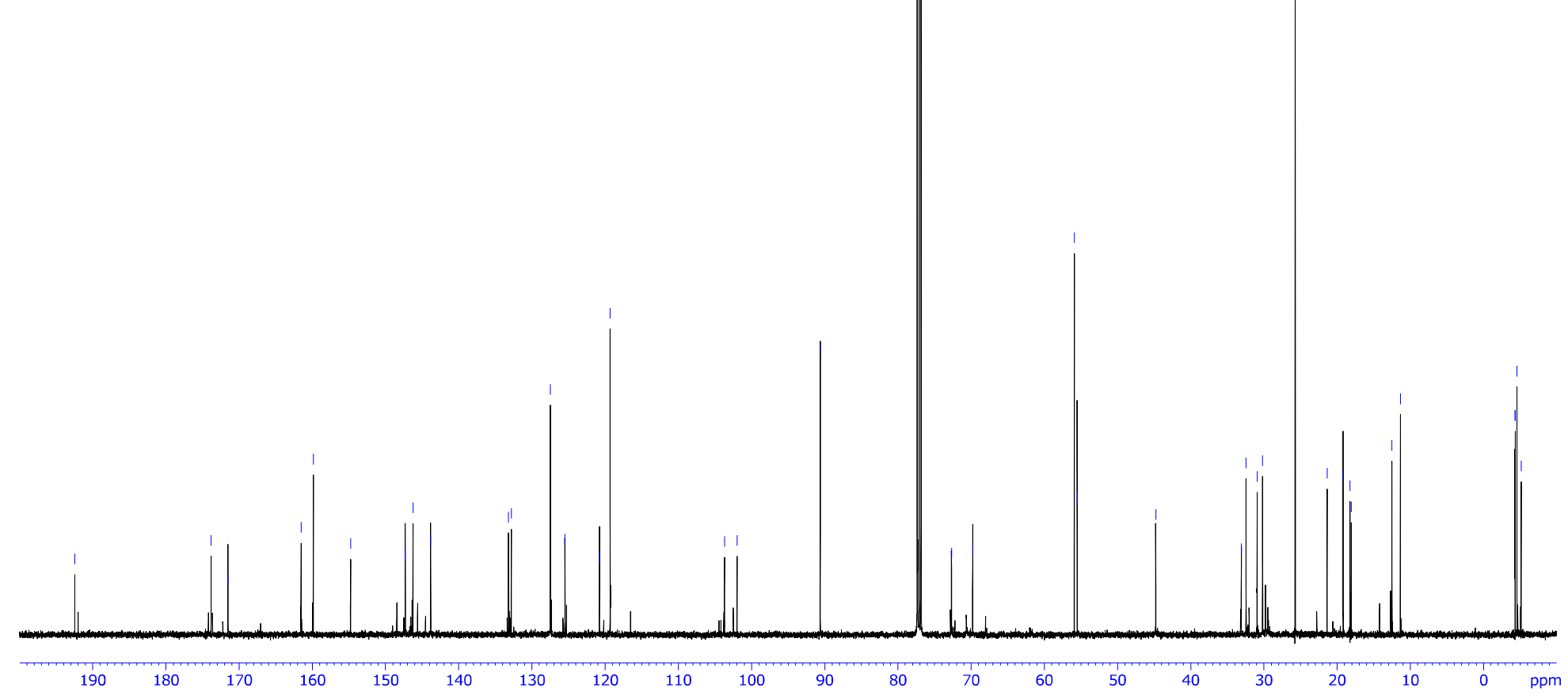


Christian Drescher and Reinhard Brückner; 3D-Structure Clarifying Total Synthesis of the (Polyenoyl)tetramic Acid Militarinon B. A Highly Acid-Labile N-Protecting Group for Amides

$(5 S)-5-\left\{(R)-\left[(\right.\right.$ tert-Butyldimethylsilyl)oxy $]\left\{1^{\prime \prime}-\left[(\right.\right.$ tert-butyldimethylsilyl)oxy)phenyl]methyl $\}-3-\left[\left(1^{\prime} Z, 2^{\prime} E, 4^{\prime} E, 6^{\prime} E, 8^{\prime} R, 10^{\prime} R\right)-1^{\prime}-h y d r o x y-6 ', 8^{\prime}, 10 '-\right.$ trimethyldodeca-2',4',6'-trien-1'-ylidene]-1-(1"',3'",5'"'-trimethoxybenzyl)pyrrolidine-2,4-dione [Z-enol-(R,S,R,R)-27]

${ }^{1} \mathbf{H}$ NMR $\left(500.06 \mathrm{MHz}, \mathrm{CDCl}_{3}\right)$ :

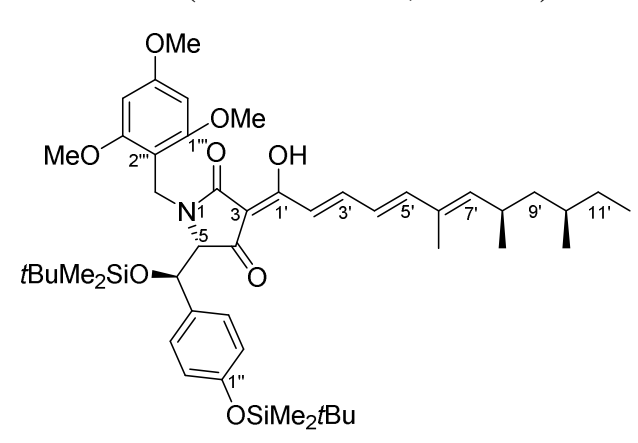

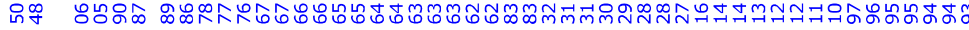

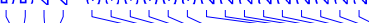

$Z$-enol-(R,S,R,R)-27 
Christian Drescher and Reinhard Brückner; 3D-Structure Clarifying Total Synthesis of the (Polyenoyl)tetramic Acid Militarinon B. A Highly Acid-Labile N-Protecting Group for Amides

(5S)-5-\{(R)-[(tert-Butyldimethylsilyl)oxy] $\left\{1^{\prime \prime}-\left[(\right.\right.$ tert-butyldimethylsilyl)oxy)phenyl]methyl $\}-3-\left[\left(1^{\prime} Z, 2^{\prime} E, 4^{\prime} E, 6^{\prime} E, 8^{\prime} R, 10^{\prime} R\right)-1^{\prime}-h y d r o x y-6 ', 8^{\prime}, 10^{\prime}-\right.$ trimethyldodeca-2',4',6'-trien-1'-ylidene]-1-(1'"',3'",5'"-trimethoxybenzyl)pyrrolidine-2,4-dione [Z-enol-(R,S,R,R)-27]

${ }^{13}$ C NMR (125.75 MHz, $\left.\mathrm{CDCl}_{3}\right)$ :

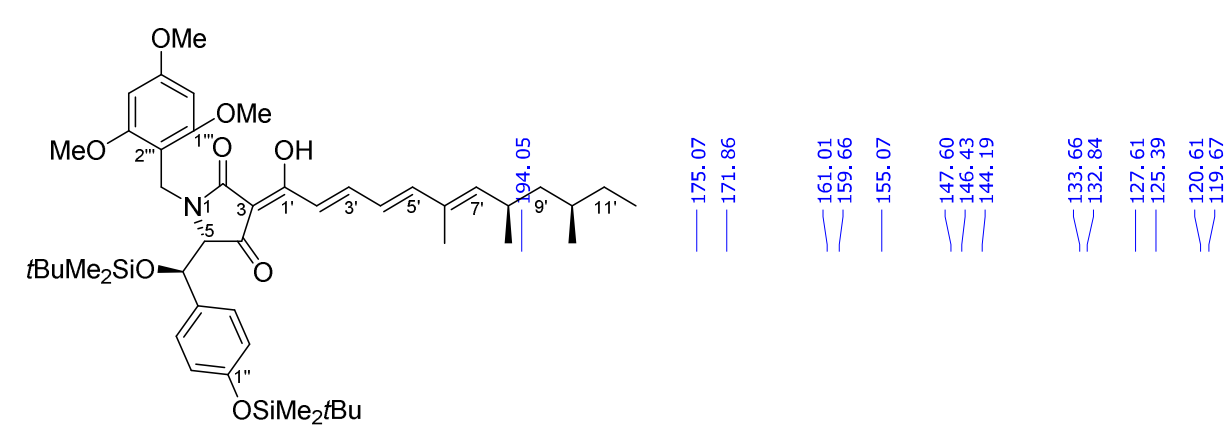

Z-enol- $(R, S, R, R)-27$

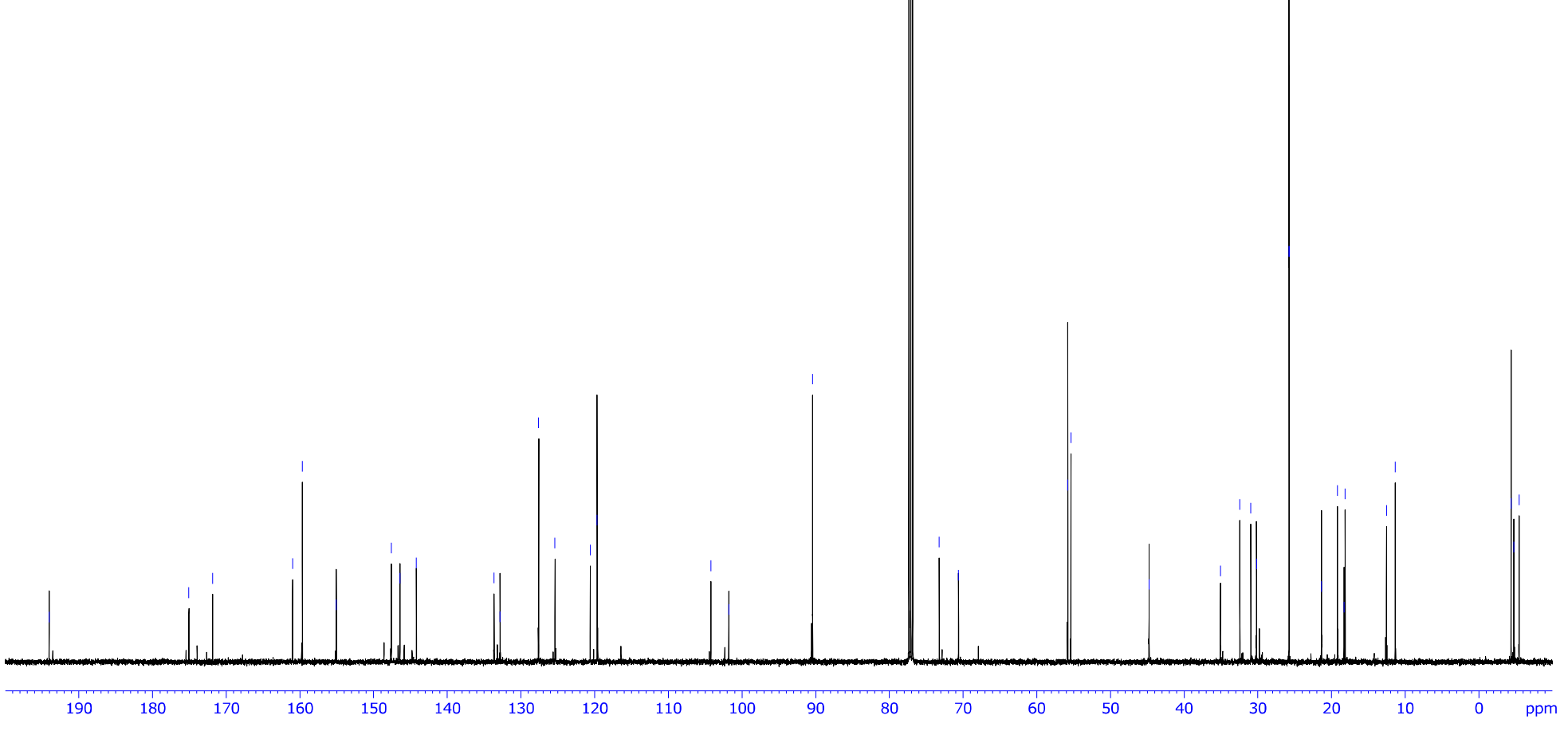


Christian Drescher and Reinhard Brückner; 3D-Structure Clarifying Total Synthesis of the (Polyenoyl)tetramic Acid Militarinon B. A Highly Acid-Labile N-Protecting Group for Amides

(5S)-5-\{(S)-[(tert-Butyldimethylsilyl)oxy] $\left\{1^{\prime \prime}-\left[(\right.\right.$ tert-butyldimethylsilyl)oxy)phenyl]methyl $\}-3-\left[\left(1^{\prime} Z, 2^{\prime} E, 4^{\prime} E, 6^{\prime} E, 8^{\prime} R, 10 ' R\right)-1^{\prime}-h y d r o x y-6 ', 8^{\prime}, 10^{\prime}-\right.$ trimethyldodeca-2',4',6'-trien-1'-ylidene]pyrrolidine-2,4-dione [Z-enol-(S,S,R,R)-28]

${ }^{1} \mathbf{H}$ NMR (500.06 MHz, MeOD):
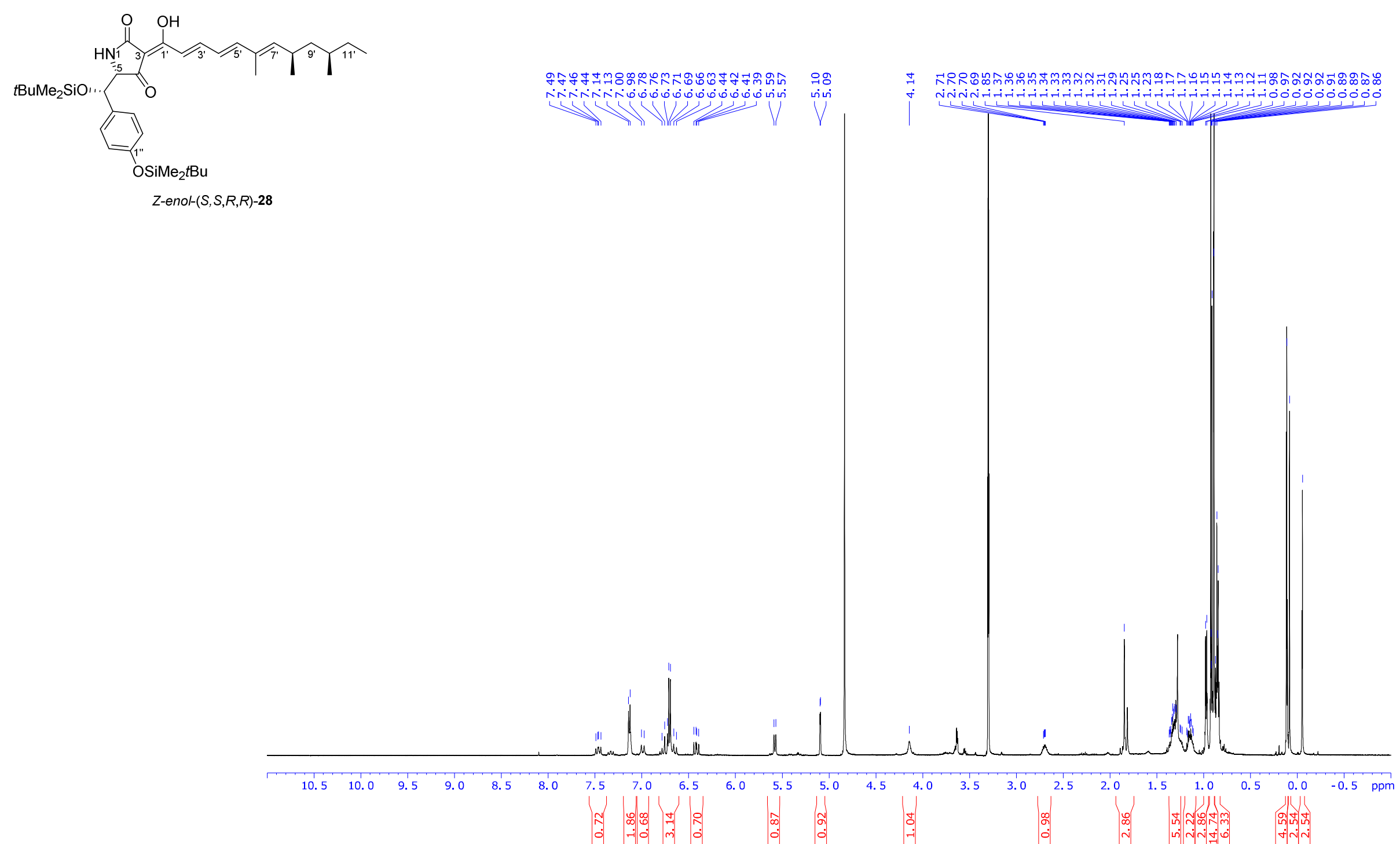
Christian Drescher and Reinhard Brückner; 3D-Structure Clarifying Total Synthesis of the (Polyenoyl)tetramic Acid Militarinon B. A Highly Acid-Labile N-Protecting Group for Amides

(5S)-5-\{(S)-[(tert-Butyldimethylsilyl)oxy] \{1'"-[(tert-butyldimethylsilyl)oxy)phenyl]methyl\}-3-[(1'Z,2' $\left.E, 4^{\prime} E, 6^{\prime} E, 8^{\prime} R, 10 ' R\right)-1^{\prime}-h y d r o x y-6 ', 8^{\prime}, 10 '-$ trimethyldodeca-2',4',6'-trien-1'-ylidene]pyrrolidine-2,4-dione [Z-enol-(S,S,R,R)-28]

${ }^{13}$ C NMR (125.75MHz, MeOD):

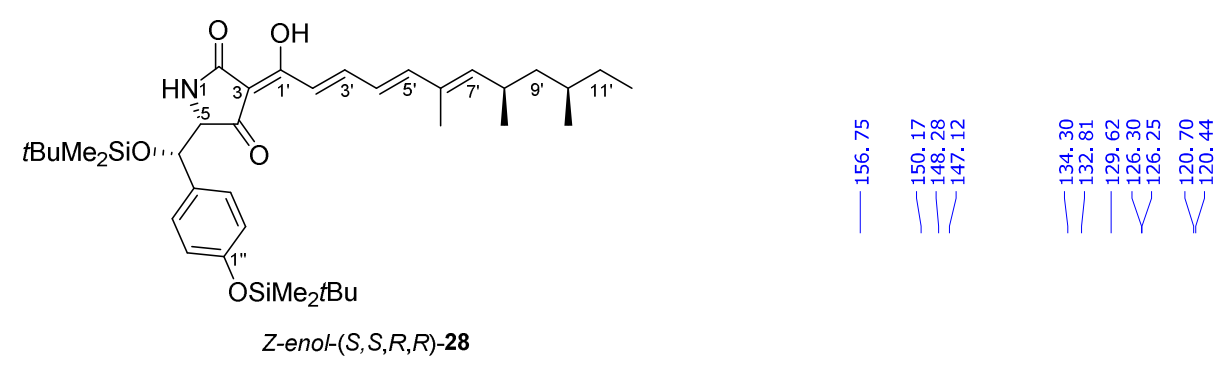

$Z$-enol-(S,S,R,R)-28 
Christian Drescher and Reinhard Brückner; 3D-Structure Clarifying Total Synthesis of the (Polyenoyl)tetramic Acid Militarinon B. A Highly Acid-Labile N-Protecting Group for Amides

$(5 S)-5-\left\{(R)-\left[(\right.\right.$ tert-Butyldimethylsilyl)oxy $]\left\{1^{\prime \prime}-\left[(\right.\right.$ tert-butyldimethylsilyl)oxy)phenyl]methyl $\}-3-\left[\left(1^{\prime} Z, 2^{\prime} E, 4^{\prime} E, 6^{\prime} E, 8^{\prime} R, 10^{\prime} R\right)-1^{\prime}-h y d r o x y-6 ', 8^{\prime}, 10 '-\right.$ trimethyldodeca-2',4',6'-trien-1'-ylidene]pyrrolidine-2,4-dione [Z-enol-(R,S,R,R)-28]

${ }^{1} \mathbf{H}$ NMR (500.06 MHz, MeOD):
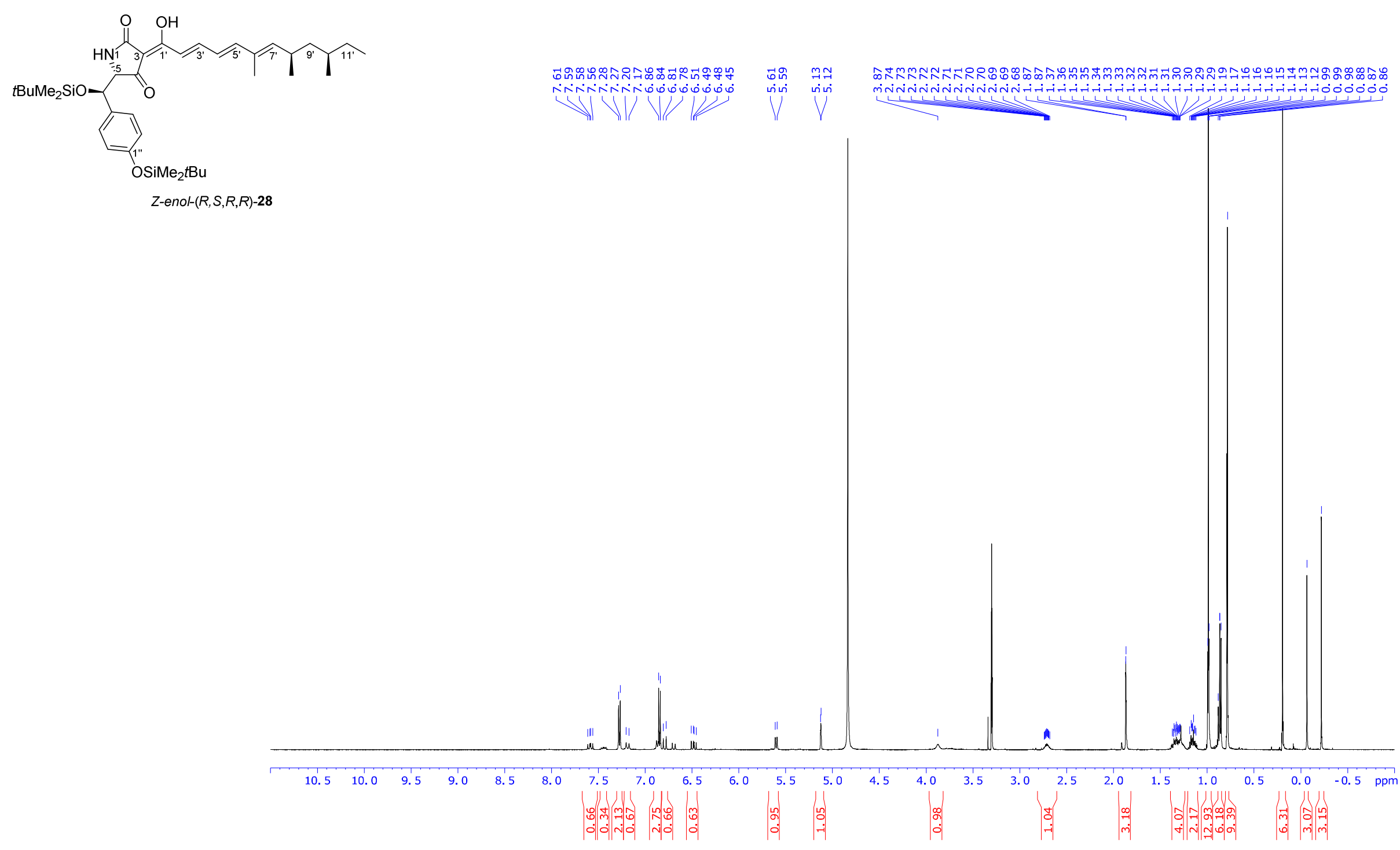
Christian Drescher and Reinhard Brückner; 3D-Structure Clarifying Total Synthesis of the (Polyenoyl)tetramic Acid Militarinon B. A Highly Acid-Labile N-Protecting Group for Amides

(5S)-5- $\{(R)$-[(tert-Butyldimethylsilyl)oxy $]\left\{1^{\prime \prime}-\left[(\right.\right.$ tert-butyldimethylsilyl)oxy)phenyl]methyl $\}-3-\left[\left(1^{\prime} Z, 2^{\prime} E, 4^{\prime} E, 6^{\prime} E, 8^{\prime} R, 10^{\prime} R\right)-1^{\prime}-h y d r o x y-6 ', 8^{\prime}, 10 '-\right.$ trimethyldodeca-2',4',6'-trien-1'-ylidene]pyrrolidine-2,4-dione [Z-enol-(R,S,R,R)-28]

${ }^{13}$ C NMR (125.75 MHz, MeOD):
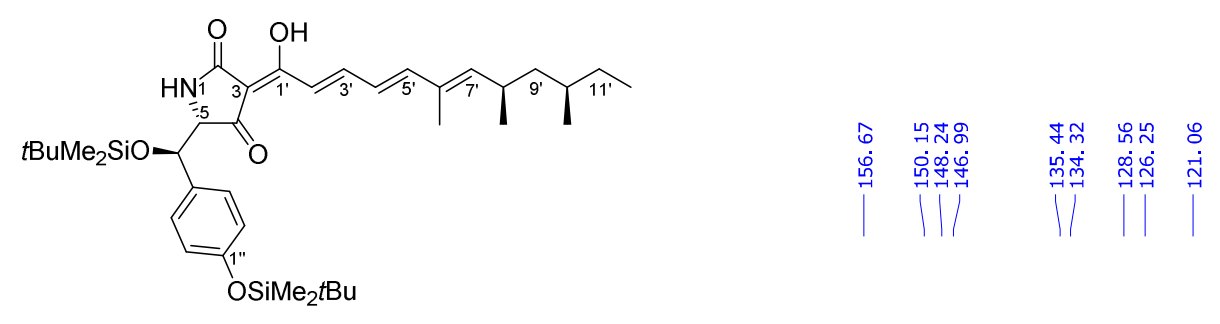

Z-enol-(R,S,R,R)-28

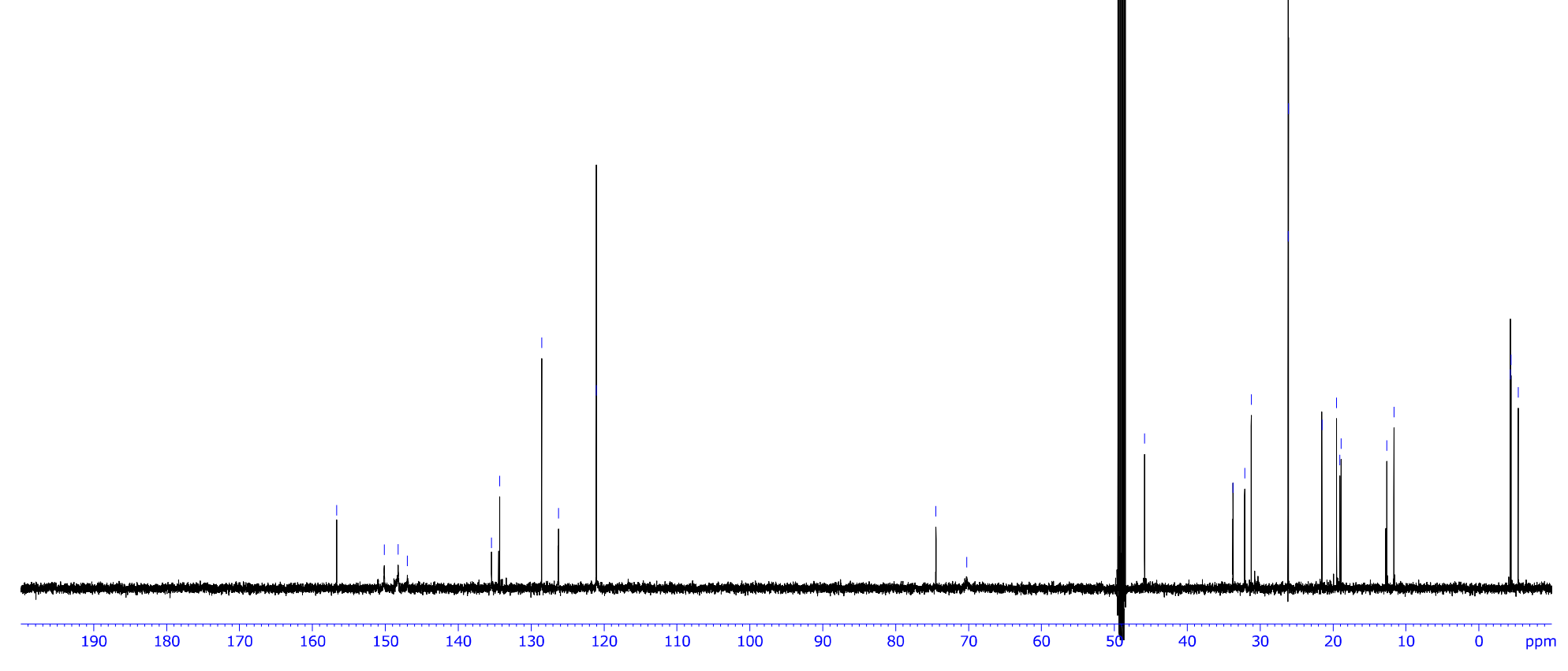


Christian Drescher and Reinhard Brückner; 3D-Structure Clarifying Total Synthesis of the (Polyenoyl)tetramic Acid Militarinon B. A Highly Acid-Labile N-Protecting Group for Amides

(5S)-5-[(S)-Hydroxy(1'-hydroxyphenyl)methyl]-3-[(1'Z,2' $\left.E, 4^{\prime} E, 6^{\prime} E, 8^{\prime} R, 10^{\prime} R\right)-1^{\prime}$-hydroxy-6', ' $^{\prime}, 10^{\prime}$-trimethyldodeca-2',4',6'-trien-1'ylidene]pyrrolidine-2,4-dione [Z-enol-(S,S,R,R)-3]

1 H NMR (500.06 MHz, MeOD):

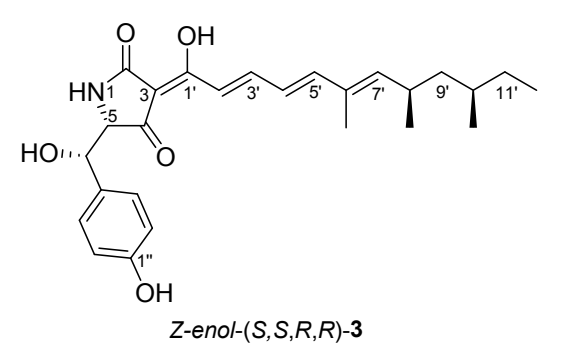

Z-enol-(S,S,R,R)-3 irivinivicionos

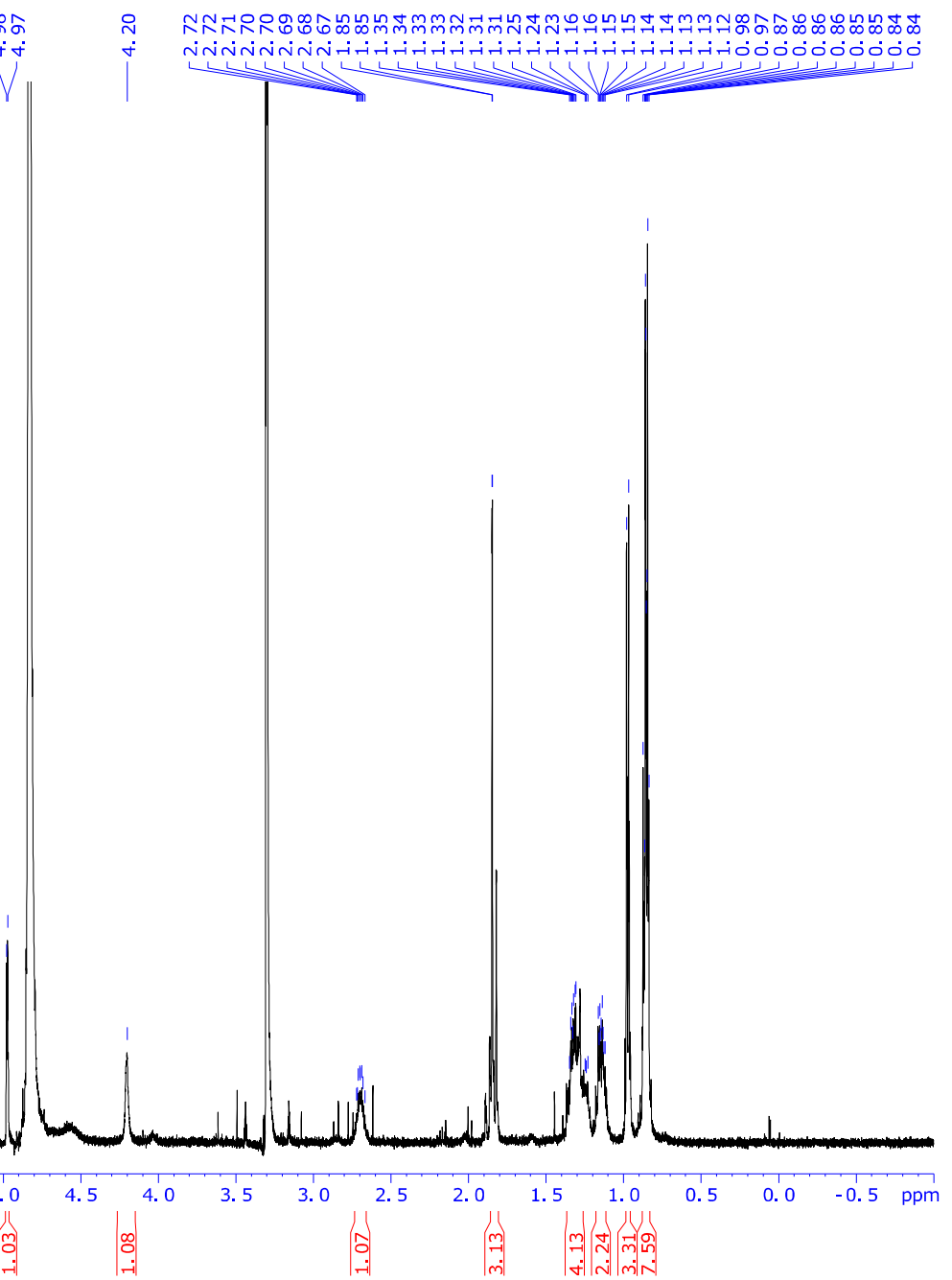


Christian Drescher and Reinhard Brückner; 3D-Structure Clarifying Total Synthesis of the (Polyenoyl)tetramic Acid Militarinon B. A Highly Acid-Labile N-Protecting Group for Amides

(5S)-5-[(S)-Hydroxy(1'-hydroxyphenyl)methyl]-3-[(1'Z,2' $\left.E, 4^{\prime} E, 6^{\prime} E, 8^{\prime} R, 10^{\prime} R\right)-1^{\prime}$-hydroxy-6', ' $^{\prime}, 10^{\prime}$-trimethyldodeca-2',4',6'-trien-1'ylidene]pyrrolidine-2,4-dione [Z-enol-(S,S,R,R)-2]

${ }^{13}$ C NMR (125.75 MHz, MeOD):
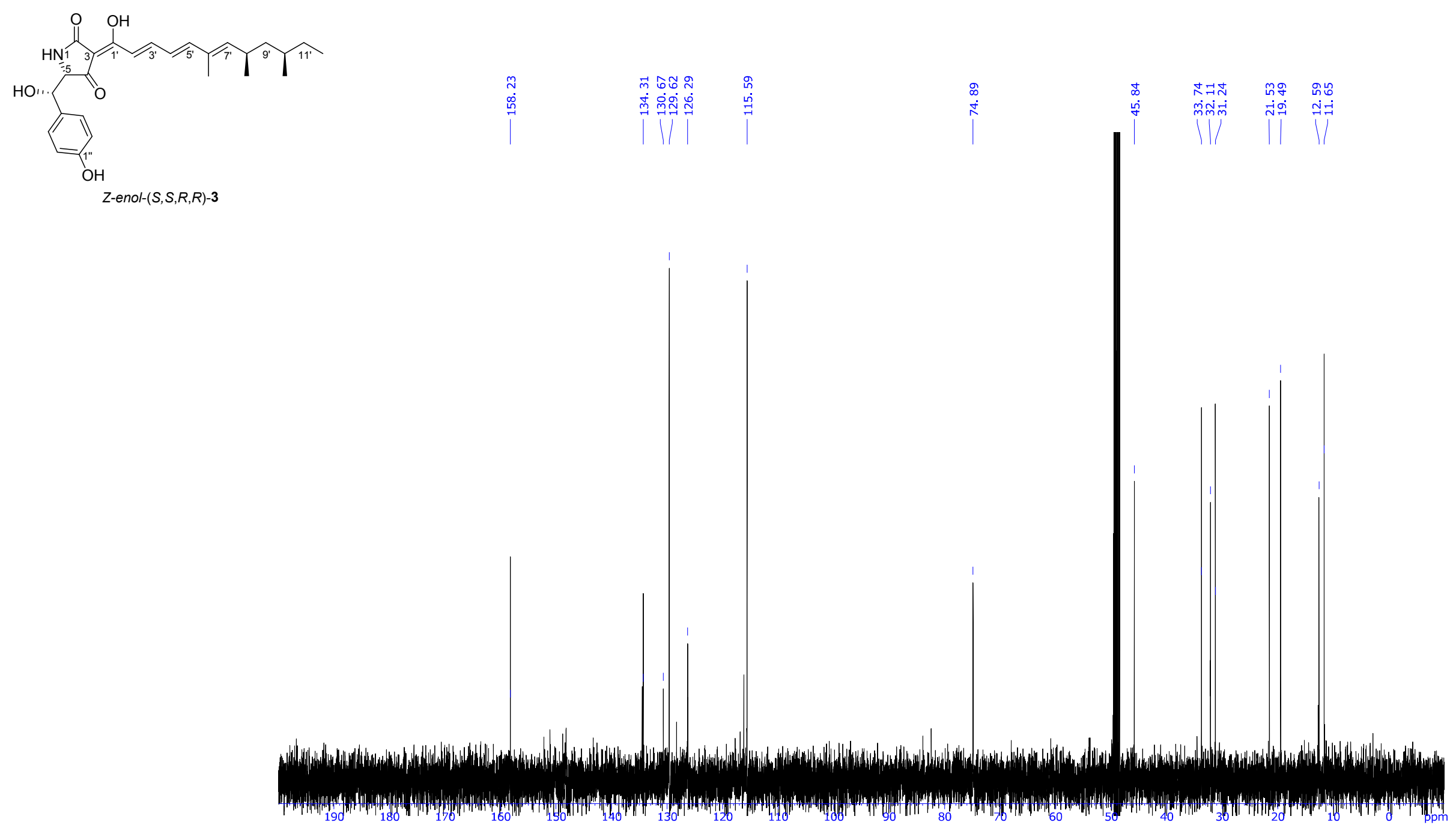
Christian Drescher and Reinhard Brückner; 3D-Structure Clarifying Total Synthesis of the (Polyenoyl)tetramic Acid Militarinon B. A Highly Acid-Labile N-Protecting Group for Amides

$(5 S)-5-\{(S)$-[(tert-Butyldimethylsilyl)oxy](1'-hydroxyphenyl)methyl $\}-3-\left[\left(1^{\prime} Z, 2^{\prime} E, 4^{\prime} E, 6^{\prime} E, 8^{\prime} R, 10 ' R\right)-1^{\prime}-h y d r o x y-6 ', 8^{\prime}, 10 '\right.$ 'trimethyldodeca2',4',6'-trien-1'-ylidene]pyrrolidine-2,4-dione [Z-enol-(S,S,R,R)-29]

${ }^{1} \mathbf{H}$ NMR (500.06 MHz, MeOD):

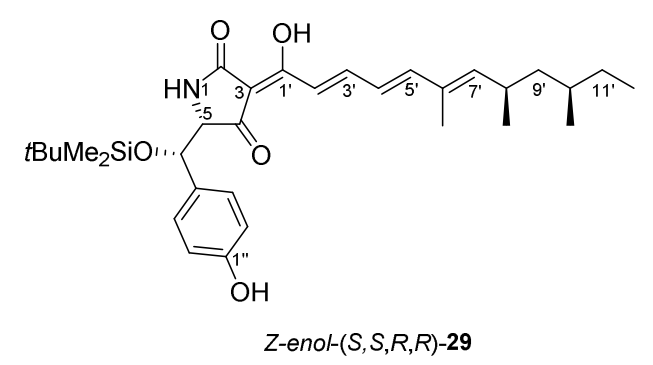

$Z$-enol-(S,S,R,R)-29
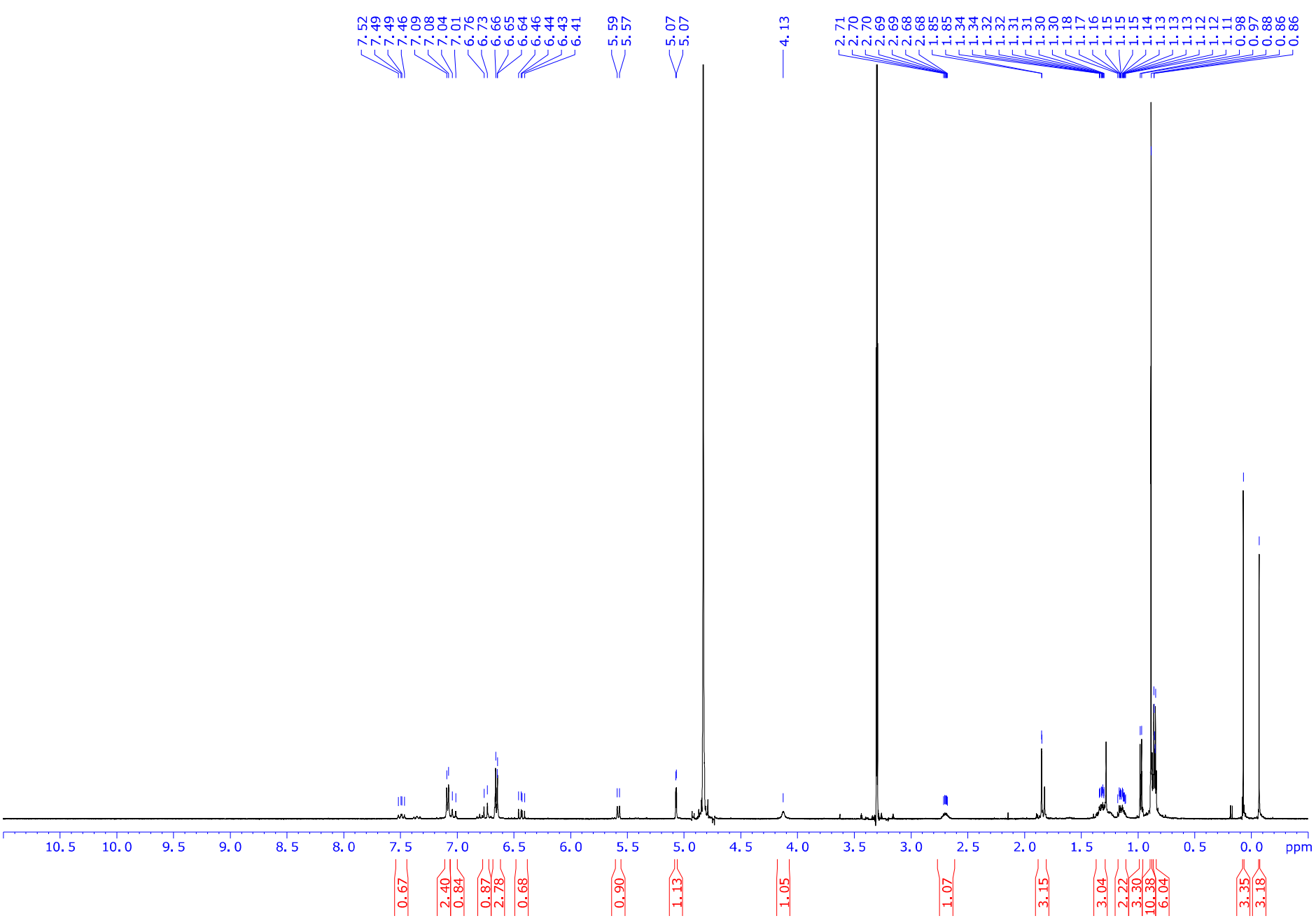
Christian Drescher and Reinhard Brückner; 3D-Structure Clarifying Total Synthesis of the (Polyenoyl)tetramic Acid Militarinon B. A Highly Acid-Labile N-Protecting Group for Amides

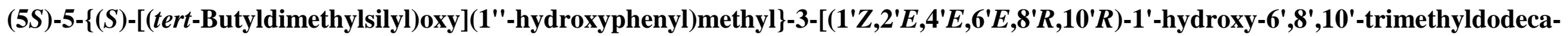
2',4',6'-trien-1'-ylidene]pyrrolidine-2,4-dione [Z-enol-(S,S,R,R)-29]

${ }^{13}$ C NMR (125.75 MHz, MeOD):
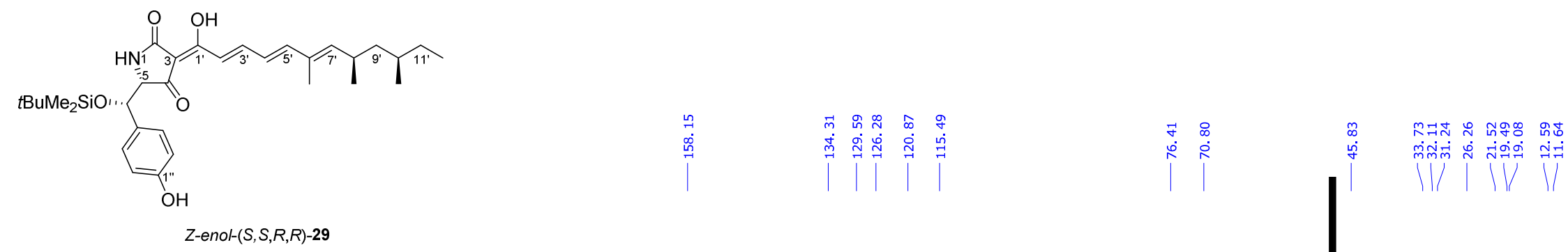

$Z$-enol-(S,S,R,R)-29

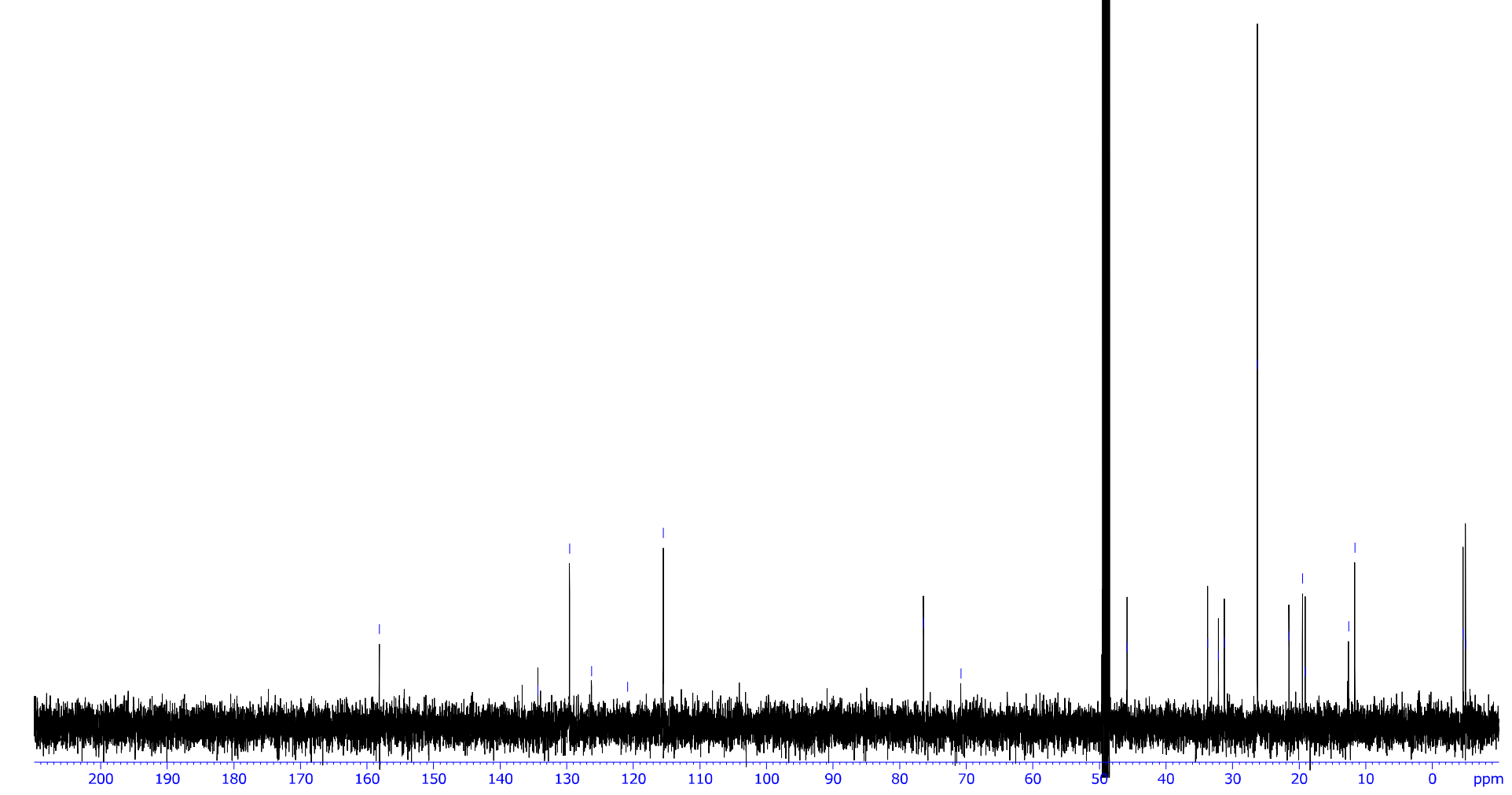


Christian Drescher and Reinhard Brückner; 3D-Structure Clarifying Total Synthesis of the (Polyenoyl)tetramic Acid Militarinon B. A Highly Acid-Labile N-Protecting Group for Amides

(5S)-5-\{(R)-[(tert-Butyldimethylsilyl)oxy](1'-hydroxyphenyl)methyl $\}-3-\left[\left(1^{\prime} Z, 2^{\prime} E, 4^{\prime} E, 6^{\prime} E, 8^{\prime} R, 10^{\prime} R\right)\right.$-1'-hydroxy-6',8',10'-trimethyldodeca2',4',6'-trien-1'-ylidene]pyrrolidine-2,4-dione [Z-enol-(R,S,R,R)-29]

${ }^{1} \mathbf{H}$ NMR (500.06 MHz, MeOD):
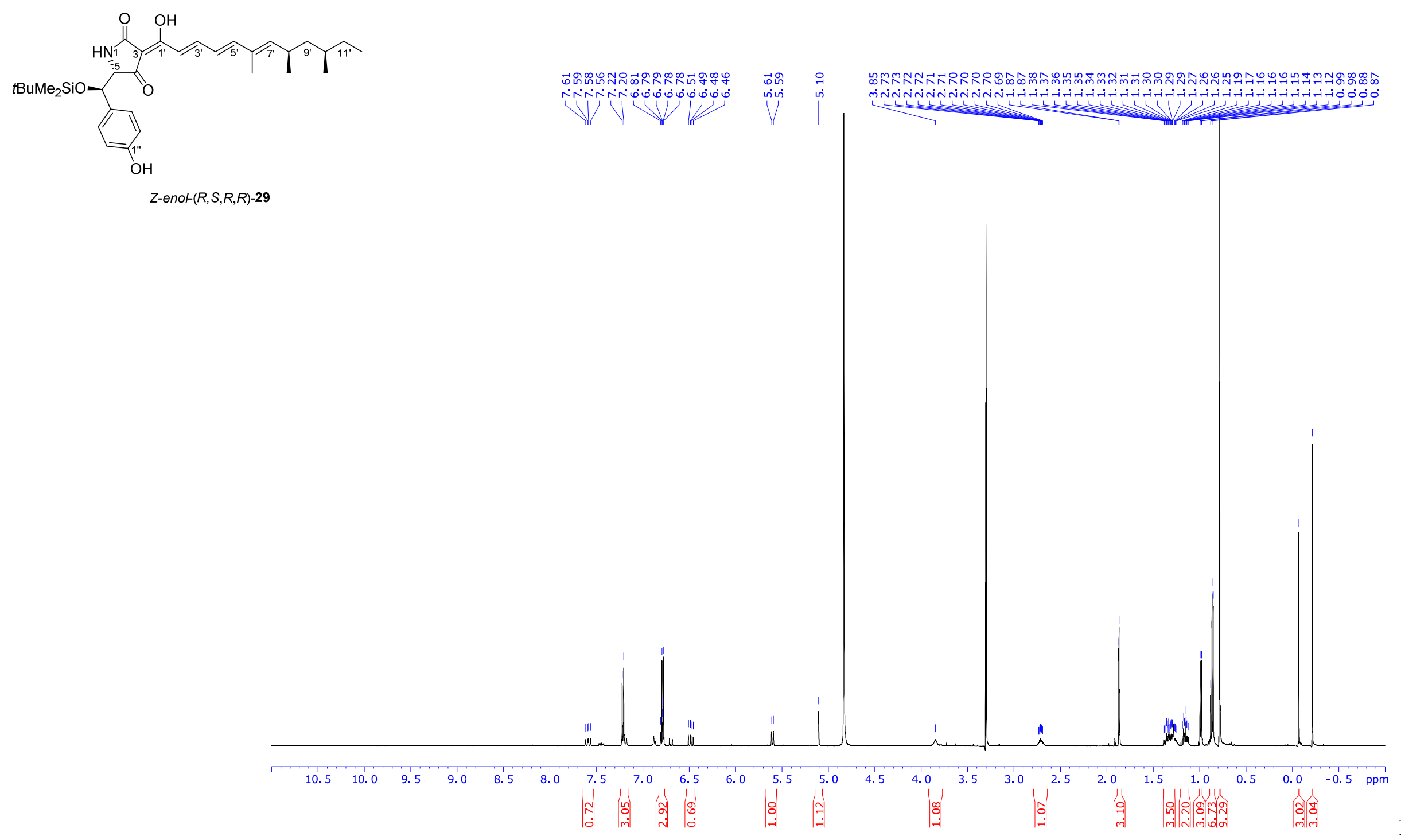
Christian Drescher and Reinhard Brückner; 3D-Structure Clarifying Total Synthesis of the (Polyenoyl)tetramic Acid Militarinon B. A Highly Acid-Labile N-Protecting Group for Amides

(5S)-5-\{(R)-[(tert-Butyldimethylsilyl)oxy](1'-hydroxyphenyl)methyl $\}-3-\left[\left(1^{\prime} Z, 2^{\prime} E, 4^{\prime} E, 6^{\prime} E, 8^{\prime} R, 10^{\prime} R\right)\right.$-1'-hydroxy-6',8',10'-trimethyldodeca2',4',6'-trien-1'-ylidene]pyrrolidine-2,4-dione [Z-enol-(R,S,R,R)-29]

${ }^{13}$ C NMR (125.75 MHz, MeOD):
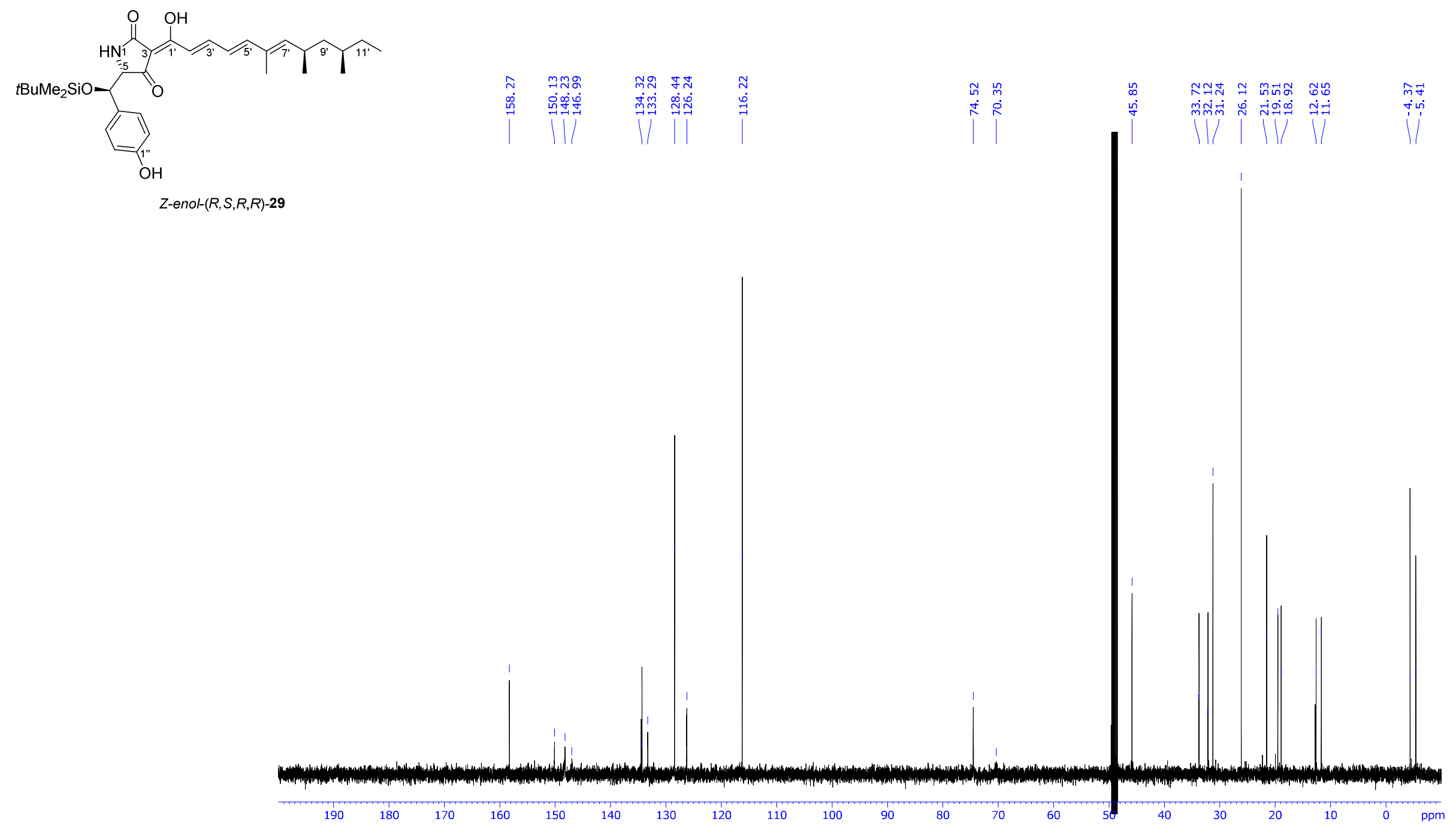


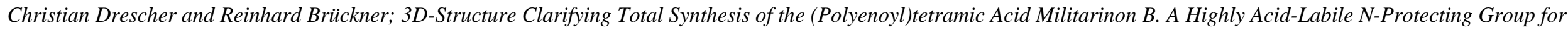
Amides

\section{$(4 R, 6 R, \mathrm{E})-2,4,6-$ Trimethyloct-2-en-1-ol $[(R, R)-8]$}

${ }^{\mathbf{1}} \mathbf{H}$ NMR (300.13 MHz, $\left.\mathrm{CDCl}_{3}\right)$ :

$\mathrm{OH}$

$\widehat{3}{ }^{5}{ }^{7}$

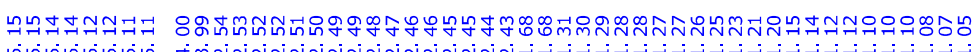

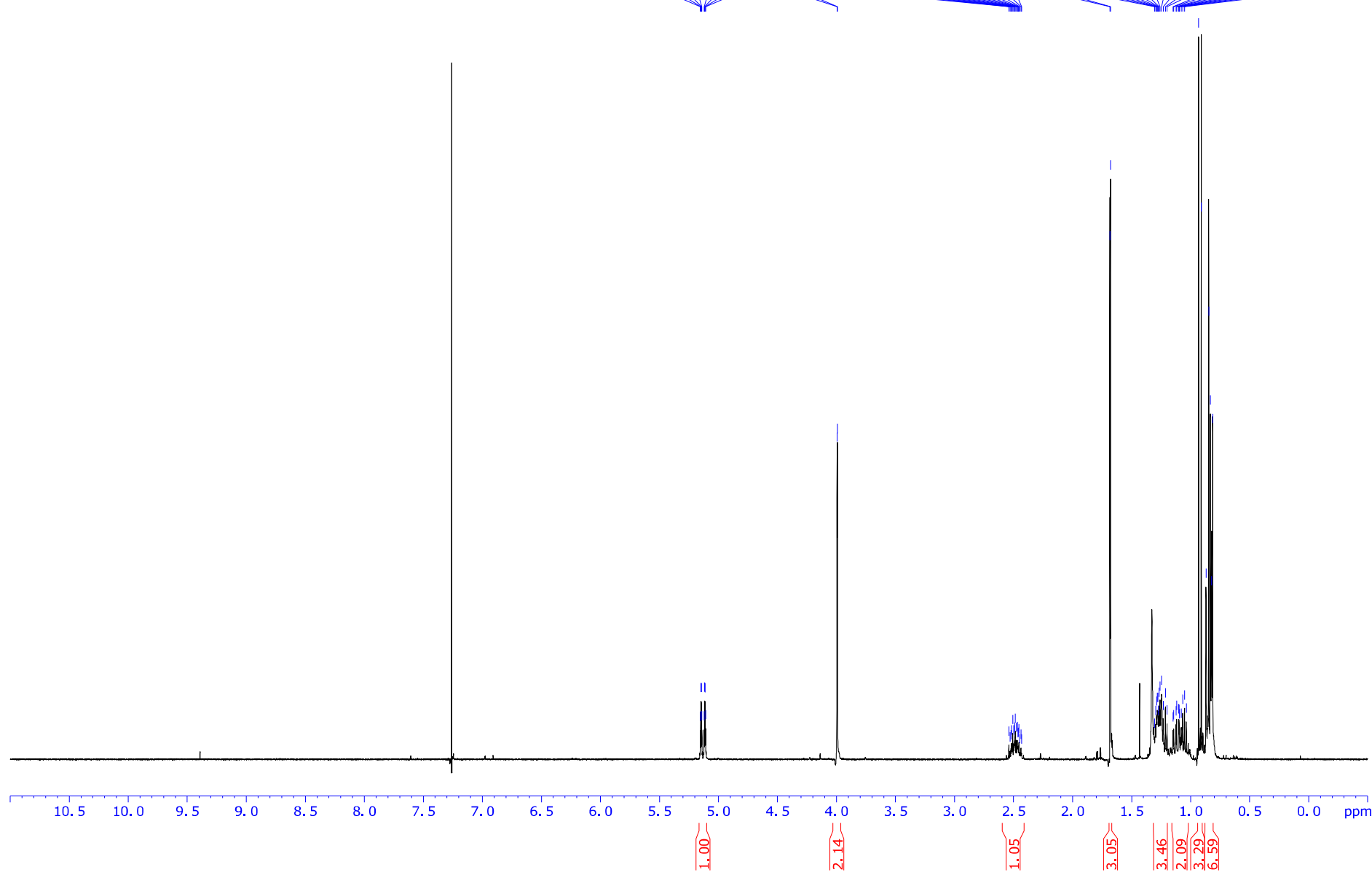

